$$
\text { - }
$$
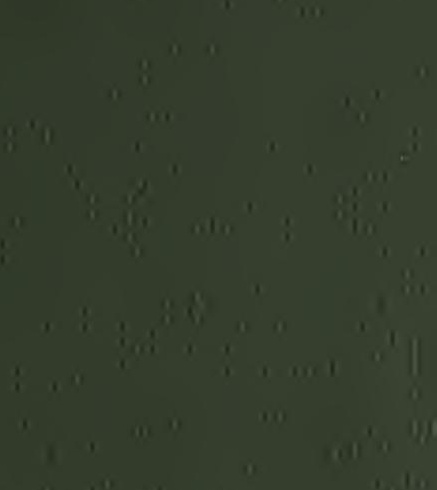

Digitized by the Internet Archive in 2007 with funding from Microsoft Corporation 



\title{
A TEXंT-BOOK
}

\author{
OF THE
}

\section{DISEASES OF THE SMALL DOMESTIC ANIMALS}

BY

\section{OSCAR VICTOR BRUMLEY, V.S.}

PROFESSOR OF VETERINARY SURGERY AND DIRECTOR OF CLINICS, COLLEGE OF VETERINARY MEDICINE, OHIO STATE UNIVERSITY, COLUMBUS, OHIO

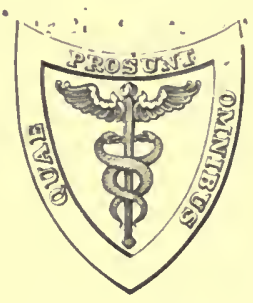

LEA \& FEBIGR

PHILADELPHIA A ND NEW YORK 
Copyright

LEA \& FEBIGER

1921

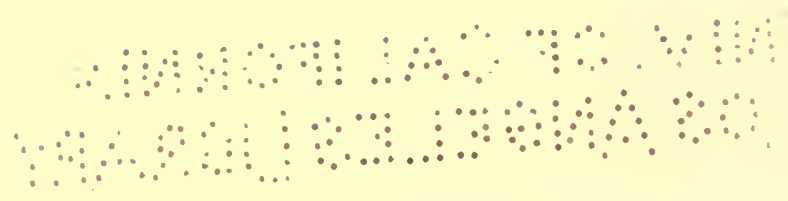

PIRTEDD IN U. S. A. 


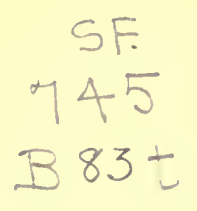

\section{PREFACE.}

THis book is intended to be a practical text on the Diseases of the Small Domestic Animals. The author feels that it is inadvisable to write an exhaustive treatise on this subject, as it is primarily intended to be a book for student use and for the busy practitioner. An attempt has been made to include most of the important diseases (medical and surgical) of small animals with which the veterinarian comes in contact. It is hoped that it will serve the purpose for which it is written.

The author does not claim originality for all the material in the book. 'The literature and standard books have been consulted freely.

For scientific reasons the metric system has been used throughout the text. A table of equivalents in weights and measures has been inserted so that the dosage can be readily determined in the other system, if desired. 'This was thought to be more desirable than including both under the treatment of each disease.

The author wishes to express his appreciation for the valued assistance rendered by his colleagues in the College of Veterinary Medicine, Ohio State University, in the preparation of the manuscript. He is especially indebted to Colonel David S. White, Dean, for his kindness in arranging and correcting the manuscript, and for his advice relative to the subject matter, and in many other ways contributing to the value of the book. Also to Dr. James Howard Snook 
for suggestions in the chapter on skin diseases and various other problems as they presented themselves. The author's thanks are extended to Dr. John Newton Shoemaker and others who have been liberal with their assistance during the preparation of the manuscript.

Columbus, Ohio, 1921.

O. V. B. 


\section{CONTENTS.}

\section{PART I. \\ DISEASES OF THE RESPIRATORY SYSTEM.}

\section{CHAPTER I.}

Examination

Diseases of the Nasal Passages.

Acute Nasal Catarrh (Coryza. Rhinitis) . . . . . . . 19

Empyema of the Infraorbital Fossa of Birds . : . . . . 20

Infectious Nasal Catarrh of Rabbits (Rabbit Plague. Rabbit Influenza. Rabbit Distemper) . . . . . . . . 20

Parasitic Nasal Catarrh of Rabbits (Rhinitis Coccidiosa). . . 22

Chronic Nasal Catarrh (Chronic Coryza. Rhinitis) . . . . 23

Epistaxis . . . . . . . . . . . 24

Parasites of the Nasal Passages . . . . . . . . . . 25

Neoplasms of the Nasal Passages . . . . . . . . . 28

Papillomata . . . . . . . . . . . . . . . 28

Polypoid Fibromata

Malignant Tumors . . . . . . . . . . . . . . . 29

Acute Laryngitis . . . . . . . . . . . . . . 30

Chronic Laryngitis . . . . . . . . . . . . . . . 31

CHAP'TER III.

Diseases of the Trachea and Bronchial, Tubes.

Examination . . . . . . . . . . . . . . . 34

Acute Tracheit is and Bronchitis . . . . . . . . . . 34

Bronchial Catarrh of Birds (Bronchitis) . . . . . . . . 38

Mycotic Pneumonia (Aspergillosis) . . . . . . . . . . 40

Animal Parasites in the Trachea and Bronchial Tubes (Syngamosis.

Gapes in Chickens) . . . . . . . . . . . . . 42

Chronic Tracheitis and Bronchitis . . . . . . . . . 44 


\section{CHAPTER IV.}

\section{Diseases of the Lungs.}

Examination . . . . . . . . . . . . 47

Congestion of the Lungs (Hyperemia of the Lungs) . . . . 49

Active Congestion . . . . . . . . . . . . . . . . . 49

Passive Congestion . . . . . . . . . . . . 50

Pulmonary Edema (Edema of the Lungs) . . . . . . . 52

Bronchopneumonia (Catarrhal Pncumonia) . . . . . . 53

Cirrhosis of the Lungs (Chronic Interstitial Pncumonia) . . . 57

Foreign Body Pneumonia (Cangrenc of the Lungs) . . . . 58

CHAP'TER V.

Diseases of the Pleura.

Pleurit is (Pleurisy) : Hydrothorax . . . . . . . . . . . . . . . 65

Pncumothorax . . . . . . . . . . . . . . . 66

Hemothorax . . . . . . . . . . . . . . 67

\section{PAR'T II.}

\section{DISEASES OF THE CIRCULATORY SYSTEM.}

\section{CHAP'TER I.}

Diseases of the Pericardium.

Examination

Jericarditis

Iydropericardium (Dropsy of the Pcricardium) . . . . . 76

Hemopericardium . . . . 77

CHAP'TER 11.

Diseases of the Mieart.

Valvular Insufficiency and Stcnosis . . . . . . . . . 78

Myocarditis . . . . . . . . . . . . 82

Acute Myocarditis . . . . . . . . . . . . 82

Chronic Myocarditis . . . . . . . . . . . . 84

Acute Fndocarditis . . . . . . . . . . . . . 85

Hypertrophy and Dilatation of the Heart . . . . . . . . . $\quad 89$

Rupture of the Heart . . . . . . . . . . . . . 92 


\title{
PAR'T III.
}

\section{DISEASES OF THE DIGESTIVE TRACT.}

\author{
CHAPTER I. \\ Diseases of tile Mouth.
}

Examination . . . . . . . . . . . 93

Stomatitis . . . . . . . . . . . . . 94

Catarrhal Stomatitis . . . . . . . . . . . 94

Uleerative Stomatitis . . . . . . . . . . . . 96

Gangrenous Stomatitis (Canker of the Mouth) . . . . 97

Phlegmonous Stomatitis . . . . . . . . . . . . . . 99

Parasitic Sto natitis (Thrush. Soor. Aphtha) . . . . 100

Benign Neoplasins of the Mouth . . . . . . . . . . 102

Papillonata . . . . . . . . . . . . 102

Fibronata . . . . . . . . . . . . . 102

Osteonla. . . . . . . . . . . . . 103

Retention Cysts (Ranula) . . . . . . . . . 103

Malignant Neoplasms of the Mouth . . . . . . . . . 104

Epithelionata . . . . . . . . . . . 104

Sarcomata . . . . . . . . . . 105

Foreign Boclies in the Mouth . . . . . . . . . . 106

CHAPTER II.

Diseases of the Teeth.

Examination . . . . . . . . . . . 107

Malforinations of the Teeth . . . . . . . . . . 107

Fractures of the Teeth . . . . . . . . . . 108

Incrustations of Tartar . . . . . . . . . . . . 108

Alveolar I'eriostitis (Pericementitis. Periodontritis) . . . . 108

Caries of the Teeth . . . . . . . . . . . 109

\section{CIIAPTER III.}

Diseases of the Tongue.

Examination

Glossitis . . . . . . . . . . . . 111

Gangrene of the Tongue (Gangrenous Glossitis) . . . . . 112 


\section{CHAPTER IV.}

Diseases of the Salivary Glands.

Examination

Parotitis (Mumps) . . . . . . . . . . . . . . 115

Submaxillary and Sublingual Glands . . . . . . . . 118

Salivary Fistula . . . . . . . . . . . . 118

\section{CHAPTER V. \\ Diseases of thile Tonsils.}

Examination

Tonsillitis and Lymphadenitis

CHAPTER VI.

Diseases of the Pharyxx.

Examination

Pharyngitis

Acute Pharyngitis .

Chronic Pharyngitis

Foreign Bodies in the Pharynx : . . . . . . . . . 126

Paralysis of the Pharynx . . . . . . . . . . . 127

Croupous Pharyngitis of Birds . . . . . . . . . . 127

Neoplasms of the Pharynx . . . . . . . . . . . 128

Polypoid Growths . . . . . . . . . . . . . . . 128

Epithelioma . . . . . . . . . . . . 129

CHAPIER VII.

Diseases of the Esophagus.

Examination .

Esophagitis

Foreign Bodies (Obstruction in Esophagus) . . . . . . . 131

Esophagismus

Stricture of the Esophagus. . . . . : . . . . . . 135

Dilatations and Diverticula of the Esophagus . . . . . . 136

Catarrh of the Crop in Birds (Soft Crop) . . . . . . . 137

Obstruction of the Crop in Birds (Hard Crop) . . . . . . 139

Neoplasms of the Esophagus . . . . . . . . . . . 141 


\section{CHAP'TER VIII.}

Diseases of the Stomach.

Examination

Gastritis

Aeute Gastritis (Simple Catarrh of the Stomach. Aeute

Dyspepsia)

Chronic Gastritis (Chronie Catarrh of the Stomaeh. Chronie

Dyspepsia)

Foreign Bodies in the Stomach . . . . . . . . . 148

Acute Dilatation of the Stomaeh . . . . . . . . . 152

Chronie Dilatation of the Stomach . . . . . . . . . 154

Ulceration of the Stomaeh (Uleus Ventrieuli) . . . . . . 157

Hematemesis . . . . . . . . . . . . . . 160

Parasites in the Stomaeh . . . . . . . . . . . . 163

Neoplasms in the Stomach. . . . . . . . . . . . 165

\section{CHAPTER IX.}

Diseases of the Intestines.

Examination

Enteritis . . . . . . . . . . . . 167

Acute Enteritis. . . . . . . . . . . . . 167

Chronie Enteritis . . . . . . . . . . . . . 173

Intestinal Hemorrhage (Enterorrhagia) . . . . . . . . 175

Diarrhea . . . . . . . . . . . . . 178

Constipation (Obstipation. Intestinal Obstruction) . . . . 182

Volvulus . . . . . . . . . . . . . . . 185

Intussusception . . . . . . . . . . . . . . . 186

Wounds of the Intestines . . . . . . . . . . . . 188

Croupous Fnteritis of Cats (Membranous Enteritis) . . . . 190

Infeetious Asthenia of Birds . . . . . . . . . . . 191

\section{CHAPTER $X$.}

\section{Parasites in the Intestines.}

Helminthiasis

Tæniæ in Dogs . . . . . . . . . . . . 195

Tæniæ in the Intestine of the Cat . . . . . . . . 197

Tæniæ in the Intestine of Rabbits . . . . . . . . . . . . 197

T'ania in the Intestines of Birds . . . . . . . . . 198 
Nematoda . . . . . . . . . . . . 201

Round worms (Ascaridse) . . . . . . . . . . 201

Rourd Worms in the Intestine of the Dog . . . . . 202

Round Worms in the Intestine of the Cat . . . . 202

Round Worms in the Intestine of the Rabbit . . . 202

Round Worns in the Intestine of Birds . . . . . 202

Strongylidax (Hookworm) . . . . . . . . . . . 206

Strongylide in the Intestine of the Dog . . . . . 207

Strongylidis in the Intestine of the Cat . . . . . 207

Strongylidis in the Intestine of the Rabbit . . . . 207

Trichinellidx (Whipworm) . . . . . . . . . . 209

Trichinellidse in the Intestine of the Dog . . . . . 210

Trichinelliclae in the Intestine of the Rabbit . . . . 210

Trichinellide in the Intestine of Birds . . . . . 210

\section{('HAP'TLR NI.}

Distases of THE: RECTUM AND ANus.

Examination

Oeelusion of the Rectun and Anus . . . . . . . . . . 212

Proctitis

Hemorrhoids (Piles) . . . . . . . . . . . . . 216

Prolapse of the Rectum . . . . . . . . . . . . . . 217

Parasites in the Reetum . . . . . . . . . . . . 221

Neoplasms in the Rectum . . . . . . . . . . . . 222

Suppuration of the Anal Glands . . . . . . . . . . 223

\section{CHAP'TER XII.}

\section{Diseases of the Liver.}

Examination

Ieterus (Jaundice)

Congestion of the Liver

Aetive Congestion

Passive Congestion

Hepatitis

Suppurative Hepatitis (Alseess of the Liver) . . . . . . 236

Atrophy of the Liver . . . . . . . . . . . . . 240

Fatty liver . . . . . . . . . . . . . 241

Amyloid Liver . . . . . . . . . . . . . . 242

Cirrhosis of the Liver (Chronic Interstitial Hepatitis) . . . . 243

Neoplasms of the Liver . . . . . . . . . . . . 244

Cholelithiasis. Gall-stones . . . . . . 245

Rupture of the Liver (Ruptura Ilepatis. Apoplexia Hepatis) . 246 


\section{CHAPTER XIII.}

\section{Diseases of the Peritoneum}

General Remarks

Peritonitis

Acute Peritonitis . . . . . . . . . . . . . 250

Chronic Peritonitis . . . . . . . . . . . . 255

Ascites (Hydrops Abdominis. Hydrops Ascites. Hydrops Peritonei).

\section{PART IV.}

\section{DISEASES OF THE REPRODUCTIVE ORGANS.}

\section{CHAPTER I.}

Disfases of the Penis and Prepuce.

Examination 263

Wounds of the P'enis and P'repuce . . . . . . . . . 263

Congenital Malformations . . . . . . . . . . . . . . 265

Preputial Catarrh (Balanitis) . . . . . . . . . . 265

Phimosis . . . . . . . . . . . . . . 266

Paraphimosis . . . . . . . . . . . . . . 267

Tumors of the P'enis and Prepuce. . . . . . . . . . . 268

Papillomata . . . . . . . . . . . . . . 269

Sarcomata, Epitheliomata and Carcinomata . . . . . 269

Venereal Granulomata . . . . . . . . . . 269

\section{CHAPTER'II.}

\section{Diseases of the Testes and Scrotum.}

Wounds and Injuries of the Testes and Scrotum . . . . . 271

Orchitis . . . . . . . . . . . . . 271

Tumors of the Scrotum and Testes . . . . . . . . 272

Parasites in the Scrotum and 'T'estes (Cuterebra Enmasculator) 273

Castration (Orchectomy) . . . . . . . . . . . . 273

Castration of the Dog . . . . . . . . . . . . . 274

Castration of the Monorchid and Cryptorchid Dog . . 275

Castration of the Cat . . . . . . . . . . . . 275

Castration of Birds (Caponizing) . . . . . . . . 276 


\section{CHAP'TER III.}

Diseases of the Prostate Gland.

Examination

Prostatitis

Tumors of the Prostate Gland (Hypertrophy of the Prostate Gland)

\section{CHAPTER IV.}

Diseases of the Ovaries.

Examination

Inflammation of the Ovaries (Oöphoritis)

Tumors of the Ovaries

Cysts

Oöphorectomy (Ovariectomy)

Oöphorectomy in the Dog

Oopphorectomy in the Cat . 286

\section{Diseases of the Uterine Tubes.}

Examination

Salpingitis

Pyosalpinx

Tumors (Cysts)

\section{CHAPTER VI.}

\section{Diseases of the Uterus.}

Examination

Metritis

Acute Metritis .

Chronic Metritis (Pyometra).

Eversion of the Uterus (Prolapse. Inversion of the Uterus).

Fibromata

Iiydrometra 


\section{CHAPTER VII.}

Diseases of the Vagina and Vulva.

Examination

Congenital Malformations . . . . . . . . . . . . . 309

Vaginitis and Vulvitis . . . . . . . . . . . . . 309

Prolapse of the Vagina . . . . . . . . . . . , 311

Rupture of the Vagina . . . . . . . . . . . . . 313

Tumors of the Vulva and Vagina . . . . . . . . . . . 313

Fibromata . . . . . . . . . . . . . . . 313

Papillomata. . . . . . . . . . . . . . . 314

Sareornata . . . . . . . . . . . . . . 314

Venereal Granulomata . . . . . . . . . . . 314

CHAPTER VIII.

Diseases of the Mammary Glands.

Examination . . . . . . . . . . . . . . . 315

Wounds and Injuries of the Mammary Glands . . . . . . 315

Congestion of the Mammary Glands . . . . . . . . . . 316

Mammitis (Mastitis) . . . . . . . . . . . . . . . 316

Tumors of the Mammary Glands . . . . . . . . . 317

Benign Tumors . . . . . . . . . . . . . 317

Fibromata . . . . . . . . . . . 317

Lipoma . . . . . . . . . . . . 318

Malignant Tumors . . . . . . . . . . . . 318

Careinomata . . . . . . . . . . . . 318

Sareomata . . . . . . . . . . . . . 318

PART V.

\section{DISEASFS OF 'THE BLOOD AND BLOOD PRODUCING ORGANS.}

CHAPTER I.

Anemia.

Leukemia . . . . . . . . . . . . . 322

Infeetious Leukemia of Chickens . . . . . . . . . . 325

Pseudoleukemia (Hodgkin's Disease) . . . . . . . . . 327

Hemophilia . . . . . . . . . . . . . . . 328

Scurvy (Seorlutus) . . . . . . . . . . . . . . . . 328 
Animal Parasites in the 1Blood. (Filarias. Metastrongulinæ) . 330

Dirofilaria Immitis (Filaria Immitis) . . . . . . . 330

Hematozoön Lewisi . . . . . . . . . . . . 332

Hrmostrongylus Vasorum . . . . . . . . . . 332

Spiroptera Sanguinolenta . . . . . . . . . . 333

\section{CHAPTER II.}

\section{Diseases of the Turroid Glands.}

Examination and General Consideration. . . . . . . . 334

Congestion of the Thyroid Glands . . . . . . . . . 334

Acute Thyroiditis . . . . . . . . . . . . . . 335

Goiter (Struma. Bronchocele) . . . . . . . . . . 336

Parenchymatous Goiter . . . . . . . . . . 336

Cystic Goiter . . . . . . . . . . . . . . 339

Fibrous Goiter . . . . . . . . . . . . . 340

Vascular Goiter . . . . . . . . . . . . 341

Malignant Goiter . . . . . . . . . . . . 342

Exophthalmic Goiter (Basedow's Disease. Graves's Disease) 343

PART VI.

\section{DISEASES OF METABOLISM.}

\section{CHAP'TER I.}

\section{Diseases of Metaiblism.}

Diabetes . . . . . . . . . . . . 347

Diabetes Insipidus . . . . . . . . . . . . 347

Diabetes Mellitus . . . . . . . . . . . . . . 350

Obesity . . . . . . . . . . . . . . 352

Gout (Porlagra. Arthritis L'rica) . . . . . . . . . 354

Feather Eating (Featlıer Pulling in Birds) . . . . . . . 357

CHAPTER II.

Diseases of Metalolism Affecting Primarily the Bones.

Rachit is (Rickets) . . . . . . . . . . . . . . 359

Osteomalacia (Brittleness of Bones) . . . . . . . . . . 362 


\section{PAR'T VII.}

\section{DISEASES OF THE ORGANS OF LOCOMOTION.}

\section{CHAPTER I.}

Articular Rheumatism (Polyarthritis Rheumatica) . . . . 363

Muscular Rheumatism . . . . . . . . . . . . . 365

Fracture of Bones . . . . . . . . . . . . . . 368

Cranial Bones . . . . . . . . . . . . 368

Fracture of the Inferior Maxilla . . . . . . . . . 369

Fracture of the Vertebre . . . . . . . . . . 370

Fracture of the Ribs . . . . . . . . . . 371

Fracture of the Scapula . . . . . . . . . . 371

Fracture of the Humerus . . . . . . . . . . . 372

Fracture of the Radius and Ulna . . . . . . . . 372

Fracture of the Metacarpal and Phalangeal Bones . . . 373

Fracture of the Pelvis . . . . . . . . . . . 373

Fracture of the Femur . . . . . . . . . . . 374

Fracture of the Patella . . . . . . . . . . 375

Fracture of the Tibia and Fibula . . . . . . . . 375

\section{CHAPTER II.}

Diseases of the Articulations.

Wounds of the Articulations.

376

Sprains and Injuries to the Articulations. . . . . . . . 377

Dislocation of the Articulations (Luxation) . . . . . . . 378

Temporomaxillary . . . . . . . . . . . . 378

Vertebral . . . . . . . 379

Scapulohumeral . . . . . . . . . . . . . 379

Humero-radio-ulnar . . . . . 379

Radio-ulnar-carpal . . . . . . . . . . . . . 380

Phalangeal . . . . . . . . . . . . . 381

Coxofemoral . . . . . . . . . . . . . 381

Patellar . . . . . . . . 382

Tibiotarsal . . . . . . . . . . . . . 383

Caudal Vertebra . . . . . . . . . . 383

Inflammation of the Synovial Membrane and Articulations.

(Synovitis. Arthritis) . . . . . . . . . . . . 383 


\section{P.ART'VIII.}

\section{DISEASES OF' 'THE URINARY SYSTEM.}

\section{CHAPTER I.}

\section{Diseases of the Kidneys.}

Examination

385

Congestion of the Kidneys (Hyperemia.) . . . . . . . 387

Acute Hyperemia . . . . . . . . . . . . . 387

Passive Hyperemia . . . . . . . . . . . 388

Inflammation of the Kidneys (Nephritis) . . . . . . . 388

Acute Nephritis . . . . . . . . . . . . . 388

Chronic Nephritis . . . . . . . . . . . . . 392

Purulent Nephritis (Kidney Abscess) . . . . . . . . 396

Inflammation of the Renal Pelvis (Pyelitis). . . . . . . . 398

Uremia . . . . . . . . . . . . . 400

Calculi in the Kidney (Nephrolithiasis) . . . . . . . . 402

Dropsy of the Kidney (Hydronephrosis. Cystic Kidney). . 405

Amyloid Kidney . . . . . . . . . . . . . . 407

Tumors in the Kidney . . . . . . . . . . . . . 407

Animal Parasites in the Kidney . . . . . . . . . . 407

Dioetophyme Renale (Eustrongylus Gigas) . . . . . 407

Other Parasites in the Kidney . . . . . . . . . . . 409

C'HAPTER II.

Diseasis OF THE BladDer.

Examination . . . . . . . . . . . . . . 410

Wounds of the Bladder. . . . . . . . . . . . . 412

Rupture of the Bladder . . . . . . . . . . . . . . . 412

Retention of Lrine in the Blarlder (Retentio L'rina Vesicalis) . 414

Incontinence of Ürine . . . . . . . . . . . . . 416

Catarrh of the Bladder (Cystitis. Urocystitis). . . . . . 416

Torsion of the Bladder . . . . . . . . . . . . . 420

Calculi in the Bladder . . . . . . . . . . . . . 420

Tumors of the Bladder . . . . . . . . . . . . . 423

Parasites in the l3lakhter . . . . . . . . . . . . 423 


\section{CHAPTER III.}

\section{Diseases of The URetirka.}

Examination

Congenital Malformations (Occlusion of the Urethra). . . . 425

Wounds of the Urethra. . . . . . . . . . . . . 426

Stricture of the Urethra . . . . . . . . . . 426

Calculi in the Urethra . . . . . . . . . . . 427

Inflammation of the Urethra (Urethritis) . . . . . . . 429

\section{PART IX.}

\section{DISEASES OF THE NERVOUS SYSTEM.}

\section{CHAPTER I.}

Diseases of the Brain.

General Considerations

Psychic Disturbances . . . . . . . . . . . 433

Sensibility . . . . . . . . . . . . 433

Hyperemia of the Brain (Congestion of the Brain). . . . . 435

Anemia of the Brain (Cerebral Anemia) . . . . . . . . 437

Meningo-encephalitis . . . . . . . . . . . 438

Cerebral Hemorrhage (Apoplexy) . . . . . . . . . . 440

Tumors of the Brain . . . . . . . . . . . . . 441

\section{CHAPTEIR II.}

\section{Diseases of the Splnal, Cord.}

General Considerations 


\title{
CHAPTER் III.
}

Diseases of the Peripheral Nervous System.

Injuries of the Peripheral Nerves . . . . . . . . . 450

Pressure upon the Peripheral Nerves (Compression) . . . . 450

Paralysis of the Peripheral Nerves . . . . . . . . 450

Facial Nerve . . . . . . . . . . . . . . 450

Trigeminal Nerve . . . . . . . . . . . . . 452

Auditory Nerve . . . . . . . . . . . . 453

Radial Nerve . . . . . . . . . . . . . . 454

Brachial Plexus . . . . . . . . . . . . . 455

Sciatic Nerve . . . . . . . . . . . . . . . . . . 456

CHAPTER IV.

Functional Nervous Diseases.

Vertigo (Megrim)

Epilepsy .

Reflex or Secondary Epilepsy . . . . . . . . . 459

Catalepsy

Chorea

Eclampsia

\section{PART X.}

\section{DISEASES OF THE SKIN.}

\author{
CHAPTER I.
}

Non-Parasitic Skin Diseases.

Examination

Dandruff .

Alopecia

Dermatit is

Acne

Eezema 


\section{CHAPTER II.}

\section{Parasitic Skin Diseases.}

Fleas . . . . . . . . . . . . . 472

Lice . . . . . . . . . . . . 473

Scabies (Mange. Red Mange. Itch) . . . - . . . . 475

Sarcoptes Mite . . . . . . . . . . . . . . 476

Demodex Mite . . . . . . . . . . . . . 480

Dermatomycosis (Vegetable Parasitic Disease of the Skin) . . 482

Herpes Tonsurans (Ringworm. Red Itch) . . . . . . . . 482

Favus (Honeycomb Ringworm. Comb Disease. White Comb) 484

\section{PAIT XI.}

\section{DISEASES OF THE EAR.}

Examination

Wounds of the Ear.

Ulceration of the Concha

Hematoma

Otitis Externa (Otorrhea)

Otitis Media and Interna . . . . . . . . . . . . 493

Tumors (Neoplasms of the Ear) . . . . . . . . . . . 493

Papillomata . . . . . . . . . . . . . . 493

Sebaceous Tumors or Cysts . . . . . . . . . . . 494

Parasites in the Ear . . . . . . . . . . . . . 494

Deafness . . . . . . . . . . . . . 495

Ear Fistula . . . . . . . . . . . . . . . . 495

\section{PART XII.}

\section{I)ISEASES OF THE EYE.}

\section{CHAPTER I.}

Diseases of the Erelids.

Examination

Wounds of the Eyelids . . . . . . . . . . . 497

Inflammation of the Eyelids (Blepharitis) . . . . . . 498 
Malposition of the Eyclids . . . . . . . . . . . 499

Entropion . . . . . . . . . . . . 499

Ectropion . . . . . . . . . . . . . . . 500

Ptosis (Blepharoptosis) . . . . . . . . . . 501

Paralysis of the Orbicularis Nerve . . . . . . . . 502

Sipasm of the Orbicularis Nerve (Blepharospasm) . : . . 503

Trichiasis. . . . . . . . . . . . 503

Districhiasis . . . . . . . . . . . . . . 504

Allhesions of the Eyelids . . . . . . . . . . . . 504

Ankyloblepharon . . . . . . . . . . . . . . . 505

Srmblepharon . . . . . . . . . . . . . 505

Lagophthalmos . . . . . . . . . . . . . . 506

Tumors of the Eyelids . . . . . . . . . . . . 506

Papillomata (Warts) . . . . . . . . . . . . 506

Chalazion (Meibomian Cyst). . . . . . . . . . 507

Pilosebaceous Cysts . . . . . . . . . . . 507

Lipoma and Sebaccous Tumors in Birds . . . . . . 508

Enlargement of the Glands of Moll . . . . . . . 508

Granulomas . . . . . . . . . . . . . 508

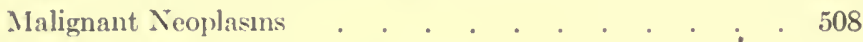

CHAP'TER II.

Diseases of the Conjunctiva.

Examination . . . . . . . . . . . . . . 510

Conjunctivitis . . . . . . . . . . . . . 510

Catarrhal Conjunctivitis . . . . . . . . . . . 511

Purulent Conjunctivitis (Blennorrhea) . . . . . . . 512

Parenchymatous Conjunctivitis (Erysipelatious Conjunctivitis) . . . . . . . . . . . 514

Croupous Conjunctivitis . . . . . . . . . . . . 516

Follicular Conjunctivitis . . . . . . . . . . . 517

Exanthematous Conjunctivitis . . . . . . . . 518

Pterygiun . . . . . . . . . . . . . . . . 519

Traumatic Lesions of the Conjunctiva . . . . . . . . 519

Foreign Bodies in the Conjunctiva . . . . . . . . 519

Wounds of the Conjunctiva $\quad . \quad$. . . . . . . . . 520

Corrosions and Burns of the Conjunctiva . . . . . . . 521

Ulceration of the Conjunctiva . . . . . . . . . . 521

Tumors and Growths on the Conjunctiva . . . . . . . . 522

Inflammation of the Membrana Nictitans . . . . . . . 522

Wounds of the Membrana Nictitans . . . . . . . . . 523

Tumors of the Membrana Nictitans . . . . . . . . . 523 
CHAP'TER III.

Diseases of the Lacrimal Apparates.

Examination

Lacrimation (Epiphora) . . . . . . . . . . . . 524

Dacryocystitis

CHAP'TER I\%.

Diseases of the Cornea.

Examination

526

526

Fieratitis

527

Superfieial Keratitis . . . . . . . . . . . 527

Vascular Keratitis (Pannus). . . . . . . . . $52 S$

Keratitis Pigmentosa (PigmentaryiKeratitis). . . . 529

Keratitis Punetata Superfieialis (Facetted Keratitis) • 529

Parenehymatous Keratitis . . . . . . . . . 530

Keratitis Punctata Profunda . . . . . . . . 532

suppurative Keratitis . . . . . . . . . . . 532

Tlceration of the Cornea . . . . . . . . 532

Abscess of the Cornea . . . . . . . . . 534

Feratitis Neuroparalytiea . . . . . . . . . 535

Kieratitis from Lagophthalmus . . . . . . . . 535

Foreign Bodies and Wounds of the Cornea . . . . . . . 535

Opacities of the Cornea . . . . . . . . . . . 536

Eetasia of the Cornea . . . . . . . . . . 537

Inflammatory Ectasia . . . . . . . . . . . 537

Staphyloma . . . . . . . . . . . . 537

Iierateetasia . . . . . . . . . . 537

Xon-inflammatory Letasia . . . . . . . . . 538

Keratoeonus . . . . . . . . . . . . 538

Iieratoglobus . . . . . . . . . . . 538

Tunors of the Cornea . . . . . . . . . . 538

CHAPTER V.

Diseases of the Iris and Ciliary Body.

Congenital Defeets of the Iris . . . . . . . . . . 539

Mydriasis . . . . . . . . . . . . . . 540

Myosis . . . . . . . . . . . . . 540

Iritis and Cyclitis (Iridocyclitis) . . . . . . . . . . 540

Cysts and Tumors of the Iris . . . . . . . . . . 541 


\section{CHAPTER VI.}

DISEASES OF THE LENS.

Examination

Cataract

Luxation of the Lens

CHAPTER VII.

Diseases of the Retina axd Chorold

\section{CIIAPTER VIII.}

Disfases of the Optic Nerve.

Papillit is .

Retrobulbar Neuritis

Atrophy of the Optic Nerve . . . . . . . . . . 547

Amblyopia . . . . . . . . . . . . . . . 547

Amaurosis

\section{CHAPTER IX.}

Diseases of the Globe and Orbit.

Panophthahnitis.

Glaucoma

Hydropthalmus .

Exophthalmus

Luxation of the Eychall . . . . . . . . . . . 550

Enophthalmus . . . . . . . . . . . . . . 551

Strabismus . . . . . . . . . . . . . 552

Nystagmus . . . . . . . . . . . . . . . . 552

Parasites of the Eve . . . . . . . . . . . . 553

Fracture of the Orbit . . . . . . . . . . . . 553

Inflammation of the Orbit . . . . . . . . . . 553

Tunors of the Orbit . . . . . . . . . . . 553 


\section{PAR'T XIII.}

\section{HERNIA.}

General Remarks . . . . . . . . . . . . . . 555

Unubilieal Hernia . . . . . . . . . . . . . . . 557

Ventral Hernia . . . . . . . . . . . . . . . 558

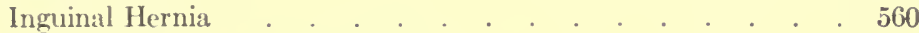

Inguinal Hernia in the Female . . . . . . . . . 560

Inguinal and Scrotal Hernia in the Male . . . . . . 561

Femoral Hernia . . . . . . . . . . . . . . . 562

Diaphragmatie Hernia . . . . . . . . . . . . . 563

Perineal Hernia . . . . . . . . . . . . . . . 563

\section{PAR'T XIV. \\ INFECTIOUS DISEASES.}

\section{CHAPTER I.}

Acute Gejeral Infectious Diseases.

Distemper of Dogs . . . . . . . . . . . . . 565

Distemper of Cats . . . . . . . . . . . . . . 577

Typhus of Dogs (Canine Typhus. Hemorrhagie Gastro-enteritis.

Dog Plague. Black Tongue) . . . . . . . . . . 579

Purpura Hemorrhagica of Dogs . . . . . . . . . . . 583

Fowl Cholera (Cholera Gallinarum. Pasteurellosis Avium. Fowl

Trphoid) . . . . . . . . . . . 584

Fowl Pest (Fowl Plague. Pestus Avium) . . . . . . . 589

\section{CHAPTER II.}

Acrte Infectious Diseases with Localization in Certain Organs.

Epitheliosis Infeetiosa Avium (Contagious Epithelioma. Chiekenpox. Diphtheria. Roup. Canker) . . . . . . . . 593 Anthrax . . . . . . . . . . . . . . . . . 599

Foot-and-mouth Disease (Aphthæ Epizoötieæ). . . . . . 600

Hemorrhagic Septieemia of Cats (Infeetious Gastro-enteritis). . 600 


\section{CHAPTER III.}

Mrfectors Diseases witu Special Involvement of the Nemous SYSTEM.

Rabies (Hydrophobia. Lyssa) . . . . . . . . . . 603

Infectious Bulbar Paralysis (Pseudorabies) . . . . . . . 611

Tetanus (Lockjaw) . . . . . . . . . . . . . 613

\section{CHAPTER IV.}

\section{Chronic Infectious Diseases.}

Tuberculosis of Birds (Tuberculosis Avium. Avian Tuberculosis) 616 Tuberculosis of Dogs and Cats .. . . . . . . . . . 620 Glanders . . . . . . . . . . . . . . . . 622 Pseudo-actinomycosis of Dogs (Streptotrichosis Canum. Actino- . myces Canis) . . . . . . . . . . . 623

\section{CHAPTER V.}

Infectiots Diseases Produced by Protozo.

Spirochætosis of Fowls (Spirochætosis Avium. Spirillosis of Chickens) . . . . . . . . . . . . . . . . 625

Enterohepatitis (Blackhead) . . . . . . . . . . 627

Infectious Diarrhea of Chicks (White Diarrhea) . . . . 629

Piroplasmosis of the Dog (Infectious Jaundice. Biliary Ferer. Malignant Jaundice) . . . . . . . . . . . 633

Table of Equivalents in Weights and Measures . . . . . 637 


\section{DISEASES OF THE SIILLL DOMESTIC MNIILALS.}

\section{PART T.}

\section{DISEASES OF THE RESPIRATORY SYSTEN.}

\section{CHAPTER I.}

\section{DISEASES OF 'THE NASAL PASSAGES.}

Examination.-The nasal passages are very small and do not almit of a free examination. In some breeds of dogs (English bull) there is an obstruction of the nasal passages which during inspiration produces a peculiar snoring sound. 'This should not be mistaken for an abnormal condition. In other breeds (Collie) where the nasal passages are longer and less tortuous respiration produces no sound. Dogs breathe freely through the mouth. In the cat the nasal passages are very small and short and can only be examined at their openings.

The examination of the nasal passages should be made as follows: Good light such as ordinary daylight or strong artificial light is necessary. A mirror to reflect light into the nasal openings is of little value as the passages are so small that the light will be reflected only a short distance. A very small nasal dilator will be found useful in some of the larger breeds. A small flexible probe with a pledget of cotton securely wrapped around one end, large enough to occlude the nostril when inserted rather high up, will at once 
induce sneezing and the character of the secretions from the opposite nostril can be more easily determined. The probe is then removed and inserted in the other nostril for a similar examination. The nose in health is moist and cool with a very thin, slight mucous discharge. 'The mucous membrane is variable in color; in most breeds dark, in others light with dark spots, and in a few breeds pink.

Abnormal conditions to be noted are the following:

Tumors.-Small papillomas on the skin and mucous membrane at the entrance to the nasal openings are frequently seen. Epitheliomas extending from the lips to form a diffuse enlargement which may partially or completely obstruct the nasal openings. Polypoid enlargenents on the mucous membrane of the nasal passages are often noted and can be accurately determined by using a small, fine wire snare to pull them toward the nasal opening.

Foreign Bodies.-Frequently in hunting dogs small pieces of straw, twigs and other foreign material will be found rather high up in the passages. 'These injure the mucous membrane and often become deeply imbedder in it and the underlying tissues.

Parasites. - The Pentastoma rhinaria (Linguatula rhinaria) is occasionally found in the nasal passages and when suspected the nasal discharge should be examined microscopically to reveal the presence of the eggs of the parasite.

Infections.-Distemper infection frequently takes place in the nasal passages and produces both local and general symptoms. Staphylococcus infection from wounds in the mucous membrane, or as secondary infection following listemper, is of common occurrence. In these conditions the nose is usually dry and hot, showing numerous fissures on the mucous inembrane and nose at the borders of the nasal openings.

Hemorrhage. - This occurs often from injuries, as blows over the nasal bones, fractures of the nasal bones; from punctured wounds through the bones and from injury to the turbinated bones.

Malformations.-Some diseases (rachitis) produce a deformity of the nasal bones interfering with the free passage 
of air through the nostrils and producing markerl cnlargement of the nose.

Discharge.-In acute and chronic nasal catarrh (coryza, rhinitis) the mucous and serous discharge from the nasal passages is greatly increased. The general condition is but little disturbed.

\section{ACUTE NASAL CATARRH.}

\section{Coryza. Rhinitis.}

Definition.-Acute inflammatory condition of the nasal mucous membrane producing a serous or mucous discharge.

Etiology.- (a) Inhalations of dust or other foreign material.

(b) Inhalations of irritating gases or chemicals.

(c) Exposure to cold (cold draughts of air).

(d) Ordinary infection (staphylococcus, etc.); specific infections (distemper, etc.).

(e) Linguatula rhinaria (early stages of invasion).

During the first stages there is hyperemia and dryness of the nasal mucosa, which is soon followed by a discharge that is serous, seromucous, or mucopurulent. 'This discharge often causes excoriation of the tissues with which it comes in contact. In non-pigmenterl membranes the congestion is more prominent.

The discharge when examined with the microscope shows epithelial cells, leukocytes, bacteria and sometimes the eggs of the parasitc, Linguatula rhinaria.

Symptoms.- Sneezing and the presence of a thin, serous discharge in the carly stages, which later is mixed with mucus, becomes dry and atheres around the nasal openings. 'The nose is rubbed against objects and wiped with the paws. Excoriations appear around the margins of nasal openings.

Diagnosis. - The presence of the above symptoms and the absence of any general disturbance charactcrize simple rhinitis.

Prognosis. Favorable.-Recovery usually takes place in about one to two weeks.

Treatment.- Iygienic.--The animal should be kept warm and protected from draughts of cold air. 
Medical.-Spray the nasal passages with a warm boric acid ( 2 per cent.) or sodium bicarbonate ( 2 per cent.) solution. Remove the dry discharge from the edge of the nasal openings. Apply vaselin or zinc oxid ointment to those parts as protective agents.

\section{EMPYEMA OF THE INFRAORBITAL FOSSE OF BIRDS.}

Definition.-A collection of pus in the infraorbital fosse which causes a distention of one or both of them.

Etiology. - This condition usually occurs during the course of infectious nasal catarrh, diphtheria or parasites (monostoma). The mucous membrane becomes infected and the purulent material collects in the fossæ greatly distending them.

Symptoms. - A warm painful swelling is noticed in the infraorbital region, which may affect one or both sides. 'The enlargement, which is quite firm, protrudes over and closes the eye.

Prognosis.-Depends upon the primary cause. When the affection is confined to the fossæ the prognosis is favorable.

Treatment.-Surgical.-An incision should be made over the cnlargement and the accumulated material which is quite hard and firm removed with a curette. 'The cavity should be washed with antiseptics.

\section{INFECTIOUS NASAL CATARRH OF RABBITS.}

\section{Rabbit Plague. Rabbit Influenza. Rabbit Distemper.}

Definition.-An infectious disease involving the mucous membrane of the nasal passages, which later produces general infection.

Etiology. - The exciting cause is a small, slender, immobile bacillus, similar in size to the bacillus of cholera in birds. It does not form spores and is Gram-negative. This organism is also pathogenic to guinca-pigs and micc, but rabbits are most susceptible. Infection takes place by inhalation during cohabitation with affected animals, or by being taken in with 
the food. The organism produces a severe inflammation of the mucosa of the nasal passages and sinuses, and later enters the circulation producing general infection, causing eleration of temperature and in most cases an inflammation of the serous membranes. The infection is often carried directly to the trachea, bronchial tubes and lungs, where it produces an acute inflammatory condition.

Necropsy. - The nasal, oral and pharyngeal mucous membranes are intensively congested, swollen and covered with a purulent exudate. In the lungs are often found evidences of a bronchopneumonia. The thoracic cavity may contain a serous or purulent exudate with fibrinous deposits on the pleura. The peritoneum will also show inflammation with some exudate in the abdominal cavity. The bacilli can be found in large numbers in the exudate and in affected organs.

Symptoms. - The incubation period is from four to six days. The first symptoms noticed are depression, a copious discharge of serous secretion from both nasal openings and severe sneezing. The temperature is usually from $104^{\circ}-106^{\circ}$ $\mathrm{F}$. and there is complete loss of appetite. This discharge soon becomes thick and tenacious, adhering around the nasal openings and on the hair of the chest and limbs, the animal frequently rubbing its nose with its paws to relieve the intense irritation. With the extension of the inflammation to the larynx and bronchi, dyspnea and coughing result. Exhaustion is soon noticed due to the general infection and anorexia.

Prognosis. - Very unfavorable in acute cases, death occurring in three to five days. In subacute and chronic cases in older animals the course is fifteen to thirty dars. Complete recovery, however, is rare; chronic nasal catarrh is a common sequel.

Diagnosis.-The rapid derelopment, high temperature, absence of coccidia (see rhinitis coccidiosa), the acute inflammation of the serous membranes and the finding of the specific bacillus in the discharges confirm the diagnosis.

Treatment.-Medical.-The nasal passages should be sprayed with antiseptic solutions (boric acid 2 per cent. or sodium bicarbonate 2 per cent.), the accumulaterl crusts removed with warm water and a protective dressing of zinc 
oxid ointment or vaselin applied. The use of internal antiseptics (salol $0.12-0.2$, etc.) is indicated to combat the general infection. Autogenic vaceines may be used.

Prophylaxis.-All material soiled by the nasal discharges as well as all animals that die should be burned and well covered with lime; all parts of the hutch and the runvays thoroughly disinfected, and a quarantine of at least two weeks imposed upon all newly acquired animals.

\section{PARASITIC NASAL CATARRH OF RABBITS.}

\section{Rhinitis Coccidiosa.}

Definition.-An inflammation of the mucous membrane of the nasal passages and sinuses duc to an infestation with coccidia.

Etiology. - This condition is due to the Coccidia perforans or the Coccidia oviformis, which gains entrance to the nasal passages and sinuses. The discharges of affected animals are infectious. Damp hutches with soiled litter and filth in them favor the spread of the disease. Young animals are more susceptible than those fully matured, the latter usually contracting the discase in a mild form.

Symptoms.-The clinical symptoms are similar to those of infectious nasal catarrh. The nasal discharge is present, being serous at first, later becoming thick and tenacious. After two or thrce days the animals become depressed, frequently gnashing the teeth and rubbing the nose. 'The temperature, however, is subnormal. In some cases the mucous membranes of the mouth and the conjunctiva exhibit catarrhal symptoms. In cases where the coccidia pass into the middle ear, the semicircular canals become involved causing the hcad to be carried to one sile, staggering gait, and in some cascs rolling over and over. Spasms of the muscles occur followed by paralysis. Paralysis, however, is a symptom of many irritating conditions in rabbits which in other animals ordinarily cause excitement. I ater a profuse diarrhea is noticed followed rapidly by exlaustion and death. 
Diagnosis.-Microscopic examination of the nasal discharges reveals the presence of the coccidia. In otodectes cynotis mange, catarrhal symptoms of the mucous membrane are absent.

Prognosis.-Unfavorable.

Treatment.-The nasal passages, eyes and mouth are cleansed with antiseptic solutions and astringents (boric acid 2 per cent., zinc sulphate 1 per cent., copper sulphate 1 per cent., creolin 2 per cent.). Small doses of sulphur (0.1-0.2) may be given every four to six hours to produce antiseptic and laxative action.

Prevention.-(See Infectious Nasal Catarrh of Rabbits.)

\section{CHRONIC NASAL CATARRH.}

\section{Chronic Coryza. Chronic Rhinitis.}

Definition.-A chronic inflammatory condition of the mucous membrane of the nasal passages with a mucopurulent discharge.

Etiology. - Usually follows repeated attacks of acute nasal catarrh; ordinary infection; tumors.

Pathology.-(a) In the productive or hypertrophic form there is a generalized infiltration of the mucosa, particularly that covering the lower portion of the turbinated bones, which leads to a more or less extensive thickening of the mucous membrane. The mucous glands increase in size, there is a thick, viscid secretion, and the nasal passages become obstructed by enlargement of the lower part of the turbinated bones. The atrophic form follows the course of the hypertrophic. The hyperplastic tissue atrophies, the epithelium of the mucosa and the glands is destroyed, and there is a collection of a yellowish, purulent material on the surface of the mucous membrane. (b) 'The discharge from the nasal passages contains bacteria, leukocytes, red corpuscles and epithelial cells.

Symptoms. - A purulent discharge varying in quantity from both nasal passages. 'This discharge in severe cases is streaked with blood and has a very offensive odor; it causes 
excoriations on the mucous membrane and skin at the nasal openings, where it drys and forms hard crusts. 'There are frequent paroxysms of sneezing. In cases where the nasal passages are occluded the animal breathes through its mouth. In some cases where infection is severe general symptoms of loss of appetite, dulness and emaciation will be noted.

Diagnosis. - The presence of the nasal discharge, the chronic course and the mild general symptoms in severe cases.

Prognosis. - Not so favorable as acute nasal catarrh. It requires several weeks for a complete disappearance of the symptoms.

Treatment. - Hygienic. - The animal should be kept in a warm place free from all irritating materials which might affect the nasal mucous membrane.

Medical.-The nasal passages should be sprayed daily with creolin (2 per cent.), boric acid (2 per cent.), or sodium bicarbonate (2 per cent.) solution; the dried crusts may be removed with warm water, and zinc oxid ointment applied to the membranes and skin at the nasal openings.

Sera-vaccine.-Cultures may be grown from the nasal discharge, and a standardized autogenic vaccine made. Two c.c. of this vaccine are injected subcutaneously every five to seven days until the purulent discharge ceases.

\section{EPISTAXIS.}

Definition.-Hemorrhages from the nasal passages.

Etiology.-(a) Injuries to the nasal mucous membrane from foreign bodies (straws, twigs, etc.); probing; injuries to the turbinated bones due to fractures or trephining the sinuses.

(b) Carbolic acid poisoning causing necrosis of the membrane extending into the nasal passages which opens the vessels.

(c) Specific and ordinary infections during the course of distemper and chronic nasal catarrh.

(d) Parasitic invasion (Linguatula rhinaria). Hemorrhage occurs during the course of the following discase conditions: 
Hemophilia, plethora, leukemia, parasitic anemia and catarrhal pneumonia.

Symptoms. - A unilateral or bilateral discharge of blood from the nasal passages. When primary the blood is of a bright red color and flows away a few drops at a time or the flow may be copious, and sometimes sufficient to produce general symptoms. When secondary to chronic nasal catarrh or distemper it is mixed with the seeretions.

As small animals lick the nose the amount of hemorrhage is not easily determined.

Prognosis. - The prognosis depends on the eharacter and amount of the hemorrhage. Most cases terminate favorably.

Treatment.-Medical.-In mild eases when treatment is necessary use injections of cold water (ice-water) or alum solution (3 per cent.) into the nasal passages. In severe cases when hemorrhage is copious and persistent use injections of adrenalin chlorid (1-1000) solution. Give internally iron and quinin citrate $(0.2-0.35)$ three times daily; or adrenalin chlorid (1-1000) ten to twenty drops, twice daily.

Surgical. - When the hemorrhage is continuous and cannot be stopped by medical treatment, it will be neeessary to use a tampon made of gauze and saturated in an alum (3 per cent.) or tannic acid (3 per cent.) solution. They should be inserted with a small flexible probe firmly and carefully as far up the nasal passages as possible. In some cases it will be necessary to use the same kind of a tampon inserted in the posterior part of the nasal passage. This is best accomplished by use of the mouth speculum and a flexible probe bent in the shape of a hook. When a tampon is inserted a free end should be exposerl to facilitate removal. As small animals breathe freely through the mouth tampons may be inserted in both nasal passages.

\section{PARASITES OF THE NASAL PASSAGES.}

Definition.-Infestation of the nasal passages and chambers by the Linguatula rhinaria.

Etiology. - IIistory.- The infestation of the dog with this parasite is rather rare in the United States. Only a few rases 
have been recorded, but in other countries (France, Germany) they are frequently found. The Linguatula rhinaria are white, the body lanceolated, elongated, vermiform and flattened above and below, the ventral surface nearly plane, the dorsal surface rounded, anterior extremity broad and rounded, posterior extremity attenuated. The thorax is short and between it and the abdomen there is no distinct boundary. The integuments show about ninety rings or segments, widest in their middle, causing the margins of the parasite to be distinctly crenated. The hooks are sharp, curved, and biarticulated. Each hook is retractile into a small sheath and is moved by muscular cords arranged in different directions. The mouth is rounded, digestive tube rectilinear. Size: Male, $18-20 \mathrm{~mm}$. long, $3 \mathrm{~mm}$. broad in front and $0.5 \mathrm{~mm}$. behind. Female, $8-10 \mathrm{~cm}$. long, $8-10 \mathrm{~mm}$. broad in front and $2 \mathrm{~mm}$. behind. The eggs are ovoid, $90 \mathrm{mmm}$. long and $70 \mathrm{mmm}$. broad.

The life cycle of the Linguatula rhinaria is as follows: The female deposits her eggs in the nasal passages of the dog; the eggs are expelled by sneezing and, being surrounded by mucus, they adhere to grasses or whatever they happen to come in contact with. 'The grasses are eaten by any of the herbivorous animals. The shells of the eggs are dissolved by the gastric juice and the embryos are set at liberty in the intestinal tract. Each embryo is provided with a median stylet and two curved hooks with which it penetrates the walls of the intestines and reaches the peritoneum, mesenteric glands, liver and the lungs where it becomes encysted. During the period of encystment in the organs and glands the embryo undergoes successive changes in its development and becomes a larva (Linguatula denticulatum). When mature the larvæ migrate by means of their hooks and the sharp spiculæ on their skin. Some of them pass into the bronchi and trachea reaching the nasal passages where they develop into the perfect parasite. Dogs become infested by eating the viscera of animals containing the larval form which passes from the stomach via the esophagus to the nasal passages where it develops. 
Necropsy.-In the early stages of the invasion of the Linguatula rhinaria they attach themselves to the mucous membrane of the nasal cavities producing an acute inflammation. The exudate is increased in quantity and later is mixed with pus covering the surface of the mucous membrane which becomes greatly thickened especially around the turbinated bones and in the nasal chambers. In the later stages necrosis of the turbinated bones and nasal septum may take place.

The discharge from the nasal passages often contains the parasites, pus, epithelial cells and large numbers of the ovoid eggs.

Symptoms. - In the early stages the symptoms are paroxysms of sneezing; obstruction of the nasal passages; a discharge which is at first serous, later becomes mucopurulent, mixed with blood, and has a very offensive odor. Accumulations of the dried discharge form crusts at the nasal openings. In animals of a nervous temperament symptoms of excitement are noted from reflex irritation of the nasal passages. Severe hemorrhage is scen from the nccrosis opening bloodvessels.

Diagnosis. - The prescnce of the parasites or eggrs in the nasal discharge.

Prognosis.-Depends upon the number of parasites and the probability of becoming reinfested.

Treatment.-Medical.-Inhalations of small amounts of chloroform, turpentine, or some other volatile oils. Spray the passages twice daily with creolin (2 per cent.), carbolic acid ( 2 per cent.), or boric acil ( 2 per cent.) solution. Remove the crusts from around the nasal opcnings with warm creolin solution and apply zinc oxid ointment to the cxcoriated membrane and skin. Give internally tincture nux vomica $(0.3-0.65)$ daily; iron and quinin citrate $(0.2-0.4)$ daily.

Surgical.-Irrigation of the nasal passages with a warm solution of creolin (2 per cent.). 'This (an be done by using a small, soft rubber tube inscrted as high up in the nasal passages as possible. Attach a funnel to the other end and pour the solution slowly into it using only a small quantity of the fluid. The nasal passages can also be irrigated through 
the posterior nares by the use of a hard nozzle bent in the form of a curved hook. Depress the head and allow the fluid to flow out through the nasal passages.

\section{NEOPLASMS OF THE NASAL PASSAGES.}

Papillomata. - These are benign tumors having a framework of fibrous tissue and bloodvessels covered by squamous epithelium. The surface of the tumor is roughened by many elevations and proliferations. Papillomata are most commonly located around the margins of the nasal openings and especially at the juncture of the skin and mucous membrane. Sometimes they extend a short distance up the nasal passages and may extend to the skin of the lips and the nose. 'They are found here in two forms: (a) A hard form which presents smooth, rounded elevations on the skin. (b) A soft form, which is pedunculated, has an irregular, broad surface and cauliflower-like appearance. The soft tumors are found on the mucous membrane. In size they vary from that of a millet seed to a walnut. There may be a large number or only a few present.

Treatment.-Surgical.-They are removed as follows: The animal should be given an anesthetic (morphin subcutaneously, or ether inhalation). Sterilize curved scissors, forceps and artery forceps; disinfect the surface of the skin, mucous membrane and tumors with boric acid solution (2 per cent.). Grasp the tumor with the forceps and cut off with the scissors, and if the hemorrhage is persistent use the artery forceps. Alum (2 per cent.) or silver nitrate solution $(0.25-0.5)$ may afterward be applied. The after-treatment consists in washing the surface where the tumors are removed with antiseptic solutions.

Polypoid Fibromata. - These occur as enlargements on the mucous membrane of the nasal passages and frequently interfere with breathing, producing complete obstruction of one or both passages.

Treatment.- These are best removed with a fine wire snare. Inject astringent solutions (alum 2 per cent.) up the nasal passages to control the hemorrhage. When this method of 
removal fails it is best to trephine the superior wall of the nasal passages and extirpate the tumor through the opening. After-treatment consists of warm antiseptic solutions injected daily up the passages.

Malignant Tumors. -Occasionally in the nasal passages are found epitheliomas, osteosareomas, and sareomas which are usually secondary to primary growths having their origin in the buceal mucosa, maxillary bones or lips (epitheliomas).

Symptoms. - Malignant growths give rise to distortions of the nasal bones, lips and often result in necrosis of the parts involved. The nasal discharge will contain necrotie material and blood and has a very offensive odor.

Diagnosis. - To make an aecurate diagnosis some of the tumors should be obtained and examined mieroscopieally.

Prognosis. - The prognosis is very unfavorable.

Treatment.-Owing to the location of the tumors, their malignant character and the tissues involved, treatment is not to be attempted. 


\section{CHAPTER II.}

\section{DISEASES OF THE LARYNX.}

Examination.-(a) With the mouth speculum, laryngoscope and good light, the anterior portion of the larynx and the surrounding tissues can be readily inspected provided the tongue is drawn well forward.

(b) By examining some of the nasal discharge collected with a dressing forceps carrying a pledget of cotton, its character is decided.

(c) By palpation, enlargements, injuries and the degree of sensitiveness may be determined.

Laryngitis.--Two forms of laryngitis are frequently observed in small animals, viz: (a) Acute, and (b) chronic.

Acute Laryngitis. - Definition. - An acute catarrhal inflammation of the mucous membrane of the larynx.

Etiology.- (a) Excessive use of the voice (barking). 'This is often seen at dog shows; in hounds after hunting; in some dogs when penned up or tied; during the course of rabies and the nervous form of distemper; excitement.

(b) Inhalations of dust, dirt, etc.; injurics (tight collars, etc.). Inhalations of gases and smoke; irritating drugs and chemicals.

(c) Exposure to cold.

(d) Infections (staphylococcus, etc.) and during the course of infectious diseases (rabies, distempcr, etc.).

(e) Extension of inflammation from adjacent parts.

Pathology.-(a) There is an acute inflammation of the mucous membrane which, in the carly stages, is covered by a thin serous exudate which later becomes turbid from admixture of lcukocytes. From the irritation produced by coughing small quantities of blood are often raised. When infection is present the secretions are mucopurulent in character. 
(b) The diseharge eontains baeteria, leukocytes, red corpuscles and epithelial cells.

Symptoms.-Hoarseness, ehange of voiee, frequent attempts at deglutition, a harsh, dry eough which later becomes softer and moist as the secretions are inereased. The mucous membranes are eongested and swollen. Pressure on the larynx, exereise, exeitement or drinking eold water induces eoughing. General symptoms are rare.

Diagnosis. - The presence of the harsh, dry cough which is aggravated by exereise and excitement and the absence of general disturbance are characteristie of laryngitis.

Prognosis.-Favorable. Reeovery usually follows in eight to ten days, unless when secondary to rabies, distemper, ete.

Treatment.-Hygienic.-Keep warm; supply plenty of fresh air.

Dietetic.-Give warm milk and warm liquid foods.

Medical.-Direet applieation of medicinal preparations to the mucous membrane is unpracticable and of little value. The following formula should be used to allay irritation and stop the coughing:

R-Morphini hydrochlorati .

Aquæ amygdalæ amaræ .

Misce et fiat solutio.

Sig.-Give teaspoonful three times daily.

Surgical.-Apply Priesnitz compress or hot antiphlogistin paek over the larynx. Renew twice daily.

Chronic Laryngitis. - Definition. - A ehronic catarrhal inflammation of the mueosa and submucosa of the larynx.

Etiology. - This is usually the result of frequent aeute attacks, and therefore the eauses enumerated under acute laryngitis are applicable to the ehronie form. Clironie laryngitis is often the result of the extension of ehronic nasal and pharyngeal catarrh; the presenee of papillomata and polypoid fibromata within the larynx; as a sequel to distemper; may be eaused by pressure on the vagus nerve (enlarged mediastinal lymplı glands, sarcomas, eareinomas, ete.); to direct irritation of the inueosa by malignant growths; enlarged thyroids. 
Pathology.-Chronic catarrh leads to hyperemia of the parts with hypertrophy of the mucosa and the submucosa, together with fibrous tissue proliferation. Localized thickenings, cither flat or wart-like, are often noticed. The submucosa is infiltrated with cells and the mucous glands are swollen and distinct, producing a granular condition.

Symptoms. - The symptoms are somewhat similar to thosc of acute laryngitis but not so severe and continuc for a longer time. 'The cough is hoarse, dry, seldom moist, and is aggravated by exposure to cold, exercise or excitement. 'The larynx is less sensitive than in the acute form when examined by external manipulation. After severe attacks of coughing the paticnts may show nausea and vomiting.

Diagnosis.-The chronic course, absence of gencral symptoms and the cough characterize the condition.

Prognosis.-Owing to the changes in the mucosa and the submucosa, improvement is slow and complete recovery seldom takes place.

Treatment.-IIygienic.-Kcep warm; supply plenty of fresh air.

Dietetic.-Give warm liquid foods (milk, soups, etc.).

Medical.-The following formulæ may be used to stop the coughing and allay the irritation:

$\mathrm{R}$-Morphini sulphatis . . . . . . . . . . 0.1

Aquæ amygdalæ amaræ . . . . . . . . 30.0

Misce et fiat solutio.

Sig.-Give teaspoonful threc times daily.

or

P-Potassi bromidi . . . . . . . . . . 10.0

Morphini sulphatis . . . . . . . . . 0.1

Aquæ distillata . . . . . . . . . . . 150.0

Misec et fiat solutio.

Sig.-Give teaspoonful three timcs daily.

For expectorant action may use the following:

Pł-Ammonii ehloridi

5.0

Antimonii et potassi tartras

Fixtraet. glycyrrhizæ .

0.5

Aquæ feniculæ

10.0

180.0

Misee et fiat solutio.

Sig.-Give teaspoonful every eight to ten hours. 
or

P-Apomorphin. hydrochloras . . . . . . . . 0.05

Acidi hydrochlorici . . . . . . . . . . 1.0

Aquæ distillata . . . . . . . . . . . 250.0

Misce et fiat solutio.

Sig.-Give teaspoonful twice daily.

Syrup of tar or syrup of wild cherry may be given in teaspoonful doses twice daily.

Inhalations of medicinal preparations (turpentine, etc.) and direct applications (silver nitrate 1, glycerin 130) may be used in some of the more obstinate cases. 


\section{CHAPTER III.}

\section{DISEASES OF THE TRACHEA AND BRONCHIAL TUBES.}

Examination.-(a) By palpation, the upper part of the trachea can be examined for enlargements, deformities of the tracheal rings, constrictions and sensitiveness to pressure. Often when inflammatory conditions are present, slight pressure on the trachea will induce coughing, attempts at swallowing and considerable uneasiness and pain.

(b) By auscultation, the condition of the mucous membrane and the character of the secretions can be determined. The tracheal and bronchial sounds are most distinct at the entrance of the trachea to the thorax. A phonendoscope held directly against the trachea at this point will greatly assist in the examination.

\section{ACOTE TRACHEITIS AND BRONCHITIS.}

Definition.-These are acute inflammatory conditions involving the mucous membrane of the trachea and the bronchial tubes.

Etiology.- (a) Small particles of foreign bodies (dust, etc.) enter the trachea and bronchial tubes producing excoriations and congestion of the mucous membrane which allow infection to take place. If these substances are putrescent a fetid bronchitis will be produced.

(b) Irritating gases (ammonia, etc.) and the fumes of acids (carbolic, etc.) may excite an attack of bronchitis by direct irritation to the mucous membrane. When inhaled in concentrated form and in large quantities they will induce inflammation extending into the smaller bronchioli producing an acute capillary bronchitis. The excretion of toxic sub- 
stances by the bronchial mucosa causes a rapid proliferation of the surface and granular epithelium of the mucous membrane and an increased secretion of mucus. 'The epithelium of the mucous membrane becomes more embryonal in character and therefore less resistant to the invasion of microorganisms. At the same time these organisms, ordinarily present, multiply more rapidly and increase in virulence, and unless the process is arrested a bronchitis is soon establisherl. Some drugs (iodin, bromin, etc.) when administered in large doses and continued for quite a time may be excreted in sufficient quantities to produce an acute bronchitis.

(c) Inhalations of smoke containing various gases and particles of foreign material will irritate the mucous membrane of the entire respiratory tract, frequently resulting in an active congestion and later in inflammation of the mucous membrane which favors the growth and levelopment of the infection which is always present.

(d) Acute bronchitis develops frequently as a secondary disease following specific infections (distemper, etc.). The specific infection reduces the normal resistance of the mucous membrane thus allowing the secondary infection (staphylococcus, streptococcus) to develop rapidly and produce the disease.

(e) Parasitic. In severe infestations of the Uncinaria canina, the larval form of this parasite, in its migrations through the body, often burrows along or through the walls of the bronchi and trachea producing an inflammation of the mucous membrane and the underlying tissues. (See Lncinariasis.)

(f) Tracheitis and acute bronchitis are often consecutive to inflammations of the nasal passages, larynx and pharynx. The infection is carried to the trachea and bronchi by inhalation where it develops, producing the inflammation.

(g) Other diseases (rcnal affections, endocarditis, diabetes, carcinomas and sareoma, valvular insufficiency) produce a disturbance of the heart action, cufeeblement of the rasomotor nerve and reduce the resistance of the mucous membrane.

It is quite evident that acute tracheitis and bronchitis 
in most cases develop from bacteria found normally on the mucous membrane.

Pathology.-The pathological changes that are liable to be found in acute tracheitis and bronchitis are somewhat diverse, the details of the process being considerably modified by the anatomical peculiarities of the parts affected and the different causes that affect them. The inflammation may be restricted to the bronchial structure but is frequently associated with other and more serious disturbances. The condition is usually bilateral, although certain parts are affected more than others. Tracheitis and bronchitis are often associated with laryngitis, peribronchitis and bronchopneumonia.

Symptoms.-Cough.-This is the most important symptom and is never absent, although it may be slight or very severe and loud, occurring as isolated coughs succeerling each other with greater or less frequency, or in paroxysms which often end in nausea or vomiting. The cough is dry and harsh in the early stages, later becoming moist as the inflamed mucous membranes produce an increased secretion. Paroxysms of coughing are often induced by excitement (visits of the owner, etc.), exposure to cold, moist air, pressure over the lower part of the trachea or by percussion over the sides of the chest. The object of the cough is to expel the accumulated discharge from the air passages.

Discharge.-This is alway's present exccpt in the early stages; it is mucous at first, becoming mucopurulent or purulent as the disease progresses. In small animals the discharge is expelled from the trachea and bronchi directly into the mouth and swallowed. Therefore it is impossible to determine the amount and character, and it becomes necessary to observe the animal while coughing to ascertain the condition of the discharge.

Dyspnea.-The degree of dyspnea depends on the obstruction of the free movement of air through the bronchial tubes. In mild cases it may not be noticed but in other cases, in which the calibre of the bronchi is materially recreased by the swelling of the mucous membrane, it may be quite distressing. 
General Symptoms.-Moderate temperature (rise of $1^{\circ}-2^{\circ}$ ), pulse quiekened, partial loss of appetite and increased thirst. In severe cases, there is eomplete loss of appetite, high temperature $\left(104^{\circ}-105^{\circ}\right)$ and general depression. On pereussion nothing abnormal is noted, except in eases where the bronehioli are affeeted and filled with an exudate where there may be local areas of dulness.

Auscultation.-In mild eases the vesicular sound is inereased. In well marked cases the respiratory sounds are harsh with some lengthening of expiration. Absence of sound may be found when obstruction of the bronchi or eollapse of lobules oecurs. Sibilant and sonorous sounds are typical signs whieh are due to the irregular narrowing of the calibre of the large and small bronehi respectively. As the secretion beeomes more liquid, moist rales are produeed in the tubes due to the liquid being driven back and forth by the air eurrents. The volume of the rale is in proportion to the size of the tube in whieh it originates. Large, moist, bubbling rales are produced in the large bronehi, and the small, moist rales arise in the smaller tubes.

Diagnosis.-From the eharaeter of the eough, auseultation and general symptoms one may readily deteet the presence of aeute tracheitis and bronehitis. Careful examination of the animal should be made, however, to determine if the disease is primary or secondary.

Prognosis.-Favorable in most primary eases; when secondary to other diseases the prognosis is not so favorable. In primary eases, the course is usually eight to fourteen day's; in secondary it is mueh longer.

Treatment. - IIygienic.-'The animal must be kept in a warm place $\left(70^{\circ} \mathrm{F}\right.$.) having good ventilation but direct currents of cold air must not reach it.

Dietetic.-Light, soft or liquid foods should be used (milk, extract of beef, soups or small amounts of raw or cooked beef). In eases where the animal will not eat, it should be given warm milk to which raw eggs have been adkled.

Medical.-For the appetite use compound tincture of gentian $(0.8-2.0)$ or tincture of nux vomica (0.3-0.(i drops). In the early stages white the cough is dry and harsh, 
expectorant formula should be used to stimulate the secretion of the mucous membrane, as

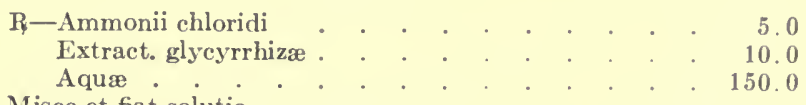

Misce et fiat solutio.

Sig.-Give a teaspoonful three times a day.

or

R-Apomorphini hydrochlorati .

Acidi hydrochlorati

1.00

Aquæ distillata

150.00

Misce et fiat solutio.

Sig.-Give a teaspoonful four times a day.

When severe cough is present, sedatives should be given to reduce the irritation to the nerve endings in the mucous membrane, in the following formula:

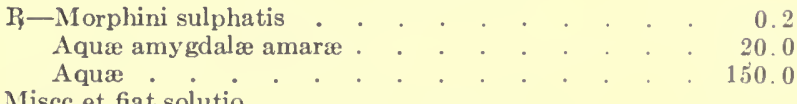

Miscc et fiat solutio.

Sig.-Give teaspoonful every thrce hours.

Counterirritants (oil of mustard and glycerin 1-20) may be applied to the walls of the thorax to stimulate the encrvation and circulation in the trachea and bronchi.

Inhalations of antiscptics and stimulants (oil of turpentine, oil of tar, creolin, etc.) may be used. 'These are best administered by adding them to hot water and allowing the animal to inhale the vapor.

In the treatment of this disease the predisposing causes should be considcred more than the infection as antiseptics cannot be applicd to produce thorough antiscptic action on the mucous membrane.

\section{BRONCHIAL CATARRH OF BIRDS.}

\section{Bronchitis.}

Definition.-An acute or chronic inflammatory condition of the mucous membrane of the bronchial tubes. 
Etiology.-This often occurs from the extension of inflammation of the nasal passages, the larynx and trachea. Exposure to dampness, cold draughts of air and to sudden and extreme changes of temperature are predisposing causes. Specific infections (infectious nasal catarrh, tuberculosis); parasites (Syngamus trachealis); fungi (aspergillus) are all exciting causes and may produce an extensive and severe bronchitis.

Symptoms. - The birds become somewhat stupid, lose appetite, show accelerated respirations and cough. Later the symptoms grow more pronounced inducing severe dyspnea, the mouth frequently held open to facilitate respiration.

Auscultation reveals a blowing or whistling sound in the bronchi, which, later, as the secretions accumulate, is bubbling or rattling.

Diagnosis. - This is made by the dyspnea, cough, stupidity of the patient, and the course of the disease.

Prognosis.-Favorable unless duc to specific infection or parasites.

Treatment.-IIygienic.-Keep the birds in a warm place which is well ventilated and free from cold draughts.

Dietetic.-Give soft and nutritious food (stale bread and milk, oatmeal and milk, meat scraps).

Medical.-Inhalations of vapors from boiling water containing sodium bicarbonate (2 per cent.), creolin ( 2 per cent.) or carbolic acid (3 per cent.).

To stimulate the bronchial secretions use the following:

R-Ammonii cliloridi
Mellis .
Aqua feniculi .

0.5

0.5

50.0

Misce et fiat solutio.

Sig.-Give tcaspoonful three to four times daily.

As a laxative and antiseptic:

\section{$\mathrm{R}$-Olei tercbinthinæ}

0.40

Olei ricini .

2.00

Misce et fiat solutio.

Sig.-Give at once and repeat every four to six lours until a laxative action is produced. 
In treating a large number of birds encourage the appetite with stomachic tonies (iron sulphate) in the food and drinking water.

\section{MYCOTIC PNEUIMONIA.}

\section{Aspergillosis.}

Definition.-A mycotic disease affecting primarily the respiratory passages, often producing bronchitis and pneumonia.

Etiology.- The exciting causes are species of fungi belonging to the genus Aspergillus. These fungi exist abundantly in nature upon all kinds of grain especially during the warm, moist weather. The spores are inhaled with dust or taken with food and lodge on the mucous membrane where they rapidly develop into the fungus, which causes the irritation. The most common species in the domestic birds are the Aspergillus fumigatus, A. nigrescens, A. glaucus and the A. candidus.

Other species are rarely found in the respiratory tract of birds. The predisposing factors are bronchitis, affections of the lungs, delicate breeds of birds, or delicate and weak individuals.

Pathology. - (a) On the mucous membrane of the trachca and bronchi is found a thick membranous mass which bears upon its surface a growth of the fungus. These masses are at first soft but become firmer with age and are yellowish or greenish in color, with some resemblance to a fibrinopurulent exudate. They adhere firmly to the mucous membrane which is thickened and congested. These growths may obstruct the air cells and the smaller bronchi, often being found as caseous or even calcareous nodules, somewhat resembling tuberculosis. The nodules vary in size from that of a pinhead to a pea and may involve the greater part of the lung tissue. Nodules may also be found in the liver, intestines, the mesenteric glands or other organs. In very aeute cases the lungs may show simply an acute inflammation with secondary conditions in other organs. . 
(b) Microscopic examination reveals the spores or filaments of the fungus in most of the lesions, either acute or chronic.

Symptoms. - In the very early stages no symptoms are noted, and it is only after the disease has progressed sufficiently to cause obstruction or irritation to the tissues that symptoms are manifested. The affected birds become weak and depressed, do not follow the flock; the plumage is rough. Respirations are accelerated and during expiration are accompanied by a rattling or snoring sound. The mouth is held open to facilitate breathing. The temperature is elevated, the appetite diminished and thirst increased. There is often a greenish-yellow discharge from the nasal passages. The birds soon become emaciated, a severe diarrhea develops, and death results from exhaustion.

Diagnosis.-Made by finding the spores or fungi by a microscopical examination of scrapings taken from the affected parts.

Prognosis.-Very unfavorable.

Treatment.-Hygienic.-Keep the affected birds in a warm, well ventilated place.

Dietetic.-Feed concentrated nutritious food.

Medical.-Treatment is unsatisfactory in well established cases. Inhalations of antiseptics as tar or turpentine are indicated. These substances may be volatilized with boiling water or the tar by placing it on a hot surface. The affected birds are allowed to inhale the fumes for a half hour every day. Potassium iodid (.05-0.1 per bird daily) is also indicated; sulphate of iron (4.0 to 1000.0) may be used in the drinking water.

Prevention. - The healthy birds should be separated from the sick ones and the premises disinfected (lime or carbolic acid 5 per cent.). The straw and grain should be cleaned before given to the birds, as dust is the common carrier of the fungus. Successful disinfection cannot be attained as the spores oecur widely distributed in nature. Cleaning of the straw and grain as suggested assists in eliminating the fungus. 


\section{ANIMAL PARASITES IN THE TRACHEA AND BRONCHIAL TUBES.}

Syngamosis. Gapes in Chickens.

Definition.-This is a condition due to the Syngarnus trachealis found most commonly in the trachea and bronehi of birds.

Etiology.-The only cause is the parasite Syngamus traehealis, genus Syngamus. This is a small, round worm, red in eolor, and the male is about $5 \mathrm{~mm}$. long and the female 5 to $20 \mathrm{~mm}$. A remarkable feature of thesc worms is permanent copulation, which is so intimate that the males cannot be separated from the females without tearing their integuments. This arrangement gives the worms a forked or Y appearance.

They attach themselves to the mueous membrane of the trachea and larger bronehi and suek the blood. By so doing they produee eonsiderable irritation. 'The ova of the syngamus are not laid but pass from the borly of the female whieh suffers rupture and usually oceurs after death. These ova are hatehed in from one to six weeks according to the temperature on damp ground or in water. 'The embryo does not need an intermediate host in order to beeome transformed into an adult worm. Birds ingest the embryos along with food or water or the adult worm coughed up by an affeeted bird may be swallowed; earth worms in infested yards frequently have embryos in their intestinal traet and when these are swallowed the embryos are liberated. They then burrow out through the walls of the esophagus and stomaeh and migratc through the tissues or possibly via the blood stream until they reach the lungs and trachea. It has been shown that fully developed parasites can be found in the trachea twelve days after feeding the ova to healthy birds.

Symptoms. - 'This disease is seen most frequently in young birds of from one to four weeks old, and is espeeially common in chieks. They have a whistling cough, and the most seriously affeeted open the beak at the same time stretehing 
the neck by a peculiar movement (gaping) in order to facilitate inspiration. The general signs are loss of appetite, dulness, wings pendant and all cvidence of cachexia. Death results from exhaustion or aspliyxiation.

Diagnosis. - The peculiar gaping movement at each inspiration, sneezing, and distressed breathing as from obstruction in the trachea. Finding the worms in the trachea or eggs in the discharge from the mouth is conclusive.

Prognosis.--Unfavorable, but depends upon the number of parasites present in the trachea. A spontaneous recovery is rare, especially in young birds.

Treatment. - All of the young birds should be treated as soon as the disease is noticed. As much garlic as they can be made to eat when chopped fine and mixed with other food, or powdered asafetida (0.25 per head daily) may be used. The volatile part of the garlic or the asafetida is eliminated with the expired air and acts as a toxicant to the parasites in the trachea. Individuals may bc treated by intratraeheal injections of sodium salicylate ( 5 per cent.) using 1 c.c. per bird and injected with a medicine dropper direetly into the trachea. This loosens the worms and they ean be coughed out. In cases of imminent asphyxia the worms may be withdrawn by the aid of a feather, having the barbules all removerl except at the cnd. Or a horsehair folded and twisted so as to form a small loop may be used. It is introluced into the trachea through the open mouth and turned round and round, the object bcing to loosen the worms. 'They may be then withdrawn witl the feather or coughed out. 'This method of ridding the chiek of the parasite is dangerous and insufficient, and can rcmove only those parasites which are slightly attached and in the upper part of the traclea.

Prevention. - Separate the sick hirds from the hcalthy and clean up all coops, pens and rumways, drinking troughs, etc. and disinfeet with carbolie acid (5 per cent.). Lime sprinkled freely throughout the house and in the coops will aid in keeping them elean. Burn all dearl birds and provide clean water and food. 


\section{CHRONIC TRACHEITIS AND BRONCHITIS.}

Definition.-This is a chronic inflammation of the mucous membrane of the trachea and bronchial tubes.

Etiology.-Chronic tracheitis and bronchitis may occur independently or secondary to some other disease. It may originate from any of the causes of acute tracheitis and bronchitis, especially when their action is persistent or frequently repeated. The common causes are:

(a) Continued inhalation of irritating dust, dirt, ete.

(b) Exposure to cold, damp air (as keeping animals in cold, damp basements having poor ventilation).

(c) Parasitic. In severe infestations with Ankylostoma canina, when the animal is being constantly reinfested, the passage of the larvæ through the tracheal and bronchial structure produces a chronic irritation. (See Uncinariasis.) Chronic tracheitis and bronchitis often result from the gradual extension of the inflammatory process from the other parts (laryngitis, pharyngitis, etc.). Many cases are secondary to special diseases such as distemper, carcinoma, sarcoma, rachitis and acute valvular insufficiency.

Pathology.-Chronic tracheitis and bronchitis are practically always purulent, and while in most particulars it closely resembles the acute form, it differs from it in the presence of a more deeply penetrating inflammation and in the production of fibrous tissue. The mucous membrane is swollen, reddened, infiltrated, and covered with purulent secretion. The walls of the bronchi are hypertrophic. Not infrequently the mucosa is thrown into little polypoid excrescences, due partly to contraction and partly to fibrous proliferation. The walls of the bronchi become thickened and there is often a fibrous peribronchitis, which in time may lead to induration of the lungs.

Symptoms. - This condition is most common in old animals where it frequently interferes with respiration producing so-called asthma. 'The most prominent symptom of this disease is the cough. It is usually moist, varying in intensity, depending upon the amount of secretion and extent of mucous membrane involved. Excitement frequently produces par- 
oxysms of coughing which are often attended by severe nausea and vomiting. In cases secondary to other diseases the cough is modified becoming short, isolated and more spasmodic. This is particularly so in chronic bronchitis due to the parasite, Lncinaria canina, and to valvular insufficiency of the heart. The discharge is mucopurulent in character, and is usually swallowed, but during paroxysms of coughing some of it mixed with mucus will be expelled from the mouth. In severe cases when the discharge is decomposed by putrefaction organisms there is a very offensive odor given off with the exhaled air.

Dyspnea.-This is always present to some extent; in old animals it is well marked. 'The dyspnea results from the emphysema and interstitial pulmonary fibrosis which always develops, and, when secretion is present in large amounts, the dyspnea is increased accordingly, producing asthmatic conditions. On auscultation there are sibilant or there may be moist rales, depending on the condition of the membranes and the character and quantity of the secretion. Vesicular sounds are increased. Percussion usually gives negative results, and only in the more severe cases are the general symptoms of emaciation, loss of appetite, etc., noterl.

Diagnosis. - The long continued course of the disease, the age of the animal, the absence of general symptoms in most cases and the characteristic cough are indicative.

Prognosis. - In most cases of chronic tracheitis and bronchitis the prognosis should be considered unfavorable as complete recovery seldom takes place. During the warm, dry months the symptoms often subside only to reappear with the return of the cold, damp weather.

Treatment. - Symptomatic treatment can be used to alleviate the cough and to modify the secretions. 'The following formulæ may be used:

R-Apomorphine hydrochlorati

Misec et fiat solutio.

Sig. - Give teaspoonful three times daily.

$\mathrm{R}$-Acidi benzoici 
In general debilitated conditions tonics and alteratives should be used as in the following formula:

R-Ferri et quinini citratis . . . . . . . . . 10.0 Syrupi . . . . . . . . . . 9 90.0

Misce et fiat solutio.

Sig.-Give teaspoonful once daily.

or

R-Tincture nuces vomicæ . . . . . . . . . 7.0

Tincture gentianiæ . . . . . . . . . . 10.0

Aquæ . . . . . . . . . . . . 60.0

Misce et fiat solutio.

Sig.-Give teaspoonful once daily.

Daily inhalations of medicated vapors (turpentine, creolin, etc.) are valuable to stimulate the secretions and assist in their removal. 


\section{CHAPTER IV.}

\section{DISEASES OF THE LUNGS.}

Examination.-The following things are essential for a complete and thorough examination:

1. The number and character of the respiratory movements.

2. The size, shape and sensitiveness of the thorax.

3. Auscultation.

4. Percussion.

1. Respiration.-In small animals the number of respiratory movements normally varies greatly. The average number while at rest is 12-24. 'This is easily and quickly increased by excitement and exercise until they may reach 60-90 per minute. During warm weather even while at rest the respirations are greatly accelerated, owing to the skin glands not being active enough to assist in the respiratory function. In order to overcome this physiological condition the animal breathes forcibly through the mouth, and the frequency of respirations is increased. Respiratory movements are also increased in the following diseased conditions: Catarrhal pneumonia, foreign body pneumonia, chronic interstitial pneumonia, usually when the temperature is elevated, in laryngitis, acute and chronic bronchitis, hydrothorax, pleurodynia, ascites, peritonitis, valvular insufficiencies, eclampsia and during the early stages of some poisonings. A lessening in the number of respirations is found in narcotic poisoning, diseases of the brain and its membranes and in the later stages of infectious diseases, septicemia and pyemia.

2. The Thorax.-In shape both sides of the chest wall should be symmetrical. Depression on one side indicates fractured ribs or pleurodynia. Distention of the thorax is seen in hydrothorax, and in fluid accumulations (ascites), tumors or when the stomach is distended, causing pressure against the diaphragin.

3. Auscultation is practised by using the phonendoscope or by covering the thoracic wall with a piece of cloth against 
which the ear is placed. With the phonendoscope the sounds are made more audible and distinct.

The normal sounds of the lungs are: (a) The vesicular; (b) bronchial, and (c) the expiratory. (a) The vesicular sound normally is a soft, regular, blowing sound caused by the air passing into the alveoli and distending them. It is most distinct in emaciated animals and where the lung tissue is in close contact with the thoracic walls. It is normally increased by excitement or exercise and is always more distinct in puppies than in older dogs. The vesicular sound is modified in the following pathological conditions: Increased in dyspnea in the healthy portion of the lung, in tracheitis and in bronchitis; decreased in stenosis of the upper air passages; in certain stages of catarrhal pneumonia; emphysema of the lungs; hydrothorax; swelling and thickening of the skin and muscles of the thorax and in certain poisonings.

(b) Bronchial respiratory sounds are best recognized by placing the phonendoscope directly over the trachea at its entrance to the chest. These sounds are normal in the larynx and trachea, but their appearance in the thorax is significant of disease. Bronchial respiratory sounds are increased by secretions in the smaller bronchi, as in catarrhal pneumonia; compression of the lungs by pleuritic exudate; laryngitis, tracheitis and bronchitis.

Irregular bronchial sounds are caused by the secretions being carried to and fro by the passage of the air. They are dry (wheezing) where there is a small quantity of mucus adherent to the mucous membrane. These sounds (wheezing) occur mostly in the smaller bronchi, while the moist, bubbling rales (sonorous) emanate from the larger bronchi where they are produced by the collection of secretions. Cavities in the lungs also produce them. Bronchial sounds are important in determining the existence, extent and character of trachcitis and bronchitis. When sibilant (wheezing) sounds are prominent it indicates that the infection extends into the bronchioli.

(c) Expiratory sounds are produced by the rapid expulsion of air from the lungs, and in normal conditions are hardly perceptible, except in puppies. This sound is increased by 
excitement, exercise, etc. In some diseases it is varied in tone, becoming louder and more prolonged.

4. Percussion.-This is performerl by the use of the percussion hammer and pleximeter or by one hand placed against the chest wall and tapped with the fingers of the other hand. 'The normal sounds of a healthy lung arc heard all over the thorax, the volume of sound depending on the thickness of the lung at the particular part being examined. The normal sound is clear, loud and resonant as distinguished from the tympanitic, dull or solid sound of disease. Tympanitic sounds are heard in the following conditions: Emphysema, $(b)$ pneumothorax, $(c)$ cavities in the lungs, (d) in collapse of the pulmonary tissue from a retraction of the lungs in the presence of pleuritic exudates. The collapsed lung floats upon the surface of the fluid above the line of dulness and on percussion emits a tympanitic sound. (e) In the first and last stages of pneumonias. Dull or flat sounds are heard over hepatized areas of the lungs, chronic interstitial pneumonia; tumors in the lungs, and hydrothorax.

\section{CONGESTION OF THE LUNGS.}

\section{IIyperemia of the Lungs.}

Definition.-An excessive or abnormal accumulation of blood in the lungs. Hyperemia may be cither active or passive.

Active Congestion.-Etiology. - It is found in the early stages of all inflammatory conditions of the lungs and pleura. Occasionally it may be due to inhalations of irritating gases, smoke, acid fumes, etc., or may result from cold.

Pathology.-The lungs are dark red in color; on crosssection blood escapes from the cut surface. The tissue will float in water.

Symptoms. - Active congestion of the lungs is charaeterized by its sudden onset, beginning as a rule witly a chill and rapidly developing dyspmea accompanied by a short, dry cough and a rapid, full pulse. 'The animal often assumes a sitting posture with elbows held outwardly, ribs elevated 
and cheeks inflated at each expiration. The temperature may rise to $103^{\circ}-104^{\circ} \mathrm{F}$., possibly higher, but it does not persist, falling by crisis in three or four days. Owing to the aëration of the lungs being diminished the visible mucous membranes become cyanotic. On auscultation the respiratory sounds will be increased, harsh and rough, and rales of various kinds may be heard. At the base of the lung fine crepitant or subcrepitant rales may be distinctly audible, while over other parts sibilant or sonorous rales may be heard. Percussion reveals only slight dulness.

Diagnosis.-Active congestion of the lungs in the beginning possesses no distinctive characteristics, but resembles bronchopneumonia. As it progresses it will be readily distinguished from this condition by the absence of hepatization and the occurrence of the crisis on the fourth or fifth day.

Prognosis. - Usually favorable but in some cases pulmonary edema with fatal results, or pneumonia may develop. 'The affection is always to be looked upon as serious and one demanding active treatment.

Treatment. - Hygienic. - The animal must be kept in a warm place and it is important that it be well ventilated.

Dietetic.-Give warm liquid foods (milk, beef tea, soups, etc.).

Medical.-Magnesium sulphate (8.0-10.0 in cold water) should be used as a hydragogue purgative. To equalize the circulation tincture of aconite $(0.01-0.1)$ or veratrum viride $(0.1-0.2)$ is beneficial and can be given every three to five hours if necessary. Oil of mustard and glycerin (1-20) can be applied to the walls of the thorax as a counterirritant. When the initial intensity of the symptoms yields, then a stimulating expectorant as ammonium chlorid should be used as follows:

P-Ammonii chloridi

Extract. glycyrrhizæ .

5.0

10.0

Aquæ

150.0

Misce et fiat solutio.

Sig.-Give a teaspoonful three times a day.

Passive Congestion. - Etiology. - This results from obstruction to the free outflow of the blood from the lungs to the 
heart. It is nearly always due to the lack of force or faulty action of the heart, as stenosis or insufficiency of the mitral valve, dilation of the right ventricle, or fatty degeneration. It may also be caused by pressure on the pulmonary veins by tumors, etc. In the latter stages of long continued diseases or in any febrile condition when the heart action is slowed, the blood gravitates to the lower part of the lung producing a hypostatic congestion.

Pathology.-The lung is somewhat enlarged, its consistency increased, of a dark red color, and on section venous blood escapes. When the condition has persisted for a long time, the lung becomes brown, dense and firm, due to a deposit of blood pigment and a proliferation of fibrous tissue in the septa of the lung, a condition known as brown induration.

Symptoms.- Dyspnea and a short, hard and usually dry cough which has a tendency to become worse with exercise or excitement. The bronchial discharge varies in amount and in some cases may contain blood. Auscultation reveals rales. On percussion only slight dulness may be noted. The membranes are cyanotic.

Diagnosis.-The dyspnea, cyanosis, characteristic cough and shallow respirations without rise of temperature are indicative.

Prognosis. - Unfavorable, depending upon the cause and extent.

Treatment.-In the treatment of passive congestion of the lungs, medicinal agents directed against the causative cardiac affection are the ones which will most favorably influence the pulmonary condition. As a heart stimulant the following formula gives good results:

$\mathrm{P}$-Extracti digitalis fluidi

Syrupus pruni virginianæ

60.0

Misce et fiat solutio.

Sig.-Give one teaspoonful once a day.

In addition to this formula strychnin sulphate (0.001 daily) may be used as a general stimulant. To relieve the cough when severe use:

P -Morphini sulphatis

Aquæ amygdalæ amaræ .

Misce et fiat solutio.

Sig.-Give a teaspoonful twice daily. 
If the animal's condition will permit the use of a hydragogue purgative, magnesium sulphate $(8.0-10.0)$ is useful to modify the circulation and remove fluid from it. This dose may be repeated in twelve hours if sufficient action has not taken place.

\section{PULMONARY EDEMA.}

\section{Edema of the Lungs.}

Definition.-An effusion of serous fluid into the alveoli and interstitial tissue of the lungs.

Etiology.-Diseases of the heart (valvular insufficiency, etc.) are the most frequent predisposing causes of pulmonary edema, although it occurs as a secondary condition to chronic pulmonary congestion, nephritis, cachexia, anemia and infectious diseases (distemper, etc.). In the latter stages of distemper pulmonary edema frequently develops when it is often the immediate cause of death. Edema of the lungs occasionally follows the administration of drugs (pilocarpin, etc.).

Pathology.-The lung is heavier and firmer than normal, pale in color and pits on pressure. When incised a thin serous fluid escapes. This may be clear or if there is congestion present it may be stained with blood. Crepitation is always lessened and small patches may be entirely airless. The bronchi contain a frothy fluid.

Symptoms. - The symptoms develop rapidly except in those cases occurring as the terminal event in exhaustive diseases in which the course is more gradual and the symptoms less pronounced. Severe dyspnea, which becomes worse as the exudate accumulates is an important symptom. The pulse is weak and small, the visible mucous membranes are cyanotic and the extremities cold. A short, feeble cough often accompanies the condition. The temperature is subnormal. 'There may be a frothy nasal discharge. I pon auscultation various kinds of rales (fine, course or bubbling) will be heard. Percussion reveals diminished normal resonance with occasional small areas of tympany, especially over the posterior part of the lungs. 
Diagnosis.-The sudden onset, severe drspnea and the absence of temperature, accompanicl by moist, bubbling rales on auscultation are indicative.

Prognosis. - Always unfarorable, depending upon the cause. Recovery may occur in mild acute cases.

Treatment. - Treatment must be directed first to relieve the edema which threatens the life of the animal, and secondarily to overcome the cause. For the first purpose use counterirritation to the walls of the thorax, oil of mustard and glycerin (1-20) or Priesnitz compress (applied hot); also strychnin sulphate (0.001 subcutaneously) as a heart stimulant. Subcutaneous injections of ether $(0.8)$, or camphor $(0.1)$ in ether (0.8) are often useful as prompt stimulants. Atropin (0.002) is of value as a heart stimulant and is espccially useful in cases of edema due to pilocarpin, being a physiological antidote.

In the treatment for the removal of the cause digitalis fluidextract $(0.1)$ is most valuable to regulate the heart action. Hydragogue purgation (magnesium sulphate 8.012.0 ) will remove fluid from the blood which tends to reduce the exudation of serum in to the lungs; also the use of a diuretic (oil of juniper $0.3-0.5$ ) for the same purpose in cascs of renal disease.

\section{BRONCHOPNEUMONIA.}

\section{Catarrhal Pneumonia.}

Definition.- This is an affection of the lungs in which the usual sequence of events is, that an inflammation of the smaller bronchioles in scattcred areas is succeeded by the involvement of anatomically related or contiguous resicles.

Etiology.- (a) 'The inhalation of foreign material in the form of small particles of dirt, dust, etc., which irritates the mucous membrane and modifies the secretions, favors the development of infection and leads to inflammation of the bronchioli and alveoli.

(b) Irritating chemicals and gases when inhalerl produce an active congestion and inflammation of the lungs. However, in most cases when inhaled in large quantities they act 
as irritants to the mucous membrane and thus favor the derclopment of infection which leads to bronchopneumonia.

(c) Cold, damp, changeable weather is a very important predisposing factor inasmuch as chilling the surface of the animal's body modifies the circulation and the secretions of the mucous membrane, reducing its resistance, as well as the general resistance of the body. This favors the growth of infection which may be already present. The effect of cold, damp weather is well shown by the greater incidence of the disease in the winter and spring months. Young puppies, especially, are quite frequently affected with bronchopneumonia when kept in cold, damp and poorly ventilated kennels.

(d) Bronchopneumonia is frequently sccondary to specific infections (distemper, etc.) which reduces the general as well as the local resistance.

(e) Inflammation of other parts of the respiratory system may produce bronchopneumonia by an extension of the inflammation. This is especially true of acute bronchitis which by extension at first produces a capillary bronchitis and later a bronchopneumonia.

(f) During the course of some diseases (nephritis, endocarditis, diabetes, valvular insufficiencies, sarcomas and carcinomas), the hcart action is disturbed, the vasomotor nerve is enfeebled, both of which reduce thic resistance to infection. Young puppies infested with parasites, which interfere with the general nutrition, frequently develop bronchopneumonia from the reduced resistance to infection.

(g) Infectious material from the mouth and the nasal passages which reach the bronchial tubes and alveoli will produce a bronchopncumonia.

Possibly in no other disease do lessened powers of resistance of the animal, from whatever cause, play such an important part in determining the inception.

Pathology.-Whe process in bronchopneumonia is associated with bronchitis, and indecd almost always starts with an inflamination of the smaller bronchioles, which then spreads to the adjacent alveoli. The exudatc is at first serous and contains a few erythrocytes, but more numerous white cells. 'There is also a large number of mononuclear cells with clear 
protoplasm, which are swollen desquamated epithelial cells from the alveolar walls. The disease usually affects both lungs, but may involve only one or even a single lobe or a portion thereof. The affected organ is heavier than normal, somewhat congested, and in its substance can be felt areas of increased consistence. These are friable, of a reddishgray, gray or grayish-yellow color, contrasting somewhat with the rest of the lung.

On pressure a turbid fluid can be expressed, in which can be seen small particles of a gray, grayish-yellow, or purulent appearance. From the sporadic distribution of the consolidated areas, the term "splenization" has been given to the condition. On section of the lung, both red and gray stages are recognized. The smaller bronchi and bronchioli show inflammation and are filled with exudate. In the alveolar spaces the exudate consists mainly of serum, a few red cells, abundant leukocytes and desquamated cells. The latter frequently contain pigment and bacteria. In the form due to inhalation of infective material, or foreign bodies', the exudate is usually purulent. Such a condition leads to a diffuse purulent infiltration of the lungs and abscess formation. Occasionally gangrene of some of the lung tissue will be noted. This is especially true in weak and debilitated animals with general circulatory disturbance.

Symptoms. - The symptoms of primary bronchopreumonia develop rapidly, usually beginning with a chill and the animal appears depressed and restless. The temperature is high $\left(104^{\circ}-105^{\circ} \mathrm{F}\right.$.) and falls by crisis. Dyspnea is pronounced, the respirations being short, shallow and very rapid, inflating the cheeks at each expiration. A short painful cough is noticed and the pulse is accelerated (180-200). On auscultation the sounds are mostly those of an acute bronchitis (impairment of the vesicular murmur, moist sibilant or sonorous rales). On pereussion dulness is noted where there is a large area of consolidation, while in cases where the consolidated areas are scattered compensatory emphysema may overcome the dulness. In the secondary forms following other diseases (bronchitis, distemper, etc.) the onset is less severe and the symptoms less pronounced. The congh and 
high temperature may be absent, but if temperature bc present it falls by lysis. Dyspnea is usually secn indicating a lack of aëration of the lungs. In the course of this secondary bronehopneumonia the symptoms are not well marked and depend largely upon the primary disease.

Diagnosis. - This is made in the primary form by the sudden onset with severe dyspnea, eough and high temperature, while in the secondary form the symptoms, not being charaeteristic, the diagnosis is more diffieult, depending mostly upon the dyspnea with its attending conditions.

Prognosis.-Unfavorable. In the primary form the prognosis is determined by the extent of the inflammatory process in the lungs, while in the seeondary form it depends upon the causative disease, and as this is usually distemper the mortality is high.

Treatment.-Hygienic. - The animal should be kept in a warm, well ventilated room free from eold draughts.

Dietetic. - The animal's strength should be sustained by the use of appetizing foods (milk, beef extract, etc.) given warm in small quantities every few hours. During eonvalescence small amounts of lean meat or other nutritious food may be given 3 or 4 times a day.

Medical.-At the onset a purgative should be given (ealomel 0.03 and sodium bicarbonate 0.5 ) to producc free purgation and stimulate the activity of the kidneys. Expectorants are useful to modify and aid in expelling the discharge from the bronehial tubes.

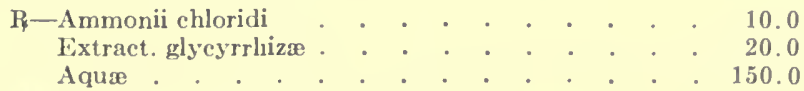

Misce et fiat solutio.

Sig.-Give teaspoonful four times daily.

Counterirritants to the walls of the thorax in the form of oil of mustard and glyeerin (1-20) or Priesnitz eompress applied hot around the chest. The heart aetion should be stimulated in severe cases by the use of digitalis fluid-extract (0.08-0.1) and for general stimulation use strychnin (0.001). Inhalations of medicated vapors (turpentine, oil of tar, ereolin in hot water) are useful to stimulate the mucous nembrane and to produce antiscptie aetion. 


\section{CIRRHOSIS OF THE LUNGS.}

\section{C'hronic Interstitial Pnenmonia.}

Definition. - A chronic inflammatory condition of the lungs characterized by an increase in the interstitial tissue and fibroid collections in the alveoli.

Etiology.- (a) Inhalation of particles of dust when continued for a long period produces fibrosis of the lungs, due to the chronic irritation of the foreign material in the tissues. The degree depends upon the amount and character of the inhaled material.

(b) Pressure upon the lungs by neighboring structures, as new growths and diverticula of the esophagus, tumors in the lung substance, all of which produce a reactive inflammation resulting in inçreased connective tissue proliferation.

(c) One of the most frequent causes in small animals is bronchopneumonia. When it terminates atypically fibroid changes occur in some of the involved lobules. In these cases the fibrosis begins as a chronic bronchitis or peribronchitis, invading later the surrounding parenchyma of the lung, which results in a great increase in the interstitial tissue. 'This is a frequent sequel to bronchopneumonia occurring during the course of infectious diseases (distemper, etc.). For some unexplained reason resolution does not occur, and the fibrinous exudate collected in the alveoli during the stage of red hepatization is displaced by fibrous tissue. The connective tissue formation necessarily begins in the alveolar walls, as from this source nust be derived the new vessels which appear in the intra-alveolar new formations. Proliferative changes in the alveolar epithelium may for a time be active during this transformation of the exudate, but eventually the new tissue within the alveoli merges with the thickened, enclosing walls, which take a relatively inactive part in the process, and the area becomes entirely fibroil. I"sually this lesion is only in parts of the lobules, but in some cases may extend into a considerable portion of the lung tissue, involving the interstitial connective tissue which is greatly increased in amount.

(d) Localized areas of interstitial pneumonia may originate 
from abscesses in the lungs, sarcomas and carcinomas, or from inflammatory reactions from the plcura.

Pathology. - Cirrhosis is characterized by the overgrowth of connective tissue in the lungs so that they become hard, traversed by fibrous bands, and more or less shrunken. The condition leads to destruction of the alveolar spaces, sometimes with bronchiectasis, and always to marked impairment of function. When due to lobular pneumonia, which is the common form of pneumonia in small animals, the fibrous tissue production follows the course of the bronchi and bronchioli. The lung is greatly increased in weight, has lost its spongy condition, and is quite hard. On cut surface it shows grayish-white color, and sometimes caseous nodules of necrosis may be seen. In advanced cases the pleura is thickened and the lungs distorted. The two layers of the pleura are often adherent and the mediastinum may be indurated.

Symptoms.-Moderate dyspnca and a chronic cough, with a discharge which may be slight or profuse, and the exhaled air has a fetid odor due to the retention of some of the discharge in the bronchiectatic cavitics. The temperature is usually normal and no general symptoms are noticed.

Auscultation reveals increased resonance and bronchial breathing. Percussion yields dulness except where there are saccular dilatations of the bronchi.

Diagnosis. - The history of the case and slow devclopment following bronchopneumonia and the presence of sevcre dyspnea will materially aid in making the diagnosis.

Prognosis. - Incurable.

Treatment.--Expectorants and general stimulants may be used to modify the symptoms.

\section{FOREIGN BODY PNEUMONIA.}

\section{Gangrene of the Lungs.}

Definition.-An inflammation of the lungs duc to the inhalation of coarsc material which usually results in necrosis of a part or of the entire organ.

Etiology.-(a) Inhalation of foreign matcrial (particles of food from the mouth, especially during anesthesia when vomition occurs, also when the pharynx and larynx are par- 
alyzed); injury to the lungs from contusions, fractured ribs, penetrating wounds through the thoracic walls or from the esophagus and stomach (swallowed neelles, sharp pieces of bone, etc.).

(b) Inhalation of irritant medicinal substances when improperly administered (by closing the nasal openings, pulling the tongue forward or holding the mouth open too wide; holding the head too high or in any position which interferes with swallowing, manipulating the larynx or pharynx).

(c) The causes enumerated above are to be considered as predisposing factors, inasmuch as infection must be present to complete the process. In addition to the ordinary infection, which is normally present in the lungs, putrefactive organisms are necessary to produce gangrene.

Pathology.-The involvement may be either diffuse or circumscribed, usually the latter, and in the form of irregular areas having a brown, greenish or black color. These are dry and surrounded by a zone of congestion and around this a zone of edema. The gangrene is of the moist variety and gives off a very penetrating odor. Cavities may occur in the lungs when the necrotic material is coughed out.

Symptoms.-Dyspnea is pronounced and the expired air has a sweet, fetid odor. 'The temperature is elevated $\left(104^{\circ}-\right.$ $106^{\circ} \mathrm{F}$.) and the pulse is small, rapid and very irregular. In the early stages the symptoms are similar to bronchopneumonia, but when cavities appear in the lungs the symptoms rapidly increase in severity. It is at this stage when the odor is a prominent symptom.

Diagnosis. - This rests upon the characteristic odlor of the exhaled air, high temperature and the rapid development. The history of the case often assists materially in the diagnosis.

Prognosis. - Very unfavorable. Most cases terminate fatally. in a few days.

Treatment.-The animal's strength shoukl be maintained by the use of highly concentrated foods (raw eggs, extract of beef, milk, etc.), given at frequent interrals.

Little can be expected of medical treatment. Inhalations (oil of tar, turpentine, or creolin) may be used to overome the odor and produce antiseptic action. 


\section{CHA P TER V. \\ DISEASES OF THE PLELRA.}

\section{PLEURITIS. PLEURISY.}

Definition.-An inflammation of the pleura accompanied by a serous, serofibrinous, hemorrhagic, or purulent exudation into the pleural cavity.

Etiology.-(a) Penetrating wounds through the thoracic walls, from fractured ribs or sharp foreign bodies in the esophagus or stomach; contusion of the thoracic walls; tumors in adjacent parts; in rare cases cysts of tapeworms. These conditions make possible the entrance of organisms directly into the pleura, or by lowering the resistance of the pleura which favors the growth of organisms that are carried to it by the lymph and blood streams. These are quite numerous and consist most often of the following: Bacillus bipolaris, Staphylococcus pyogenes, Leptothrix buccalis, and Streptothrix canis.

(b) Occurs in cases of nephritis from an accumulation of waste products in the blood or by bacterial toxins which are carried to the pleura by the blood and produce direct irritation to the serous membrane.

(c) Exposure to cold and cold dampness are common predisposing factors as they reduce the general resistance to infection.

(d) Pleuritis frequently follows bronchitis and pneumonia, the organisms present enter the lymph and blood streams and are carried to the pleural cavity. In some cases of pneunonia where abscesses develop and open' into the pleural cavity a purulent pleuritis is produced.

(e) Extension of inflammation of neighboring tissues, viz: Mediastinitis during the course of infectious diseases; acute peritonitis; inflammation of abdominal organs. 
(f) Pleuritis is very often found during the course of infectious diseases. In the dog it occurs frequently as a secondary disease following distemper, septicemia and pyemia, the infection being carried by the blood and lymph. It is also seen during the course of rheumatic conditions.

(g) Other predisposing causes are extreme exertion (hunting dogs, greyhounds), or living under adverse conditions (bench shows, during transportation, etc.) which reduce the natural resistance.

Pathology. - The involvement in pleuritis may be acute or chronic, local or general, and according to the variety of exudate, serous, serofibrinous, hemorrhagic or purulent. A single case of pleuritis may pass through all of the above stages. In all cases the pleura becomes hyperemic, rough and dull. The roughness interferes with the free movement of the gliding surface producing pain and the characteristic frictional sounds. Later exudation takes place into the pleural cavity. In the fibrinous form there is soon an exudate of fibrin, forming a thin, yellowish layer on the surface, and as this increases it causes the pleural surfaces to adhere together slightly. The exudate is composed of flakes and masses of fibrin containing leukocytes. The exudate may be absorbed completely, but if there has been much fibrin formation adhesions of varying density result. These adhesions later are organizcd into connective tissue which is at first delicate but soon becomes very dense, and in some cases so extensive as to obliterate almost completely the pleural cavity; or they may be in scattered areas only.

In serofibrinous pleuritis there is a large anount of serous as well as fibrinous exudate. It may originate as the fibrinous form, but usually begins with a serous outpouring. This exudate is denser than the transudate seen in hydrothorax, and contains the usual inflammatory products. 'The amount of fluid will vary with individual cases and in the different small animals from 100 c.c. to 5000 r.e. When present in large quantities the lung is pushed backward and the neighboring organs pressed upon. 'This pressure on the lungs of the dog and cat is often sufficient to completely occlude the passage of air into the right posterior lobes. 
Hemorrhagic pleuritis is generally the result of infectious diseases and malignant growths on the pleura. The exudate is chiefly serous, with red blood cells present, but at times may be almost pure blood.

Purulent pleuritis (pleuritis purulenta; pyothorax; empyema) is the result of infection by some one of the many pyogenic organisms (staphylococcus, etc.). It may begin as a purulent pleuritis or it may follow infection of the serofibrinous form. In the pleural cavity is found a varying amount of cloudy fluid which contains a great number of pus cells. It may be greenish in color at times, but is usually yellowish. The pleuræ are generally thickened and congested and covered with flakes of fibrin or degenerated endothelium. The pus may be completely absorbed and the two surfaces unite with dense adhesions; or it may become caseated and undergo calcareous infiltration. The changes are most marked in the visceral pleura, which becomes greatly thickened, at first being soft and edematous while fluid is present, but as.this disappears it becomes indurated. During the course of purulent pleuritis there is always more or less involvement of the lungs which in some cases may result in pleurogenic pneumonia. In some chronic cases of pleuritis (pleuritis granulosa) there are numerous papillomalike enlargements distributed over the surface of the pleura, and these by becoming confluent may produce extensive masses which interfere with the function of the neighboring tissues and organs.

'The serous exudate contains endothelial cells, white corpuscles and small particles of fibrin. The infected exudate contains numerous pus cells, endothelial cells, and various kinds of detritis. A rather high percentage of albumin is found in the serous exudate.

Symptoms. - In the early stages of pleuritis the symptoms manifest themselves in various ways, depending largely whether the disease is primary or secondary, local or general. In acute, primary pleuritis the disease usually begins abruptly with pronounced general symptoms, viz: A chill, the animal seeking a warm place, twitching and trembling of the muscles, followed in a few hours with an elevation of temperature 
$\left(103^{\circ}-105^{\circ} \mathrm{F}\right.$.) and a small, weak and thready pulse. Cough appears early, is usually dry, and on account of the pain, is partially suppressed. The gait is stiff and they show considerable pain when moved. 'There is little or no appetite, but as a rule the thirst is intense. The visible mucous membranes are reddened and congested, and in cases where there is much exudation the membranes are cranotic. Constipation is often noted, the feces being quite dry and hard. 'The urine, while the exudate is forming, is roided in small quantities and contains albumin. Later as it is absorbed the urine is increased in amount and of very light color.

Dyspnea is quite marked in nearly all cases of pleuritis. In the early stages when the pleura is dry, the respirations are superficial, rapid and painful, but after exudation takes place they are less painful but labored. When bilateral the animal usually assumes a sitting posture, but when only one side is affected, will lie on the affected side. These positions are assumed to assist in fixing the intercostal muscles to relieve the pain which accompanies the elevation and depression of the ribs. 'The abdominal type of respiration is used, the elbows being held outwardly and the abdominal muscles and the diaphragm brought into action. There is less expansion on the affected side on account of the pain; when both sides are affected the ribs are held in a fixed position. Palpation of affected parts produces acute pain.

Percussion. Dulness which gradually rises as the fluid accumulates in the cavity. The upper line of dulness is horizontal and varies with the position of the animal. Above the level of the accumulated fluid tympany will be noter.

Auscultation. In the early stages when the inflamed membrane is dry, frictional sounds are characteristic and the vesicular murmur is increased, but as the exudate collects the sounds become less distinct or blowing in character, and finally only the bronchial tones will be audible.

In the chronic form all the acute symptoms are modified with slight, if any general disturbance, although the temperature may be variable, changing daily from below to above normal $\left(100^{\circ}-103^{\circ} \mathrm{I}\right.$. . $)$.

Auscultation gives negative results. Pleuritic adhesions cannot be diagnosed during life. 
Diagnosis.-The diagnosis depends upon the painful respiration, pain on palpation, abdominal type of respiration, and the presence of fluid in the thoracic cavity. History of injury may also aid materially.

Prognosis. - Usually favorable in mild, acute cases or when local; the generalized purulent forms rarely recover.

Treatment. - Hygienic. - It is necessary that the animal be kept in a warm and well ventilated place but free from draughts of cold, moist air.

Dietetic.-Concentrated food should be given 3 or 4 times daily, using warm milk, eggs or extract of beef.

Medical.-In the very early stages of pleuritis when the frictional sounds are present, cold applications to the walls of the thorax are indicated (cold water compresses, ice packs, etc.). The refrigeration from these cold applications penetrates the walls and contracts the vessels, thus relieving the congestion of the pleura. Later (two or three days) warm, moist compresses are indicated to increase the circulation which will modify the inflammatory process and hasten the absorption of the fluid. In addition to this, counterirritants (oil of mustard and alcohol 1-20, tincture of cantharides, etc.) could be used to continue the same process. When the cough is severe, narcotic agents (morphin sulphate 0.025 daily) can be used to allay the irritation. Quinin sulphate ( 0.2 three times daily) may be used to control the temperature and pulse if they become too high. Encourage diuresis by using fluidextract of digitalis (0.1-0.2 daily) or diuretin (0.2-0.5 three times daily). If constipation be present catharsis may be produced by the use of calomel (0.05-0.5 daily) or magnesium sulphate $(5.0-10.0)$ to produce a hydragogue action. When fluid is present absorption may be induced by the use of potassium iodid $(0.2-0.3$ three times daily) or pilocarpin (0.005-0.1) except in cases of feeble heart action when they must be used sparingly.

Surgical.-The effusion will require aspiration under the following conditions: (a) When it excites much dyspnea; (b) when in large quantities; $(c)$ when purulent; $(d)$ when it remains unabsorbed after ten to twenty days' treatment.

Thoracentesis is performed by the use of a thoroughly 
sterilized trocar and cannula or aspirating needle. It is best done with the animal in either a sitting or standing position. Remove the hair from and disinfect a small area on the side of the thorax so that the instrument may be inserted (in a forward direction) at the anterior border of the sixth, seventh and eighth ribs and as low in the cavity as possible. 'The cavity is reached as soon as resistance to the passage of the needle has ceased. 'The fluid flows out in a continuous stream at first, then synchronous with respiration. After some of the fluid is out, the air may rush in during inspiration, and to prevent this hold the finger over the tube at the end of each expiration. If the flow ceases suddenly it is due to plugging with flakes of fibrin which can be forced away by reinsertion of the trocar. The amount of fluid removed depends upon the heart action. When it becomes weak and rapid, or when coughing suddenly develops, the operation should be discontinued. This can be done daily at a different site of puncture until all of the fluid is removed.

\section{HYDROTHORAX.}

Definition.-A collection of serous fluid in the thoracic cavity without inflammation of the pleura.

Etiology.-This condition always oceurs as a secondary process and is a symptom of many affections (insufficiency of the heart; nephritis; chronic diseases of the lungs). It usually accompanies ascites, hydropericardium and edema of the skin; also due to obstruction of ressels (vena azygos); follows a general anemia, hydremia, chronic infectious diseases, carcinomas and sarcomas. These latter diseases damage the endothelium of the vessels and allow the transudation of fluid from them.

Pathology.- The transudate is light or reddish-yellow in color and contains a few flakes of fibrin. If the condition develops very rapidly, the transudate contains many red corpuscles. 'The specific gravity and albumin content are less than that of blood serum. T'he pleura may be thickened and pale. 
Symptoms.-Hydrothorax develops rapidly and on both sides of the thoracic cavity. Dyspnea results from compression of the lungs. In severe cases this compression may bc sufficient to cause edema of the lungs. The temperaturc is normal. The shape of the chest is unchanged except when a very large quantity of fluid is present causing the lower part of the thorax to be depressed.

Auscultation reveals hard respiratory sounds due to increased respiration. Percussion over the lower part of the chest reveals dulness, the upper lcvel of which changes with the position of the animal. Above this level tympany is pronounced.

Diagnosis. - Dyspnea with sudden onset in the absence of other symptoms; percussion, and exploratory puncture.

Prognosis.-Depends upon the causative disease.

Treatment.-Medical.-Salinc purgatives (magnesium sulphate, 8.0-12.0) may assist in the removal of the fluid by eliminating fluid from the circulation.

Surgical.-Thoracentesis (see plcuritis), when respiration becomes distressed, and cyanosis is evident. Repeat as often as necessary, using a new site for puncture each time. Further treatment is to be directed to the primary causc.

\section{PNEUMOTHORAX.}

Definition.-Air in the pleural sac.

Etiology.-Mechanical.-Perforating wounds from the exterior (injuries, exploratory puncture, etc.); perforation through the diaphragm by abscesses of the liver, stomach or esophagus; perforation from the lung by abscesses or rupture of air vessels in the normal lung. Gas-producing organisms (B. aërogenes capsulatus) in the pleural exudates are occasional causes.

Pathology.-Pneumothorax rarely occurs by itself, usually being associated with serofibrinous (hydropnemmothorax) or infectious pleuritis (pyopncumothorax), due to infection being carricd in with the air. A serous or purulent fluirl is found in the pleural sacs and the membrancs are inflamer. 
Symptoms.-Dyspnea is usually quite pronouncerl, the mucous membrane cyanotic and the pulse rapid and feeble. The physical signs are very distinctive. The affected side shows marked enlargement and the heart beat is displaced.

On percussion the resonance is usually tympanitic, and depending upon the degree of tension there may be flat tympany, or a full hyperresonant tone like emphysema, while in others with extreme tension dulness will be noticed. There is usually dulness at the lower part due to the effused fluid, which can readily be made to change the level by changing the position of the animal.

On auscultation the normal lung sounds are suppressed on the affected side and exaggerated on the other side, which is very suggestive. The rales have a peculiar metallic sound.

Diagnosis. - The dyspnea, enlargement of the affected side, small amount of effusion in the cavity and cyanotic membranes.

Prognosis.-Depends upon the cause but is usually favorable.

Treatment. - In pneumothorax with extreme tension immediate aspiration (see thoracentesis) should be performed. Penetrating wounds through the thoracic walls should be occluded (adhesive plasters, bandage, etc.) to prevent further entrance of air and infection into the cavity. A small amount of it will be readily absorbed and if infection has not been carried into the cavity recovery will be prompt.

\section{HEMOTHORAX.}

Definition. - An effusion of pure blood into the pleural sace. Etiology. - Traumatism, rupture of the vessels from overexertion especially when the walls of the vessels are diseased (carcinomas, sarcomas, and infections).

Pathology. - Presence of pure blood in the thoracie "avity" and the injury to the ressel wall.

Symptoms. - The symptoms are those of an acute anemia accompanied by a rapid filling of the thoracic cavity. 
Diagnosis. - The above symptoms in addition to thoracentesis confirm the diagnosis.

Prognosis.-Depends upon the extent and nature of the injury.

Treatment.-Medical.-The administration of internal astringents (ergot fld. ext. 2.0-4.0) or adrenalin chlorid (0.6-4.0 of 1-1000 sol.). These can be repeated in one or two hours if necessary. Intravenous injections of normal salt solution to maintain the blood pressure may also be used. 


\title{
PART II. \\ DISEASES OF THE CIRCLLATORY SYSTEII.
}

\author{
CHAPTER I. \\ DISEASES OF THE PERICARIDIUN.
}

Examination.-An examination of the circulatory system in small animals is of importance in determining diseases affecting primarily the heart and bloodvessels, and also for assisting in the diagnosis of a number of acutc infectious and non-infectious diseases.

A systematic examination from a clinical standpoint includes the following: (1) The pulse, and (2) the heart.

1. The pulse is best examined in the dog by slightly pressing the femoral artery with the index finger, or the radial artery inside the forearm. In small dogs this is often difficult, and in such cases the hand should be placed against the thorax on the left side just behind the elbow, and at the lower third of the cavity, where the heart beat is readily distinguished. In cats and rabbits this method is the most satisfactory. In birds the pulse is difficult to recognize owing to the vessels being wcll protected and the heart very small and surrounded with fat and the other organs. A phonendoscope is best used to detect the heart beats. Clinically wc must consider: (a) The frequency, $(b)$ the rhy thm or cadence, and $(c)$ the quality of the pulse.

(a) The frequency of the pulse varies considcrably in different species of small animals and bircls, and also in individual animals of the same species. 'The size, age, sex, breeding, temperature, time of day, ete., all have a decided 
influence upon it. The average frequency for small animals is as follows:

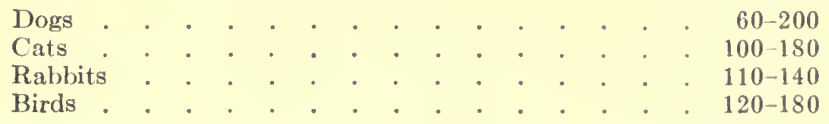

An abnormal inerease in the pulse is found in all elevations of temperature, in severe injuries (fraetures of bones, etc.), in mental exeitement, valvular defeets, severe hemorrhage, and in heart weakness from any eause.

A decrease in frequency, or slow pulse, oecurs in the later stages of some poisonings, diseases of the brain (chronic and subacute hydrocephalus), tumors in the brain, icterus gravis, collapse, etc.

(b) The rhythm of the pulse should be regular, espeeially in eats, rabbits and birds; in the dog an irregular pulsc is found so frequently in apparently healthy animals, that it should not be looked upon as an abnormal eondition; in faet, an irregular pulse in the $\operatorname{dog}$ is the rule. An irregular or arhythmic pulse is therefore a physiological condition in some of the small animals. However, in many pathological conditions a pronouneed irregularity oeeurs as, during eonvalescence from infeetious diseases, severe gastro-intestinal disturbances, severe weakness, and in some ehronie eonstitutional diseases. An irregular pulse is obscrved often after large doses of digitalis.

(c) The quality of the pulse. The pulse beat should be of cqual volume, and it varies with the different species of animal. In all small animals the pulse is rapid, strong and hard. In the $\log$ an unequal pulse is frequently observerl. The pulse is full and distended after exercise, during the early' stages of a number of diseases; empty after severe hemorrhage, intense heart weakness and collapse. The pulse is hard and full in severe pain, peritonitis, and aeute brain discases.

The venous pulse is often observed in old dogs and is usually indieative of some ehronie heart affeetion, or general heart weakness. 
2. The Heart.-This is best examined in small animals, by (a) palpation, (b) percussion, and (c) auscultation.

(a) Palpation. The heart beat is reaclily felt in all small animals by placing the hand over the cardiac region (between the fourth and seventh ribs, lower third of thorax on either side). The heart beat can be felt on both sides of the chest. This method of determining the frequency of the heart beat is practical especially in very young or small animals, and, as noted, also gives us the frequency of the pulse.

The force of the heart beat depends largely upon the condition of the animal (emaciated or fat), and whether taken during exercise, excitement or at rest.

The force of the heart beat is increased in the following conditions:

In hypertrophy of the heart, acute myocarditis, endocarditis, and pericarditis; some poisons, such as aconite and digitalis; after severe exertion or excitement; after considerable loss of blood; where the temperature is elevated. The heart beat is weakened in the following: Degeneration of the heart muscle; the later stages of acute infectious diseases; some poisonings; compression of the heart in hydrothorax, hydropericardium, pneumopericardium; emphysema of the lungs, and in the effusion stage of pleuritis. In unilateral pleuritis the normal force of the beat may be more plainly noted on the healthy than on the diseased side.

(b) Percussion. 'This is of little value in diagnosis in small animals. The zone of cardiac dumess is between the fourth and seventh ribs. 'This zone is normally about two to two and one-half inches in diameter.

The zone of carliac dulness is increased in hypertrophy of the heart; hydropericardium; tumors and induration of the lungs (chronic interstitial pneumonia). The zone is decreased in emphysenta of the lungs and pneumothorax. Pain is observed on percussion in acute pericarditis and myocarditis.

(c) Auseultation. 'This method of examination is of great value in determining the condition of the heart. It is best done in the small animal by placing the patient in a standing position on a table, covering the cardiar region with a cloth, 
and placing the right ear directly over it. A phonendoscope may be used which materially assists in differentiating the heart sounds, the instrument greatly intensifying them. Normally, there are two heart tones. One of these occurs at the moment of contraction of the heart (systolic), and the other at the dilatation of the organ (diastolic). In most small animals the rapid pulse makes it difficult to distinguish between these sounds. It is therefore best to compare the pulse with the heart beat. 'The interval between the systolic and diastolic sounds is shorter than the one between the diastolic and systolic. In very fat animals it is difficult to distinguish the heart tones with any degree of accuracy. In rabbits and cats the heart tones are often so rapid, the sounds following each other in such rapid succession, that they are indistinguishable. This condition occurs in all animals after excitement or exercise. In very young animals it is normal. In birds the heart sounds are almost impossible to recognize.

Both of these heart sounds are increased in hypertrophy of the heart, anemias, and thickening of the lung tissue around the heart.

The second or diastolic sound is increased in distention of the arteries, often the result of a congestion of the pulmonary circulation combined with hypertrophy of the heart.

A decrease in the volume of the heart sounds is observed in heart weakness, such as degeneration of the heart muscle, in hydropericardium, and in emphysema of the lung. In many of these cases the heart sounds are imperceptible. In small animals the various heart bruits (abnormal sounds) are often difficult to recognize.

It will require careful and persistent effort on the part of the student to familiarize himself with even the more common bruits which are the following: (1) Endocardial, and (2) pericardial.

\section{Endocardial Bruits are divided into: (a) Organic} endocardial bruits which are caused either by a narrowing (stenosis) of the valves of the heart, or changes in the valves which interfere with their proper closing (insufficiency). (b) Inorganic endocardial bruits occurring without any 
particular alteration at the orifices or valves of the heart, and are found in the different forms of anemia in animals.

2. Pericardial Bruits. -These bruits consist of friction sounds due to the pericardium becoming rough and dry. These sounds can be distinguished, as they do not occur synchronously with the heart sounds and are often indepenent of them. They are noted in pericarditis where there is not sufficient fluid to keep the membranes separated. 'They must be distinguished from pleural friction sounds, and can be quite readily, as they occur entirely independent of the respiratory movements.

From a clinical standpoint, in small animals, further differentiation of the various heart bruits cannot be made.

\section{PERICARDITIS.}

Definition.-An acute or chronic inflammation of the pericardium.

Etiology. - Inflammation of the pericardium may originate primarily or secondarily.

Primary pericarditis may result from: (a) 'Trauma, which may originate from within, such as the passage of foreign bodies (needles, pins, pieces of bone, etc.) through the esophageal walls. This occurs most commonly in the log and cat from swallowing needles, pins, etc., during play. It may also result from trauma from without, such as puncture wounds through the thoracic walls, or from falling, being run over by vehicles, etc. In such eases the pericardium is injured directly or indirectly by being pressed upon by the displaced thoracic wall or adjacent organs.

(b) Infective processes which in some cases may be primary or at least involve principally the pericardium. Staphylococci, streptococci and the specific virus of distemper are the most common organisms found. Primary infection, however, is not a common etiological factor.

(c) Cold has frequently been mentioned as one of the causes of pericarditis. 'This should be considered simply' as a predisposing factor in lowering general resistance, which allows the development of infection, etc. 
Secondary Pericarditis.-'This form may result from a number of conditions: (a) Catarrhal pneumonia, which is common in the dog and cat; simple pleuritis; endocarditis, myocarditis, cte. It may also follow diseases of the mediastinal lymph glands, the ribs, sternum, and even in some cases the abdominal viscera. (b) In septic processes, such as suppurating wounds, puerperal septicemia, ctc. (c) In specific febrile diseases, such as distemper in the dog and cat, pncumonia, chicken cholera, etc.

Necropsy.-On postmortem are recognized: (a) Acute fibrinous pericarditis, (b) pericarditis with effusion, and (c) chronic adhesive pericarditis.

(a) Acute Fibrinous Pericarditis.-This may be local or general. In the mild form dull, rough, lusterless masses of exudate cover the surface of the pericardium with a thin coating of fibrin which is readily peeled off. In the more severe form the exudate is more abundant, the masses of organized fibrinous deposits giving the surface a rough, shaggy appearance. In this form there is usually found a small quantity of fluid in the meshes of the fibrin. 'The heart muscle is not affected, except in the more severc form where it will be found pale and turbid.

(b) Pericarditis with Effusion.-This effusion may.be serofibrinous, hemorrhagic or purulent. It is most commonly serofibrinous. In this casc the pcricardial surfaces are covered with a thick fibrin and a collection of serous fluid fills the pericardial sac. The hemorrhagic form is usually found in acute cases which have terminated fatally following injuries, ctc. The pericardial sac will contain a rarying quantity of scrous fluid mixed with blood. When pus is present the pericardial surfaces will appear rough, occasionally eroded and of grayish color. This form occurs via metastasis or from internal trauma introducing infection into the pericardial sac.

(c) Chronic Adhesive Pericarditis.-Chronic allhesive pericarditis is found occasionally on postmortem. It is characterized by marked thickening of the inembranes, with adhesions betwcen the membranes themselves, and the adjacent organs. 
Symptoms.-Mild eases of pericarditis present, as a rule, no symptoms by which an aceurate diagnosis can be made. In the more severe forms, fever is usually present, but the elevation of temperature depends as much upon the primary disease as upon the pericarditis present. Temperature is usually $103^{\circ}-104^{\circ} \mathrm{F}$. The respirations are increased, especially when the animal is moved, the slightest exertion causing a marked dyspnea. Palpation over the cardiac region often reveals a distinct fremitus from the pericardial folds rubbing together.

Auscultation. The friction sound due to the rubbing together of the pericardial surfaces is distinctly audible, and is the most important diagnostic symptom. 'The examiner is greatly aided by the phonendoscope, which brings out the friction sounds more prominently compensating for their indistinctness due to the rapid heart beat.

As the disease advances, the heart action becomes interfered with, and all the symptoms of valvular insufficiency will present themselves. I)ropsical conditions from defective arterial tension will be in evidence, especially ascites, and dropsy of the extremities. There are general symptoms of impaired appetite, emaciation and general weakness. Death may occur suddenly or the condition become chronic and last for weeks, depending very largely upon the cause.

Diagnosis. - An accurate diagnosis in small animals presents some difficulties, and a thorough examination is always necessary. The frictional tones are characteristic, but they are determinable only in the acute fibrinous pericarditis.

Prognosis. - In the miller cases of acute fibrinous pericarditis and serofibrinous pericarditis the prognosis while favorable, should always be guarded at least until the etiological factor can be established. Other forms are alway's to be considered unfarorable.

Treatment.-Iygienic.-The animal should be placerl where there is absolute quiet. Prevent excitement, such as by the visits of the owner, or strangers. Have the room moderately warm and well rentilaterl.

Dietetic.-Nutritious food (lean meat, raw or cooked; milk, etc.) should be given to maintain the general comdition of the animal. 
Medical.-Heart tonics and stimulants are indicated to tone up the action of the heart, and to assist in the elimination of fluid from the body. Digitalis is of value for this purpose, given to dogs in doses of $0.1-0.2$ of the fluidextract once or twice daily depending upon the condition of the patient.

Laxatives (magnesium sulphate 8.0-12.0, or calomel 0.050.1 ) are indicated occasionally to regulate the bowels and also to assist in the elimination of fluids.

Surgical.- When the exudate accumulates to the extent of greatly interfering with the heart action, indicated by dyspnea, small rapid pulse, cyanosis, etc., paracentesis of the pericardium should be performed at once. 'The operation is not very difficult and is as follows: A rather long, small exploring trocar or aspirating needle is thoroughly sterilized. Shave the hair over the left cardiac region, wash thoroughly with soap and water, and follow with alcohol; then paint the surface with tincture of iodin. The needle should be inserted at the lowest point in the pericardial sac so as to be able to remove all the exudate and lessen the danger of injury to the heart. Insert the needle slightly downward and forward, and not too deep. 'The fluid will flow out in a steady stream. Repeated operations on successive days are often necessary. 'Ihe skin wound should be protected by covering with flexible collodion.

\section{HYDROPERICARDIUM.}

\section{Dropsy of the Pericardium.}

Definition.-A collection of serous fluid (transudate) in the pericardial sac not due to inflammation.

Etiology.-This condition most of ten accompanies general hydropsy resulting from valvular defects (insufficiencies), myocarditis, diseases of the coronary arteries, chronic nephritis, etc. It also occurs from pericarditis (see Pericarditis).

Symptoms.-'The symptoms are similar to pericarditis (see Pericarditis) except it runs a longer and more chronic course. There is absence of temperature, and frictional bruit is heard on auscultation.

Treatment.--Same as for serofibrinous pericarditis, which see. 


\section{HEMOPERICARDIUM.}

Definition.-A collection of blood in the pericardial sac.

Etiology.-This condition is found in aneurysm of the aorta, cardiac wall, or coronary arteries, and in rupture and wounds of the heart. Dogs and cats are frequently affected from gunshot wounds, being run over, etc.

Symptoms. - The principal symptoms are those of rapid heart weakness, paleness of the mucous membranes, rapid weakness and in most cases death in a very short time from compression of the heart. In slight hemorrhages the animal may live for several hours or days with a progressive heart weakness, dyspnea and all the physical signs of effusion in the pericardial sac.

Treatment.-Treatment in most cases is impossible, and in the slow progressive cases usually unsatisfactory.

Other diseases of the pericardium, such as pneumopericardium, are of no importance clinically. 


\section{CHAPTER II.}

\section{DISEASES OF THE HEART.}

\section{VALVULAR INSUFFICIENCY. AND STENOSIS.}

Definition.-A pathological or anatomical defect in the valves and openings of he heart leading to an irregularity in the circulation of the blood. These conditions are very common in small animals, especially the dog, where often quite extensive alterations in the valves are found on autopsy which failed to produce any marked symptom during life. However, when severe forms of insufficiency or stenosis occur, they are characterized by a marked disturbance in the heart action, circulation of the blood and the general condition of the animal. It is often very difficult and sometimes impossible to differentiate clinically between the various valvular and ostial defects which are found on autopsics.

Insufficiency.-In general, this condition occurs in two forms: (a) Imperfect closing of the valves which permits a portion of the blood at the contraction of the heart muscle to flow back into the chamber from which it came. This defect may exist in the atrioventricular valves or in the semilunar. Improper closing of the semilunar valve allows a portion of the blood which has becn forced into the artery to again return to the chamber during cliastole; or imperfect closing of the mitral or tricuspid valves during systole allows a portion of the blood to flow back into the auricles again from whence it came. In the early stages of insufficiency, owing to certain compensatory processes, no marked symptoms will be observed. However, as soon as the heart is not capable of performing the increased labor from lack of nutrition, increased disturbance in the valves, or general weakness from anemia, cachexia, etc., marked clisturbance in the general circulation will soon become evident. 'These are 
manifested by ascites, dropsical effusions, etc., in different parts of the body.

(b) Stenosis or contraction of the openings. In this condition the opening is narrowed so that the chambers of the heart are improperly filled. At the period of diastole, the blood is held back at the entrance of the affected chamber, and therefore normal filling of the chamber is interfered with. This condition may occur at any of the openings of the heart and will always induce imperfect heart action. Every interference with an arterial opening causes imperfect ventricular contraction; likewise defective venous openings lessen the auricular contraction and power. This abnormality in the action of the heart produces more or less general disturbance in the circulation of the blood, and as a consequence the reserve force of the heart is called into action until compensatory hypertrophy can take place to keep the circulation as near normal as possible. As soon as the reserve energy is used, or the compensatory hypertrophy fails to keep up the circulation, serious general symptoms of defective circulation appear as is often observed in old dogs.

Etiology. - Valvular defects are produced by a variety of causes:

(a) Endocarditis is the most common cause (see Etiology of Endocarditis). Endocarditis produces an inflammatory thickening of the margins of the valves leading to their imperfect closure. If the chronic inflammations persist it leads to cicatricial contractions (stenoses), and often there are found in long standing cases in old animals, deposits of calcareous matter on the valves and around the openings.

(b) Dilatation of the heart or weakness of the heart muscle will sometimes affect the openings, the dilatation preventing contact of the valve margins and a complete closure.

(c) Certain poisons and toxins are causes which lead to alterations in the structure and ultimately the action of the valves.

(d) Atheromatous processes may produce the comlition. However this is not common in small animals.

(e) Occurs during the course of some diseases, surde as anemia, pernicious anemia, ete. 
Necropsy.-It has been found that the mitral and aortic valves are the ones most commonly affected in the dog. In the early stages the edges of the valves are slightly thickened and invaded with small nodules. Later are found, as the sclerotic changes increase, contractions of the fibrinous tissue, producing thickening and deformity of the segments of the valves, the edges of which become round, curled and cannot be closed perfectly. In some severe cases the valves become very much thickened, with numerous hard nodules (which may be calcareous), giving them a marked uneven surface. The chordæ tendineæ are often found thickened and contracted. The apices of the papillary muscles frequently show fibrated or calcareous change.

Symptoms.-In small animals the general syinptoms of valvular deficiency are of greatest importance from a clinical standpoint, as it is very difficult during the life of the animal to distinguish with any degree of accuracy the separate valvular insufficiencies. However, some of them present some symptoms which are rather characteristic, and therefore, a brief description of the most common ones will be given.

As general symptoms, the following are the principal ones observed which are common to all valvular defects at some period in their course: Increased heart action; rapid and irregular pulse; palpitation of the heart; venous pulse (observed in the jugular at its entrance to the thorax); dyspnea; cyanosis of mucous membranes; hydrothorax; ascites; edema along abdomen, pectoral region, extremities; general emaciation, partial or complete loss of appetite with marked digestive disturbance. The condition gradually becomes aggravated until there is a general nutritive disturbance, weakness, and death from exhaustion. Careful auscultation will reveal the valvular insufficiency. Palpation over the cardiac region will determine the irregular and rapid pulse, and often a distinct fremitus. The temperature in the early stages is usually elevated $\left(103^{\circ}-104^{\circ} \mathrm{F}\right.$.); it later becomes normal or even subnormal as the disease progresses.

Symptoms of insufficiency of the mitral valves: This is of 
frequent occurrence in the dog. It is often accompanied by dilatation or hypertrophy of the heart. Pulse rapid and irregular; systolic bruit and increase of the diastolic sound on auscultation; dyspnea; cyanosis; general weakness; dropsical conditions (ascites, etc.). A careful examination is necessary to make a differential diagnosis.

The direct effect of aortic insufficiency is the 'regurgitation of blood from the artery into the ventricle, causing a distention of the cavity and a reduction of blood pressure in the artery. The amount returning varies with the size of the opening. This regurgitation eventually leads to dilatation and finally hypertrophy. In this way the valve defect is compensated for, and as with each ventricular contraction a larger amount of blood is forced into the arterial system, the regurgitation of a certain amount for a time during diastole does not interfere with the nutrition or with the general circulation.

The condition is characterized clinically by: A full pulse; strong heart beat; dyspnea; dropsical conditions (ascites, etc.); cough from congestion and edema of the lungs; increased area of dulness in the cardiac region; diastolic bruit, etc.

Insufficiencies of other valves are impossible to recognize during life in these animals.

Diagnosis. - The faulty lueart action is not difficult to recognize, but to determine the location of the lesion is very difficult in small animals. In some cases, however, by carefully observing the symptoms and using the phonendoscope a differentiation is possible.

Prognosis.-Complete recovery camnot be expected even in mild cases of insufficiency, therefore the prognosis is unfavorable. However, the condition may exist for a long time in individual cases without producing any serious symptoms provided adequate compensation exists.

Treatment.-(a) l)uring the stage of compensation no medical treatment is indicaterl. Kierp the animal quiet as possible, avoid all undue cxcitement and exercise, and grive nutritious food to maintain the general condition.

(b) Stage of broken compensation, when the simptoms of weakness, edema, palpitation, etr., appear, leart tonics 
are indicated. Digitalis fluidextract $(0.05-0.15)$; tinct. strophanthus (0.5-1.5 twice daily); or caffein citrate (0.1-0.2 twice daily). By the use of these preparations an effort is made to reëstablish a compensatory action, and should this take place the symptoms of edema, ascites, palpitation, etc., will gradually disappear. Should this treatment be unsuccessful, a symptomatic treatment may be tried such as the use of diuretics and catharties to relieve the effusions, and in severe palpitation, sedatives (morphin). In cases of severe weakness, wherc the pulse is very weak and irregular, direct heart stimulants (camphor, ether, etc.) must be given to afford temporary relief.

In disturbances of the digestive tract with loss of appetite, tincture of nux vomica $(0.2-0.8)$ or tincture of gentian (1.0$2.0)$ should be given twice daily to tone the digestive tract. Other symptoms that develop must be treated according to their importance.

\section{MYOCARDITIS.}

The following forms of myocarditis are observed in small animals: (a) Acute inyocarditis, and (b) chronic myocarditis.

Acute Myocarditis.-Definition. - An acute inflammation of the heart muscle (myocardium). From a pathological standpoint two distinct types of this condition are recognized, viz.: The acute parenchymatous and the purulent myocarditis. From a clinical standpoint such a differentiation cannot usually be made. Therefore, both will be considered under acute myocarditis.

Etiology.-(a) From severe excrtion, such as hunting dogs on long runs, continuous stud service, hard pulling of draft dogs, etc.

(b) Exposure to cold, or sudden chilling of the surface of the body, resulting in an unequal distribution of the blood, producing a congestion of the bloodvessels of the heart and occasionally resulting in an inflammatory reaction.

(c) Secondary to infectious diseases, such as distemper, pyemia, septicemia, tubcrculosis, cholera, and cnterohepatitis in fowls, etc. During the course of the infectious discases toxins are formed and carried by the blood to the 
heart muscle leading to inflammation and fatty degeneration. The other organs in the body are often similarly affected during the course of infectious diseases.

(d) From poisons (arsenic, phosphorous, silver, mercury, etc.) being absorbed, carried through the circulation to the heart muscle, producing irritation, congestion, and some of them (phosphorous, arsenic) later fatty degeneration of the muscle.

(e) Often results by spread of the inflammation from the endocardium and pericardium, especially in ulcerous endocarditis and suppurative pericarditis, the infection reaching via blood the heart muscle.

(f) Direct injuries to the heart which occur quite frequently in small animals from foreign bodies perforating the esophagus or chest wall; also by being run over, the heart muscle becoming contused.

Necropsy. - Pathologically numerous conditions are found, the changes in the myocardium are quite varied, and in acute myocarditis may be classed under two hearls as follows:

(a) Acute Parenchymatous. Iy yocarditis.-'This as a prinary condition, is not very common, and is usually associated with acute inflammation in other organs. In this form there is found interstitial infiltration, inflammation of the muscle fibers, which are colored reddish-gray, yellowish-white, or even white. The muscle fibers are indistinct and show a more or less homogeneous structure.

Microscopically, the fibers show a loss of striation, with small cellular infiltration in the interstitial connective tissue. Large numbers of white corpuscles are found between the fibers.

(b) Purulent Myocarditis. - This form occurs quite commonly in the dog, less commonly in cats and birds, and is found mostly in pyemic conditions. Small abscesses are distributed through the connective tissue varying in size from a millet seed to a pea. Sometimes by confluence the abscesses are much larger.

Symptoms. - In the early stages the symptoms are similar to those of insufficiency or weakness of the heart. 'The heart beat is very rapid, three to four times normal, throbbing, and 
in the dog can be seen quite a distance from the animal, the tumultuous throb of the heart shaking the whole body. The heart action soon becomes irregular and very weak. The pulse is very rapid, weak, small, irregular and in later stages imperceptible. The pulse usually becomes so rapid that it is impossible to count it. Respirations are rapid, labored, and the animal soon becomes prostrated.

The temperature in the early stages is elevated, the degree depending upon the etiological factor; later it becomes subnormal.

In some cases myocarditis takes a very rapid course, the animals dying suddenly from rupture or paralysis of the heart.

Diagnosis.-This is difficult as the symptoms are quite similar to endocarditis, pericarditis, etc. A careful examination should always be made. When insufficiency of the heart action exists without frictional sounds or bruits, myocarditis may be suspected.

Prognosis.-The prognosis is unfavorable, especially in secondary myocarditis, due to infection, or during the course of infectious diseases. Even in primary acute myocarditis there are always degenerative changes in the heart muscle which are impossible to entirely overcome. If recovery occurs it is as a rule only partial (chronic myocarditis).

Treatment.-Hygienic.-The animal should be kept in a quiet place, avoiding all excitement or handling.

Medical.-The heart weakness should be treated by using heart stimulants and tonies, such as dilute alcohol (2.0$4.0)$; fluidextract digitalis $(0.075-0.10)$; caffein citrate (0.5-1.0 subcutaneously every six to ten hours); ether (2.04.0 subcutaneously); oil camphor (1.0-4.0 subcutaneously); or atropin sulphate $(0.04-0.075$ subcutaneously). The use of these preparations will depend largely upon the needs of the case.

In very acute cases there is no treatment that will produce satisfactory results.

Chronic Myocarditis.-Definition.-A chronic inflammation of the myocardium. This condition occurs frequently in small animals but is rarely recognized during life. It is often confused with other heart affections. 
Etiology.-(a) Occurs commonly from mild, acute attacks, especially in those cases where the interstitial connective tissue is primarily involved.

(b) During the course of chronic muscular or articular rheumatism in old dogs; also from chronic nephritis, tuberculosis, pericarditis, endocarditis of dogs, cats and birds.

(c) Chronic poisoning by chemicals, tuxins, etc., often will produce the condition by interfering with the circulation through the coronary arteries.

Necropsy.-Throughout the heart muscle appear circumscribed masses of fibrous tissue which are white in color and of firm to hard consistency. They occur most conspicuously near the apex of the left ventricle. The fibrous areas may be quite dense, like a cicatrix, constituting the so-called "heartscar." Pigment and calcareous deposits may be present in them. The affected heart wall becomes very thin in places and may bulge forming a so-called aneurysm. Fatty degeneration may be present. The heart may show hypertrophy with dilatation.

\section{ACUTE ENDOCARDITIS.}

Definition.-An acute inflammation of the endocardium, especially involving the part covering the valves. This condition occurs very commonly in small animals, especially in dogs and birds.

Etiology.-1. The acute endocarditis in the majority of cases results from infection, the toxins of a number of infectious diseases irritating the endocardium. The organisms (pus-producing organisms, bipolar bacillus, colon bacillus, Bacterium tuberculosis, etc.), or thcir toxins, are the oncs most commonly accused. An acute, primary, infectious endocarditis is observed occasionally; in the majority of cases, however, it occurs secondary to other diseases, pyemia and septicemia usually producing it. It develops often also after abscesses, wounds on the skin, abscesses in the internal organs, septic metritis, sapremia, etc. The organisms or their products (toxins) are carried by the blood to the endocardium where they become lorgerl, especially along the 
edge of the valves. They propagate, produce irritation, and assisted by the mechanical artion of the valves, an active inflammatory reaction is soon established. The inflammation spreads to the other parts of the endocardium and may cause a general endocarditis. 'The rapidity of the process depends upon the virulency of the infection or the amount of the toxins present.

2. Spread of the inflammation from adjacent structures or organs may produce the condition, such as from a myocarditis, pericarditis, pleuritis, or a pneumonia.

3. There are several predisposing conditions which should be considered: (a) Chilling the surface of the body, such as exposure to cold, or cold baths, carelessness in thoroughly drying the skin of animals after baths, etc., make them more susceptible to infection by reducing resistance.

(b) In old animals legenerative changes of the heart muscle, valves, etc., make them less resistant.

(c) Young puppies, chicks, kittens, from hereditary influences, often favor the development of the condition.

(d) During convalescence from long-continued diseases there is a predisposition to endocarditis.

(e) 'Traumatic conditions over the region of the heart (kicks, blows, fractured ribs, etc.).

4. Mineral poisons (mercury, phosphorus, etc.) or some drugs administered in large doses, or for too long a period, produce direct irritation to the endocardium, or prerlispose to the condition.

In the etiology of enlocarditis, infection must always be regarded as the principal causative factor.

Necropsy.-Macroscopically two forms of acute cnlocarditis are recognized, viz.:

(a) Endocarditis I alvularis Verrucosa.-This is characterized by small cnlargements, about the size of a millet seed, on the free edges of the valves, on the tendons and papillary muscles. 'They are nodular, wart-like in appearance, grayish-white or whitish-yellow in color, and their surface covered with blood-stained or colorless stratified epithelium. 'There grou ths may develop into large polypous proliferations, which often cause stenosis of the openings 
and insufficiency of the valves of the heart. The cndocardium in general is swollen, opaque, reddened somewhat, and covered with a thin coagulum; small hemorrhagic foei are found often in the subendocardial tissue, as well as between the fibers of the heart muscle.

(b) Endocarditis Ulcerosa.-This is the more severe form of the condition, characterized by necrosis and ulceration of the margins of the valves and other portions of the endocardium. The ulcers are usually well defined, isolated and vary considerably in size from a pea to several times larger. They are covered with friable, discolored, necrotic masses. These masses are often torn loose by the circulating blood and are carried to the other organs, even to the heart itself, forming metastatic abscesses. In dogs endocarditis verrucosa is very commonly found on postmortem and although the lesions may be extensive no marked symptoms were noted during life. 'The aortic or bicuspid valves are most often affected.

Symptoms. - The symptoms of acute cndocarditis vary considerably, depending to a great extent upon the cause, and the nature and extent of the inflammatory process.

The early symptoms are those of a greatly disturbed heart action, which is at first palpitating and irregular; later the - beat is diffuse. 'The number of heart beats sometimes exceeds that of the pulse (Fröhner). 'The heart beat is often so tumultuous, especially in $\operatorname{dogs}$, that the entire borly is shaken and can be observed some distance from the animal.

The pulse is very rapid, intermittent, irregular, and in the later stages becomes imperceptible. 'The frequency in dogs will be 120-300, and in other animals even more rapid. 'The heart muscle is at first normal, but soon becomes abnormal, the sounds often blenderl to a single somnd. Iater, characteristic endocardial bruits are heard, a blowing, stenotic (systolic) noise oceurring with the first heart somnd, and at times a prolonged rustling and vibrating sound oceurs at diastole.

The temperature in the early stages is always high $\left(10: 3^{\circ}-\right.$ $105^{\circ} \mathrm{F}$.), but in the dog it becomes normal or subnormal. Cats have a high temperature, which remains high for at 
longer period than in the dog. 'The respirations are accelerated, the dyspnea similar to that of pneumonia, a disease with which it is sometimes confused in making a diagnosis.

In the later stages of the condition, circulatory disturbances are quite prominent. These are manifested by cyanosis, venous pulse, edema of the lungs, etc. In the septic form a hemorrhagic diathesis appears with hemorrhages from the membranes, bloody urine, etc. As general symptoms, depression, weakness, etc., come on rapidly, the animal often shows complete prostration.

Diagnosis.-Acute endocarditis is quite difficult to recognize as the symptoms are similar to other diseases of the heart. It may be confused with any acute febrile disease having a sudden onset (septicemia, inflammation of the brain, pneumonia, etc.). A careful examination of the heart is always necessary for a diagnosis. It is often very difficult, and in some cases quite impossible to distinguish between acute endocarditis and myocarditis, with which it is very closely associated. Between the two forms of endocarditis it is almost impossible to differentiate. In the ulcerous form the onset is more rapid than in the verrucose, and sometimes the primary seat of infection can be located.

Prognosis.-The prognosis is unfavorable, especially in the ulcerous form. Complete recovery is rare. The valves are usually left permanently impaired resulting in valvular insufficiency.

The course of the disease varies. It is sometimes very acute, ending in death in a few hours or days (endocarditis ulcerosa). Or the condition may last for several days or weeks, eventually developing into the chronic form (endocarditis valvularis verrucosa).

Treatment.-The animals should be kept in an absolutely quiet place avoiding all excitement or morement.

In the early stages (in dogs especially) coll compresses should be applied over the region of the heart (ice-bag or cold water compress). These should be (hauged as often as necessary.

Regulate the action of the heart by the use of rigitalis, or if the heart is very weak, it should be stimulated by the use 
of alcohol, caffein citrate or ether subcutaneously. 'To reduee the temperature acetanilid ( $\operatorname{dog} 0.2-0.5$; cat $0.05-0.1$ ) should be given twice daily.

In endocarditis from rheumatic conditions, salieylic acid or sodium salicylate ( $\operatorname{dog} 0.2-0.5$; cat $0.05-0.1$ ) should be administered twice daily.

When general weakness is well marked, stimulants must be administered according to the needs of the patient. Camphor, ether, or atropin are best for this purpose.

\section{HYPERTROPHY AND DILATATION OF THE HEART.}

Definition.-Hypertrophy is an enlargement of the heart due to a thickening of its musculature; dilatation is an increase in the size of the heart from an enlargement of its cavities. From a clinical standpoint a distinction between them cannot be made as they nearly always coexist. Hypertrophy is an active enlargement of the heart, while dilatation is a passive one.

Etiology.-Generally speaking the causes of hypertrophy of the heart are those conditions which interfere with the circulation of the blood and thus increase the blood pressure. The following are the most common:

(a) Increased physical exertion. 'This occurs in dogs used for hunting (fox hounds, greyhounds).

(b) Adhesions between the pericardium and heart interfering with the heart aetion.

(c) Obstruction to the free eireulation through the arteries, such as aneury'sms of the aorta, stenosis of the aorta, thrombi, atheromatous and arteriosclerotic processes, etc.

(d) Defects in the valves of the heart (ehronie endoearditis).

(e) Diseases of the lungs, such as chronic interstitial pneumonia (common in the $\operatorname{dog}$ ), adhesions, exulations, abscesses, chronic bronchitis, which increase the blood pressure through the right heart.

(f) Chronic inflammation of the liver, kidneys, etc., by impeding the free eirculation of blood (common in old dogs).

(g) Dilatation of the heart usually follows hypertrophy, 
the hypertrophied heart eventually growing weaker and becoming distended by the abnormally increased blood pressure. It may also develop in an acute form after acute diseases of the lungs, as the circulation through the right heart is greatly interfered with.

Necropsy. - In both hypertrophy and dilatation the heart is enlarged, in hypertrophy the increase in the thickness of the walls, in dilatation in an enlargement of its cavities.

(a) True hypertrophy of the heart appears in several forms, depending upon whether it is general or local, $i$. $e$, involving a whole ventricle, or circumscribed affecting only some of the papillary muscles. The left heart is most frequently affected. In hypertrophy the shape of the heart will vary. When the left heart is involved it appears elongated, cylindrical; when the right heart, it is flatter and broader than normal. If the whole heart is hypertrophicd it assumes a round or oval form and is increased in size. The walls are usually two or three times the normal thickness, the muscles firmer, toughcr, darker red in color, and the interstitial connective tissue occasionally shows marked prolifcration. Sometimes areas of fatty degeneration are noted on the surface.

(b) In dilatation of the heart the cavities are found much larger than normal, and the walls thinner and weaker. In the active form of dilatation the walls are stronger than in the passive where they are relaxed and distended. Dilatation appears oftener in the right heart than in the left. The structurc of the muscles may be normal. Isually, howcver, the organ is anemic, friable, the musculature yellowishbrown in color, often very thin, and due to a complete atrophy of the muscle, in some areas the wall is almost transparcnt.

Symptoms. - In true hypertrophy of the heart, especially compensatory, the development is often so gradual that it may exist for a long periorl without producing marked symptoms. In severe cases, when accompanying other diseases, it is characterized by a strong, full pulse, very loud, clear heart sounds, and an increase in the area of cardiac dulness. Duc to the coexistence of dilatation the symptoms which characterize hypertrophy are rarely noted in practice. The most 
prominent symptom of hypertrophy with dilatation is the extension of cardiac dulness, which in dogs may reaeh as far back as the last rib, or even to the false ribs.

Dyspnea, palpitation, disturbances in the eireulation, dizziness, etc., are resulting symptoms. A prominent sign is the throbbing of the heart, which often shakes the whole body and may be seen quite a distanee from the animal. The heart sounds are usually irregular, the first sound loud, metallic and sometimes vibrating; the second sound very weak and often imperceptible. The slightest exertion will produce a very rapid heart action. The pulse is weak. A venous pulse is common in old dogs. ['ltimately, due to insufficient heart action, general eyanosis, aseites, hyrdrothorax, etc., develop.

Diagnosis. - Care should be taken not to eonfuse hypertrophy and dilatation with diseases of the lungs, and other affeetions of the heart. The increase in the area of cardiac dulness, the abnormally loud, systolic sound, arhythmie pulse, and tendency for ascites and edemas are all charaeteristic of dilatation of the heart. A hard, full pulse with increased areas of dulness points to hypertrophy of the heart.

These conditions usually can be distinguisher from valvular defects by the absence of the characteristic bruits.

Prognosis. - Favorable in simple hypertrophy of the heart. Unfavorable in dilatation of the heart, exeept in the very acute cases.

Treatment.-Absolute rest and quiet should ahways be insisted upon. It is important to remove the causes of these conditions if possible.

(a) In hypertrophy of the heart with hyperkinesis, seclatives such as potassium iodid (dogs $0.2-0.8$; ( ats 0.0$) \tilde{j}-0.10$ ) to act as a general sedative may be used for one or two doses.

(b) In hypertrophy complieated by dilatation, with a weak, feeble heart action, cardiac tonics and stimulants are indieated. I)igitalis fluidextraet ( $\operatorname{logs} 0.1-0.3$, eats half the quantity) is probably the most important drug for this condition. 'These doses may be repeated as often as neressary. Good, nutritious food should be allowed at all times. General stimulants are to be used when necessary. 


\section{RUPTURE OF THE HEART.}

Etiology. - (a) Traumatic influences (kicks, fractured ribs, being run over, falling, etc.). (b) Diseases of the muscular walls of the heart (abscesses, fatty degeneration and infiltration, endocarditis and myocarditis, atheromatous degenerations of the aortic walls at their origin, etc.). (c) Shock from operations, and other conditions and diseases.

Symptoms. - In most cases the animal dies apoplectic. In cases where the rupture is very small, symptoms of internal hemorrhage are noticeable. Death in these cases, however, usually occurs in a few hours.

Treatment.-No treatment can be given in this condition. 


\title{
PART III. \\ DISEASES OF THE DIGESTIVE TRACT'.
}

\author{
C HA P T E R I. \\ DISEASES OF THE MOU'TH.
}

Examination. - The examination of the oral cavity requires good light (daylight) or in some cases artificial light (electric bulb with reflector) is necessary where a careful examination is to be made. In docile animals the mouth can be opened by pressing the lips against the tecth above and below causing the animal to open the jaws. For protracted cxamination it is necessary to hold the mouth open by the use of tapes placed just back of the canines, one tape above and one below, which are grasped by an assistant; or a mouth speculum may be used. In vicious animals an anesthetic should be emploved (morphin for dogs; ether for cats). For examination of the posterior part of the cavity, the tongue should be depressed with a spatula, or pulled well forward with the fingers or blunt forceps. In examining the mouth, the following should be observed:

(a) Odor.-An offensive odor is noted from retained or decomposed food; ulcerative or gangrenous stomatitis: gangrene of the lungs; fetid bronchitis; acutc and chronic gastritis; caries of the teeth; roup in birds. 'The odor is often characteristic and readily distingnished. In some poisonings the odor of the drug is evident (cartolic arcid, hydrocyanic acid, etc.).

(b) Secretions.--Secretion is diminished in all acute fobrile conditions; in some poisonings (belladonna, at ropin). Secre- 
tion is increased in parotitis; inflammatory conditions of the mouth; injuries; foreign bodies; poisons (calomel); eruption of teeth in puppies; following injections of pilocarpin. An abnormal quantity of saliva is noted in the mouth in dysphagia. 'The saliva which flows from the mouth is in clear strands or in the form of foam from masticatory movements.

(c) The Mucous Membranes.-An anemic or pale condition is found in chronic constitutional diseases; intestinal parasites; skin parasites, especially in birds; severe hemorrhage. A hyperemic or congested condition is noticed in all acute inflammatory conditions; in acute infectious diseases with elevation of temperature; occurs during the course of gastritis. Cyanosis occurs from chronic heart diseases; interference with respiration or the local circulation. A blue line is seen around the gums in lead poisoning.

(d) Foreign Bodies.-A carcful examination should be made for foreign borlies which often become imbedded in the mucous membrane or around the tongue or forced in between the teeth. In cats fishboncs, needles or pins are often found in the posterior part of the mouth.

(e) Neoplasms.-Papillomata are frequently seen on the margins of the lips or on the mucous membrane in various parts of the mouth. Retention cysts often occur under the tongue (submaxillary gland), inner surface of the lips and cheeks (buccal glands). Other tumors are occasionally found. In all cases where the mouth is held open, whether from complete or partial paralysis or foreign bodies, dumb rabies should be suspected and the examination made with care.

\section{STOMATITIS.}

Several varietics of stomatitis are met with in practice as follows: (a)-Catarrhal; (b) ulcerative; (r) ungrenous; $(d)$ phlegmonous; (e) parasitic.

Catarrhal Stomatitis.-Definition.-An arute or chronic inflammatory condition of the murous mombrane of the mouth.

Etiology.-Mechamical.-Injuries from foreign boties or sharp material in the food (bones, etc.); from irritation due 
to tartar around the teeth; dentition; weed hairs penetrating the membrane (seen in hunting dogs after rumning in fields).

Chemical.-Irritating medicinal agents administered in concentrated form; poisons (carbolie acid, arsenic, mercury, etc.); decomposed food which has been retained in the mouth; internal administration of calomel or lead compounds in too large doses or for too long a time.

Thermic.-Hot food or drink.

Infectious. - It occurs in the suckling young of bitches affected with infectious mammitis; aceompanies infectious diseases (septicemia, distemper in dogs and cats, diphtheria and roup in birds); usually present during the course of gastritis, some of the toxins when absorbed into the cireulation are secreted with the saliva and thus cause irritation to the oral mucous membrane; produced by extension of inflammation from other parts (pharynx, larynx and salivary glands).

Chronic constitutional diseases (rachitis, anemia and leukemia) are predisposing factors.

Pathology. - There is at first a superficial redness and dryness of the mucous membrane followed by an increased secretion and swelling. 'This secretion collects around the teeth and on the tongue in the form of a dirty gray or brown coating. The lips often become fissured and ulcerated.

Symptoms. - The animal shows pain during mastication and has an increased thirst. On clirect examination the mucous membranes are seen to be red, swollen, and eovered with mucus. 'The redness may be in spots or diffuse, depending on the cause. Saliva often flows from the mouth or hangs in strings from the lips. The swelling of the mucous membrane may occlude duets of the buceal glands, forming cysts which appear as small gray nodules on the imner surface of the lips. Quite frequently there is an erlematous condition of the mucous membrane. The upper surfaee of the tongue shows a brown or greenish-brown diseoloration. In cats it is slightly yellow, and the papillae are enlarged and quite prominent. In birds the epithelium is thickened, tongue dry, and a pseudomembrane is often noticerl.

Diagnosis. - The diagnosis is made by the absence of greneral symptoms, the inflamed and sensitive mucons membrane and the characteristic conditions noted above. 
Prognosis.-Favorable in primary cases, healing occurring in about one week; in secondary cases it depends on the causative disease.

Treatment.-Dietetic.-The animal should be fed warm liquid food in small amounts.

Medical.-The use of antiseptic mouth washes is indicated (boric acid 2 per cent., alum or tannic acid 1 per cent., vinegar and water 1-10); in the severe chronic form direct application of silver nitrate (1-2 per cent.) may be found useful. Tincture of myrrh applied direct to the gums is very useful as a deodorant and antiseptic.

Surgical.-Remove foreign bodies and tartar from around the teeth, using a curette.

Ulcerative Stomatitis. - Fetid Stomatitis. Stomacace. Sore Mouth.-Definition. - An acute inflammation of the mucous membrane of the mouth resulting in the formation of ulcers, which appear most commonly on the margins of the gums.

Etiology.-This disease is found in weak, anemic dogs and cats. It also frequently develops during the course of distemper, rachitis and other constitutional diseases. It is found commonly in old dogs and cats with diseases of the teeth (caries), especially when these animals are insufficiently nourished. The exact causes producing this necrosis of the tissues are not definitely known. The character of the disease process points to infection. In man a similar disease is contagious. The Bacillus septicus has been isolated from the diseased area in dogs, and the Bacillus necrosis and Bacillus coli communis in cats, but have not been proved to be the specific cause. Lack of cleanliness in the mouth, diseased teeth and accumulations around them favor the occurrence of the disease. The internal administration of mercury in large or long continued doses, may produce a similar condition of the gums.

Pathology.-The gums at first are swollen and dark red in color, but soon become pale yellowish and necrotic. The epithelium is destroyed, deep ulcers form, suppuration ensues and the teeth may become loose and fall out.

Symptoms. - In the early stages it begins as a serere stomatitis, the gums bleed freely, are swollen and partly 
envelop the teeth, but as their margins ulcerate and recede the teeth become more and more exposed. The ulcerative process may spread to the contiguous parts destroying much tissue. Salivation is profuse and the odor of the breath very fetid. Chewing and swallowing are difficult. The temperature is usually slightly increased. In severe cases the afferent lymph glands are enlarged and symptoms of septicemia may be noted.

Diagnosis. - This condition should be distinguished from scorbutus. The chief differential features are the absence of general symptoms and the free bleeding of the gums, both of which are more marked in scorbutus. The anamnesis will usually differentiate ulcerative stomatitis from poisons; the characteristic general symptoms of the latter are also evident.

Prognosis. - A favorable termination of the disease is only to be expected in young, healthy animals with mild affection. In such cases, the course is from one to three weeks. In severe cases in weak anemic animals the prognosis is unfavorable. Complete healing is, however, rare; death may occur suddenly from septicemia.

Treatment.-Medical.-Potassium chlorate seems to be nearly a specific for this condition. It may be administered internally $(0.3-0.6)$ three times daily, and also applied as a mouth wash. As potassium chlorate is toxic, its use should not be prolonged. When there is much fetor, a solution of potassium permanganate ( 2 per cent.) can be used as a mouth wash and silver nitrate ( 2 per cent.) applied to the ulcers. In the mercurial stomatitis, a subvariety of the ulcerative, the treatment consists in the removal of the cause and the use of antiseptic mouth washes.

Surgical.-Examine the teeth carefully and remove all incrustation. Loose teeth should be extracted.

Gangrenous Stomatitis.-Cenlier of the Mouth.-Definition.-A clisease of the mouth tharacterized by a rapidly progressing gangrene, starting on the gums or lips and producing extensive sloughing.

Etiology.- This discase is usually seen in yomer anminals (puppies, kittens, etc.) which have been kejt under very unsanitary conditions; or in older animals convalescent 
from infectious diseases. It is evidently an infectious disease, probably due to the Bacillus necrophorus. The lack of resistance especially in young animals favors the development of the organisms. Accumulations of filth in the kennels and injuries to the tissues are predisposing causes.

Pathology. - The gangrenous area has the appearance of a corroded surface under which the mucous membrane seems transformed into a dry, finely granular or firm mass. It is grayish-yellow in color and bordered by a zone of thickened tissue, slightly reddened and somewhat granulated. The necrotic tissue is very adherent and can be only partially peeled off. The condition may extend to the underlying tissues and even involve the bones.

Symptoms. - Slight salivation and a disinclination to take food are the first symptoms noticed. An examination of the mouth at this time may show an area of inflammation or possibly an erosion. The latter rapidly increases in size and depth, forming a sharply circumscribed, or at times diffuse area of necrosis, which continuing to spread, may involve any of the adjacent tissues. It of ten perforates the cheeks forming a fistulous opening, or it may penetrate the hard palate and produce a greenish-yellow nasal discharge: With the involvement of the nasal passages, the larynx or trachea respiration is disturbed. When life is prolonged for a week or more, necrotic foci may be established in the lungs, giving rise to symptoms of bronchopneumonia. As the disease progresses, salivation becomes profuse, deglutition difficult and the swollen tongue often protrudes from the open mouth. A very offensive odor is exhaled. When the infection becomes general (septicemia), the temperature is elevated $\left(104^{\circ}-\right.$ $106^{\circ} \mathrm{F}$.) and the animal shows extreme weakness. Diarrhea is not uncommon and indicates an invasion of the gastrointestinal tract.

Diagnosis. - This is made by the rapid spread of the disease in the tissues of the mouth, fetid odor and the general symptoms.

Prognosis.-Ordinarily this disease shows no tendency to a spontancous recovery, and if untreated death usually results. If taken early, however, it usually responds to 
treatment. Inder such favorable conditions the prognosis is good, recovery occurring in twelve to fifteen days.

Treatment.-Dietetic.-As the animal refuses food on account of the pain when swallowing it should be forced to take some nourishment (warm milk can bc given puppies and kittens; milk and soups to older animals).

Surgical.-In those cases where the lesions are accessible, the treatment consists in removing all the necrotic tissue with a curette. This exposes the causative agent, an anaërobc, to the air which inhibits its growth and development.

Medical.-The skin around the head, eyes and mouth must be thoroughly cleaned with antiseptic washes (boric acid 2 per cent., potassium permanganate 1-250). The direct application of carbolic acid (5 per cent.), or Lugol's solution to the exposed areas has proved quite beneficial. In obstinate cases silver nitrate (2 per cent.) may be used.

Prevention. - Prevention of this disease consists in a thorough disinfection once daily for a few days, of the mouth and nose of those animals that have been exposed and are predisposed by the eruption of the first teeth or the shedding of the milk teeth; or through association with affected animals. All filth should be removed from the kennel and disinfectants freely used.

Phlegmonous Stomatitis.-Definition. - In acute phlegmonous inflammation of the mucous membranes of the mouth, lips and tongue.

Etiology.-Mechanical.-Foods containing irritating materials. In hunting dogs sharp projections, such as thorns, spikes, nettles, hairs, etc., on grasses and weeds are causes.

Chemical.-Carbolic acil, alkalies, ammonia, croton oil, etc., when concentrated, produce an intensc inflammation and swelling of the nembranes.

Infectious.-It is produced secondarily during the course of diseased processes of neighboring organs (infectious pharyngitis); also secondary to infectious diseases (distemper in dogs and (ats and diphtheria in birds).

Pathology. - The mueous nembrane is reddened, adematous and covered with a thick tenacious mucus. 'The subcrltaneous tissues are infiltrated with sermm. I) squamation of the epithelium is often noticed from the intense irritation. 
Symptoms. - The disease begins with swelling, redness and a very painful condition of the mucous membrane. The local temperature is accelerated. The lips and cheeks become swollen, the lower lip hangs down and strands of saliva hang from the corners of the mouth. Later the mucous membranes on the inner surface of the lips, cheeks and the back part of the mouth are bluish-red in color. The gums are swollen and dark red, the tongue becomes much thickened which interferes with deglutition and forces the mouth open. In severe cases following infectious diseases, the submaxillary and sublingual lymph glands are swollen and very painful to the touch. The general symptoms depend largely upon the primary condition.

Diagnosis. - Is made by the acute inflammatory condition of the mucous membranes with the swelling of the lips, cheeks and tongue. The anamnesis in some cases will materially assist in making the diagnosis.

Prognosis.- Usually favorable; in the secondary cases it depends on primary condition.

Treatment. - Dietetic.-All solid food should be withheld for a few days and small quantities of liquid foods given (milk, meat broth, etc.).

Medical.-The mouth should be thoroughly cleansed twice daily with antiseptic and astringent solutions (alum 1-250; tannic acid 1-250; potassium permanganate 1-250).

Parasitic Stomatitis.-Thrush. Soor. Aphtha.-Definition. -An inflammation of the mucous membrane of the mouth produced by the Oirlium albicans.

Etiology.-The Oïdium albicans (Monilla candida, Saccharomyces albicans) is the exciting cause of this disease which is found occasionally in healthy young animals, most frequently in birds. 'This fungus is widespread in nature, occurring especially on decaring vegetable matter as a saprophyte. The mycelia are composerl of cylindrical cells, 1-4 microns wide and 10-20 microns long. The filaments show branching and the outer ends are rounderl off' or club shaped. 'The rounded ends often contain oval, highly refractive bodies, the gonidia or spores, which are also found free between the filaments. If the free spores come in contact with the oral 
mucosa, in which there are slight epithelial defects, they mav develop and lead to the formation of thrush spots and pseudomembranes. In some cases the filaments and spores may penetrate deeper into the tissues, or by metastasis involve the internal organs. Frequently in birds, the spores are taken into the crop where they develop and produce typical thrush lesions. Damp, warm rooms poorly ventilated and filthy, favor the growth of this fungus.

Pathology.-The affection begins with diffuse redness of the mucosa and the formation of a glistening or shiny adhesive exudate of grayish appearance. Small white or yellowish dots next appear, which stand out promincntly against the hyperemic background. These patches may be quite large and when removed the underlying mucosa is congested and eroded. The disease usually begins on the tongue or inner parts of the cheeks, from where it spreads to other parts of the mouth. In severe cases it may cxtend to the pharynx, esophagus, or even in fowls to thc crop and intestines. The microscope rcveals filaments, spores, epithelial cells and pus.

Symptoms. - The general symptoms are depression, emaciation and loss of strength. On direct examination of the mouth an acid odor will be detected and the characteristic lesions or spots will be seen on the mucosa. 'These may be in the form of white or yellowish spots, or they may coalesce forming a superficial felt-like membrane which can be rcadily scraped off. Similar membranes form in the esophagus and crop interfering with the appetite and nutrition. 'This condition leads to cachexia and death, which is often preceded by convulsions.

Diagnosis. - The discase is readily distinguished from other forms of stomatitis by the absencc of acute inflammation. The diagnosis is made positive by the finding of the filaments or spores of the fungus in the deposits on the membranes. The clinical appearance may be confused with the condition due to the aspergillus fungi. (See Aspcrgillosis.)

- Prognosis.-When the diseasc is localized on the mucous membrane of the mouth, the prognosis is favorable, but when it extends to thc esophagus and crop, treatment is unsatisfactory. 
Treatment.-Dietetic.-Good nutritious food should be given (small meat scraps, oatmeal and milk, etc.) to sustain the strength.

Surgical.-Carefully curette all the arcessible lesions and wash with antiseptic solutions (boric acid 2 per cent., mercuric chlorid 1-1000, the latter used with care that it may not be swallowed). When the disease extends to the crop, give enough boric acid ( 2 per cent.) solution to distend the latter, then massage it thoroughly with the head held down so the contents will be forced out of the mouth. Repeat this daily until the appetite is improverl.

\section{BENIGN NEOPLASMS OF THE MOUTH.}

Papillomata. - These tumors occur on the mucous membrane of the mouth and occasionally on the margins of the lips and sometimes they apparently assume a contagious character.

Symptoms.-They are noticed as small, isolated or confluent growths, usually pedunculated, of a whitish color and often rough (cauliflower-like) on their surface. 'They are found most commonly at the juncture of the mucous membrane and the skin, and on the inner surface of the lips, but may be generally distributed over the oral mucosa. 'They rarely interfere with mastication, but when present in large numbers or masses they may cause some salivation and from the decomposed food collecting around them a very disagreeable odor is emitted.

Treatment. - These tumors frequently disappear in a short time without any treatment. However, it is best to remove them by clipping off with scissors. The mouth should be washed for a rlay or two with antiseptic solutions (alum $1-250$; potassium permanganate $1-250)$. Tannic acid (2 per cent.) applier direct to small papillomata often causes them to slough off after a few applications.

Fibromata.-These tumors are composed of connective tissue elements which may be a pure or mixed fibrous growth 
containing cartilage and sometimes spicula of bone. 'They have their origin in the submucous tissue or the periostcum of the bone, and usually occur as a single growth.

Symptoms. - The tumor is found growing at the crlge of the gums in the form of a hard, reddish enlargement varying in size from a pea to a walnut. Its growth is slow often requiring months to develop into sufficient size to be noticeable. The slow growth and firm consistency assist in distinguishing it from any of the malignant growths.

Treatment. - Complete extirpation and cauterization of the wound with silver nitrate or thermocautery are curative.

Osteoma. - These tumors are found growing from the periosteum of the maxillæ. 'They are composed of osseous material and frequently devclop after injuries to the periosteum.

Symptoms.-Osteomas appear as very hard enlargements firmly attached at their base. As a rule the skin or mucous membrane is not arlherent over them. 'Their hardness and slow growth rearlily distinguish them from malignant tumors.

Treatment. - Expose the enlargement by an incision through the soft tissues and dissect down to the base, when it may be removed if not too diftuse, by the use of bone forceps or a small bone chisel. Curette the surface until smooth and suture the skin over it. After-treatment as an ordinary wound, using antiseptics to cleanse it rlaily.

Retention Cysts. - Ramula.-These are enlargements appearing in the buceal cavity from a stoppage of the duets of glands which discharge their secretions into the mouth. (Cohesion of openings occurs from inflammations of the mucous membrane, swelling, ete., partially or eompletely closing the duct. At the same time the glands continue to secrete their fluids which distend the ducts forming the enlargements. Closure or obstruction of Wharton's duct produces an extensive enlargement under the tongue. The most common retention cysts, howcrer, are from the ducts of the buccal or sublingual glands. When the obstruetion is complete the secretions, as they collect, burrow downward along the neck producing an enlargement which appears subcutaneously in the submaxillary region, or may extent to the supe- 
rior part of the neck, appearing as a soft fluctuating enlargement. There is an absence of inflammatory symptoms and the skin is not adherent over it. 'These cysts develop slowly in contrast to a rapidly developing hematoma; the skin is adherent in the latter. 'The contents of the cysts can be obtained by the use of a large aspirating needle or trocar and examined. The fluid which flows out very slowly is a thick, viscid, honey-like material which makes the diagnosis positive.

Treatment.-Surgical.-Complete removal of the cyst is hardly possible by excision, as the glandular secretion continues, and when adhesion of the edges of the wound takes place, another cyst will develop. The most satisfactory treatment consists in aspirating all of the contents and the injection of Lugol's solution or tincture of iodin to destroy the cyst wall and the secreting gland. The injection should be sufficient to distend the cyst so that some of it will be forced to gravitate into the gland to destroy it. 'This should be done every second or third day. As soon as the gland is destroyed, the secretions will stop, the gland will atrophy and the enlargement disappear. This usually takes place in three to four weeks.

\section{MALIGNANT NEOPLASMS OF THE MOUTH.}

Epitheliomata. - These tumors appear most commonly on the margin of the lips, involving the mucous membrane, subcutaneous tissue, muscles and skin. They occur most often in old animals.

Symptoms.- In the beginning they are olsserved as small flat growths, which later have a tendency to show ulceration on the surface. 'The surface has a roughened, granulating appearance often covered with a thin dried mass. It gradually develops in size until it invades the entire lip and occasionally the maxillæ. Secondary enlargement of the cervical and submaxillary lymph glands is of common occurrence. Epitheliomata are diagnosed clinically by their rapid, progressive growth, roughened irregular surface and invasion 
of the entire lip. In cases where a satisfactory diagnosis cannot be made clinically a small portion can be removed for microscopical examination.

Treatment.-Surgical.-Complete excision early as possible. When the tumor is small and involving the lip, a "V"-shaped incision should be made through the entire lip removing all of the tissue affected. The wound should be carefully sutured to bring the edges in direct apposition when healing will readily take place. In more diffuse and extensive growths complete excision is difficult but should be attempted if at all possible. When the lymph glands are involved treatment is usually unsatisfactory. After-treatment consists in the use of antiseptic washes.

Sarcomata. - This tumor most commonly affects the maxillæ, usually the superior maxilla, as it originates either in the periosteum, the medulla, or the endosteum of the bone. They are frequently quite large and often invade the nasal passages, the orbits and the sinuses. Metastatic processes are common in the adjacent lymph glands and occasionally a generalized sarcomatosis is produced.

Symptoms. - The growth first appears as a rather firm, oval, reddish colored enlargement in close proximity to the maxilla. Later the surface becomes irregular, lobulated and covered with thickened mucous membrane. The tumor usually has a broad base rather firmly attached, but may in some cases be pedunculated: The growth is quite rapid, beginning as one enlargement around which eventually numerous secondary ones develop; later by confluence the base becomes broal. 'The teeth are often hidden by the growth or in some ases elevated from their position.

Treatment. - The removal of the entire growth should be done as early as possible. Inder general anesthesia, dissect out the tumor, using bone forceps, chisel or curette, being careful to get out all the affected tissue. When the alveoli are affected, extract the tooth and curette and cauterize the cavities to destroy, as far as possible, all the sarcomatous cells. When the lymph glands and the sinuses are involved treatment is practically impossible. 


\section{FOREIGN BODIES IN THE MOUTH.}

Foreign bodies in the mouth consist principally of such objects as are taken in the mouth with food or during play. Fragments of bone are most common. These either penetrate the soft tissues or are firmly lodged in between the teeth, or, if larger, may be between the rows of teeth. Splinters of wood and pieces of wire are usually found imbedded in the mucous membrane. Needles, pins and fish bones are also common, especially in cats. Birds often have grains of corn, sunflower seeds, etc., lodged under the tongue in the floor of the mouth. Hunting dogs when running through fields often have pieces of twigs or weeds forced in the mucous membrane of the mouth. Porcupine quills are common in localities where these animals abound.

Symptoms. - When the foreign body causes much inconvenience, the animal makes persistent attempts to remove it by pawing at the mouth with the feet and shaking the head. Masticatory movements may be continuous or the mouth may be held open, with some salivation. Smaller objects as needies, pins, ete., may not produce prominent symptoms at first and the former may only be noticed by the presence of a thread attacher. Food and drink are either entirely refused or fceble attempts made at eating. Thorough inspection of the mouth reveals the foreign body or the wound in the membrane where it entered.

Treatment.-Ordinarily objects lodged betwcen the teeth may be readily removed with forceps. Imbedded objects should be observed closely to determine their direction of entrance and removed in the opposite direction. Vicious animals, especially eats, should be etherized to render the opcration safe. 


\section{IDISEASES OF THE TEETH.}

Examination.-Examination of the teeth ean be done by elevating the lips, whieh readily exposes them, or by the use of tapes or a mouth speeulum to hold the mouth open (see examination of the mouth). (Careful inspeetion should be made for malformations, fraetures, inerustations of tartar and disease eonditions.

\section{MALFORMATIONS OF THE TEETH.}

These are not eommon in small animals, only oeeasionally' being seen as distortions of the skull bones so affeeting the jaws that the teeth do not meet in proper relationship. 'This may' result in exeessively long teetlr. A few instances of defeetive dentition are reeorded. 'They are important only when they' interfere with mastication and subsequent nutrition. Breeding has been carried to such an extent with sone breeds (English bull, ete.) as to amount almost to a malformation of the maxillæ with unusual relationship of the teeth in some individuals. Birds oeeasionally have a deformity of the beak rendering prehension of food diffieult.

Treatment. - Surgical.-Surgical intervention is possible only in rare eases to improve the appearance or eondition. Preseribe a suitable diet when the animal is unable to masticate solid food (meat, ete., should be given in small pieces). Long teeth may be removed or the points clipped off with bone foreeps. 'The long points of malformed beaks should be so shaped, using a sharp knife, that they will assume a more normal form. 


\section{FRACTURES OF THE TEETH.}

Fractures of the tecth with exposure of the pulp cavity sometimes occur, especially in dogs, and are usually due to fighting, falls on hard surfaces, kicks (horses, ctc.), being hit with hard objects; often occur during play in attempting to catch a ball or stone when thrown; also due to biting iron bars in their attempts to escape from cages.

Treatment.-If the fractured tooth is painful, extract it. It is possible to have it filled.

\section{INCRUSTATIONS OF TARTAR.}

'Tartar occurs quite commonly on the teeth of dogs and it is due to the precipitation of the carbonates, phosphates and some organic substances from their solution in the mouth secretions. It collects around the neck of the tooth close to the gingival border which is the least exposed part of the tooth, and therefore the deposits are not worn off by eating. By constant accretion, these deposits increase, producing an irritation to the gums and inflammation. As the process continues, it separates the gums from the teeth and often causes the tceth to loosen and fall out. In some severe cases, the tooth may be completcly covered with the incrustations. The gums are reddened, swollen and painful, later showing ulceration. A foul odor is emitted from the mouth.

Rcmove all deposits from the teeth with a curette or scaling instrument, being careful not to injure the gums. Extract all loose teeth. Tincture of myrrh applied to the gums is useful as an antiscptic, astringent and reodorant. As this condition usually persists, these cases should have attention every few wecks.

\section{ALVEOLAR PERIOSTITIS. -}

P'ericementitis. P'eriodontitis.

Definition.-This is an inflammation of the alveolar periosteum.

Etiology.-It begins in most cases from the irritation produced by a collection of tartar around the teeth. As the 
incrustation gradually increases, it causes separation of the gums from the teeth and this learls to suppuration of the peridental membrane at the neck of the tooth, and, as the process continues, the entire membrane becomes involved.

Pathology.--The affected tooth is loosened, slightly raised from its alveolus, and, from disturbance to the vessels and nerves leading to loss of nutrition, it becomes discolored (dark or yellowish.) Injury to a tooth with exposure of the peridental membrane may also lead to a similar condition but in this case only one or a few teeth are affected while in the former several, or in severe cases the entire set is lost.

Symptoms. - The animal either refuses foor or takes only a small amount and this very carefully. Saliva flows freely and the mouth emits a foul odor. The gums are dark red (livid), swollen and bleed easily. Tlcerative stomatitis often accompanies this condition.

Treatment.-Extract all loose teeth and remove incrustations from the others. Disinfect the mouth daily with antiseptic solutions (potassium permanganate 1-250), or by direct application of tincture of myrrh to the gums. Extraction may be accomplished by the use of dental forceps, a mouth speculum being necessary to open the mouth when molars are to be extracted but not necessary for the incisors. Grasp the tooth as far up the root as possible and for single fanged teeth, loosen with half turn twist each way, and molars with a pressure alternately inward and outward, care being taken to avoid breaking the roots. General anesthesia should be used when the animal is vicious.

\section{CARIES OF THE TEETH.}

Definition.-Caries is the true decomposition or disintegration of the dental tissues. It is rare in small animals.

Etiology.-The process always commences on the surface of the tooth where the enamel is inferior or lamaged, or at protected parts of the tooth where food particles lodge and decompose.

Pathology.-The point where this begins may or may not be discolored, and as the dentin is disintegrated more 
rapidly than the enamel, a cavity is soon formed within the tooth but having a small opening through the enamel. As this process continues it opens the pulp cavity which becomes infected. As long as the opening through the enamel is free the pus will be discharged without further complication but if it becomes closed with food particles, etc., an abscess develops at the root and the pus burrows out into the adjacent parts, usually into the sinuses or may break down the sinus wall resulting in a maxillary fistula. The disintegration of the dental tissue is brought about by chemical action from the lactic acid fermentation in the mouth.

Symptoms. - This condition will be noticed only by careful inspection until the disintegrating process reacles the pulp cavity when it gives rise to sharp pain, depression of the head toward the affected side and careful mastication. When a fistula results the pain is not pronounced. Empyema of the sinuses often causes a distortion of the affecterl side and a dull sound when percussed. Suspect all fistulæ opening in the maxillary region as having their origin in a carious tooth.

Treatment. - Extract diseased tooth and cleanse the alveolus with antiseptic solution (boric acid 2 per cent.). If a fistula is present, it is readily irrigated when the tooth is removed. 'Thorough irrigation through the entire tract should be done daily. Healing is usually rapid. 


\section{CHAPTER III.}

\section{DISEASES OF THE TONGLE.}

Examination. - This can be readily done by opening the mouth (see examination of the mouth) and pulling the tongue forward as far as possible with a blunt forceps or by a piece of tape wrapped around the free end of the tongue, using gentle traction.

In paralysis of the tongue, it will be relaxed and protrude from one side of the mouth. The examination should be made for $(a)$ foreign bodies, splinters of bone, needles, pins, etc., which often penetrate the tongue. (b) Rubber bands, pieces of bone or cartilage becoming fixed around the free portion of the tongue. (c) Inflammation of the tongue (glossitis). (d) Necrosis of the free portion of the tongue. (e) Ulcerative processes extending from the mucous membrane of the mouth. (f) Edema of the tongue occurring during the course of infectious diseases (distemper, etc.). (g) Inflammation of the glands in the posterior part of the tongue. ( $h$ ) Deposits on the tongue.

\section{GLOSSITIS.}

Definition. - An inflammation of the tonguc.

Etiology. - Mechanical.-Injuries by foreign bodies (pieces of bone, needles, etc.) which penetrate it; biting the tonguc or being bitten by other animals or insects, cats being frequently bitten by rats or mice.

Chemical.-Medicinal substances taken in too concentrated form (carbolic acid, anmonia, etc.) or given for too long a period or in too large doses will lead to severe glossitis.

Thermic.-Hot foodstuff's or the tip of the tongue touched against hot objects. 
Infectious.-Occurs during the course of infectious diseases (distemper, etc.). Glossitis also usually accompanies the various forms of stomatitis.

Pathology.-The mucous membrane is reddened, swollen and covered with a grayish-white membranous deposit. When foreign bodies are present at the point of entrance, the tongue is swollen and edematous, later becoming a dark bluish color. In severe cases foci of gangrene are seen.

Symptoms. - The animal refuses food or eats very carefully; the mouth is often held open; saliva, sometimes streaked with blood, runs from the corners of the mouth. The swelling may be so great that the tongue is protruded. Direct examination reveals the swollen and reddened condition of the tongue and quite often foreign bodies are found imbedded in it or around the free end. As a rule no general symptoms are noticed except when due to infectious diseases.

Prognosis.-The majority of cases terminate in recovery, depending somewhat on the cause and extent of the injury. When due to infectious diseases, the prognosis is less favorable.

Treatment.-Dietetic.-When the swelling interferes with mastication, soft or liquid foods should be given (milk, beef broth, etc.).

Medical.-Antiseptic washes (boric acid 2 per cent., potassium permanganate 1-250, potassium chlorate 1-100) may be used several times daily. When there is severe swelling, astringent solutions are also indicated (alum 1 per cent.).

Surgical. - In very severe cases or where abscesses develop, make deep incisions in the substance of the tongue followed by the use of antiseptic solutions.

\section{GANGRENE OF THE TONGUE.}

\section{Gungrenous Glossitis.}

Definition. - This is a gangrenous condition of the tongue which may involve the entire free end or appear as small rapidly: spreading ulcers. 
Etiology.-Mechanical.-Obstruction to the circulation from foreign bodies is not uncommon. Rubber bands are occasionally slipped over the tongue by children; rings of cartilage from the trachea or aorta from cadavers upon which the dog has been feeding, have been found around the tongue.

Infectious. - Gangrene of the tongue may be caused by the Bacillus necrophorus and often is associated with gangrenous stomatitis.

Chemical.-The action of concentrated drugs may be so severe as to cause a gangrene.

Pathology. - When the tongue is encircled by foreign bodies which shut off the blood supply that part of the tongue anterior to the foreign body becomes swollen and in a few hours very dark and gangrenous and will slough off in three or four days. In cases of infection, the process begins as a small ulcer which rapidly extends and may involve the entire organ. Chemicals usually cause only small areas of gangrene which do not show a tendency to spread.

Symptoms. - The animals refuse food and drink, and saliva flows freely. If the tongue is much swollen it may protrude from the mouth. The inconvenience causes the animal to appear depressed. General disturbance is not noticed in the early stages but later, from the absorption of the gangrenous toxins, an elevation of temperature and symptoms of sapremia are seen.

Diagnosis. - The presence of the foreign body around the tongue partly obscured by the swelling, or in infection the rapidly spreading ulcer.

Prognosis.-This depends on the degree of compression by the foreign body, and the length of time it has been on the tongue. Complete obstruction of the blood supply for a few hours (4-6) will of ten result in loss of the tongue. The infectious form is favorable if treated early.

Treatment.- Remove the cause if a foreign body. When due to infection or chemieals, remove the nearotic material with a eurette and thoroughly eleanse with antiseptic solntions (see (rangrenous stomatitis.) Ifter removing the foreign body from around the tongue a few hours should 
be allowed for the establishment of the circulation and if it does not occur in that time, the affected part should be amputated. This must be done under general anesthesia. Fix the jaws open with a mouth speculum and draw the tongue forward, using blunt forceps or a tape suture through the healthy tissue to hold it. A pair of dull, heavy scissors can be used to remove the diseased part. A small écraseur will also do. Control the excessive hemorrhage by twisting the artery with forceps or by ligation. An animal with part of the tongue removed will have difficulty in drinking, and water should be supplied in a vessel of sufficient depth to allow the mouth to be submerged or from a faucet placed at the height of the head. 


\section{DISEASES OF THE SALIVARY GLANDS.}

Examination. - 1. The glands can be examined by palpation for: (a) Enlargements (cysts, abscesses, tumors, etc.); (b) inflammations; $(c)$ wounds; $(d)$ fistulæ.

2. The character and the amount of the secretions. The normal secretion is a mixture of secretions from the parotid, submaxillary, sublingual and the mucous glands of the mouth. It is a thin, slightly viscid, opalescent fluid, having a feeble alkalin reaction and a specific gravity of $1005-1008$.

An increase in the salivary secretion is noticed in the following conditions: 'The different forms of stomatitis; dentition; chorea by reason of the increased masticatory movements; gastric ulcers; nausea; helminthiasis; severe pain; direct nerve stimulation either central or peripheral; uremia; mercurial poisoning; drugs such as pilocarpin which produce direct stimulation to the secretory nerve.

The secretion of saliva is diminished in the following conditions: During the course of fevers (pneumonia, septic fever, etc.); after the use of atropin or belladonna; fright and excitement; severe diarrheas; cirrhosis of the liver when ascites is developing; atrophy of the salivary glands.

\section{PAROTITIS.}

\section{Iumps.}

Definition.-An acute or chronic inflammation of the parotid gland.

Etiology.-Mechanical.-Direct injuries to the gland by being run over by vehicles; struck with stones; kicks, etc.; pulling back when tied and the collar injuring the grland or by being caught in a door.

Chemical.--The internal administration of potassium iodid in too large doses or the absorption of iodin from local appli- 
cations will often produce it. Lcad when given in large doses will sometimes cause acutc parotitis which may result in a chronic induration of the gland. Inflammations of the mucous membrane of the mouth from chemicals will reflexly and by absorption produce an inflammation of the glands. Obstruction of the ducts from chemical action or from other causes of stomatitis of ten leads to a chronic parotitis. From the retention of the secretions, the glands become enlarged and hard and may resemble tumor formation.

Infectious.-The disease sometimes appears in the form of an epizoötic which no doubt is caused by a specific organism. A diplostreptococcus has been isolated from the gland and from Steno's duct during such epizoötics. Other organisms have also been found but as yet none of them has proved specific. During the course of infectious diseases (distemper, etc.) the parotid is frequently infected resulting in an acute inflammation. Parotitis occurs as a secondary disease in pharyngitis, stomatitis and other local inflammatory conditions. Chronic parotitis results from repeated acute attacks or from obstruction to the ducts.

Pathology.-The acute parotitis in the early stages begins with a swelling, congestion and serous infiltration of the interlobular conncctive tissue. Later small abscesses develop which become confluent forming one large abscess. In chronic parotitis there is a thickening of the connective tissue and atrophy of the glandular substance which produce a liard, fibrous condition (indurative parotitis).

Symptoms. - The acute infectious parotitis begins with a swelling in one or both glands, with a collateral edema in the surrounding tissues. The swelling usually develops rapidly, is very painful to the touch and changes the appearance of the head and neck. The head is hold away from the affected side or if bilateral is cxtenderl. The temperature is elevated $\left(103^{\circ}-105^{\circ} \mathrm{F}\right.$.), the animal slows depression, partial or complete loss of appetite, and mastication is slow and careful. 'The saliva is usually increased in quantity and runs from the corners of the mouth in strands. In a few day's abscesses develop, producing a fluctuating enlargement which diseharges reddish eolored pus when opened. In 
parotitis produced by injuries, lesions are often found on the skin. In most cases only one gland is affected and general disturbance will not be noted. When resulting from pharyngitis and stomatitis, the symptoms are modified by the swelling of the mucous membrane and connective tissues. There is quite an extensive edema of the lips, tongue and in the tissue around the gland. Chronic parotitis is characterized by a firm enlargement of the gland, acute symptoms being absent. Steno's duct may be distended from a closure of the buccal opening.

Diagnosis. - Acute parotitis may be confused with enlargement of the lymph glands and therefore must be examined carefully. The position, shape and nature of the enlargement are to be considered in making the diagnosis.

Prognosis.- I'sually favorable. When abscesses develop a fistula may result from the opening of some of the ducts. In chronic indurative parotitis, the prognosis is less favorable.

Treatment.-In the early stages when the glands are enlarged endeavor to hasten resolution, or abscess formation. Later a stimulating liniment (soap or white) may be used with good results. When abscesses derelop, they should be opened early so as to secure good drainage. When the incision is made, it should be only through the skin, and the tissues then separated with the fingers in order not to injure the gland any more than is absolutely necessary. Irrigate the cavity daily with antiseptic solutions (boric acid 2 per cent., etc.). In cases where the discharge is persistent and abundant, tincture of iodin or Lugol's solution injected into the gland gives good results (see Salivary Fistula).

In chronic parotitis recovery may be hastened by the application of iodin ointment orer the surface of the glands. Lugol's solution may be injected directly into the gland. Insert the needle rather leep into the gland substance, being careful that it does not enter a bloodressel, which will be shown by the free flow of blood. Injections may be made at different parts of the gland and repeated in a few days if necessary. Small doses of potassium iodid (0.05-0.10) givell once daily will assist in the absorption of the deposits in the gland. 


\section{SUBMAXILLARY AND SUBLINGUAL GLANDS.}

'l'hese glands owing to their position are not as often injured as the parotid, therefore inflammation due to traumatism is rare. Occasionally infection develops in the glands by gaining entrance through the ducts and producing an acute inflammation.

Symptoms. - Enlargement of the glands, profuse salivation with the head held extended. Abscesses often form in the glands which open and discharge a reddish colored pus. 'The opening may be through the skin or into the mouth.

Treatment.-See Parotitis.

\section{SALIVARY FISTULA.}

Definition.-A fistula which discharges secretions from the salivary glands.

Etiology.-Mechanical.-Injuries (cuts, bites, etc.) in which Steno's duct or any of the smaller ducts are opened so as to permit the escape of saliva; often follows operations for the removal of a calculus from Steno's duct.

Infectious.-Abscesses which develop within the gland may rupture and lcavc an opening through which the saliva escapes.

Pathology.- When the injuries or abscesses open a duct, the overlying tissues heal until there is only a small opening left through which the saliva continues to be discharged. In some cases, however, the hcaling of the skin is completc and then the accumulated secretions burrow along under the skin. When there is enough fluid to cause necrosis of the skin by the pressure, it opens and thus forms a fistula. 'This in turn may heal only to reopen in another place. Such openings have been found in the lumbar region.

Symptoms. - A small opening will be noted partially covered with matted hair and from which is discharged a thin serous fluid (saliva) and occasionally a small amount of pus. When probed, it will be found to be only subcutaneous unless in the region of the gland when it will be deeper and lead to the gland or duct. A history of thesc 
fistulæ appearing from time to time may extend over several months. In fistula of the duct, the saliva flows freely and increases where food is offered or taken.

Diagnosis.-The chronicity and the character of the discharge are usually sufficient to make a diagnosis.

Prognosis.-Unfavorable.

Treatment.-When the fistula is distant from the gland, probe carefully until the origin is reached. Open the skin at this point and apply treatment direct to the opening in the duct or gland. 'Tincture of iodin applied direct to the opening leads to swelling which occludes the opening.

In obstinate cases the thermocautery should be used. When Steno's duct is open, suturing may be attempted. When all treatment fails complete destruction of the gland by repeated injections of iodin directly into the gland or extirpation should be practised. 


\section{CHAP T E R V. \\ DISEASES OF THE TONSILS.}

Examination.-The tonsils can be readily examined in docile animals by opening the mouth (see examination of the mouth) and pulling the tongue well forward. In vicious animals, partial or complete anesthesia is advisable. The tonsils should be examined for acute or chronic inflammations, deposits of mucus on their surface, abscess formation, tumors and foreign bodies. The adjacent lymphatic tissues are frequently involved producing a diffuse enlargement of all the surrounding parts.

\section{TONSILLITIS AND LYMPHADENITIS.}

Definition.-An acute or chronic inflammation of the tonsils and the adjacent lymphatic tissues.

Etiology.-Tonsillitis is not of very frequent occurrence. Some breeds (Boston terrier), however, are occasionally affected.

Exposure to wet and cold and bad hygicnic surroundings appear to have a direct influence in producing the disease.

Chemical. - Carbolic acid, arsenic, ete., may produce it by direct irritation.

Mechanical.-Foreign bodies (sharp pieces of bone, needles and pins, ete.) often penetrate the glands and lymph tissue resulting in acute inflammation. Inflammation of contiguous parts will of ten produce the condition by spread of the inflammatory process (stomatitis, pharyngitis, etc.).

Infections. - The tonsils and lymphatic tissues undoubtedly take up a lot of micrö̈rganisms, the most common of which are the streptococci and the staphylococei. These organisms by their rapid development in the tonsils produce an acute 
inflammation, and from there, they or their products may enter the general circulation and produce symptoms of a general infection, or toxemia. Further, this condition may appear during the course of some diseases, as distcmper, rabies, endocarditis, etc.

Pathology.-The tonsils and the lymphatic tissues become swollen, reddened, and later covered with a thick, tenacious mucus. Vesicles are frequently formed, and in some instances even membranous exudation, forming a pseudomembrane. The lacunx of the tonsils become filled with a cheesy mass of exudation, often becoming confluent, forming small abscesses. The contents of the lacunæ are composed of epithelial débris and micrococci. In the chronic form, the tonsils become hypertrophied due to a multiplication of the glands mainly involving the lymphoid; or in some instances the fibrous stroma is increased and the tonsils become hard and swollen.

Symptoms. - In the early stages the temperature is clevated $102^{\circ}-104^{\circ} \mathrm{F}$., respirations and pulse accelcrated. When the glands become much enlarged, the patient breathes with difficulty, holding the mouth open, producing a peculiar snoring sound. Direct examination reveals the reddened and enlarged tonsils. The cervical lymph glands are also frequently enlarged from the infection. In severe cases the tonsils become very much enlarged forming abscesses. However, in the majority of cases the inflammation subsides within a week, the temperature becomes normal, and the local condition rapidly disappears. In the chronic form the symptoms are milder and are usually overlooked.

Prognosis. - In most cases favorable; depends somewhat upon the cause. The course is rarely longer than one week or ten days for the acute form, while the chronic form may continue for several weeks.

Treatment.- Iocally the tonsils and the lymph tissucs may be treated with a 5 per cent. sodium hicarbonate solution. Astringent and styptic preparations (iron, alum, zinc and silver nitrate) may be found useful. Borax in glycerin (2 per cent.) or thymol in glycerin (3 per cent.) (an be userl as a deodorant when the mouth becomes offensive. Ahscesses 
should be incised freely to allow drainage and antiseptic solutions used. In chronic hypertrophy of the glands and lymph tissues, it often becomes necessary to remove the enlargement surgically. This can readily be done under anesthesia with the aid of a mouth speculum to fix the jaws open when with a sharp curette the growths can quickly be removed. After-treatment with antiseptic solutions (boric acid 2 per cent.) should be continued for a few days. 


\section{CHAPTER VI.}

\section{DISEASES OF THE PHARYNX.}

Examination.-The pharynx is easily exposed to view by opening the mouth and pulling the tongue forward. For a more careful examination the mouth speculum may be used to immobilize the jaws, while in vicious animals it is always best to use an anesthetic.

\section{PHARYNGITIS.}

Pharyngitis is divided into $(a)$ acute, and $(b)$ chronic.

Acute Pharyngitis.-Definition. - An acute inflammation of the pharynx.

Etiology.-Mechanical.-Sharp foreign bodies (needles, pins, sharp pieces of bone, etc.) may penetrate the mucous membrane and produce an acute local inflammation.

Chemical.-Inhalation of gases (smoke, ammonia, etc.) will produce inflammation in the plarynx as well as in the larynx. Drugs administered in concentrated form.

Thermic.-Very hot liquids or foodstuff's when swallowed frequently produce in dogs a severe pharyngitis. Exposure to cold when the body is heated will produce a congestion in the pharyngeal mucous membrane and this may result in an acute pharyngitis.

Infectious.-Many of the above causes predispose to infection, the most common of which are produced by the streptococcus and the Bacillus necrophorus. A severe form of infectious pharyngitis is occasionally seen in wcek-old puppies and kittens, in some cases amounting almost to an enzoötic, affecting the entire litter. Pharyngitis is secondary to infectious diseases as rabies, distcmper, infectious nasal catarrh and cholera of birds. It is often produced by an extension of inflammation from the adjacent organs and tissues (nasal catarrh, bronchitis, stomatitis, etc.). 
Pathology.-(a) Acute pharyngitis is characterized by redness and swelling of the nuncous membrane which has a glazed appearance due to the collection of mucus on the surface. Iater there is an abundant discharge of a thick mucus or a mucopurulent exuclate. Occasionally it may be tinged with blood. In severe cases small erosions appear on the posterior part of the pharynx. The lymph follicles are enlarged and appear as small, round, elevated, reddish nodules projecting through the membrane.

(b) The discharge contains bacteria, leukocytes, blood cells and desquamated and degenerated epithelium.

Symptoms.-One of the first symptoms is difficulty in swallowing food. Frequent attempts at swallowing with the head extenderl are often noticed in the early stages. Later, in the more severe cases, abundant salivation results from increased secretion, and inability to swallow, while retching and sometimes romiting will be seen in some cases from the irritation. The head is usually held extended and palpation of the pharynx produces pain. 'The submaxillary and retropharyngeal lymph glands often become enlarged and may produce abscesses. Occasionally in severe cases the salivary glands become involved. On examination of the pharynx the mucous membrane will be found reddened, congested, and covered with mucus or mucopurulent exudate, depending upon the stage of the development of the disease, and in severe cases swelling and congestion of the adjacent tissues. Cough is absent and only occurs when the inflammatory process extends to the larynx. In all cases where infection develops, the temperature is elevated $\left(103^{\circ}-105^{\circ} \mathrm{F}\right.$.). In mild cases when the general symptoms are absent, the appetite remains good although the animal takes food slowly and with care. In severe cases there is a complete loss of appetite.

Diagnosis. - The symptoms of acute pharyngitis are very characteristic: 'The extended head, difficult swallowing and salivation; while direct examination and the temperature readily distinguish it from foreign bodies, tumors and paralysis of the pharynx.

Prognosis. - In older animals it is farorable, recovery oceurring in one to two weeks. In young animals, when it occurs as an enzoötic, the mortality is high. 
Treatment.-Dietetic.-Soft liquid foods (rice soup, milk, extract of beef, etc.) should be given in preference to solids to avoid irritating the mucous nembrane.

Medical.-Local treatment in the form of astringent and antiseptic solutions (2 per cent. silver nitrate solution; 5 per cent. alum solution; iorlin and glycerin 1-30) applierl directly to the membrane by using a pledget of cotton held in dressing forceps. In milder cases a solution of potassium chlorate ( 2 per cent.) or iron sulphate in the drinking water is recommended. A Priesnitz compress applied over the pharynx and followed with mild stimulating limiments (soap liniment, etc.) is often beneficial. Internally unild purgatives such as castor oil or cascara should be used.

Chronic Pharyngitis. - Definition. - A chronic inflammation of the mucous membrane of the pharynx.

Etiology.-This condition may follow repeated acute attacks, or is frequently associated with chronic nasal catarrh. It also occurs secondary to inflammation of adjacent tissues. Dogs constantly barking from a nervous temperament or during shows are often affected from the spread of the inflammation from the larynx.

Pathology. - The mucous membrane is relaxed, the lymph tissue becomes proliferated forming small, round elevations, red or bluish-red in color which project above the surface of the membrane, and is known as pharvngitis granulosa. The secretions are lessened producing a dry, glistening condition of the pharyngeal mucosa.

Symptoms. - The symptoms are similar in many respects to those of acute pharyngitis, but milder in most cases. 'The swelling of the adjacent tissues and lymph glands is hardly noticeable. The difficulty in swallowing is especially marked when a large quantity of food is taken or when the food is very hot or very cold. Direct examination of the pharynx reveals the bluish-red color and the elevations over its surface.

Prognosis. - Considered farorable in most cases, depending somewhat on the possibility of removing the camses. In the milder cases it is rery often overlooked.

Treatment.-I)irect applieation of Lugol's solution to the mucous membrane has been found useful in most case's. 
Tannic acid and glycerin (1-30) may also be used. Chlorid of iron (1-10) in water is of value owing to its astringent and antiseptic qualities. The application of the preparations should be made daily until the symptoms subside.

\section{FOREIGN BODIES IN THE PHARYNX.}

Foreign bodies may find lodgment in the mouth or in the pharynx. When dogs are ravenously hungry large particles of food or food containing foreign bodies are swallowed which may lodge in the pharynx, producing choking. The condition often terminates fatally in a short time. Cats when fed on fish are liable to have fish bones lodge in the pharynx. These bones frequently penetrate the mucosa, producing an edema of the pharynx and larynx, resulting in death from asphyxia. Birds, when eating large seeds (sunflower seeds, corn, etc.) often have them lodge in the pharynx, producing serious symptoms. Various kinds of foreign bodies have been found in the pharynx, the most common being needles, pins, bones, hard food masses, meat skewers, cartilage, etc.

Symptoms. - The symptoms vary somewhat according to the size and character of the foreign body. Dribbling of saliva from the corners of the mouth; frequent attempts at swallowing; clawing at the mouth with the forefeet, and sometimes retching and vomiting. When the foreign bodies are large they often interfere with the respirations.

Diagnosis. - The diagnosis is made by direct examination of the pharynx (see examination of the pharynx), the sudden development and the characteristic symptoms.

Prognosis.-Depends upon the size and character of the foreign body. Where the foreign bodies are small and the animal can be treated at once, the prognosis is favorable. In other cases where cdema occurs or where the foreign body is quite large, death may terminate before assistance can be given.

Treatment. - Surgical. - A mouth speculum is used to keep the mouth open and the foreign body often can be easily removed with a long curved throat forceps. Holding the tongue well forward will materially assist in locating accu- 
rately the foreign body. When the foreign body is in the form of a threaded needle, which frequently occurs in cats, the sharp point is usually toward the mouth. Therefore care should be used in removing it to prevent laceration of the tissues. By grasping it with the forceps and pushing it downward until the sharp point is free from the tissues, it can then be readily removed. Large food masses often can be broken between the fingers and extracted or pushed down into the esophagus. Where edema of the tissues results from laceration and symptoms of suffocation develop, tracheotomy should be performed at once. (See Traclieotomy.) No after-treatment is necessary except when severe injury to the tissues has taken place; in these conditions the pharynx should be treated direct with antiseptic solutions (boric acid 2 per cent.), using a dressing forceps with a pledget of cotton firmly attached. In birds the mouth is held open and a small pincette used to dislodge the foreign body.

\section{PARALYSIS OF THE PHARYNX.}

Pharyngeal paralysis occurs mostly during the course of specific diseases (rabies, distemper) and will therefore be described under those diseases which it accompanies.

\section{CROUPOUS PHARYNGITIS OF BIRDS.}

Definition.-A croupous inflammation affecting the pharyngeal mucous membrane of birls.

Etiology.- This condition is produced by a flagellated infusoria, the Monocercomonas gallinæ. 'This parasite is round or discoid in shape, of a pale color and from 14 to 25 $\mathrm{mm}$. in length and 5 to $7 \mathrm{mmm}$. in breadtl. 'The disease affects young birds, especially pigeons. Unsanitary conditions and cold, damp quarters are predisposing factors.

Pathology.-.-Small white elevations are found on the mucous membrane of the pharynx, often extending into the esophagus and crop. Occasionally these lesions are noted at the base of the tongue and on the palate. Surrounding these small elevations are found zones of acute inflammations with considerable swelling and congestion of the membrane. 
Symptoms. - Ioss of appetite, dulness and general weakness are the first symptoms observed. The plumage becomes rough, the wings are pendant and the mouth held open. 'The odor from the mouth is offensive. Fxamination of the' posterior part of the mouth and the pharynx reveals the presence of the small white elevations. A microscopic examination of scrapings made from these white spots will confirm the diagnosis by finding the infusoria.

Prognosis. - It should be considered unfavorable especially in very young birds. Death occurs in many cases from loss of appetite and exhaustion.

Treatment.-Where only a few cases are observed, they should be separated at once from the healthy birds and the runways and roosts thoroughly disinfected with lime, or carbolic acid ( 5 per cent.). 'The mouth and pharynx should be cleansed with a solution of boric acid (2 per cent.) or creolin to destroy the infusoria. Remove the small elevations with a blunt curette and apply to the surface chlorid of iron (1-10) in water or a solution of tamic acid (5 per cent.) in water, to arrest the hemorrhage and to produce antiseptic action.

\section{NEOPLASMS OF THE PHARYNX.}

Polypoid Growths.-Polypoid growths (myxomas) are found occasionally projecting from the pharyngeal mucous membrane. 'They vary greatly in size from half an inch to four inches in length. From severe or sudden exertion or swallowing they are often forced into the esophageal opening, interfering with the prehension of food and rlrink.

Symptoms.-Sudden interference with deglutition; symptoms of suffocation; often rapirl recovery; periodic recurrence of the symptoms and direct examination of the pharynx will reveal the presence of the polypoid growths.

Treatment.-Surgical. - The mouth speculum should be used, the tongue pulled well forward and a small wire écraseur used to remove the enlargement. It should be removed as close to the base as possible. No after-treatment is necessary. Recovery takes place promptly. 
Epithelioma.-These are found occasionally in old animals, and occur in the pharynx as a primary condition or may be due to metastasis. 'The retropharyngeal and submaxillary lymph glands are nearly always involved. This condition has been seen secondary to malignant goiter, having extended to the lymph glands by metastasis and from there to the pharynx.

Symptoms. - Difficulty in swallowing; fetid odor from the mouth; often profuse salivation. Direct examination shows the presence of an ulcerated enlargement on the pharyngeal mucosa. The general condition is in most cases disturbed. Emaciation, especially when due to metastasis; loss of appetite. A small portion of the enlargement should be obtained and examined microscopically to confirm the diagnosis.

Treatment.-No satisfactory treatment can be given. 


\section{CHAPTER VII.}

\section{DISEASES OF THE ESOPHAGUS.}

Examination.-The cervical portion of the esophagus is readily examined by palpation over its course along the upper surface of the trachea. The probang affords a means of examining the interior for foreign bodies, strictures, etc., along its entire length.

\section{ESOPHAGITIS.}

Definition.-An acute inflammation of the mucous membrane of the esophagus.

Etiology.-(a) Mechanical.-Irritation from foreign bodies (bones, needles, splinters of wood, etc.), passing of sounds, etc., lacerating the mucous membrane. External injuries to walls.

(b) Thermic.-Eating very hot foodstuffs or drinking hot liquids.

(c) Chemical.-Alkalies and acids, ammonia and corrosive medicinal agents frequently in their passage through the esophagus produce an acute inflammation.

(d) Infectious.-Occurs during the course of infectious diseases (rabies, distemper).

Secondarily it is produced by the spread of the inflammation from the pharynx or stomach. It occurs sometimes spontaneously in very young suckling animals.

Pathology.-Redness of the mucosa is rarely seen except after injuries or severe chemical irritants. The epithelium is thickened, desquamated and the surface covered with a fine granular substance. 'The mucous follicles are swollen and sometimes erosions may be seen. In phlegmonous inflammation, the mucous membrane is swollen, with a 
purulent infiltration in the submucosa. This condition is usually seen around foreign bodies when they penetrate the membranes, and, as a rule, remains localized. Gangrene of the membrane is sometimes seen where the injury or infection has been severe.

Symptoms. - In the milder forms of esophagitis the symptoms are unobserved. In more severe forms there is great difficulty in swallowing which act is often soon followed by vomiting. The vomitus contains blood, the solid particles covered or streaked with it. Frequent attcmpts at swallowing, constantly extending the head or moving it from side to side. In some cases where corrosives have been swallowed fragments of the inucous membrane will be ejected with the vomitus. Profuse salivation, blood is often mixed with the saliva. Palpation along the cervical portion of the esophagus produces severe pain. When localized in the cervical portion the esophagus should be palpated carefully for foreign bodies.

Course and Prognosis.-In the majority of cases, recovery takes place in one to two weeks. In very severe inflammation complications are liable to occur. Strictures or abscess with perforation of the walls of the esophagus may result. In the latter when the thoracic portion is involved the termination is fatal from infectious pleuritis.

Treatment.- In the early stages cold milk, or tamnic acid (1-2 per cent.) in cold water is indicated to allay the inflammation and to produce astringent action. Cold compresses to the cervical portion of the esophagus often have a beneficial action. Inflammations from caustic substances should be treated as early as possible with the proper antidote. When severe pain is shown small doses of morphin should be achministered subcutaneously. Tincture of opium in dilute solution may be given per orem. In very severe cases where foods cannot be given via the mouth, enemata should be employed.

\section{FOREIGN BODIES. OBSTRUCTION IN ESOPHAGUS.}

Etiology.-Obstruction in the esophagus occurs most frequently in dogs. The body lodges immediately posterior to 
the pharynx; at the lower extremity of the cervical portion at its entrance to the thorax; or near the cardiac orifice (at this point the lumen of the esophagus is less than at any other place along its course). Owing to the habit of these animals of taking food in large pieces and without mastication the majority of the obstructions oecur just posterior to the pharynx. However, sharp bodies may be found anywhere along its course. In the dog a great variety of substances have been found producing the obstruction, as these animals during eating or at play swallow many substances that would not be found in other animals. The most common substances which are liable to produce the obstruction in dogs are: Bones, cartilage, hard food masses, pieces of tendon, needles, rubber balls, meat skewers, stones, etc. In cats fish bones are frequently found which they get from eating scraps of fish given them without removing the bones. As a rule fish bones lodge in the anterior portion of the esophagus just behind the pharynx. Young kittens while playing occasionally attempt to swallow threarled ncedles, or pins which lodge at some point along the esophageal wall. Large bodies when indefinitely retained often produce a pressure necrosis with perforation of the walls.

Symptoms. - The early symptoms are those of refusing food, pain during swallowing, paroxysms of ehoking with retching and in some cases vomiting, salivation, and scratching at the mouth and neck. The head is lield extended, the respirations become labored, and the patient evinces severe pain. In cats the mouth is held open, there is profuse salivation, and when the foreign body has penetrated the walls severe nervous symptoms are produced. The foreign body when located in the cervical portion of the esophagus will at once be recognized by the painful swelling which appears along its course, especially in the case of large objects. There is frequently edema of the surrounding tissues which may extend for some distance from the point of injury. Palpation usually reveals the size and character of the obstruction. Often when the objects are located just posterior to the pharymx by opening the mouth and pulling the tongue well forward they can be seen or felt with the finger. In cats, 
needles and fish bones are often seen by this nethod. In cases of small obstructions located in the thoracic portion of the esophagus, the symptoms are not so pronounced. Loss of appetite, emaciation and occasional vomiting are the most prominent manifestations of the condition. However, when sharp objects penetrate the walls at this point, various complications of a serious nature may be produced. The passage of a sound (horse catheter) is often a valuable aid in arriving at a correct diagnosis. In valuable animals, Roentgen rays may be used to assist in locating hard or metallic substances.

Diagnosis. - This is made positive by a careful examination together with the above mentioned symptoms. Care should be taken to exclude rabics as the symptoms are similar. (See Rabies.) Always beware of the dog with "bone in the throat.'

Prognosis.-Foreign bodies located in the cervical portion of the esophagus can usually be removed which is followed by rapid recovery except in those cases where necrosis is produced from pressure upon the walls, or extensive phlegmonous inflammation from perforation. It sometimes happens that needles and pins will penetrate the walls and become encapsuled in the adjacent tissues without producing any further disturbance to the animal. Foreign bodies located in the thoracic portion of the esophagus should always be considered unfavorable owing to their location and the danger of injury to the organs in the thoracic cavity or a purulent pleuritis resulting from perforation.

Treatment.-Obstructions to the esophagus are removed by: (a) Ise of throat forceps; (b) by propulsion with the sound into stomach; (c) by emesis; (d) by esophagotomy; (e) by gastrotomy and sound forcing the foreign body out via mouth.

(a) The throat forceps can often be used to an adrantage when the object is located in the posterior part of the pharynx or in the anterior part of the esophagus. 'The mouth is held open with the speculum, the tongue pulled well forward, and the object grasped with the forceps and removed. Care should be taken to prevent laceration of the tissues in case of a sharp object or one of an irregular shape. 
(b) 'The sound is to be used in those cases where the obstruction is located fartler down the tube and cannot be reached with the throat forceps. Various kinds of instruments have been devised for this purpose. 'The horse catheter will answer in a large number of cases. It is introduced by using the mouth speculum, dcpressing the tongue. Having oiled the instrument, it is passed, holding it firmly against the roof of the mouth and following the postcrior wall of the pharynx, into the esophagus. When the instrument reaches the pharynx the animal will invariably swallow, which greatly assists in its introduction into the esophagus. 'The sound is then brought in contact with the object which is carefully pushed into the stomach. When there is much resistance and the object is firmly fixed, the catheter should bc removed and other methods used to dislodge it. 'The bristle probang is often used to advantage in extracting foreign bodies which do not entirely close the lumen of the esophagus. It is introduced closed so that it may pass the object, and then opened so that the bristles will completely fill the lumen when it is withdrawn bringing the foreign body out in front of it.

(c) 'The act of vomiting, which is easily induced, will often displace the foreign body. This is best brought about by the use of apomorphin (dogs 0.0016-0.006; cats 0.001-0.003). The administration of castor or linseed oil to lubricate the mucous membrane will often assist in removing the obstruction.

(d) Esophagotomy is performed in the following manner: Secure the animal in the dorsal position with the head extended. (lip and shave the hair over the field of operation and cleanse thoroughly with antiseptic solutions (bichlorid of mercury 1-2000; boric acid 2 per cent., etc.). Under general ancsthesia, using morphin (0.016-0.21), ether, etc., make a skin incision over the obstructing body and between the muscles, being carcful to avoid the large vessels of the neck. When the esophagus is exposed make a longitudinal incision through it of sufficient length to allow the foreign body to be rcmoved. Care should be used to prevent laceration of the mucous membrane. When the incision 
is very long, one or more interrupted sutures should be made in the esophagus and the wound packed with gauze saturated in a boric acid solution ( 2 per cent.), retained with sutures through the skin. Allow this to remain in position for twenty-four to forty-eight hours, then remove pack, the sutures in the esophageal wall and treat as an open wound, cleansing it daily with boric acid solution (2 per cent.) until healing is complete. Withhold all food and drink for twentyfour hours and then give only liquid foods for a few days. Rectal feeding of milk, eggs, etc., may be used when the swelling of the mucous membrane is sufficient to obstruct the esophagus.

(e) As a last resort when the firmly fixed object is located in the thoracic portion of the esophagus and cannot be removed by the methods mentioned, gastrotomy should be performed (see Diseases of Stomach), and the catheter introduced to propel the foreign body out via the mouth. Should the object be located in the cardiac portion of the esophagus, a small dressing forceps is often useful to grasp it and remove it via stomach incision.

\section{ESOPHAGISMUS.}

Definition.-A spasmodic contraction of the esophagus.

Etiology.-This condition occurs occasionally during the course of some diseases. It has been observed in chorea, epilepsy and in the early stages of rabies. Sometimes foreign bodies, by irritating the membranes, produce a spasmodic contraction of the walls of the esophagus.

Symptoms. - Very similar to foreign bodies in the esophagus; often foreign bodies are also present.

Prognosis.--In most cáses favorable.

Treatment.-The passage of the sound is usually sufficient to overcome the condition except in the case of foreign bodies being present. (See Foreign Bodies in the Esophagus.)

\section{STRICTURE OF THE ESOPHAGUS.}

Definition.-A constriction of the esophagus due in most cases to cicatricial contraction of the walls reducing the size of the lumen. 
Etiology.- The most common causes of this condition are the following: (a) Cicatricial contraction of healed ulcers, usually due to corrosive poisons; injuries by foreign bodies, etc.; esophagotomy. (b) External pressure by enlarged lymph glands, enlarged thyroids, other tumors and occasionally pericardial effusion. (c) The growth of tumors in the walls of the esophagus (metastatic sarcomas and carcinomas). The stricture may occur in any part of the esophagus, and in severe cases may involve the entire tube, but usually it is found either near the pharynx or the stomach.

Symptoms.-Difficulty in swallowing and only small quantities taken. Severe pain immediately after eating. Retching and vomiting are often noticed. Gradual emaciation due to the interference with deglutition. Examination of the esophagus in the cervical region by palpation or the passage of the sound to the thoracic region will usually reveal the constricted condition of the esophagus.

Prognosis. - Should be considered unfavorable in all cases of long standing. Recent cases, depending upon the cause, may recover sufficiently not to interfere much with the animal's general condition.

Treatment.-By surgical means the cause of the condition should be removed if possible. The passage of the sound, well lubricated, daily for a time will in some cases overcome the constriction. In severe constrictions where a large portion of the walls is involved no treatment can be applied that will be of any service.

\section{DILATATIONS AND DIVERTICULA OF THE ESOPHAGUS.}

Definition.-I)ilatation is a diseased condition whereby the lumen of the esophagus is enlarged (ectasia nesopliagi). A diverticulum is a saccular distention of the esophageal wall at a given point along its course (diverticulum oesophagi).

Etiology.-Dilatation may develop secondary to stenosis of the esophagus, from pressure of food inasses retained above the stenosis resulting in paralysis or atony of the muscular wall; from foreign bodies remaining in the esophagus for sufficient lengtl of time to produce paralysis; injuries; 
esophagotomy. I)iverticula may result from overdistention of the esophagus with rupture of the muscles allowing the mucous membrane to protrude; also due to cicatricial contractions following inflammatory adhesions to lymph glands.

Symptoms. - An enlargement will be noticed along the region of the esophagus if it be in the cervical portion. Difficulty in swallowing; saliva increased; regurgitation of small amounts of undigested and decomposed food covered with mucus; fetid odor from the mouth; the probang may pass without difficulty although it may be obstructed by the stenosis or stricture at the distal end. The symptoms of a diverticulum are very obscure except when large and in the cervical portion of the esophagus. The retention of food gives rise to retching and vomiting, or from the ensuing ulceration may result in the formation of a fistula.

Diagnosis.-Careful observation of the symptoms; the absence of gastric secretions in the vomitus; palpation reducing the enlargement which will become refilled after feeding. The use of the probang may assist in the diagnosis by determining the presence of the stenosis or spasm.

Prognosis.-Diffuse dilatation, or a diverticulum of the thoracic portion of the esophagus, should be considered incurable. The prognosis depends upon the completeness with which nutrition can be carried on. It is always unfavorable as there is a tendency to ulceration and perforation of the walls with formation of a fistula.

Treatment. - No satisfactory treatment can be given except in diverticulum in the cervical portion of the esophagus which may be operated (see Esophagotomy) and the diverticulum reduced by suturing.

\section{CATARRH OF THE CROP IN BIRDS. SOFT CROP.}

Definition. - A catarrhal inflammation of the mucous membrane of the crop.

Etiology. - Irregular feeding is a common causative factor. Birds fed irregularly are quite liable to overload the crop, resulting in distention and partial paralysis of the muscular walls. This condition interferes with the secretion of the 
glands resulting in fermentation or deeomposition of the erop contents whieh irritate the mucous membrane often producing a severe eatarrhal inflammation. Eating indigestible or decomposed substances (feathers, putrid meat, etc.) often produces catarrh of the crop.

Poisons (arsenic, phosphorous, salt, etc.), when taken aceidentally or given intentionally, often produce a very severe form of the disease. The presence of parasites (Dispharagus nasutus, etc.) in the erop will in most eases produce a catarrhal inflammation, the severity of whieh depends upon the number present. In pigeons fermentation of an overabundant secretion after the loss of the young will produce a serious eatarrhal inflammation of the erop and other parts of the digestive tract. Crop catarrh also occurs during the course of some diseases (diphtheria, thrush, eholera).

Symptoms. - Distention of the crop is at first the most noticeable symptom. On palpation the erop will be found to be soft and fluetuating, due to an aecumulation of liquid, gaseous and solid food material. Loss of appetite, eructations of gases, sometimes retehing and vomiting are symptoms. By pressure upon the crop the contents will be expelled via mouth, emitting a very offensive odor. The birds may die from exhaustion.

Diagnosis. - This is determined quite aecurately by a careful examination of the erop and the eharacter and eondition of the eontents.

Prognosis. - When not a symptom or complieation of some other disease (eholera, thrush, ete.), the prognosis is usually eonsidered favorable and the majority respond readily to treatment. When a large number of a flock is affected, indieating a severe eatarrhal inflammation, the prognosis is less favorable owing to the difficulty in applying prompt remedial measures. Repeated return of the eondition often produces the so-ealled hanging crop or dilation.

Treatment. - Hygienic. - The birds should be provided with a elean, dry place free from extremes in temperature.

Dietetic. - In mild eases where the appetite is not entirely lost, solid food should be withheld for a day or so, only a small quantity of liquids being allowed. 
Medical.-Before administering medicinal agents it is necessary to thoroughly empty the crop which is done in the following manner: The bird is held with its head pendant, and the crop carefully manipulated and compressed between the thumb and fingers, when the contents will flow out via mouth. The crop should be thoroughly compressed from behind toward the mouth until it is completely emptied. Ten to 30 c.c. of a bicarbonate of soda solution ( 2 per cent.) is then administered to neutralize the acidity of the retained contents and to dissolve the accumulated mucus from the membrane. For a few days small doses of sodium bicarbonate $(0.15)$ or bismuth subnitrate $(0.10)$ may be administered daily. Only small quantities of food should be allowed for at least four to five days.

\section{OBSTRUCTION OF THE CROP IN BIRDS. HARD CROP.}

Definition.-An impaction of the crop.

Etiology. - The obstruction of the crop is often seen from overfeeding, especially with dry foods (oats, rye, corn, peas, etc.) or with quantities of straw, leaves, grasses, and other indigestible substances swallowed by the bird. In water birds it occurs from eating large quantities of certain grasses (triticum repens, etc.). 'Turkeys are most commonly affected by eating large quantities of insects (grasshoppers, etc.), straw, grains and grasses. Further, swallowing various kinds of foreign bodies with the food (metallic substances, large stones, pieces of glass, bones, egg shells, or large numbers of very small stones, etc.) often produces impaction of the crop.

In some mild cases of catarrh with irritation of the mucous membrane the birds often eat large quantities of indigestible substances leading to an impaction. Animal parasites when in large numbers will produce the same result.

Symptoms. - The birds are dull, stupid, show rough plumage, pale comb and wattles, with a constant opening and closing of the beak; or the beak is helk open to facilitate breathing which is often interfered with by pressure of the enlarged crop upon the trachea. A disagreeable odor emanates from the mouth, and often quantities of fermented or 
decomposed liquids and food particles escape from the mouth and nostrils. 'The appetite in the early stages is lessened and later entirely lost. 'The crop when examined will be found greatly distended, hard and more or less firm on palpation. In some cases it is very hard, producing the so-called "hard crop." When metallic substances are present, it is possible in some cases to palpate them, or in case of sharp objects they are sometimes found projecting through the muscular walls and skin. With the aid of the Roentgen rays the character and size of the foreign body can often be revealed.

Prognosis.-This depends upon the character of the impacted material, its cluration and the condition of the bird. In most cases if allowed to continue it will result fatally. In some cases, the crop becomes enormously distended, producing the so-called "hanging crop."

Treatment.-The early indication in the treatment is to massage the crop and try to remove the contents via mouth. This is best done by suspending the bird head downward, and massaging and kneading the crop carefully which crushes the crop contents so that they may be gently forced into the mouth. Often the entire contents can be removed in this manner. 'The administration of a small quantity of linseed oil to lubricate the esophagus will often assist when applying the above method.

Where this methor will not suffice, it will be necessary to open the crop and remove the contents through the incision. The feathers should be pulled out over a space about $1 \frac{1}{2}$ inches wide and $2-3$ inches long at the point of greatest protrusion. The field of operation should be thoroughly disinfected. The incision can be enlarged sufficiently to admit of the crop contents being easily removed. A blunt curette or pincette is useful in assisting removal. The crop should be thoroughly irrigated with a sodium bicarbonate solution ( 2 per cent.), and the edges of the wound disinfected with a boric acid solution (2 per cent.). The wound is then stitched with a single row of interrupted sutures sufficiently close together to prevent the escape of any contents. The stitches should not be removed for at 
least eight to ten days. All food and liquids should be withheld for twenty-four hours and for the next four or five days only a small quantity of liquid food given.

\section{NEOPLASMS OF THE ESOPHAGUS.}

Tumors of the esophagus are very rare and those occurring most commonly are: Epithelioma, sarcoma and carcinoma. These occur most frequently via metastasis. Retention cysts are found at the anterior part of the esophagus.

Symptoms. - Tumors produce a narrowing of the esophagus similar to stenosis (see Stenosis). When this occurs in the cervical portion the condition may be determined by palpation. A differentiation from foreign bodies, diverticula and dilatation is often made possible by careful palpation.

Diagnosis. - The kind of tumor can only be determined by obtaining some of the enlargement for microscopical examination.

Prognosis.-When malignant tumors (carcinoma, sarcoma, epithelioma) are present, the prognosis is very unfavorable; retention cysts unless they interfere with nutrition are more favorable. Small ones may be present without being noticed for a long time.

Treatment.-No treatment should be attempted for malignant tumors. Retention cysts may be rechuced by pressure from the outside or esophagotomy (see Esophagotomy) may be performed and the cyst wall clestroyed. 


\section{CHAPTER VIII.}

\section{DISEASES OF THE STOMACH.}

Examination.-The stomach can be examined as follows: 1. By the character, condition and quantity of the contents, which may be obtained: (a) By the use of an emetic (apomorphin $0.0016 .-0.003$ etc.), (b) by natural vomiting, (c) by the use of a stomach tube or $(d)$ through gastrotomy (see Foreign Bodies in the Stomach).

2. Palpation over the region of the stomach will cause pain in acute inflammatory conditions, although this may be confused with painful conditions of the liver, peritonitis and enteritis.

3. By performing laparotomy and making a direct examination of the stomach. The mucous membrane may also be examined directly by performing gastrotomy.

\section{GASTRITIS.}

Gastritis is an inflammation of the stomach. The following forms are recognized: (a) Acute, and (b) chronic.

Acute Gastritis.-Simple Catarrh of the Stomach. Acute Dyspepsia.-Definition.-An acute catarrhal inflammation of the mucous membrane of the stomach which may involve the entire wall.

Etiology.-Acute catarrhal gastritis is usually due to errors in diet. The ingestion of more food than can be digested, irregular feeding, or eating unsuitable food, which is partially decomposed containing ptomaines (cadavers, garbage, etc.), and hard pieces of bone, cartilage and tendon are very common causes of this condition. Injuries to the epigastrium (kicks, blows, and being run over by vehicles, etc.).

Chemical.-Various chemical substances (phenol, arsenic, mercury, phosphorus, etc.) when taken accidentally or 
given intentionally will produce a very serious form of gastritis (toxic). An excess of sodium chlorid given in the food to birds will often produce a severe gastritis.

Thermic.-Very hot, solid foods and liquids, or very cold, frozen foods will sometimes produce a gastritis. Rabbits eating frozen vegetables are thus often affected.

Infectious.-Infectious gastritis occurs during the course of most infectious diseases (distemper, rabies, etc.).

Parasites.-See Parasites of the Stomach.

Pathology.-Acute catarrhal gastritis is characterized by a swelling and hyperemia of the mucous membrane which is often corrugated, and intensely red with small ecchymoses appearing over the surface. Small superficial erosions are occasionally noticed.

Symptoms. - Vomiting occurs early and is the most prominent symptom often being quite frequent and in severe cases very persistent. The vomitus in the beginning consists of quantities of undigested and decomposed food material covered with mucus and sometimes streaked with blood, with a very disagreeable odor. When due to chemical causes it often has the characteristic odor of the poison. Later the vomitus consists almost entirely of small amounts of frothy mucus, and when the vomiting is severe there is often an admixture of bile. 'The appetite in severe cases is completely lost and in mild cases it is variable and vitiated, the animal eating unnatural material. Bowel complications (see Enteritis) invariably follow severe cases of gastritis.

On examination the animals show pain on palpation over the region of the stomach, which manipulation often induces vomiting. There is a grayish-white deposit over the dorsal surface of the tongue (furred tongue); the temperature is elevated in the early stages of severe cases $\left(103^{\circ}-105^{\circ} \mathrm{F}\right.$.), later the temperature is subnormal $\left(97^{\circ}-100^{\circ} \mathrm{F}\right.$.). In milder cases the temperature shows but little variation.

The general symptoms in the early stages of severe gastritis are those of uneasiness and intense pain (howling, etc.), stiffness and considerable pain which is shown when the patient is moved about. In milder cases the only symptoms noticeable are occasional vomiting and variable appetite. 
Diagnosis.-Acute non-infectious gastritis is not very difficult to diagnose as the symptoms are very characteristic and quite often the anamnesis is of value. 'The primary infectious form, however, may be confused with that due to specific infectious diseases (distemper, etc.), but this can be distinguished by the general symptoms, especially the temperature which in the latter cases rises more abruptly and shows less variation than in the former.

Prognosis.-In primary acute gastritis, duc to errors in feeding, the prognosis is usually favorable.

When due to poisons, foreign bodies, and injuries, it depends largely upon the extent of injury to the stomach and the possibility of removing the cause. In infectious gastritis, when not due to specific infection, the prognosis is usually favorable, but if accompanying a specific disease it depends on the primary disease.

Treatment.-Dietetic. - In strong animals all food should be withheld twenty-four to forty-eight hours and then only a small amount of easily digested food given at frequent intervals until recovery takes place. In weak individuals easily or predigested food may be allowed unless it induces persistent vomiting when nourishment should be given via the rectum.

Medical.-Mild cases usually recover promptly following the use of a purgative (ol. ricini, $\log \mathrm{s} 15.0$ to 60.0 , cats 5.0 to 20.0; (alomel, dogs $(0.3-0.4$, cats $0.01-0.005$; cascara fld. ext. (logs, 5.0-10.0, cats 1.0-5.0).

In scvere cases unless romiting has occurred an emetic, such as apomorphin hydrochlorate (dogs $0.005-0.01$, cats $0.002-0.005)$ given subcutaneously is indicated to cxpel irritating material and foreign bodies which may be present. Following vomiting, irrigation of the stomach with a sodium bicarbonate solution ( 2 per cent.) is arlvisable to remove irritating material from the surface of the nucous membrane. This can be arcomplished by the use of a rubber tube or horse catheter inserted into the stomach. 'The warm sodium bicarbonate solution $(500.0-1000.0)$ may be introluced into the stomad hy elevating the free end of the tube and using a fummel. Allow the tube to remain in the stomach for a few 
minutes, then depress it to allow the fluid to flow out again. Repeat this two or three times. To stimulate the functions of the stomach, stomachic tonics are indicated:

$$
\text { For Dog. }
$$

R-Ferri et quinnæ citratis . . . . . . . . . 4.0

Pepsini . . . . . . . . . . . 1.0

Saeehari albæ . . . . . . . . . . . 2.0

Misce et fiat pulv. No. XX.

Sig.-Give a powder every twelve hours.

$$
\text { For Cats. }
$$

One-half the above dose.

or

$$
\text { For Dog. }
$$

R-Tineturæ nueis vomieæ . . . . . . . . . 7.0

Tineturæ gentianæ . . . . . . . . . . 10.0

Aqua eommunis . . . . . . . . . . . 60.0

Misee et fiat solutio.

Sig.-Give teaspoonful twice daily.

$$
\text { For Cats. }
$$

$\mathrm{P}$-Tineturæ nueis vomieæ . . . . . . . . . 1.0

Syrupi auranti . . . . . . . . . . . 60.0

Misee et fiat solutio.

Sig.-Give teaspoonful onee daily.

or

R-Aeidi hydroehloriei . . . . . . . . . . 2.5

Tineturæ gentianæ eomp. . . . . . . . . 10.0

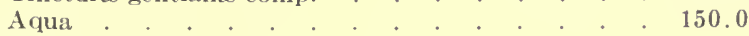

Misee et fiat solutio.

Sig.-Give teaspoonful three times a day.

Vomiting is often present in these cases but it is usually stopped by the removal of the irritating material through irrigation of the stomach (lavage), but if it persists it may be controlled by using sedatives.

$$
\text { For log. }
$$

R-Bismuthi subnit ratis .

()pii pulverati

Succhari alba

0. 2

1. (1

Misce et fiat pulv. No. VI.

sig. - Give one powder crery four to six hours unt il roniting is reduerel. 
When due to chemicals the proper antidote should be administered (see Poisons).

Surgical. - When foreign bodies are present and cannot be expelled by emesis or purgation, gastrotomy must be performed (see Foreign Bodies of the Stomach).

Chronic Gastritis. - Chronic Catarrh of the Stomach. Chronic Dyspepsia.-Definition. - A chronic catarrh of the stomach with a disturbance in digestion, increased mucus formation, changes in the gastric secretions, partial paralysis of the muscular walls and alterations in the structure of the mucosa.

Etiology.- ('hronic gastritis is a rather common condition especially in the dog. It results very frequently from several repeated attacks of acute gastritis and is therefore produced by causes similar to those found under acute gastritis (see Etiology of Acute Gastritis). It often occurs as a secondary complication to various diseases, such as ulceration of the stomach, gastric tumors, diseases of the liver, chronic constitutional diseases (anemia, chlorosis, chronic nephritis, etc.). Parasites, by constant irritation to the membranes for a long period, will produce chronic gastritis (see Parasites).

Pathology. - The stomach is usually cnlarged, the mucous membrane pale, becomes gray in color and its surface covered with a thick, tenacious mucus. 'The veins are found distended and small hemorrhagic erosions and ecchymoses are seen distributed over the mucous membrane. In the later stages the mucous membrane becomes greatly thickened, especially toward the pyloris, and the mucous glands large and indurated from the constant irritation. Nicroscopically there is every evidence of a parenchymatous and an interstitial inflammation.

Symptoms.-'This affection persists for an inclefinite period and like most chronic conditions changes from time to time. In the dog, the animal most commonly affected, the appetite is variable, sometimes greatly inpaired and at other times very good. Vomiting and retching are frequent symptoms and are especially noticeable a short time after eating. The vomited material consists of undigested food particles covered with a thick, tenacious mucus and has a very sour, disagree- 
able odor. After severe and prolonged paroxysms of vomiting, the mucus is frequently mixed, with blood from the rupturing of small bloodvessels at the seat of the erosions. The chemical analysis of the vomited material shows the presence of abnormal acids, such as butyric or sometimes acetic in addition to lactic acid, while the hydrochloric acid is either absent or greatly reduced in quantity. Digestion, therefore, is delayed and decomposition and gas formation favored which in some cases greatly distends the stomach. Constipation is usually present, but in some cases there is diarrhea and the undigested food passes rapidly through the bowels. The urine is often reduced in quantity, has a high color and a very disagreeable odor. There are general symptoms of disturbed nutrition, resulting in cmaciation and general weakness.

Diagnosis. - A diagnosis of chronic gastritis is not always easy as the symptoms present are also indicative of other conditions. However, by a careful analysis of the symptoms and considering the condition of the animal and a carcful examination of the contents of the stomach, the diagnosis may be made with a certain degree of accuracy.

Prognosis. - A complete recovery in this condition is practically impossible. Howerer, a great number of cases will improve with careful diet and treatment. As a rule an unfavorable prognosis should be made owing to the pathological changes which have taken place in the mucosa and muscular walls of the stomach.

Treatment.-Dietetic.- The dietetic treatment is of greater importance in the majority of cases than the use of medicines. A careful regulation of the diet is always to be insisted upon. Only small quantities of easily digested food should be given for the first few days (beef extract, rice soup, white of eggrs, small quantities of milk, oatmeal gruel). In cats rery small amounts of milk or rice soup show the best results.

Medical.-A thorough lavage of the stomach with warm water or a sodium bicarbonate solution (2 per cent.) should always be the first thing attempted. It can be done readily in the following manner: The animal is placed on a table and held by an assistant; a stomach tube is then intro- 
duced into the stomach (see Foreign Bodies in Esophagus) and the free end depressed to allow some of the stomach contents to flow out. Should the contents be too thick to pass out through the tube, some of the 2 per cent. soda solution (about 500 c.c.) is introduced into the stomach by elevating the free end of the tube. Attach a funnel to the tube and gradually pour in the solution. After it has entered the stomach by gravity, allow it to remain for a few minutes, lepress the tube and allow it to flow out again. This operation should be repeated until the liquid flows out freely and is clear of food particles and mucus. It is advisable in severe cases to repeat this treatment daily for three or four days and after this at biweekly intervals.

Bitter stomachics (tincture gentian compound 4.0 ; or tincture of colombo 2.0) are indicated before feeding to stimulate the mucous membrane and the muscular walls of the stomach. Small doses of pepsin and hydrochloric acid are indicated in severe cases to assist in digesting the food. These should be arministered shortly after feeding to obtain the best results. Artificial Carlsbad salts (8.0) given twice daily is valuable as a stomachic and anticatarrhal agent for the mucous membrane. Further, in cases where there is considerable fermentation, salicylie acid $(0.2-0.5)$ or creosote $(0.1)$ is useful to arrest the fermentation. These drugs should be repeated at each meal to obtain the desired results.

\section{FOREIGN BODIES IN THE STOMACH.}

Etiology.-1) ogs often swallow foreign bodies with the food, or during play, which pass into the stomach and produce symptoms of a serious nature. Most commonly the foreign bodies consist of small rubber balls, glass marbles, peach stones, stones, needles, pins, and meat skewers. During rabies (see liabies), owing to the vitiated appetite, dogs eat large quantities of foreign material, sueli as splinters of wood, pieces of cloth, stones, straw, ete.

('ats often take with the food, neelles, pins, fish bones, fruit stones, which enter the stomach and remain for some 
time producing alarming symptoms. Birds often pick up metallic substances with the food (nails, pins, etc.).

These substances after entering the stomach may remain for a long time without producing any marked disturbance. However, as a rule they produce an acute or chronic gastritis and sharp objects may penetrate the walls of the stomach producing an acute inflammatory condition, or peritonitis. Hair balls are occasionally found in the stomach of rabbits and $\operatorname{cog}$ s.

Symptoms. - In a largc percentage of cases where foreign bodies are taken there are no symptoms noticeable, especially where the foreign bodies are small and regular in outline. These will often pass out via the intestinal tract or be vomited up without interfering with the function of the stomach or producing general symptoms. When the foreign bodies are of large size, irregular in outline, or sharp, they often produce symptoms of acute or chronic gastritis (see Acute and Chronic Gastritis). Very commonly the foreign bodics produce extensive irritation of the mucous mcmbrane, penetrate the walls of the stomach or obstruct the pylorus producing severe vomiting (whicl is persistent), complete loss of appetite, intense thirst, hematemesis (from the erosions on the mucous membrancs or some of the bloodressels being injured by the sharp objects). Where extensive injury is produced in the membranes, the animals often show excitable symptoms, howling and other symptoms similar to rabies (sce Rabies), stiffness in gait, and walk with the back arched. By palpation over the region of the stomach (especially in cats, rabbits and in some breeds of (logs), the forcign bodies can often be detceted and their character determined. When perforation of the walls of the stomach is produced by sharp objects, symptoms of acute peritonitis derclop (see Peritonitis). Lastly, under symptoms of general weakness, subnormal temperature, and very weak, imperceptible pulsc the animals often die from exhaustion.

Diagnosis. - The characteristie symptoms, the anamuesis and carcful palpation make the diagnosis rather casy when foreign bodies are present in the stomach. Dircet palpation of the walls of the stomach (explorative laparotomy) 
is to be recommended in some cases where the diagnosis is in doubt. In other cases the Roentgen rays will assist in loeating the foreign body. Ilowever, this method is rather diffieult owing to the location of the stomach and its relationship to the adjaeent organs.

Prognosis.--The prognosis depends upon the character, size and eondition of the foreign body, the general eondition of the animal and the possibility of its removal. In most eases the prognosis should be made unfavorable.

Treatment.-Medical.-Emeties are often of value in removing foreign bodies from the stomach. Subeutaneous injeetion of apomorphin hydroehlorate (0.005-0.01) should be administered and the vomitus carefully inspeeted to determine whether or not the foreign body has been ejeeted. Should this method fail, surgical means should be employed as early as possible.

Surgical.-This eonsists in performing laparotomy and gastrotomy as follows:

Laparotomy.- This operation should be performed at the median line whenever it is possible to do so. When the incision is made through the linea alba, healing may not be quite so rapid as if it were made just to one side through the muscular tissue. The former position is to be preferred, however, as there is little or no hemorrhage to interfere with the operation, and, as both sides of the wound are alike, the edges ean be more evenly approximated by suturing.

The animal being secured in the dorsal position, and under general anesthesia, the skin at the field of operation is elipped and slaved of all hair and cleansed, first with soap and warm water and then witl antiseptic solution (boric aeid 2 per eent.; ereolin 2 per eent.; mereurie bichlorid 1-2000).

When the operation is not extremely urgent, it is best to apply an antiseptic pack (borie acid powder) over the field of operation after cleansing for the twenty-four hours preceding in order to ensure better antisepsis of the skin. Sterilize the following instruments: Sicalpel, probe pointed bistoury, scissors, artery forceps, a full eurved needle and suturing material. 'The incision is made with a sharp scalpel through 
all the tissues down to the peritoneum. Considerable eare should be taken in going through this in order to avoid puneturing the abdominal organs. It is best to grasp a small part with foreeps and elip it out with the seissors, making an opening large enough to insert the probe pointed bistoury and with this enlarge the ineision the desired length whieh depends on the operation to be performed and on the size of the operator's finger. One and one-half inehes is suffieient for most eases, although it may be made large enough in a large dog to adinit the entire hand. Absorbent cotton or a pledget of gauze moistened with antiseptic solution is useful to keep the wound free from blood, but none of the antiseptie fluids should be allowed to enter the peritoneal eavity. Sterile watcr should be used to cleanse any portion of the viscera or peritoncum exposed. 'To suture the laparotomy wound, use medium-sized silk or linen suture material and make interrupted sutures about one-quarter to three-eighths of an ineh apart depending upon the thiekness of the abdominal wall. I'sing a long, slender, full eurved needle, start about one-quarter of an inch from the margin of the wound, and pass it through the muscle and peritoneum, having the index finger inserted in the wound to prevent the needle entering the viscera, particularly the intestine or bladder, and bring it out on the other side at the same distanee from the margin so that when tied, and swelling occurs, one edge will not be foreed above the other. 'The surgeon's knot, $i$. e., wrapping the first half of the knot twice, shoull be used, and if omentum is forced ont when suturing it may be pushed back with blunt forceps as the knot is being tied. To avoid a hernia following this operation, be sure that the sutures pass through all the layer's of the abdominal wall, $i$. e., skin, musele, aponeurosis, and peritoncum.

Gastrotomy.-Perform laparotomy, as above, in the median line and about one-quarter of an ineh posterior to the xiphoid eartilage, making the ineision about two and a half inches long. Grasp the stomaeh with the thmmb and finger and draw it out as far as possible. l'ack it securely witl sterile eotton squeezed out with sterile water, or with dry sterile gauze to prevent any of the stomach contents from entering 
the peritoneal avity when the incision is made. Have an assistant support the stomach by grasping it at either end with thumb and fingel. Make the incision at the greater curvature and at right angles to the long axis of the stomach, and at a point where the bloodvessels are few and small. The organ is best opened by puncturing with a sharp pointed bistoury through the muscular and mucous coats and then enlarging it with the scissors, the length of incision depending upon the size of the foreign body to be removed. 'The removal of large sharp pointed bodies must be done with care and, if possible, they should be crushed to avoid making too long an incision. Blunt dressing forceps are useful to grasp small objects. The division of the muscular coat allows it to contract while the mucous coat projects out through the excess of mucous membrane so that its edges may be evenly approximated. Suture the mucous membrane with interrupted sutures one-eighth of an inch apart, then suture the muscular coat in the same way, being careful to bring the edges in close contact. A Lembert suture is then applied over the wound to invert it. This is made by inserting the needle through a sinall portion of the muscular coat on one side of the wound, carrying it over and inserting the same way on the other side, and these when tied will invert the wound and bring the peritoneal surfaces of either side of the wound in apposition, which favors an early adhesion and prevents the escape of the stomach contents. 'These sutures will be encapsulcd and are not to be removed. Cleanse the surface of the stomach and return to the abdominal cavity. All food should be withheld for twenty-four hours, after which time a small amount of liquid food (milk, beef broth) may be fed for a few days when the regular diet may be given.

\section{ACUTE DILATATION OF THE STOMACH.}

Etiology.-This condition is observed morc frequently in dogs and rabbits than in other small animals. It is most frequently produced by overloading the stomach, especially when large quantities of indigestible or dry foods are taken. Such substances as potatoes, bread (dry), dog biscuits, blood, 
some green foods, grains, etc., are the ones most liable to produce the condition. I ogs when very hungry, will often eat an entire bird, rat or mouse, without masticating it. When the mass reaches the stomach it will causc a sudden dilatation.

Debilitating Condition.-Both general and local, such as rachitis, anemia, toxic conditions, infections, diseases of the heart, etc. 'These conditions may' be the result of various influences which alter the tone of the muscular wall, either indirectly by affecting nervous control, or more directly by toxic action on the muscle.

Previous gastric diseases often predispose to the condition, usually through fermentation, distending the walls leading to the gaseous form of acute dilatation. Excessive secretions at the same time will assist in the dilatation.

Injuries. - Such as blows on the abdomen, being run over by vehicles, spinal injuries, abdominal operations (ovariotomy) have been known to produce dilatation.

Pathology.- The stomach is usually of enormous size, extending back nearly to the pelvis. The color is bluish, purple-red, or pale, and the wall is often very thin. The dilatation not infrequently involves the duodenum and in some cases may extend to the other small intestines. At times the stomach will be found distended with hard, undigested food masses, and in rabbits rupture of the stomach at the greater curvature often takes place from the distention with food and gases.

Symptoms. - Tomiting is one of the early symptoms and the romitus consists in many cases of particles of hard food masses, fluids, etc. Owing to the act of romiting taking place early, some of the material will be ejected, so that in many cases this will be the principal symptom noticeable. Howcrer, in some cases whcre the stomach contents are not removerl by romiting, more serious symptoms develop. There is profuse ptyalism, accelerated pulse, dyspnea, evidences of severe pain such as howling, excitement, etc. In serere cases fermentation of ten takes place in the intestinal tract, prohucing severe dyspnea and death in a short timc. In rabhits and some breeds of dogs, by palpation, the stomach will often be found greatly 
distended with food masses, gas, and fluids, which can readily be distinguished through the abdominal walls. Very often the symptoms of this condition are similar to acute gastritis (see Symptoms of Acute Gastritis).

Diagnosis. - A correct diagnosis requires a careful and accurate anamnesis. 'This condition is often mistaken for some acute abdominal disease, such as peritonitis, acute gastritis or intestinal obstruction. However, by carefully observing the symptoms, and by palpation, together with the anamnesis, the diagnosis is usually made without much difficulty.

Prognosis. - In those cases, duc to overloading, the prognosis is considered favorable; when due to other causes it is unfavorable as complications are liable to ensue which often terminate fatally.

Treatment.-Medical.-When due to overloading the stomach or from foreign material, emetics are indicated early to remove it. Apomorphin (0.005-0.01) should be administered subcutaneously, and the stomach carefully kneaded to break up the food masses and to mix the contents with the secretions so that they will be more easily ejected. After the material has been removed by vomiting it is advisable to administer sodium bicarbonate solution ( 2 per cent.) to remove irritating material from the surface of the mucous membrane. (See Treatment of Acute Gastritis). In cases where therc is a lack of tone in the muscular walls from pressure by retained food masses, gas, etc., or defective nervous control of the muscle, the administration of strychnin sulphate $(0.0005-0.001$ daily), or tincture of nux vomica (0.3-0.6 twice daily) has been found advantageous.

Surgical.-In severe cases where the food particles cannot be removed by emesis, or where foreign material is present, it is advisable to remove it by performing gastrotomy. (See Surgical Treatment of Foreign Bodies in the Stomach).

\section{CHRONIC DILATATION OF THE STOMACH.}

Etiology. - While not common in small animals it is occasionally observed, and consists of a dilatation of the 
stomael, which is very eommonly produeed by food masses remaining in the stomath for a long period, produeing pressure on the walls of the stomaeh leading to a lack of tone in the muscular wall. This allows further aeeumulation of fluids and gases from the fermentation of the food eontained and eonsequently the stomach becomes dilated. In some individual animals the walls of the stomach are relatively. weak, and after eating, the food will remain in the stomaeh for too long a period, and gradually produee a ehronie dilatation. Many of the eauses enumerated under aeute dilatation, if eontinued for some time, will result in the ehronic eondition.

Stenosis of the pylorus is one of the most frequent eauses of this eondition. The etiologieal factors producing the stenosis are the following: Spasm, gastric uleer, erosions of the mucous membranes resulting in cieatrix formation. Foreign bodies whieh may remain in the stomach for a long period, and produce by their constant irritation hypertrophy of the mueosa of the pylorus, resulting in a narrowing or eomplete closing of the pylorie opening. Tumors, either malignant or benign, extending into the pylorus. Tumors, outside of the stomaeh (earcinomas, sarcomas) and assoeiated with adjacent organs or tissues. 'These by produeing pressure upon the duodenum will narrow the humen and result in a stenosis. Inflammation in organs adjacent to the stomaeh as the liver and panereas; or omental adhesions which oecur eommonly after abdominal operations, or by being injured, as blows to the abdomen or being run over by vehieles. 'The stenosis whieh is produced by the above eauses will interfere with the passage of the food from the stomach where it remains too long eventually leading to ehronie dilatation. In old dogs it frequently results from chronie obstipation. Further, debilitating diseases will often produce it by interfering with the nervous eontrol of the stomach.

Pathology. - The stomach is mueh enlarged and in many' eases distorted. When clue to stenosis or obstruction to the pylorus, the stomach walls are greatly thickened. 'T'he musculature is hypertrophic and the mueous nembrane is often 
sereral times its normal thickness, with all the evidences of a ehronic inflammation. When no stenosis exists, the dilatation being produced by other causes, the muscular wall is very thin and atrophic. In some cases the nucous membrane shows areas of atrophy and hypertrophy alternating. The interstitial tissue is often infiltrated with fibrous tissue.

Symptoms. - In dogs the synuptoms are very similar to chronic gastritis. There is a partial or complete loss of appetite, intense thirst, especially if the pylorus is obstructed, the patients drinking large amounts of water which is afterward vomited.

Constipation is also quite marked in most cases of chronic dilatation. As no water ean pass from the stomach to the intestines, the contents of the intestinal tract become hard and dry to be passed with diffieulty, or retained as a hard mass in the posterior part of the bowels. When dilatation occurs without stenosis of the pylorus large amounts of water will pass into the intestinal tract producing diarrhea, which may alternate with eonstipation. Emaciation is usually quite marked, especially in advanced cases, because no nutriment ean be absorbed. In somc eases where there is only a partial stenosis the emaciation will be more gradual, as some food will be passed to the small intestines. Severe pain, the animal often showing exeitable symptoms, howling, etc., is shown a short period after eating, due to the particles of food passing through the narrowed lumen of the pylorus. Where no stenosis exists there is but little pain. Vomiting is a constant symptom, especially in obstruction of the pylorus, and usually oceurs shortly after feeding. The patient will sometimes eat the ejected mass again only to repeat the aet of romiting. This symptom is quite charactcristic coming as it does shortly after eating and continuing in somc cases in the same manner for days or even weeks.

Gradually the animals become weak, anemic, the temperature subnormal, and dic from inanition.

Palpation over the region of the stomach will often reveal the enlarged organ with its eontents; percussion gives a tympanitic sound. 
Diagnosis.-Diagnosis is rather difficult in most cases. The chronic course, the characteristic symptoms, and the findings of an explorative laparotomy suffice to make the diagnosis.

Prognosis. - The prognosis should be carefully guarderl until the causes and condition of the stomach are thoroughly understood. Complete recovery is possible in both forms of this disease, provided the wall of the stomach is not permanently damaged and the general condition of the animal capable of improvement. However, in stenosis of the pylorus, it should always be considered unfavorable when tumors are present.

Treatment.-Dietetic.-Small amounts of easily digested food are to be given at short intervals during the day rather than large quantities at long intervals. In some cases where romiting is persistent, rectal feeding should be employed giving albumen or predigested foods.

Medical.-In dilatation accompanied by lack of tone of the muscle stomachic tonics, such as tincture of nux romica $(0.3-0.6$ twice daily $)$ or strychnin sulphate $(0.0005-0.001)$, may be used.

Massaging or kneading the abdominal wall over the stomach is a valuable adjunct to increase the muscular tone. When stenosis exists surgical interference is the only thing that will give relief; it should be resorted to as early as possible. (See Gastroduodenostomy).

\section{ULCERATION OF THE STOMACH.}

\section{Clcus Ventriculi.}

Definition.-This condition is a more or less progressive destruction through necrosis beginning in the mueosa and often extending to and through the deeper lavers of the stomach wall. True ulcers appear only where the gastric juice flows. They are found in the dog at the extreme lower end of the esophagus, in the stomach wall itself, and in at portion of the duorlenum above the opening of the bile duet. The ulcerations may be acute or ehronic. Sometimes a tendency to cicatrization and healing is shown. At the point 
where the stomach tissue is destroyed an oval or round opening or depression with irregular margins appears. The more chronic the process the greater the irregularity in outlinc.

Etiology.-Simple ulceration of the stomach is due to a destruction of the gastric epithelium caused chiefly by a disturbance in blood circulation in the stomach and hyperacidity of the gastric juice. It is often brought about by inflammation of the mucosa or hemorrhages, resulting from poisonous substances, caustics, drugs (when administered in too concentrated form); sharp foreign bodies; during the course of infectious diseases (distemper, etc.), or from an invasion by the Bacillus necrophorus (puppies and kittens), or other local infections. General infection (pyemia) may too be a causative factor. These infections produce an extensive inflammation of the mucosa, often leading to a disturbance in the circulation, interfering with nutrition, and ultimately leading to an ulcerative process. Wounds of the mucous membrane from external violence, partial rupture of the stomach walls or parasitic invasion are causes. They are often contributory to the beginning of the ulcerative process. Embolism is also a cause, producing infarcts in the mucosa and submucous tissue. Hemorrhagic erosions and hemorrhages occurring and associated with chronic diseases of the heart, liver or kidneys are probable causes. Hyperacidity may produce gastric ulcer when from any cause the mucous membrane is injured, the acid acting upon the ends of exposed vessels by contracting them, thus inducing local anemia and eventually necrosis.

Pathology. - The typical ulcer is round or oval, extending morc or less deeply into the mucous membrane or the wall of the stomach. They have a characteristic funnel shape, and when acute form a rather regular outlinc, while in ulcers of long standing the margins become very irregular. When chronic ulccrs are present, the entire wall is usually thickened. Sometimes a coalescence of the ulcerations or erosions occurs. When ulceration becomes severe and the submucosa is involved, adhesions exist witl adjacent organs.

Symptoms. - In quite a large percentage of cases of mild ulceration or erosions the symptoms are never observed 
during the life of the animal. In more advanced ulcerations the symptoms are often very similar to chronic gastritis (see Chronic Gastritis). Bloody vomiting is a characteristic symptom. The amount of blood ejected varies depending upon the extent of the injury to the vessels done by the ulcerative process. This condition usually persists for a long time with exacerbations and remissions. When the ulcer perforates the wall of the stomach symptoms of acute peritonitis develop rapidly and a fatal termination soon follows.

General symptoms of emaciation, weakness, and disturbances of the intestinal tract are usually observed.

Diagnosis. - An accurate diagnosis is very difficult, and can only be made by a careful observation of the symptoms. When ulceration is suspected laparotony can be performed (see Foreign Bodies of the Stomach) to determine the condition of the stomach. However, when the ulcerative process is slight or only erosions are present, even this examination may not suffice for diagnosis.

Prognosis. - The prognosis is always unfavorable, as only the advanced cases are recognized clinically.

Treatment.-Dietetic.-Only small quantities of easily digested food (beef broth, milk, small amounts of lean meat finely divided) should be allowed, as there is danger of the -wall rupturing at the point of ulceration.

Medical.-Astringents and hemostatics are indicated (bismuth subnitrate $0.3-0.5$, or silver nitrate $0.05-0.1$ well diluted in distilled water). These preparations can be administered two or three times daily, depending upon the action desired. Where severe hemorrhage takes place, adrenalin chlorid (1.0-2.0 of a 1-1000 sol.) may be given several times daily until the hemorrhage stops. To neutralize the acid secretions in the stomach Carlsbad salts (2.0-4.0) can be used twice daily. In cases where romiting is persistent small doses of tincture of opium (0.2-0.5) are administered every two hours until vomiting ceases.

Surgical.-In some cases where medical treatment does not afford relief, laparotomy should be performerl (see lioreign Bodies of the Stomach) and the ulceraterl area extirpated (see Gastrotomy, under Foreign Bodies of the Stomach). 


\section{HEMATEMESIS.}

Definition.-Hematcmesis is the vomiting of blood. It is merely a symptom. 'The hemorrhage does not always come from the stomach itself, but from struetures eontiguous to the stomach. In small animals hematemesis is mueh more common than is usually recognized, but unless large quantities of blood are vomited, it eseapes notiee. Often seant hemorrhages go unreeognized, the determination of whieh would be valuable for the diagnosis of some diseases.

Etiology. - In hematemesis there may be no anatomieal lesions on the mueous membrane (diapedesis); or the lesions may be very small but numerous and the hemorrhages eopious eoming from a large surface of the mueosa. On the other hand the lesion in the mueosa may be quite marked (rhexis; uleer, ete.). In the dog hematemesis is quite eommon and has the following eauses:

Local causes: Clcerations on the mueosa; erosions on the mueosa; aeute and chronic gastritis; tumors; trauma whieh may be direet (foreign bodies, injury from stomach sound) or indircet (straining during vomiting, stomach operations); chemicals (poisons, purgatives, emetics).

Indireet local causes are further: Obstruetion to the portal circulation; pressure on the portal vein; thoraeie diseases. disturbing circulation; organie heart lesions, ete.

Hematemesis may oecur also from many general causes whieh produce at the same time hemorrhage from several of the mueous membranes. The following are the most common conditions: Septieenia; autotoxie eonditions, uremia, ete.; blood dyscrasias and diseases, hemophilia, scurvy, pernieious anemia, leukemia. The blood may have its origin outside the stomach and come from contiguous abscesses rupturing the stomach wall, or from ulcers and fistulie whieh may open a suppurating traet into the stomach.

Hematemesis also occurs during the eourse of infectious diseases (distemper, rabies).

Pathology.- The pathologieal findings depend upon the amount of the hemorrhage. When the hemorrhages prove fatal there is a general pallor of all the viscera; where the 
hemorrhages have been slow and continuous fatty degenerations of the heart, liver, kidneys, gland cells are observed. The original cause will in part determine the nature of the pathological changes. The mucous membrane of the gastrointestinal tract is pale, and remains of the hemorrhage will be noticed along its course, which is of a dark color and more or less tarry. At the seat of the hemorrhage will be found eroded vessels, and sometimes imperfect clot formation. Other lesions found are those of ulceration, foreign bodies, depending upon the original cause of the hemorrlage.

Symptoms. - These depend very largely upon the amount of hemorrhage and rapidity with which it flows. When the amount of hemorrhage is small the syinptoms may be entirely overlooked. The romited blood may be only in small quantities often poorly mixed with the ejected food. The color of the blood depends upon the amount and length of time remaining in the stomach. When fresh, the color is bright and the cells are unchanged; when retained in the stomach some time before it is ejected, it is dark, the oxyhemoglobin being changed to hematin, and resembles coffee grounds. A microscopic examination of the ejected mass will at once reveal the presence of large numbers of red corpuscles unless the blood has been retained in the stomach long enough for the red corpuscles to become entirely disintegrated.

When severe hemorrhages take place in the stomach, some of the blood will pass into the intestinal tract to be passed out with the feces. The feces will be of a dark tarry consistency, with a disagreeable odor. If examined the blood pigment will be found in large quantities.

General symptoms of internal hemorrhage are noticed in the more severe cases. The mucous membranes sucklenly. become pale and colorless, the pulse rery weak and often imperceptible, dyspnea and general weakness appear. If repeated hemorrhages do not result fatally the patient suffers from chronic anemia.

Diagnosis. - This depends upon the presence of the blood mixed with the vomitus. ('are should be taken in making the diagnosis in the dog as they often eat large quantities of 
clotted blood, or lick blood from bleeding surfaces which may produce vomiting. When this is suspected the general symptoms should be carefully considered and a careful anamnesis obtained if possible.

A microscopic examination of the contents from the stomach and bowels will in most cases assist in arriving at an accurate diagnosis. It may be differentiated from hemorrhage of the lungs by the presence of food particles, and the absence of air mixed with the material, and from the fact that it usually has an acid reaction from contact with the gastric juice.

Prognosis. - This depends very largely upon the cause, and to a less extent upon the severity of the hemorrhage. When resulting from wounds in the mucosa when not too extensive it should be considered favorable. However, when due to ulceration of the mucosa, or resulting from general causes the prognosis is unfavorable.

Treatment.-The early indication in the treatment is to keep the animal in a quiet place free from all excitement or noise. 'The administration of cold water (ice water) internally or cold applications to the epigastrium in the form of cold water or ice are beneficial. 'To control the hemorrhage when severe, adrenalin chlorid solution (1.5-2.0 of $1-1000$ solution), or ergotin $(0.2-1.0)$ subcutaneously should be given every two or three hours until the hemorrhage stops. Gelatin given via the mouth has been highly recommended for the same purpose. In less severe cases mild astringents (tannic acid, lead acetate, tannoform, alum) are to be used. Where general weakness is well markerl subcutaneous injections of strychnin sulphate $(0.0005-0.001)$, or caffein citrate $(0.06-0.19)$ are to be used to stimulate the heart action, and as a general stimulant. Saline infusions given as rectal injections, intravenous injections, or intraperitoneally are indicated in severe hemorrhages to replace the loss of blood in the body. When hematemesis is due to other diseases, the cause should be determined and treatment applied accordingly.

All food should be withheld for at least two days, and then allowed only in small quantities. Milk or beef broth is useful in this regard. 


\section{PARASITES IN THE STOMACH.}

The parasites that derelop in the stomach of the dog are very rare. 'The Spiroptera sanguinolenta, a small blood red worm from 5 to $8 \mathrm{~cm}$. long, is the most common. It forms small, tumor-like masses in the mucosa. Occasionally the larve of the Gastrophilis intestinalis are found attached to the mucous membrane. Ascarides and tænia commonly migrate from the intestines. Trenia are often found in the stomach of the cat, the Trenia tæniæformis being the most common. The Ollulanus tricuspis, however, is the only parasite proper to the stomach of the cat.

The adult Ollulanus tricuspis is about $1 \mathrm{~mm}$. long and has three points at the caudal extremity. The adult worm lives in the gastric mucous membrane and when present in large numbers, seriously interferes with the function of the membrane. The embryos are quite large compared with the adult parasite. Some of these embryos are passed with the feces while others burrow through the walls of the intestines and wander to various parts of their host, particularly the pleura, diaphragm, liver and lung, where they form cysts. When the free embryos are ingested by rodents they migrate into the muscles and become encrsted. It is probable that their development is completed in the digestive canal of the cat that feeds upon the infested rodent.

Rabbits are chiefly infested with the Strongylus strigosus, often found in large numbers, and give rise to a fatal anemia. This parasite is 8 to $16 \mathrm{~mm}$. long, body blood red, filiform and transversely striated.

The parasites most common in the anterior portion of the digestive (anal of birds are the Dispharagus spiratis, D)ispharagus nasutus and the 'Trichosomum contortum. The dispharagi are from 7 to $9 \mathrm{~mm}$. long and the trichosome 12) to $17 \mathrm{~mm}$. and are found either entirely embedded in the mucous membrane or fixed in it by one extrenity and the other floating free in the carity of the organ. 'The Trichosomum contortum is frequently found in adjacent structures, but is common in the walls of the esophagus. 
Symptoms. - The parasites which are proper to the stomach of small animals and to the anterior part of the digestive canal of birds do not as a rule produce any marked symptoms unless present in very large numbers. The Spiroptera sanguinolenta of the dog may give rise to a chronic gastritis with frequent vomiting, an irregular appetite and resulting emaciation. The tumor-like masses in which the parasites live may perforate the peritoneum and occasion a fatal peritonitis. Large numbers of the Ollulanus tricuspis in the cat's stomach cause a thickening and ecchymosed condition in the mucous membrane with severe gastric disturbance. As the larvæ of this parasite migrate into the adjacent tissues, they can produce extensive inflammatory processes and give rise to pleuritis, peritonitis, bronchitis, etc. The Strongylus strigosus produces a severe anemia in rabbits by abstracting blood from the mucous membrane of the stomach, and by so doing produces a disturbance in the function of the stomach with inanition as a result. In warren rabbits where they are continually subjected to reinfection, this anemia often terminates fatally, at times being epizoötic in its extent.

The parasites of birds produce the most serious disturbance by burrowing in the walls of the esophagus and crop. The food when swallowed becomes impacted through loss of propelling action of the organ and dilatation results often with fatal termination.

Diagnosis. - An accurate diagnosis of the stomach parasites of the dog and cat is difficult. A microscopical examination of the feces and vomitus for the larvæ may reveal their presence. When the general symptoms are indicative of parasitism laparotomy and possibly gastrotomy should be performed, and a direct examination of the tumor-like masses madc. In rabbits and birds, as several are usually affected, a postmortem examination of one or two of the most typical cases is recommended.

Prognosis.- Infarorable in all cases of severe infestation. Mild cases in dog, cat and rabbit often recover spontaneously or with ordinary treatment. In birds, however, the injury to the walls soon leads to dilatation and quite often to a fatal termination. 
Treatment. - No satisfactory treatment can be given for those parasites living within the tumor masses or burrows in the mucous membrane. When such parasites are diagnosed, all the affected individuals should be removed and a thorough cleaning given all the houses, runways, and grounds to which they have had access. (For ascarides and tænia see Parasites of the Intestines.)

\section{NEOPLASMS IN THE STOMACH.}

Tumors in the stomach espeeially primary growths are very seldom found in small animals. Oecasionally secondary carcinomas or sareomas occur.

Symptoms. - The symptoms observed are very similar to chronic gastritis (see Chronic Gastritis). The growth of the tumor is often sufficient to increase the size of the abdomen and can usually be palpated through the abdominal walls. Other symptoms are persistent vomiting, icterus, and loss of appetite. When a tumor of the stomach is suspected laparotomy should be performed and a direet examination made.

Prognosis.- L'nfavorable.

Treatment. - No attempt should be made to operate malignant growths. Tumors involving the anterior part of the stomach are inoperable owing to the loeation and elose attachment of the stomach. Fortunately, however, tumors of the stomach usually involve the pylorie end, and in such cases gastroduodenostomy should be performed removing the entire part involved. Gastroduodenostomy must be performed with eonsiderable care to prevent infection from the stomach contents and hemorrhage from the large vessels, and also to so place the ligatures as to avoid eutting off blood supply from any part of the stomach not removed. The modus operandi is as follows: Thoroughly anesthetize the animal and seeure in the dorsal position. Cover the field of operation with sterile cloths and provide plenty of suture material (linen maehine cord No. 40) and mediunsized, straight sewing needles (Emmet's bowel needles). Perform laparotomy at the median line from the xiphoid 
cartilage to the umbilical scar. Grasp the stomach and draw it up 'through the opening. I igate the right and left branches of the gastric arteries, the splenic and the gastrohepatic arteries. The location of the ligatures along the arteries depends on the location of the excision. In all cases the ligatures must be close to the excision to avoid having an area of the stomach left without blood supply. Place a gastrectomy forceps, the jaws protected with rubber tubing, across the stomach just back of the point of each excision, and two others across the part to be removed far enough from the others to allow cutting between them with shears. The forceps protect against hemorrhage and escape of contents while the latter prevent escape of material contained in the part to be removed. Remove by cutting between the clamp with scissors and suture both ends with continuous suture over and over the cut edges to control hemorrhage, and invert the ends by putting in Lembert sutures. Remove the forceps and take up a part of the anterior wall of the stomach and apply the forceps, having the part that projects through the forceps about two inches long by one-half inch through. Do the same with the duodenum about three inches from the pylorus. Place the forceps close together and apply sutures through the muscular coat close to the lower jaw of each forceps, and do not cut off the suture. Then make an incision in the stomach close to the line of sutures and one in the duodenum to correspond with the one in the stomach. Then with a new suture join the distal edges of the wound with Connell's sutures, the forceps being loosened but left in place to support the stomach. These latter sutures invert the cut edges and the first suture can now be continued over the Connell suture as a Lembert suture. Return the organs and suture the laparotomy wound. After forty-eight hours feed liquid food for several days. 


\section{CHAPTER IX.}

\section{DISEASES OF THE INTESTINES.}

Examination.-The intestines can be easily palpated through the abdominal wall in most small animals, when they are not too fat, and this greatly assists in making an accurate diagnosis, especially of foreign bodies, fecal accumulation (coprolith), etc. This is best done by placing the animal in the standing position using both hands, one on either side of the abdomen. When necessary to make a more careful examination, as for perforating wounds, volvulus, etc., explorative laparotomy should be employed. The intestines are best reached by making the incision at the median line in the iniddle of the distance from the xiphoid cartilage to the anterior border of the pelvis, and of sufficient size to permit of a thorough examination of the entire length of the intestine. The Rocntgen ray's can be employed to detect foreign bodies.

The feces should be carefully examined for the following: Frequency, color, odor, quantity, eonsistency, presence of blood, parasites or their ova, mueus, foreign bodies, undigested food. The peristalsis is important and can be examined by placing the animal on its side, covering the abdomen with a towel or cloth and applying the ear. 'The phonendoscope is of great assistance for this purpose. Percussion is important in differentiating accumulations of fluid or gas in the abdomen or intestines.

\section{ENTERITIS.}

Two forms of enteritis are recognized in small animals, viz.: (a) Acute, and (b) chronic.

Acute Enteritis. - Definition. - This is a catarrlal inflammation of the mucous membrane of the small intestine, as well 
as the upper portion of the large bowel. In small animals it is impossible from a clinical standpoint to recognize the condition as affecting different parts of the bowels as: Duodenitis, ileitis, jejunitis, etc. Enteritis very frequently accompanies acute gastritis, the causes producing acute gastritis often extending into the intestines.

Etiology.-The etiology is in many respects similar to that of acute gastritis: (a) Foreign bodies which irritate, such as sharp pieces of bone, needles, pins, etc. These when swallowed pass through the stomach into the intestines, often producing extensive irritation to the mucosa, resulting in an acute inflammation. Enteritis may result also from operations, such as laparotomy when the bowels are roughly handled, rectal injections and manipulations, etc. Powdered glass is sometimes given intentionally to small animals, and it produces a very severe form of enteritis.

(b) Chemical substances (carbolic acid, arsenic, phosphorus, mercury, etc.) produce a very severe form of enteritis by their corrosive action on the mucous membrane. Chemical irritants contained in food when eaten by the animals will produce the same condition. Sometimes excessive quantities of foods, or digestive disturbance may occasion fermentation in the intestinal tract and the formation of irritants that directly excite an inflammatory condition. Also foods (meat, fish) may contain preformed toxic bodies, such as ptomaines or other products of bacterial action, which may occasion violent inflammation of the bowels. Cats are frequently affected by eating fish partially decomposed. Birds, from eating substances containing rather large quantities of sodium chlorid, suffer from a severe form of inflammation of the bowels as the result.

(c) Exposure to cold, or sudden cooling of the surface of the body may produce an enteritis by interfering with the innervation and circulation in the intestinal walls; also taking large quantities of cold water, frozen or very cold foods will induce it.

(d) Bacteria probably rarely cause inflammation of the bowels directly, but their action upon the intestinal contents causing fermentative changes are causes of enteritis. Ente- 
ritis is an essential complication in certain infections diseases (distemper, enterohepatitis, etc.).

(e) Severe infestations with animal parasites can produce acute enteritis by the irritant action of the parasites upon the mucosa producing congestion and in some cases by attaching themselves to the membrane or burrowing into its walls. The severity of the inflammation produced depends very largely upon the number and species of parasites present. This condition is observed most often in puppies and kittens. Acute enteritis also occurs secondarily to other diseases, such as septicemia, septicemic diseases of birds, peritonitis, coccidiosis of rabbits and birds, etc.

Pathology. - The mucous membrane of the bowel may be involved in its entire length with almost equal severity, but usually certain portions are more seriously affected than others. The mucosa is swollen and reddened, the surface usually covered with mucus, which may be tinged with blood, and sometimes the submucosa is edematous with hemorrhagic extravasations. The lymphatic follicles are enlarged and project out from the mucosa prominently as light patches against the inflamed surface of the mucosa. In severe cases pseudomembranous or diphtheritic inflammations of the mucous inembrane of the intestines may be seen occurring usually secondary to infections originating elsewhere or from the action of corrosive poisons which are very common in dogs and birds. In all of these cases the mucous membrane is covered with an extensive, dirty yellow or gravish deposit, appearing as a coating over the surface or as a firmly attached pseudomembrane. This pseudomembrane may be found in the entire length of the bowel. Areas of the necrosis and deep ulceration are often seen where the pseudomembrane has been dislodged. Hemorrhages are often found under and from the mucosa, the blood mixing with the intestinal contents forming a dark red mass. 'The feces are very thin even in the large intestine and have a disagreeable odor. The mesenteric limph glands are swollen and edematous.

Symptoms. - The clinical symptoms in enteritis vary with the intensity of the cause and the part of the intestinal tract 
involved. In milder cases the symptoms are those of diarrhea due to functional disturbances of the bowels. In severe cases the symptoms come on suddenly and are of great severity. In dogs therc is usually constipation in the early stages followed in a few hours by diarrhea. 'The fcces, as a rule, arc liquid, brownish in color and of more or less offensive odor, depending upon the extent of the putrefactive changes and the intensity of the inflammation. In severe inflammations due to corrosive poisons, etc., the feces are mixed with blood, sometimes passed in quite large quantities, and in the form of clots. When due to poisons, the odor of the poison is often noticed in the feces.

Colicky pains are noted and in severe cases intense abdominal pain is one of the most prominent symptoms. The abdominal muscles are tense, contracted, often giving the animal a "tucked up" appearance. In severe inflammation of the bowels the dog will often lie stretched out on its abdomen, showing intense pain by howling, crying, nervousness, etc. Sometimes the nervous symptoms will be prominent enough to simulate rabies.

Vomiting is a common symptom provided the stomach is involved. The vomitus usually consists of food particles, mucus, blood, bile, and in rare cases feces from the small intestines. 'There is loss of appetite except in the very mild cases. Fever is more or less high in the early stages, while later in the dog it becomes subnormal. The temperaturc, as a rule, cannot be depended upon to dctermine the severity of the condition, as it may be increased in slight cases, and in severe, be normal or subnormal. The general symptoms are those of weakness, in proportion to the degree of inflammation, complcte prostration or collapse. 'The surface of the body becomes cold, the mucous membranes cyanotic, there is well marked dyspnea and a wcak, rapid pulse. In cats the diarrhea is a very prominent early symptom. The feccs are liquid and often mixed with blood. Complete prostration takes placc early under symptoms of dyspnea, general weakness, and a very rapid weak pulsc.

In birds enteritis begins with diarrlea. 'The feces are of a thin consistency, of a yellowish or greenish color, and 
very offensive odor. 'The feathers in the region of the cloaca become soiled, matted together, retaining a quantity of the feces which oftcn becomes dried and forms a hard mass around the anal opcning. There is usually loss of appetite, general weakness, the wings are held pendant and the patient completely prostrated. In the more severe cases due to ptomaines, toxins, etc., the symptoms develop very rapidly, and there is but little noticed except subnormal temperature, narcosis and coma. Death occurs in a large percentage of those cases duc to ptomaines, toxins, etc.

Diagnosis.-'The recognition of acute enteritis offers no great difficulties in most cases. The anamnesis, sudden onset, abdominal pain, and the severe diarrhea are all quite charactcristic. Only when diarrhea is absent would the diagnosis be difficult. A carefal examination should always be made to determine if possible the cause of the enteritis, whether it is a primary condition or secondary to some other disease. Examination of the feces should be made both macro- and microscopically to determine their condition, the presence of blood, foreign material, or of parasites.

Prognosis. - In ordinary cases of enteritis the prognosis is usually favorable. However, it depends very largely upon the cause, and all cases should be considered serious until the cause can be determined. When due to corrosive poisons, ptomaines, toxins, etc., the prognosis should be considered very unfavorable depending upon the amount of the material ingested. In cats the prognosis should be guarded as these animals are very sensitive to intestinal disturbances.

Treatment.-IIygienic.-The animals should be placed in a warm, dry place which is well ventilated and free from all noise and excitement. Not too many birds or small animals should be allowed in onc room, and the floors, roosts, etc., should be thoroughly cleaned (daily) and disinfectants, such as carbolic acid ( 5 per cent.) frecly used. The drinking water should be kept fresh, and the food, when allowed, free from dirt, dust, and not contaminated with bowel discharges. Collections of feces on the hair and feathers shomkl be removed daily with warm water or bicarbonate of soda solution (2 per cent). 'The mouth may be washed with clean 
water to remove all food particles which might remain to decompose and eventually be swallowed to perpetuate the intestinal disturbance.

Dietetic.-In most cases of acute enteritis, all food is withheld for at least twenty-four to forty-eight hours. After this time, when improvement begins to be noticeable, dogs may be given small amounts of lean. meat finely divided, milk, beef tea, rice soup, etc., once or twice daily. Rabbits should be fed small quantities of roasted oats, corn or barley, or roasted bread. For birds small amounts of cooked rice, oatmeal, cornmeal or bread and milk may be given once daily. Green foorls should be witheld at first and only allowed in small quantities several days after improvement is noted. 'They tend to increase the diarrhea by their laxative action.

Medical.-In mild cases of enteritis, due to errors in feeding or irritating foodstuffis, etc., small doses of laxatives are indicated to remove the irritating material from the bowels. Castor oil (dogs, 15.0-40.0; cats, 3.0-15.0; rabbits, 5.0-10.0; birds, 1.0-8.0), or calomel (dogs, $0.3-0.4$; cats, $0.1-0.15$; rabbits, 0.2 ; birds, $0.05-0.2$ ) can be administered. Magnesium sulphate (dogs, $10.0-15.0$; cats, $1.0-5.0$; rabbits, 1.0-3.0; birls, 1.0-2.0) is frequently used for the same purpose owing to its increasing the fluids in the bowels and flushing them out more promptly and thoroughly. It is best administered in solution with warm water.

In more severe cases where the liarrhea is persistent after the use of laxatives, it is necessary to arminister styptics, such as opium ( $\log$ s, $0.1-0.5$; cats, $0.005-0.2$; rabbits, $0.05-$ 0.1 ; birds, $0.05-0.1)$. In cases where severe pain is present, especially in dogs, morphin sulphate is indicated. It may be given (for $\operatorname{logs}, 0.02-0.15$ ) as a subcutaneous injection dissolved in water $(5.00)$.

Astringents are sometimes of value in controlling the persistent diarrhea and tannic acid is often arministered for that purpose in the following dosage: Dogs $(0.1-0.5)$, cats $(0.05-0.2)$, rabbits $(0.05-0.2)$, birds $(0.01-0.05)$.

Where fermentative processes continue in the bowels risinfectants may be arministered: Lysol (0.5-2.0), creolin (1..5-2.0), salol (0.2-0.8). 
It is often advisable in dogs to wash out the intestinal tract with bicarbonate of soda solution (1 to 2 per cent.) followed by alum, tannie acid or iron sulphate solutions (1 to 2 per cent.). This can be done quite readily in the dog by the use of a flexible rubber tube inserted as far up into the bowels as possible. The free end is elerated and a fumnel attached. 'The solution is pourcd into the fumnel and allowed to gravitate slowly into the bowels. When there is no obstruction the fluid will flow into the stomach and be ejected through the mouth. In birds it is best introduced with a small syringe, being careful to aroid using too much force. In very severe cases, where general weakness is apparent, stimulants are adrisable. Subcutaneous injections of caffein citrate $(0.1-0.5)$ may bc used as a heart stimulant. Normal salt solution, at the body temperature $\left(100^{\circ} \mathrm{F}.\right)$, is of great value in severe yeakness from hemorrhage or narcosis. It may be introduced intrarcnously, subcutaneously, intraperitoneally or per rectum.

Chronic Enteritis. - Definition.-This is a chronic inflammation of the small intestines involving the upper part of the large intestines, but often extending to all parts of the intestinal inucosa. Frequently there is extensive ulceration at different places along the intestinal tract. In some cases the stomach mucosa is involver from the same causes that produce the chronic enteritis.

Etiology.-Chronic enteritis most commonly develops from repeated acutc attacks or from the same causes acting mildly producing gradually a chronic inflammatory condition. (See Acute Enteritis.)

('hronic enteritis also oceurs secondary to other diseases, such as chronic diseases of the heart, lungs, and liver which cause interference with the circulation in the walls of the intestines. Chronic infectious diseases, such as chronic distemper, chronic constitutional diseases, rachitis, ctc., have at times chronic enteritis associated with then. P'arasites in the intestinal tract are (ommon ranses.

Pathology. - The intestines always present evidences of long-continued inflammation of the mucesa, the surface being covered with excessive secretions of mucus or muco- 
purulent material. There are extensive areas of thickening in the mucous membrane; erosions and atrophy may too be present. Ulcerations and cicatrices are often noted. Ulcerations may lead to perforation of the bowel wall producing a localized area of adhesive peritonitis, or in some cases a generalized peritonitis. The mucous membrane is usually bluish-red and at various points will be noted light and dark points due to the pathological changes in the structure of the membrane. 'The intestinal contents are usually liquid, of a slate gray color and cmit a very offensive odor. Sometimes when constipation is present the feces will be dry and hard in the large bowels, and of a grayish color.

Symptoms. - The most conspicuous symptom is a continuous or intermittent diarrhea. IIowever, diarrhea may alternate with constipation. The general symptoms are dulness, gradual emaciation, rough hair coat and inanition which is marked owing to the lost or variable appetite and the interference with digestion and food assimilation. From time to time the bowels are distended with gases from the fermentative processes in the intestinal tract. This is especially marked when constipation is present and the peristalsis reduced. At other times large quantities of gases, feces mixed with mucus and often streaked with blood, are passed during the period of diarrhea.

In most cases there is little evidence of abdominal pain, except when ulceration of the bowel wall takes place and an adhesive or a general peritonitis is produced. 'Then all the evidences of inflammation of serous membranes are present. (See Peritonitis).

Diagnosis.-The long-continued course, the intermittent diarrhea, the anamnesis and the general condition of the animal will be sufficient in most cases to make a comparatively accurate diagnosis. However, a careful and thorough examination should always be made to determine if possible the cause of the enteritis. 'This is valuable especially for a proper prognosis and rational therapeutics. 'The feces should be carefully examined for parasites and their ova, as these form a frequent cause. 
Further, as this condition is often secondary to other diseases, a differential diagnosis should always be attempted. Look for chronic constitutional diseases, diseases of the liver, heart and lungs and chronic infectious diseases. In birls an examination should be made for infectious asthenia and parasites.

Prognosis.-Chronic enteritis whether of primary or secondary origin should be considered unfavorable owing to the pathological changes which have taken place in the mucous membrane. Complete recovery can hardly be expected even in mild cases. In mature animals the prognosis is considered more favorable than in young animals. When it occurs secondary to other diseases the prognosis depends largely upon the primary disease.

Treatment. - A careful regulation of the diet (see Acute Enteritis) is of great importance and should be thoroughly impressed upon the attendant.

Medical.-The action of the bowels should be kept as regular as possible by the use of castor oil, magnesium sulphate, etc. (See Acute Enteritis.) When diarrhea becomes persistent astringents are indicated as in acute enteritis.

Washing out the bowels (see Acute Enteritis) is especially valuable in chronic enteritis to remove irritating material, mucus, fecal matter, etc.

If parasites are present proper anthelmintics should be employed. (See Parasites in the Intestines.)

To encourage the appetite and to aid in digestion, bitter stomachics (gentian, nux romica) are indicated. In the treatment an attempt should always be made to remove the cause; when secondary to other diseases treatment should be given to promote the removal of the fundamental disease.

\section{INTESTINAL HEMORRHAGE. ENTERORRHAGIA.}

Definition.-This is a condition where hemorrhage takes place from the intestinal mucosa. It may be due to a definite 
change in the structure of the mucosa or occur during the course of certain diseases attended by hemorrhagic diatheses.

Etiology.--The causes of intestinal hemorrhage are quite numerous: (a) Mechanical agents, such as sharp foreign bodies which penetrate the intestinal mucosa and injure the bloodvessels, producing a more or less severe hemorrhage. Parasites, by their irritating action on the mucous membrane, or by burrowing into the mucosa, will produce the same result. Injuries, such as being run over by vehicles, kicks, blows, etc., over the region of the abdomen will often rupture some of the vessels in the intestinal mucosa, resulting in hemorrhage. Powdered glass, when administered to destroy animals, will produce severe hemorrhage by the fragments of glass penetrating the inucosa and opening the vessels.

(b) During the course of poisonings by chemicals or caustic substances, severe hemorrhage often takes place due to the corrosive action on the mucous membrane. Drugs, when administered in large doses, may produce a congestion of the bloodvessels of the mucosa, resulting occasionally in overdistention and rupture.

(c) Hemorrhage takes place sometimes from an engorgeinent of the intestinal circulation from diseases of the heart and liver, hemorrhagic infarction of the bowel from embolism or thrombosis of the mesenteric vessels, intussusception or strangulation of the bowel, or hernia. In these cases the hemorrhage may result from an overdistention of the bloodvessels.

(d) During the course of some diseases severe hemorrhage in the intestinal tract occurs, as in anemias, severe infections (distemper) and certain degenerations.

(e) Tumors (carcinomas) and ulcerations on the mucous membranes may lead to severe hemorrhage by destruction of some of the bloodvessel walls. Hemorrhoids, collections of feces, etc., may produce hemorrhage in the large bowels.

Symptoms.- Ilemorrhage in the intestinal tract is often difficult to recognize, unless the quantity of bloor is sufficient to distend the bowels, or be ejected through the rectum. In mild cases of hemorrhage only general weakness and paleness 
of the nucous membranes may be recognized. When the hemorrhage has been severe from the small bowels the feces will be very dark and of a tarry consistency; from the large bowels and rectum the color is a bright red the blood being well preserved. In severe hemorrhage the symptoms are those of extensive hemorrhage from any source. Sudden paleness of the membranes, rapid weakness, etc., are the most prominent symptoms observed. Repeated small hemorrhages, such as occur during ulceration, action of parasites, etc., may produce different grades of anemia, depending upon the extent of the hemorrhage.

Diagnosis. - This can only be made after a careful examination of the animal and the discharges from the bowels. When slight hemorrhages take place, a microscopic examination of the discharges may be necessary to determine the presence of blood.

Prognosis. - This depends upon the cause, and the extent of the hemorrhage. In most cases bowel hemorrhage should be considered serious, as it is often impossible to determine the cause or extent of the hemorrhage.

Treatment. - The cause should be determined if possible, as the treatment depends very largely upon this factor. Opium is often of great value to suppress peristalsis. The tincture may be used in the following dosage: For dogs $(0.5-1.0)$, cats $(0.2-0.5)$, rabbits $(0.2-0.5)$, birds $(0.1-0.2)$. Astringents, such as tannic acid, etc., are also indicated. They should be administered in rather large doses, and repeated every hour or so. Adrenalin chlorid is most useful in severe hemorrhage: The dose for dogs is 2.0 of a $1-1000$ solution given every hour; other animals one-half the quantity. Ergot may also be used. Hemorrhage from the rectum or large bowels is best treated by injections of gelatin dissolved in hot water, astringents (alum $2-5$ per cent.), or cold water. The injections should be repeated as often as necessary to control the hemorrhage.

When there is great loss of blood, normal salt solution $(500.0)$ should be administered intraperitoneally or per rectum. Further treatment depends upon the symptoms that derelop. 


\section{DIARRHEA.}

Definition.- This term is applied to a too frequent discharge of more or less fluid feces. The condition may vary greatly in small animals from a slight increase in the daily evacuations to repeated evacuations of large quantities of liquid feces. It is important to note that often repcated evacuations of small amounts of liquid feces may occur even when an actual obstruction of the bowels '(fecal impaction) exists.

Etiology. - The essential condition in diarrhea is an abnormal increase of fluid in the feces. The causes, thercfore, will be found to be those which occasion a rapid evacuation of the bowel contents, or a reduction in the absorption from the bowels; or an oversupply of fluid in the bowels either from the ingestion of large quantities of water or any overabundant secretion from the mucous membrane.

Normally the contents of the small bowels are fluid. They pass from the stomach to the large bowels in a few hours. The contents of the large bowels become gradually hardened from the absorption of the water during their passage through this part of the intestinal tract, which requires in small animals, from twelve to twenty hours. Any conditions therefore that hasten the movements of the contents through the large bowels would tend to make the feces softer or liquid. 'The same result may occur from a variety of conditions which disturb the process of absorption and keep the feces in a liquid state. Occasionally conditions that influence the absorption from the mucous membrane, such as irritations of various kinds, at the same time cxcite the secretion of considerable quantities of fluids. These same agents may also increase peristalsis and thus hurry the contents through the bowels. The most common causes of diarrhea therefore are: (a) I) rinking excessive quantities of water or eating large quantities of fat meat, larrl, or butter will result in diarrhea. The unabsorber fat or its decomposition in the intestinal tract acts as an irritant and greatly increases intestinal activity.

(b) Foorls will sometimes produce diarrhca by their irritating aetion on the nurous membrane. Such substances as table scraps containing fruit stones, parings, or fish, 
.cheese, milk, etc., may undergo fermentative changes in the intestinal tract or from their decomposition contain preformed toxic substances which excite diarrhea. Excessive amounts of food may set up a diarrhea as a result of derangement of the gastric digestion and secondary bacterial fermentations.

(c) A large number of purgatives, when administered in large doses, will produce diarrhea by acting upon the muscle of the bowel and the nervous mechanism stimulating peristalsis and thus hastening the contents through the bowels before absorption can take place. Salines (magnesium sulphate) increase the amount of fluid in the bowels, and also stimulate peristalsis. Some foods in which bacterial decomposition has taken place will have an action similar to salines greatly increasing the amount of fluids in the bowels by exciting secretion from the glands in the mucosa.

(d) In some animals (cat) the nervous mechanism of the stomach will be greatly influenced by shock, fright, excitement, etc., and severe diarrhea may result.

(e) Diarrhea may result from diseases of other organs. In diseases of the stomach, when undigested food is passed into the intestines, the resulting irritation may induce severe diarrhea. In valvular insufficiencies or other heart lesions a congestion of the mucous membrane of the bowel results and an excessive secretion follows. Diseases of the kidney may also produce diarrhea by vicarious excretion of urea via intestinal tract. The urea decomposes producing free ammonia which irritates the mucosa causing a severe form of diarrhea.

(f) Various infections in the bowels (distemper, etc.) are associated with a more or less intense diarrhea. Infections with lesions at some distance from the bowels may have diarrhea as a consequence due to the elimination of toxic substances from the intestinal mucous membrane, which can result in sevcre diarrhea. An overproduction of bile during the course of some diseases of the liver can have the same effect.

(g) Organic discases of the bowels (catarrhs, ulcerations) often lead to diarrhea from the irritation and resultant products of decomposition. 
(h) The normal contents of the bowels contain numerous varieties of organisms, which under abnormal conditions may multiply sufficiently or increase in virulence as to bec me pathogenic, producing diarrhea.

(i) Various specific organisms (coccidia, etc.) produce diarrhea. 'They will be considered under the diseases caused by them.

Symptoms.-The clinical symptoms of diarrhea depend largely upon the part of the intestine affected and the nature of the causative agent. When due to errors in feeding and the upper part of the intestine is involved, diarrhea may not occur if the posterior bowels remain normal. In diarrhea the discharges from the bowels consist of portions of undigested food, mucus, etc., and are vellowish or greenish in color, due in part to unchanged bile or to pigment derived from fermentation. Excessive acidity, which has not been sufficiently neutralized in the small intestines, may produce inflammation in the lower bowel, due to its irritant action upon the mucous membrane. In cases where both the small and the large bowels are involved the discharges are more abundant and liquid. 'The feces will be very thin and of a yellowish or a dark brown color. When the large bowel is particularly affected, the animal shows frcquent attempts at defecation, and only a small amount of feces and blood mixed with quantities of mueus will be passed.

In the more severe forms of diarrhea colicky pains are often observed and in some cases the pain becomes intense. There are frequent attempts at defecation, and tenesmus is not unusual. When severe diarrhea is present it frequently produces extreme prostration and in cats complete collapse. The cxtremitics become cold, the inucous membranes at first pale, later cyanotic and every evidence of extreme weakness appears.

The temperature as a rule becomes subnormal; however, in the very early stages it is often elevated one or two degrees.

'The severity of the symptoms and the course depend very largely upon the causative agents producing the diarrhea. In mild cases it lasts only a few hours; in the scverc forms several day's. 
Diagnosis. - This is established mainly by detemining the cause. The history of the case is of great service in this regard. A thorough examination of the animal and feces is necessary for a differential diagnosis to distinguish this condition from specific diseases of the bowels.

Prognosis. - The prognosis is favorable in most cases but depends largely upon the cause. Diarrhea is a symptom and not a disease. Until the cause is determined an accurate prognosis cannot be made.

Treatment.-Dietetic.-All food should be withheld for at least twenty-four to forty-eight hours, especially where but little weakness or prostration is apparent. Should weakness develop small amounts of milk, or milk and egg beaten together, should be given every four to six hours. Later when the symptoms of diarrhea begin to disappear a little lean meat may be allowerl. ('ats may have small quantities of rice and milk, or salmon. Birds should be allowed only small quantities of oatmeal or seeds. Avoid the use of laxative foods, or foods containing irritating material of any kind.

Medical. - The first indication in the treatment of diarrhea is the evacuation of the irritating material from the intestinal tract. In a large percentage of cases the spontancous evacuations are sufficient to rid the bowcls of this material. Howcver, as a general rule, it is advisable to assist nature by the administration of a laxative to ensure the prompt removal of all irritating ingesta. Castor oil (dogs, 15.0-40.0; cats, $5.0-10.0$; rabbits and birds, $1.0-4.0$ ) is valuable. After thorough evacuation of the bowels opiates are indicated to control the excessive peristalsis and sccretions. Iscful is tincture of opium (dogs, 0.5-1.0; cats, 0.2-0.5; rabbits, 0.2-0.5; birds, $0.1-0.2$ ). These doses can be repcated every few hours if necessary.

Sinall doses of lime water are of value to neutralize excessive acidity which is often a common condition in small animals.

Where severe pain is present, which is rather common in the dog, small doses of morphin sulphate $(0.05-0.10)$ may be given subcutaneously:

Where the diarrhea becomes persistent, it should be troated as in acute or chronic enteritis. 


\section{CONSTIPATION. OBSTIPATION. INTESTINAL OBSTRUCTION.}

Definition.-An infrequent or difficult evacuation of feces which are abnormally dry or hard; or may be retained.

Etiology.-'The common causes of constipation in dogs are lack of exercise and feeding dry food. Dogs kept in the house or kennel often withhold the feces until they become hard and dry. Isually in old dogs the peristalsis becomes slowed and there is lack of tone in the muscular wall with some dilatation of the large bowel which causes constipation. The passage through the intestine may be blocked by fecal masses (coproliths), foreign bodies, calculi, parasites or hair balls, the latter being more common in eats as they lick off and swallow loose hair. 'The lumen of the intestine may be narrowed as a result of chronic inflammation, cicatricial contraction, stenosis, tumors within the wall, or hemorrhoids. Causative conditions external to the bowel are hernias, fibrous adhesions or tumors. Other causes are chronic inflammation of the liver with suppression of bile; chronic diseases of the spinal cord; enlarged prostates in old dogs; chronic inflammation of the anal glands; agglutination of the hair with feces at the anus in long-haired dogs. Rabbits are commonly affected by cating too much dry food. Hair balls or masses of hair and feces arc often found in these animals. Birds occasionally swallow feathers which accumulate in the intestines interfering with the passage of the contents. The most common causes are dry feed and foreign borlies.

Pathology. - 'The feces are dry, hard, usually of a light gray color and sometimes covered wi.h mucus or streaked with bloor. Coproliths or caleuli or impactions of large size often result in necrosis of the intestinal wall with perforation and peritonitis.

Symptoms. - Mild cases slow only difficult defecation at long intervals (two to four days). The feces are very dry, hard and may be streaked with blook from injuries produced in the rectal mucosa.

In severe cases the retained feces decompose producing 
toxins which are absorbed and intoxicate the patient. The animal will show depression, loss of appetite, increased temperature $\left(103^{\circ}-104^{\circ} \mathrm{F}\right.$.), and thirst. Frequent efforts at defeeation are made, the attempts inducing eries of pain. 'There is a characteristic carriage of the tail whieh is arched as when defecating, and a fulness of the abdomen. Vomiting is not uncommon especially in the more aeute cases, the vomitus containing some bile and feces. Rabbits often show paralysis of the posterior parts. Birds make frequent attempts to pass feces and usually sit or stand in an upright position. By palpation large hard masses in the posterior bowel can be determined.

Digital examination per rectum or over the abdomen will often reveal sensitiveness and the impacted fecal masses.

Diagnosis. - The frequent attempts to defecate, the condition of the evaeuated material, and the presence of fecal masses on digital examination will readily irlentify the condition.

Prognosis. - Favorable in mild eases when due to errors in feeding; otherwise depends entirely upon the causes.

Treatment. - Mild cases of constipation nay be relieved by the use of a purgative given in full closes (castor oil I). 15.0-40.0, ( . 5.0-10.0; calomel 0.05-0.15), while in chronic cases after these purgatives clear the bowel small doses of laxatives (extract of cascala sagrada, 2.()-10.(); tincture rhei, $5.0-10.0)$ must be given for some time to prevent a recurrence of the condition. 'Tineture of nux vomica $(0.3-0 .(i)$ or stryehnin sulphate $(0.001)$ is useful to stimulate peristalsis, especially when there is enervation from diseases of the cort, and to increase the tone of the inuseular wall in dilatations. In obstinate cases large quantities of warm soapy water, or oil and water, introduced with a soft rubber tube and irrigator well up into the rectum, will stimulate peristalsis, lubricate and soften the fecal masses and farilitate removal. A blunt irrigating curette can be used to brak down the hard masses. Manipulation of the ablomen while irrigating is often beneficial.

Rabbits may be relieved by the use of purgatives (castor oil, 2.0-4.0; tincture rhei, $0.3-0.6$ and a diet of green forkls, vege- 
tables, etc., should be supplied. ('hickens may be treated with enemas of glyecrin, or warm soapy water, and massaging over the abdomen. Fecal masses, calculi or foreign bodies that cannot be evacuated by any of the above methods may be removed by laparotomy and enterotomy. This operation should be performed early before nccrosis occurs in which case enterectomy must be resorted to and the necrotic portion of the intestine resected (see Enterectomy). The modus operandi is as follows: Perform laparotomy (see Laparotomy) at the median line just posterior to the umbilicus and withdraw the intestine containing the obstruction. Ligate the bowel with a heavy suture or tape on either side of the obstruction to prevent escape of contents and control hemorrhage by ligating all vessels supplying the part to be operated. Make an incision lengthwise of the bowel opposite the blood supply and of sufficient length to remove the obstruction which must be done carefully. Cleanse the bowcl, and suture the mucous membrane, and then the muscular coat with continuous sutures applied very close to the cdge and then apply continuous Lembert sutures over these. By suturing the two layers separately, there is less tissuc in the part to be folded on by the Lembert sutures. Remove the ligatures from around the bowel and the vessels, cleanse and return to the cavity.

Constipation due to cicatricial contraction or stenosis may bc alleviated by entero-enterotomy. Pcrform laparotomy as for entcrotomy, withdraw the part of the intestinc involved and apply a long bowel clamp lengthwise across a curved portion of the bowel, clamping off about three inches above the affected arca, and another below, having not less than eight inches between the two clamps. Bring the clampedoff portions in apposition, making a circular loop in the bowel, thus having the peristalsis in the same dircction. Apply continuous sutures through the muscular and serous coat of the clamped off portion just above the clamps, bringing the two parts in contact and leave the suture. Make an incision from onc to one and a half inches long of same length and position in each bowel. Apply continuous suture over the free edges in contact. Loosen clamps but leave in posi- 
tion, and continuing with the latter suture, apply ('onnell's sutures elosing the opening. 'Then beginning with the first suture apply lembert sutures around to the point of beginning. Remove vessel ligatures, cleanse and return to the abdominal cavity.

Following these operations all food must be withheld twenty-four to forty-eight hours and then only small amounts of liquid food for several days. Tumor formations in the bowel are rare but when causing trouble must be removerl by enterectomy (see Enterectomy).

\section{VOLVULUS.}

Definition.-An intestinal obstruction due to a twisting or knotting of the bowel.

Etiology.-This condition is rare in dogs owing to the short mesentery. It may occur following falls or rough handling by children during play, or by a portion of the bowel passing through an opening in the mesentery caused by an injury or following operations (enterectomy).

Pathology. - Volvulus of the bowel is more or less obstructive owing to twisting or kinking which it produces. In other cases a loop of intestine is twisted about another like a bow knot or slipped through an opening in the mesentery which partly obstructs the passage of the contents. Gas formation soon dilates the bowel further obstructing it and also the bloodvessels. When the obstruction is complete the bowel above is much distended while the part actually involved, having the circulation arrested, will appear dark red and later become necrotic leading to peritonitis.

Symptoms. - As the condition is acute the symptoms appear suddenly and are very pronounced. They are abdominal pain, accompanied by vomiting, great prostration and a hard, rapid pulse. Palpation of the abdomen may or may not produce much pain; sometimes no change in the intestine can be detected.

Diagnosis.-The acute abdominal pain, the sudden onset, rapidly increasing severity of the symptoms, vomiting and 
rapid pulse, serve to identify volvulus and differentiate it from other more chronic forms of intestinal obstruction.

Prognosis.- Cnfavorablc unless recognized early when a prompt operation affords relief.

Treatment. - Injections of large quantitics of warm water or of air into the rectum may be bencficial in some cases. However, owing to the rapid changes taking place in the bowel, laparotomy should be performed early, and an attempt made to reduce the volvulus. Should the involved part of the intestine be necrotic, enterectomy must be employed to remove the entire part affected.

\section{INTUSSUSCEPTION.}

Definition.-The invagination of a part of the intestine into an adjacent portion.

Etiology.-The exact causes are not known, although from the conditions under which it occurs and by experiments it is believed to be due to irregular innervation by which a given spot is contracted while immediately below it there is a dilatation, thus permitting the latter to invaginate the former. It may be produced by supercatharsis, incrcased production of bile (ictcrus), or the taking of cold water immediately following severe exercise, all of which cxcitc inncrvation or produce sudden increase in peristalsis. Predisposing causes are constipation; dilatations following removal of fecal masses by enterotomy; end-to-end cnterectomy, the circular cicatrix being incapable of contraction or lilatation which favors invagination; tumors in the intestinal wall. Intussusception may involve any part of the small intestine. The small intestine may pass in the ileocecal valve. Invagination of the colon or rectum may also occur.

Pathology.-Intussusception is a condition in which onc part of the bowel slips into another forming a sausage-like enlargement of varied length. 'The enlargement is slightly' curvel from tension of the mesentery. As a result of the constriction, blood circulation is interfered with and often entirely arrested. In the early stages therc is slight reddening, later the parts are swollen, congested and of bluish color. 
Adhesions oceur between the adjacent layers in a few hemrs and finally the parts become necrotic and may perforate leading to peritonitis. 'The rapidity with which these changes occur lepends upon the extent of the intussusception as the farther the invagination the greater the pressure on the bloodvessels; if the circulation be completely obstructed, necrosis will occur in a few hours.

Symptoms. - Intussusception manifests itself early by abdominal pain, the tension on the mesentery producing the first symptoms. It has been noticed in a few cases that the animal will lie on its back in order to ease the pain from mesenteric tension. Later symptoms of acute enteritis become prominent, the feces frequently show the presence of blood, there is tenesmus, eolicky pains and oceasionally vomiting, and the vomitus may be mixed with feces. Palpation of the abdomen will often reveal an elongated enlargement of the bowel and slight pressure will cause pain. Palpation is difficult in very fat animals or those having a very thick-iralled abdomen.

Diagnosis. - The presence of the painful enlargement of the bowel, bloody stools, tenesmus and the sudden occurrenee are the principal diagnostic features.

Prognosis. - I nfavorable in all cases not operated early. If recognized early and reduced or the portion excised (enterectomy) a good recovery may be expected. Spontaneous healing may oceur by sloughing of the invaginated portion and adhesion at the anterior part.

Treatment. - Intussusception of the posterior part of the bowel may be reduced in the early stages by dilating the bowel with rectal injections of large quantities of warm water, using as much pressure as can be applied safely. Air may also be used in the same manner. Purgatives or specifie stimulants to peristalsis should not be used as they only serve to inerease the invagination. Operative measures should be at once resorted to when other efforts fail. A laparotomy should be performed at the meelian line just posterior to the umbilical sear, the enlarged portion of the bowel sought and attempts male to reduce it by careful manipulation. A small blunt probe or sealped latulle may. 
be inserterl between the arlhered peritoncal surfaces to break (lown the allhesions which hold the two layers together. If this is impossible cnterectomy must be performed. Place ligatures of heary suture material around the bowel a short distance above and below the affected area and cut off the bowel with scissors making the incision diagonally across the intestine. Cut off the mesentery supporting the part to be removed, ligating each ressel as it is reached. Bring the parts of the bowel in end-to-end contact and suture with Connell's sutures or if preferred a lateral anastomosis may be used. After-treatment is the same as for enterotomy.

\section{WOUNDS OF THE INTESTINES.}

Definition. - Wounds of the intestines frequently occur in small animals, especially the $\mathrm{dog}$, which is morc subject to injury than the cat, rabbit or bird.

Etiology. - The most common causes are: Falling from a great height, being run over by vehicles, kicks, or severe blows over the abdomen. Punctured wounds of the abdomen often penctrate the intestines, such as gunshot wounds or those produced by sharp objects (forks, etc.) which penetrate the abdominal walls. P'enetrating wounds should always be considered serious, as the extent of the injury in the abdominal carity is difficult to determine. There is also danger of infection being carricd into the abdominal cavity from without, or from within, the bowel contents escaping into the cavity and causing septic peritonitis.

Symptoms.-Injuries to the intestines arc often difficult to recognize, especially those produced by kicks, blows and other forms of external violence. 'The most prominent symptoms of intestinal wounds are hemorrhage and collapse. Often the abdomen will become rlistended with blood when the bowel is ruptured. 'The anamnesis, paleness of the mucous membranes and the sudden enlargement and distention of the abdomen usually suffice for diagnosis. However, an accuratc diagnosis cannot be made, unless an explorative 
laparotomy is performed, and the eavity most thoroughly inspeeted. Other organs in the abdominal eavity may also be injured by the eauses mentioned. 'The symptoms produced by punetured wounds depend very largely upon the externt of injury to the intestines or other organs. Gunshot wounds when of small ealiber will not produee any very marked symptoms other than tenderness over the abromen. Healing may follow readily. Small wounds may not penetrate farther than the mueosa whieh may poueh outward through the opening and prevent the eseape of the intestinal eontents. Later adhesions take place preventing septie peritonitis. Larger wounds, however, usually prove fatal under symptoms of eollapse from hemorrhage or septie peritonitis, unless an aeeurate diagnosis is made and the proper treatment immediately applied. When punctured wounds are observed, the animal's general eondition should be noted at onee. Avoid the use of probes owing to the danger of earrying infeetion into the cavity.

Treatment. - The first indieation in the treatment of intestinal wounds is to perform laparotomy (see Laparotomy) as early as possible. A generous ineision should be made in the median line to allow a eomplete and thorough examination of the entire intestinal traet and other organs. The intestine should be thoroughly examined the entire length, and any wounds found closed with Lembert sutures. If the wound is extensive, as a rupture of the bowel, it should be closed as in enterotomy. In some eases where the bowel wall becomes torn or has an uneven surface, a portion shoukl be removed. (See Enterectomy.)

'The organs should also be inspected and any wounds in them sutured.

The abdominal eavity should be thoroughly irrigated with a borie aeid solution (2 per eent.), or normal salt solution, espeeially in those eases where intestinal contents have escaped or blook is present.

Should the animal be weak from loss of blood, stimulants shoukd be given, such as strychnin sulphate (1).(1)1) repeaterl as often as necessary. 


\section{CROUPOUS ENTERITIS OF CATS.}

\section{Membranous Enteritis.}

Definition.-A croupous or membranous inflammation involving the mucous membrane of the intestines.

Etiology.-Croupous enteritis is observed most frequently in kittens occurring as an epizoötic in certain districts during the winter and spring seasons of the year. 'The exact etiological factor has never been determined. It is possibly due to a virulent form of the colon bacillus aided by a reduction in resistance from exposure to cold, irregularities in diet, parasites, etc. Older animals are also occasionally affected but not in such large numbers.

Pathology.-The principal pathological changes are noticed on the mucous membrane of the bowels, the muscular walls, and the mesenteric lymph glands. The mucous mcmbrane is highly congested, reddened, swollen, and the surface covered with a thick membranous exudate. Often the epithelial surface and even the submucosa will become loosened from the other tissues. 'The wall of the bowel is edematous, and the serous covering shows inflammation. The mescnteric lymph glands are enlarged, edematous, and show acute inflammatory changes. The liver, spleen and kidneys show. similar changes. The contents of the bowels are liquid, and contain considerable blood.

Symptoms. - The carly symptoms are: Vomiting and a severe diarrhea which come on suddenly and usually affect several animals at the same time. There is a complete loss of appetite, depression, and in twenty-four to forty-eight hours the animals will become very weak, comatose and die from exhaustion.

Diagnosis. - The epizoötic character of the disease, the sudden onset and the age of the animal affected are indicative. It can be easily mistaken for various kinds of poisonings; the anamnesis therefore should be carefully ascertained to assist in the differential diagnosis.

Prognosis، - Should be considered unfavorable; the largest percentage of cases terminatc fatally. 
Treatment. - Treatment of croupous enteritis is as a rule unsatisfactory. Little can be done except the administration of general stimulants and bowel disinfectants. As prophylactic measures, all feeding pans should be thoroughly cleaned and sterilized, and the discharges of the bowels carefully taken care of to prevent further contamination of the food.

\section{INFECTIOUS ASTHENIA OF BIRDS.}

Definition.-This is a chronic disease found in birds, which affects primarily the duodenum in the form of a chronic catarrhal inflammation.

Etiology. - The cause is a microörganism known as the Bacterium asthenix. It develops principally in the duodenum, producing an irritation to the mucous membrane, which gradually leads to chronic inflammation.

Pathology.-The characteristic lesions are: Extreme emaciation of the muscular system with an almost complete absence of fat. The bowels are empty, containing only a small quantity of slimy mucus. The walls of the duodenum are reddened, thickened, and show all the evidences of a catarrhal inflammation of the mucous membrane. The duodenal feces will contain large numbers of the Bacterium asthenix.

Symptoms.-Infectious asthenia is observed most commonly in young birds one to six months old. It is characterized by extreme emaciation, variable appetite, paleness of the comb, wattles and membranes. The course is chronic and the termination usually fatal in about three months. The extreme emaciation which comes on gradually has led to the term "going light" being given it. l)uring the course of the disease the birds become weak, anemic, the plumage rough; there is general depression which causes them to sit in one position for a long time. Death oceurs from cachexia.

Diagnosis.- The large number of birds affecterl, the age of the birds, the long chronic anrse, and the chariacteristic lesions found on postmortem are usually sufficient to estab- 
lish the diagnosis. The finding of the Bacterium asthenix in several of the birds will confirm the diagnosis.

Prognosis.-Owing to the infectious nature of the disease, the chronic course, and the pathological changes which have taken place in the duodenal mucosa, the prognosis is unfavorable. Where the disease has reached an advanced stage it is advisable to destroy the bird and thoroughly disinfect the premises.

Treatment.-Medical.-Small doses of laxative, castor oil $(1.0-4.0)$ or calomel $(0.01-0.05)$ should be given.

As a stomachic and tonic iron sulphate may be given in the drinking water (1-1000).

The aromatic seeds (fennel, coriander, anise) may be used once or twice daily with the food to stimulate the secretions of the stomach and intestines.

Prevention.- The healthy birds should be separated from the sick ones at once. All parts of the houses (roosts, floors, etc.) should be thoroughly cleaned and disinfected with a carbolic acid solution (5 per cent.). 


\section{CHAPTER X. \\ PARASITES IN THE INTESTINES.}

\section{HELMINTHIASIS.}

In small animals parasites in the digestive tract are very numerous. They pass rapidly through the anterior portion of the digestive tract, which prevents their becoming fixed; also in this portion of the canal secretions for their proper development are lacking. In the stomach, owing to its acidity, they do not remain long, but are cither destroyed or pass into the small intestines. In a fow instances, however, they may burrow underneath the mucosa of the stomach. The intestines are favorable for the development of parasites, owing to their length, the presence of an abundance of fluid, and the slow peristaltic movement of the bowels which does not materially interfere with their fixation and development. The majority of the parasites are found in the small intestines; fewer are found in the cecum, colon and rectum. Each species of parasite has its particular location, and unless in unusual numbers, or under abnormal conditions, they will be found infesting an exclusive portion of the bowcl. Parasites are frequently found in very large numbers, and produce scrious disturbances in the intestinal tract, depending upon the species of parasite and the animal infested. Thie most common parasites found in the intestinal tract belong to the animal kingdom, and are: Cestoda; Nematoda; Acanthoccphala; Coccidia.

\section{TAENIASIS.}

\section{restodte.}

'The dog is the favorite host of the tieniar. They oremr in this animal in considerable numbers, sometimes so as to 
excite wonder at the continued good health of the host. More than 50 per cent. of the dogs examined harbor worm parasites.

It is essential for the tænix to have an intermediate host to complete their life cycle. The following animals serve as intermediate hosts for most of them: Sheep, ox, pig, horse, goat, rabbit, and even man.

The head (scolex) of a tænia is generally expanded and supplied with suckers; some species in addition are provided with hooks. From this head, by proliferation, is formed the neek, a thin non-annulated constriction, which, continuing and becoming wider produces the body or strobila. The body is in the form of a long, narrow band divided into more or less distinct segments, and as these become mature they are cast off gradually from the distal end of the parasite as new segments develop from the head. Each of the segments contain numerous eggs (ova) which pass out with the feces. Before becoming detached the segments can also liberate ova through an opening on the lateral wall or ventral surface, called the genital pore, forming two ways of disseminating the ova. When deposited in a suitable medium, preferably warm, moist soil, or filth, the ova undergo a series of complieated changes which finally result in the first larval form. In this form they are ingested by the intermerliate host, most commonly with the fool or water. They burrow through the intestinal walls and migrate into the adjacent tissues, or with the blood stream are carried to remote parts where they develop into cysts. I) the various organs serious conditions, such as " gid" in sheep, echinococcus disease of man, ete., may arise in the new host. As the log is the harborer of the parent trenia, treatment should be given not only to reduce the disorders they occasion in other hosts but also to lessen the injury they produce in the dog. 'The cyst form is the limit of development in the intermediate host. The life cycle can be completed only in case the cyst is ingested by and reaches the digestive tract of another host in which it can develop. I)ogs harboring the adult tania cannot become reinfested by ingesting the larval form, but the larval form inay migrate from the intestines 
into adjacent tissues and there develop new cysts. 'This is the limit of their growth, however, in the host of the adult worm.

Tæniæ in Dogs.--The most common tæniæ found in the dog are as follows: (a) Dipylidium caninum (Tonia cucumerina), a worm 30 to $40 \mathrm{~cm}$. and $3 \mathrm{~mm}$. at its greatest breadth. Its club-shaped head is provided with four suckers and four rows of very small hooks. The neck is long and narrow. The first segments are narrow, the others are longer than they are wide and like melon seeds in form (cucumis). Genital pore double and opening toward the middle of each side of the segments on a slight prominence. Ova are globular, from 37 to 46 microns in diameter, and pass from the segments massed in a small group enclosed by a capsule (cocoon). The cyst form is the Cryptocystis trichodectis and the intermediate hosts are the dog flea (Ctenocephalus canis), dog louse (Trichodectis canis) and the flea that lives on man (Pulex irritans). These intermediate hosts become infested $\mathrm{ky}$ ingesting the ova of the txuix which are always present on the skin or hair of the $\log b y$ having fecal discharge mixed with the bedding in the kennel. 'The cysts develop usually in the abdominal eavity of the intermediate hosts which are in turn swallowed by the dog with water and food, or while licking or biting the skin to relieve the irritation which they produce. They then attach themselves to the walls of the intestine where they derelop into the ardult worm.

(b) Toenia pisiformis ('T'ania serrata).- This parasite is about 1 meter long, head a little broader than the nerk, and armed with 24 to 38 hooks. Segments at first are mucli shorter than hroad, about square in the middle portion while the mature segments are 10 to $17 \mathrm{~mm}$. long by + to $6 \mathrm{~mm}$. broar. Genital pore on the lateral border, and rery prominent, causing the border to appear convex and the segurent to be wider in the middle than at the ends. Posterior borders straight and the angles uneven which gives the strobilat at saw-like apprarance. Eggrs are ovoid and 30 to f(l mircrons long and 31 to :36 microns broald. 'The rest form is the ('ysticereus pisiformis and is frequent in tlie peritoneal carity 
of hares and rabbits. Dogs become infested by eating the viscera and in twelve days the tæniæ are 2 to $3 \mathrm{~cm}$. long, and matured in two months. The ripe ova given to rabbits become cysticerci.

(c) Tonia hydatigena (Tania marginata).-This is the largest trenia found in the dog, being 1.5 to $2.0 \mathrm{~m}$. long. The head is scarcely wider than the neck and has 30 to 44 hooks. The segments are nearly square with the genital pore on the lateral border which begins to develop in the segments about one-fourth of the distance from the head. The posterior border of the segments is slightly wary, and received into the succeeding segment. Mature segments when detached are about $15 \mathrm{~mm}$. long by $7 \mathrm{~mm}$. wide. The ova are almost spherical and from 30 to 36 microns in diameter. The cyst form is the Cysticercus tenuicollis and is commonly found in the peritoneum, pleura and even in the pericardium of domesticated animals, especially ruminants. It requires four to five months to fully develop into the mature worm.

(d) Multiceps multiceps (Tania canurus).-This worm rarely exceeds $1 \mathrm{~m}$. in length. Head small, slightly broader than the neck and armed with 22 to 32 hooks. Segments narrower than any of the preceding species, becoming square with the genital pore developed about the 125th segment, 15 to $20 \mathrm{~cm}$. from the head. Mature segments 10 to $12 \mathrm{~m}$. long, 3 to $4 \mathrm{~m}$. wide. Eggs spherical 31 to 36 microns in diameter. The cystic form is the Multiceps multiceps (Conurus cerebralis) which is developed in the cerebrospinal cavity of sheep; more rarely in other domesticated animals, causing the disease commonly called "gid." The cyst is polycephalic, the ingestion of one cyst producing numerous tænix. The tænia requires about two to three months to reach maturity.

(e) Multiceps serialis (T'ania serialis). -This parasite is 45 to $75 \mathrm{~cm}$. long, head wider than the neck and having 26 to 32 hooks. Segments similar to those of the M. multiceps. The ripe segments are 10 to $16 \mathrm{~mm}$. long and 3 to 4 $\mathrm{mm}$. wide, the posterior border being straight. Eggs ovoid and 34 microns long and 27 microns wide. The cyst form is 
the Multiceps serialis and its intermediate liosts are sone of the rodents and warren rabbits. This tania develops more rapidly than the others requiring but a few weeks.

(f) Echinococcus granulosus (Toria echinococcus).-Whis species is distinguished from all others by its size. It is only 4 to $5 \mathrm{~mm}$. long, and composed of 3 to 4 segments, the last of which contains the mature ova. The head is armed with a double row of $2 S$ to 50 hooks. Eggs ovoid, 34 by 25 microns. The cyst form is the Echinococcus granulosus (E. polymorphus) and is found in most of the organs of the herbivora and even man, but is more common in the liver and lungs of ruminants and pigs. It requires one month to fully develop. 'This cyst is polycephalic and polysomatic.

Tæniæ in the Intestine of the Cat.-Three species of tæniæ have been found in the cat.

(a) Tania taniaformis (Tania crassicollis). - This is the most common tænia found in the cat. It attains a length of 15 to $50 \mathrm{~cm}$. and in appearance is similar to those found in the dog. The head is armed with a double crown of 26 to 52 hooks and is about as wide as the neck. The posterior segments are 8 to $10 \mathrm{~mm}$. long and 5 to $6 \mathrm{~mm}$. wide. Ova are globular and 31 to $37 \mathrm{~mm}$. in diameter. 'This tienia is represented in the vesicular or bladder form by the Cysticcrcus fasciolaris which inhabits the liver of rats, mice and other rodents. This cysticercus, which is always coiled up in a cyst it has itself produced, is elongater in form, the body composed of segments, and from 3 to $20 \mathrm{~cm}$. long, while the vesicle is ovoid and frequently no larger than a pea.

(b) Tania elliptica and (c) Tania pseudo-elliptica have been described as a variety of the Dipylidium caninum of dogs; they are unimportant.

(d) The Bothriocephahus felis has also bcen mentioned. Little is known about it and no disturbance in the cat lias been ascribed to its presencc.

Tæniæ in the Intestine of Rabbits.-Cestoles are rare in these animals and all belong to one species, the (Cittotania denticulata (Moniezia denticulata). 'These may attain the length of $8 \mathrm{~cm}$., head small and supplied with suckers. segments $1 \mathrm{~cm}$. broad and not so long. 'Two genital pores are 
in the posterior part of the segment. The eyst form and intermediate hosts are unknown.

Tæniæ in the Intestines of Birds. - In the intestines of the fowl, nine species of cestodes have becn found-eight trenia and one bothriocephalus. 'The treniæ are difficult to distinguish from one another, and as they are rare, little work has been done to establish their complete history. Outbreaks do occur, however, in which so many fowls are infested as to amount to an epizoötic treniasis.

(a) Choanotania infundibuliformis, length 20 to $130 \mathrm{~mm}$., armed and supplied with suckcrs. Intermediate host said to be the common house fly.

(b) Dicranotonia sphenoides, length 2 min., head armed and supplied with suckers. Intermediate host the earthworm.

(c) Davainea proglottina, length $1.0-1.5 \mathrm{~mm}$., head armed, and supplick with suckers. 'The segments of this tænia are cast off' as soon as mature. They continue to live and develop, greatly increasing in length. Intermediate hosts are various species of inollusca.

(d) Daxainea cesticillus, length 9 to $45 \mathrm{~mm}$., head unarmed. Intermediate host unknown.

(e) Davainea echinobothrida, length 50 to $100 \mathrm{~mm}$., head has suckers and is armed. Intermediate host unknown.

(f) Davainea tetragona, length $25-100 \mathrm{~mm}$., head has suckers and armed. Intermediate host unknown. Tery little is known of the other species.

(g) Trnia cantaniani, the only tænia found in turkeys. It is $14 \mathrm{~mm}$. long, hcad unarmed, but provided with suckers, and has no neck. Life history unknown.

(h) Dacainea crassula very rarely infests pigeons. Length 30 to $40 \mathrm{~cm}$., breadth $4 \mathrm{~mm}$., head armed with double crown of 60 hooks. First segment short, posterior ones long, genital pores unilateral. Ova very large, ovoid, $2 S$ microns long, and arrangerl in groups.

Pathology. - Trenire are extremely frequent in dogs, but the various species are not equally distributed, and the variation seems to pertain to different countries and also to the different sections of the country. The frequency of trenia is also 
directly related to that of the cystic or blackler worms infesting ruminants, rabbits, and other intermediate hosts. The number of individuals by which each of these trenix may be represented in the same dog is also variable. 'The Dipylidium caninum varies up to 360 , the $\mathrm{T}$. pisiformis as high as 64 , the ' $\mathrm{T}$. hydatigena and the M. multiceps usually' less than 10, but the Echinococcus granulosus from one to several thousand. This variation is due in part to the fact that some cyst forms give rise to but one adult parasite (monocephalic), while others produce many (polycephalic). The common location of the tænia is in the small intestine; only occasionally does it migrate to other parts of the digestive canal. The head is attached to the mucosa by suckers or hooks, and as the body may be folded on itself many times long parasites may only occupy a short distance of the bowel. Large numbers are often massed together almost completely occluding the lumen of the bowel. The mucosa is hyperemic, thickened and covered with mucus. Some of the glands are hypertrophied. Rare cases of perforation of the walls by tonice have been reported, but as a rule the pathological changes are those of a chronic enteritis.

Symptoms. - Notwithstanding their extrcme frequeney, the tania often have no apparent influence on the health of the dog. Sometimes, however, by their accumulation and intestinal obstruction, they cause abdominal pain or a chronic enteritis. The appetite is often irregular, but while the patient may eat well its condition remains bad. Growth is checked, emaciation often develops, and the hair coat loses its normal luster. Young logs are uneasy, change their position frequently and show a desire to bite the skin orer the abdomen. 'They may have epileptiform attacks, which are periodical, with normal intervals between. When frequent, these attacks may be followerl by a gradual sinking and rleath.

'The 'Tania taniaformis of cats is frequently found in large numbers in the small intestines and occasions serions disease. The appetite gradually declines. A slight diarrhea is an early symptom followed by constipation; salivation is abundant; great prostration; nervous phenomena as loss of sight 
and hearing, and epileptiform convulsions which oceur at intervals.

Rabbits exhibit symptoms similar to the cat, except the former usually have a partial posterior paralysis instead of convulsions.

Chickens lose their appetite, become emaciated, are dull and feeble and a few show diarrhea. The presence of segments on the surface of the feces is often the only symptom of tæniasis.

Diagnosis. - The poor or emaciated condition of the animal, diarrhea or constipation, would indicate the presence of intestinal parasites, but an accurate diagnosis of tæniasis can only be made by finding the segments or ova mixed with the feces. The ova can only be detected by the microscope. A microscopic examination is readily made by shaking some of the feces in a test-tube, one-half full of water, until the mass is broken up. Allow it to stand for a few minutes, and with a pipette withdraw a quantity from the center of the fluid. Place a few drops on a slide, cover with a cover-glass, and examine first with low, then with high power. The ova of the tæniadæ all appear about the same size. While the variations peculiar to each species make differentiation possible, it is unnecessary to consider them as the prognosis and treatment are similar for all the species. For an accurate diagnosis of the species, the entire parasite, especially the head, must be obtained and examined microscopically.

Should the feces be soft or liquid, smear slides can be made and examined with as good results. A negative opinion should not be given without examining several slides. Usually, however, one finds 20 or 30 ova on a single slide.

Prognosis. - Favorable in mild infestations, but when large numbers are present chronic catarrh, or death from obstruction is always probable.

Treatment. - As truix, even in mild infestations, inconvenience the lost more or less, it is advisable to treat them. A further reason is the possible infestation of man and herbivorous animals with the cyst form. Hogs, sheep, cattle, show and hunting dogs not only improve in condition when the tænia are removed, but the further propagation of tæniasis is correspondingly reduced. 
The administration of a temiafuge should be preceded by a purgative and a twenty-four hour fast. Nany preparations have been employed with good results, but inale fern is probably one of the inost reliable. The oleoresin (small dogs, $0.5-1.0$; large dogs, 2.0-5.0; cats, $0.2-0.5$ ) may be used. An excellent mode of administration is to mix it with a dose of castor oil. The purgative action of the oil assists in the evacuation of the parasites. Kamala (dogs, 3.0-10.0; cats, $1.0-4.0$ ) is good; it also produces a purgative action. Areca nut powder is often used and is given in doses of two grains per pound weight of the animal. It may be given with soup, ordinary food or milk, after the bowels have been emptied by a purgative. Areca nut powder may be repeated in a few days. Rabbits may be given kamala (0.5-1.0) in the feed. Birds are best treated for tæniasis by mixing areca nut powder (chickens, 2.0; geese, 4.0; young chiekens, 1.0) with the feed. Oil of anise is also good, and can be arlministered in 0.9-1.0 doses to adult birds.

\section{NEMATODA.}

Round Worms. - Ascarida. - Round worms are quite eommon in dogs and cats, espeeially in puppies and kittens two or three months old. Fully 50 per cent. are infesterl. In one animal ten to thirty parasites are usually present; eighty were found in one subject.

Round worms do not require an intermediate host to complete their life cycle as do the tæniæ. The ova deposited by the adult worm with the feces are passed out, and in warm, damp ground or in other suitable places undergo certain ehanges, leading to the formation of embryos. Such changes may occupy a few days to several weeks, depending upon the eonditions of moisture and temperature. "The embryos when ingested by their partieular host, rapidly develop into the adult parasite. Puppies and kittens beeome infested as soon as they begin nursing, provided these parasites are present in the mother or other animals in the kennel. 'The arlult worms pass continuously a large number of ova, and embryos are present in infested soil which adhere to the 
mammary glands and are swallowed by the young while nursing. Birds are less commonly affected with round worms, and as a rule they harbor only a few specimens. Occasionally, however, round worms are found in birds in large numbers, especially in certain localities where this form of parasitism may be enzoötic. Pigeons are the common victims; in some cases whole flocks succumb to the infestation.

Round Worms in the Intestine of the Dog.-Belascaris marginata.-This species is similar to the B. mystax of the cat, and is somewhat larger. The body is white or reddish, head usually curved and provided with two membranous lateral wings, which cause it to look like an arrow head. Male 5 to $10 \mathrm{~cm}$. long, tail curved. Female slightly longer, tail obtuse. Ova almost globular and 75 to 80 microns in diameter. Capsule thick and showing circular striations; granular center with small clear space between it and the capsule

Toxascaris limbata (T. marginata).-This parasite is similar to the above; found in intestinal tract of dogs; rare in United States.

Echinorhynchus canis,-Occasionally found in the dog in certain districts (Texas).

Round Worms in the Intestine of the Cat.-Belascaris mystax.-This round worm is slightly smaller than the B. marginata of the $\mathrm{dog}$, the male being 4 to $6 \mathrm{~cm}$., the female 4 to $10 \mathrm{~cm}$. long. Ova slightly smaller than B. marginata, 60 to 75 microns in diameter. Similar to B. marginata only smaller.

Round Worms in the Intestine of the Rabbit.-Oxyuris ambigua (Passolurus ambigus).-This is a white fusiform worm, male 3 to $5 \mathrm{~mm}$. long, female 8 to $11 \mathrm{~mm}$. The body' of both sexes terminates in a suddenly tapering tail, $0.22 \mathrm{~mm}$. in length. It infests the large intestine and cecum. It is not so common as the round worm of the dog and cat, nor does it occur in as large numbers. Ova globular and very small, with thick capsule similar to $\mathrm{B}$. marginata.

Round Worms in the Intestine of Birds.-Heterakida.(a) Ileteralis papillosa (IIeterakis vesicularis).-This is a common species infesting chickens, and oceasionally ducks. 
Male is 8 to $9 \mathrm{~mm}$., female 11 to $15 \mathrm{~mm}$. long. Mouth surrounded by three distinct lips. 'The body is gradually attenuated toward the posterior part, and has two unequal spicules. This parasite is found exclusively in the cæca and occasionally in large numbers. Ova are elongated, capsule with full granular center.

(b) Heterakis differens.-This species is slightly larger than the Heterakis papillosa attaining a length of 15 to $20 \mathrm{~mm}$. The mouth has no apparent lips. Has two spicules of equal length and the posterior extremity of the female terminates in a sharp point. It is usually found in the posterior part of the intestine of chickens. Ova similar to H. papillosa.

(c) IIeterakis inflexa (IIeterakis perspicillum). - This is not very common, and when present is found in the small intestines. In length and appearance it is similar to the $\boldsymbol{H}$. differens. The host is the ordinary fowl.

(d) IIeterakis compressa is similar to above; occasionally found in the small intestines of chickens.

(e) IIeteralis muculosa.-This is the common parasite of pigeons and of ten oecurs in such large numbers as to prevent their being reared, 400 to 500 are sometimes found in an individual. The body is white and attenuated at both ends. Male is $20-25 \mathrm{~mm}$. f female $2(0-25 \mathrm{~mm}$. long.

( $f)$ IIeterakis crassa. - This parasite is common in the duck. The male is 12 to $15 \mathrm{~mm}$. long and the female 40 to $50 \mathrm{~mm}$. Tail is thin, conical, and straight.

(g) Heteralis lineata.-Heterakis lineata is rare; found in the intestine of the domesticated duck. Its length is from 6 to $10 \mathrm{~cm}$.

(h) Heteralis dispar.- This species found in small intestine of geese is closely related and similar to the II. papillosa. Mouth has three very small lobes. Two lateral wings on the neck becoming narrower toward the tail. Male 12 to $18 \mathrm{~mm}$., female 16 to $23 \mathrm{~mm}$. long. This parasite is not very common.

Pathology. - Young animals which have died from the disorder caused by the ascarides, show on antopsy large numbers of this parasite which almost orchule the lumen of the bowel. The stomach may also contain many of them. They are found seattered along the intestines or coiled up in masses. 
The mucous membrane shows a severe enteritis with numerous ecchymoses and many small ulcerations. 'The intestines contain no food, only a slimy mucus in which are found the parasites. In older dogs the parasites are fewer in number and are found scattered along the entire length of the intestine and rarely cause much change in the mucosa.

Symptoms.-Puppies and kittens when infested show symptoms of inanition at three to five weeks of age. They are stupid and do not play as such animals usually do at this age. Vomiting is common and quite often some of the parasites are thus expelled. Emaciation increases, the mucous membranes are anemic, and the abdomen appears enlarged ("pot bellied"). Diarrhea is not uncommon, often alternating with constipation. Quite frequently the patients, especially kittens, show epileptiform or rabiform symptoms. These are probably due to the irritation of the nervous system produced by toxins excreted by the parasites as well as by the irritation of the intestines they produce. These symptoms gradually become more severe, and finally food is refused, followed by weakness, coma and death in five to eight weeks. The temperature is only slightly elevated in the early stages, later as coma comes on it is subnormal.

In mild cases or in older dogs the symptoms are less intensive, and often unnoticed. The appetite remains good, often voracious, but the general condition is not the best; the hair coat dull and rough and the growth impaired. Young birds infested with round worms show diarrhea, emaciation and depression, finally resulting in death. In older birds the condition becomes chronic with marked emaciation.

Diagnosis. - This can only be positively made by finding the ova in the feces or the parasites in the feces or vomitus; or on postmortem cxamination. (See Diagnosis of Tæniasis.) The general symptoms are similar to those of tæniasis. Ascariasis is, however, more common in very young animals which have had no meat. When several animals or birds are affected one or more of the typical cases should be killed and a careful autopsy made. 
Prognosis.-Severe infestation in young animals is very unfavorable. Ascariasis causes greater loss among puppies and kittens than any other disease. Entire litters of ten succumb at four to eight weeks of age, and in some kennels it is almost impossible to rear young animals rlue to this parasite. Chicks and young birds usually succumb; while older birds do not die, they grow emaciated and droopy and become an easy prey for other diseases.

Treatment.-Medical.-It is advisable to administer a vermicide to all puppies and kittens when three to five weeks of age, and repeat every two to four weeks until screral months old. Treatment should be given as soon as symptoms appear. Santonin (puppies, 0.016 ; small dogs, $0.05-0.2$; large dogs, 0.2-0.5; kittens, 0.008-0.025; cats, $0.06-0.2$ ) is the most efficient agent used to remove the ascaridx. It is best administered in small doses, repeated for three or four tays, than in a single, large dose. 'This is especially true for all young animals, as they are rery susceptible to the toxic action of this drug. Older animals are rarely affected even with enormous doses. Santonin may be administered in castor oil in sufficient amount to produce catharsis, or the oil may be given an hour or two later. Tablets of santoniı and calomel of various proportions may be had and are convenient to use with the food. Other anthelnintics are employed with good results, such as areca nut powder (dogs, $0.5-4.0$; cats, $0.1-2.0$ ) which is easily given with milk; benzine (1.0-7.0 in oil); kamala (2.0-8.0). Birds may be treated with arcca nut powder (chickens 3.0, pigeons 1.0 each), mixed with moist ground feerl. Oil of anise $(0.4-(0.4)$ or benzine $(0.2-0.6)$ in castor oil has been used with grood results.

Prevention. - In order to rear young animals free from these parasites energetie measures must be elirected toward the rcmoval of all ascarida from the older animals and a thorough disinfection of all the premises to which they have access. 'This is best done in the early winter, the wouther conditions at this time being unfarorable to their derelopment. Frequent cxaminations of the feces should be made and treatment applied when necessary. All additions to 
the kennel or flock should be examined and treated before being allowed with the other animals or birds.

Pregnant animals should be entirely freed of all parasites, and thoroughly washed to remove all ova or embryos that may be on the hair or skin. Afterward remove to a clean plaee that has not been used for animals for some time where the mother and young should be kept for several weeks. The feed and water, and all reeeptaeles must not be permitted to be soiled by other animals which harbor parasites. In this way it is possible to rear puppies, kittens or birds without experiencing the trouble with parasites.

Strongylidæ.-Hoohworm.-This speeies of the nematodes inhabits the small intestines, preferring the anterior half, and oeeasionally is found in the stomaeh. They attach themselves to the intestinal walls, wound the mueosa, eat the epithelium, and suck the blood. Aeeording to present evidenee they produce a poisonous substance whieh inhibits the coagulation of blood and possibly also injures the host. Inasmueh as these parasites frequently move from place to plaee, wounding the mucous membrane in many different places, from whieh hemorrhage continues for some time, a severe anemia is soon produced. These eontinued injuries to the mucosa soon result in a severe enteritis with all of the symptoms of inanition.

The arlult parasite in the intestine lays numerous eggs which are passed out with the fecal material. After a short time (eight hours to several days), the period varying aceording to conditions of heat and moisture, an embryo develops in eaeh egg. The embryo soon breaks through the shell. In the soil it undergoes a change in two or three days, and another in about a week, during which time it also becomes larger. 'This stage is known as the infesting stage. The parasite may live in this condition for five months or longer. Infestation may orcur via the mouth, the embryo being taken with the food or drink; also by burrowing through the skin or mucous membrane and by following the blood stream finally reaching the intestines. In experimental cases the worm has been found in the intestines in eight to fourteen days after skin infestation. 
Strongylidæ in the Intestine of the Dog. $-(a)$ Ankiylostoma canina (Uncinaria trigonacephala; Dochmius trigonacephalus). - This parasite is small, being 10 to $15 \mathrm{~mm}$. long, the body white, mouth slightly expanded, the ventral border or jaw terminating in four hook-shaped projections arranged in pairs, and usually called lips, by means of which the worm attaches itself to the mucous membrane. Within the mouth on the merlian line is a conical tooth-like projection, on the summit of which a gland opens. On the dorsal border there are in addition, two small straight teeth. The tail of the male is expancled, while that of the female terminates in a blunt point. The vulva is at the posterior third of the body. Ova ovoid, 74 to 84 microns long by 48 to 54 microns broad.

(b) Uncinaria stenocephala (Anhylostomum stenocephalum). - This species of the strongylidæ is smaller than the above and less common. Body is thinner than that of the Ankylostomum canina. Head is narrow, buccal capsule having on each side of its ventral aspect a sharp bordered "lip," back of which is a hook-shaped tooth. The dorsal border has no teeth. The male is 6 to $8 \mathrm{~mm}$. long, female 8 to 10 mm. Ova ovoid, 63 to 67 microns long by 32 to 38 microns broad.

Strongylidæ in the Intestine of the Cat. Ankylostomum trigonacephalum.-This parasite has been described under the name of Dochmius balsami. It is ahnost, if not, identical with the Ankylostoma canina of dogs as described above. 'They often occur in larger numbers in cats, and may' localize at one particular part of the small intestine.

Strongylidæ in the Intestine of the Rabbit.-(a) Strongyloides longus. - This species inhabits the anterior part of the small intestines. It is a very small worm, and the mouth has no hooks. Eggs are ovoid, 40 microns long and 20) microns wide.

(b) Strongylus strigosus. - This is a rare species and inhabits the cecum and colon of the rabbit. In rabbits the parasite has not been known as yet to produce any serious pathogenic conditions. 
Pathology.-On autopsy dogs which have died from ankylostomiasis show cachexia. In addition the mucous membrane of the small intestines and cecum is very much thickened. Small hemorrhagic areas are scattered over it, and often so extensively as to be visible on the peritoneal surface of the intestine. The villi are quintuple their normal size, are highly injected and closely packed together. In the relatively healthy parts are seen a multitude of small hemorrhagic points, and in their centers or vicinity are noted one or more of the parasites, often coupled. More parasites are found in dogs that have been ill only a short time than in those in which the malady is chronic. In the latter only a few parasites may be found in the ileum, a circumstance which may raise a doubt as to the anemia being due to them. Only a small amount of very dark or black fecal material will be found in the intestines.

Symptoms. - The symptoms are those of a severe anemia. At first there is only debility and wasting, although the appetite remains good, though at times variable. The animals affected appear dull and indifferent, the hair coat is rough and staring, and the skin scaly or reddened with erythematous patches, especially at the stifles and nose. The latter is tumefied, cracked, rough and excoriated, with a mucopurulent discharge from the nostrils. Attacks of epistaxis occur at intervals of a few to several days with a loss of two or three ounces of blood. Another important symptom is edema of the limbs. It is at first intermittent, but finally becomes quite permanent. In the latter stages the diarrhea, at first intermittent, becomes continuous, and the appetite, which was capric ous, disappears. The animals, feeble and emaciated, remain in a lying position, grow comatose and die sometimes in convulsions. Death takes place in from a few months to a year.

Diagnosis - Ankylostomiasis is easily mistaken for nonparasitic anemia. The diagnosis really depends upon the finding of the ova or the parasites. When several animals in a kennel or pack are affected, an autopsy will remove all doubt as to the nature of the malady. 
Prognosis. - If the condition is recognized early and treatment administered, the prognosis is favorable. In those cases showing extreme emaciation and exhaustion the prognosis is bad.

Treatment.-Treatment in ankylostomiasis must be directed toward the removal of the parasite. The common anthelmintics used for intestinal parasites have little or no effect on the strongylidx. Thymol $(0.06-0.6)$ is probably the best for this species, and good results follow its use in daily repeaterl doses. It is advisable to precede the treatment with a dose of Epsom salts to free the intestines of food and the mucus with which the parasites are usually covered. The object of the treatment is to have the finely pulverized thymol pass, only slightly dissolved, through the entire length of the intestines, and coming in contact with the parasites, destroy, or so disable them, that they may be evacuated. As thymol is very soluble in alcohol, fats or oil, and serious results follow its absorption, all medicines containing aleohol as well as all fatty foods (fat meat, milk, butter, etc.) should be avoided during its use. It must be administered in a capsule thoroughly mixed with three times the amount of sugar to prevent the thymol collecting in a mass, as it would otherwise do as soon as liberated from the capsule and pass through the intestine with little or no effect on the parasites. Should absorption occur with the production of toxic symptoms, a subrutaneous injection of magnesium sulphate (0.3) will often overcome it. Nutritious and easily digested food (lean meat, rice soup, cooked regetables) may be given during the treatment after which the best of foor that the dog will eat should be allowed. Stomachic tonics as iron, quinine citrate $(0.2-0.3)$, tincture gentian compound (1.0-4.(1) are useful to stimulate the appetite. These should be continued for some time. In a few weeks make another examination for parasites or ora, and, if present, repeat the treatment. Thorough disinfection of the kennels is important.

Trichinellidæ.- IThipnorm.--'lhis parasite lives in the ceemn and large intestines, and is less common than the species deseribed. It is also much slower in development, 
requiring three to four months to produce embryos, which, when introduced into the digestive tract, attain complete development in two to three months. The trichuris does not require an intermediate host; embryonic development takes place entirely outside the body and the parasite must pass into the digestive canal while still enveloped in its shell. This species is often found in animals affected with severe anemia, but it does not appear to play other than a secondary part in the development of the disease. When present in eonsiderable numbers in the cecum, however, it may cause a chronic inflammatory condition by its repeated injuries to the mucosa.

The ova differ considerably from those of other intestinal parasites, being ovoid, 70 to 80 microns long and 30 to 35 microns broad, of a distinct yellow color, and have at each end a small rounded projection which appears clear under the microscope.

Birds are occasionally infested but the parasite is rarely present in large numbers, and the anemia it produces is only secondary to the chronic enteritis which results.

Trichinellidæ in the Intestine of the Dog. - Trichuris depressiusculus.-This species is often spoken of as the whipworm, owing to the resemblance of the body to a whip. It is 45 to $75 \mathrm{~mm}$. long. The anterior half of the body is much smaller than the posterior part and the tail is usually curved.

Trichinellide in the Intestine of the Rabbit.-Trichuris unguiculatus. - This species is rare and is only oecasionally found in the large intestine and cecum of the wild rabbit and the hare. The male is 3 to $4 \mathrm{~cm}$., the fomale 3 to $5 \mathrm{~cm}$. long.

Trichinellide in the Intestine of Birds. - (a) Trichosomum retusum.-Male $13 \mathrm{~mm}$., female $19 \mathrm{~mm}$. long. Borly white or yellowish-white, tail blunt.

(b) Trichosomum annulatum.-Male $15 \mathrm{~mm}$., female 80 $\mathrm{mm}$. long. Body white and very attenuated at its anterior extremity and markerl with close annular stripes.

(c) Trichosomum collare.-Male 8 to $10 \mathrm{~mm}$., female 9 to $12 \mathrm{~mm}$. long. 
(d) T'richosomum tennissimum.--This species has been found in pigeons. It is 10 to $18 \mathrm{~mm}$. long.

(e) Trichosomum brevicolle. - Malc 10 to $12 \mathrm{~mm}$., fomale 20 to $24 \mathrm{~mm}$. long. This parasite is found occasionally in the large intestine of geese.

Pathology. - These parasites are usually found in the cecum and large intestines but rarely in large numbers. In several cases of parasitie anemia in which whipworms oceur other parasites are usually also prescint. 'The whipworms are found partly coilcd up and attached to the mucous membranes of the posterior bowel. 'The mucous membrane is slightly thiekened and shows small arcas of inflammation from the injury produced by the parasites.

Symptoms. - Gradual emaciation and anemia are the prineipal symptoms. The appetite may be variable, and constipation and diarrhea alternate.

General symptoms are rarely noted, unless the infestation has been severe when general anemia occurs. Birds are more often seriously affeeted than other animals.

Diagnosis. - This depends entirely upon finding the parasites or ova in the feces. (See Fecal Examination for Parasites.)

Prognosis. - Favorable if the condition is recognized, treatment applied early, and before extreme emaciation occurs.

Treatment. - Thymol, same as for the strongylidx. 


\section{CHAPTER XI.}

\section{DISEASES OF THE REC'TLM AND ANLS.}

Examination.-These parts are quite readily examined in all small animals. The anus by inspection and palpation for enlargements and abscess formation in the anal glands, congenital occlusion in puppies, pseudocoprostasis, inflammation at the anal opening, foreign bodies and parasites.

The rectum is best examined as follows: (a) Direct palpation. The index finger is inserted as far as possible to determine the condition of the mucosa, the presence of foreign bodies, parasites, fecal matter, blood, etc. (b) A rectal speculum is used to dilate the anus and rectum. By using reflected light (mirror) the mucosa can be directly examined for inflammations, tumors, parasites, foreign bodies etc. (c) Palpation through the abdominal walls will admit of an examination of the anterior portion of the rectum. It can easily be distinguished from the other tissues, and quite readily inspected in this location for fecal accumulations, foreign bodies, etc. (d) Laparotomy when performed just anterior to the pubis, in the median line of females, and to either side of the penis in males, will allow direct inspection of the rectum for inflammations, tumors, fecal accumulations, etc.

\section{OCCLUSION OF THE RECTUM AND ANUS.}

(a) A congenital occlusion of the rectum and anus has been observer quite frequently in puppies. Imperforate anus is most common. This condition exists at birth and is the result of improper development during fetal life. The rectum is formerl from the hypoblastic and mesoblastic embryonic layers while the anus is dereloped by the invagination of the epiblastic which, as the development progresses, joins the rectum by absorption of the intervening septum. Anything 
which interferes with the normal development would produce imperforate anus. Sometimes the fetal development will be interfered with sufficiently to produce occlusion of both the rectum and anus.

(b) An artificial occlusion of the rectum and anus (pseudocoprostasis) occurs occasionally in long haired $\operatorname{logs}$ (poodles), birds and cats (angoras) from the hair or feathers becoming agglutinated with fecal matter which becomes dry and forms a firm film or plaster over the anal opening interfering with defecation. Also occasionally foreign bodies (splinters of bone, needles, pins, etc.) are found which have successfully passed other portions of the alimentary tract only to become lodged at or near the anal opening interfering with the passage of the feces.

Symptoms.- The congenital occlusion is seldom observed until there is persistent and ineffectual attempts at defecation. Puppies when examined carefully will be found to have the rectum distended with feces and an absence of an anal opening, the skin being pouched out where the anal opening should be. However, should the rectum and anus both be imperforate the enlargement will be absent. The abdomell becomes distended, and they refuse to nurse. A careful examination will at once reveal the condition. There is more difficulty in recognizing an occlusion of the rectum, but by passing a small probe or sound the condition can be definitely determined.

In artificial occlusion (pseudocoprostasis) the principal symptom is the persistent attempts at defecation without the passage of fecal matter. A careful inspection of the anal region will at once reveal a collection of feces and the matterl hair or feathers. 'This condition if persistent will procluce symptoms similar to constipation or obstipation. (Coe Constipation.)

Diagnosis. - The diagnosis is quite readily established hy a careful inspection of the parts involved.

Prognosis. - Favorable, except in comgenital deformity of the rectmm.

Treatment. - In imperforate anus, an X-shaped incision should be made over the point distended by the feries. Carre 
should be taken to prevent injuring the sphincter muscle. The flaps of skin should be either trimmed off to form a circular opening, or stitched back to prevent adhesions taking place. The passage of a small sound daily, keeping the edges of the wound well lubricated with vaseline, or the direct application of silver nitrate every day or two, will prevent adhesions. In cases where the rectum is also involved, treatment is not to be attempted.

Artificial occlusion from collections of feces can be removed by clipping away the hair or feathers from around the anal opening, softening the hardened mass by the use of warm water, and administering a purgative or allowing laxative foods for a few days.

When foreign bodies are present, a careful examination should be made to determine their size and character. They should be removed carefully to prevent laceration of the tissues.

A purgative is advisable, and if the foreign body has led to atony of the walls of the lower bowels, sinall doses of strychnin sulphate $(0.0005-0.001)$ should be administered daily.

\section{PROCTITIS.}

Definition.- An acute inflammation of the mucous membrane of the rectum.

Etiology. - Proctitis is observed quite commonly in the dog and cat and results usually from the same causes that produce inflammation of other parts of the alimentary tract. Also, it occurs frequently from direct injuries, such as insertion of the thermometer, careless manipulation with the finger, frequent passing of catheters or sounds, or the injections of too strong antiseptic solutions or soapy water, etc. All of these conditions will produce a more or less severe proctitis depending upon the amount of injury done to the mucosa.

Pathology.-The num(ous membrane becomes reddened and congested, especially at the apex of the folds; hemorrhages and erosions are often observed. Hemorrhages take place from the mucous membrane, and occasionally small or 
copious quantities are ejected from the rectum. In severe forms, due to poisons, infections, etc., the epithelium becones desquamated, and quantities of it will become loosened from the submucosa. Further, when due to injuries, the lesions are usually confined to the lower part of the rectum, and depend upon the extent of injury.

Symptoms.-1)ifficult and painful defecation with frequent attempts at defecating and only a small quantity of feces being passed. The feces are streaked or covered with blood. Edema of the mucosa, which can be seen slightly pouching out through the anal opening. Direct examination reveals the painful, inflamed mucous membrane. I)igital examination produces severe pain. Through the rectal speculum the mucous membrane will be found highly congested, swollen and the surface covered with dark, bloody fecal matter.

Diagnosis.- This is not difficult as a direct examination will readily detect the inflammatory condition.

Prognosis. - Usually favorable when localized in the rectum. However, a careful cxamination should be made of the other portions of the digestive tract before a positive prognosis is made. It depends also somewhat upon the cause and the extent of injury to the mucosa and the adjacent structures.

Treatment.- The cause should first be found if possible, and removed, to prevent further injury and irritation to the mucous membrane.

Rectal injections of mild astringent and antiseptic solutions (alum 1-2 per cent., boric acid 2 per cent.) are indicated. These should be introduced with a syringe having a blunt, well rounded nozzle, and the injection made slowly only a small quantity at each time. The fluid should be at or near the body temperature to avoid straining.

Tincture of opium is indicated as an injection when pain is severe, to allay irritation and to prevent straining. I solution of one part tincture opjum to thirty parts water will be found useful for this condition. 'This injection shonld be repeated as often as necessary.

In some cases it is adrisable, where irritants are stlspected of being present, to irrigate the rectum with warm water or a bicarbonate of sola solution (2 per cent.). 


\section{HEMORRHOIDS. PILES.}

Definition.-Hemorrhoids are varicose or dilated veins of the hemorrhoidal plexus. According to their location they are termed external, internal or mixed. External hemorrhoids are located outside the sphincter ani and in the subcutaneous tissue, while internal hemorrhoids are located inside the sphincter muscle and under the mucous membrane. The mixed variety consists of both of the above appearing at the same time.

Etiology. - This condition is commonest in old dogs, and results most frequently from obstructions to the portal circulation, through constipation, congestion of the liver, proctitis, enlargerl prostate glands, or chronic cardiac diseases. All of these conditions, from a defective circulation to the parts involved, lead to a venous stasis with a resultant distention of the reins of the hemorrhoidal plexus.

Pathology. - The extcrnal variety is usually made up chiefly of hypertrophied perirectal connective tissue, appearing as small cutaneous projections, involving the external veins, which become distended or rupture, forming a soft tumor-like mass. 'The internal variety consists of numerous distended ressels, increased connective tissue formation, which often show an ulcerating surface, and are sometimes found projecting through the anal opening. Hemorrhages often take place readily from them, through irritation by the passage of fecal material.

Symptoms.--The act of defecation is usually very painful, the feces being covered with blood, or a quantity of blood passed following the feces. Sometimes defecation is stopped entirely from the severe pain which is induced by it. Pruritus is also a prominent symptom, the animal biting or licking the parts, or sliding the anus along the floor. Direct inspection of the parts reveals the presence of the hemorrhoidal enlargements, which appear as bluish-red knots encircling the rectum. If external, the enlargements will be noticed on e ther side of the anal opening. Rectal examination is very painful (different from rectal polypi, or other ncoplasms, which are occasionally found in this location). 
Diagnosis. - This is usually not difficult, as the parts can be readily inspected.

Prognosis. - When appearing in old animals a complete recovery seldom takes place as the causes are difficult to eliminate. However, in recent cases, or in younger animals, the prognosis is more favorable, as a number of the cases are amenable to treatment.

Treatment.- The early indications in the treatment are to regulate the kowels by the use of saline laxatives (magnesium sulphate, $\operatorname{dog} 8.0-12.0$ ), and laxative foods (soups, etc.) to overcome constipation.

Enemas of cold water are also useful to relieve the congestion and irritation. 'Zinc oxid ointment will also be found valuable for its astringent and lubricating properties.

Should the internal hemorrhoids protrude into the canal or through the anal opening, they should be grasperl with the forceps, drawn out through the anal opening, and ligated (see Prolapse of the Rectum). In some cases where ligation is impracticable, the rectum is dilated with a speculum and the actual cautery applied, care being taken to prevent injuring the adjacent tissues.

In external hemorrhoids, it is advisable to operate by dissecting around them carefully, ligating them firmly at the base with sterile silk or linen, remoring the ligated portion and suturing the skin wound. Should blood clots or abscesses form they should be opened and treated with antiseptic solutions.

\section{PROLAPSE OF THE RECTUM.}

Definition. - An erersion of the rectal mucosa, or the entire walls of the rectum through the anal opening.

Etiology. - An eversion of the rectal mucosa is very commmon in the rlog and cat, and oceurs most often from a local hyperemia just anterior to the anal opening, the resultant swelling forcing the mucosa out through the opening. (Or it ma! come from straining fluring constipation, diarthere or the presence of parasites, or foreign bolies in the rectum. Licral prolapse is observed very often in puppies and kittrus, due in part to straining incident to catarh of the howeds. presence 
of parasites, etc., and also in part to a weakness of the sphincter muscles or the supporting tissues of the rectum.

Prolapsus of a part or the entire rectum is also frequently observed, due mainly to excessive straining during constipation, in diarrhea, enteritis, etc. This is common in old dogs from unduly straining during the course of chronic constipation, diarrhea, hemorrhoids, urethral stricture, enlarged prostate glands, rectal tumors, labor pains, or after the use of irritating or hot rectal injections or infusions. Sometimes these causes not only lead to prolapsus or intussusception of the rectum, but other portions of the bowels may be involved, and protrude out through the anal opening. (See Intussusception.)

Symptoms.-Mild cases of eversion of the rectal mucosa are hardly noticeable, except when the animal strains, when the enlargement becomes visible. In more severe cases, the rectal mucosa will appear as a rounded, congested enlargement, protruding out through the anal opening. The condition of the mucosa will depend greatly upon the length of time it has been everted. On close inspection, the mucosa will usually be found to be everted only from one side of the rectum, or in rare instances may be the entire mucosa. It will be found congested, of dark color, hemorrhagic, the external covering desquamated and often hanging in thin shreds. Often the surface bleeds when the parts are manipulated. Necrosis may result from exposure, or from the blood supply becoming redueed by the swelling. An eversion of the rectum will at once be recognized by the curved, eylinderlike bowel which protrudes. Some feces will be passed, and will collect around the orifice of the canal. Longer exposure (twenty-four to forty-eight hours) will often lead to induration, with foci of ulceration, gangrene, beginning as a rule at the apex of prolapsed portion. 'The progressive changes which develop will depend very largely upon the length of time the bowel remains exposed.

Diagnosis. - This is not diffieult as a close inspection of the parts involved will at once reveal the condition.

Prognosis. - An eversion of the inucosa is always considered favorable, recovery taking place promptly. However, if the 
causes persist, in somc cases the eversion of the mucosa may be followed by a prolapsus of the rectum.

The prognosis in prolapsus of the rectum is usually favorable, unless the bowel becomes nccrotic, or complications higher up in the bowel take place from infection, etc. 'Thereforc, the prognosis depents very largely upon the length of time the bowel has been protruded. When treated early and before pathological changes have developed in the exposed bowel, the prognosis should be consitered favorable.

Treatment. - In the mild cases of everted mucosa, when taken early, applications of cold water or astringent solutions, such as alum (2-5 per cent.), are usually efficient in reducing the size of prolapsed portion so that it can be returned to its proper position. Further application of astringents will assist in keeping it in position. Only small quantities of the solutions should be employed, owing to the danger of inducing undue straining. Should this method fail in keeping the mucosa in its proper position, it can be grasped with small dressing forceps, pulled out gently and cauterized with the thermoeautery, making two or three longitudinal lines, being eareful to prevent going through the mucosa. Should the prolapsed mucosa show necrosis, it is best to remove it in the following manner: The prolapsed portion should be grasped firmly with dressing forceps and drawn out until the normal membrane appears; it is held in this position and a double suture applied through the base and tied either way around the enlargement. The ligature should be applied rather firmly to prevent hemolrhage and retraction of the tissues which would loosen it. The ligated portion is then removed with the scissors. The base is returned to the rectum and treated with antiseptie and astringent solutions.

Prolapsus of the rectum is often quite diffieult to reduce. When taken early, before much swelling has taken place, it "an usually be pushed lack into place, by digital kneadiug, or by the use of a bougie or a well romulerl sound. Placing the animal in a pendant position with the head downard, will assist in the replacement. When the parts are in position, it is advisable to resort to some methoul of retaining them in place for a time, otherwise they are puite liable to be 
thrown out again by the animal straining. A tobaeco-pouch suture, which is used to constriet the anal opening, is often used successfully, making the constrietion just suffieient to hold the parts in position, and to allow soft or liquid feces to be passed. However, as soon as this suture is removed the prolapsus often reeurs.

Slould these methods fail to replace the prolapsed reetum, or hold it in position, laparotomy should be performed (see I aparotomy), and the prolapsed bowel returned to its proper position by gentle traction. 'The bowel when returned to the eavity should be held in place by suturing same to the abdominal wall (ventrofixation). The sutures should be applied carefully so that they only pass through the serous and muscular coats of the bowel. Several of these sutures should be applied to firmly fix the bowel in position. Laxatives or laxative foods are indieated to prevent constipation and pressure on the posterior bowels. Should the prolapsed portion be mueh swollen, necrotie, or severely inflamed, this method is not advisable, as it would aet as a foreign body and induce severe straining, resulting in "a repetition of the former condition. Should the prolapsed portion show evidences of marked pathological ehanges, it is advisable to resort to amputation, which is done in the following manner: 'The animal is given a general anesthetie, plaeed on the table in a ventral position, and the parts involved thoroughly eleansed with an antiseptie solution. 'The prolapsed bowel is then grasped with a bowel elamp, gentle traction used until normal tissues appear, and a previously sterilized sinall. rubber tube applied close to the anus, to aet as a tourniquet. A circular ineision should be made through the external intestinal wall, a short distance posterior to the tourniquet, and parallel to the anal margin. Seize the severed external tube at the anal margin with sinall hemostatie forceps, to prevent its retraction and draw it out gently to bring its serous coat in contact with the serous coat of the internal tube. These two surfaces should be sutured using sterilized cat-gut, silk or linen, making interrupted sutures the entire eireumference of the incised portion. Care should be taken in inserting these stitehes, as they should only pass through 
the serous and muscular coats. When this is completerl, the outer tube is cut off with the scissors distal but close to the row of stitches. The inucous surfaces are then approximated with continuous or interrupted sutures, and the stump thoroughly cleansed with antiseptics and returned within the anus.

Should hemorrhage occur during the operation all ressels should be ligated, as the persistent hemorrhage interferes with the application of the sutures. The after-treatment consists in the daily injection of small quantities of antiseptic solutions.

Another successful method of procedure is to insert a sound of proper size, depending upon the size of the animal, into the lumen of the canal; apply a tourniquet rather firmly around the prolapsed portion close to the anal margin to control the hemorrhage, and to prevent the wall from retracting. 'The prolapsed portion is then amputated rather close to the tourniquet. A continuous suture is applied around the margin of the incised portion, the stitches placed close together and including enough of the tissues so that the serous coats will be brought in apposition. The tourniquct and sound are removed and the stump replaced within the anus. Antiseptic and astringent solutions should be user for a few days.

\section{PARASITES IN THE RECTUM.}

Parasites in the rectum are not very numerous, although one specics is found inhabiting the posterior bowels. Other species arc found quite often in their exit from the intestinal tract, especially tæuix, ascarides, etr.

'The parasites inhabiting the rectum belong to the Venatorla family, Oxyurida. The following are the ones most often found:

(a) Oxyuris vermicularis, found in the dog. 'The male is $2 \mathrm{~mm}$. to $3 \mathrm{~mm}$. long, the female $9 \mathrm{~mm}$. to $10 \mathrm{~mm}$., the color being white and the body expanded anteriorly.

(b) Oxyuris compar, found in the cat in the small and large bowcls, also in the rectum. The female is 8 mm. to $1.7 \mathrm{~mm}$. long. Same characteristics as those fouml in the dow. 
(c) Oxyuris ambigua, found in the rabbit. 'The male is $3 \mathrm{~mm}$. to $5 \mathrm{~mm}$. long, and the female $8 \mathrm{~mm}$. to $11 \mathrm{~mm}$. They are white fusiform worms the body terminating in a sublated or suddenly tapering tail.

Symptoms. - These parasites, by producing irritation to the rectal mucosa, induce severe pruritis, causing the animals to bite or lick the anus and often slide along on the floor to relieve the intense itching. 'The parasites are often seen projecting from the anal opening, or observed being passed with the feces. Aside from the disagreeable symptoms induced they do not effect much change to the mucosa, and no general symptoms are observed.

Treatment.-Oleaginous or saline infusions injected into the rectum with a syringe are usually sufficient to destroy them. These injections may be repeated every few days until the symptoms disappear.

\section{NEOPLASMS IN THE RECTUM.}

Tumors of the rectum and around the anal opening are occasionally observed, more commonly in dogs than in any of the other small animals. The most common tumors found in the rectum are adenocarcinomas or adenomas. Epitheliomas also occur locatè outside the anus and originating from the skin. Occasionally are found fibromas and sarcomas originating from the periproctal connective tissue.

Symptoms. - The arlenomas or adenocarcinomas found. on the rectal mucosa or margin of the anal opening interfere with defecation and cause straining. They usually appear as fungus-like growths with ulcerated, bleeding surfaces. 'Their' rapid development is characteristic. Epitheliomas occur as a wart-or cauliflower-like growth with a rough uneren surface situated in the shin aromnd the margin of the anal opening and often involving the anus, skin and periproctal connective tissue. These vary in size, may appear singly or multiple and often show an ulcerated surface, with a fetid diseharge. Fibromas are usually quite large and grow from the periproctal tissue on one side of the anus. 'They may be two or three inches in dianeter, and are characterized by their 
slow growth, smooth surface and firm consistency. 'I'hey greatly interfere with defecation.

Diagnosis. - While an approximate diagnosis may be made from the symptoms, the growth and the general appearance of enlargement, an accurate diagnosis depends upon the microscopical examination.

Prognosis.-Obviously the prognosis in all malignant tumors is unfavorable, beeause of the danger of recurrence and the difficulty of a complete removal. Benign tumors when not too extensive take on the other hand a favorable prognosis.

Treatment. - Tumors should be extirpated early. Linder general anesthesia, and striet antiseptic precautions, they should be carefully disseeted out, including some of the normal tissue to be sure the entire growth is obtained. A ligature is applied firmly around the base of them to control the hemorrhage, when they may be removed with the seissors or knife. Sometimes it is advisable to eauterize the base of the tumor with a thermocautery. Other methods of procedure in the removal of these growths will depend very largely upon the location, size and character of the tumor.

\section{SUPPURATION OF THE ANAL GLANDS.}

'T'ris condition has been observed quite frequently' in the log. 'The anal glands secrete a grayish or brownish material of fetid odor and acid reaction, discharged througl a eireular opening on either side of the margin of the anus. In old animals the secreting membrane often becomes inflaned or irritated from constipation, foreign bodies, infection, etc., which dhanges the diaracter of the secreted material. From infection, the secretion becomes purulent and the orifice partially or completely closed giving rise to a retention of the secretion, and swelling. In some eases an incrase of the secretion may result eausing discharge which collects on the margin of the anus or soils the hair around the anal region. cometimes when the orifices hecome closed, and no outlet is left for the escape of the discharge, the skin perforittes over the enlargement forming an exit for the escalpe of the 
discharge. 'Thus a fistulous tract may form. Painful defecation is noted, or, if the pain is great, severe constipation results. There are frequent attempts at defecation, and pruritis. Direct examination reveals the presence of a hot, sensitive, fluctuating enlargement or there may be a fistula present. The discharge is noted when it collects on the hair around the anus and tail which it stains, and an offensive odor is emitted.

Diagnosis.-Lsually not difficult as a direct examination will reveal the condition.

Treatment. - When the enlargement is present, the contents of the sac should be expelled by pressure with the thumb and finger. This should be repeated daily for several days in case the sac show's a tendency to refill. If necessary to stop the discharge, Lugol's solution or tincture of iodin should be injected with a hypodermic syringe, enough of either to slightly distend the sacs. This treatment can be repeated in a few day's if necessary.

Should a fistulous tract be found, it should first be thoroughly cleansed with an antiseptic solution (boric acid 2 per cent.) to remove all of the secretions, etc., then injected with Lugol's solution to destroy the secreting membrane.

This treatment should be repeated every few day's until the discharge ceases. 


\section{CHAPTER XII.}

\section{DISEASES OF THE LIVER.}

Examination.-The liver is examined by:

(a) Palpation.-This method is not very satisfactory owing to the well protected position of the liver, and the thickness of the abdominal muscles over it. However, when the liver is much enlarged from acute inflammation, etc., it is possible to palpate it through the walls.

(b) Laparotomy. - When a thorough examination of the liver is desired this method is much more satisfactory than by palpation. The incision is made just posterior to the xiphoid cartilage, same as for gastrotomy, and long enough to admit of a thorough examination. The liver should be examined for inflammations, atrophy, cirrhosis, foreign bodies, abscesses, tumors, injuries, ete.

\section{ICTERUS. JAUNDICE.}

Definition. - Icterus (jaundice) is a term applied to staining of the tissues with bile pigments. It is characterized clinically by a yellowish or greenish-yellow discoloration of the skin, mucous membranes, and the presence of the bile pigments in the urine. 'This is not a disease in itself, but merely' a symptom of a number of conditions, indicative of a disturbance in the secretion or excretion of bile, which is significant of a disease of the liver.

Etiology.-Icterus evidently results from two general conditions: (a) An obstruction at some point in the course of the biliary ducts in consequence of whiels the bile becones absorbed by the lymphaties or the bloodvessels, producing ieterus by stasis or hepatogenous ieterus. This form of ieterus is very common in the dog and has a number of 
etiological factors, which are: Catarrhal inflammations of the mucous membrane of the stomach and duodenum, the inflammation being sufficient in a number of cases to close the orifice of the duct by the swelling of the mucosa; forcign bodies in the duct producing irritation and swelling; parasites; gall-stones; inflammatory conditions of the duct; neoplastic formations in the duct; cicatricial stenosis of the duct; compression of the duct from without by tumors on or in the stomach, intestine, lymph glands or mesentery. Adhesions after operations (gastrotomy), fecal accumulations, aneurysms of the arteries, interference with the outflow of bile by torsion of the ducts which sometimes occurs during pregnancy, tumors in the abdomen, etc.; localized inflammatory processes in the liver (abscesses), and primary and secondary new growths in the liver are further etiological factors. Obstruction of the bile ducts causes the bile to be retained in the liver, the hepatic cells continue to secrete bile and to convert into bile pigments the free hemoglobin brought thither. From the resulting accumulation the pressure in the smaller biliary capillaries increases causing them to rupture into the lymph spaces to be eventually carried through the lymph vessels to the general circulation.

(b) Disturbance in the function of the liver cells, diverting the bile from the biliary capillaries to the lymphatics or bloodvessels producing icterus by hemolysis or hemohepatogenous icterus. This form of icterus occurs most commonly from the following: In many infections, such as the different types of infectious icterus; distcmper (partly obstructive, by producing a catarrhal inflammation of the duodenum); pyemia; in the lifferent forms of intoxications, poisonings by ptomaines, mineral poisons (phosphorus, arsenic, coal-tar products, ctc.); pernicious anemia; hemoglobinemia; disturbance of the circulation, such as passive congestion; some nervous discases, whereby the function of the liver is modified.

Pathology.- Tellow discoloration of all the organs and tissucs by the bile pigment with the exception of some of the nerwous and corneal tissue, characterizes the postmortem. The discoloration varies from a slight tinge of yellow to a deep grcenish ycllow, depending upon the amount of bile 
pigment deposited. Catarrhal inflammation of the stomach and duodenum is often observed, the mucous membrane swollen, the bloodvessels congested, and as a rule the mouth of the hepatic duct will be found closed from the swelling of the nucosa. The duct itself is often found occluded from a swelling of its membranes from infection, parasites, foreign borlies, gall-stones, etc. The duct is usually partially filled with a thick, syrupy, or semisolid mass of bile and mucus. The liver is usually found enlarged, and varies in color from a rliffuse light yellow to yellowish-brown, or the color may be irregularly distributed causing a mottled appearance. The bovel contents are light gray or slate gray in color, owing to the absence of bile, and emit a fetill odor.

Symptoms. - The early manifestations of icterus depend largely upon the underlying causes of which jaundice is merely a symptom. All of the tissues and organs, with the exception of the nervous, are stained witl biliary pigments; in very severe cases where infection is the cause the nervous sy'stem may also be stained. The discoloration is most noticeable in the skin and mucous membrane.

Mucous Membranes and Slin.-Icterus is first manifested by a yellowish discoloration of the eye involving the conjunctiva and selera. In rery mild cases a slight tinge of yellow noticed on the conjunctiva may be the only symptom of the condition. As a rule, as the disease progresses the otler visible mucous membranes will also show the rellowish discoloration. In the dog the entire membranes of the mouth will be colored yellow. The skin, especially if nonpigmented, becomes a characteristic light yellow, or greenishvellow (lepending upon the amount of bile pigment distribut(al. The discoloration is seen early on the skin of the abdomen, thighs, and nltimately over the entire body. In dark skinned animals the eondition ean atso be observed, the skin assuming a dark olive green color. The color of the skin may assist somewhat in arriving at the possible etiological factor, as the discoloration is usually light in the toxic or hemolepatogenous icterus, while it usuatly is darkest when the ducts are completely obstructed-hepatogenous icterms. The intensity of the external simptoms, therefore, is in pro- 
portion to the completeness of the obstruction to the ducts and to the extent of the rupturing of the biliary capillaries. The symptoms develop on the external membranes, as a rule, in a few hours, although in some cases of slow development three to four days may be required depending upon the degree of infection or obstruction of the ducts.

The Lrine.-This is changed in color to a yellowish-green, dark green, yellowish-red, or greenish-brown, depending upon the amounts of bilirubin, biliverdin (oxidation products), or urobilin (reduction product). These bile pigments are often observed in the urine before any discoloration takes place in the tissues, therefore the urine furnishes an early important symptom. The urine when it comes in contact with organic matter will stain it the color of bile, or upon shaking or agitating the urine it will form a foamy liquid, which is quite characteristic. Gmelin's test may also be used for recognition of icteric urine. In addition to the bile pigments the urine often contains a number of hyaline casts (indicating nephritis) and desquamated epithelial cells.

Gastro-intestinal Tract.- The absence of bile from the intestinal tract results in most cases in the passage of pale, grayish, clay colored, or slate gray feces, having a fetid odor, and containing undigested fat, and hydrobilirubin. The pale color is lue partly to the absence of bile, and partly to the imperfectly digested fat which may be increased from the normal 20 to 50 per cent. The fetid odor of the feces is ascribed to the absence of bile which when present limits fermentation of the intestinal contents. Constipation is the rule in the majority of cases. However, diarrhea may be present in some cases due to the imperfect digestion of the fats and to the laxative action of free fatty acids which are formed. The constipation is due no doubt, when present, to defective motility of the muscular walls of the bowels, from lack of the normal stimulating properties of the bile. Increased acidity of the stomach is observed in many cases, the obstruction to the flow of hile apparently causing an increase in the activity of the gastric secretion.

lerems System.- Serious nervous symptoms are often ohserved. 'The bile salts present in the circulation are at 
once carried to the nervous sistem, and, when aceumulated in sufficient quantities, will produce by irritation of the nerve cells marked symptoms of active delirium, convulsions, etc. This period of excitement and delirium, as a rule, lasts only' a very short time, terminating in depression, muscular debility, somnolence, and eventually deep coma and (leath from a general paralysis

The general symptoms (loss of appetite, increased thirst) are noticeable from the beginning. Usually a subnormal temperature $\left(96-100^{\circ} \mathrm{F}\right.$.) exists, (lepending upon the severity of the condition. Examination of the liver by palpation is unsatisfactory, as pain or enlargement of the liver is rarely noted.

Diagnosis - The recognition of this condition offer's no difficulties, as the simptoms are very characteristic. However, the cause in many cases is hard to determine. In dogs: digestive disturbances, often resulting from errors in lict, etc., produce the largest percentage of ases. Here the anamnesis is raluable. From infectious jaundice a rlfferentiation can in most cases be made from the fact that this condition occurs mostly in young dogs (puppies) and in an enzoötic form, affecting all the puppies of a litter.

Prognosis. - The prognosis in icterus is generally speaking unfavorable. A large percentage of the rases in the dog terminate fatally. In mild cases, due to catarrhal inflammation of the mucous membranc of the duodenum, recovery usually takes place. 'The cause, if possible, should he ascertained, and the conditions thomongly ansirlened before making a prognosis.

Treatment.-Dietetic.-In the milder "ases where the appetite is retained small quantities of leam meat slombl be given, aroidling all fats for a few dass. In severe cases to conscrve the animal's strength eggs, given in suall quantitics of milk, are nseful. I) uring convaleseence foods should be allowed only in small quantities, alvolding fats and irritating foods as much as possible.

Mediral. - When icterus is the lesult of cat a rrhal inflammattion of the duodenum, it is advisable to irrigate the stomadels with bicarbonate of sola solution (2) per cent.), repeating this 
opcration until all nucus is dissolved and the liquid flows out clear. 'This treatment should be used at least onee or twice daily. Following the stomach lavage Carlsbad salts $(0.5-2.0)$ are useful to stimulate seeretions and to dissolve the mucus aceumulated on the mueous membrane of the stomach and duodenum. In some cases this will be suffieient to allow the escape of the bile into the duodenum.

Injeetions of warm water or warm bicarbonate of soda solution ( 2 per cent.) into the reetum as high up as possible are often valuable to stimulate peristalsis, eneourage defecation, and to produce alkalinity of the intestinal contents.

Should constipation be present calomel ( $\operatorname{dog}, 0.3-0.4$; cat, $0.1-0.15$ ) should be given and repeated in twelve to fifteen hours if catharsis has not been established. Magnesium sulphate ( $\log$, 8.0-16.0; eat, 1.0-4.0) or castor oil (dog, 15.0-16.0; cat, 5.0-20.0) may be used for the same purpose.

In severe cases it is advisable to try to overeome the obstruction to the duet and empty the gall-bladder, either by inechanically eompressing the liver by manipulation or by the use of emctics. 'The latter method has proved to be the most satisfactory. The action of the emetic by contraeting the abdominal muscles will of ten exert enough pressure upon the liver and gall-bladder to force the bile out into the bowel. An obstruction, such as mucus, parasites, foreign bodies, etc., can thus also often be removed.

When the bile pigments are deposited in the body in large quantities, or the blood contains a large amount of undeposited bile salts, its elimination should be encouraged by the use of diuretics. P'otassium aectate or nitrate (dog, 0.200.50 ; cat, $(0.0,5-0.10)$ is to be given twice daily.

General stimulants, such as camplor or ether, are indicated when general depression and coma are observel.

In severe cases normal salt solution given as an intravenous injection is useful to assist in the elimination of bile and to produce general stimulation.

Faradization of the liver has been tried but its usefulness is questionable. 


\section{CONGESTION OF THE LIVER.}

Two forms of this disease are distinguished: (a) Active, and $(b)$ passive.

Active Congestion.-Definition.-An engorgement of the liver with blood resulting from an increased circulation through the portal vein or hepatic artery.

Etiology.-There are a number of etiological factors in active congestion of the liver: (a) During the process of digestion there is a physiological increase in the amount of blood carried to the liver by the portal vein. 'This, however, usually subsides after digestion is completed. In small animals, owing to the great variation in the amount and quality of food taken, and the fact that the food often contains irritating material, toxins, ptomaines, etc., all of which increase the functional activity of the liver, a more or less permanent active congestion results. (b) Various poisons provoke a severe form of congestion of the liver. These include many autogenic poisons carried to the liver from the intestinal tract by the portal vein, or certain ptomaines preformed in the food before it is ingesterl. Mineral poisons (arsenic, mercury, phosphorus) can produce it. Some of the toxic products of infections, which develop in the intestinal tract, will sometimes be carried to the liver in sufficient quantities to excite an acute congestion. (c) Congestion of the liver may also result from the specific products of certain infections (virus of distemper, staphylococei and streptococci) that may reach the liver via the general circulation. (d) Dogs, when kept indoors, fed on highly nutritious food, and not receiving the proper amount of exercise, will often develop active congestion of the liver.

Many of the causes mentioned are also the chief etiological factors in producing inflammation of the liver (hepatitis) of which congestion is the first stage.

Pathology.-The liver is enlarged, feels firm or hard, contains an increased amount of blood, and is of a dark red or reddish-brown color. On cut surface, the blood drips or flows off freely. 
Symptoms. - I) ue to the fact that excretion is interfered with, which learls to a gencral intoxication of the body, general symptoms of stupidity, depression, loss of appetite, etc., appear. Nausca and vomiting arc often observed. Constipation is the rule. The feces are clay colored and have a fetid odor. Jaundice, which is nearly always present, is first noticed in the conjunctiva; the urine is stained yellow with bile pigment. The liver is usually enlarged and by palpation it may be distinguished through the abdominal walls; also is oftcn quite painful to the animal when compressed by digital pressure.

The urine is highly colored (often green), of high specific gravity, and shows precipitates of urates and uric acid. The body temperature is either normal or subnormal.

Diagnosis. - An accurate diagnosis presents some difficulties. 'The causes (history), the disturbance of the digestive tract, the jaundicc, and the cnlarged and painful liver, should be considered in arriving at a definite conclusion. In atypical cases an accurate diagnosis is impossible, although enough symptoms may develop to suspect the acute congestion.

Prognosis. - The prognosis is usually farorable, except in those cases produced by poisons and infections. In these the prognosis will depend largely upon the character of the infection or the nature and amount of the poison.

Course.-In most cases the course is short, rarely lasting over two to four days.

Treatment.-I)ictic.--All irritating food should be withheld. 'The diet should consist almost entirely of milk given only in small quantitics with long intcrvals between meals. Lime water adderl to the milk, to make it alkalinc, will have a beneficial action on the mncosa of the stomach and intestines.

Medical.-Free purgation is indicated early. Magnesium sulphate (dog, 10.0-1i).(); cat, 1.()-5.0) has proved to be the most satisfactory. 'Thesc doses should be repeated until free catharsis has been established. ('alomel nay also be used, but is not as good as magnesium sulphate, as its action is more cholagogic which would be contraindicated where congestion of the liver exists. When nausea and vomiting are present, indicating an irritated condition of the stomach and 
intestinal mucosa, sodimu bicarbonate (dog, ().j-1.0; cat, $0.20 .8)$ given three to four times daily is beneficial. Ammonium chlorid ( $\log , 0 . \overline{5}-1.2$; cat, $0.2-() . \overline{5})$ gireen three times daily will assist in the excretion of the urea, uric acid, etc., and relieve the intestinal catarrh. I) uring convalescence bitter stomachics are indicated to stimulate sceretions and to assist in digestion. Intestinal antiseptices, such as salol, are also often indicated.

Passive Congestion. - Definition. - A congestion of the liver due to some impediment in the efferent circulation of the blood in the liver.

Etiology.-This condition may result from the following: (a) I)efective heart action, whether it be acute or ehronic, such as valvular insufficiencies. (b) I)uring the course of some diseases of the lungs which increase the work of the right side of the heart, eventually weakening it. Examples are emphysema, chronic bronehitis (common in old dogs), compression by pleural exudates, adhesive pleuritis, tumors of the mediastinum, ete. (c) Local obstructions to the circulation of blood through the hepatic reins and posterior rena cava. The most common are: Pleural or peritoneal cftusions (when in large quantities, displacing the heart or eompressing the veins), tumors of the liver (carcinomas and sarcomas in older animals), adhesions around the liver from abdominal operations, injuries, ete.

Pathology.-The congested liver in the early stages is somewhat increased in size, depending upon the amount of blood contained. 'The organ is firm, lense and of a bluish or dark purple color.

Cut section shows a more or less uniformly congesterl. dark-blue or purplish color, and presents a nottled appearance with light areas. In the advanced stages there is an excess of blood, and the liver presents the characteristic's of the "nutmeg" liver"; the intrabobular and sublobular" venules being distended and filled with blool, appearing as dark blue, purplish or reddish spots, while the liver rells are pale yellowish, or whitish, showing fatty infiltration and biliary pignentation, which gives the marked rontrast in color " "nutmeg" liver). In the most advaneed stanges, the 
liver becomes smaller, and may be smaller than normal. It is firm and dense, but still retains the characteristic nutmeg appearance. Connective tissue develops around the central veins; the adjacent hepatic tissue is atrophic and pigmented, and invading it arc fine fibrous connective tissue trabeculæ. The capsule is often thickened and opaque.

Symptoms. - The symptoms vary greatly, depending largely upon the causes. When due to primary cardiac or pulmonary disease, the symptoms are complicated with these conditions, those of the primary condition usually predominating. The local symptoms are principally loss of appetite, disturbance in digestion, nausea, vomiting, and more or less obstinate constipation. Jaundice is a common symptom. It may be moderate. The cyanosis which is present in the conjunctiva with the jaundice produces a peculiar bluishgreen color of the mucous membranes. In cases where infection develops rapidly, the jaundice increases, often producing nervous symptoms such as excitement, convulsions, etc.

Ascites is a common symptom in the later stages resulting from the extensive interference with the circulation. A large amount of fluid is often found in the abdominal cavity, especially in dogs. In the carly stages the liver is enlarged, while in the more advanced cases it may be atrophied. Examination of the liver, therefore, by palpation may not reveal any characteristic condition. An accompanying gastro-intestinal catarrh develops which interferes with digestion; the chronic course lcads to general weakness and emaciation.

Diagnosis.-The diagnosis depends upon finding the primary disease of the heart or lungs, the condition of the liver, and the locil symptoms of icterus, gastro-intestinal catarrh, etc. An accurate diagnosis is somewhat difficult. A carcful examination of the patient and the prolonged chronic course of the disease will assist in arriving at an accurate conclusion.

Prognosis.-As a rule unfarorable, especially in the dog. It depends upon the primary condition, the stage of the disease and the condition of the animal. 
Treatment. - Dietetic.-Treatment affords only temporary relief. Eneourage the animal to eat, by giving small amounts of lean meat, milk, etc., which may be given in conjunction with alkalies (sodium bicarbonate) to conserve the strength.

Medical.-For defective circulation, due to diminished heart action (valvular insufficiency), digitalis fluidextract (dog, $0.1-0.3$; cat, $0.025-0.05$ once or twice daily) is the most efficient drug, especially when used in the early stages.

Magnesium sulphate should be given in constipation. Stomachic tonics (gentian, nux romiea) are also indicated.

\section{HEPATITIS.}

Definition. - An acute or chronic inflammation of the liver. This comprises a series of most diverse eonditions varying from aetive congestion, acute or chronic inflammation, to localized foci of neerosis or to the different forms of icterus gravis.

Etiology. - Hepatitis may result from the many causes enumerated under aetive congestion of the liver (see Congestion). The only real difference between the eonditions is in degree. A clinical differentiation, therefore, may be difficult. Acute hepatitis is most commonly due to the absorption of toxins during the course of infections or speeific infectious discases. It may also result from the absorption of toxic materials, sueh as poisons, from the intestinal traet (common in $(\operatorname{logs})$.

('hronic interstitial hepatitis may develop from the acute or from valvular disease of the heart.

Pathology. - In acute hepatitis the pathologieal changes are varied. The whole phenomena of inflammation ('ongestion, (houcly swelling, focal necrosis, etr.) may be present. In mild cases the liver appears as in active congestion with (louly swelling. In serere alses the pathologioal chamger are intensified. 'Tle liver is enlargerl, swollen, softenerl, and rather pale in color; the cut surface is pale, opaque, and shows mottling depending upon the degree of inflammation.

Symptoms. - The symptoms are very similar in many respeets to those of aetive eongestion of the liver of which acute 
hepatitis is a more advanced stage. As a rule the symptoms are more intensive than in aeute congestion. Nausea and vomiting are more pronouneed and usually more persistent; the vomiter material often contains a quantity of bile eoloring the material a greenish color. Blood may be vomited up along with the other material, from the irritation of the mucous membrane. ('onstipation is nearly always present. At certain periods in the eourse of the disease diarrhea mav: appear. The fecal discharges are very fetid, yellowish or clay colored. Icterus, noticeable on the conjunctiva, mucous membrane of the mouth, and sometimes in the nonpigmented skin, will be a prominent symptom. 'The liver is found enlarged and sensitive on palpation.

The urine is usually coneentrated, highly colored, of increased specific gravity, and contains a high percentage of urates and uric aeid as well as bile pigment.

The temperature in the early stages of acute hepatitis is usually quite materially elevated $\left(10 ; 3-104^{\circ} \mathrm{F}\right.$.). 'The fever temperature assists in differentiating the condition from simple congestion. However, in the later stages, the temperature may be found normal, or even subnormal, due to retained toxins, bile salts, etc. 'The general srmptoms are dullness, intense thirst, and gastro-intestinal disturbance.

Diagnosis.-'The diagnosis depends on the causes, the elevation of temperature, the enlargement and sensitiveness of the liver, and the general srmptoms. To distinguish between acute congestion and inflammation of the liver is diffieult and depends upon the severity of the symptoms.

Prognosis.- The prognosis is unfavorable. In some of the milder cases reeovery takes place, but when advaneed it nearly always proves fatal.

Treatment. - The treatment for aetive congestion of the liver is applicable. (See Active ('ongestion of the Iiver.)

In chronic hepatitis treatment is valueless.

Suppurative Hepatitis. - Lbscess of the Liver.-Definition. - An inflammation of the liver resulting in abseess formation, which oceurs under at variety of cireumstanees and in several forms. Liver abscesses a re commonly divided into two kinds : (a) The large single abscess; (b) the small multiple abscess. 
Fundamentally, however, the two kinds may not differ from each other, since the large single abscess may lecome multiple by infecting adjacent liver tissue giving rise to the development of secondary abscesses. By coalescence a number of small abscesses may by peripheral extension form a large single abscess.

Etiology. - Liver abseess is always the result of infection by microörganisms (staphỵlococci, streptococei) which reach the liver in one of several ways: (a) Traumatism. In small animals injuries, direct or indirect, frequently give rise to abscess of the liver. Direct injuries, such as punctured wounds, gunshot wounds, etc., permit the entrance of pyogenic organisms. Indirectly contusions or rupture of the liver, which reduce the resistance to infecting organisms which may be cireulating in the hepatie or portal blood. Such usually produces a single, small or medium-sized abscess.

(b) Diseases of contiguous organs often occasion the formation of abscess in the liver. Examples are: Crastric or duodenal ulcer with perforation; abdominal organs which have been operated and infeeted, and suppurative conditions of adjacent organs.

(r) Infection vin the portal or hepatic aireulation. Multiple alscesses result from gangrene or abscess of the lungs. purulent pleuritis, purulent and fetil bronchitis, etc. 'These processes give rise to many infectious emboli which lodge in the liver forming abscesise.s.

(d) Infection ria the biliary ducts. Here abseress formation is due to the infection carried in through the bile ducts. or by direct exteusion of ulcerative and suppurative processes in the biliary tract to the adjacent liver tissue.

(e) In some cases infertion may take place through the Jimphatios.

Pathology. - The appearance of the liver will vary greatly, depending upon the morle of infection, the virulence of the infecting material, and the location and munber of alswessen.

Abseresses resulting from tranmatism, ulecrative and suppurative processes in ad jacent organs are unally single. small or of moderate size, and mostly superfictial. 'These abscesses are in isolated areas, a foreus of intlammation sur- 
rounded by a zone of intense hyperemia. In or near the center liquefaction necrosis of the exudation begins which spreads by peripheral extension until a smaller or larger area of softened or fluid purulent material, surrounded by a more or less well defined zone of limitation, results. 'The softened material consists of leukocytes, red cells, necrotic and degenerated liver tissuc, infection, etc. The abscesses are very commonly situated near or on the surface of the liver. The surface of the liver is, therefore, involved (perihepatitis), and extensive adhesions may bind to it the contiguous organs. In some cases (especially after operations) the abscess is on rather than in the liver (suprahepatic, infrahepatic).

When small, multiple, metastatic abscesses are present, the liver is usually enlarged, swollen, opaque, and shows evidence of parenchymatous degeneration, or cloudy swelling. On section the organ reveals numerous grayish or yellowish, softened spots surrounded by hyperemic zones; the spots vary considerably in size depending upon the stage of development of the condition. In some cases the numerous small abscesses, by peripheral extension, become confluent and form a large abscess which may involve a whole lobe or in some cases the entire liver. The purulent contents are usually thick, creamy, yellowish, or thimner and seropurulent, or stained with blood or bile; the surrounding liver tissuc is in most cases stained a greenish-yellow tint. The pus often has an offensive odor espeeially when due to gastric or duodenal ulcers.

Symptoms. - The early manifestations are not very characteristic. Therefore, unless the condition is well established involving a large portion of the liver, or interfering severely with its function, it is apt to be overlooked.

In traumatic abscess, and abscesses due to sprear of infection from adjacent organs, the patient usually shows pain in the region of the liver, especially when the animal is handled or moved about. Jaundice, due to compression of the biliary ducts, and enlargement of the liver can usually be determined by palpation; fluctuation may also be evident. In multiple abscess the liagnosis is difficult, as there are 
no eharaeteristic symptoms. 'The temperature is variable, usually slightly elevated; ehills may be present. Examination of the blood often reveals leukoeytosis, which is not always present, especially in ehronie, well eneapsulated abseesses. The urine is coneentrated, highly eolored, speeifie gravity increased, with an abundant deposit of urates and urie aeid. When there is mueh destruetion of the liver tissue the amount of urea is diminished, and albumin is often present.

Spontaneous rupture of the abseesses often takes place, especially in those eases resulting from traumatism, causing serious symptoms to suddenly develop. As in most eases the rupture oecurs into the peritoneal eavity, a generalized peritonitis follows whieh soon leads to death.

Diagnosis. - An aeeurate diagnosis is usually quite diffieult. 'The most suggestive signs are progressive enlargement and tenderness of the liver, jaundice, ehills and fever, leukoeytosis, and the eonsideration of the etiologieal faetors.

When an abseess is suspeeted an aceurate diagnosis can be made with safety (especially in the $(\log$ ) by making an explorative laparotomy .

Prognosis. - Abseesses of the liver even in the single suprahepatie form should alwars be eonsidered unfarorable. The small multiple abseesses are almost always fatal, deatl oceurring in one to two weeks.

Treatment.-'The early indications in the treatment are surgieal. Inder general anesthesia and strict antiseptic precautions, an explorative laparotomy should be performed. The ineision in the abdominal wall should be made large enough to admit of a eareful and free examination of the liver. Should the abscess be suprahepatic, or single in the liver substance, that portion of the liver is carefully brought out through the incision. It is very important that the liver be carefully manipulated to prevent mupturing the abscess. Should the abscess be of large size, it is often advisable to aspirate most of the contents hefore it is nanipulated to prevent rupture of the sac and also to farcilitate its withdrawal through the alolominal incision. When the affected portion of the liver is withdrawn, it should be sur- 
rounded on either side with sterile gauze to prevent any of the pus entering the cavity while operating. A free incision is made directly over the abscess its full length, the contents of the cavity thoroughly washed out with boric acid solution ( 2 per cent.) and the abscess wall thoroughly curetted to remove all of the necrotic tissue and detritus. If necessary some of the tissue is removed with the scissors or knife to straighten the erlges of the wound, and also to be sure to remove all of the necrotic tissue. The wound in the liver is sutured with a deep continuous suture. Should the hemorrhage interfere it should be controlled by ligating the larger vessels.

Should the abscess be suprahepatic or infrahepatic, the affected parts should be carefully withdrawn, the adhesions broken down and the entire wall of the abscess completely extirpated. All exposed portions should be thoroughly irrigated before returning to the abdominal cavity.

When multiple abscesses are found surgical treatment is rarely of valuc. 'The external wound is sutured as in gastrotomy. (See (iastrotomy.)

\section{ATROPHY OF THE LIVER.}

Definition. - A term generally applied to a reduction in the size of the liver. In most conditions where there is a reduction in the size of the liver it is due to degenerative changes.

Etiology.-Atrophy of the liver occurs most commonly from the following:

(a) Pressure upon the liver, by tumors, enlargement of adjacent organs, passive congestion, amyloid disease, ete. These causes may produce a true atrophic condition of the liver with subsequent replacement fibrosis, or atrophy and degeneration (fatty degeneration and neerosis) as in passive congestion and anyloirl lisease.

(b) From a stenosis or occlusion of the portal vein, general lepatitis, alvanced passive congestion, etc., a general atrophy of the liver may develop).

(c) A reduction in the size of the liver, which is not a true atrophic condition, follows many degenerative processes, 
such as poisoning by phosphorus, arsenic, mercury, chloroform, etc.

(d) Reduction in the size of the liver frequently occurs, to which the term atrophy is applicable, in inanition, cachexia, etc. In this case the liver participates in the general atrophic process.

Pathology. - The liver is smaller than normal, dark in color, dense, of increased specific gravity, and dry. These changes are the result of the increase in the connective tissue and the decrease in the parenchyma. On cut section the surface is dry, and the liver substance very dense and firm.

Symptoms. - The symptoms are not very characteristic. There will be noticed inanition, cachexia, etc. The patient shows digestive disturbances, and the feces are light colored. The liver is very small; owing to its location it is difficult to palpate.

Diagnosis. - An accurate diagnosis is in most cases impossible during the life of the animal. The etiological factors may assist somewhat in making a diagnosis.

Prognosis.-The prognosis should always be considered unfavorable, owing to the structural change which has taken place in the liver, and the difficulty of renoving the causative factors.

Treatment.-Satisfactory treatment is hardly possible, although treatment for the removal of the cause would be indicated.

\section{FATTY LIVER.}

Definition. - A term applied to the excessive amount of fat in the liver. It includes fatty infiltration, in which there is an excessive deposit of fat without the liver cells becoming much altered, and also fatty degeneration, in which the liver cells are converted into fat cells.

Etiology.-The causes are: (a) Feeding of animal for a long perior on fats and carbolydrates, without the proper amount of excrcise. 'This tends to produce obesity (which is common in house dogs), of which fatty liver is a common symptom. 'The fatty deposit is probably due to the incomplete oxidation of the excessive amounts of fool. (b) Anemia, 
and cachexia, occurring in the later stages of chronic diseases, carcinomatosis and sarcomatosis, general inanition, etc. These conditions result in an insufficient supply of blood, therefore incomplete oxidation. (c) Passive congestion of the liver due to valvular insufficiency of the heart. $(d)$ Poisonings, such as phosphorus, arsenic, mercury, etc., which are very common in small animals. Ptomaines from meats will produce a similar effect. (e) Infections developing in the intestinal tract, as distemper, infectious diarrheas, etc., the toxins of which are carried to the liver through the portal vein. General infection, such as by pyogenic organisms, will produce the same effect on the liver.

It is quite evident that the majority of cases of fatty liver develop through a deficient oxidation.

Pathology.-The liver is enlarged, of ten twice the normal size, the specific gravity lessened, and the resistance reduced. It is pale yellow in color, or yellowish areas or streaks are seen on the surface. The surface is smooth, and the edges somewhat rounded. On cross-section it is usually pale, anemic, and fat globules which adhere to the knife are often noted. In cases of passive congestion the characteristic appearance of nutmeg liver is observed (dark center and light periphery of the lobules).

Symptoms. - The symptoms of fatty liver are very obscure, and not sufficient in most cases to make an accurate diagnosis intra vitum.

\section{AMYLOID LIVER.}

Definition.-A degenerative process of the liver characterized by the conversion of the proteins of the tissues into a structureless, homogeneous substance called lardacein.

Etiology. - This condition results most commonly in animals from the absorption of the toxins of pyogenic organisms. It requires for its production a long time and a persistent and continuous infection. It is not a very common condition in animals, and usually accompanies a general amyloid degeneration of other organs.

Pathology. - The liver is enlarged, sometimes two or three times its normal size, and the edges are rounded or blunt. 
The color is grayish-brown, and on cut section shows white points. Microseopic examination reveals the anyloid degeneration.

Symptoms. - Difficult to recognize during the life of the animal, and is only of importance to the pathologist. Other degenerative conditions, whieh have been ohserved on postmortem in small animals are only of importance to the student in pathology and will not be deseribed here, as a diagnosis can only be made on postmortem.

\section{CIRRHOSIS OF THE LIVER.}

\section{C'hronic Interstitial IIepatitis.}

Definition.-A chronie inflammation of the liver with an inerease in the interstitial eonnective tissue.

Etiology.--The exact eauses are not definitely known. Infection no doubt play's an important role, bacterial toxins from the intestinal tract being carried direct to the liver. It may be produeed seeondarily from other diseases of the liver, sueh as acute and chronic hepatitis, congestion, etc. Parasitic invasion has been known to prochuce it in certain distriets.

Pathology. - In the early stages the organ is usually enlarged; in later stages often atrophic. The liver maintains its shape, the surface is smooth, or in some cases granular. The color varies somewhat from a light green to a dark olive green, and the liver nodules are separated by connective tissue. 'The consistency of the liver is greatly increased, and when incised it euts hard and grates uncler the knife, dhe to the exeressive amount of connective tissue. The bile passages are misually found normal.

Symptoms. - The simptoms are those of a dironice condition, with which is associated a disturbance in the intestinal tract. Jaundiee, which is usually mild, prochecing only a slight tinge of yellow in the mucous nembranes; bile in the urine, giving it a greenish color; namsen and vomiting are often observer in the dog. An entargenent of the liver can be readily detered by palpation. In the advanced statges of 
the disease there is often ascites, enlargement of the spleen, and eventually general cachexia.

Diagnosis.-In animals a diagnosis is very difficult. The condition is usually first noted on postmortem examination. An explorative laparotomy in the dog is recommendable.

Prognosis.-The prognosis is unfavorable owing to the changes which have developed in the structure of the liver.

Treatment.-Dietetic.-Small quantities of easily digested food (raw, lean meat, milk, etc.) should be given to sustain the condition of the animal.

Medical.-Salines (magnesium sulphate, dog 8.0-12.0) are indicated to produce a laxative action.

Should ascites be present diuretics are indicated to assist in the removal of the fluid from the abdominal cavity.

Surgical.-Thoracentesis abdominis is indicated.

(Sce Ascites.)

\section{NEOPLASMS OF THE LIVER.}

Tumors of the liver are not very common, except secondary to malignant growths in other organs. These consist mainly of carcinomas and sarcomas, which have become generalized (carcinomatosis and sarcomatosis). Benign tumors are occasionally met with, viz.: Adenomas and angiomas.

Symptoms.-Tumors of the liver are difficult to recognize during life, and only in those cases where they become very large, producing distention of the abdomen, will they bc recognizable. Palpation, when done carefully, will often reveal the tumor which will be freely movable in the cavity. If the tumor is malignant (sarcoma and carcinoma) it will learl to symptoms of anemia and cachexia. An accurate diagnosis can only be made after laparotomy.

Treatment. - When malignant tumors are found, no treatment is of value. Benign tumors may be removed by carcfully ligating all vessels, and preferably removing an cntire lobc of the liver where affectcd. Hemorrhage is usually severe when the tissue of the liver is incised. Thercfore opcrations on the liver are always considered serious. 


\section{CHOLELITHIASIS. GALL-STONES.}

Cholelithiasis is quite rare in animals. Only a few cases have been reported where free concretions were found in the biliary ducts and these occurred most commonly in the gall-bladder and ductus choledochus. Gall-stones are the result of a catarrhal inflammation of the membrane of the duct or gall-bladder causing a desquamation of the epithelium and a collection of bile salts, gradually forming coneretions. Infection or parasites gaining entrance via the duct and producing irritation are common causes.

Symptoms. - Gall-stones may exist for some time without producing any marked symptoms, depending upon the location of the concretion. When in the gall-bladder but little disturbance will be produced, but when the concretion passes out into the rluctus choledochus, scvere colicky symptoms are observed, and by completely blocking the exit of the bile, obstruction or hepatogenous icterus is prorluced. (See Icterus or Jaundice.) A lliagnosis is difficul: and, when suspected, an cxplorative laparotomy should be performed.

Treatment.-Surgical means should be employed early. Lnder general anesthesia laparotomy is performed, as for gastrotomy (see Gastrotomy) making the incision longer, if necessary. 'The portion of the liver containing the gallbladder and duct is brought out through the incision. A careful examination should be made to determine the location of the concretion. When located, an incision is made down upon the concretion just large enough for its removal. Care should be taken to prevent the bile from running into the cavity. After all the concretions are removed the wound is stitched carefully with a fine suture using a small, straight needle. A continious suture is first used to bring the edges of the wound in apposition, and then a Lembert suture to completely close the opening and to bring the serous membranes together for rapirl healing. 'The parts should be thoroughly clcansed before returning to the ablominal cavity. The laparotomy wound is sutured in the regular manner. (See Laparotomy.) 


\section{RUPTURE OF THE LIVER.}

\section{Ruptura Ilepatis. Apoplexia IIepatis.}

Etiology. - Owing to the structurc of the liver being very friable and easily torn and its blool supply great, it is not uncommon that rupture of this organ takes place. The common causes are injuries, such as being run over by vehicles, penetrating rib fractures; severe exertion (running, jumping, falling, etc.), or during the course of infectious discases when there is a severc congestion or inflammation of the liver. Predisposing factors are: I)egenerative processes in the liver, such as fatty liver, amyloid liver, etc., or diseases of the heart and ressels.

Pathology.--The postmortem lesions depend largely upon the extent of the rupture. Small hemorrhages are often fomml showing through the capsulc of the liver, greater may occur in the form of a large hematoma. In most cases, however, the capsule also becomes torn and the blood is allowed to flow out into the abdominal cavity. The liver when examined will reveal the rupture. The other tissues and organs will be pale and anemic.

Symptoms. - Small hemorrhages in the liver will not proluce any marked symptoms. If sevcre there will be all the simptoms of intermal hemorrhage, such as paleness of the mucous membranes, general weakness, anxious expression, weak rapid pulse, and dyspnea; the extremities and skin become cold, trembling of muscles, and finally coma. Death often takes place very suddenly or within ten or twelve hours, depending upon the extent of the rupture. Sinall and contimuous hemorrhages will be observerl where the rupture is very small or where a hematoma is forming showing symptoms of weakness, paleness of the membranes and of ten some icteric simptoms. Such cases often recover in a few days or death may ocrur from exhaustion in four to ten days.

Diagnosis. - An ac'(nIrate diagnosis is very difficult. The anamnesis may assist in arriving at a definite conclusion.

Prognosis. . Should be considered unfavorable as most (ases, especially" where the hemorrhage is severe, terminate 
fatally. Where slight hemorrhage takes place, the prognosis is more favorable, although if there is some disease of the liver present, fatal hemorrhage may recur at any time.

Treatment.-The treatment must be given as early as possible and is the same as for any internal hemorrhage.

Subcutaneous injections of ergotin (dog, 0.1-0.3) or adrenalin chlorid (0.5 to 1.0 of a 1-1000 solution) are useful. These doses can be repeated in a half to one hour if necessary.

To stimulate the heart action strychnin sulphate $(0.001)$ or caffein citrate (0.1-0.3) should be given every few hours. Otherwise the treatment is symptomatic. 


\section{CiH A P T E R X II. \\ DISEASES OF THE PERITONEUM.}

General Remarks.-The peritoneum is a serous sac, and, considering all of its reflections and fossæ, it covers a surface very nearly as great as that of the skin. In the female it differs from other serous cavities in that it has an indirect external opening through the uterine tube; in the male there is no opening.

The peritoneum through the lymphatics and bloodvessels has great power of absorption as has been demonstrated on numerous occasions. The dog or rabbit will absorb fluid equal to 10 per cent. of the body weight in a half hour. Fluids and soluble substances are readily taken up and carried away by the blood, while insoluble substances, including microörganisms, are taken up by the lymphatics with the aid of the phagocytes. In health, the secretion of fluid into the peritoneal cavity and the absorption therefrom is just sufficient to keep the surfaces moist and free from infection. In disease this normal equilibrium becomes disturbed, and either produces a dry condition of the membrane, from absorption bcing greater than secretion (acute inflammation), or secretion being in excess of absorption (ascites). The presence of the fluid in the peritoneal sac has a further action than preventing friction to the surface, and that is by exerting a bactericidal action.

The lymphatic absorption is carried forward by the lymphatic trunks to the mediastinal glands. Experiments have shown that microörganisms can be removed from the peritoneal sac via the lympl stream, and carried into the mediastinal glands in six minutes after their injection into the abdominal cavity. The peritoncum covering the diaphragm and the omentum is most active in this process of 
absorption, removing inert bodies and also microörganisms from the peritoneal cavity. The omentum is, further, an important factor in preventing peritonitis, in that it removes the microörganisms from the abdominal cavity before they can produce their pathogenic action. The omentum also has the great faculty of localizing inflammation and infection by attaching itself to any inflamed organ or possible source of infection. It becomes fixed around the margin of the inflammatory or infected area, where it adheres, preventing generalization. The omentum is an important protective mechanism after surgical work on the organs in the abdominal cavity. Another point worthy of mention is the fact tliat abscesses which develop following operations, puncture of abdominal walls, etc., usually open to the outsirle rather than in the abdominal cavity, due no doubt to the great defensive powers of the peritoneum as compared to the other tissues.

The visceral peritoneum is not very sensitive to pain while the parietal is very sensitive. Therefore, the reason for extreme sensitiveness to pressure over the abdomen in acute peritonitis. The visceral peritoneum even in inflammatory conditions is not very sensitive. The natural resistance of the peritoneum varies greatly in the different animals. The dog seemingly has the greatest resistance, and, therefore, abdominal surgery can be practised on this animal with a much greater degree of safety than in other animals. The cat is slightly less resistant than the dog. Ilowever, it must always be remembered that there is a great variation in the natural resistance of individuals of the same species depenting to a great extent upon their physical condition, etc.

As a summary, the defensive powers of the peritoneun are of great importance and are: (a) Its great absorbing power, removing organisms before they can multiply sufficiently, produce toxins, and excite inflammations. (b) 'The phagocytic action exerted by leukocytes, polymorphonuclear leukocytes and the endothelial cells. (c) 'The faculty of the' omentum in walling off local infections and inflammations preventing diffuse peritonitis. (d) The antitoxic and bactericilal properties of the peritoneal fluid. Thi- fluiel is increased when necessary. 


\section{PERITONITIS.}

Definition.-An inflammation of the peritoneum. From the standpoints of intensity and duration peritonitis may be classified into: (a) Acute, and (b) chronic.

Acute Peritonitis.-Definition.-An acute inflammation of the peritoneum. From a clinical standpoint acute peritonitis may be divided into: (a) Circumscribed or localized, and $(b)$ general or diffuse. Other divisions are hardly recognizable during the life of the animal. Even the most severe cases of peritoneal infection often show the least evidence of inflammatory rcaction. The inflammatory reaction which takes place in peritonitis is often a salutary process, by preventing excessive absorption from the peritoneum, leading to the destruction of microörganisms that have gained entrance, and by the formation of fibrin and adhesions preventing the spread of infection to the entire serous membrane.

Etiology.-From the standpoint of cause acute peritonitis may be divided into: (a) Primary, and (b) secondary.

(a) Primary, acute peritonitis is applied to those cases where therc is no local focus in the abdomen to account for the infection of the peritoneum; it is assumed that the infection has reached the abdomen by the blood or lymph stream, or from some injury to the abdominal wall, such as blows, kicks, gunshot wounds through the abdominal walls, bowels, etc., or operations of various kinds on the organs in the abdominal carity.

(b) Secondary, acute peritonitis is due to infection at some localized area in the abdomen, or in the immediate neighborhood, which thevelops rapirly producing a diffuse or circumscribed inflammation. This form of peritonitis is very common in animals and results from a number of conditions. It may be due to infection from the abdominal viscera, following perforation of their walls, or to inflammation and the infeetion passing through the walls. The following conditions arc frequent causes: Perforation of gastric or duodenal ulcer; acute toxic gastro-enteritis; mycotic gastroenteritis; traumatic rupture of the stomach or bowels; strangulation of the bowels, volvulus; intussusception; 
foreign bodies in the bowels; impaction of the bowels; rupture of abscesses in the liver, spleen, omentum, lymph glands, etc.; rupture of the bladder when inflamed; aeute phlegmonous, or gangrenous eholeeystitis; metritis and parametritis after parturition; injuries to the uterus dluring parturition; abscess of the prostate gland, etc. These conditions allow the free entrance of microörganisms, or reduce the resistance of the peritoneum so that organisms develop readily. Numerous organisms are found producing peritonitis, such as staphylocoeci, diplocoeci, streptococci, Bacillus bipolaris, streptothrix eanis, Baeillus pyogenes, Bacillus coli communis. In chickens, peritonitis is often found as a prominent condition in cholera, and also from different species of fungi, such as Aspergillus fumigatus, Aspergillus glaucus, etc. A fungoid peritonitis has also been observed in the dog produced by the Sporotrichum beurmani. Parasites (Pleurocereoides bailletis) when severe invasions take place will often produce peritonitis. Exposure to cold, and unsanitary conditions are often predisposing causes, especially in dogs and birds.

Pathogenesis. - As soon as the defensive powers of the peritonem (the phagocytic action of the enclothelial and other cells, the bactericidal power of the peritoneal fluid depending on the presence of antibodies, and absorption which destroy and remove organisms) are neutralized by any of the etiological factors mentioned, the membrane becoming dried and exposed during operations, etc., or the presence of solicl bodies, particles of fool, blood clots, etc.. the resistance of the peritonem is lowered and its absorbing and bactericidal power interfered with. This allows the organisms to develop, produce their toxins and an arcute peritonitis.

Pathology. - According to the character of the exuclation, preritonitis can be rlassified as fibrinous, serofibrinous, fibrinopurulent, suppurative and hemorrhagic. 'The character of the inflammation depends greatly on the nature of the infection, In cases of low virulence, such as are oceasionally observed, the serous surfaces present little more than a slight loss of luster, with some slight deposits of delicate fibrin. In 
the ordinary form, in the early stages, there is marked congestion of the serous membrane, later the serous surface beeomes dull, lusterless, and the eontiguous surfaces beeome slightly adherent through the depositing of yellowish-white flakes of fibrin (peritonitis fibrinosa). In some instances there is but little effusion of fluid, but in most cases there is a copious effusion of exudation, somewhat turbid containing floeculent masses of a yellowish color (peritonitis serofibrinosa). The quantity of fluid varies considerably from a few cubic centimeters to several liters. In other eases the exudation is more turbid and eontains purulent material (peritonitis purulenta). The peritonitis following rupture of the bowels is very virulent, the exudation is purulent and contains bowel contents. In the abdominal cavity a quantity of dirty, brown, turbid fluid of offensive odor is found. In all cases of peritonitis with effusion more or less blood is always present (peritonitis hemorrhagica). When the peritonitis results from rupture of the bladder, urine will be present in the abdominal cavity, and the odor will be deteeted in the abdominal eontents.

Symptoms.-Acute, diffuse peritonitis, when due to injuries, rupture of the bowels, abscesses, etc., usually develops rapidly under symptoms of marked general disturbance. In the most severe infections the symptoms are principally those of a septicemia or toxemia. In the beginning there is abdominal pain, restlessness, stiff unnatural gait, and "tucked up" abdomen. Palpation over the abdomen reveals intense contraction of the abdominal muscles, and eonsiderable sensitiveness. When the peritonitis is diffuse the sensitiveness is noted over the entire abdomen; when eircumscribed only local areas of tenderness are evineed. The pain is often severe enough in small patients (dog and eat) to cause them to groan and cry. Pain is a prominent symptom, and is usually continuous, except in cases where a general toxemia exists. 'The respirations are of the costal type. In most cases they are diminished, but if fluid is present there is severe lyspnea.

Vomiting is an early and characteristic symptom of peritonitis in the dog and cat. It is usually one of the first 
symptoms, and is very persistent. 'The vomitus rousists mainly of mucus, foorl particles, bile, and, if severe, of fecal matter.

There is complete loss of appetite noted early in the course of the disease. 'The urine is decreased in quantity, highly colored, and contains a large amount of indican. There is often severe straining as if to urinate (tenesmus vesica).

In the early stages there is diarrhea followed later by constipation with considerable tympany. In the very early stages the temperature in the $\operatorname{dog}$ and cat is elevated (104$106^{\circ} \mathrm{F}$.). The temperature, however, remains high for only a few hours when it drops rapidly and becomes subnormal $\left(96-100^{\circ} \mathrm{F}.\right)$. In small animals the temperature in peritonitis is usually normal or subnormal.

'The pulse is rapid, small, hard and often wiry. In the later stages the pulse becomes very weak, irregular, and finally imperceptible. 'The extremities grow" cold, the mucous membranes cyanotic, and there is every evidence of a deficient heart action.

Effusion of fluid (ascites) is usually present except in some of the more acute cases which are rapidly fatal. 'The percussion sound is flat, the area of dulness shifting as the patient's position is changed. A friction sound may be present in the early stages, but due to the effusion which forms early, soon disappears.

In some cases of peritonitis, due to serere septic infection (rupture of abscesses), the course is very rapid and the general symptoms of toxemia are the only ones noticed. Peritonitis following rupture of the stomach or bowel, from severe injury, such as being run over, kicks, etc., runs a very rapid course. (ieneral weakness, coldness of the extrenities, and coma may be the only symptoms noterl.

The svmptoms of eircumscribed peritonitis are similar to those of diffuse, except that they are so mild at times as to be overlookerl.

Course.-The acute, diffuse peritonitis nsmally terminates in deatl. The most intensive forms nsually produre death in thirty-six to seventy-two hours; however, most commonly death results in five to eight darys. Fonne of the miller cases 
terminate in recovery, or chronic peritonitis, which runs a long chronic course.

Diagnosis.- In typical cases the sudden onset, the sensitiveness over the abdominal region, the fever, the wiry pulse, the development of effusion, the collapse and the vomiting, present a rather characteristic picture. In some cases of rapid devclopment the diagnosis is very difficult and is hard to rlfferentiate from septicemia or toxemia.

In the latter stages of the disease, where deep coma is present, an accurate diagnosis is impossible. Often circumscriberl, acute peritonitis is overlooked. A careful examination, therefore, is necessary to determine the exact condition.

Prognosis. - The prognosis in acute, diftuse peritonitis is unfavorable, especially if it follow rupture of the stomach, bowel or abrlominal abscesses. Such cases invariably terminate in death. In circumscribed fibrinous or serofibrinous peritonitis the majority of cases make a complete recovery. Circumscribed peritonitis, however, due to local infection should be looked upon as dangerous, as the abscess inay rupture into the abdominal cavity evcntually terminating in death from diffuse peritonitis.

Treatment.-Medical.-In the early stages, diarrhea is present and the peristalsis active. In order to prevent friction between the peritoneal surfaces, which tends to sprear the inflammation, small doses of opium ( $\log , 0.1-0.3$; cat, $0.05-0.1)$, or morphin sulphate ( $\operatorname{dog}, 0.016-0.12)$ subcutaneously are indicated.

Cold applications, if applied early to the walls of the abdomen, are indicated (cold water compress or ice pack) to relieve the intense congestion of the serous membrane. I ater counterirritants may be used in the form of oil of mustard mixed with olive oil (1-10). Apply by mbloing well into the skin of the abdomen. Hot water applications may also be used. Should constipation be marked laxatives should bo given, such as castor oil ( $(\log , 15.0-30.0$; cat, 5.0-8.0) or magnesium sulphate ( $\log , 10.0-14.0$; cat, 2.0-5.0). Warm water infusions into the rectum will be uscful to remore feces and also to produre a soothing action on the membranes. Gencral stimulants (strychnin sulphate, $\log 0.001$; cat, $\frac{1}{2}$ 
the quantity subcutaneously') are enployed to combat symptoms of general weakness and coma. Alcoholic stimulants or camphor may also be used for the same purpose.

Surgical.-When severe infection is present, and in the early stages, it is advisable to irrigate the abdominal cavity in the following manner: Laparotomy should be performed (see Laparotomy) and a sufficient amount of sterile, normal salt solution introduced at the borly temperature to thoroughly irrigate all parts of the cavity. This should be followed by a boric acid (2 per cent.) or a salicylic acid solution (2 per cent.). The value of this method will depend largely upon the thoroughness of the application. Before irrigating a thorough examination of the organs and tissues in the cavity should be made for ruptures, etc., and if found, proper treatment should be applied. When an excessive amount of effusion is present it should be removed. (See Treatment for Ascites.)

Chronic Peritonitis.-Definition.-A chronic inflammation of the peritoneum which may be either diffuse or circumscribed. As a rule chronic peritonitis is rarely found equally well marked over the entire abdominal cavity. From a clinical standpoint it is difficult to separate the two conditions, therefore, they will be described as one.

Etiology. - Chronic peritonitis may be due to a number of different causes, the most important of which are: Intraabdominal lesions, such as diseases of the liver (hepatitis, abscesses), the kidneys, spleen, etc., which mar reduce the resistance of the peritoncum; or from gastric or duoclenal ulceration providing a focus for peritoneal infection. In these cases a general chronic peritonitis results instead of a tocal inflammation, owing to the reduced resistance of the membrane, or the low virulency of the infection. Chronic venous engorgentent from defective heart action would produce much the same effect. ('hronic peritonitis maly result from the acute fibrinous form when complete resolution does not take place, or the infection is mild.

In some cases it may result from disease of the plental hy spreading through the diapliragm. ('hronic peritonitio ma! also result from disturbances of the intestinal tract. 
Aseites, when due to disease of the heart, may produce ehronic peritonitis by lowering general resistance, or through organisms which gain entrance during paracentesis abdominis. Parasites (Linguatula denticulatum, Pleroeereoides bailleti, Echinoeoceus granulosus) in the dog and cat may cause ehronie peritonitis by the constant irritation to the peritoneum they prokuee.

Pathology. - The postmortem lesions vary somewhat depending upon the eauses, and the extent of the process. The peritoneum is covered by a thiek membrane, which is dull white or glistening, pearl-like in eolor; pigmentation is sometimes present. In very severe cases of long standing this membrane beeomes very thiek, especially over the visceral peritoneum, and ean be peeled off from the organs. Adhesions between the folds of the viseeral peritoneum are often found, which may bind together several organs into one mass. The formation of this membrane is due mainly to an organization of the exudation and not to hyperplasia of the peritoneum itself.

Symptoms.--The symptoms are somewhat similar to ascites. The onset is gradual; usually no symptoms are noted until distention of the abdomen develops. There is no pain or tenderness on manipulation. On pereussion a dull sound is emitted, and on auseultation, espeeially if the abdomen be tapped on the opposite side with a finger, the presence of fluid ean be letected by the splashing sound produced.

There are usually general symptoms of dullness, and lack of energy, shown by the animal lying down a great deal and refusing to move about. The patient has, as a rule, a good appetite unless constipated. Respirations are increased owing to the pressure against the diaphragm.

The charaeter of the fluid varies somewhat. It has a specifie gravity of about 1015, is of a yellowish or turbid color, from the presence of large numbers of eells, and when allowed to stand it usually beeomes thiek and forms large amounts of floeeular.

The temperature in the dog and eat remains normal or slightly subnormal. 'The pulse is_often sapid and irregular. 
Diagnosis. - The character of the fluid in the abdomen is one of the chief diagnostic symptoms, and some of it should be obtained and examined for its specific gravity, cells, etc. A careful examination of the animal must be made to distinguish this from ascites due to other causes. The anamnesis may also assist in making the diagnosis.

Prognosis.-The prognosis is unfavorable, the course chronic. Complete recovery is hardly to be expected, although a number of cases have been reported where apparent recoveries have taken place. Death is usually the result of heart failure.

Treatment. - The cause should be ascertained if possible, and the treatment given accordingly. If the cause cannot be determined, symptomatic treatment is given. Diuretics, such as caffein citrate $(0.1-0.3)$ or diuretin $(0.15-0.4)$ are indicated to assist in removal of the fluid.

Paracentesis abdominis (see Ascites) should be performed whenever necessary to remove the fluid from the cavity. The $\mathrm{dog}$, as a rule, responds more readily to treatment than the cat.

\section{ASCITES.}

IIydrops Abdominis. IIydrops Ascites. Hydrops Peritonci.

Definition. - A collection of serous fluid in the abdominal cavity. This condition is quite common in dogs, and also occurs in cats and birds. Mild cases of ascites are often overlooked during life, and are only found on postmortem or during operations on the abdominal cavity.

Etiology.-1. Local Causes. - (a) Chronic inflammation of the peritoncum, either simple, carcinomatous, sarcomatous, or by cysts of parasites (Plerocercoides bailleti, Linguatula denticulatum, etc.). (b) Obstruction to the portal vein, cither in its terminal branches in the liver, such as by cirrhosis, chronic, passive congestion, etc., or by compression of the vein in the gastrohepatic oncutum, such as by proliferative peritonitis, abscesses, tumors (sarcomas, carcinomas, etc.), or by ancurysm. (c) Thrombosis of the portal rein. (d) 'Tumors in the abdominal cavity in general. 
Cysts of the ovaries (common in cats).

(f) Occurs in the secondary stage of acute circumscribed or diffuse peritonitis.

2. General Causes.-Ascites often occurs as a symptom of general dropsy, the result of mechanical effects, as in heart diseases, chronic indurative or interstitial pneumonia. In some heart diseases the effusion is confined to the abdominal cavity, in which case it is no doubt due to secondary changes in the liver. Ascites also occurs in chronic diseases of the liver. In young dogs (puppies) ascites is frequently observed, and often disappears as the animal develops, apparently without any particular cause being found.

Pathology. - The presence of fluid in the abdominal cavity, of varying quantity from a few cubic centimeters to 15-20 liters. This fluid has a specific gravity of 1012-1015, a light or yellowish color, clear, and contains, as a rule, but a slight amount of fibrin, or flocculent precipitate. The chemical reaction of the fluid is alkaline or neutral; the albumin content is about 2 to 5 per cent. Sometimes the fluid will be of a reddish color, due to slight hemorrhages, or to some of the red cells passing out with the serum. This is especially noticeable in obstruction to the portal vein. A greenish cast is noticed when the liver is secondarily affected. The precipitate when examined will be found to contain a small number of leukocytes, fatty endothelial cells, flakes of fibrin, and sometimes red cells, and in rare cases numbers of small cysts of parasites. In dogs and cats the fluid often contains numerous fat cells and has a milky appearance.

The pcritoneum is usually pale, glistening, thickened, espccially in cases of long standing or those due to chronic peritonitis. The organs in the cavity are usually anemic, dull on the surface and sometimes atrophic.

Symptoms. - An enlargement of the abdomen is usually the first indication of the disorder. Until the accumulation of fluid becomes great enough to cause a distention of the abdomen, the symptoms will not be positive enough to make a diagnosis. As the amount of fluid in the abdomen varies greatly, the symptoms will vary considerably in individual cases. The fluid distends the abdominal wall, causing the muscles and skin to become tense, and the abdomen to assume 
a characteristic pear shape. When the animal assumes a standing posture, the fluid collects in the lower portion of the abdomen and the amount can be rather accurately determined by percussion. Above the line of dulness will be noticed a tympanitic sound. Clanging the position of the animal causes a shifting of the horizontal line which marks the upper limits of the area of dulness.

On palpation the resistance is fairly uniform. By placing one hand on the side of the abdomen, and tapping gently on the other side with the other hand, a plain undulation will be felt. As the amount of fluid increases, pressure is produced on the diaphragm, interfering with the function of the organs in the thoracic cavity. Severe dyspnea with cyanotic nembranes is noted in some cases. The pulse is weak and rapid. As the cases progress, emaciation appears. The appetite is impaired, the digestive tract disturberl and occasional attacks of vomiting occur. The temperature is normal; in the later stages it may be subnormal. 'The urine is reduced in amount, and often highly colored in the dog; otherwise it is normal. 'The animal may die from general exhaustion, or from asphyxia.

Diagnosis.- The diagnosis is not difficult, provided a careful examination is made of the patient, its history obtained, and all the symptoms carefully noted. However, there are quite a number of conditions with which ascites might be confused. It might be mistaken for acute or chronic peritonitis with effusion. By puncturing the ablominal wall with an explorative trocar and obtaining some of the fluid, a differential diagnosis can be made.

Excessive accumulation of fat (obesity) might be confusing. Invever, obesity oceurs mostly in old amimals, and the slrape of the abdomen is more apple (broad back, well romeled barrel) than pear shaped.

Advanced pregnaney an be differentiated by calreful palpation which will reveal the presence of the fetuses.

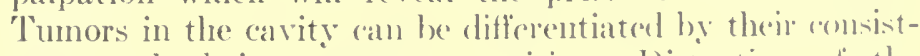
eney and their permanent position. Distention of the bladkler produces an enlargenent which remains in the sime position at all times, and when palpated ean be felt at al 
large body within the cavity. Passing the catheter will reduce the enlargement by removing the urine.

Rupture of the bladder, when distended, will produce a sudden distention of the abdomen, and general symptoms of stupor and coma often with convulsions. Puncturing the abdomen will reveal the presence of urine.

Prognosis. - The prognosis depends largely upon the cause. As a rule, it is unfavorable.

Treatment.-Medical.-The cause should first be determined, if possible, and measures taken to eliminate it. Should the heart action be deficient, digitalis would be indicated ( $\mathrm{dog}, 0.05-0.10$; eat, $\frac{1}{2}$ quantity) to stimulate the heart and overcome venous stasis. This drug is further of great value owing to its diuretic action, which assists in the elimination of fluid from the body. Caffein citrate (dog, $0.1-0.5$; cat, $0.05-0.1$ ) has a similar action. The resorption of the exudate may also be aided by the use of diuretics: Potassium acetate (dog, $0.5-0.8$; cat, $0.05-0.1$ ); bulbous scillæ in powder form (dog, 0.05-0.2; cat, 0.01-0.05); diuretin (dog, $0.5-1.0$; cat, $0.01-0.05$ ) every two or three hours, or agurin (dog, 1.0-2.0; cat, 0.02-0.08) daily. Laxatives should be administered occasionally to keep the bowels open which also assists in removing the fluid. Magnesium sulphate ( $\operatorname{dog}, 8.0-14.0$; cat, 1.0-4.0) is best for this purpose. Diaphoretics may also be administered, although not so important in small animals as in large ones, as the skin glands are less developed. Pilocarpin hydrochlorate (dog, 0.005-0.01) can be used, but there is some danger of edema of the lungs or paralysis of the heart from its use.

Surgical.-If the fluid produces severe dyspnea and interferes with the action and function of abdominal and thoracic organs, it should be removed surgically by performing paracentesis abdominis. 'The operation is as follows: The animal is placed in a standing position, or on its side on the operating table and the hair shaved from a small area at the most pendent portion of the abdomen, usually near the umbilicus. The surface should be thoroughly disinfected by washing with biclilorid soap, followed by alcohol, and tincture of iodin painted over the surface where the puncture 
is to be made. A small exploring trocar is used, which should be sterilized, and inserted through the abdominal walls. When the stylet is withdrawn, the fluid will usually flow out in a stream. Sometimes the end of the cannula becomes blocked by omentum or bowel. This can be overcome by moving the cannula slightly or by re-inserting the stylet. The fluid should be collected in a vessel to note its character. The amount of fluid to remove depends largely upon the condition of the animal. Should, however, symptoms of dyspnea, cyanosis, and rapid, weak pulse appear, the cannula should be at once removed. The operation may be repeated next day. The punctured wound resulting from the operation should be protected by covering with collodion and a small pledget of cotton. Death occurs occasionally from collapse following the operation. Therefore the patient should be carefully watched and the removal of the fluid stopped, and general stimulants given, when signs of collapse appear. 



\section{PART TV. \\ DISEASES OF THE REPRODUCTIVE ORGAIS.}

\section{CHAPTER I.}

\section{IDSEASES OF THE PENIS AND PREPLCE.}

Examination. - The prepuce should be examined by observing the discharge at its opening, and the condition of the mucous membrane. Frequently there will be found a more or less extensive mucopurulent discharge which indicates a catarrhal inflammation of the prepuce. The preputial opening should be dilated and the mucosa exannined for foreign bodies, tumors, ulcerations, secretions, etc.

The penis may be examined in the following manner: The animal should be placed in the dorsal position, and either held by assistants, or securely fastened to the table with hopples. With the left hand, the prepuce is gently pushed downward and backward, exposing the free end of the penis. The penis is then grasped with the fingers of the other hand and pulled as far out of the prepuce as possible. A piece of tape should be placed around the penis just posterior to the glans, and with gentle traction the penis can be exposed for a considerable distance. The penis should be examined for inflammations, tumors, venereal granulomatat, rubber bands, wounds, fractures of the os penis, etc.

\section{WOUNDS OF THE PENIS AND PREPUCE.}

There are quite a variety of injuries which are fouml involving these structures. Principally; injuries result from 
the bites of other animals, and different degrees of the conditions are found, from sinall cuts of the prepucc to extensive laceration of the tissues. Rubber bands are occasionally found on the penis just back of the glans. These, if left on very long, result in extensive edema and necrosis of a portion of the penis. Injuries also occur during coition as the penis is retained in the vagina until the semen is ejaculated, which requires several minutes in the dog, and during which time the movement of the female will often cause torsion or twisting of the penis with subsequent edema. Frequently dogs are roughly handled at this time and the penis lacerated or cut. The prepuce is often found split from being cut by sharp objects, or perforations occur which allow the penis to prolapse through the opening.

Symptoms. - The symptoms depend to a great extent upon the kind of injury and the amount of damage done to the tissues. Attention to wounds of the prepuce will be drawn by the hemorrhage and swelling. Examination reveals the extent of the wound. In edemas of the penis, the animal shows difficulty in urination, the penis often projecting from the prepuce, swollen, reddened, and if strangulated will be of a dark color (venous stasis). Thorough examination should be made to determine the degree of injury, and to discover any foreign bodies which might be present.

Prognosis. - Favorable in most cases, as wounds in this location respond to treatment readily. Not so favorable in cases of strangulation of the penis with subsequent necrosis.

Treatment.-The parts inust be thoroughly cleansed, washed with an antiseptic solution, and the character of the injury determined. Wounds in the prepuce, such as cuts or perforations, are sutured after thorough disinfection and all ragged edges removed with the scissors. Injuries to the penis should be looked after carefully, as they sometimes lead to sufficient swelling to interfere with the discharge of urine. In such cases, the catheter is introduced and the urine withdrawn. Should this procedure fail, make an opening in the urethra at the ischial arch to temporarily take care of the discharge of urine. Applications of antiseptic solutions for a few days will reduce the inflammation and. 
infection. When the penis is strangulated and neerotie, it is advisable to amputate the affected portion. 'This is done in the following manncr: The animal is anesthetized, placed on the table in a dorsal position and sceurely hoppled. The parts should be thoroughly cleansed with soap and water and disinfected. Pull the penis out of the yrepuce as far as possible, and apply a rubber tourniquet at a point above the seat of operation to control the hemorrhage. Incise the penis with a scalpel, and if it is necessary to amputate through the os penis a saw should be used for this portion. The urcthra is protceted by a catheter previously inscrted, and left about one-half ineh longcr than the stump of the penis. It is split with the seissors on its dorsal surface back to where the penis was ineised. The flaps of the urethra are then securely stitched to the stump of the penis to prevent a stricture forming at this point. All vessels should be ligater, and the parts again washed with an antiseptic solution. 'The tourniquet is removed and the penis allowed to retraet back into the prepuce. Cleanse daily with antisepties.

\section{CONGENITAL MALFORMATIONS.}

Malformations of these structures are not common. Arrester development of the penis or prepuec is seen occasionally. ITermaphrodites are not common among dogs. Congenital closure of the prepuce is observed sometimes in puppics, which nust be opened with a scalpel and the edges of the skin stitched back to prevent adhesions.

\section{PREPUTIAL CATARRH.}

Balanitis.

Definition. - A catarrhal inflammation of the murous membrane of the prepuce.

Etiology. - Preputial catarth may be produced by sereral different factors:

(a) Principally local infection.

(b) Develops in a number of eases seconding to other diseases; venous stasis; phimosis; paraphimusis; injuries, or forcign borlies. 
(c) Sequel to specific infectious diseases, as distemper.

(d) Follows in some cases of mange and eczema.

In all of the above causes we find that the condition is brought about by infection either as a primary cause, or due to reduced resistance of the tissues by other diseases which allows secondary infection to take place. In some, accumulations of dirt will favor injury to the mucosa with subsequent infection.

Symptoms. - The mucosa of the prepuce will be injected, swollen, and there is present at the preputial opening a discharge of pus of a yellowish or greenish color. Some of the material accumulates around the preputial opening agglutinating the hair, drying and forming crusts. Examination of the mucosa shows a marked catarrhal inflammation.

Prognosis. - The condition is not serious as in most cases it remains local. When treatment is applied, recovery takes place after a few weeks.

Treatment. - The parts are thoroughly cleansed and all long hairs removed with the scissors. The prepuce should be washed thoroughly once or twice daily with antiseptic and astringent solutions (alum, 2 per cent.; silver nitrate, $\frac{1}{4}$ per cent.; zinc sulphate, 1 per cent.).

\section{PHIMOSIS.}

Definition.-A contraction of the prepuce in front of the glans penis, preventing the projection of the penis, interfering with coition, and in some cases the escape of urine.

Etiology.-Phimosis is a congenital defect in puppies. It may cause in some cases almost complete occlusion. It results frequently from trauma of various kinds, the subsequent cicatricial tissue formed leading to constriction. Acute infectious inflammation (balanitis) with severe swelling will prorluce a temporary closure, and in some cases, owing to the change in the structure of the tissues, a permanent contraction.

Symptoms. - In puppies it will be noticer that urine cannot be discharged; there may be frequent attempts at inicturition, with only a small quantity passed or there may be complete 
suppression. In mature animals the same symptom of difficulty in passing urine is often present. 'There is a narrowing of the preputial opening; sometimes it is exceerlingly small. Animals suffering from phimosis are unable to (o)

Treatment.-In phimosis surgical relief should be given promptly. There are two methods employed:

(a) The patient is placed in a rlorsal position on the table and the parts disinfected. A longitudinal incision of sufficient length is made on the inferior portion of the prepuce to allow the penis to protrurle. The two portions are temporarily stitched back to the skin to prevent adhesions and a recurrence of the condition. 'The wound should be treated daily with antiseptics.

(b) The animal is placed in the same position as above and local or general anesthesia given. A circular incision is made completely around the prepuce, removing about onequarter to one-half inch of its end. 'The hemorrhage is usually slight. The mucosa should be stitched to the skin for the entire distance around the prepuce. This will insure a preputial opening of sufficient size. ('are must be taken in this operation not to remore too much of the prepuce, as it would allow prolapsus of the penis. After-treatment consists in cleaning and disinfecting the parts dailı.

\section{PARAPHIMOSIS.}

Definition. - I contraction of the prepuce around the penis, preventing the penis from assuming its normal position.

Etiology. - Paraphimosis can result from coition. 'The prepuce becones inverted when the penis retracts, but the resulting swelling prevents complete retraction. Is a result of roition, with subsequent swelling, the penis becomes ton large to pass through the preputial opening. It is further observed in some cases of paralysis of the penis. organic changes having taken place in the tissues from exposure. Veoplasms, such as granulomata, are sometimes proluctive of the same conclition. 
Symptoms. - Tlle cxposed penis becomes swollen, edematous and discolored and when strangulated, gangrenous. The animal will have difficulty in voiding urine due to the 'swelling and compression of the urethra. There is stiffness in walking. Examination of the parts will at once reveal the exact nature of the condition.

Prognosis.-In early cases, resulting from coition, the prognosis is favorable. In cases of long standing, after strangulation, or in paralysis, the prognosis is unfavorable.

Treatment. - In early cases of paraphimosis, following coition and before much swelling has taken place, it is possible in most cases to correct the inversion of the prepuce and return the penis to its normal position. This is best done by cleansing and disinfecting the parts thoroughly, lubricating the penis and preputial ring with some bland oil (olive), and by manipulating the parts by pressing on the glans penis. If at the same time the prepuce is pressed forward, the parts will often be reduced without much difficulty. Should this procedure fail, it is advisable to reduce the congestion of the glans penis by bathing in cold water, applying alum solution (2-5 per cent.), or by applying tape tightly around the glans. After the congestion has been reduced, manipulation as above will often reduce the condition. These manipulations will not suffice in cases which lave become greatly swollen or edematous. It is then neccssary to resort to an operation, which consists in simply splitting the prepuce longitudinally on its lower surface, making a slit of a sufficient length to relieve the strangulation and allow the penis to retract. Where gangrenous conditions have developed, amputation of the penis must be resorted to. After-treatment in these cases consists in applying antiseptic solutions freely:

\section{TUMORS OF THE PENIS AND PREPUCE.}

Various forms of tumors, both benign and malignant, are found in this location. The following varieties are most common: 
Papillomata.-These in most cases involve the prepuce. They are small, benign growths, appearing usually as pedunculated warts. Sometimes they are found in large numbers with irregular or cauliflower-like surfaces and are usually found at the juncture of the skin and mucous membrane.

Symptoms.- They produce, as a rule, no inarked symptoms of any kind, except in some cases a catarrhal inflammation of the prepuce (balanitis).

Treatment.-Tumors should be removed with scissors as close to the base as possible. Cauterize the base with silver nitrate. A recurrence is unusual.

Sarcomata, Epitheliomata and Carcinomata.-'These are occasionally found involving the prepuce and penis. 'These tumors are malignant and show much the same characteristics from a clinical standpoint.

Symptoms. - In most cases these tumors involve the prepuce. 'They are characterized by their sudden development, irregular outlinc, degenerative changes, and tendency to spread into the adjacent tissues. They usually present a raw, ulcerating surface, show a tendency to bleed, etc.

Diagnosis.- The diagnosis depends upon the characteristic growth, and the microscopic findings.

Treatment.-In case a diagnosis of malignant tumor is positively made, the results of treatment are only temporary. Removal of the tumor is advised, and the incised portion should contain not only all of the malignant growth but also include a small portion of adjacent normal tissue. Malignant tumors are liable to recur in a short time.

Venereal Granulomata.-This is a specific, infectious tumor formation affecting the penis and prepuce of the $\log$. It is transmitted in most cases by the act of copulation. Affected stud dogs transmit it diring coition. It may he transmitted in rare instances by intermediary agents. It is far more common in Europe than in the I nited States where it has bcen introduced by imported sturl doges. The growth seems to confine itself to the prepure penis, and immediately adjacent tissues with involvement of the afferent lymphaties.

Symptoms. - Venereal gramulomata are lisually fomml only in the best bred dogs. 'The carly symutoms are redness, 
swelling and a reddish colored discharge from the prepuce. Micturition is frequent, and the penis is protruded from the prepuce. On palpation the prepuce will be found swollen and irregular in outline. On exposing the penis, it will be tumified, purplish or lark red in color, and on its surface presents a number of vegetative growths. 'These growths may be found also on the prepuce. 'They are characterized by their soft friable condition, tendency to bleed at the least touch; they may be sessile or pedunculated. The growths first appear in the form of small vesicles, which soon develop into pimples at first of a firm consistency, but as they grow become softer and more friable. Their growth is slow. In six months to one year they can involve most of the prepuce and penis. Examination of the inguinal lymph glands often revcals enlargement and secondary changes.

Diagnosis. - This must be based mainly on the infectious character of the growth (its spread by coition), and its characteristic development.

Prognosis.- The tendency to reappear after removal and the spread to other animals make the prognosis unfavorable. Often when the growths are removed from one area thcy reappear on another.

Treatment.-Complete removal of the growths surgically is recommended when possible. On account of the tendency to recur, they should be completely dissected out, even including some of the normal mucosa. It is advisable to cauterize the surface after their removal, and to treat the wounds with antiseptics. In all cases the patient must be closely observed and at the first sign of recurrence of the growths, promptly operated. In advanced cases, with extensive involvement of both the penis and prepuce, and where general symptoms of emaciation and weakness are present, the penis should be amputated even including a portion of the prepucc. 


\section{CHAPTER II.}

\section{DISEASE OF 'THE 'TESTES ANI) SCROTTYI.}

\section{WOUNDS AND INJURIES OF THE TESTES AND SCROTUM.}

In the dog and cat wounds and injuries of these organs are of frequent oceurrence, partly aceidental and partly intentional. Attempts at castration with the escape of the animal before completion of the operation is a common condition. There will be incised wounds of the scrotum and in some cases even exposure of the testes. Bites from other animals, such as dogs, cats, and rats, produce a variety of wounds and injuries to the testes. Being run over by vehictes is al common source of injury to these organs.

Symptoms. - Hemorrhage from the scrotum will be noted in the ease of wounds, which should alwars be examined carefully to determine the extent of the injury. Contused wounds or bruises are alway's eharacterized by swelling, stiffness, straddling gait, and pain on manipulating the testes.

Prognosis. - Wounds in this location are not as a rule unfavorable, although, if extensive, castration of the animal may be necessury. Slight wounds heal ralpidly.

Treatment. - Small, incised wounds of the serotum should be cleansed thoroughly with antiseptics, at least once darily: If the wound is recent it stould be cleansed, the hemorrhage controlled, and sutured. ('over the sutures with an imporvious dressing like flexible collodion.

In rase the testicles ane exposed, they should be remored. (Ser (astration.) ('ontusions must be treated as in orehitis.

\section{ORCHITIS.}

Definition.- In inflammation of one or both testicles.

Etiology.-Orehitis oc'enrs in the dog and eat from bruise's, or bites of other anminals. Being rme orer by vehicten is a 
cause. Orchitis may be a sequel to wound infection, to distemper in the dog and cat, and the spread of infection from adjacent organs and tissues.

Symptoms. - The first symptoms noticed are those of enlargement of one or both testes; pain on manipulation of the organs; and a stiff, straddling gait. We should not mistake thickening of the scrotum, a common condition in old dogs, for orchitis. If due to infection there will be more or less elevation of temperature, and other symptoms of the primary condition. The local temperature is also increased.

Prognosis. - In orchitis from injuries, most cases terminate favorably. In the infective form the gland may be destroyed, or becomes chronic and the patient impotent.

Treatment. - In the early stages cold packs should be applied, as cold water or a small sack filled with cracked ice. The ice or water pack can be held in place by a bandage passed around the abdomen just anterior to the pelvis, and fastened over the back and between the limbs. It should be renewed after a few hours to keep up the refrigeration. After the swelling and pain have subsided, the pack can be gradually discontinued.

In subacute or chronic cases of orchitis, hot packs are indicated, best in the form of hot water, applied in the same manner as above. Massaging with a small amount of antiseptic ointment is recommended, following the hot packs. In the infective form hot packs should be used with hot water to which has been added some antiseptic. Abscesses must be opened and treated with antiseptics. Should they involve the testicles it is best to remove them. (Sce Castration.)

\section{TUMORS OF THE SCROTUM AND TESTES.}

Several forms of tumors are found in these organs:

(a) Fibromata.-In old dogs a marked thickening of the serotum is common. It is due either to an eczema, or from the constant irritation from sitting on the testicles. 'The latter is characterized by absence of hair on the scrotum, the 
presence of a dark, thickened pad of tissue, and the increase in the size of the scrotum.

Fibroid thickening of the stroma of the glands is observed as a sequel to orchitis of different forms. 'The glands have lost their normal resiliency, are hard and non-sensitive. After a time the animal becomes impotent and the glands atrophic.

(b) Retention cysts are found occasionally in the testicles of the cat and dog. They are characterized by the increase in size of the testicles, and by their soft, elastic feel. A small exploring needle can be used to determine their contents.

(c) Sarcomata and carcimomata oceur occasionally in the testicles, as secondary growths from other parts of the body. These may develop as unilateral or bilateral tumors. Most cases observed have been unilateral. 'They are characterized by their rapid growth, their irregular outline, adhesions between the testicles and the scrotum, and their enormous size in some cases. They are always accompanied by acute inflammatory synıptoms.

Treatment.- Castration is the best means of relief, even in the malignant form.

\section{PARASITES IN THE SCROTUM AND TESTES.}

\section{('uterebra Emasculator.}

In some localities a fly (Cutcrebra emasculator) has been observed which makes punctures in the scrotum in which its eggs are deposited. The larva develop and induce a reactive inflammation which leads eventually to swelling and destruction of the testicles. 'The infestation las been observed in several species of animal; it is quite common in squirrels in certain districts. ("astration of the aminal, or if found carly the removal of the larra, is recommenderl.

\section{CASTRATION. ORCHECTOMY.}

In the dog and cat castration is performed in some cases to reliere pathologieal conditions of the testicles; in ofluers as a renedial measure in enlargement of tle prostate grand (chronic prostatitis). It has been proverl quite conchusively" 
that enlarged prostates in the dog are materially reduced by castration. The operation is also quite extensively practiced in cases where the organs are normal, but to correct vicious habits (onanism) or a disposition to wander away from home.

In chickens castration, or caponizing, is becoming quite general in certain sections. It not only changes the disposition and habits of the birds, but makes them develop more rapidly. They become much larger, and the texture of the flesh much finer. Hence the operation is of considerable economic importance.

Castration of the Dog.-The operation can be performed at almost any age, but is less dangerous before the dog reaches maturity. Between the ages of three to ten months the operation is safest as it causes less constitutional disturbance during the early period of the animal's life. The disposition of the animal is changed less, and there is not the tendency to obesity when castration is performed at an early age. However, when pathological conditions, onanism, etc., exist the operation may be performed at any time.

When the testicles are found in the scrotum, the operation is as follows: The animal is anesthetized by using morphin 0.06-0.2 subcutaneously, or chloroform inhalation. Place the patient in a dorsal position, with head lowered, and hind limbs spread apart on the table. This exposes the testicles and makes them easy of access. The scrotum should be thoroughly scrubbed with soap and water, followed by liberal use of antiseptics. The testicles are grasped between the thumb and index finger of the left hand and gentle pressure used to make the scrotum tense. With a scalpel or castrating knife in the right hand, an incision is made parallel to the long axis of the testicle, cutting through the tunica propia. The testicle is grasped with the right hand which exposes to view the epididymis and tunica reflexa. 'There are several methods used to remove the testicles. The cord may be ligated above the epididymis and tunica reflexa by using a sterile, silk ligature (No. 10). This is a safe method, as it removes the possibility of hemorrhage, provided the ligature is securely applied. The other testicle is removed 
in the same manner. The emasculator also gives excellent results. The instrument should be allowed to remain in position for a few moments after the cord is severed. Occasionally hemorrhage will follow the emasculator, but is usually not serious. If severe, the vessels should be ligated. After the testicles are removed the wound in the scrotum may be left open, or a retaining suture put in to prevent the entrance of foreign material. The incisions in the scrotum should be carried forward sufficiently to afford thorough drainage. The scrotum should be kept clean for a few days, and the wound washed with antiseptics. Union takes place in a week or ten days. It is well to observe the animal, note the temperature and pulse for a few days, and if the temperature is found elevated, examine the scrotal wound for retention of pus and secretions.

Castration of the Monorchid and Cryptorchid Dog.-In monorchids one testicle is removed the same as in ordinary castration. The retained testicle in the abdominal cavity must be removed by making an incision through the abdominal walls about trio to four inches anterior to the pubis, and to one side of the penis (note the side of retention!). 'The incision is made large enough to admit the index finger freely. The cord is then searched for in the sublumbar region, and when found, withdrawn through the opening, the testicle following. 'The cord may be ligated or the operation performed with the emasculator. The abdominal wound is approximated with two or three interrupted sutures. An antiseptic pack and bandage should be applied to protect the wound and changed daily until healing takes plaee. The handlage is applied so that it does not interfere with inicturition. Cryptorchids are operated in the same manner; botl testicles may be removed througl one opening, or two abdominal incisions made if found neressary. 'The patient should be properly prepared by dieting and administering a laxative twenty-four hours before the operation.

Castration of the Cat. - The same rules in regard to age, ete., apply as in the clog. ('astration of the cat is hest performed under complete anesthesia. 'The animial is placerl on the table in the clorsal position, well hoppled and stretened 
out so that it cannot squirm loose. Ether is administered until complete anesthesia is established. The hair is clipped off with a scissors, and the scrotum washed with soap and water and boric acid ( 2 per cent.) solution.

The testicles are grasped between the thumb and index finger of the left hand, and with the other hand a scalpel is used to make the scrotal incision. Two methods of removal are used:

The first method is to make the incision down to the testicle exposing it, pulling it out of the scrotal sac, and removing it with the emasculator or by ligation. Care should be taken in this method to pull the testicle up far enough to include the epididymis and tunica reflexa. The other testicle may be removed in a similar manner.

The second method is to perform the "covered" operation. This is done in much the same manner as the other operation, except that the incision is made down to the tunica vaginalis which is not incised but drawn out with the testicle. The cord, including the tunica vaginalis, is then ligated above the epididymis, and the testicle and its enveloping tunic removed. Hemorrhage and infection are not so liable to follow this method.

Castration of Birds.-Caponizing.-Castration is commonly practiced on male fowls. A castrated rooster is called a capon. The best results are obtained by operating on the early hatched birds as it gives them more time for development. Two to three months is the best age to operate. When the bird reaches the age of four or five months the mortality is much higher and the male characteristics more pronounced.

The fowl is restrained on an operating table, barrel, or box with a cord looped around the wing and one around the legs, to each of which a one or two pound weight is attached. 'The bird is then laid on its right side; the weights serve to hold it in position. Other mechanical devices made and used for this purpose have proved satisfactory. The instruments for this operation are made up in special sets known as caponizing sets. A set consists of a scalpel for making the abdominal incision, spreaders or retractors for keeping the abdominal 
womml open, and a special, spoon-like hook for removing the testicles.

The technic of the operation is as follows: 'The feathers are plucked from the field of operation, which is between the last two ribs extending from the cartilage of prolongation of the ribs to a point about one-half to one and a half inches from the back. The skin over this region slrould be disinfected, best by painting with tincture of iodin. The incision is then made through the abdominal wall observing the following points:

The skin should be pulled to one side before the incision is made, so that following the operation, the skin and deeper wounds will not cover one another.

The incision should follow closely the border of the last rib in order to avoid cutting the intercostal artery. ('are must be taken to avoid injuring the cartilages of prolongation of the last ribs. Should they be cut, which is easily done, the healing of the wound will be delayed. Further, the incision through the walls should be marle carefully to aroid puncturing the lungs.

After the retractors are placed in position, and the incision dilated, the testicles are located just anterior to the kidney. The upper one will be found without difficulty; the lower one is brought into view by separating the mesentery with the handle of the scalpel or other blunt instrument immerliately below the upper testicle. Remove the lower testicle first. The spoon-hook or scoop is applied aroumd the testicle by being slipped over tle spermatic cord. ("are slowld be taken to prevent injury to the large bloodressels. 'The testicle is removed by torsion. A few turns of the instrument will sever the testicle from its attachnents when it can be lifterl out. 'The other testicle is then removed in a similar manner. "The entire testicle should be removed, for if a portion is left in the fowl will (levelop into what is commonly known as a "slip" and the object of the operation defeated. After the spreaders or retractors are renoverl the ribs will assume their normal position closing the incision. No further treatment of the wound is necessary; it closes without difficoulty in a werk or 
ten days. The operated birds must be kept quiet for a few days following the operation, and fed a light diet. The wound should be observed occasionally until complete union takes place. In a few cases collections of air under the skin, forming "wind puffs," will develop. If these occur they are opened and the air forced out. The mortality from caponizing should not exceed 1 per cent. 


\section{CHAPTER III.}

\section{DISEASES OF' 'THE PROS'TATE GLANI).}

Examination. - In the dog this gland is best examined by digital palpation, and in some cases, when the gland is much enlarged, by palpation through the abdominal walls. The finger is inserted in the rectum and if any enlargements are present they can be distinctly felt by pressing in a downward direction; if acute inflammation is present the slightest pressure will produce severe pain.

\section{PROSTATITIS.}

Definition. - An acute or chronic inflammation of the prostate gland. In the dog this diseasc is nearly always found to be of a subacute or chronic type. Acute prostatitis is very rare in these animals.

Etiology.-Prostatitis is produced by microbic invasion, either via the urinary tract, the blood or lymph streams. It inay occur from the spread of the inflammation from other portions of the urinary tract.

Symptoms. - Prostatitis usually develops gradually. The early symptoms are painful defecation and micturition. The animal will make frequent attempts at urinating, the urine voided in small quantities, or there may be complete suppression. Defecation is painful; sometimes impossible. The bladder is found distended. 'The passage of the catheter is often difficult duc to the pressure of the enlarged glands upon the urethra. Gentle pressure on the catheter will cause pain, but in most cases it will grarlually pass through the constricted portion into the bladder. 'The urine will then flow out.

1)igital examination with the index finger inserted in the rectum will reveal the enlarged glands. Should pus be present in the glands they will be somewhat soft and fluctuating. 
The abscesses sometimes rupture, either into the urethra, the abdominal cavity or through the skin in the perineal region.

More or less irregularity in the temperature is noted.

Prognosis.-Although complete recovery cannot be hoped for, owing to the changes which have taken place in the glands, partial recovery is possible.

Treatment.-Medical.-Purgatives should be administered at once, such as castor oil (15.0-30.0) or magnesium sulphate (8.0-15.0). Small doses of morphin may be given in case pain is severe.

Surgical. - The bladder is examined and if found distended, the catheter should be passed and the urine withdrawn. If this is impossible, on account of the compression of the urethra, immediate puncture of the bladder with a trocar may be made. The prostate gland must be examined carefully, and if found fluctuated, indicating abscess formation, make an attempt to relieve it by pressure with the finger inserted into the rectum. This method is often satisfactory. Should this fail, a long exploring trocar is introduced into the gland either through the rectal wall or through the perineum. In either case the finger should be retained in the rectum to guide the insertion of the trocar, and also to exert some pressure on the gland to force the contents out. Prostatic abscesses are sometimes found with an opening out through the perineum. The opening should be enlarged sufficiently to afford good drainage, and syringed out with an antiseptic solution. Rupture of the abscess into the peritoneal cavity results in peritonitis, and death in a short time.

\section{TUMORS OF THE PROSTATE GLAND.}

\section{IIypertrophy of the Prostate Gland.}

Hypertrophy is common in old dogs, and is occasionally observed in young animals. In hypertrophy the normal glandular tissue is gradually replaced by fibrous connective tissue, which leads to an atrophy of the tubules and muscle fibers greatly increasing in the stroma of the gland. 'The gland gradually loses its function of secretion, becomes much 
larger, and firmer than normal. In the dog the prestate gland lies at the neck of the bladder, almost surrounding the urethra, and when hypertrophied it causes eompression of the urethra making the passage of urine difficult.

Symptoms. - The most pronounced symptoms of hypertrophy of the prostate gland are: Obstruetion to the pasisage of urine. 'The animal makes frequent attempts to urinate but either only a small quantity or none at all is passerl. 'The straining induced may cause hematuria. The hemorrhage results from the pressure on the venous plexus of the gland exerted by the hypertrophic tissue. The bladker will be found distended and the animal showing considerable distress. There is always danger of rupture of the bladder from overdistention. A eomplication of conditions is often found in these cases of long standing, such as hyctronephrosis, erstitis, etc. ('onstipation is practically a constant symptom owing to the interference with defecation. No febrile symptoms are observed in hypertrophy of the gland. Passing the catheter will reveal the urethral obstruction. This may he so pronounced that it will be impossible to get the atheter beyond the prostate. It is possible to palpate the enlarged gland by inserting the finger in the rectum, or in very thin subjects the gland may be felt through the abdominal walls.

Diagnosis. - The diagnosis is male by observing the symptoms, passing the catheter, and digital palpation. We must ilfferentiate hypertrophy from absecess and inflammation of the gland. 'The difference in the temperature, the age of the animal, the size and consisteney of the ghand, and the chronicity of the process are indirative.

Prognosis. - The prognosis is unfavorable.

Treatment. - The bladder should be cxamined, and if found distended, emptied either by passing the atheter or by the use of the trocar.

Laxatives should be given (see Inflanmation of the P'rostate (Bland). Internal administration of potassimm iodid (0.1-1).2) daily hats been used with sureeses in some catses. . It the sance time injections of Lugol's solution direrely inte the gland may be cmployed. The injertion is malde with at small calibered, hypodermie needle, which is inserted intw the 
gland, either through the rectum or perineal region, the syringe attached and the injection made. 'Two to four c.c. of the solution are sufficient.

Castration is distinctly remedial as it is followed by a reduction in the size of the gland. Obviously it cannot be employed in stud dogs. (See Castration.) Experimentally it has been proved that in a short time following castration the gland begins to atrophy. The activity and function of this gland depends to a large extent upon the function of the testicles. Some few cases have been successfully treated by castration.

Sarcomata and carcinomata have been found in this gland, but are not common. When found, no treatment can be given. 


\section{CHAP'TER IV. \\ DISEASLE OF" 'THE OVARIES.}

Examination. - Sereral methods of examination are used to detect abnormal and pathological conditions of the ovaries.

(a) By abdominal palpation. 'This method has not proved very satisfactory on account of the small size of the ovaries, and the amount of tissue necessary to palpate through. In emaciated subjects or when glands are enlarged, palpation is useful. In cats with large ovarian cysts, the cysts may be felt through the abdominal walls. For abdominal palpation the animal is placed in a standing position. The manipulator should stand either immediately in front of or in the rear of the animal with one hand on either side of the abdomen; the ovaries may be felt in the sublumbar region. 'This method is of value in a general way, but for accurate diagnosis it does not suffice.

(b) $\mathrm{By}$ observing the animal to note any symptoms of excitement, ete. Cats with ovarian cysts will often show evilences of nervous excitement, epileptiform convulsions, etc.

(c) I)irect inspection of the ovaries can be marle with but very little danger. 'Therefore in doubtful cases laparotomy should be performed and the ovaries examined for inflammation, tumors, cysts, etc.

\section{INFLAMMATION OF THE OVARIES.}

\section{Oöphoritis.}

Definition.- An acute or chronic inflanmation of one or both ovaries. Ö̈phoritis is not observed very often in animals. It shoukl not be mistaken for the normal liyperemia of the glands during the estral period. IIowerer, ateute and chronic inflammations are found involving these glands. 
Etiology.--liesults from injuries, sueh as being run over by rchicles. 'The compression of the organs may be sufficient to crush them, or it may lead to inflammation.

Extension of the inflammation from adjacent organs and tissues, as from the uterus and uterine tubes.

Infection of the ovaries may take place in some of the infectious diseases (distemper in the dog and cat), or it may be due to some non-specific infection carried to the ovaries by the circulatory system.

Symptoms. - In dogs oöphoritis may not be noticed. The patient will show stiffness in walking and pain on palpation over the glands. In cases where infection has taken place in the glands, abscesses may develop, and febrile symptoms be present. In subacute or chronic inflammations no marked symptoms will be observed beyond an enlargement of the glands. In cats epileptiform convulsions may result.

Diagnosis. - In the mild forms an accurate diagnosis is difficult without making an explorative laparotomy. In cats it should be differentiated from ovarian cysts. A careful examination must be made in all cases.

Prognosis. - Farorable, except when produced by infection. In the chronic form sterility is a common sequel.

Treatment.-Not much treatment is needed. In the severe forms, or when abscesses are present, it is advisable to remove the ovaries (oopphorectomy).

\section{TUMORS OF THE OVARIES.}

Cysts. - Cystie formation in the ovaries is of very frequent oceurrence, and perhaps much more common in cats than in any of the other animals. They consist in most instances of unruptured Graafian follicks, and are found either single or multiple. Inless they are of considerable size they do not produce any marked symptoms. In fact, where they are frequently found as multiple cysts, and when regeneration of the ovary has taken place, marked ncrvous symptoms will be noterl. These are excitcment, prolonged estrum, and in some instances cpileptiform convulsions. 
Diagnosis. - The diagnosis is difficult in most cases. ['nless the cysts are of large size, and the nervous symptoms marked. the eondition is usually not suspeeted. Laparotomy should be performed and the ovaries inspeeted to make the diagnosis positive. The cysts appear as enlargements projecting from the ovary. Their eonsistency is fluctuating; their contents transparent.

Prognosis. - The prognosis is unfavorable so far as relieving the condition and preserving the ovary are concerned. The sy'mptoms can be relieved by removal of the glands.

Treatment. - The treatment is surgieal and consists of the removal of the diseased gland. (See Orariectomy.)

Other tumor formations in the ovaries are uneommon. Dermoid cysts have been found in a few instances. Adenoma and adenocarcinoma oeeur as seeondary growths. When found the entire gland or glands should be extirpated.

\section{OÖPHORECTOIYY. OVARIECTOMY.}

Oöphorectomy is extensively practiced to correet eertain pathological conditions which are found in the ovaries to suppress the sexual desire and prevent the female from becoming pregnant, and to make more desirable house dogs and pets as they are more eontented and peaceful. (ats, when operated during the first few months of their life, beeone much larger, and are much more desirable animals to have about the house.

All female animals should be operated if possible before the advent of the first estrual period, as it has been proved by experienee that some females will contimue to show sexual desire following complete removal of the glands. Such cases are observed in older females, especially those that have given birth to young, and these that have estruated normally for some time. However, we must take into eonsideration that in a number of eases where estruation follows removial of the glands, is due to the faet that a small portion of the ovarian tissue has been left in, which develops and frequently becennes. cystic.

The effeet of this operation on females in worthy of mote. 
Young animals operated before the periods of estrum are present, show no appreciable change in their development. They are active, develop regularly, and in every way make very desirable animals. The most marked change occurs when older animals are operated. They often become fat, lazy, and inactive. Certain breeds of animals show these changed characteristics more than others. For this reason the operation should be performed before the animal reaches sexual maturity.

Oöphorectomy in the Dog.-This is one of the most frequent operations performed on dogs. It is a safe operation provided the following precautions are taken into consideration: (a) The operation should be performed when the dog is about three to five months old, and before it has had an estrual period. (b) The animal should not be operated during estruation, notwithstanding the prevailing belief of the laity to the contrary. As the ovaries and other portions of the generative tract are congested at this time, the danger of hemorrhage and inflammation is greater. One should wait two or three weeks after the period of estruation so that the organs can return to their normal condition. (c) The preparation of the animal before operating is of special importance. The bowels should be empty. For this purpose castor oil (15.0-60.0) should be administered twenty-four hours previous to the operation. All solid food is withheld, but small amounts of milk and water only may be allowed. 'The field of operation should be prepared twenty-four hours previously; the hair removed, the skin washed with soap and water, and an antiseptic pack applied. This pack is best made of cotton or gauze with boric acid; it is applied to the operating field and held in place by a special baudage mate of a wide pieee of muslin so as to extend from in front of the forelimbs to a point back of the field of operation, and tied over the back. This pack should be kept in position until the animal is ready' for the operation. 'This will ensure a sterile operating field. As a last precautionary measure, just previous to making the incision, the operating field is painted with tincture of iodin. An anesthetic or nareotic should be given. In the dog various methods of anesthetiza- 
tion and narcotization have been employed witl cqually good results. Morphin given as a subcutaneous injection about twenty to thirty minutes previous to the operation has been used with most excellent results. The value in this method of narcotization lies in the fact that it will cause vomiting in most cases, defecation in some, removing material from the stomach and bowels. Further, it will keep the animal quiet for several hours following the operation. It may be considered a perfectly safe narcotic, which to dogs can be administcred in largc doses. The amount to be administered will depend to a great extent upon the size of the animal. Lsually from $0.1116-0.2$ are to be given.

('hloroform and ether may be used as a general anesthetic; both are safe when administered properly. After anesthetization the animal should be placed in a dorsal position, well hoppled and the table tilted to lower the head as much as possible. This assists the operator, as the bowels will descend toward the diaphragm which lessens the tendency for them to protrude through the incision. 'The incision may be made either at the median line or in the flank region. There are good reasons for choosing the median incision. There is less hcmorrhage at this location, it is much easier to locate the cornua and the removal of both ovaries through one opcning can be done with less difficulty. The exact location for the incision in the median line is at a point about one to one and a half inches posterior to the umbilicus. 'The incision should be made of sufficient length to allow the index finger to be inserted, or it may be enlarged so that light can be thrown into the abdominal cavity. The incision is made through the skin, separating the muscles down to the peritoneum. In making the incision through the peritonemun it is best to pick up a small portion of it with a forreps, nick with the scissors and enlarge with a probe-pointed knife. By this procedure injury to the bladder or other abdominal organs is avoided. The index finger of the right hand is then inserted through the opening; follow the left abdominal wall pushing the boweds back until the finger reaches the botton of the cavity. At this point the finger will come in contart with the left cormu, which is hooked over the finger and etrawn out of 
the carity. Be careful to keep the finger in constant contact with the abdominal wall so that the cornu will not slip off. When once outside of the cavity the ovary can be located easily by following the course of the cornu. The ovary is distinguished by its consistency. In most cases in the dog it will be found imbedded in a capsule of fat. There are two methors of removing the ovary, and the choice of them will depend somewhat upon the condition of the gland. In young females, before the ovaries have fully matured, and in older ones between the periods of estrum, it is safe to remove them with an emasculator, unless considerable congestion is present. Where there is danger of hemorrhage, the ligation method is used. When this method is employed, sterile silk or linen is best which should be put on securely so that it will not slip off after removing the ovary. Ligation should be made at two points, around the cornu posterior to the ovary, and around the ressels and the broad ligament anterior to the ovary. Care must be taken that all of the ovarian tissue is removed. Otherwise the females will again estruate, and the success of the operation will be incomplete. When removing the gland with the emasculator it must be pulled up sufficiently so that it will include all of the ovarian tissue. It is best to allow the instrument to remain in position a few moments. 'The cornu is then returned to the cavity. The other ovary is obtained by inserting the index finger of the left hand and following along the right abdominal wall as ras done on the opposite side.

The location of the oraries is not difficult when the animal is properly prepared. Sinall, fat patients, with a short abdominal cavity, will present the greatest difficulties. 'The introduction of a probe or catheter through the ragina may be userl by the beginner.

'The abrominal incision is clcansed thoroughly and approximated by using two or more interrupted sutures. Suturing the abrominal walls may be done by using two rows of sutures one row including the peritoneum, and the other the skin and muscles, or may be closed by a single row including all of the tissues. This method has proved satisfactory. The abdominal walls should be aceurately approximated as it facilitates adhesions and healing. 
An antiseptic pack is applied and the regular bandage used to hold it in place. I)ress the wound daily and in four to six days the sutures may be removed.

Sometimes animals are observed to estruate following this operation, and in such cases they should be reoperated, as it results in most instances from a small amount of ovarian tissue being left in. (y'sts will form in such cases leading to a continuance of the estrual period.

Oöphorectomy in the Cat. - Cats are operated to correet pathological conditions, particularly crstic formations in the ovaries, which are very common in these animals, and also to prevent them from becoming pregnant. 'They should be operated if possible prior to sexual maturity, and the best time is between the ages of three and seven months.

The same preparation should be made for the eat as in the dog. The most satisfactory anesthetic is ether. The operative technie is the same as in the dog. It has been claimed that this operation is more dangerous in the eat than in other animals, but when done under proper conditions the mortality is very low.

\section{POULARDIZING THE FEMALE CHICKEN.}

This operation is performed on the female fow to prevent egg formation, and to ensure quick growth and a finer quality of meat. The operation is not practiced as extensively in this country as in some of the European comntries. Indoubtedty, however, the operation will become more popular than at present.

The best age to operate is after two to three months. The same preparation should be made as in eaponizing. (See ('aponizing.)

The operation is performed as follows: Securely fasten the bird on a table or other suitable place, and remove the feathers from the field of operation, which is between the last two ribs. Thoroughly rleanse and disinfect. The incision is made between the last two ribs, ming the silne precantions as in caponizing. Retractor's are nosed to spread the incision. The egre cluster will rome into view at conce. 
A section of about one to one and a half inches of the oviduct should be removed with the forceps and scissors. Care must be taken to prevent injury to the large bloodvessels which lie in close proximity to the egg cluster. The removal of a portion of the oviduct prevents further egg production. After-treatment is the same as for caponizing. 


\section{CH.IP'TER V. \\ DISEASES OF THE T'TERINE 'TU BBS.}

Examination.- Examination of the uterine tubes is quite difficult, except by explorative laparotomy. 'They should be cramined for inflammation, tumors, cysts, and pus aceumulations. In some cases, when the animal is much emaciated, and the tubes large, it is possible to palpate them through the abrominal wall.

\section{SALPINGITIS.}

Definition. - Inflammation of the uterine tubes.

Etiology.-Salpingitis occurs as a secondary condition following inflammation of other portions of the generative apparatus.

Symptoms. - The diagnosis is difficult unless laparotomy is resorted to. Other reproductive organs are usually also involved complieating the symptoms.

Treatment. - Very little can be tone exrept complete extirpation of the affected tubes.

\section{PYOSALPINX.}

Pyosalpinx is a purulent inflammation of the uterine tubes. It is secondary to other diseases of the reproductive organs. Removal of the uterine tubes is recommonterl.

\section{TUMORS. CYSTS.}

These are found occasionally and when present should be extirpated. 


\section{CHAP'TER VI.}

\section{DISEASES OF 'THE U'TERLS.}

Examination.-There are three principal ways in which an examination of the uterus may be made: (a) By abdominal palpation; $(b)$ by obtaining the discharge from the uterus and noting the condition of the vulva and vagina; $(c)$ by laparotomy.

(a) In abdominal palpation it is possible to determine various conditions involving the uterus. The patient should be placed in a standing position. With one hand on either side of it over the postero-inferior abdominal region, the operator by gentle pressure with the finger can feel the uterus when distended, as an elongated, suspended body, within the abdominal cavity. Palpation may be used to detect pregnancy, pyometra, hydrometra, tumors and inflammation. Sometimes in order to differentiate between these conditions it is necessary to make a general examination of the animal.

(b) In some of the conditions involving the uterus, there is a discharge from the vulva. The discharge should be colleeted and examined carefully, noting whether or not it consists of blood, mucus, pus, membranes, bacteria, etc. 'The microscope may be employed if necessary.

(c) By laparotomy it is possible to make a direct inspection of the uterus. It is advisable when there is evidence of serious involvement of the organ, and where the diagnosis is in doubt. The incision through the abdominal walls is made in the median line just anterior to the pubis, extending forward a sufficient distance to allow the uterus to be drawn out. 'The uterus may be enlarged. The external or serous covering should be observed for evilences of inflammation, hemorrhage, and rents or tears in the walls. Note 
the relative size of the two cornua, as compared to the size of the body of the uterus, their position and attachments. 'Tumors, pregnancy, etc., should be looked for. The consistency of the organ is important as it is modificd by the character of its contents, whether fluid, or solid material. The entire organ should be carefully palpated for differential diagnosis between pregnancy, tumors, proliferative or fibroid endometritis, pyometra, hydrometra, etc. After a careful examination the uterus may be returned to the avity or operated as the condition indicates.

\section{METRITIS.}

Definition. - In inflammation of the uterus which may be (a) acute, or (b) chronic.

Acute Metritis. - Definition.-An acute inflammation of the uterus. In most instances it is to be regarded as an acute inflammation of the mucosa (acute endometritis). In some cases the musculature and serous coverings are involved (metroperitonitis).

Etiology.- Netritis is a condition in which infection of various kinds is found to be the primary etiological factor. There are various conditions which oc'ur in small animals that favor uterine infection and ultimately lead to an acute infiammation. 'These are: (a) The retention of the fetal envelopes. If not expelled after the normal time has elapsed they constitute a somre of danger to the animal, as they form a favorable medium for the growth of bacteria. 'The' retained membranes keep the cervix of the uterus open, which favors the introduction of bacteria, and interferes with the normal involution of the organ. Retained placenta is not so common in small animals as the membranes ares usually passed with the fetus.

(b) Infection is introduced into the uterus at the time of parturition by the use of infected instruments or fingers: nsed in cases of dystocia.

(c) Wounds of the mucosa of the vagina and uterus greatly facilitate the entrance of infertion. Depending upon their depth, wounds may lead to metroperitonitis. 
(d) The retention of a fetus or fetuses which decompose, irritate the mucosa, and, if allowed to remain for a long period, often produce grave simptoms of local inflammation and sapremia. In some cases the uterine mucosa is greatly changed by the infection. The uterus may be converted into a cavity filled with pus (pyometra).

(e) Slowness in the involution of the uterus from lack of muscular tone favors the introduction and derelopment of infection. For the same reason individuals weakencd from delayed parturition, systemic diseases, etc., are predisposed.

(f) In bitches and cats that are kept in cold, damp kennels, metritis is occasionally observed, and no doubt results from the general reduction in resistance, and from the weakened condition of the highly sensitive reproductive organs. 'This favors the development of microörganisms.

(g) In small animals injuries of various sorts are common, such as being kicked, run over by vehicles, or roughly handled by persons, especially during the latter stages of pregnancy. The uterus may be injured, sometimes torn or lacerated, and inflammation with infection is the common sequel.

Pathology. - In fatal cases of acute metritis, marked pathological changes are observed in the generative tract, and particularly in the uterus. The uterus is dark colored, in some cases almost black, the mucosa showing neerotic areas. In some instances the necrosis cxtcuds to the other tissues of the walls of the uterus causing perforations. The walls of the utcrus are thickened, edematous; the serous covering is often inflamed as are the adjacent organs and tissues in contact with it. Occasionally abscesses are found in the uterine walls, or in the surrounding tissues. In the virulent cases of metritis there will be found evidences of thrombosis of the bloodvessels of the uterus, leading to embolisms in the vessels in distant parts of the body, producing in some cases pyenic arthritis, etc. The vulva and vagina are swollen and necrotic, and a greenish colored exudate of offensive odor is present. The other organs and tissues will show the usual postmnortem lesions of septicemia or pyemia. The blood is dark colored and fails to coagulate. 'The kidneys and liver 
are soft and congested. 'The musculature in general is pale, friable and soft.

Symptoms. - The first indication of metritis is a marked swelling and congestion of the vulva. On, digital examination the parts will be found sensitive, very hot, and present on the mucosa a greenish or brown or blood-stained discharge which has a very fetid odor.

The vagina is swollen, very hot, and in the early stages, reddened and congested. Later it becomes dark or dark bluish, and in some cases almost black in color, with a foul smelling exudate. This discharge is more copious at times, as it is forced out of the uterus at different intervals.

Frequently the animal shows marked symptoms of straining, the abdominal muscles become tense, and quantities of a thick, dark colored exudate are discharged from the vulva.

The temperature during the early stages is elevated $\left(106^{\circ} \mathrm{F}.\right)$. Later, as the toxins are absorbed, the temperature drops to normal, often subnormal.

There are general symptoms of suppression of appetite, vomiting, general stiffness in walking and pain on palpation over the region of the uterus. The animal in most instances assumes the recumbent position.

In mild cases the symptoms will gradually disappear and terminate in complete recovery, or in chronic metritis.

Diagnosis. - The condition appearing as it does following parturition, with the characteristic discharge from the vulva, and the painful and sensitive condition of the uterus, makes the diagnosis rather easy. Careful examination should be made in all cases to establish a correct diagnosis and especially to differentiate acute metritis from puerperal septicemia and pyometra.

Prognosis.-In small animals, owing to the difficulties encountered in the treatment, and the retention of the exudate in the cornua, the prognosis is unfavorable. 'The milder cases recover but there is always danger, even in the mild cases, of chronic metritis or pyometra developing.

Treatment.-Medical.-Owing to the small uterus and the long cornua, irrigation is a more difficult problem than in 
larger animals. Ilowever, it should be attempted as good results often follow thorough irrigation. Boric acid (2 per cent.); creolin (1 per cent.); lysol (1 per cent.); or theropogen (2 per cent.) may be used as follows: A small metallic catheter or flexible human male catheter is inserted into the uterus and a rubber tube and funnel attached to the free end. The antiseptic solution is allowed to flow into the uterus by gravity. After $\frac{1}{2}$ to 1 pint of the solution is introduced the tube should be lowered and the fluid allowed to flow out. The catheter is introduced into each cornua. This treatment should be applied every three or four hours to keep up the antiseptic action, to remove the exudate and to prevent absorption and the resulting general symptoms.

Sinall doses of ergot or other ecbolies should be administered once daily to stimulate the uterus and to hasten its involution.

Surgical.--In severe forms of acute metritis, and in cases where medicinal trcatment does not relieve, it is advisable to rcmove the uterus and ovaries by performing laparohystero-oöphorectomy.

Chronic Metritis. - Pyometra.-Definition. - A chronic inflammation of the uterus, characterized by the formation and collection of pus in the uterine cavity. In case the cervical canal becomes closed, retaining the pus, the uterus may be converted into a veritable abseess. ('hronic metritis occurs quite frequently in the bitch; less commonly in cats. It makes its appearance in most cases following parturition, at any period in the animal's life, but may be found in females that have never given birth to young.

Etiology.-(a) Commonly a sequel to acute metritis, the acute symptoms disappearing, leaving behind bacteria of a low virulence, which keep up a constant irritation to the mucosa resulting in chronie inflammation with pus formation.

(b) Infection gaining entrance to some portion of the reproductive organs which may find its way to the uterus direct, or by extension of the process from other parts or adjacent tissues, leading to a primary inflammation of chronic type.

(c) Following parturition it frequently happens that a 
small portion of the placeita is retained leading to a slowly developing inflammation with pus formation.

(d) Injuries of a mild character during parturition, or at other periods, reduce the general resistance of the animal and particularly the local resistance of the uterus allowing infection to develop. In these cases we will often find that the female has never been pregnant. Such cases tend to develop into uterine abscess.

(e) Anything which reduces the resistance of the reproductive organs or the animal's general resistance has a tendency to favor the formation of this condition. Exposure is a predisposing factor.

Pathology. - The presence of pus in varying quantity in the uterus. The pus is thick, viscid, dark or reddish, sometimes reddish-gray in color and of offensive odor. The walls of the uterus are much thickened, dilated, and in some cases enormously distended with pus (uterine abscess). The mucosa is dark in color, soft, spongy, and shows numerous elevations of various sizes. The process can extend into both cornua. In gencral the animal will show emaciation, the muscles are palc, soft and friable. Secondary abscesses are often observed in the kidneys, liver and lungs.

Symptoms. - The most prominent symptom is the chronic discharge from the rulva, which varies in quantity, being more copious at certain times than at others. 'This is due to the fact that it accumulates in the uterus until a certain distention of the organ is reached, when it will be ejected. The discharge is grayish-red, or dark red in color, and of fetid odor. It soils the tail, limbs, and hair around the vulva. In uterine abscess, with occlusion of the cervical canal, the discharge will be absent.

Enlargement of the Abdomen.-In all cases, and particularly in uterine abscess, there is a marked increase in the size of the abdominal cavity which may simulate pregnancy.

Careful palpation should be made especially in distention of the uterus with no discharge from the vulva (uterine abscess).

General Symptoms. - In all cases of chronic metritis systemic disturbances are noted. They are general cmacia- 
tion, weakness, rough hair coat, a variable appetite and temperature.

Diagnosis.-Diagnosis does not present much difficulty as the symptoms are quite characteristic. Uterine abscess should be differentiated from pregnancy, distention of the bladder, ascites, tumor's, etc. In doubtful cases, laparotomy is advisable.

Prognosis.-Usually favorable, especially so when the animal's condition is still good, and proper treatment possible. Such animals cannot be used for breeding as the condition in the uterus, which cannot be entirely relieved, prevents conception.

Treatment. - Medical treatment is of little value. Irrigation of the uterus with an antiseptic solution may be tried. However, surgical treatment is necessary in order to properly overcome the general symptoms. An early removal of the uterus and ovaries will prevent metastatic abscesses developing in the kidneys, liver, etc. 'The operation is as follows: The animal should be given a general anesthetic and placed on the operating table in the dorsal position with head lowered. 'The incision is made in the median line, beginning just anterior to the pubis and extending forward a sufficient distance to allow the uterus to be drawn out of the abdominal cavity. Sterile silk ligatures (No. 10) are applied around the broad ligament at the distal portion of the ovary, and around the uterus just anterior to the cervix. The ligature around the uterus should be placed in sections, and then around the entire part to prevent it from slipping off and fatal hemorrhage resulting. The entire portion between the ligatures is then separated with knife or scissors, and the stumps returned to the abdominal cavity.' 'The abdominal incision is cared for in the regular way.

In case of collapse or weakness following the operation small dloses of strychnin (0.001) should be given.

\section{PUERPERAL SEPTICEMIA.}

Definition. - A common disease in small animals in which either bacteria or their products are introduced into the 
general system. Puerperal septicemia accompanies to a greater or less extent most forms of septic infection of the reproductive organs during the puerperal state.

Etiology.-Puerperal septicemia results invariably from retention of placenta, a fetus or fetuses, or from wounds acquired during or following parturition. Putrefaction changes in the retained placenta occur rapidly, other infection is introduced, and the products of bacteria or the bacteria themselves are taken into the circulation producing a general septicemia or sapremia. This disease may follow normal hirth, dystocia, etc.

Pathology. - The changes in the reproductive organs are often slight as compared to the severity of the symptoms. The blood is thin, does not coagulate readily. The muscles are pale, soft and friable. Ecchymoses are observed on the serous membranes, particularly in the abdominal cavity. Parenchymatous degeneration is noted in the liver, kidneys and spleen.

Symptoms. - The symptoms develop rapidly, usually in from one to three days following parturition. There is great depression, the animal lying down most of the time; elevated temperature $\left(106^{\circ}-107^{\circ} \mathrm{F}.\right)$, increased respirations and pulse. Later appear great prostration, subnormal temperature, cold extremities, etc.

locally the generative organs show marked changes in some cases, such as swelling of the vulva, the mucosa of the vagina; in others little change will be found. There is nearly alway's a copious discharge from the vulva, consisting of a greenish colored pus, containing shreds of placenta and other material. 'This discharge has a very offensive odor.

The bowcls are irregular, in some cases constipation will be present and in others a screre diarrhea. 'The symptoms usually increase in intensity, the animal reaching a stage of coma or collapse. In the milder forms of infection, and particularly in sapremic conditions, the symptoms gradually disappear and the animals make a completc recovery.

Diagnosis. - This is made on the sudden onset, the severe general symptoms, the high temperature in the early stages, and the characteristic involvemcnt of the reproductive organs. 
Puerperal septicemia should be differentiated from acute and chronic metritis which cau be made by a careful examination of the patient.

Prognosis. - The prognosis is unfarorable, most cases terminating fatally. Nild cases often recover; more severe ones may recover if treatment can be applied early.

Treatment.-Hedical.-In cases of marked collapse, subnormal temperature, etc., stimulants, such as aromatic spirits of ammonia, spirits of camphor, oil of camphor, or strychnin should be administered early.

Surgical.-As local applications in the form of irrigation with antiseptics have proved unsatisfactory, an early operation is advised. Before operating obviously the genital tract should be flusherl with antiseptics. The animal should be anesthetized and operated as in chronic metritis. (See Chronic Metritis.) (are must be exercised in preventing infection of the serous membrane of the abdominal cavity. The stump of the uterus should be inverted and thoroughly disinfected before it is returned to the abdominal carity.

The after-treatment is very important, and prompt remedial agents (stimulants) should be administered.

Irrigation of the vagina should be done at regular intervals to control local infection. Normal salt solution should be used in cases of collapse following the operation. It is best injected intraperitoneally (2:50 to 500 c.c.).

\section{EVERSION OF THE UTERUS.}

\section{Prolapse. Intersion of the L'terus.}

Eversion of the uterus is not common in the small, multiparous animals, particularly in the dog and cat. 'The small uterus and long cormua present an anatomical arrangement which tends to prevent eversion cxcept in rare instances.

It is occasionally observed in the bitch following parturition. The eversion in most ases consists of an invagination of the anterior extremity of the cornu into the succeeding portion, and should the process continuc, it will appear at the vulva or even project outside. In most cases the prolapsed portion will consist of one cornu and a portion of the 
uterus. However, in a few instances there will be found a complete eversion of both cornua, the body of the uterus and a portion of the vagina. The prolapse of one cornu through the utcrus usually prevents the other one following.

Symptoms. - The early indications are the expulsive efforts of the animal, which are very similar to those noted in parturition. The animal becomes uneasy, looking at its sides, licking the vulva, etc. When such symptoms occur following parturition, the uterus should be examined. The local symptoms after the uterus appears at the vulva, are quite characteristic. There is a rounded enlargement between the lips of the vulva, at first only slightly congested and swollen, later considerably swollen and changed in color to a clark red or almost black. When the organ has been prolapsed for some time, the mucosa becomes darker, covcred with a thick greenish or purulent exudate, and in some cases extensively gangrenous.

General symptoms of anxiety, restlessness, dyspnea, increased labor pains, and later septicemia are observed.

Diagnosis.- In small animals care should be taken to differentiate crersion of the uterus from prolapse of the vagina or its mucosa, tumors, etc. This can be done by inserting the finger around the periphery of the enlargement to determine its point of origin. Further, the characteristies of the prolapsed portion will assist in the diagnosis. In later stages, when necrosis has developed with much swelling of adjacent tissues, the diagnosis is more difficult.

Prognosis. - Several things tend to alter the prognosis. More favorable are those cases of recent development, and cspecially before extensive pathologieal changes have taken place in the uterus. The prognosis is unfavorable if amputation of the uterus is necessary on account of the danger of septicemia. 'The prognosis is unfavorable from the standpoint of breeding.

Treatment. - In case the prolapse is of recent development reposition should be attempted at once. The parts should be thoroughly cleansed with antiseptics and astringents. Reposition should be attempted by gentle pressure on the prolapsed portion. Patience is often necessary to effect 
reposition. In case this does not succeed, it is advisable to perform laparotomy under anesthesia, and pull the uterus back into position. Care should always be taken to avoid tearing the tissues, and it is best to have an assistant manipulate the parts in the vulva, and at the same time exert some pressure so that the entire prolapsed portion will at the same time go back into position. It is advisable to suspend the uterus to the abdominal wall after it has been withdrawn in order to prevent further prolapsus. This can be done easily by simply including the serous and muscular coats of the uterus in the sutures when closing the abdominal walls.

In case the prolapsed portion is necrotic or gangrenous it should be amputated at once. The parts are cleansed thoroughly with antiseptics, and the prolapsed portion drawn out from the vulva until healthy tissue appears. A ligature is applied around the entire mass as high up as possible. It should be placed in position by drawing it tight to avoid postamputation hemorrhage. The mass is then removed with a scissors or knife. Hemorrhage should be controlled, if present. The stump is thoroughly washed with antiseptics and returned to the vagina. The vagina should be irrigated with antiseptics for a few days following the operation. The ligated portion will slough away in a few days.

\section{TORSION OF THE CORNUA UTERI.}

'This is a condition occurring occasionally in biteles previous to or at the time of parturition. A twist oecurs at the junction of the cornua with the body of the uterus, preventing the birth of the fetuses.

Symptoms. - No special symptom of torsion of the cornua will be observed. It may be necessary to make a careful examination to reveal the exact condition; in some case's an explorative laparotomy must be made.

Treatment.-The torsion can be reduced by performing laparotomy. In case it is found that the circulation has been so disturbed as to cause necrosis, it will be best to amputate the entire organ. 


\section{RUPTURE OF THE UTERUS.}

Rupture of the uterus has been observed in both the bitch and cat. It may be due to unequal uterine contractions, or to the rough use of instruments at parturition.

The rupture may be small, simply allowing some of the fluids from the uterus to escape into the abdominal cavity, or may be of sufficient size to allow the fetus to pass through. This is usually a serious condition on account of the infection getting into the abdominal cavity, producing septic peritonitis.

Symptoms. - The stoppage of the labor pains, and the sudden prostration of the animal are the most characteristic symptoms. Examination should be made by inserting the finger through the vagina into the uterus and at the same time with the other hand pushing upward and backward on the fetus. If there is membrane between the fetus and finger, one should suspect that the fetus is in the abdominal cavity. Laparotomy should be performed at once to make a positive diagnosis.

Prognosis.-The prognosis is unfavorable owing to the danger of peritonitis.

Treatment.-Prompt surgical treatment should be given. The fetus and membranes should at once be removed, and the abdominal cavity flushed thoroughly with normal salt solution. The rent in the uterus is closed with Lembert sutures. Gauze is placed between two of the sutures in the abdominal wall to afford drainage. In thirty-six hours this may be removed.

\section{TUMORS OF THE UTERUS.}

Various forms of tumors have been observed involving the uterus in the bitch and cat. The usual varieties are: Fibromata, myomata, cysts, and hydrometra. Malignant neoplasms are very rare.

Fibromata.- These are benign tumors found involving the muscular wall of the uterus. In the majority of instances they consist in part of fibrous tissue, and myomatous ele- 
ments. In older animals there is a preponderance of fibrous tissue. 'They develop gralually within the muscular walls, and project into the lumen of the organ in some cases, while in others the growth is mainly toward the peritoneal cavity. When extensive, and when the growth is towarl the lumen of the organ, they may be found projecting through the os into the vagina. 'They are characterized by their slow development.

Symptoms. - The first indication of the presence of fibromata will be an increase in the size of the abdomen, simulating pregnancy. However, on examination by palpation, the difference will be apparent at once. Frequently on inserting the finger into the vagina the tumor will be recognized, and it should be observed whether the tumor projects from the os, or is attached to the vaginal wall. No general disturbances are noted, except when the tumor becomes of sufficient size to interfere with the function of the abdominal or thoracie organs.

Diagnosis. - The enlargement of the abdomen, which comes on gradually, the lack of general symptoms, and the location of the enlargement differentiate it from pregnancy. Further, through laparotomy the uterus may be examined direct.

Prognosis. - Favorable.

Treatment.-Surgical.-Surgical interference by complete removal of the uterus and ovaries is indicated. When the tumors are found projecting through the os into the vagina, they should be withdrawn into the abdominal cavity and extirpated.

Myomata.-In myomatous tumor's of the muscular walls of the uterus, the symptoms, diagnosis, and treatment are the same as for fibromatous.

Hydrometra.-Definition.-A collection of transudate or other sterile fluid in the uterus.

Etiology.-Occhusion of the cervix, or any portion of the uterus which prevents the escape of fhuicl.

Inflammation of the cervix (endocervicitis) resulting from wounds and injuries during parturition.

Pressure of inguinal hernia producing ocelusion.

It may he produced by ligation of the uterus, as is done 
occasionally to prevent pregnancy. It may develop as a simple hydrometra, in which case there will be no serious effects upon the animal.

Symptoms.-Distention of the abdomen which becomes pronounced and simulates pregnancy. Its persistence and the absence of lactation differentiate it from pregnancy, however. 'There are no general symptoms, no pain on manipulation of the abdomen, and no change in the other parts of the reproductive organs.

Diagnosis.-Hydrometra should not be confused with pregnancy, tumors of the uterus, ascites, and pyometra. A laparotomy may be necessary to make an accurate diagnosis.

Prognosis. - Favorable.

Treatment. - If due to an inguinal hernia, a surgical operation is necessary for relief. In other cases the entire uterus together with the ovaries should be removed.

\section{DYSTOCIA.}

Definition.-Difficult parturition. Dystocia is of frequent occurrence in the bitch and cat.

Etiology.-Many etiological factors produce dystocia:

1. In a mechanical way we find numerous obstacles which interfere with the expulsion of the fetus.

(a) Lack of expelling power of the uterine muscles. This is frequently due to general weakness, anemia, etc.

(b) A narrow and undilatable pelvic canal, which is too small for the fetus to pass.

(c) Constriction of the os uteri, which prevents the passage of the fetus.

(d) 'Torsion or displacement of the uterus, closing the uterine exit.

(e) Abnormal development of one or more of the fetuses which are too large to pass through the pelvic canal.

( $f$ ) Malpresentation or position of the fetus which prevents it entering or passing through the pelvic canal.

(g) Deformity or abnormality in the development of the fetus. In some instances, as in hydrocephalus, ascites, or 
monstrosities, they are too large to pass through the pelvic canal.

(h) There is frequently a narrowing of the pelvic canal from tumors in the vagina, fractures of the pelvic bones with enlargement, ete.

2 . It has been observed frequently that environment has a great deal to do with producing dystocia in the bitch. Animals that are kept closely confined, fed highly nutritious food, and are not exercised, are more predisposerl to parturition difficulties. Some breeds are more often affected than others, perhaps due in most cases to the manner in which they are cared for.

3. Nating animals of extremes in size, particularly a small female bred to large male. The young will be too large in some cases to be expelled.

4. Young females at the first birth are more commonly affected with dystocia than at later periods.

5. Females when bred before reaching complete maturity often will have difficulty at the time of parturition.

Symptoms. - In normal parturition it requires from three to thirty-six hours for the birth of the young. It depends somewhat on the number of fetuses, and the condition of the animal. Therefore it is sometimes difficult to determine accurately the time at which dystocia begins. 'The principal simptoms of dystocia are: Extreme restlessness; severe labor pains at first, later their cessation; discharge from the ragina; general weakness.

Examination of the patient will determine the condition. The following examination should be made in these cases:

Note the general condition in regard to pulse, respiration and temperature. Note the phrsieal condition.

Palpate the abdominal region to determine whether any young are present. Differentiate between the presence of fetuses and other enlargenents commonly found in this location.

Note the condition of the external genitals; the discharge from the vulva. 1)isinfect the fingers and palpate through the vagina. If the fetus has been presenter at the pelvic inlet, it can be felt. If still in the uterus it may not be determined 
by raginal palpation. In palpating note the condition of the pelvic eanal, whether constricted, tumors present, etc.

Diagnosis. - If the labor pains are normal and no impediment to the passage of the roung through the pelvic canal is apparent, and the animal is in good physical condition, we should allow more time to elapse before assuming it to be a case of drstocia. Howerer, if the animal is weak, the general condition disturbed, labor pains absent, etc., we are justified in diagnosing dystocia.

Prognosis. - Favorable in most cases. Will depend upon (a) the condition of the animal, $(b)$ the length of time in labor, and $(c)$ the condition of the fetus and membranes.

Treatment. $-A$ thorough examination of the patient should be made at once to determine the proper treatment to use. There are three lines of treatment recommended in dystocia.

Medical.-This is indicated in cases when there is no apparent impediment to the passage of the fetus, and when the labor pains are weak and insufficient. Extract of ergot $(0.5-2.0)$ or pituitrin $(3.0-10.0)$ depending on the size of the animal may be given. These doses may be repeated in a few hours if necessary. P'ituitrin is being used quite suecessfully in such cases.

Forced Extraction of the Fetus.-Examination is made of the condition of the birth canal and the position of the fetus noted. Various forms of instruments have been recommended for this work. Perhaps the most satisfactory ones are the smallest forceps, a rather blunt vulsellum forceps, and a wire snare. These instruments should be thoroughly disinfected, and the ragina washed with an antiseptie and lubricant solution (ereolin, 2 per cent.; lysol, 1 per cent.). The methorl of manipulation will depend upon the position, presentation, and condition of the fetus. All manipulating with the instruments should be done earefully to avoid injuring the vaginal mueosa. An assistant who exerts pressure on the abdominal walls in a backward direction will often help in keeping the fetus in position until the instrument is firmly attacherd. Gentle traction should be useerl. This methorl when done carefully will often overcome the difficulț. 
Hysterotomy. - After examination of the patient it is found that it is impossible for the fetus to be born, or after the other methods of treatment have failed, it is advisable to perform hysterotomy as early as possible. Delay in performing the operation is often fatal on account of the infection in the uterus from resulting sapremia or septicemia.

The animal is anesthetized, placed on the table in a dorsal position, well hoppled. An incision is made through the abdominal walls in the median line just anterior to the pubis and extended forward about three to four inches. The uterus will at once be seen as a voluminous body. It is withdrawn carefully from the cavity well surrounded by sterile gauze to prevent fluids from flowing back into the abdominal cavity. An incision is made through the walls of the uterus of sufficient size to allow the fetus or fetuses to be withdrawn. If there should be any fetus in either of the cornua they can be removed through the same opening. The membranes and any other material should be removed from the uterus. The incision in the uterus is closed with Lembert sutures and returned to the abdominal cavity. The abdominal incision closed as usual.

General stimulants should be administered following the operation.

Hysterectomy is advisable in cases where there is evidence of puerperal infection. (See Chronic Metritis.) 


\section{CHAPTER VII.}

\section{DISEASES OF THE VAGINA AND VULVA.}

Examination.-It is possible to make a thorough examination of the vagina and vulva by direct inspection. The animal should be placed in a dorsal position with the hind limbs hoppled forward. The vulva can be inspected directly by separating the labia with the fingers. The condition of the mucosa should be observed, any wounds or injuries carefully examined to determine their depth and extent. Note the color of the mucosa. It should be remembered that in the bitch there is often a normal pigmentation of the mucosa which should not be mistaken for some diseased condition. Papillomata and fibromata are commonly found at the juncture of the skin and mucous membrane. The vagina may be inspected by using a speculum to dilate the vulva and a portion of the vagina so that the mucosa can be seen. It is best to use an artificial light with a reflector to observe the mucosa farther into the pelvic canal. The mucosa should be examined for wounds and injuries which are common sequels to dystocia, inflammation, tumors, prolapsus, constrictions, etc.

\section{CONGENITAL MALFORMATIONS.}

Various forms of malformations have been observed in the bitch and cat. Stenosis of the vagina is seen occasionally. Imperfect development of the vulva, vagina and anus during fetal life has been noted. These conditions may interfere with copulation and impregnation. When found an attcmpt should be made to correct them surgically.

\section{VAGINITIS AND VULVITIS.}

Definition.-An acute or chronic inflammation of the vagina and vulva. 'These are very common conditions found in both the bitch and cat. 
Etiology. - Mechanically there are a number of conditions which bring about inflammation of these parts. Anything causing bruising of the mucosa will result in an inflammatory condition varying in degree and depending upon the extent of the injury. Iacerations and abrasions of the mucosa make possible the entrance of bacteria with resultant inflammation. I uring dystocia, wounds, and lacerations of the vulva and vagina are very common from rough manipulations and sharp instruments. At this time the animal's general resistance is materially reduced and serious infection and infiltration of the tissues can take place. Infection is frequently introluced by infected fingers or instruments. Another factor of importance in these cases following dystocia is the fact that in the extraction of the fetus, or the discharge of the secretions from the uterus, the vagina is further exposed to infections material.

Foreign bodies finding their way into the ragina will produce inflammatory conditions clepending upon the kind of foreign body and the extent of the injury done by the same. 'Tumors in the vaginal wall usually produce a chronic inflammation.

Symptoms, - Acute inflammation of the vulva is apparent from the swelling of the labia and the congestion of the mucous membrane. The color of the nucosa is at first red, later of a bluish, or greenish-black, depending upon the stage of the inflammatory process. It first there is no discharge, but later a murcous or mucopurulent disclarge is observed. In acute vaginitis the murosa will be red in color, swollen, and in most cases the seat of the injury or infection will be observed. The animal will be restless, of ten shows symptoms of straining as if to urinate. General symptoms of elevation of temperature, disturbances in the circulation and respiration are often observed from the absorption of the toxins, or the presence of micrö̈rganisms in the blool. In chronic vaginitis the principal simptom is the chronic, whitish, purulent discharge from the vulva. Examination reveals the chronic inflammatory changes of the mucosa of the vagina and vulva. In most of these cases it is rifficult to make a distinction between the two ronditions as they are nearly always associated except in injuries. 
Diagnosis. - This is made by a direct inspection of the mucosa.

Prognosis. - Favorable in most cases, unless general symptoms of septicemia are present, or in some cases of extensive laceration of the mucous membrane.

Treatment.- The parts should be thoroughly cleansed with mild antiseptics (lysol, 2 per cent.), followed by weak solutions of astringents (silver nitrate, 0.25 per cent., or silver citrate 0.5 per cent.). These applications should be made daily.

In case any foreign bodies are present, they should be removed and antiseptic treatment applied. When the vulva or vagina is found lacerated the extent of the wound should be determined by probing, all loose fragments removed with the scissors, and antiseptics used as above.

In gangrenous conditions of the vulva or mucosa, all such portions must be removed promptly, and the surface treated thoroughly with antiseptic solutions. It is sometimes necessary in recent wounds of the vulva to apply one or more sutures to properly approximate the torn edges to prevent improper union.

\section{PROLAPSE OF THE VAGINA.}

A true prolapsus of the ragina is uncommon in small animals. A hypertrophic condition of a portion of the mucosa which protrudes through the vulvar opening is frequently mistaken for prolapsus. This, however, is not a true prolapsus, but inasmuch as it simulates the condition, it will be described with prolapsus.

Etiology. - Prolapse of the vagina often results from injuries during copulation, the penis, which is forcibly withdrawn before ejaculation has taken place, pulling the vaginal mucosa out with it.

Results also from serere straining, and from inflammation of the mucosa. Hypertrophy of the mucosa is observed frequently in some of the larger breeds, Great Danes, St. Bernards, and becomes a chronic and especially prominent following the estrual period. It consists of simply a chronic - inflammation which is most marked during estruation. 
Symptoms. - The condition is characterized by an enlargement appearing at the vulvar opening. At first the prolapsus is a red, congested mass, which later, on exposure, becomes dark in color and gangrenous. In hypertrophy of the mucosa it appears as a rounded enlargement of rather firm consistency projecting through the viulvar opening and coming from one side of the vaginal wall. In some cases it will remain outside of the vulva, becoming dark in color and gangrenous on the surface; or it may not protrude beyond the vulvar opening and appear only at intervals. Usually there are no general symptoms. There may be some interference with micturition.

Diagnosis. - This is not difficult in most cases. A thorough manual examination should be made to determine the exact conditions present.

Prognosis. - Favorable.

Treatment. - In prolapsus of the vagina an attempt should be made to replace it. The parts should be cleansed, disinfected and if there is much congestion, astringents (alum, 2 per cent.) may be used. In some cases this will be sufficient, in others when there is a recurrence of the condition without necrosis or gangrene, it should be returned and if necessary held in place by temporary sutures through the labia of the vulva. They should be placed so that the urine can be voided. Remove them in twenty-four to forty-eight hours. When gangrene sets in, amputation of the prolapsed portion becomes necessary. This is done by grasping the mass and withdrawing it until the normal mucosa appears. If the prolapsus involves only a portion of the circumference of the vagina it may be ligated. The ligature should be inserted through the base of the mass and drawn securely to control hemorrhage and stop absorption. In case the vagina is prolapsed, throughout its whole circumference sectional suturing will be necessary. Care must be taken in all cases to avoid injuring the urethra. In hypertrophy of the mucosa amputation of the mass is necessary. 'This is done by thorough cleansing and disinfecting the parts, withdrawing the mass from the vulva, and ligating through its base. 'The mass should be removed with the knife or scissors and the 
stump returned to the vagina. The vagina should be cleansed daily with antiseptics until the discharge has ceased.

\section{RUPTURE OF THE VAGINA.}

I) uring dystocia from rough manipulation, or from sharp instruments the vagina may be torn or ruptured making at once an opening into the abdominal cavity. This will allow septic material to gain entrance which usually produces peritonitis. In some cases after rupture of the vagina, and when straining is induced, or still present from the dystocia, there may be a prolapsus of the bladder through the rent. When this occurs the bladder becomes displaced and projects from the vulva. It will be recognized as a fluctuating enlargement appearing suddenly between the labia of the vulva. An exploring trocar may be used to determine its contents if the diagnosis is in doubt. Laparotomy should be performed at once (see Laparotomy) and the displaced organs returned to their normal position. If possible close the opening in the vagina. If septic infection has developed little can be done.

\section{TUMORS OF THE VULVA AND VAGINA.}

The majority of the neoplasms found in the vulva and ragina are benign growths consisting in most instances of fibromata, papillomata, or a mixture of fibromata with myxomatous, myomatous, or lipomatous elements. Malignant tumors are uncommon in the ragina, but do occur occasionally as secondary growths, or in the form of venereal granulomata.

Fibromata.-These are found in most instances projecting from the walls of the vagina, or from the cervix. 'They' appear as hard, firm enlargements, usually smooth on the surface, show no tendency to degencration or necrosis, except when they project through the rulva and become irritated from exposure.

Diagnosis.-Made by the character of the enlargement, its slow growth and finally by a microscopic cxamination.

Prognosis.-Favorable in most cases. 
Treatment. - Complete amputation should be done as early as possible. Ligation, the same as for hypertrophy of the mucosa, is perhaps the most satisfactory method.

Papillomata.-'These occur at the juncture of the skin and inucous membrane of the vulva. 'They appear as small, rounded, pedunculated (usually) tumors. 'They often have a roughened surface.

Treatment.-Papillomata should be removed with the scissors, and the bases cauterized with silver nitrate. They rarely reappear.

Sarcomata.-These are found occasionally on the vaginal. mucosa. They are characterized by their rather rapid growth, uneven surface, and tendency to spread to adjacent structures. If possible a small section should be obtained for microscopic examination.

Treatment.-Treatment is unsatisfactory and should not be attempted.

Venereal Granulomata. - Venereal granulomata have been described under venereal granulomata in the male animal. In the female they appear on the mucosa of the vulva and at the posterior portion of the vagina. They consist of progressive neoplasms varying in rapidity of growth.

Treatment.-Complete removal should be attempted, except in very adranced cases. 


\section{H A P TER VII I.}

\section{DISEASES OF THE MAMMARY GLANDS.}

Examination.-These glands may be examined by observing their size, condition, and by palpation to note their consistency or the presence of wounds, inflammation, abscesses, etc.

\section{WOUNDS AND INJURIES OF THE MAMMARY GLANDS.}

In the bitch and cat, wounds and bruises of the mammary glands are quite common. They result in most instances from being run over by vehicles, by falling, from bites of other animals, or the glands may be punctured by sharp objects. The degree of injury will vary greatly. In some cases it will consist in a simple, slight contusion of the glandular substance; in others bruising with hemorrhage into the gland producing a hematoma, and in some cases abscesses result. A careful examination should be made to determine the degree and extent of the injury.

Treatment.-The treatment will depend to a great extent on the condition of the glands. In slight contusions but little treatment is necessary, while in more extensive bruising, warm applications should be applied in the form of a warm antiseptic pack containing lysol (2 per cent.). In open wounds their extent should be determined and treated with antiseptics. An antiseptic pack may be applied when the animal interferes with the wound by biting or licking it excessively.

When abscesses derelop in the gland, they should be opened freely to allow good drainage and the wound treated as above. In most cases recovery takes place promptly. If fistula or necrosis of the gland should develop, it may be amputaterl. 


\section{CONGESTION OF THE MAMMARY GLANDS.}

A normal condition occurring at the end of the gestation period, and during lactation. It has been observed in nonpregnant and virgin animals. The glands become enlarged, hot, and sensitive. No treatment is necessary.

\section{MAMMITIS. MASTITIS.}

Definition.-An inflammation of the mammary glands. The inflammation may involve one or more of the glands. Mastitis is a common condition in bitches and cats shortly after parturition.

Etiology.-In the majority of cases mastitis is due to infection. Pyogenic organisms enter usually through the teat canal to the acini of the gland, and from this point spread to the perilobular lymphatics.

Premature removal of the young seems to be a predisposing factor as it permits the milk to collect, congesting the gland as is often noted in cats. Streptococcus infection of the mammary glands of the cat has been quite often observed. It frequently has the appearance of a specific disease appearing as an enzoötic in catteries. Wounds and contusions of the gland will produce mammitis and the degree will depend upon whether or not there is infection. Chronic mammitis is observer occasionally in the bitch and cat, resulting either from acute mammitis, or occurring independently.

Symptoms. - One or more of the mammary glands are swollen, hot, and very sensitive on palpation. As there are no milk cisterns in the gland, the condition extends immediately to the glandular tissue producing marked inflammation and edema of portions of the gland. On palpation the portion affecterl can be detected. Later as the infection develops the milk will be found changer into a grayish, purulent mass sometimes mixed with bloor. Abscesses, often multiple, frequently develop. 'They open and discharge a reddish, purulent mass. General symptoms are quite marked in some cases, especially in cats. 'The toxins absorher prorhe general intoxication which is often fatal. 
In the chronic form the glands become indurated and the nilk canals obliterated. 'The gland tissue becomes fibroid in character.

Prognosis. - The prognosis is favorable in animals other than the cat. When general symptoms are present the prognosis should be guarded.

Treatment.-The milk is removed, and the glands thoroughly massaged to remove as much of the infective inaterial as possible. Hot antiseptic packs or fomentations of hot antiseptic solutions should be applied. These should be changed every few hours if feasible. Avoid coal-tar products in cats.

Should abscesses derelop in the glands they are incised to grive free drainage. Follow with antiseptics. Small doses of castor oil, or magnesium sulphate are indicated to assist in the elimination of toxins. In chronic mammitis with fibrosis which may also involve the teat, it is best to remove the gland. An anesthetic should be given, and the animal placed on the table in the dorsal position. The hair is shaved from around the gland and the skin thoroughly disinfected. The gland is then dissected out, which is not difficult, and the vessels ligated. The skin should be trimmed so that the edges approximate accurately. A regular bitch bandage is applied to protect the wound. Recovery is prompt.

\section{TUMORS OF THE MAMMARY GLANDS.}

Neoplasia of the mammary glands is of frequent occurrence in bitches. Both benign and malignant types are inct with in practice.

Benign Tumors.-Fibromata. - A very common form of benign tumor found in the mammary gland of bitches. It consists of fibrous tissue elements proliferating around and into the glandular acini, isolating portions of the gland cells. Their derclopment is slow hut progressive, ultimately involving the entire gland. The tumor may appear as a pure fibroma or be mixed with other varieties. 
Symptoms.-Fibromas occur as hard, sharply defined enlargements which show no inflammatory symptons nor tendency to degenerate. The size of the tumors varies from a pea to several inches in diameter. When mixed with other tumors, they are softer and take on somc of their characteristics.

Prognosis.-Favorable.

Treatment.-Extirpation of the gland.

Lipoma.-Fatty tumor found in old bitehes, especially in those afflicted with obesity.

Symptoms.-They are soft, well-defined tumors of the gland.

Prognosis.-Favorable.

Treatment.-Removal of the gland is advisable.

Malignant Tumors. - Carcinomata. - A very common form of malignant growth found in the mammary gland. 'They' are frequently mixed tumors, appearing as adenocareinoma, fibrocarcinoma, etc.

Symptoms.-Carcinomas are characterized by their growth, slow at first but with sudden, rapid development, lobulated appearance, and tendency to degeneration and abseess formation. A small portion of the tumor should be examined mieroscopieally.

Prognosis.- Enfavorable as they are apt to recur.

Treatment.- Removal of the gland should be done as early as possible. When metastasis has taken place, no treatment is successful.

Sarcomata. - Sarcomata occur usually in conjunction with other varieties of tumor, as fibrosarcoma, adenosarcoma, ctc.

Symptoms. - Sarcomas develop rapidly witl acute, inflammatory simptoms.

Diagnosis.-A diagnosis can be made only by microscopical examination.

Treatment. - Same as for carcinoma.

Other varieties of tumors which involve the mammary glands are rare and of minor importance. 


\title{
PART V. \\ DISEASES OF THE BLOOD AND BLOOD PRODUCING ORGANS.
}

\author{
CHAPTER I.
}

\section{ANEMIA.}

Definition. - A reduction in the total volume of blood or of its corpuscles, oligocythemia, or of certain of its more important eonstituents, such as albumin and hemoglobin. Two forms of anemia are reeognized, viz.: (a) Acute, and (b) chronic.

Occurrence.- Tery frequently observed in dogs and cats. The most common form is the acute. The ehronie form following various diseases is also of eommon oecurrenee.

Etiology.-- (a) Many cases of acute anemia are the direct result of loss of blood. 'The condition develops rapidly' following epistaxis, intestinal hemorrhage, rupture of bloodressels in the lungs, hemorrhage of the uterus, parenehymatous hemorrhage, or any external, severe hemorrhage.

(b) Chronic anemia develops slowly and gradually. several different causes are found producing this type: Insufficient food, or food of poor quality in whieh the essential nutritive elements are deficient; diseases of metabolism in whieh the nutritive proeesses are modified and the food elements not utilized in the body. In small animals anemia often follows diseases of the digestive tract (eatarrhal inflammation) producing either a loss of appetite or an interferenee in the digestion and assimilation of the food. This may 
occur following distemper in dogs, presenee of parasites in large numbers, or other diseases affeeting the mueous membranes in a similar manner. Large numbers of ehickens are sometimes found with anemia from infeetious asthenia, and other diseases of the digestive traet. Parasites of the blood are oceasionally the eause. Young chicks are frequently affeeted with white diarrhea resulting in a severe form of anemia. Many of the general diseases are aeeompanied by anemia.

Pathology.-The most eharaeteristie feature of acute anemia is the paleness of all the tissues and the absence of blood. The respiratory passages show evidence of lack of blood by their pale, pink eolor. The heart and bloodvessels are only partially filled with a loose eoagulated blood. In chronie anemia the membranes are found pale and eolorless, the blood present in the vessels and tissues low in coloring matter, and usually redueed in total volume. Fatty degeneration of the heart, liver, kidneys and other organs is often observed. Owing to the general weakness and emaeiation, transudation of serum takes plaee and is found present in the thoraeie and abdominal eavities in varying quantities. Lesions of the primary condition produeing anemia are often apparent.

Symptoms.-(a) Acute Anemia.-The symptoms of aeute anemia, when due to hemorrhage, come on suddenly, and depend upon the amount of blood lost. 'The patient beeomes very weak, may be unable to stand, or if standing does so with difficulty. In attempts at walking the animal will show museular ineö̈rdination and frequently falls down. Depression, subnormal temperature and inereased respirations are prominent symptoms. The mueous membranes are pale or colorless and the leart aetion may be very weak or impereeptible. In some instanees evidenee of the hemorrhage will be present by the discharge of bloor either from the nasal passages, mouth or wound. Many cases terminate fatally in a few minutes, or hours, when the hemorrhage is severe.

(b) Chronic Inemia.-In ehronic anemia the symptoms (ome on more slowly and gradually with more or less emaeiation and general debility. The hair coat or plumage beeomes 
rough, and the eyes sunken. The animal is easily fatigued, and unsteady in its movements. It will lie down much of the time and refuses to move or get up when called. The mucous membranes are pale or colorless. The heart action is irregular, the pulse weak, and the respirations shallow and accelerated. The appetite becomes variable and entirely suppressed in some cases; in others it is retained but weakness and emaciation continue. The blood when examined will show a reduction in hemoglobin content and the number of red corpuscles diminished in proportion to the number of white. Later as the disease progresses edematous swellings are found along the abdomen, under the neck, and on the limbs. Other symptoms may be present depending upon the complicating conditions present.

Course. - The course of acute anemia is short, lasting in the majority of cases only a few hours. In the chronic form the course is much longer lasting for several weeks or months. In this case the course will depend very largely upon the cause of the condition.

Diagnosis.-The diagnosis does not present any difficulty. This is particularly true in the acute form. A differential diagnosis is necessary in some cases to distinguish it from diseases of the heart, or leukemia. In the former the examination of the heart will reveal the difference, while in the latter case an examination of the blood will at once make clear the distinction between the two conditions.

Prognosis.-The acute form, providing the animal does not succumb from the hemorrhage, will disappear promptly. The elements of the blood will soon be normal from the drinking of large quantities of water and ingesting nutritious foods.

In the chronic form the prognosis is not considered very favorable. It will depend largely upon the primary factor producing the anemia. A careful examination should be made in all cases to determine if possible the actual conditions. Many patients will recover completely after the elimination of the causative factor.

Treatment.-In acute anemia when hemorrhage is taking place an attempt should be made to arrest it at once. If it is 
external, the vessels should either be ligated or, if this is impossible, pack the wound and apply a bandage to compress the vessels. Internal hemorrhage may be controlled by administering hemostatics. Adrenalin chlorid solution (11000), using 1-2 c.c. intravenously. This may be repeated in thirty minutes if necessary. Ergot and fluidextract of hydrastis may also be used.

Rectal injections of normal salt solution are recommended. Inject the solution as high up into the rectum as possible. The solution should be at or near the body temperature. Intravenous injection of salt solution in small animals is unsatisfactory. In the chronic form determine the cause of the condition if possible and apply treatment to correct it.

The diet is an important thing in the treatment of anemia. Foods should be given that are rich in protein. Meat and meat scraps, milk and eggs have proved of great value. These substances should be given frequently and in small quantities to obtain the maximum benefit. Later the amount and the time betwecn the feeding periods can be increased. Numerous medicinal preparations have been used, but the iron compounds have given the best results. Iron and quinin citrate in doses of $0.1-0.5$ twice daily are of great value as a general tonic and altcrative. Reduced iron, saccharated carbonate of iron, and sulphate of iron have been used successfully. Small doses of these preparations can be given for a few weeks if necessary. Fowler's solution of arsenic in doses of 0.1-0.S daily is excellent. Carlsbad salts should be given in small doses along with the iron preparations. Good hygienic conditions should always be observed.

\section{LEUKEMIA.}

Definition. - A disease characterized by an increase in the white corpuscles, together with changes in the spleen, lymph glands, or bone-marrow. Two forms are recognized, viz.: (a) Myelogenous, and (b) lymphatic. The distinction is based on whether there is an increase in the leukocytes or lymphocytes. Combination of the two conditions may be 
found, or variations and degrees of either of the two forms may be present.

Occurrence.- This disease occurs most frequently in dogs, oceasionally in cats.

Etiology.-Nothing is definitely proved relative to the cause of leukemia. The disease is probably of an infectious origin, but experiments conducted along this line have given negative results. Toxic agents have been given as the cause, as have injuries and various other factors.

Pathology.-On necropsy in leukemia it is often difficult to make a distinction between the two forms as the lesions of both oecur concomitantly in the majority of cases. It is characterized by enlargement of the spleen, lymph glands, liver, kidneys and distinct ehanges in the marrow of the bones.

The spleen is enlarged (in some instances three to four times its normal size), dense and often easily torn. Frequently nodules are seen projecting from its surface. The color is dark red, and on cut surface dry, and shows numerous whitish colored, enlarged follicles each of about the size of a wheat grain. 'The stroma of the gland and the capsule are thickenerl.

The lymph glands are found enlarged. They are harder or softer than normal, of a whitish or gray color, sometimes showing small red points over a cut surface. 'The surface when seraped gives off a yellowish, creamy material. The majority of lymph glands will be found affected. The bonemarrow is of a dark red or gray color, and soft consistency. On examination the bone-marrow will be found very rich in white corpuscles.

The liver is enlarged and show's numerous small nodules of tymphoid tissue. 'The kidneys are enlarged in the same manner. Simall nodes will be found throughout the serous membranes, lungs and other tissues in the body.

Symptoms.- The carly development of leukemia is usually not observed. Very often the disease is not recognized until the symptoms become prominent and it has reached the arlvanced stage. The early symptoms are very similar to those of anemia, and the differential diagnosis may be very 
difficult until decided changes take place either in the blood or lymphatic system. The mucous membranes are very pale or white in color, the animal becomes weak and edématous swellings may appear. The enlargement of the lymph glands comes on gradually and is found involving practically all of the palpable glands. The enlargements vary in size, but stand out in some cases very prominently. The glands are firm, non-painful and well circumscribed or defined. The glands in the submaxillary space, at the pharynx, chest and inguinal regions show most enlargement. The movement of the animal may be interfered with on account of the increase in size of the lymph glands. Respiratory disturbances may be present when the glands become large enough to compress bloodvessels or nerves. Extensive edemas arc often present from the same cause. Ascites is a common symptom in dogs from enlargement of the mesenteric glands. The spleen is enlarged but difficult to palpate on account of its position; the enlargement of the abdomen may be the result of the enlargement of the spleen or liver, or both. Percussion of the abdomen may assist in determining the character of the enlargement.

Owing to the involvement of the bone-marrow the animal is lame and shows stiffness and soreness in movement. Pressure on the long bones frequently shows marked sensitiveness and pain.

Characteristic alterations are found in the blood. It appears pale red, or even brown, indicating a reduction in hemoglobin. When allowed to stand and coagulate (which it does slowly) it separates into two layers, the lower consisting of red corpuscles, while the upper is composed of white corpuscles and fibrin. The number of white corpuscles is always increased; in some cases equal in number to the red ones. A decrcasc in the number of red corpuscles can be demonstrated in most cases. A differentiation may be made between the two forms of leukemia by the blood cxamination. In lymphatic leukemia the lymphocytes are found increased, while in myelogenous leukemia the leukocytcs are found in much larger numbers. Clinically the disease develops gradually, emaciation more prominent, and 
more or less extensive hemorrhages occur in the various organs.

Course.-The disease is usually chronic. The acute form is very rare in small animals. The course usually extends over a long period, and complications are common.

Diagnosis. - The characteristic involvement of the lymph glands, spleen and liver, and the increase in the number of white corpuscles, will make the diagnosis comparatively easy. A microscopic examination of the blood is necessary in order to make the diagnosis accurate. The differentiation between the forms of leukemia is determined definitely in this inanner.

Prognosis. - Very unfavorable. When the disease is once established there is little hope of recovery.

Treatment.-Owing to the pathological changes present not much can be expected in the way of treatment. In some cases the patient's general condition may be improved by allowing plenty of nutritious food and administering alteratives and tonics. Iron and quinin citrate $(0.2$ to 0.4 twice daily) have given the best results. Transfusion of blood has proved unsatisfactory in small animals.

\section{INFECTIOUS LEUKEMIA OF CHICKENS.}

Definition.-An infectious disease of chickens probably produced by an ultramicroscopic virus. It is characterized by an increase in the number of leukocytes, an atrophy of the marrow of the bones, and an increase in the size of the spleen and liver. In many cases the number of red blood corpuscles is reduced and the whitc ones increased.

Occurrence. - Lp to the present time the disease is found only in chickens. Other fowls are not affected. It often appears in an enzoötic or epizoötic form when large numbers in a flock or community will be affected.

Etiology.-From rather extensive investigations made with this disease it is evidently due to a virus found in the affected organs, viz.: The spleen, lymph glands, and bonemarrow. 'The disease is easily transmitted by intraperitoncal or intravenous injections, while subcutaneous injec- 
tions prove negative. Animals other than chickens are not susceptible to the disease.

Pathogenesis.-The method of development of infectious leukemia has not been definitely proved. The virus of the disease by irritation produces an increase in the number of white blood cells in the capillaries of the spleen, bone-marrow and liver.

Pathology. - The most pronounced lesions are found in the spleen, liver and marrow of the bones. The spleen and liver are much enlarged, and the bone-marrow reddened and congested. The lymph glands are also enlarged occasionally. The blood is lighter in color than normal (less hemoglobin) and contains a larger proportion of leukocytes than normally. General anemia is apparent by the wasted tissues.

Symptoms. - The period of incubation is approximately thirty to sixty days. The disease develops very slowly and insidiousty. Some cases may assume an acute form, the birds succumbing in about two weeks. The early indications are anemia as indicated by the paleness of the comb and wattles and a decided loss in weight. Later as the disease eontinues there is a tendeney for hemorrhages to take place even from slight wounds, indicating a hemorrhagic diathesis.

Examination of the blood reveals the true condition. There will be found a great increase in white corpuscles and a naterial reduction in the number of red. The latter may be reduced to one-fourth their normal number. The hemoglobin content of the blood is also reduced giving it a pale color and reducing its staining properties.

The condition when chronic develops gradually and the chicken often lies from exhaustion.

Course.- The course of the disease is quite variable. Many cases apparently run a very rapid course after the appearance of the first symptoms, lasting from one to thrce weeks. Others may linger longer or from one to four months.

Diagnosis. - The diagnosis can be establisherl only by necropsy and a nieroscopie examination of the blood to determine the ratio of the white to the red corpuscles. I)ifferential diagnosis may be difficult, but considering the lesions 
and the fact that a number of fowls may be affected at the same time, assist in arriving at the diagnosis.

Prognosis.-Very unfavorable. Recoveries are very rare.

Treatment. - No treatment has proved of any value. Dis-. infection of the premises and destruction of all affected birds are advisable.

\section{PSEUDOLEUKEMIA. HODGKIN'S DISEASE.}

Definition.-A disease characterized by a progressive enlargement of the blood-forming organs (spleen, liver, lymphatic glands) and nodular growths in these and other organs. It resembles leukemia in many respects. A notable exception is that the white corpuscles are not increased as in leukemia.

Occurrence.-The disease appears most commonly in dogs, cats and chickens. It is more common, however, in dogs and chickens than leukemia.

Etiology.-The true character of the disease is not known. The etiological factors are believed by some authorities to be identical with leukemia. Others are not in accord with this belief.

Pathology.--The lesions found in pseudoleukemia resemble very much those found in true leukemia. Enlargement of the spleen, liver and lymph glands is observed in most cases.

Symptoms.-Progressive anemia and enlargement of the lymph glands are the prominent early symptoms. Examination of the blood will show that the ratio between the red and white corpuscles is nearly nornıl. Other symptoms are practically the same as in leukemia.

Diagnosis. - A diagnosis can be made only by a microscopic examination of the blood. It should be differentiated from leukemia, malignant tumors and tuberculosis.

Prognosis. - I'nfavorable.

Treatment. - Treatment is unsatisfactory. Iron and quinin citrate $(0.2$ to 0.4 twice daily), or Fowler's solution of arsenic (0.2 to 0.6 once daily) may be tried. Potassium iodid in sinall doses is also recommended. 


\section{HEMOPHILIA.}

A constitutional defect in which there is a tendency for uncontrollable hemorrhage from slight wounds. It may occur after slight injuries, congestions, or apparently spontaneously. The coagulation of the blood is retarded or absent. Hemophilia is very rare in animals. For further information the reader is referred to other works.

\section{SCURVY. SCORBUTUS.}

Definition.-A disease characterized by marked debility and weakness, spongy and ulcerative condition of the gums with bleeding and a tendency to hemorrhage in the various organs.

Occurrence.-Scurvy is not very common in animals. A few cases have been found in dogs. Ulcerative stomatitis is often mistaken for true scorbutus.

Etiology.-There are several factors which contribute to the production of this disease, viz.: (a) The diet, when restricted to certain foods for a comparatively long time, will produce the disease by disturbing general metabolism, the body being deprived of food elements necessary for its normal maintenance. (b) Unsanitary conditions, such as damp cellars, badly kept kennels, exposure to cold and dampness are important predisposing causes. (c) One of the most important causes in dogs is ptomain poisoning, resulting from eating spoiled meats, fish, etc. (d) There is some question as to the disease being produced by infection. Infection is, of course, a possible cause.

Pathology. - Small hemorrhages are found in the tissues and organs of the body. These are most noticeable under the skin, in the muscles, on mucous and serous membranes, in the joints, liver, spleen and kidneys. Along the margin of the gums will be found distinct ulcerative processes. The gums are dark, almost black, or reddened, and show separation from the teeth. Shreds of the membrane may be removed easily with the forceps. The spleen is enlarged and of a soft consistency. The lymph glands of the mesentery 
arc enlarged and congested. Changes in the blood may leave it thin and pale in color.

Symptoms. - Anemia and emaciation are early symptoms of the disease. The patient becomes very languid, and does not care to exercise or move about. The appetite is suppressed either partially or completely, and the thirst is increased. Marked changes are observed on the visible mucous membranes, those of the mouth showing the most pronounced lesions. The mucous membrane around the margin of the gums becomes discolored red, later dark bluish-red, and even almost black. Hemorrhages are present in many cases, or the least manipulation of the gums causes bleeding. The membranes become swollen and very sensitive to the touch. The patient when eating will often stop abruptly and show marked pain from the food irritating the involved membranes. Distinct ulcerative processes are observed in the later stages, the gingival membrane becoming separated from the teeth and often the teeth themselves become loose and fall out. Hemorrhages are also found on the conjunctival membranes, in the nasal passages, and under the skin.

Vomiting is a common symptom. The vomited stomach contents are often mixed with blood indicating gastric hemorrhage. Bowel discharges may also contain blood. The disease is progressive and the symptoms increase in intensity until the animal is exhausted or some complication, such as septicemia, pneumonia, or extensive hemorrhage develops.

Diagnosis. - A differential diagnosis is necessary in dogs in order to distinguish scurvy from ulcerative stomatitis. The main points of difference arc: (a) The absence of gencral symptoms and hemorrhages in ulcerative stomatitis. (b) 'The localization of the condition in ulcerative stomatitis, while in scurvy other parts of the body are affected. In long-standing cases of ulcerative stomatitis the differcntiation may be somewhat difficult.

Prognosis. - This will depend a great deal upon the progress the disease has made. If the cause can be removed in the very early stages, the prognosis is more favorablc than in 
cases where the ulcerative processes are well established. When general symptoms of anemia and cachexia are evident the prognosis is bad.

Treatment.-This disease can bc readily prevented when a variety of food is allowed and good sanitary conditions prevail.

The early indications in the treatment are to change and regulate the diet. Give the patient nourishing food, such as meat, milk, eggs, etc. Iron preparations (iron and quinin citrate, pulverized iron, saccharated carbonate of iron) are the most satisfactory for the anemia which is nearly always present. Tineture of nux vomica ( 0.3 to 0.5 daily) or tincture of gentian ( 0.5 to 0.8 twice daily) is useful as a tonic.

Local applications should also be used. Swab the gums and other affected mucous membranes of the mouth with tincture of myrrh once or twice daily.

When hemorrhages arc present they must be controlled by appropriate treatment.

\section{ANIMAL PARASITES IN THE BLOOD.}

\section{Filarida. Metastrongulina.}

Several species of animal parasites have been found infesting the blood and circulatory organs in small animals. 'The dog is most frequently affected. In several districts of the Inited States, ('anada and Mexico, the condition has bcen reported a number of times. 'The Dirofilaria immitis, Hematozoön Iewisi, Hæmostrongylus rasorum and the Spiroptera sanguinolenta are the most important ones found.

Dirofilaria immitis (Filaria immitis).-Occurrence.-This blood affection has been observed very commonly in the dog, particularly in Japan, (hina, and in some sections of North and south America. The Inited States is fairly free from the infestation, but it is quite possible that many cases have been overlooked.

Etiology.- The I)irofilaria immitis is a long, white worm, the male being 12 to $18 \mathrm{~cm}$., and the femalc 20 to $30 \mathrm{~cm}$. long 
'The posterior portion of the male parasite shows a curvature or spiral bending. The female gives birth to living embryo.

The inature parasites live in heart (right half) in the majority of infestations. The left heart and bloodvessels harbor them in some cases. The number of parasites vary from a few to several hundrerl. They are usually found entangled in a round ball-like mass. The females give birth to embryos which are each about $\frac{1}{4} \mathrm{~mm}$. long, and are set free in the blood in large numbers. The sexually mature parasites may be found at the same time in other parts of the body. The manner in which infestation takes place has not been fully proved. It is probably from the drinking water which contains the larve. These embryos reach the heart before they mature. They may be discharged from the animal either in the urine, nasal discharge, blood, or with the feces and contaminate food or water again.

Pathogenesis. - The mature parasites located as they are in the heart or large vessels produce more or less of a mechanical disturbance in the action of those organs, or direct interference in the flow of blood through them. Formation of emboli in the ressels, which often results, brings on various complications in part due to necrotic foci in the lungs or other organs. 'The presence of the embryos in the blood ultimately leads to anemia with leukocytosis.

Pathology.-On necropsy the right heart is usually found to be partially or completely filled with the parasites surrounderl by a coagulated mass of bloor. The endocardium is found more or less thickened and inflamed. 'The heart is often dilated, the walls thin, and some eases ruptured. Very frequently the lungs show small foei of necrosis, or nodules in the center of which embryos are found. Similar lesions occur in the liver, kidneys, skin and muscular tissues. General anemia is present.

Symptoms. - The symptoms will depend a great deal upon the number of aldult parasites and embryos infesting the circulatory system. When sinall numbers are present the animal may not show any particular manifestations. When the number is great, anemia and marked emaciation develop. Hemorrhages are proverl to appear in the mucous membranes, skin, lungs, intestinal tract and other organs. 
Various complications will be observed from the emboli affecting the different functions in the body. The most pronounced complicating symptoms are in connection with the heart, lungs and brain. The blood shows evidence of anemia and leukocytosis. There is absence of fever, and a gradual progressive anemia which may continue for several months.

Diagnosis. - A correct diagnosis can be made only by a microscopic examination of the blood for embryos. These are easily recognized by their shape and peculiar snake-like movements.

Treatment.--Very little can be done in the way of treatment. Nutritious food and iron preparations are recommended. Atoxyl in small doses has been used successfully to reduce the number of embryos in the blood.

Complicating symptoms are treated as they develop.

Hematozoön Lewisi.-This parasite is found infesting the circulatory system in much the same manner as the Dirofilaria immitis. Dogs are most commonly affected. A microscopic examination of the blood is necessary in order to establish the diagnosis. The embryos attach their heads to the cover-glass and move their bodies very rapidly when viewed under the microscope. Little is known concerning the life history of this parasite. It is supposed to be taken into the body from fleas and lice in which the larvæ of this parasite have been found.

Treatment is the same as for I)irofilaria immitis.

Hæmostrongylus vasorum. - This is a fine, thread-like worm infesting the right ventricle of the heart and the pulmonary artery and its branches. The male parasite is about 13-16 $\mathrm{mm}$. long, and the female $17-20 \mathrm{~mm}$. It forms small nodules in the lining membrane of the vessel wall and thrombi in the smaller vessels. The female parasite lays eggs which lodge in the finer vessels, which, like the embryos, lead to the formation of small nodes. 'The embryos migrate into the bronchi and are coughed up and taken in by other animals.

Symptoms. - The symptoms are mostly those of a progressive anemia and are not characteristic. 
Diagnosis. - The diagnosis is only made by finding the parasites on autopsy or the cggs and embryos in the expcctoration or blood.

Treatment.-Lnsatisfactory.

Spiroptera sanguinolenta.-This is a small, thread-like worm found in different parts of the body, but morc particularly in the heart and large arterial branches. It is of minor importance as the infestation is of rare occurrence in small animals. The symptoms are those of anemia and heart weakness and are not characteristic. A microscopic examination or a necropsy is necessary in order to confirm the diagnosis. 


\section{CHAP'TER 11 .}

\section{I)ISEASES OF' 'THE THY'ROII GLANISS.}

Examination and General Consideration.-Diseases involving these glands are of considerable importance in dogs in which animals all forms of goiter are found. Other small animals are very seldom affected. The two thyroid glands, one on either side of the neck, are situated in the upper third of the cervical region. They are normally small but easily palpable. When diseased they usually grow larger and tend to descend downward toward the thoracic inlet. The position of the diseased glands will vary, therefore, with their size and the breed of dog.

An important thing to be considered in connection with the thyroid glands is the presence of small glandules closely related to them. These glandules are often found in close contact with the thyroid glands occurring in the adjacent tissue or they may be quite a distance removed from them. They consist of small, nodular glands, composed of true thyroid or lymphoid tissue, and are considered accessory glands to the thyroids. Normally they are very small, but usually become much enlarged when the thyroid glands are involved. 'These small glandules seem to be closely' associated with the thyroid glands in function, and will replace to a certain degree the secretions necessary, when the true glands are discased or destroyed.

\section{CONGESTION OF THE THYROID GLANDS.}

At or near the time of puberty the glands are often found enlarged; during the estrual priods the same condition is eommonly observed. 'This temporary congestion will disappear in a few days in the majority of cases and the glands 
assume their normal size and condition. A temporary congestion may also occur from collars being too tight, or dogs pulling on the leash, which disturbs the circulation in the glands. Swelling of one or both glands may result from this and last for several days simulating goiter. As soon as the cause is removed the circulation will return to its normal state and the congestion gradually disappear. Direct injuries to the glands are not uncommon and congestion and edema result. Examination will often reveal the presence of a wound or a contused area. Permanent enlargement of one or both glands may result from injurics, due to fibrous formation.

\section{ACUTE THYROIDITIS.}

Definition.-An acute inflammation of one or both thyroid glands. 'This condition has been observed in dogs.

Etiology.-The majority of cases are secondary to other diseases, such as distemper, local infection in the adjacent tissue, or from direct injuries.

Symptoms.-The glands are first noticed to be prominent, enlarged, sensitive on palpation, and the local temperature elevated. In severe congestion the gland will often show pulsation. A differentiation should be made between this condition and the various forms of goiter. The sudden devclopment, local heat, and marked reaction in the glands will assist in making the differentiation. The history of the condition following distemper or injuries should also be taken into consideration in making the diagnosis.

Prognosis. - The termination of acute thyroiditis is usually favorable. The inflammation subsides and the gland resumes its normal function. Some few cases terminate in a degeneration and suppurative condition which may destroy a part or the whole gland. It is possible also to have the condition become chronic and produce a firm fibrous growth simulating some forms of goiter.

Treatment.-During the acute stage cold applications should be applied either in the form of cold water or ice packs. This followed by a lot Priessnit\% compress and continued until resolution takes place or degcneration and abscess 
formation oeeur. Should the function of the gland be disturbed for a prolonged period either potassium iodid in small doses or thyroid extract has proved to be of considerable value. When abscesses oceur they should be opened, drainage effected, and an iodin pack applied.

\section{GOITER. STRUMA. BRONCHOCELE.}

Definition. - A hypertrophy or enlargement of the thyroid glands which occurs sporadically. It is very frequent in dogs. In some instanees goiter is congenital and whole litters of puppies are affected. The enlargement of the glands in these cases may be of suffieient size to interfere seriously with parturition. Very often the glands will be six to ten times larger than normal, seemingly as large as the puppy itself. The lobes and isthmus of the glands are so intermingled that no distinction can be made between them, the whole forming one large mass in the inferior eervieal region.

The disease is also acquired in a great many eases, coming on at different periods in the development of individual animals.

Occurrence.-The occurrence of goiter in the various breeds of $\operatorname{dogs}$ seems to be in about the same ratio, although in certain districts pointers, bull dogs, and bull terriers, seem to be affected in larger numbers than other breeds.

Many forms of goiter are recognized in dogs, viz.: Parcnchymatous; $(b)$ cystic; $(c)$ fibrous; $(d)$ vascular; $(e)$ malignant; $(f)$ exophthalmic.

Parenchymatous Goiter.-Definition.-A diffuse parenchymatous enlargement of the gland with an increase in the stroma and a collection of gelatinous colloid material in the follicles.

Occurrence.-This form is by far the most common and occurs more frequently in puppies or young dogs.

Symptoms. - The first symptom noticed is the enlargement of one or both thyroid glands. 'The enlargement may not be equal in both glands. One is frequently much larger than the otler. This form of goiter often develops suddenly 
and the glands assume enormous proportions. Puppies are often born with this particular form or develop it during the first few weeks of their lives. In many cases no marked symptoms of any general disturbance are observed, the animal developing apparently normal except the presence of the enlarged thyroid glands. In other cases, when the glandular secretion is materially interfered with, cretinism and myxedema are prominent symptoms. This is evidenced by marked nervous disturbances and degeneration of the various tissues in the body. The animal becomes emaciated, weak, a mere shadow of its former condition. Young animals as a result of this glandular disturbance do not develop normally, the head may be larger than normal, and other parts of the body undeveloped, or vice versa. Respiratory disturbances often result from the enlarged glands pressing upon the trachea, or from compression of the vagus and sympathetic nerves. 'The latter condition no doubt accounts for the larger number of cases in which labored respiration and spasm of the glottis are prominent symptoms. Hemiplegia laryngis is produced in a similar manner by pressure on the recurrent nerve. ( irculatory irregularities are not so common in parenchymatous goiter. I)irect examination of the glands will reveal the enlargement, its smooth regular outline, moderately firm consistency, and absence of local heat or pain. The isthmus of the glands can be palpated, except when the glands are greatly enlarged. In many cases no general svmptoms of rleranged appetite, elevation of temperature, or cachexia will be noticed.

Diagnosis. - The diagnosis of goiter in general is quite easy, but in some cases it is difficult to make a definite distinction between the various forms. Goiter should be differentiated from abscess, hematoma, and various cyst formations. This (an be done as a rule very easily after carefully palpating the enlargement. In order to make a positive diagnosis of the variety or form of goiter present, it is necessary to consider the character of the enlargement, its consistency, the condition and age of the animal. Palpation of the glands to determine their form, whether regular and smooth, or irregular and lobulated, and their consistency, is a very 
important means of making the differentiation between the various forms of goiter.

Prognosis. - In parenchymatous goiter most cases recover. The glands grow smaller until of normal size or near it, and any general symptoms disappear. However, in cases where the glands are enormously enlarged and symptoms of cretinism and myxedema are present the prognosis is not favorable. A recurrence of this form of goiter, while rare, is always probable.

Treatment.-Iodin medication both externally and internally has proved to be of great value. Some remarkable results have been obtained in the rapidity and degree of reduction after its use. For internal administration it has been definitely proved that small doses give the best results. The dose should be regulated somewhat according to the age and size of the animal. One-fourth grain, 0.016 of potassium iodid for puppies, or $0.05-0.08$ for older animals, given once daily, has given the best results. When given in large doses there is danger of too rapid depletion of the body and paralysis of the heart. 'These small doses should be continued daily for one to three weeks. For external application colorless tincture of iodin may be applied. Where staining the hair does not matter the regular tincture should be employed. An application can be made daily, or cvery second day. Thyroid extract ( 0.15 daily) has been used with excellent results. Surgical interference has not proved successful in relieving this form of goiter. Complcte unilateral thyroidectomy might be tried in case one gland is enormously enlarged and the other nearly normal. The operation is performed under general anesthesia and strict antiseptic precautions. 'The gland is dissected out carefully and multiple ligation of the pedicle is necessary so that the tissue will not retract and allow the ligature to slip off. The gland is then removed about $\frac{1}{2}$ inch from the ligature and the wound packed with antiseptic gauze for a few days. The ligature is then removed cautiously and the wound covered with an antiseptic dusting power. It is very important that the wound be kept free from infection during the first few days, and the ligature kept in position to avoid fatal hemorrhage. 
Cystic Goiter.-Definition.-An enlargement of one or both thyroid glands, characterized by the formation of cysts which may be single or multiple. In cases of long standing, calcification of the cyst wall may take place. The cause of cystic goiter has never been clearly proved. When hemorrhage occurs in the gland follicles they are distended, certain changes in the contents result, and there is left a more or less clear serous fluid. Further, through disturbance in the glandular activity, from injury, nervous influences, or hyperemia, the circulation of the glands is abnormal leading to extravasation of serum into the follicles which may result in a permanent cystic condition. Cysts commonly complicate parenchymatous goiter.

Symptoms. - An enlargement of one or both of the thyroid glands is the most prominent symptom. Many cases develop rather suddenly while others require considerable time before the glands reach a sufficient size to become noticeable. As a rule, unless complicated with other forms of goiter, no general symptoms will be present. Should the cystic formation involve both glands it is quite possible that the normal function of the glands will be disturbed, then general symptoms will result. Pressure on the vagus, sympathetic, and recurrent nerves may lead to complications as in parenchymatous goiter. In the majority of cases the course of this goiter is chronic, and aside from the unsightly enlargement they produce, the animal will not suffer any inconvenience. Examination of the gland by careful palpation will reveal the soft, fluctuating swelling, the absence of inflamination, and a consistency differing from other forms of goiter.

Diagnosis. - The diagnosis can be made positive by the examination, and if necessary by the use of an exploring trocar to obtain some of the yellowish or clear serous fluid.

Prognosis.-Favorable when proper and prompt treatment is used. Further, as this is in most cases a local condition confined to the gland, remedial measures can be applied more satisfactorily.

Treatment. - This is of two kinds, viz.: (a) Medical, and (b) surgical. 
Medical.-Medical treatment in the form of potassium iodid (0.03-0.06) is of value to assist in replacing the loss in the normal secretion of the gland, especially when both glands are extensively involved. Thyroid extract in 0.15 doses daily ean also be used.

Surgical.-This eonsists in either aspirating the serous fluid from the eystie follieles and injeeting a small amount of Lugol's solution to stop further filling up, or opening the eysts and paeking the eavity temporarily with iodin gauze. After-treatment eonsists in the use of antisepties. The wound will heal leaving but slight enlargement.

Fibrous Goiter.-Definition.-An enlargement usually of one of the thyroid glands (rarely both) eharacterized by a hypertrophy of the stroma and an atrophy of the glandular tissue. It is most frequent in old animals. 'This form of goiter results in most eases from injuries, aeute and ehronie inflammation, and in a few eases no doubt from some of the other forms of goiter. The interstitial tissue is gradually increased exerting abnormal pressure on the gland substanee, which leads to pressure atrophy.

Symptoms. - The presence of the enlargement confined in most eases to one gland. Oeeasionally both glands will be found affeeted. 'The size of the enlargement varies greatly from very slight to extreme thiekening of the entire interstitial tissue. No general symptoms are observed in the majority of eases as it is eonfined to one gland and of long standing. When both glands are extensively involved, interfering with the funetion of the glands, emaeiation, weakness and general eaehexia may be prominent symptoms. Respiratory and other disturbances are only observed when the enlargement presses on the trachea or nerves.

On palpation of the gland it will be found exceedingly firm, resistant, smooth in outline, non-inflammatory, and non-sensitive. The isthmus of the gland is easily determined.

Diagnosis.-This should not be diffieult as the outline of the enlargement and the hard, firm consisteney, with the other symptoms, are characteristic of fibrous goiter.

Prognosis. - Favorable. Many cases, when not exceeringly large, are not treated as they eause but little ineon- 
renience to the animal. Reduction in the size of the gland is possible, or it can be extirpated.

Treatment.-Injections of iodin into the gland substance liave given good results. Inject iodin tincture (2.0 to 5.0 ) into the gland being careful to aroid introducing the needle directly into a bloodvessel. The needle should be inserted unattached to determine this fact before the injection is made. The injections may be repeated after the acute simptoms subside. It is sometimes necessary to make sereral applications before reduction is complete. Extirpation of the enlarged gland is recommended when of considerable size and the fellow gland normal. The operation is performed in the same manner as in parenchymatous goiter. When both glands are enlarged and general symptoms of athyrea are present, potassium iodid, or thỵroid extract in small doses is recommended.

Vascular Goiter.-Definition.-An enlargement of one or both thyroid glands, characterized by dilatation of the bloodvessels without the formation of new glandular tissue, and the absence of any marked general simptoms. This condition is not to be confused with exophthalmic goiter. It is the result of a local dilatation of the bloodressels supplying the glands and may originate from the same causes as those producing congestion and inflammation of the glands. 'The exact etiology is not known, but has been observed as a sequel to distemper, prolonged estrual periods, and sexual diseases.

Symptoms.- One or both glands will be found enlarged and engorged with blood. Distinct pulsation of the arteries and even the glands can be seen at a distance. In some cases rupture of some of the ressels results, the blood accumulating in the adjacent tissues, producing an extensive enlargement along the course of the neck. The srmptoms, as a rule, subside after a few weeks and may recur at more or less regular intervals. Nany cases recover spontaneously. Very little general disturbance is present.

Diagnosis. - The dilatation of the arteries and engorgement of the gland will easily differentiate it from all forms of goiter, except exophthalnic. In exophthalınic goiter, tarhycardia, 
and nervous disturbances present should make the clinical picture complete and different from vascular goiter.

Prognosis.-Complete recovery is possible in most cases. In the milder forms it often disappears without any treatment.

Treatment. - An examination of the animal should be made carefully and if any general disturbance is observed it should be treated. Owing to the increased activity of the gland locally, iodin preparations are contraindicated. Small doses of tincture of opium ( 0.2 to 0.4 daily) can be tried.

Should the glandular activity become too pronounced, as indicated by hyperthyrea, ligation of a portion of the blood supply to the gland would be indicated. However, in the majority of cases this is not necessary as the symptoms will subside in the course of a few weeks.

Malignant Goiter.-Definition.-An enlargement of one or both thyroid glands due to a malignant neoplasm. 'The malignant growth is either a sarcoma or carcinoma. The growth tends to invade the adjacent tissues and lymph glands, or even to the extent of involving secondarily the lymph glands of the thoracic cavity, the lungs, and other organs and tissues. The condition may become generalized. It is found mostly in old animals.

Symptoms. - The malignant growth may be confined to one gland, but in many cases occurs in both. When the enlargement once begins, its development is quite rapid, and is characterized by an uneven, irregular form of the gland, the presence of acute inflammatory symptoms, very sensitive, and in some cases areas of degeneration and abscess formation are found. Within a short time general symptoms are prominent. Emaciation and general cachexia develop rapidly: The rapidity of its development and the fact that it is usually an old animal affected would assist in making the diagnosis.

Diagnosis. - This is made by careful examination. 'The characteristic enlargement and condition of the gland with secondary involvement of adjacent lymplı glands, together with tle general symptoms of emaciation and cachexia, and 
the age of the animal, slould make the differentiation from other forms of goiter comparatively easy.

Prognosis. - Lnfavorable.

Treatment. - But little can be expected of treatment. In the early stages, before involvement of the adjacent tissues takes place, extirpation of the gland may relieve the condition for a time but no permanent results can be hoped for.

Exophthalmic Goiter.-Basedow's Disease. Graves's Disease.-Definition. - A disease characterized by exophthalmos, functional disturbances of the circulatory system, and more or less enlargement of the thyroid gland. This disease is not of frequent occurrence in animals, but is found occasionally in the dog.

Etiology.-The exact etiological factor has been in dispute, but is evidently a pure neurosis as indicated by some of the more recent investigations made. It has also been claimed that it is due to a central lesion in the medulla oblongata. A certain amount of evidence has been produced to partially substantiate this claim. The fact that the primary result of the disease is a hyperthyrea would indicate that it might be a special involvement of the glands. However, this has not been proved and the best evidence seems to prove the former etiological factor the most likely.

Pathology.-On examination the thyroid glands show enlargement, congestion, the production of newly formed tubular spaces and a collection of mucinous fluid. They show every evidence of hyperactivity.

Pathogenesis. - It is quite important from every standpoint to note that this form of goiter is a primary hyperthyrea while most forms are just the opposite (athyrea). 'The developinent of the disease is the result of the increased secretion of the thyroid glands leading to a general toxic condition. The iodothyroidin content of the secretion is greatly increased which no doubt accounts for the intoxication.

From an experimental standpoint much the same condition can be produced in animals by administering large and continued doses of thyroid extract. Further, when thyroid extract or iodin is administered in exophthalmic goiter the condition becomes rapidly aggravated. 
Symptoms.-13oth the acute and chronic forms have been observed. In the acute form the discase develops very rapidly. The following symptoms are most prominent:

(a) Exophthalmos.-A prominence of the eyes which may be unilateral or bilateral. This symptom is readily recognized by the protrusion of the eyeballs, and the prominence of the sclera of the eye. The lids do not cover the cye completely. - It is important to note that in some breeds of dogs the eyes are very prominent and this should not be mistaken for exophthalmos. The normal sight is retained in most cases. In severe cases it is possible to have a panophthahnitis which would destroy the sight temporarily or permanently. Llceration of the cornea is common.

(b) Enlargement of the Thyroid Glands.-. Some cnlargement of the glands is present in practically all cases. The common expression "inward goiter" has bcen given to this condition when the glands are not much enlarged and the other symiptoms are prominent. The enlargement may be general or orly one lobe affected.

(c) Tachycardia.-The heart action is rapid and the pulserate is usually so much increased that it cannot be counted accurately. The action of the heart at first is regular, but during the later stages of the disease becomes very irregular. The throbbing of the heart is often so intense as to shake the animal's body. Acute dilatation of the heart with dyspnea, cough, etc., is a prominent symptom in the later stages.

Distention of the arteries and pulsation of the entire gland is easily seen from a distance. On palpation a distinct throbbing can be felt. The gland may be more or less firm, or soft and flabby, depending on the degrec of involvement.

(d) Tremors.-Tremors or trembling of the muscles is of ten observed. General symptoms of restlessness, whining and crying, and spasms are present in some cases. Emaciation comes on rapidly, and general exhaustion is very commoll.

The chronic form is characterized by similar symptoms but milder in character and lasting for several wecks or months. 
Diagnosis.-The diagnosis is not difficult when all the symptoms are taken into consideration. A differential diagnosis should be made from the other forms of goiter, but aside from vascular goiter this should be easy. In rascular goiter the general symptoms are usually absent.

Prognosis. - Not considered favorable. Some cases recover rapidly under proper treatment.

Treatment.-Owing to the hyperthyrea present, iodin preparations and thyroid extract are contraindicated. The treatment indicated is the reduction of the hyperactivity of the thyroid glands, and regulating the heart action. The hyperactivity of the glands is best controlled by ligation of a portion of the blood supply to them which will immediately stop the function of that particular part and reduce the total volume of secretion. It is recommended that the anterior arteries be ligated. This should be done under strict antiseptic precautions and morphin anesthesia. Immediate good results are obtained in many cases. Extirpation of a portion of the gland is often done in the human subject but is not so practicable in animals. The heart action should be controlled by small doses of digitalis fluidextract $(0.1$ to 0.2 twice daily), or aconite tincture (0.1 to 0.2 two or three times daily). The animal should be kept in a quiet place. Other conditions arising must be treated accordingly. 



\title{
PART VI. \\ DISEASES OF MET'ABOLISH.
}

\author{
CHA P'TER I. \\ DISEASES OF METABOLISM.
}

\section{DIABETES.}

Definition.-Diabctes is a disease ordinarily characterized by an excessive secretion of urinc. It occurs in two forms: (a) I) iabetes insipidus; (b) diabetes mellitus.

Diabetes Insipidus. - Definition. - A chronic disease characterized by the passage of a large volume of urine of a low specific gravity and containing no albumin or sugar. There should be a distinction made between diabetes insipidus and polyuria. 'The latter is a symptom of some other disease. In polyuria, often confused with diabetes insipidus, the volume of urine passed is not constant. The disease is evidently a functional disturbance of the kidneys in which large quantities of water are voided and equal amounts taken into the system. The solid content of the urine is naturally reduced.

Occurrence.-1)iabetes insipidus is not observed very often, but perhaps the larger number of cases is found in dogs. l'olyuria is frequently seen in rabbits from improper feeding. ('ertain foods tend to produce it, which, if continued, may result in a chronic condition resembling diabetes insipidus. 'There is no doubt but that it is often not diagnosed.

Etiology. - Several things have been recorded as having to do with the causc of diabetes insipidus: (a) Observed dur- 
ing the convalescent stage of acute diseases (distemper).

Accompanying diseases of the nervous system, such as tumors of the brain, lesions of the medulla, cerebral hemorrhage, inflammation of the membranes of the brain or spinal cord. (c) Subjection to cold; drinking large quantities of cold water have been thought to cause the diseasc. 'Trauma of the liver; vertebral column. (e) The administration of diuretics either when given in large doses or continued over a long period of time in small doses will produce it. $(f)$ There is a possibility that the disease is of infectious origin; however, this has never been proverl.

The production of diabetes insipidus is no doubt due to a rasomotor disturbance of the renal vessels resulting in a great increase in the secretion of urine. 'The above montioned conditions lead to this disturbance through direct irritation, or to functional irritation of the center in the medulla, which brings about a constant state of renal congestion.

Pathology.-The necropsy findings are unimportant in the majority of cases. Quite often very slight or no changes in the tissues are found. In some cases the kidneys are enlarged and hyperemic. Dilation of the ureters and pelvis of the kidney's is occasionally seen. Certain changes will also be found in the ncrvous system, liver and lungs. 'The musculature is pale and anemic in advanced cascs.

Symptoms. - The disease may come on suddenly resulting from irritation to the nervous system or kidncys, or it may be gradnal in its development. In rabbits it may develop suddenly after feeding mouldy food, etc. Many cases are not observed until the symptoms of anemia and general eachexia are present. 'Two very prominent symptoms are: (a) Copious secretion of urine; (b) intense thirst.

(a) A large quantity of urine is passed each day; in dogs as much as two to four liters in twenty-four hours, and in rabbits one-fourth the quantity. The urine has a low specific gravity (1.001 to 1.003), is colorless, almost odorless, and voided without any difficulty execpt when the condition is accompanied by a catarrhal inflammation of the mucous membrane of the nrethra or bladder. The urine contains 
neither albumin nor sugar. Normal solids are very much rerluced in quantity.

(b) The animal will show intense thirst; large quantities of water are consumed each day, in dogs as much as four to ten liters. Often animals will show a vitiated thirst and will drink contaminated water or even urine. The appetite is usually normal during the first stages, but gradually diminishes and becomes variable. During the later stages the appetite may be lost entirely, the animal becoming einaciated, and various complications develop. The temperature remains normal until the very latest stages when it inay be subnormal.

Course.-When polyuria results from the food it may disappear in a short time following the change in food. This has been observed particularly in rabbits. In cases of real diabetes insipidus the course is chronic, the disease lasting one or two year's.

Diagnosis. - A differential diagnosis must be made between diabetes insipidus, polyuria and diabetes mellitus. Polyuria is distinguished from diabetes insipidus by the symptoms disappearing when the food is changed. The symptoms of polyuria are also more variable. Diabetes mellitus is distinguished by the increased specific gravity of the urine and the sugar content.

Prognosis. - While in polvuria the prognosis is usually favorable, in real diabetes insipiclus it is bad, the disease terminating fatally after a prolonged course.

Treatment. - An investigation of the food should be made, and if found of poor quality, containing moulds or other objectionable substances, it should be discontinued and other foods substituted. Regulation of the diet is an important factor during the early stages of the disease. Limiting the water supply does not have any appreciable effect on true diabetes insipidus, but if it is a simple polyuria it would be well to restrict the patient to a small amount of water.

Medical treatment has not been satisfactory. Small doses of ralerianate of zinc ( 0.2 to 0.4 ) rlaily, may be tried; $\mathrm{Ol}^{\circ}$ vasoconstrictors used, such as fluidextract of hydrastis, or fluidextract of ergot. Stomachics and general tonics nay be 
of some value, tincture nux vomica $(0.3$ to 0.6$)$, once or twice daily.

Diabetes Mellitus.-Definition.-Diabetes mellitus is a chronic, nutritive disorder in which grape-sugar accumulates in the blood and is excreted in the urine. The condition may be permanent or occur periodically. It is generally considered that in order for a case to be true diabetes mellitus, the sugar eliminated must be grape-sugar, and it must extend over a rather long period of time.

Occurrence. - The disease is not very eommon in animals. A few cases have been found in dogs. The cat and rabbit are very seldom affeeted.

Etiology. - The exaet etiology of diabetes mellitus is not known. It is quite evident that several factors may have to do with the production of the disease. Nervous diseases, especially those of the central nervous system, and diseases of the liver and pancreas probably produce the larger number of cases. The pathogenesis of diabetes mellitus is not quite clear, but the condition may be brought about by a disturbance of the liver or pancreas, in which the grape-sugar taken into the body is not converted into glyeogen but taken up as grape-sugar by the circulation. and eliminated in this form.

Pathology.- Many cases present no anatomical changes. 'There may be hemorrhages in the nervous system, tumor formations, etc. Very often fatty degeneration of the liver is present. The pancreas may be enlarged by eonnective tissue proliferation and an atrophy of the glandular substance. The other structures show cmaeiation and cvidences of eachexia due no doubt to the chronicity of the disease.

Symptoms. - Until the patient shows emaciation, notwitllstanding good appetite, the disease is usually not recognized. 'The general symptoms are: 'The animal fatigues easily, becomes dull and listless and loes not move about in a normal way, shows increased thirst and appetite. 'The mucous membranc of the mouth becomes dry, and gingivitis is often present. The most characteristic symptoms are found in eonnection with the urinary organs. 'The urine is increased in amount, sometimes louble the normal quantities voided, is palc, colorless, of a swectish odor (aceton) and acid in 
reaction. 'The specific gravity is increased (1040 to 1060). An examination of the urine should be made to determine the sugar content. From 3 to 5 per cent. of sugar has been found. The amount of sugar content will depend to quitc an extent on the kind of food given the patient. The feeding of carbohydrates materially increases the per cent. while nitrogenous foodstuffs diminish it. (For testing for sugar in the urine, see Diseases of the Kidneys.)

As the disease progresses certain other symptoms are uniformly present. Opacity of one or both cornea (keratitis), or of the lens (cataract) is often found, gradually producing blindness. Other portions of the eyc may also be affected. I"lceration of the cornea has been observed. Secondary involvement of the respiratory organs takes place in the form of a catarrhal inflammation of the larynx, trachea, bronchial tubes and lungs. Pneumonia and gangrene of the lungs may develop. Cardiac weakness is noted during the last stages. Other symptoms, such as vomiting, diarrhea and constipation, may be seen. I'lceration of the skin, falling out of the hair, found in some cases, indicate the general disturbance in metabolism. I uring the last stages the patient becomes very weak, emaciated, cachectic, and dies from exhaustion.

Course. - The disease is characterized by its chronicity and may last from several months to two years. Sugar is sometimes present in the urine for a long time before the disease is recognized.

Diagnosis.- This disease should be differentiated from diabetes insipidus. In the former the presence of sugar in the urinc, the gradual emaciation and the retention of the appetite would be sufficient to make a positive diagnosis.

Prognosis.- Is always unfarorable. The diseasc is a progressive one, in which complications follow each other making the condition hopeless.

Treatment.- Very little can be accomplished except by regulation of the diet and limiting the carbohydrates fed. Dogs should be ferl on a diet rich in fats and proteids (eggs, fat meat, or oat meal with fat meat). Large doses of sodium bicarbonate should be given (1.5 to 2.5$)$ daily to reduce the acidosis which is apt to develop. 'The amount may be 
increased if necessary. Other alkaline agents, such as ('arlsbad salts and ammonium carbonate, may also be administered. Tincture of opium in large doses (0.6 to 1.0) once or twice daily is highly recommended as it tends to reduce the amount of sugar. 'The treatment at best can only bring about temporary relief, and no permanent results can be hoped for.

It is important that the patient be kept quiet and its general strength maintained.

\section{OBESITY.}

Definition.-An excessive fat accumulation in the body which may be general throughout the entire organism, or eonfined to certain localized areas, such as under the skin. The condition is very commonly observed in small animals, particularly in dogs. ('ertain breeds, especially the smaller, are most subject. Castrated animals, especially if castrated after maturity, tend to become obese.

Etiology,-One of the chief factors in the production of obesity is the taking in of larger quantities of food elements than are utilized in the body. Pet animals which are fed highly nutritious foods (candies, cakes, etc.) and are not given sufficient exercise become over-fat. It often happens that an animal will aceumulate fat rapidly when only the normal amount of food is allowed. This occurs in most cases from insufficient exercise, the elements taken in not being utilizerl. 'The feeding of large quantities of earbohydrates, or restricting the diet to substances containing a large proportion of such elements will bring it about. The temperament of animals no doubt las also some influence. Animals having a plilegmatic temperament tend to become obese through insufficient activity and exercise. Castration of animals changes their disposition, tends to make them phlegmatic and lazy, and therefore fit subjects for the derelopment of this condition. Should animals be castrated hefore maturity this tendenry for obesity is not so noticeable.

Anemia ly reducing muscular energy may cause obesity, providing no organic lesion is present and the proper amount 
of food is allowed. 'The majority of cases can be ascribed to hyperalimentation and lack of exercise, rather than any constitutional disturbance of metabolism. 'Through the disturbance of glandular secretions (thyroid) and its regulation by certain organs (adrenals, pancreas, etc.) oxidation is diminished and constitutional obesity may develop.

Pathology. - No anatomical changes are noted except a general accumulation of fat under the skin, in the muscles, liver, around the heart and other organs. The changes in appearance of the organs and tissue are due to the fatty deposits.

Symptoms. - The condition is characterized by the rapid increase in weight, the change in contour of the body, and in the disposition of the animal. The most common locations of observable fatty deposits are in the panniculus adiposus, neck, shoulders and abdomen. Palpation of the parts will reveal the condition. The animal shows lassitude, fatigues easily, and often shows dyspnea on exercise. This is no doubt the result of impeded action of the organs from the fatty accumulations. Later, during the progress of the condition, disturbances of other organs and functions will develop. I)igestive disturbances are often quite pronounced and the heart action may be interfered with. The nucous membranes are pale, showing anemia.

Course. - The course is chronic. Mikd degrees of obesity do not interfere particularly with the animals' health or usefulness.

Prognosis.-Depends a great deal upon the extent of the condition. If the diet can be regulated and restricted, the prognosis is quite favorable. In pet animals, however, it is very difficult to restrict the diet as the owner will invariably break the rules laid down in this regard.

Treatment.-Reduction in the amount of food, systematic and forced exercise are important. At first the reduction in food should be slight but continuous until only a sufficient amount is allowed to maintain the strength of the patient. The composition of the foodstuff's given is also important. Limit the amount of carbohydrates and fats and allow a greater proportion of nitrogenous foods. A 
reduction in the amount of drinking water should be attempted.

The medical treatment consists in administering small doses of magnesium sulphate (5.0 to 12.0) daily, or Carlsbad salts (4.0 to 10.0 ) once or twice daily. Care should be taken to avoid a too severe laxative action over a long period of time. Thyroid extract is of value in increasing the oxidation of fats. Thyroidinum depuratum $(0.2$ to 0.75$)$, or iodothyrin (0.1 to 0.2 ) administered once daily has given the best results. 'The proteid food elements should be increased slightly when these medicinal agents are administered. Complications, should they arise, are given appropriate treatment.

\section{GOUT. PODAGRA. ARTHRITIS URICA.}

Definition.-A disturbance of nutrition with an excess of uric acid in the blood. It is characterized clinically by attacks of acute arthritis, a gradual accumulation of sodium biurate in and adjacent to the articulations, and with the development of irregular constitutional symptoms. 'The condition may involve the internal organs, or both the organs and the articulations.

Occurrence.-Old dogs are most commonly affected. Birds, especially chickens, pheasants and pigeons, are also common sufferers. Expcrience has shown that it is far more common in fowls than in any of the other small animals.

Etiology.-The real factors in the production of gout are not definitcly known. 'The following, probably predisposing causes, arc the most important: (a) The dict evidently plays an important role in the production of the disease. Foods rich in albumin, particularly nucleoproteids, seem to favor the development of the condition. Chickens and other birds when fed on mcat, and meat scraps, are often found witl the diseasc. Feeding offal from slaughter houses has the same effect. (b) Certain chemical substances, such as lead, chromic acid, corrosive sublimate, carbolic acid, acetone, etc., seem to have some effect in bringing on the disease. (c) It may be duc to the change in the metabolic processes. 
(d) Fungi on grains and grasses have been found to be the cause in fowls. (e) Diseases of the kidney's and ureters, in which there is an interference in the elimination of the uric acid compounds, have proved to be important factors in its production. ( $f$ ) Lack of exercise, confinement of the animals or birds, together with the feeding of highly nitrogenous foods are no doubt the cause of quite a number of cases of gout. Older dogs, and the larger breeds of chickens are most commonly affected.

Pathogenesis.-The development of gout is probably due to an increase in the production of uric acid, and an inhibition in the elimination of urates, which allows its accumulation in the blood, and in certain cases to be deposited in connection with the tissues. Other theories have been advanced for the development of the disease and have supporters. It is possible also to have the disease develop in cases when there is no increase in the formation of uric acid, but other diseases being present to interfere with the elimination of the uric acid compounds.

Pathology.-The characteristic lesions found in articular gout are enlargement of the joints of the toes, or wings, and a chalky-like deposit found in the joint proper or in the adjacent tissue. Examination of the bone-marrow, and the ligaments and tendons around the joint will often reveal the same condition. Several joints may be found affected. In visceral gout, the serous membranes of the cavities, the pericardium, air sacs, and the liver are covered with small nodules containing the same white chalky material. 'The kidneys contain small white foci, and the ureters are often found filled with a white, pasty mass. Examination of this material under the microscope will reveal the crystals of sorlium biurate.

Symptoms. - The first indications of the condition are usually enlargement of one or more joints, difficulty in walking about, and lameness. The joints most commonly enlarged are the wing joints (birds), and the metatarsal and phalangeal joints in dogs and birls. The animal will stand quietly and refuse to move, but when forced to do so shows considerable pain and lameness. Fowls often assume a 
crouching position standing on one limb or shifting from one leg to the other. Birds will walk or flap their wings with a minimum use of museles owing to the pain induced. Examination of the wings will show enlargement of one or more joints. At first the swellings are soft and sensitive, but later beeome more defined, firmer and not so painful. The size of the nodules will vary in different birds but is usually from that of a pea to a hickory nut. Considerable deformity of the joints of the wings and limbs may be present. The toes are often spread apart from the enlargement. In some eases the joints beeome anchylosed, while in other's degeneration takes plaee with neerosis and a diseharge of a whitishyellow soft mass. In the latter ease there will be an ulcerative proeess left which often exposes the joint. Small nodules are found in other locations in the skin, muscles, and tendons: The general symptoms of rough plumage, pendent wings, paleness of the eomb and wattles, and general emaciation develop gradually. The bird will frequently die from exhaustion.

Dogs show enlargement of the joints of the toes, metatarsus ribs, ete. Lameness and stiffness are quite prominent symptoms. A eareful examination should be made in all eases. Internal or visceral gout will not be recognized except when aceompanied by enlargement of the joints.

Course. - The disease is practieally always chronic. A few cases have been reeorded of the aeute form. The disease develops irregularly from one joint to another and therefore runs a protraeted eourse.

Diagnosis.-The charaeteristic symptoms of enlargement of several joints, mieroscopie examination of the contents of the nodules, and elemieal examination will make the diagnosis positive.

Treatment.-Dietetic.-Foods rich in nitrogenous substanees should be withheld. If neeessary reduce the total feed ration for a few days to a week.

Medical.-Alkaline agents, sueh as sodium bicarbonate should be used freely in the drinking water of the fowls. Give $\operatorname{logs} 1.5$ to 2.0 two or three times daily. The alkaline preparations will assist in the elimination of the deposited 
urates. P'iperazin is highly recommended to prevent the derelopment of the urates in the tissues (chickens, 0.2 to 1.0 ; pigeons, 0.1 to 0.4 ; dogs, $0 . \bar{j}$ to 1.0 ). Small doses are to be given three times daily and continued for several lays. Internal administration of sodium salicylate has been tried with fairly good results. Local applications of menthol, or salicylic acid should be used. Effect a solution and apply to affected joint on absorbent cotton. When the nodules become large they should be opened, curetted and an antiseptic pack applied.

\section{FEATHER EATING. FEATHER PULLING IN BIRDS.}

A condition observed very commonly in caged birds, canaries and parrots, but also in chickens, turkeys and pigeons.

Etiology.-(a) It may be the result of improper food. Feeding the same materials for a long period, or foods in which there is a deficiency of certain nutritive elements is a cause. Confinement of birds in close quarters predispose to it, partly through lack of exercise, and partly to a narrowing of the feed ration. (b) It is often considered a vice or habit. Fighting or picking at each other starts the vice. (c) Results from skin diseases, especially from parasitic conditions (lice, -fleas, mange mites). 'The irritation from the parasite leads to picking at the skin and feathers. Feathers will drop out as a result of certain diseased conditions.

Symptoms.-Birds are observed to be pulling out their own feathers or of those confined with them. Bald spots will be noticed on different parts of the body. Often large areas are lenuded. Careful observation of a flock reveals the situation. In skin diseases the feathers fall ont which can easily be distinguished from feather pulling. 'The skin lesions will assist in making the differentiation.

Treatment.-Birls confined, especially chickens, turkeys and pigeons, should be given freer range. ('omplete change of food will have an important bearing on overcoming the condition. Feed plenty of oyster shell, bone meal, meat scrap, dried blood, etc., with green foods to supply the 
elements lacking. Parrots and canaries are difficult to relieve from the habit. Change in the food, with plenty of green materials will often help. Examine them carefully to be sure they are free from lice, or other parasites. Bitter solutions applied to the feathers may stop it. Feather eating is difficult to control, but change of food as described above, and the administration of small doses of apomorphin hydrochlorid $(0.0005-0.001)$ have proved to be of value in a number of cases. 


\section{CHAPTER II.}

\section{I)ISEASES OF METABOLISM AFFECTING PIRIMARILY THE BONES.}

\section{RACHITIS. RICKETS.}

Definition.-A disease of young animals, characterized by impaired nutrition of the entire body and disturbanees and ehanges in the growtl of the bones leading to marked deformity.

Occurrence.-The disease is rather widespread, appearing in nearly all eountries. Animals in some eountries are very commonly affeeted, while in others the disease is not so prevalent. Young animals, as a rule, beeome affeeted during the first few weeks of life, or at about weaning time. The condition is observed more eommonly in dogs than in any of the other small animals. Cats and rabbits are not very often affeeted. Fowls occasionally suffer from the disease, espeeially ehickens and pigeons. It is commoner in the larger breeds of dogs and fowls, perhaps due to their more rapid growth during early life.

Etiology. - Various faetors have been given as the eause of raehitis: (a) I) ue to a defieieney of lime salts in the organism. 'This is no doubt one of the most important causes of the condition, as it develops most frequently in the breeds that grow rapidly, and at a time in the animal's life when there is apt to be a defieieney in the amount of lime salts ingested. Clinical and experimental evidence tend to prove the aeeuraey of this statement. (b) Improper assimilation of lime salts due to a laek in the acid content of the gastrie juice preventing the lime salts from being dissolved and eonsequently not absorbed. Foods rich in potassium salts reduce the hydrochlorie aeid eontent in the stomach leading to an insuffieient 
solution or absorption of the lime salts. (c) Digestive disturbances (gastric and intestinal catarrh) reduce the amount of lime salts absorbed by increasing the normal elimination of calcium compounds from the intestines. In young animals this condition of ten occurs at the time of weaning when the character of the food is suddenly changed. (d) Inflammatory conditions of the bones in which the bloodvessels are enlarged and the circulation increased preventing the depositing of the lime salts in the new bone tissue. This process keeps the lime salts in solution instead of being deposited in their normal manner. (e) The possibility of infection having to do with the production of rachitis has becn considered, but not proved. Various toxic agents (poisons) will bring about a disturbance in the function of the normal metabolic processes in the body and possibly bring on the condition or predispose to it. ( $f$ ) Absence of sunlight, dark, damp and unsanitary cellars are no doubt predisposing causes. Heredity has been montioned also as having some influence in the development of the disease. It is quite evident that the exact etiological factor in the production of rachitis has not yet been ketermined.

Pathology.-The deformity occurring in rachitis is found mainly in connection with the long bones. The diaphyses of the bones are. shortened, softened, become curved. 'The epiphyses are thickened, more or less spongy, and much enlarged. The bones are much softer than normal and have the consistency of decalcified bonc. 'They' can be cut easily' with a knifc. 'The periosteum is thickencel, reddened and when remorcd from the bones may include some of the bone substance. In many cases the interior of the bones is soft and the canat abnormally large. The short bones are found spongy and easity separater. Marker deformity occurs in the skull bones which are often separated. 'The patient in general will show evidences of malnutrition.

Symptoms.-1)uring the early stages of the discase, before deformity of the boncs is present, there are gastric and intestinal disturbances (catarrh), and evidences of malnutrition. Weakness, loss of appetite, diarrhea and tendency 
for bloating are observed in the carly stages. Very often the disease is not noter until the change in the form of the bones begins. I) formity appears in various places. The bones of the head and face show a bulging which gives the animal a peculiar appearance. The limb bones show curvature and enlargement at the joints. Palpation along the ribs at the attachment of the bones to the cartilage will reveal small, rounded enlargements. 'Twisting and curvature of the spine are quite commonly observed. The teeth become loose and displaced, the gums soft and spongy and blecd casily. Deformity of other bones may be present. General cmaciation, weakness and irregular development of the bones, with marked gastric and intestinal disturbances ale prominent symptoms.

Course.- The course of rachitis is nearly always chronic, lasting for several months. Occasionally the condition runs an acute course when complications are present.

Prognosis. - When the disease is observed early and treated, the prognosis is rather favorable. After there is marked deformity and evidences of emaciation and malnutrition very little can be hoped for.

Treatment. - The first consideration in the treatment should be the diet. I ogs should be allowed plenty: of meat with the addition of ground bone. In birds meat scraps and ground bone seem to produce the best results. Gastric and intestimal catarrh should be treated, if present, by alkalies and mild laxatives. 'The lime deficiency must be relieved by the administration of line salts. Sirup of ealeium lactophosphate has been very satisfactory for this purpose (dogs, 8.012.0; chickens, 1.0-2.0). 'This should be given twice daily. and continued for a few weeks. Clialk (calcium rarbonite), or ealcium phosphate is also to be recommended in doses of 1.0 to $\$ .0$ daily. In some cases there will also be a defieiency of phosphorus which can be replaced with ealcinm phosphate. Phosphorus in a bland oil (dogs, $0.001-0.0(0) 2$ in 10.0 oil; birds, 0.00 - -0.001 in 2.0 oil) is also recommenderl. sinall doses of hydrochloric acid, well diluted, will assist in the solution and assimilation of lime. 
In dogs, when the limb bones become soft and deformed, splints and bandages are useful to act as a support which reduces the deformity to a minimum.

\section{OSTEOMALACIA. BRITTLENESS OF BONES.}

Osteomalacia occurs so rarely in small animals that the reader is referred to other works for the discussion of it. 


\title{
PART VII.
}

\section{DISEASES OF THE ORGAIS OF LOCOMOTION.}

\author{
CHA P'TER I.
}

\section{AR'TITIAAR RHELMA'TISM. POLYARTHRITIS RHEUMA'ITC.}

Definition. - Articular rheumatism is evidently an infectious febrile disease in which several joints are affected. It is a serous or serofibrinous inflammation of the joints. It may suddenly shift from one joint to another.

Etiology. - The actual cause of the disease has never been definitely proved, but all indications point to infection. Teveral factors no doubt have considerable influence in bringing on the attacks, such as (a) subjection to cold and chilling the surface of the body; $(b)$ keeping animals in cold, lamp cellars, or forcing them to sleep out in extreme cold weather; (c) infection following birth of the young. The close association of the disease with endocarditis and other diseases of serous membranes further suggest its infectious nature.

Pathology. - The joint capsule is more or less distended with a yellowish gelatinous-like fluid mixed with the synovia. 'The connective tissue is infiltrated with the same material. Fxamination of the synovial membrane itself reveals swelling, injection and hemorrhages. The cartilage of the joint is bluish or yellow in color and the surface roughened. Chronic cases show marked thickening of the periarticular tissue. Similar changes are often found in the tendon sheaths. 'The musculature shows atrophy. 
Symptoms.- The condition of ten begins suddenly involving one joint or sereral at the same time. The affected ones are very painful, the animal refusing to place any weight on the limb and holding it in a flexed position. The joints will be hot and swollen. Owing to the pain small animals usually remain in a recumbent position and refuse to arise. 'The acute symptoms often subside after a week, or ten days, and eventually develop into a chronic inflammation with deformity of the joints. Favorite locations of the inflammation are in the carpal, stifle, and phalangeal joints. In the early stages of the attack there is fever, which may reach $103^{\circ}-105^{\circ}$ F. The respirations are increased, the pulse double the normal number. 'The animal refuses to eat in most cases. 'The urine is reduced in quantity and dark in color.

In the chronic form the disease assumes the character of a serosynovitis; the joint capsule is very much thickened and adhesions take place between the joint surfaces in a number of cases. Ankylosis, however, is rare. There is usually but little fever in this form but general emaciation and cachexia will be prominent. In a few cases complications develop. Inflammation of the serous membranes is the most common. 'This is indicated by a greater rise in temperature and by the specific symptomis of the complicating condition.

Course.-In the acute form the disease usually runs for two or three weeks. Remissions often occur at different intervals for several months to one year. In many cases the attack disappears quite suddenly which may be for only a short time or permanently.

Diagnosis.-This may be difficult. A careful examination must always be made to differentiate it from other diseases affecting the joints. It should be borne in mind that articular rheumatism comes on primarily while that produced by other liscases shows the primary condition elsewhere before the joints become affeeterl.

Prognosis. - Shoull not be considered too favorable. Howerer, in the log they may recover completely. Very often the rondition becomes chronic leading to permanent reform- 
ity of the joints. Complications may also follow, such as endocarditis or inflammation of other serous membranes.

Treatment.-Treatment in small animals is often satisfactory by the use of large doses of sodium salicylate (1.0 to 2.0 daily). After two to three davs the temperature falls almost to normal and the general condition will improve. Other preparations have been recommended, as salicylic acid (intravenously), salol, or acetanilid. Local applications to the joints will assist in giving relief from the pain, and in the absorption of the deposits. Iodin liniments, camphor oil, or mercuric iodid ointment, any one of which may be applied alternately with hot water applications. 'The animal should be placed in a moderately warm room, where it is quiet, and provided with a soft bed. In the chronic form massage the joints thoroughly daily with Lugol's ointinent.

\section{MUSCULAR RHEUMATISM.}

Definition. - A primary affection of the muscles (myositis) probably of infectious origin. It occurs commonly in dogs. Owing to the fact that it is very difficult to make a distinction between muscular rheumatism and so-called "soreness" and "stiffness" of the muscles, they are usually classed as muscular rheumatism. The number of cases of muscular rheumatism would no doubt be decreased if a careful examination were made in all cases and a more accurate diagnosis established. There has been a tendency no doubt to use the term inuscular rheumatism in a too inclusive sense.

Etiology.-The immediate cause of muscular rheumatism is probably an infection, the nature of which has not been determined up to the present time. Several factors have to (lo with the development of the disease: (a) I)amp, cold kennels, and exposure to cold have proved decided factors in bringing on the attacks. Very commonly observed in humting dogs following their exposure on hunting trips. This is especially true in those animals that have been pampered and kept in warm buildings and quarters. (b) Ptomain poisoning and intoxication from toxins absorbed from the intestinal tract will also produce it. (c) Certain infectious 
diseases of serous membranes will bring on attacks of muscular rheumatism in a similar manner as articular rheumatism. (d) Injuries to the museles, overexertion without previous exercise, will produce similar symptoms. This, however, should not be classed as muscular rheumatism.

Pathology.-On examination the muscles will show serous inflammation with infiltration of the intramuscular connective tissue. Disintegration and fatty degeneration are found in most cases. The muscular tissue shows evidence of hyperemia and swelling. In chronic cases the fibrous connective tissue elements are thickened.

Symptoms.-Pain is always a prominent symptom. This may be constant in severe attacks or in the milder forms periodic and recurrent. In dogs the condition very often comes on suddenly without any prodromal symptoms. The attack is usually transient lasting from a few hours to several weeks, and is very apt to reeur. 'The symptoms of muscular rheumatism will depend somewhat upon the groups of muscles affected. Sometimes the muscles of the head and neck are involved; in this case the head and neck will be more or less fixed, the animal holding the head in a rigid position. When manipulated or the animal made to move indications of severe pain are noted. In other cases the muscles over the scapula will show the primary seat of the disease. This is often unilateral, but may be present on both sides. The anterior limb will be relaxed or the animal show marked lameness when weight is placed on it. When bilateral the animal may be found in a recumbent position, with the limbs straightened out, and refuses to get up. The patient cries out and whines when the muscles are palpated.

In involvement of the muscles of the back (lumbago) rheumatica) the animal assumes a rigid attitude, very cautious in all its movements, frequently refusing to move and when forced to do so great pain is manifest. Occasionally the patient will be found lying down. Manipulation of tlie muscles over the region produces intense pain. When the muscles of the extremities are primarily affected the animal lies on its back with the feet upward, whining and crying. Feces and urine are often retained when the abdominal 
muscles are affected. The various functions of the body are interfered with owing to the pain from the disease. The sensibility of the skin is either normal or hypersensitive. As a rule no appreciable rise in temperature is noted. Thc respirations are increased and may be shallow when the respiratory muscles are affected. 'The pulse may be increased inaterially, due to the pain present. The general condition of the animal is good and the appetite retained. In severe cases animals become emaciated from the disturbance of the functions. Complications occur occasionally in the form of acute inflammation of serous membranes, and disturbances in the digestive tract.

Course.-The acute attack, as a rule, lasts only a few days to one week. The symptoms may disappear entirely. Recurrence of the condition is to be expected.

Prognosis.-Complete recovery without periodical recurrences of the disease can hardly be hoped for. Unless complications develop the patient will apparently recover from the attack.

Treatment.-The early indications in the treatınent are: Protection of the patient from exposure, relief from the pain and the administration of antirheumatic agents. 'The animal should be placed in a moderately warm, dry place with a soft bed. All further exposure to cold and dampness should be avoided. Local applications to the affecter muscles arc recommcnded. Massage the muscles with soap lininent, camphor liniment, or chloroform liniment. These should be applied once or twice daily. Hot towels applied to the affected area often give relief promptly. Internally administer sodium salicylate or salieylic acid in large doses (0.9) to 1.5$)$ once daily. When the pain is intense it is arlvisable to control it by subcutaneous injections of morphin sulphate $(0.1$ to 0.2$)$. 'This may be repeated in twelve to eighteen hours if necessary. Conplications as they arise should be treated. In cases that tend to become chronic, potassium iodid is highly recommended $(0.2$ to 0.4$)$. Plcthoric animals should be depleted by administcring saline laxatives (magnesium sulphate 8.0 to 12.0 ). 


\section{FRACTURE OF BONES.}

As a result of injuries from various causes small animals are particularly prone to fractures of different kinds. 'The number of fractures and the bones affeeted are mueh greater than in the large animals. All kinds of fractures (partial, complete, compound, or comminuted) are observed. The larger number of fractures naturally take place in the extremities, although fractures of the skull bones, inferior maxilla, ribs and vertebre are not uncommon. The causes of fractures are varied, but the greater number of cases result from traumatism (being run over by vehicles, kicks, falls, jumping, gunshot wounds, bites from other animals). In a few cases cxtreme muscular contraction will fracture the bones. Various diseases of the bones, and senility predispose to fractures. The following are the more eommon fraetures met with:

Cranial Bones. - This form of fracture oceurs oceasionally in dogs. The writer has observed six cases during the past ten years.

Etiology.-Practically always results from traumatism. Being struck by sharp or blunt objects, run over by automobiles, kicks, etc., are the most frequent causes.

Symptoms. - Tarious kinds and degrees of fractures are recognized, from a simple fissuring of the bones to penetrating, depressed or compound comminuted fraetures. The symptoms vary with the degree and kind of fracture. Simple fissuring of the bones may not produce any marked symptoms unless the concussion has been sufficient to eause hemorrhage or injury to the membranes of the brain. The presence of the wound and on pressure slight movement between the bones may be observed. P'enetrating wounds with fracture often produce complications of liemorrhage into the cranial cavity or direct injury to the structures within. Examination of the external wound and carefully probing will at once disclose the actual conditions. Fraetures with depression of a fragment of bone cause more or less brain disturbance depending upon the location of the fraeture. The psychical disturb- 
ances and the presence of the wound would assist in making tlie diagnosis. If there is doubt in regard to the exact conditions the external wound can be opened sufficiently to examine the parts direct. Compound and comminuted fractures show the presence of the wound and the opening through the cranial bones. Fragments of bone may be found projecting out through the opening or downward into the cranial cavity. Probing will reveal the conditions. The concussion from the injury may produce unconsciousness for a time even though but little damage has been done the bones themselves. Death occurs suddenly in many instances when the brain is severely injured or sudden severe hemorrhage takes place. Careful examination should be made in all cases in order to establish a positive diagnosis.

Prognosis. - The prognosis should not be considered too favorable on account of the danger of brain complications.

Treatment. - The treatment will depend upon the kind of fracture. In simple fissuring of the bone no treatment is necessary, except for the contusion of the skin and muscular tissue covering the bone. Penetrating fractures are best treated by opening the external wound, examining the fractured portions and elevating them carefully with a scalpel or stiff probe. Clean the wound thoroughly and suture the skin covering it with iodoform collodion.

Depression of the skull bones should liave prompt treatment to relieve the pressure from the brain and membranes. 'l'his is best done by making external incision, and if necessary a small opening through the bones so that an instrument can be inserted to elevate them to their normal position. Strict aseptic precautions should be observed in the operation. suture the outside wound. In compound comminuted fractures all fragments of bone should be removed, conserving as much as possible, and a protective dressing applied to the parts.

Fracture of the Inferior Maxilla.- liracture of this bone occurs rery often in dogs and cats. The points of fracture are usually at the symphysis, in front of the premolars, or posterior to the molars. Frequently the fracture is bilateral, but in most cases involves one side. 
Etiology. - Traumatism is often the cause of the fracture. It can result from extraction of teeth. It may follow alveolar periostitis, or exfoliation of a portion of the bone weakening the jaw at this point. Extreme pressure from biting on hard objects (stones of large size) can be a cause. Diseases of the bones (fragilitas ossium) predisposing them to fracture.

Symptoms.-Difficulty in eating is the first symptom noticed. Other symptoms are displacement of the maxilla at the point of fracture, excessive salivation, and enlargement on the side affected. On examination crepitus and movement between the portions of bone will be recognized at once.

Prognosis.-Owing to the complications of infection, and the difficulty in keeping the parts quiet, the prognosis is unfavorable.

Trealment.- Various methods of treatment have been tried. In fissuring without laceration of the mucous membrane it is advisable to try wiring them together with silver wire using the teeth as fixed points. In compound fractures when infection takes place treatment is usually unsatisfactory. Fixing the maxilla by the use of a leather pad or muzzle in contact with the superior maxilla, disinfecting the mouth twice daily, and giving liquid foods have proverl the most satisfactory treatment.

In comminuted fractures where fragments of bone must be removed but little can be expected of any treatment.

Fracture of the Vertebræ.-The points at which fractures most commonly occur are in the cervical, lumbar, and coccygeal segments.

Etiology. - Most cases result from traumatism.

Symptoms. - The symptoms will depend upon the kind of fracture, whether it is confined to portions of the vertebra outside of the canal or involves the canal with the spinal cord. repitus and pain on manipulation of the parts are prominent symptoms. Should the fracture be confined to the lateral processes no other symptoms will be observed. In complete fracture of the cervical vertebre with involvement of the cord, death may result in a few minutes, or hours. Complete paralysis exists posterior to the point of the fracture. Fract- 
ure in the lumbar region produces paraplegia and paralysis of the posterior portion of the body. Differentiation between hemorrhage in the canal, edema of the cord and fractures is often difficult and can be determined only by the progress of the case. Fractures of the coccygeal vertebre are easily determined by crepitus and the increased mobility of the parts.

Prognosis.-Complete fracture of the vertebra is unfavorable. Fracture of the lateral processes or coccygeal vertebrax is favorable.

Treatment.-No treatment is possible in fracture of the cervical and lumbar vertebræ. The animal should be placed in a quiet, comfortable place. Give nourishing food. If necessary artificial feeding should be carried out. Laxatives are also necessary. Simple fracture of the coccygeal vertebræ is best treated by bandaging. If complications arise or a compound fracture is present amputation may be necessary at the point of fracture, or slightly above.

Fracture of the Ribs. - This is found most commonly in $\log$ s and is of very frequent occurrence.

Etiology. - Produced in many cases by being run over by vehicles, struck by objects, or kicked.

Symptoms.-One or two ribs may be fractured without producing marked symptoms. This is especially true in partial and in simple fractures. Some will show displaceinent and enlargement at the point of fracture. Crepitus is hard to distinguish in all patients. ('ompound fractures may (ause injury to the pleura or lung tissue. Examination should be made carefully.

Prognosis.-Favorable unless complications involving the pleura and lungs develop.

Treatment.- Most cases do not require any treatment. Keep the animal quiet. A bandage applied around the thorax to assist in keeping the parts fixed might be used. (Compli(ations involving the pleura and lungs should be treated accorling to the conditions.

Fracture of the Scapula.--This is not of frequent oceurrence. In dogs the points of fracture most common are through the neck and glenoid cavity. In young animals 
separation takes place frequently between the epiphyses and diaphyses.

Etiology.-From injuries and diseases of the bones.

Symptoms. - Sudden development of lameness. 'The limb usually hangs inert and cannot be moved upward and forward. Examination will reveal the fracture.

Treatment. - No satisfactory treatment can be applierl owing to the location of the scapula. I)ogs, when well cared for and protected, will recover without special treatment. Dogs protect the limbs better than any other animal. Stimulating liniments might be applied over the area (soap liniment, iodin liniment).

Fracture of the Humerus. -Owing to the exposed position of the humerus it is frequently fractured. In young animals separation of the epiphyseal and diaplyseal portions is not uncommon. It has been observed that the lower end of the bone is more often fractured than any other portion. Fracture of the condyles occurs with considerable regularity.

Etiology. - Traumata of various kinds produce the majority of cases. It occasionally happens that both the humerus and scapula are fractured at the same time.

Symptoms. - When unilateral fracture of either the ejoiphyseal or diaphyseal portions of the bone takes place the animal camnot support any weight on the limb. It will hang free and limp. Should both be fractured the animal rests its weight on its haunches, or assumes a recumbent position.

Diagnosis. - Examination of the limb will at once make the diagnosis positive. It is very rare to find a compound fracture.

Prognosis. - Favorable.

Treatment. - The application of bandages is very difficult. 'This is especially true when the fracture occurs in the upper portion of the bonc. Experience has proved that good results follow without splints or bandages. Occasionally a false union of cartilage or fibrous connective tissue will result forming a pseudo-articulation.

Fracture of the Radius and Ulna. - This is the most frequent of all fractures in the anterior limb of the dog. All small animals are affected in a similar manner. Fracture 
usually occurs in both bones simultaneously. When one bone is fractured it is usually the olecranon portion of the ulna. 'The lower third of the bones is the seat of the majority of fraetures.

Etiology. - Practically always result from injuries. Some few cases are due to diseases of the bones.

Symptoms. - Inability to place weight on the limb and angularity at the point of fracture. There are swelling and distortion of the limb. When the olecranon is fractured the distortion will be marked at the point of the elbow. Crepitus and free movement between the fractured portions will be apparent on manipulation.

Treatment. - Wrapping the limb with ordinary linen bandage is recommended until the swelling subsides. Plaster bandages are unsatisfactory during the first few days owing to the change in the size of the parts from swelling. A pad of cotton should be applied to protect the skin from pressure necrosis and to allow for swelling to take place, then strips of cardboard softened by placing in warm water for a few minutes, and over this a linen bandage. The patient should be observed to determine whether the bandage has been drawn too tight or has become loose. Should the foot be found swollen it indicates that the bandage is too tight and free circulation of blood interfered with. It should be loosened. After a few days a plaster bandage can be applied if thought advisable. It should remain in position for a few weeks to ensure complete union. I'niting the bone at point of fracture with silver wire has been tried with varying degrees of success. Radial fractures usually make prompt and complete recoveries.

Fracture of the Metacarpal and Phalangeal Bones. - These hones are fractured from the same causes as mentioned under the others.

Diagnosis.- This is made by careful palpation.

Treatment. - Bandage as in fracture of the radins and ulna. In compound fractures it is sometimes necessary to amputate a portion of the limb or toes.

Fracture of the Pelvis. - Fracture of this bone is quite common in small animals, and presents a variety of condi- 
tions. 'The most common points of fracture are througl the symphysis, external angles of the ilium, or through the acetabulum. Both unilateral and bilateral fractures are observed. Complications are common following fracture of the pelvis, owing to the injury of adjacent structures. Injury to the nerves or bloodvessels is most common.

Etiology.-Pelvic fractures are produced very frequently by being run over, struck by objects, kicks, or falling.

Symptoms.--The symptoms will vary somewhat depending on the degree of fracture, its location, and the complications. Deformity of the pelvis is present in fracture of the external angle of the ilium. Crepitation and movement of the parts can be detected. Fracture through the symphysis is evidenced by the umnatural gait of the animal, the abduction of the limbs, and the separation which can be detected on palpation between the fractured portions. It is sometimes difficult to make a positive diagnosis in fracture at other points on account of the smallness of the openings through which the examination must be made. Complicating symptoms should be examined for in all cases.

Prognosis.-- - Unless complications of hemorrhage and destruction of some of the nerves occur, the prognosis is considered favorable. Different degrees of deformity will be met with which should be considered in the prognosis. The condition is serious in breeding animals on account of the deformity narrowing the pelvic canal. Such animals should be given a careful examination in this regard.

Treatment.-But little can be done in the way of treatment. Inion usually takes place promptly by giving the animal protection for a few weeks. Various methods have been tried to approximate fractures of the pelvis but with little success.

Fracture of the Femur.-Fracture of the femur occurs often in dogs, cats and rabbits. The majority of cases are found involving the lower third of the bone. Some few cases are observed in the upper portion, even involving the head of the femur.

Etiology.-Injuries and accidents account for the larger number of cases. Diseases of the bone (fragilitas ossium and osteomalacia) predispose to fracture. 
Symptoms.--In practically every case the limb is held off the ground, freely moved in all directions, and appears shorter than normal. Care slould be made to differentiate between fracture and dislocation of the head of the femur. Examination of the limb will reveal the point of fracture.

Diagnosis. - Fissuring of the bone may present some difficulty but in other cases the diagnosis is easy.

Prognosis.-Favorable. Enion of the bones may result in some permanent distortion, such as shortening the limb, or a certain amount of angularity.

Treatment. - An attempt should be made to bandage the limb to keep the bones in apposition as nearly as possible. A temporary muslin and splint bandage should be used until the swelling subsides. Later a better and more permanent dressing may be applied. As a rule union takes place quite promptly and without much distortion.

Fracture of the Patella. - This bone is very rarely fractured in small animals. Careful palpation over the region would reveal the condition. No treatment other than bandaging can be applied.

Fracture of the Tibia and Fibula.-These bones are the seat of all types of fractures. Very common in dogs and rabbits. The symptoms, prognosis and treatment are similar to those given for the radius and ulna. 


\section{CHAPTER II.}

\section{DISEASES OF THE ARTICLLA'TIONS.}

THe various articulations in small animals are subjected to a variety of conditions.

\section{WOUNDS OF THE ARTICULATIONS.}

Punctured wounds and various kinds of incised wounds are frequently met with. In some cases the adjacent structures may be severely injured complicating the condition. Further classification should be made into aseptic and septic wounds. From a practical standpoint it is important to examine the wounds carefully to make these distinctions.

Etiology. - It is selfevident that these wounds occur through various accidents and injuries to which the animals are subjected.

Symptoms. - The animal will favor the joint affected and refuse to walk on the limb. A thin, serous discharge (synovia) is noted coming from the wound. The amount of pain and swelling will not be marked in the early stages. However, if infection is introduced, these symptoms develop very rapidly. Pus soon makes its appearance, the joint becomes vcry hot and sensitive, and general symptoms of fever, loss of appetite, are observed.

Diagnosis. - This is made by carefully examining the discharge and by probing the wound with a sterile probe.

Prognosis. - Should not be made very favorable in any casc, owing to the possibility of destruction of the joint, or ankylosis. Non-infected wounds usually heal without difficulty.

Treatment.-After learning the facts concerning the involvement of the joint, strict antiseptic precautions should be taken, and all means employed to prevent infection. 
Shave the hair fom the area and disinfect the surface with bichlorid of nereury and glycerin (1 to 500$)$. Saturate gauze or cotton with this solution and apply under a bandage, if possible. When infection is already present it is advisable to open the wound so that adequate drainage will be brought about. Cse the same solution as above and inject it into the articulation. Apply a protective dressing. Repeat this treatment at least twice daily.

\section{SPRAINS AND INJURIES TO THE ARTICULATIONS.}

In the larger number of injuries occurring in small animals it frequently happens that the structures around the joints are torn or otherwise injured without an external wound. 'This condition may occur in any of the articulations. As soon as the injury occurs the synovial secretion is increased in amount, and the adjacent tissues are often edematous.

Symptoms.-The condition develops suddenly following injury, or violent exercise. 'The articulation soon becomes enlarged, very hot and painful to the touch. The patient refuses to bear any weight on the limb. Careful examination must be made to differentiate sprains from fracture or dislocation. Distortion, increased movement, and crepitus found in fractures are absent in this condition. 'The normal position of the bones would cxclude dislocation. In severe cases the swelling may be so extensive as to interfere with making a positive and early diagnosis.

Prognosis.-Most cases of sprains recover.

Course.-'The course depends upon the degrec of injury.

Treatment.-The animal shouid be confined in a quiet place for several days. Cold applications in the form of cold water, or better, ice packs, should be applied during the first twenty-four to thirty-six hours. 'The packs are clianged frequently to keep up the refrigeration. Follow this treatment with hot water, or liniment (iodin liniment, white liniment) applied once or twice daily. Massage the parts thoroughly. 'The latter treatment sliould be continued for' two to thrce wecks, if necessary. 


\section{DISLOCATION OF THE ARTICULATIONS. LUXATION.}

I)islocation of the various articulations in dogs, cats and rabbits is a common occurrence. Birds of ten have luxation of the joints of the wings.

The following are the most common dislocations found in small animals:

Temporomaxillary. - This occurs more frequently in dogs than in any other animal.

Etiology.-Resulting in most cases from extreme opening of the mouth, either from being forcibly pulled open, or from large objects being taken into the mouth. The condition may be either unilateral or bilateral.

Symptoms. - Fixation of the lower maxilla either laterally (in case of unilateral luxation), or downward and forward (bilateral luxation). Saliva flows from the mouth freely, the animal is unable to move the maxilla, or only slightly, and shows considerable anxiety and pain. Pawing at the mouth with the forefeet, rubbing the parts against objects, and carrying the head in abnormal positions, are prominent symptoms. Patients showing these symptoms should be handled carefully, as in some respects they are not unlike dumb rabies. Examination will easily reveal the differential features.

Prognosis.- Csually favorable, except when the case is of too long standing and fracture of the bones is present. There is always the possibility of a recurrence.

Treatment.-Reduction of the dislocation should be made early. The animal should be anesthetized (morphin sulphate, 0.1 to 0.2 ), placed on a suitable table in the dorsal position, and by using a fulcrum inserted between the teeth, the jaws are forced apart by bringing the incisors together. $\mathrm{By}$ careful manipulation the bones are replaced in their normal position. Considerable patience is often necessary to accomplish this. If fractures are present but little can be done. Ise artificial feeding of liquid foods for a few days. It is adrisable to tape the mouth shut for a few days until the articulations are normal again. 
Vertebral.-1)islocation unattended by fracture is rare. 'The articulations are so arranged anatomically that luxation without fracture is not liable to occur. Partial luxation is met with occasionally and is diagnosed by the position of the head and curvature of the vertebre. Straightening the head and neck should be attempted.

Scapulohumeral. - Owing to the position of the articulation and its anatomical structure complete luxation is not common. The articulation is capable of rather extensive movement without dislocation.

Etiology. - It is brought about by excessive flexion of the joint from injuries and accidents. The luxation is usually forward and inward.

Symptoms. - The condition occurs suddenly and distortion of the joint is apparent at once. The joint is held in a flexed position and distention is practically impossible. The limb is much shorter than normal. Examination of the artieulation reveals the head of the humerus forward producing an enlargement anteriorly and a depression posteriorly. Animals show pain on manipulation of the joint.

Prognosis. - In complete luxation the prognosis is favorable when taken early but later when swelling takes place reduction is difficult, and the joint capsule will rarely resume its normal condition. Partial luxations are favorable.

Treatment. - The animal should be anesthetized (morphin sulphate, 0.1 to 0.2 ), placed on the table in lateral recumbency with the affected articulation presented. Extend the humerus and push backward on the head of the humerus. Usually replacement will take place without much difficulty. Apply a bandage for a few days to protect the part. Massage and a stimulating liniment should be applied around the joint.

Humero-radio-ulnar. - Luxation of this articulation may be partial or complete. Various conditions ean be found owing to the anatomical structure of the joint. The dislocation may be between the liumerus and radius or between the radius and ulna. In some cases all of the struetures are involved. The dislocation may take place to the inside or outsicle depending upon the cause of the condition. When dislocation occurs there is nearly always a tearing or stretch- 
ing of the ligamentous attachments around the joints. I eongenital disfocation is sometimes observerl.

Etiology.- The condition is brought about by injuries, such as blows from the outside or inside, extreme flexion or extension of the joint, falling, or jumping. Frequently the limb is given a sudden twist by the foot being eaught when the animal is in motion, or pulling backward to free itself.

Symptoms. - Distortion of the articulation is noted, and the lower limb in an abnormal position, either abdueted or adducted. 'There is an enlargement present on one side and a corresponding depression on the opposite. The limb appears short, and the animal refuses to place any weight on it when in motion or at rest. Examination reveals the joint to be hot and painful when manipulated. The displaced bone ean easily be palpated, and the luxation deterinined.

Prognosis. - Not very favorable owing to the injury to the annular ligaments, and the possibility of a return of the condition.

Treatment.--IReduction of the dislocation should be made under general anesthesia. The method of procedure will repend upon the dislocation. As a rule but little diffieulty will be experieneed in bringing the parts back to their normal position. After reduction of the luxation the parts should be bandagerl to hold them in position. The bandage should be left in place for two or three weeks, or until the annular ligaments are united. Congenital luxation is unsatisfactory from the standpoint of treatment. Wiring of the bones might be trierl in eases that cannot be kept in position by baundaging.

Radjo-ulnar-carpal. - From violent injury luxation of this articulation may take place. The annular ligaments beeome torn allowing the artieular surfaces to be displaced.

Symptoms.-I)istortion of the articulation (ither forward or backward is most common.

Diagnosis.- The diagnosis is not rlifficult when a careful examination is made.

Treatment.- Rerluetion of the luxation is easy, but it recuires several weeks before the animal will be able to use 
the limb. Protect the joint by placing a pad of cotton under a bandage. Keep the bandage on for two or three weeks.

Phalangeal.-Dislocation is very common and results from traumata. A careful examination should always be made to diffcrentiatc luxations from fractures, and to definitely determine the joint displaced. They should be reduced as early as possible and a bandage applicd. Keep the bandage in position for two to three weeks.

Coxofemoral. - This is onc of the most common dislocations in $\operatorname{logs}$, cats and rabbits. It may be partial or complete. When partial the damage is mainly in connection with the capsular ligament, while in complete luxation the cintire joint structure and the adjacent tissues are involved.

Etiology. - Being struck by objects, run over by vchicles, and having the foot caught in traps, etc., are the most common causes. Extreme extension of the limb from any cause may bring it about.

Symptoms. - The most frequent form of luxation is forward and upward. The animal is found with the limb fixed in a hackward, adducted position, and refuses to placc any weight on it. An enlargement will be noted just anterior to the normal position of the joint, and on palpation the head of the fenur can be felt. IIanipulation of the limb will at once reveal the condition. Luxation into the foramen ovale is not common hit occurs in a small percentage of cases. The limb appears longer than normal, is slightly abducted, and the stifle joint is turned outward. Adduction of the limb is difficult and inpossible to the normal degree. P'alpation of the parts will easily diagnose the condition.

I hackward, upward luxation occurs occasionally and the position of the limb is forward and inward, with an enlargement present on the posterior aspect of the joint. ('areful (examination will retermine the relationship of the parts. In partial luxation the animal is able to move the limb and slows lameness but very little deformity. Chronic conditions become accommodated to the movements of the anim through the formation of a pseudo-articulation.

Prognosis.--Rather unfarorable for a complete recorery. Larly cases are much more favorable than those of a few days or weeks standing. 
Treatment.-Treatment should be applied as early as possible. Anesthetize the animal and effect replacement by manipulation of the limb according to the character of the luxation. The principle involved is to extend the articulation in whatever direction is nccessary so that the head of the femur can be returned to the acetabulum. Very littlc can be done in after-treatment except keeping the animal quiet for screral days. Mechanical appliances to hold the bones in position have not proved satisfactory. Little can be expected in the treatment of chronic cases.

Patellar. - This occurs most frequently in the dog. 'T'he ligaments of the patella are not very well developed outside of the middle one. Therefore, displacement is more liable to occur laterally. Experience has shown that internal displacement is by far the most common.

Etiology. - The anatomical structure of the patellar ligaments and the joint in particular make displacement casy from excessive exertion or injuries. In some breeds the internal femoral ridge of the patellar groove is very small; thercfore internal displacement is of common occurrence.

Symptoms. - The limb is held in a flexed position, and the patient has difficulty in supporting weight. The flexion is most apparent at the stifle joint. The position of the limb is somewhat characteristic, the stifle joint adducted, the hock rotated outwardly, and the lower portion of the limb carried toward the median line. Examination of the stifle joint will reveal the misplaced patella. When the condition is bilateral the animal will show a crouching attitude, and will have considerable difficulty in maintaining the standing position. The animal moves with great difficulty in bilateral dislocation.

Prognosis. - The prognosis should not be considered favorable owing to the difficulty in keeping the parts quiet and the possibility of a recurrence of the condition. Very often therc is a tendency for the condition to become chronic.

Treatment.-Replacement of the patella presents very little difficulty; therefore the problem of kccping the patella in position until the ligaments assume their normal condition is to be the principal aim in treatment. Replacement is 
aflected by extension of the joint which will allow the patella to be pulled back to its normal position. Apply linen bandage in order to retain the parts in their normal position. The animal should be kept quict for several days. A plaster bandage might be used to advantage.

Tibiotarsal. - This is not very common in small animals. When it does occur it is accompanied by rupture of the ligaments or fracture of some of the bones. A careful examination should be made to determine the conditions as accurately as possible. Linen bandage should be applied for the first few days, followed by a plaster bandage if necessary.

Caudal Vertebræ.- The larger breeds of dogs with long tails (Great I)ane, greyhound, etc.) are the most frequent sufferers. Examination of the parts will reveal the condition. I bandage applied rather firmly and kept in position for several days will correct the dislocation.

\section{INFLAMIMATION OF THE SYNOVIAL MEMBRANE AND ARTICULATIONS. SYNOVITIS. ARTHRITIS.}

Definition.-An inflammation of the synovial membrane and articular surfaces. 'The conditions may be acute or rhronir. Very of ten the entire joint will be inflamed (synovitis and arthritis). It is very difficult to make a distinction botween these two conditions.

Etiology. Most cases result from sprains and contusions of the joint. Few result from infection (pyogenic). 'The infection usually gains entrance through wounds of the synovial membrane.

Symptoms.- Enlargement of the joint, extreme sensitiveness, and increased local temperature are characteristic sinptoms. 'The animal cannot use the limb to any extent. Fxamination shoukd be made carefully to differentiate it from articular rhenmatism.

Prognosis.- Favorable in ases resultiug from sprains and bruises; unfarorable in infected joints.

Treatment.--I Juring the earty stages of the condition cold applications are applied either in the form of eold water or ice parchs which should be arried out conseientiousty if the 
best results are to be expected. 'This treatment may be followed by hot applications and liniments (soap liniment, white liniment, iodin liniment). I)uring treatment the animal must be confined to ensure rest to the affected joints. In case pus is present in the articulation, it should be opened and antiseptics directly injected. (See Wounds of Joints.) Chronic cases are not amenable to treatment. 


\title{
PART VIII. \\ DISEASES OF THE [RINARY SYSTEM.
}

\author{
CHAPTER I.
}

DISEASES OF THE KIDNEYS.

Examination. - Examination of the kidneys consists of abdominal palpation, direct inspeetion and exploration, and examination of the urine.

(a) Abdominal palpation is best done with the animal in a standing position, using the fingers of both hands. By gentle digital pressure, abnormalities in the dimension and loeation of the kidneys will be noted. 'This method of examination will prove satisfaetory only in eases where a marked enlargement oeeurs, or in emaciated animals.

(b) Direct inspection of the kidneys may be made, especially in dogs, by performing laparotomy under anesthesia (sce Laparotomy). 'This method can be done safely and will be found useful in determining aecurately the size, eonsistency, and location of the kidneys. In cases of cysts or other enlargements an exploring needle may be used to obtain some of the contents for examination.

(c) The examination of the urine should be made chemically and physieally. Chemically tests should be made for albumin and sugar. The tests for albumin are made as follows: Heller's ring test is a very valuable one as a very small percentage of albumin can be demonstrated with it. It is made in the following manuer: A small amount of urine is filtered, poured into a test-tube containing eoncentrated nitric acid. At the point of contact of the two liquids 
a well defined white ring forms, the depth of which depends upon the amount of albumin present.

The boiling point may also be used. Take a few cubic centimeters of urine in a test-tube and heat to boiling, acidulate by adding 5 to 10 drops of concentrated nitric acid.. A precipitate which has been formed by earthy phosphates or carbonates will then dissolve, but one due to coagulated albumin remains.

The metaphosphoric acid test is made by adding an aqueous solution of metaphosphoric acid to the urine. 'The urine becomes cloudy in case albumin is present.

The acetic acid-ferrocyanid of potassium test is made by adding 2 per cent. of acetic acid to the urine and then a 5 per cent. solution of ferrocyanid of potassium, the latter drop by drop avoiding an excess. If albumin is present a decided turbidity or flocculent precipitate appears.

The quantitative detcrmination of albumin is made with Esbach's albuminometer. Acidulated urine is filled into the sign $\mathrm{L}$, the reagent up to $\mathrm{R}$ ( 1 part of picric acid, 2 parts of citric acid, and 100 parts distilled water), the tube is closed with a rubber stopper and slowly turned orer several times without shaking; let stand at room temperature for twentyfour hours, the precipitate has settled and may be read off. The figure indicates the proportion of albumin in grams pro mille. Lrine containing a large percentage of albumin must be first diluted with water and the indicated figure must then be multiplied with the figure of the dilution to obtain the exact amount of albumin present.

When it has been demonstrated that albumin is present in the urine, then it must be determined whether the albumin originates from the kidneys, from the urinary passages, or from the nearby genital organs. If the urinary sediment contains no organic form elements, or form elements derived from the kidneys, and if the presence of dissolved coloring matter of the blood may be excluded, then it is positive that it is renal albuminuria. If there is a large amount of organic sediment then it is not likely to be renal albuminuria. In some cases it is possible to have a mixture, coming from the kilneys and also from other parts of the urinary system. 
The ehemical test for sugar in the urine is best marle by using Trommer's test, which is as follows:

A few eubic centimeters of urine are put into a test-tube, after removing any albumin which might be present, dilute it with an equal volume of water, render it alkaline with a small quantity of sodium hydrate, then add drop by drop a 4 per cent. solution of copper sulphate until the liquid is clear and the sediment dissolved, then heat until it boils. If sugar is present, a reddish-yellow vapor appears at the surface of the fluid. The bismuth test is often used for the same determination. The albumin is removed from 10 parts of urine and added to this is 2 parts of subnitrate of bismuth, 4 parts Rochelle salts, and 100 parts of a 10 per cent. solution of sodium hydroxid. This mixture is boiled for five minutes. It becomes black if sugar is present.

'To determine the quantity of sugar present the saecharometer is used. The urine is fermented by adding a small quantity of yeast. Graduated glass tubes or other apparatus are necessary to determine the exact quantity of sugar present.

Phy'sically the urine varies in color and amount, depending a great deal upon the kind and amount of food, the condition of the kidners, and the speeies of the animal.

\section{CONGESTION OF THE KIDNEYS. HYPEREMIA.}

This condition is divided into two forms, viz.: (a) Acute hyperemia (arterial hyperemia), (b) passive hyperemia (venous hriperemia).

Acute Hyperemia. - Etiology. - The causes of this condition are usually the same as those produeing aente nephritis. Irritating foodstuff's, ehemicals and various diuretic compounds, are the most common things producing acute hyperenia. It may oecur during the course of infeetious diseases (distemper, rabies). Plethoric animals frequently have active 1. percmia of the kidneys.

Pathology.-The kidneys are enlarged, swollen, softened and highly redklened. 'The arteries and eapillaries are found 
distended. Small hemorrhages appear under the capsule. The capsule is easily removed.

Symptoms. - This condition may not produce very marked symptoms. The most noticeable is the increased amount of urine, the specific gravity of which is much lower than normal. Sensitiveness over the region of the kidneys, and stiffness in walking are also common symptoms.

Prognosis.-Depends upon the cause. There is always danger of the condition resulting in an acute inflammation of the kidneys.

Treatment. - Acute purgation is indicated. Administration of magnesium sulphate, or calomel to remove the waste material through the bowels. Avoid irritating foodstuffs. Give milk for a few days. The cause should be removed if possible.

Passive Hyperemia.-Etiology. - This condition is brought about as a secondary disease following valvular defects, diseases of the lungs, pleura, etc. In some cases it is produced by pressure on the renal vessels by tumors, etc. In all instances the circulatory disturbances lead to an engorgement. or passive congestion of the kidneys.

Pathology.-In passive hyperemia the kidneys are of a dark bluish-red color, larger than normal. On cut section the venous blood oozes out. In old cases there will be found considerable connective tissue thickening.

Symptoms. - In this condition the quantity of urine is decreased, and albuminuria is present.

Prognosis.-Depends upon the primary condition.

Treatment. - Medical.-Digitalis administered in small doses daily. Animals should be given gentle exercise. Give nourishing food which is non-irritating (milk and eggs).

\section{INFLAMMATION OF THE KIDNEYS. NEPHRITIS.}

Acute Nephritis-Definition.-Anacute inflammatory condition of the kidneys, which is characterized either by nutritional disturbances of the renal epithelium with only a slight change in the interstitial connective tissue (parenchymatous nephri- 
tis), or an involvement of both the renal epithelium and the interstitial tissue without the formation of a purulent exudate (nephritis acuta diffusa).

Etiology. - This disease is quitc common in small animals and birds in which it frequently develops from infectious diseases and poisons.

Acute nephritis occurs secondary to infectious diseases. The organisms circulate via the blood stream, become lodged in the glomeruli and in the intertubular bloodvessels, and at the point of lodgment injure the tissues. In this way the disease occurs cluring the course of distemper, chicken cholera, septicemia, etc. Bacterial toxins in passing through the rellular clements of the kidneys, produce in them and the bloodvessel walls ecrtain degenerative processes, which eventually lead to an acute inflammation. The bacterial toxins probably produce the disease in the majority of cases, as the absorption of toxins takes place in a number of conditions, such as in diseases of the alimentary tract, diseases of the peritoncum, etc., and in this manner we may account for the development of acute nephritis during or following such diseases. In like manner acute nephritis may occur following any organic disease. In small animals it frequently follows the ingestion of decayed foods, the preformed toxins are absorbed and eliminated through the kidneys, producing a severe form of acute nephritis. The same condition may result from absorption of products from wounds, from mange, erzema, etc., especially when extensive.

Acute ncphritis also kevclops from regetablc and mineral poisons, especially when introduced in rather large quantities. Such substances, as cantharides, carbolic acid, arsenic, oil of turpentine, extract of male fern, mercury preparations, ete., are eliminated via the kidney's and excite an acute inflammation. Birds (turkeys) often cat various insects, such as grasshoppers, catcipillars, the acrid substances of which are absorbed from the intestinal tract and induce the inflammation. 'This same condition has been observed in cats from eating large numbers of insects (grasshoppers).

Injuries in small animals are very common, such as blows across the back in the region of the kidneys, being run over 
by vehicles, falling, etc., which may result in direct injuries to the kidneys and eventually in acute inflammation. Subjection to extreme cold has been considered one of the etiological factors: It probably reduces the general resistance of the renal tissue so that infectious substances have a greater effect upon it. This has been observed, especially in puppies and kittens that have been kept in damp, cold kennels. The same thing is found in hunting dogs after being forced to wade or swim through cold water.

Pathology.-This is usually divided into three classes as follows:

(a) Parenchymatous nephritis, in which the primary inflammatory changes are most pronounced in the parenchyma of the kidney. This is characterized by only slight swelling, the capsule more easily removable, and on cut section a grayish or dull color is noted. Sometimes there will be observed grayish-red or yellowish-brown spots. 'The medullary substance is hyperemic, often dark red in color and the Malpighian bodies are quite prominent, while the balance of the organ is either normal or of a soft consistency.

Microscopically the veins and capillaries are enlarged, congested, granular and fibrous deposits between the uriniferous tubules, and epithelial casts, cells, fatty degeneration with swelling and opacity.

(b) Ilemorrhagic parenchymatous nephritis, which is characterized by a large number of red points giving the external appearance of the organ a dark red color, dull gray on section, blotehes of red and in the medullary portion a deep red.

Microscopically the veins and capillaries are found engorged with blood, hemorrhages appearing in the adjacent tissues.

(c) Diffuse Acute Nephritis.-Very noticeable is the increase in size and weight of the organ, sometimes two or three times larger than normal. The tissues are soft and friable. 'The capsule is easily removed, and the external surface a bright red color with some yellowish or gray patches.

Microscopically there will be found enlargement of all vessels with extensive extravasation of blood into the tissues, leukocyte casts in the dilated urinary ducts, and extensive 
cellular infiltration between them. The glomeruli are often filled with blood and covered with bloody extravasations.

Symptoms. - The general symptoms are loss or suppression of the appetite, slight elevation of the body temperature, pulse strong and hard at first, later weak and rapid; frequently romiting in the dog and cat. Constipation during the early stages, followed later by diarrhea, is prominent in most cases.

Most animals have difficulty in walking (stiff gait) as the morement of the body tends to compress or move the kidneys, hence pain is induced. During the early stages of the disease there are frequently noticed paroxysms of pain, especially when the animal is first moved, or palpated over the region of the kidncys. On standing they assume a stretched attitude in order to relieve the tension on the diseased organ. Frequently in walking the limb on the side affected will be dragged or the forward step shortened. It has been observed in male animals that one testicle will be drawn higher than the other.

The changes in the urine form the most characteristic symptom. Usually at first there is suppression, with very scanty flow of urine, highly colored, containing some blood, albumin and tube casts. The total quantity passed in twentyfour hours is greatly reduced, specific gravity high, of a thicker consistency than normal, often slimy and turbid. Hematuria may develop.

Microscopically the urine is found to contain urinary casts in large numbers, white and red blood corpuscles and numerous epithelial cells. The urine is voided a few drops at a time, espccially in the dog, with pain (strangury).

Tremic symptoms are noted in somc cases. The stoppage of the flow of urine from the swelling of the tissue of the kidneys, compression and filling of the ducts with exuded casts, causes a retention of waste products and a lack of secretion, hence an accumulation of urea and uric acid, and other decomposition products sufficient to produce marked symptoms. In the dog these symptoms develop rather rapidly in the form of weakness, staggering gait, convulsions, irregular temperature and coma. 
Diagnosis. - This condition is very frequently mistaken for inflammation of some other abdominal organ, especially peritonitis, enteritis, cystitis, or metritis. It is possible to make a differential diagnosis by a careful examination of the animal, by observing the character and amount of urine voided and a microscopic examination of the urine.

Course.-The course is usually acute; occasionally the disease terminates in chronic nephritis.

Prognosis. - On the whole the prognosis is unfavorable, the patient often dying in six to ten days. When the symptoms of uremia are present, the prognosis should be considered very unfavorable.

Treatment.-Dietetic.-For the dog and cat a milk diet is of the greatest importance. Avoid the giving of irritating foods and drugs.

Medical.-Establish diaphoresis as early as possible. 'This can be done by the use of warm baths, steaming the animal, and rubbing the skin. Wrap the animal in warm blankets. Diaphoretics, such as pilocarpin, are not very satisfactory in small animals.

Purgatives are to be recommended. Magnesium sulphate (10.0-16.0) every four hours until active catharsis takes place is useful. Small doses of arecalin $(0.003-0.005)$ may be given to the dog to hasten early evacuation of the bowels.

Calomel, owing to its prompt action, is excellent for dogs. In heart weakness, during the secondary stages of the disease, digitalis fluidextract $(0.1-0.15)$ should be used. Alkaline diuretics, as potassium acetate, are to be used in small doses. Diuretin has been found to be valuable as a diuretic.

In convulsions following uremia, potassium bromid or morphin for dogs should be administererl. Tannic acid $(0.1)$ is highly recommenderl.

Chronic Nephritis-Definition. - A chronic inflammation of kidney, which the may be divided clinically into two groups, viz.:

(a) Chronic parenchymatous nephritis, characterized by marked dropsy and luring the early stages of the disease, on postmortem, by the large white kidney. In the later stages 
of the disease the kidney usually is small-small white kidney.

(b) Chronic interstitial nephritis, characterized by cardiovascular changes which are pronounced, but only in a few instances will dropsical conditions appear.

Etiology. - It has been observed that chronic nephritis in some instances follows the acute form of the disease. This is true no doubt only in the subacute or milder acute cases.

It may develop gradually as an insidious disease without any apparent cause.

Injurious substances in the form of irritants, which may be either parasitic or chemical in nature, in being eliminaterl via the kidneys, may produce sufficient irritation to induce a chronic inflammation. Certain drugs when administered for some time (turpentine) or absorbed from the skin, as in the treatment of mange (coal-tar compounds), will produce irritation resulting in chronic nephritis.

It may follow some of the infectious diseases, such as distemper in the log and cat. The excess of waste products together with the various toxins formed are eliminated in such quantity that they irritate the renal tissue.

Subjection to extremes in temperature (coll or heat) disturbs metabolism, which increases the action of the kirlneys, and the amount of albuminous decomposition may be a cause. In other cases no apparent cause can be found.

Pathology. - (a) In chronic parenchymatous nephritis several varieties have been recognized.

The large white kidney is characterized by enlargement, the capsule very thin. When cut longitudinally the cortex is swollen and yellowish-white in color, mottled on surface with a number of opaque spots. The pyramids of the kidney are deeply congested.

'The small white kichey in which the connective tissue is found thickened, and a gradual reduction in the enlargement of the parenchymatous tissue. On cut surface the resistance is much greater than the other type, the cortex is much smaller and contains a number of white or whitish-yellow spots. 'These whitish-yellow spots represent areas of fatty degeneration. 'The interstitial tissue is changed, enlarged, 
many of the glomeruli destroyed, degeneration of the epithelium in the convoluted tubules, and the arteries are much thickened. Microscopically the epithelium is found granular and fatty; the tubules of the cortical substances are enlarged and filled with tube casts. Hyaline changes arc found in the epithelial cells. The glomeruli are found enlarged, the capsules are thicker than normal, and the capillaries show some hyaline changes. The interstitial tissue is increased to some extent.

(b) In chronic interstitial nephritis the kidneys are small, contracted, and may be reduced to nearly one-half their normal size. The capsule of the kidney is thickened, very much adherent, and when stripped off carries with it some of the cortical substance. Small cysts are often found on the surface. On cut section the kidney is found reddish or dark red in color. The cortex is very thin. The surface of the kidncy is uneven with numerous small projections. Sometimes the kidneys are lobulated, the consistency is hard, and the general texture almost semicartilaginous. 'The interstitial connective tissue is far in cxcess of the parenchymatous structure.

Microscopically there is noted a great increase in the connective tissue, a degencration and atroplyy of the scereting structures, both glomerular and tubal. 'The increase in the fibrous elements is widely distributed throughout the kidney, although in most cases found more extensively in the cortical portion. The glomerular changes arc found marked, numbers of them being completely degenerated into hyalinc substances. The tubules show changes in thc epithelium, in solnc instances greatly atrophied, in others the epithelium has cntircly disappeared.

The bloodressels (arteries) in the advanced cases show advanced selerosis. 'The changes takc place in the entire vessel wall. In chronic ncphritis we find in a great many eascs in dogs organic clianges in the heart (lyypertrophy).

Symptoms. - This condition docs not present very definite symptoms until the rlisease is quite well advanecd. 'The first symptoms noted are those of a general nature, such as partial or complete loss of appetite, wcakness, fatiguc, ctc. 
In parenchymatous nephritis the secondary symptoms are charaeterized by dropsieal swellings appearing on the limbs, breast, and particularly ascites. The animal shows general emaciation, pale membranes, and all the signs of general cachexia. A careful examination must be made in sueh cases to distinguish from circulatory disturbances. The urine should always be carefully examined. We will find in these cases the amount of urine decreased and its specifie grarity increased. The urine will also be found to contain numerous casts, epithelium, fat cells, and in some cases red blood corpuscles. The pulse will be found accelerated, tense and hard; the heart beat is strong, palpitating, and in smaller breeds may shake the entire body. Marked dulness over the region of the heart is noted indicating hypertrophy. 'The temperature is slightly elevated until in the later stages of the disease when it will be found to be subnormal. In the very late stages symptoms of uremia appear, with rapid emaciation and exhaustion, and the animal soon succumbs.

In chronic interstitial nephritis the symptoms are somewhat different. The most noticeable difference is in the charaeter and eomposition of the urine. The amount of urine is inereased, the speeifie gravity very low, and the atbumin content is greatly diminished. There is compensatory hypertrophy of the heart, and if this compensating artion is sufficient, the animal may live for some time. However, sooner or later there will be insufficient heart action, the pulse will become weak, feeble, and ascites and edemas appear. The animal gradually becomes weaker and finally dies from exhaustion.

Diagnosis. - This is only possible in cases where a careful examination is made of the urine, together with a painstaking general examination. We must differentiate chronie nephritis from primary circulatory disturbances.

Prognosis. - In both forms of ehronic nephritis, the prognosis should be eonsidered unfavorable, because in the majority of aases the condition is quite well advanced before a diagnosis is made. Even in apparently mild eases marked pathological changes in the kidneys are often found. 
Treatment.-Dietetic.-Improve the general condition of the animal by giving plenty of milk and easily digested food. Avoid highly nitrogenous foods.

Medical.-The first thing to be considered from a medical standpoint is to sustain and strengthen the heart action by giving small doses of fluidextract digitalis $(0.1$ to 0.15$)$ daily. As a diuretic, administer diuretin (0.2 to 0.4$)$ twice daily. Calomel is useful in dogs to keep the bowels open and assist in the elimination of waste products.

In dropsical conditions (ascites) small doses of pilocarpin ( $\operatorname{dog}$ and cat $0.003-0.01$ ) may be administered once daily. Sinall doses of potassium iodid $(0.06-0.2)$ are to be administercd as a resorbent once daily to dogs.

Surgical.-When ascites threatens the life of the animal the fluid should be removed with a trocar. (See Abdominal Puncture.)

Purulent Nephritis.--Kidney Abscess.-Definition.-An inflammation of the kidneys resulting from infection and characterized by the formation of either numerous small purulent foci, or larger abscesses.

Etiology.-A very common cause of this condition is the infection reaching the renal tissue from the blood stream (hematogenous). This mode of infection in most cases follows diseases of other organs, or pyemia, such as cndometritis pucrperalis in the bitch and cat; mammitis, pneumonia, phlegmonous pharyngitis in the cat; purulent bronchial catarrh, distemper, and, in puppies, infection at birth. It has been found that purulent nephritis will be produced in animals without any particular focus of infection. In cases of general reduced resistance the organisms nay find their way to the renal tissue, develop and form abscesses.

Traumatic causes are quite common in small animals as injurics over the region of the kidneys are frequent. 'These may bring about the condition by reducing the local resistance and the accompanying inflainmation makes a favorable place for the development of orgánisms which are present in the blood stream.

Irogenic causes are perhaps the most frcquent. The 
infection spreads to the organ via the bladder, ureters, and pelvis of the kidney.

In small animals subjection to extremes in temperature has been mentioned as an indirect factor in bringing about the condition,

Pathology.- We recognize two forms of purulent nephritis on postmortem:

Diffuse Purulent Nephritis (Nephritis purulenta punctata, diffusa).-In this form the kidney's are enlarged; numerous small white spots or yellowish dots are present which are surrounded by a reddish zone. On close examination the purulent masses may be easily removed. These foci of infection may be found quite generally distributed in one or both kidneys.

Mieroscopically there is found a large number of pus cells in the foci of infection, cellular infiltration with large numbers of bacteria present in the tissue surrounding them. The epithelial cells show fatty degeneration, the glomeruli surrounded by pus, and the tubules partially filled with leukocytes, red blood cells, and casts.

Nephritis Apostematosa (Renal absress).- We find in this form abscesses of varying size, which may be a single abscess formed from an embolus, or the kidney tissue break down to form a very large abscess (pyonephrosis). The connective tissue increases around the abscess forming a thick wall.

Symptoms. - Clinically it is quite difficult to recognize this condition. In cases which originate by metastasis we will observe the symptoms of the primary condition, such as pyemia, endometritis, etc.

Should the disorder develop rapidly, there will be found practically the same symptoms as in acute diffuse nephritis. (See Acute Nephritis.)

In dogs the patient becomes very stiff, refuses to move, slows pain on palpation over the region of the kidneys; in some cases a distinct enlargement on one or both sides high in the lumbar region can be determined. In examining such cases the animal should be placed in a standing position, both hands used, one on either side of the animal, and the 
palpation performed with gentle pressure. Micturition is painful, very often only small quantities of urine being voided. As the disease progresses more pronounced general symptoms of weakness, exhaustion, and uremia will be observed. Animals often die very early from general sepsis.

Diagnosis. - An accurate diagnosis is very difficult. In many cases the disease is not recognized until a postmortem examination is made. The symptoms, including careful palpation coupled with examination of the urine, usually suffice for the diagnosis.

Prognosis.-The prognosis is bad as the disease is usually in an adranced stage before being recognized.

Treatment. - In cases of advanced renal abscess there is little that can be done. 'The operation of removing one kidney does little good as both are usually diseased. Tremia commonly follows the removal of the kidney when both are involved. Symptomatic treatment is about all that can be done. Heart stimulants, laxatives, etc., may be tried. (See Acute Nephritis.)

\section{INFLAMMATION OF THE RENAL PELVIS.}

\section{Pyelitis.}

Definition.-Inflammation of the pelvis of the kidney.

Etiology. - Prelitis may result from the spread of inflammation from adjacent parts or organs, such as from the kidney (pyelonephritis) or from the bladder (pyelocystitis).

The presence of urinary calculi in the pelvis of the kidney mechanically irritates the membrane and leads to an inflammation.

In the course of infectious diseases (distemper in the dog and cat; cholera in birds) this condition develops on account of the infectious or poisonous matter excreted from the body via the kidneys irritating the mucous membrane.

Excretion of toxic materials (poisons of different kinds) would act in a similar manner. On account of the frequency of poisoning in small animals this is a very common cause.

Parasites often cause prelitis, especially in clogs (I) ioctophyme renale). The parasites cause an intense inflan- 
mation of the pelvis of the kidney which may involve the entire organ.

Retention of urine from either disease of the ureters or the bladder. This leads to an inflammation of the pelvis of the kidney from decomposition of the urine.

Pathology.-Catarrhal inflammation of the pelvis of the kidney is characterized by swelling and redness, some hemorrhage, and later, as the condition becomes chronic, the membranc becomes thickened, lighter in color and covered with thick mucus or pus. In severe cases numerous hemorrhagic foci will be observed, with sometimes extensive hemorrhage, or, if the irritation has been severe, ulcers will be found (pyeloulcerosa). In cases which have resulted from obstruction to the flow of urine, we may find dilatation of the pelvis of the kidneys, with the presence of urine (hydronephrosis).

Symptoms. - This disease does not present a clear clinical picture; it can easily be confused with inflammation of the kidneys or adjacent parts. 'The general symptoms are a disturbed gencral condition of the animal, frequent micturition, which is more or less painful, stiff, painful gait, loss of appetitc and slight elevation of temperature. The urine shows changes which should be considered. It will be found to contain much mucus, pus, organic sediment, long-tailed epithelial colls, having pointed projections on the ends, which come from the membrane of the renal pelvis. 'The urine should be examined for parasite eggs; this will of ten assist in locating the seat of the inflammation. Small granules are found when calculi are present. Careful palpation as in chronic ncphritis may assist in locating the inflammation.

Diagnosis. - The microscopic cxamination of the urine is the best means of making an accurate diagnosis. 'The presence of the peculiar, molar-shaped epithelial cells may be considered significant. Pyelitis must be differentiated from inflammation of the mucous membrane of the bladder or ureters. 'The presence of parasite eggs (I)ioctophyme renale) in the urine will be indicative of involvement of the renal jelvis.

Prognosis.- 'The prognosis depends to some extent upon the causative factor. In most cases it is not very favorable. 
Treatment.-Dietetic.-Milk should be the principal food. No irritating materials should be given.

Medical.-Diluents in the form of water or milk should be administered frequently ( 2 or 3 times daily) to assist in increasing the volume of fluids eliminated via kidneys to remove accumulated products.

Disinfectants in the form of sodium salicylate (dog, 0.12.0); cat, $0.05-0.1$ ) should be given twice daily. Urotropin $(0.5)-1.0)$ three times daily with plenty of water or milk is useful.

\section{UREMIA.}

Definition.-A toxemia developing during the course of certain diseases, such as nephritis or in conditions associated with retention of the urine. The nature of the poisons retained in the body is not definitely known. They may be normal urine compounds, or the result of abnormal metabolism.

Etiology. - Cremia is produced by the retention in the body of waste materials which should be eliminated by the kidneys. In the derelopment of rertain diseases, such as acute and chronic nephritis, or obstruction to some part of the urinary passages, the urine is not properly excreted but is retained in the bloorl. If due to stoppage of the outflow, the back pressure produced inhibits further secretion, hence the products of metabolism accumulate in the body.

A cause is rupture of some of the urinary organs (kidneys, ureter or bladdler) which is of frequent occurrence in the dog from injuries. 'The urine will flow out into the adjacent tissues, or peritoneal cavity, to be absorbed by the circulation, producing in the course of a few hours marked symptoms of acute poisoning.

Symptoms. - Clinically we recognize two forms: $(a)$ Acute uremia, and $(b)$ chronic uremia.

Acute Cremia.-In the dog the symptoms usually begin with chills, trembling of the muscles, staggering gait, followed in a short time by stupor, the animal finally lapsing into complete unconsciousness. lirequently there will be noted 
during the development of uremia certain nervous symptoms, such as contractions of groups of muscles (clonic spasms, epileptiform convulsions) followed by unconsciousness. Yelping or howling is often a prominent symptom during the nervous attacks.

The respirations are slow, often difficult; edema of the lungs usually exists.

The temperature is at first elevated, but later becomes subnormal, sometimes as low as $96^{\circ} \mathrm{F}$.

Tomiting in the dog and cat is a frequent symptom as is diarrhea. The discharges from the stomach and bowels usually have a uriniferous odor.

Chronic Uremia.-Chronic uremia derelops from chronic diseases of the urinary organs, particularly chronic nephritis, and from long standing cases of partial urine retention due to some obstruction in the urinary tract.

'The most prominent symptoms of ehronic uremia are: Digestive disturbances, such as gastro-intestinal catarrh, diarrhea, romiting, etc., without any apparent cause; disturbances in the nervous system as dulness, and at times epileptiform convulsions, which are usually mild and recurrent.

Diagnosis.-This is made by careful examination of the patient, observation of the symptoms, and examination of the urinary organs.

Prognosis. - The prognosis depends upon the primary condition producing the uremia. In acute uremia it is unfavolable, most cases terminating fatally.

Chronic uremia is not so immediately fatal. The long course of the disease, which suffers exacerbations and remissions, eventually, however, terminates in death.

Treatment.-.Medical.-Medical treatment consists in the arlministration of laxatives to assist the elimination of urea compounds via the bowels. Diuretics are used except in those cases where there is some impediment to the outflow of urine.

In chronic cases, after the uremic symptoms begin to disappear, tonics and alteratives are indicated.

Surgical.-Where an obstruction exists in some portion of the urinary tract, which interferes with the outflow of 
urine an operation may be indicated. Rupture of the bladder or ureters should receive immediate attention and the torn or injured part sutured.

\section{CALCULI IN THE KIDNEY.}

\section{Nephrolithiasis.}

Definition.-The presence of urinary stones in the pelvis of the kidney.

Etiology.-Calculi in the renal pelvis are not frequent in small animals. They are far more common in the bladder. The principal condition for the formation of urolithic deposits is that the urine contains excess of salts, or that insoluble or slightly soluble salts are formed in it. In the elimination of these salts they become gradually deposited around some forcign material. The center or nucleus of a calculus is usually an epithelial cell, bit of mucus, pus, a blood cell, cast, etc. In some cases the food which is rich in salts of various kinds will hasten the formation of calculi. In certain districts where the water is rich in mineral substances calculi are more common, no doubt due to the excess salts taken in and eliminated. Inflammatory diseases of the urinary passages, or in retention of urine from any cause, will often learl to the formation of urinary calculi by changing the character of the urine and the salts contained therein.

Pathology.-Stones in the pelvis of the kidney, if small, may not produce much change in the mucosa, except slight abrasion and irritation. When larger they may fill up the entire calices or the whole renal pelvis, and can lead to marked pathological changes such as extensive inflammation, hemorrhage, and sometimes marked distention of the pelvis. In small animals the calculi usually consist of ammoniacal magnesium phosphate, small quantities of calcium phosphate or carbonate, some uric acid and its salts. ('ystic calculi are also occasionally seen. They are small, soft, and have a shiny surface.

Symptoms.--The clinical picture of kidney stones is very similar to that of pyelitis, except the pain is usually more 
severe. 'The condition may be entirely' overlooked during the life of the patient. 'The most pronounced symptoms are sudden attacks of colic, which come on after running, jumping, or falling, which dislodge the stone and occlude the ureter. The colicky ssmptoms are howling, whining and crying, which continue until the stone either passes into the bladder or back into the pelvis of the kidney. A prolongation of the retention of urine may result in uremic symptoms. Periodic recurrence of the colicky pains is somewhat charaeteristic of this condition. 'The urine when examined microscopically will be found to contain pus cells, epithelial cells, and often very small fragments of stones. Blood cells are also common as small hemorrhages frequently take place. In the $\log$ a direct examination can be made by performing a laparotomy which permits of the kidney being seen and felt. If alculi are present, they will be easily recognized by their hardness and shape.

Diagnosis. - The general symptoms are not sufficient for an accurate diagnosis. It should be differentiated from other discases of the kidney. 'The urine should be carefully examincd. In doubtful cases a laparotomy may be performed.

Prognosis. - The prognosis depends on the size and number of stones present and whether or not the condition is uni- or bilateral. When the stones are small and the condition confined to one kidney, the prognosis is much more farorable. Owing to the difficulties encountered in removing the stones the prognosis is usually unfarorable.

Treatment. - Dietetic. - Food should be given that contains but a small quantity of salts. Plenty of water should be allowed but the same precaution must bo observed in regard to the salt (o)ntent.

Medical. - The various compounds used to dissolve calculi lave not proved very satisfactory. Iarge quantities of (arbonated water may be tried; it has proved of value in some cases.

Surgical.-Surgical treatment has proved of practical importance in the dog, and las been suecessfully arecomplisher in a number of cases. If, after an explorative laparotomy of the kidney, it is found normal, showing no evidence 
of hydronephrosis, nephrolithotomy should be performed. There are two methods used in this operation, as follows:

(a) A longitudinal incision is made at the convex border of the kidney, at which point the vascularity is at a minimum, into the pelvis. In case hemorrhage is severe, clamping the renal artery will control it. By compressing the kidney longitudinally the incision will be held open and with a blunt curette the stones are removed. Care should be taken to be sure that the renal pelvis is freed of all the calculi, and a blunt probe of small caliber inserted into the ureter to be certain of a free passage into the bladder. Normal salt solution, which has been previously sterilized, is used to cleanse the cut surface. The wound is then stitched with sterile silk, using two or three interrupted sutures. Care must be taken not to use too much force in drawing the wound together as the resulting swelling will tear out the sutures. Return the organ to its proper position. Healing should take place per primam.

(b) The other method is to open the pelvis of the kidney direct. Make an incision at one side of the pelvis, in about its middle portion, of sufficient size to remove the calculi. After all the stones have been removed and all the fluids absorbed by sterile gauze, the wound is stitched carefully, using plenty of sutures so that the edges of the wound will be thoroughly approximated. This must be done carefully to avoid fistula following the operation. If, however, the kidney is found diseased (hydronephrosis) nephrectomy should be performed, as follows: Laparotomy is performed. The kidney is then freed of its covering, gently pulled toward the wound, and doubly ligated at its pedicle, so as to prevent serious hemorrhage. Care should be taken to get the ligature securely in place to prevent its slipping off after the abdominal wound is closed. Double ligation is safest, ligating the vein and artery separately.

After-treatment consists in the administration of general stimulants, and restricting the diet for four or five days to milk, or milk and eggs. 


\section{DROPSY OF THE KIDNEY.}

\section{Hydronephrosis. Cystic Kidney.}

Definition.-A chronic condition in which urine collects in the pelvis of the kidney or the kidney proper, leading to functional disturbances of the organ.

Etiology.-(a) Mechanically by some impediment to the flow of urine from one or both kidners. The urine is dammed up in the pelvis of the kidney with a gradually increasing pressure. As the pressure of the urine increases, the loss in the secreting power of the organ is more manifest, and eventually if the pressure is constant or increased, the function of the organ may be entirely lost. The parts of the urinary passages affected by the impediment gradually dilate and the renal tissue atrophies, so that the condition anatomically stops as no more urine is secreted.

(b) A number of conditions which cause partial stoppage of the flow of urine will cause cystic kidney. The most frequent are: Catarrhal inflammation of the ureters, bladder, or prostate glands; the presence of calculi in some portion of the tract which interferes with the passage of urine.

(c) Sometimes the condition is congenital; there is either no opening through the ureter, or it is otherwise anatomically deficient. This allows the urine first formed to accumulate until the backward pressure is sufficient to stop renal secretion.

(d) Compression of the urethra and neck of the bladder by adjacent new growths. The new growths interfere with the flow of urine and cause it to accumulate in the pelvis of the kidney.

(e) Paralysis of the urinary bladder which allows the urine to collect and lead to back pressure.

(f) In chronic inflammation of the kidneys some of the urinary tubules become constricted at certain points by the contraction of the interstitial tissue, which undergoes atrophy, causing the canals which are attached to the Malpighian bodies to become dilated. If the urine continues to be secreted it accumulates and forms small retention cy'sts. 'These cysts may' be found singly or in large numbers in the 
kidneys. 'This type of the condition is not found as often as the other form.

Pathology. - We find rarying degrees of cystic killney. In the earlier cases will be noted only a dilation of the calices and pelvis of the kidney with mild pathological changes in the lining membrane. In the more advanced cases will be noted distention of the pelvis of the kidney with compression of the renal tissue so that the atrophy is well marked. In some cascs the renal tissue will be practically destroyed, and the kidney will be represented by a soft undulating mass. In dogs the kidnevs may be so enlarged that distention of the abdominal wall appears.

Sympioms. - Frequently on autopsy cystic kidney involying onc of the kidneys, will be found which has not been noticed during the life of the animal, the other kidney having performed the function of both. When both kidners are involved, however, a change in the quantity of urine will be noticeable. On careful examination of the patient (dog) very frequently one of the kidneys, or in rare cases both of them, will be found much enlarged and can be easily palpated through the abdominal wall; cases appearing where the abelominal enlargement is noticeable by observing the standing patient from the rear and carefully comparing both sides. (ieneral symptoms of weakness, stiffiness, etc., are shown in the more advanced cases. Generally, however, cystic kidney does not produce characteristic symptoms during life.

Diagnosis. - This is made by eareful examination of the patient, observing the flow of urine, and finally where necessary an explorative laparotomy.

Prognosis. - Favorable when unilateral as it may not affect the general health of the patient; unfarorable when bilateral.

Treatment.- The early indication in the treatment is to relieve the imperiment to the flow of urine. The ureters, bladker, and urethra shoukd be examined and if diseased proper treatment should be given. If the outflow of urine "an be reëstablished, the disorder will be relieved. If this is impossible, nephrectomy should be performed if the condition is milateral. (For Nephrectomy, see Renal Calculi.) No other treatment las been found of value. 


\section{AMYLOID KIDNEY.}

This condition is of no practical importance except to the student in pathology. It is not common in the small animals and the symptoms are very obscure.

\section{TUMORS IN THE KIDNEY.}

In small animals the kidneys are not commonly affected by primary tumor formations. There will be found: Sarcomata, carcinomata, and adenomata, occasionally resulting as secondary growths from some other organ or tissues. 'Tuberculosis of the kidney has been noted in a few cases in the dog. It may be either metastatic or urogenic in origin.

It is not usually possible to recognize tumors during life. 'They sometimes produce disturbance in kidney function and may become metastatic.

Treatment. - Treatment must be symptomatic.

\section{ANIMAL PARASITES IN THE KIDNEY.}

Dioctophyme renale (Eustrongylus Gigas).-This is the most common parasite found in the kidney of small animals where it is confined almost exclusively to the dog. Its location is usually in the pelvis of the kidney where in some cases it produces marked clinical symptoms; in others but little disturbance results considering the degree of pathological change induced.

1)ioctophyme renale is a blood-red worm with a number of fine transverse stripes, tapering slighty at the extremities. The mouth is triangular in shape, and surrounded by six small papillex. The male is $13 \mathrm{~cm}$. to $40 \mathrm{~cm}$. long, and $4 \mathrm{~mm}$. to $(i$ mm. in diameter; tail is obtuse, terminated by a patelliform, membranous, entire pouch without radlixe, and traversed by a very slenter, single spicule. Female, $20 \mathrm{~cm}$. to $1 \mathrm{~m}$. long, and jo mm. to $12 \mathrm{~mm}$. in diameter; tail is obtuse and slightly curved; a single ovary; vulva very near the mouth. () va ovoid and brownish, (is to $\mathrm{s}(0)$ microns long, and 40 to 43 microns broad. (Neumant.) 
The life cycle of the Dioctophyme renale is not entirely known. The ovum is expelled from the borly of the host, and undergoes further development in water or damp soil. It requires some time for these changes to take place (from three to six months). The embryo may live and be active after one year to eighteen months. The embryo is 240 microns long and 14 microns broad, cylindrical, and gradually tapering posteriorly; the head is pointed, mouth terminal and not provided with papillæ, but with a small projection which no doubt serves the embryo as an organ of penetration. The intermediate host has not been fully determined. The dog takes the embryo into the intestinal tract, where it undergoes further development, and the embryo migrates to the organs and tissues. It may also be introduced into the urinary passages direct. The favorite seat is the kidney, although it may be found in the abdominal cavity, liver, etc. There still exists some doubt as to the exact method of migration of the embryo.

The parasite after reaching its destination, develops and produces marked changes in the pelvis of the kidney, and in a number of instances completely destroys the organ. In cases of early invasion there will be found inflammation with hemorrhages, and purulent material. More advanced cases will reveal a secondary inflammation with destruction of the renal tissue, often transforming the kidney into a sac with thick walls. The worm or worms will be found coiled up in this sac. The number of parasites found will vary. In most cases only one; in others two or more, even as high as four have been found.

Symptoms. - There may be no symptoms during life. In a case of the author's in which four parasites were found, and the renal tissue completely destroyed, the dog showed no symptoms. The worms were found on autopsy. The animal was in excellent condition and apparently in the best of health. However, in some cases, symptoms are observer. Severe pain, restlessness, nervousness and even rabiform phenomena have been observed. The dog may show spinal curvature due to pain on the affected side. The general condition is often interfered with, the animal becoming 
enaciated and exhausted. 'The urine is voided with difficulty, containing blood and pus. Fxamined microscopically it will reveal a prelitis, and the eggs of the parasite. In some instances after the renal tissue is completely destroyed, the parasite will pass into the urethra where it becomes lodged. It may burrow through the urethral wall and ultimately lodge in either the pelvic fascia or work forward into the abdominal eavity. Eventually it will produce inflammation in the new location, resulting in abscess, perforation and external fistula. Peritonitis may result following its entrance into the abdominal cavity.

Diagnosis. - An accurate diagnosis can be made only by finding the eggs in the urine by microscopic examination. The symptoms would be much the same as those in pyelitis, etc.

Prognosis.- When the parasite has produced sufficient pathological changes to bring about emaciation and exhaustion, the prognosis is unfavorable.

Treatment.-Medical.-Anthelmintics are advisable, and especially those which will be eliminated via the kidners. Turpentine ( 5.0 to 10.0 ) repeated in forty-eight hours, may be given, if necessary.

Surgical.-Laparotomy may be performed, and direct examination made. If the renal tissuc is destroyed, nephrectomy would be indicated. (See Renal Calculi.)

Other Parasites in the Kidney. - A few other speeies of parasites are seen occasionally, but as they produce no effect upon the host they are of no clinical inportance.

(ysticercus cellulosie and several forms of coccidia have heen found on autopsy.

Birds are not uncommonly affected by coccidia. 'The Eimeria aviun (Coecidium tencllum) is the one most commonly found in poultry. Some general disturbances, snch as depression and loss of appetite, have been noted. 'Thle birds die of uremie poisoning or exhaustion. 


\section{CHAP'TER II .}

\section{DISEASES OF' THE BLAIDER.}

Examination. - A thorough and complete examination of this organ may be made, especially in the $\log$, by palpation through the reetum, vagina, and abdominal walls; by laparotomy (direct examination) and by examination of the urine.

Palpation can be done satisfactorily only in the larger brceds when not too fat. When palpating through the abdominal wall place the animal in a standing position, and, with one hand on either side of the lower abdominal wall, just anterior to the brim of the pubis, exert enough pressure to feel the bladder through the walls. In case inflammation is present pain will be evinced. 'The bladder will be felt as a pear-shaped enlargement just anterior to the brim of the pubis, which is movable and extends forward a varying distance depending upon its distention. Care must be taken to differentiate between a distended blatder and other abnormalities which are commonly present in the abdominal cavity, such as ascites, fecal stasis, neoplasms, etc.

Rectal palpation is done by first thoroughly cleansing the hand and the anal region with soap and water, followed by an application of boric acid solution ( 2 per cent.). The index finger is inserted through the anal opening as high up in the rectum as possible. The bladder can be felt as a distended body, projecting back into the pelvie inlet. I)ifferentiation must be made between a distended bladder and chronic or acute prostatitis which is quite common in old dogs. This ean be done by considering the difference in position and density of the two bodies. Inflammatory ("onditions of the bladder, which are painful on pressure, and other enlargements such as tumors may be found in this location. 
Vaginal palpation is possible in the larger breeds, and is performed in much the same manner as the reetal. The index finger should be inserted as far as possible, and if the bladkler is rlistended it ean be felt at the pelvic inlet, or if it is inflamed slight pressure will be very painful. Vaginitis, fecal accumulations in the rectum, and tumors should be differentiated.

Laparotomy. - In both the male and female dog it is quite possible, safe, and practical, to perform this operation under strict antiseptic preeautions, so that a direct inspection of the bladder may be made. In the female the incision should be made just anterior to the pubis in the median line, and in the male to one side of the penis but close to the pubis. 'The incision should be made large enough ( 2 or 3 inches) so that the blarkler may be exposed to view. Care should be (n)server in eutting through the peritoneum so as not to incise the bladkler, as this organ when clistended will extend forward in some cases beyond the umbilieus. 'The bladder is examined for distention with urine, inflammation (aeute and (hronic), calculi, ulcerations, paralysis, etc. The laparotomy wound should be closed as usual. (see Laparotomy.)

Examination of the Lrine-A sample of urine is best obtained by passing a catheter, provided there is any urine present, or by catching the urine in some receptacle as it is passeel by the animal. (atheterization has been found to be the most practical method in these animals. 'This is done in the male animal by placing it in a dorsal position; restrain with hobbles. (Choose a small sized human catheter, soften and disinfect by placing it for ten to fifteen minutes in warm lysol solution (2 per cent.). Expose the penis by pushing hack the prepuce with the left hand, and with the right hand insert the catheter into the urethral opening. 'Two normal ohstructions will be noted as the catheter is inserted: 'The first one as the ratheter reaches the bone of the penis; the other as the catheter reaches the isehial arch. 'The former obstruction may be overcome hy gentle pressure; to pass the ischial areh it will sometimes be necessary to partially romove the stilet, and with the finger direet the catheter over this point. 'The stilet should be grarlually removed 
as the catheter is inserted. When it reaches the bladder, if urine is present, it will begin to flow out at once. In the female animal the eatheter is passed without much difficulty. It is best to place the animal in a ventral position, securely fastened with hobbles; the same catheter as for the male animal can be used, but best to use a special metallic catheter, as it can be sterilized. 'To insert the catheter a vaginal speculum is used to dilate the vagina, which facilitates insertion into the urethral opening.

'The urine should be examined particularly for epithelial cells, pus, bacteria, red blood cells, etc. A differential examination should be made to determine whether the abnormal constituents of the urine come from the bladder or some othcr urinary organ. Note the reaction of the urine, its specific gravity, color, odor, consistency, etc.

\section{WOUNDS OF THE BLADDER.}

'The bladder is the seat of several conditions produced by trauma in the small animals: Traumatie or spontaneous rupture, penetrating wounds from bullets or other objects, accidental cutting of the bladder during surgical operations.

\section{RUPTURE OF THE BLADDER.}

Rupture of the bladder is most often brought about by the animal being run over by vehicles, being kicked, falling, or may be due to overdistention when there is some impediment to the flow of urine. It can also occur as the result of the walls being weakened by ulceration and other destructive processes. It has been observed in well broken house dogs when confined for too long a period, the bladder becoming distended and finally paralyzed, the continuance of the secretion eventually leading to rupture.

Symptoms. - In rupture the symptoms develop in the course of a few hours. They are complete suppression of micturition, general symptoms of collapse, uriniferous`odor of the exhaled air, subnormal temperature. The history is quite important, as often the history of an injury will assist 
in the diagnosis of rupture. Passing the catheter will reveal the empty bladder. Examination by performing laparotomy should be done as early as possible in all cases where rupture is suspected.

Prognosis.-After the development of general symptoms of collapse, subnormal temperature, etc., the condition is considered unfavorable. In cases of rupture where the diagnosis is established early, or, in accidental cutting through the walls of the bladder during surgical operations, the prognosis is quite favorable, provided prompt treatment is given.

Treatment. - Medical. - It is always advisable to adıninister stimulants at once. Strychnin in small doses (0.001).

Surgical.-Prompt surgical treatment is absolutely essential for a successful termination.

The animal should be properly prepared for laparotomy (see Laparotomy), and the operation begun as soon as possible. The abdominal cavity should be emptied of all the retained urine, by flushing thoroughly with normal salt solution, which should be repeated two or three times to be sure that all the urine is removed. Locate the wound in the bladder and suture with interrupted and Lembert stitches. A milliner's needle will be found to be the best suturing needle. Place the stitches quite close together. Suture the abdominal wound in the usual manner. The after-treatinent consists in placing the animal in a warm place and using stimulants for the first ten to twelve hours. Thoroughness in treating these cases will often bring exccllent results.

Wounds of the bladder, such as gunshot wounds, injury by compression without complete rupture, puncture by fragments of bone, etc., are found in the $\log$, and their seriousness depends upon the degree of injury. In very small punctured wounds and small bullet wounds, aside from the symptoms of cystitis, and stiffness, no serious complications set in and the animals make prompt recoveries. In the other forms when urine escapes into the peritoneal carity and some hemorrhage takes place, the ease will soon assume serious complications. (See Rupture of the Bladder.) 


\section{RETENTION OF URINE IN THE BLADDER.}

\section{Retentio Urince Tesicalis.}

Definition.-A collection of urine in the bladder, with subsequent distention of the bladder, ureters and pelvis of the kidney. Retention of urine is a symptom of a number of indcpendent conditions or diseases which are found in the log and cat, often leading to inflammation of the bladder, rupture, peritonitis, and uremia.

Etiology.-Commonly eaused by foreign bodies in the urethra or neck of the bladder interfering with the voiding of urinc. These foreign bodies consist mainly of calculi of different kinds, sediment, fibrin, blood coagula, etc.

In paralysis of the bladder, the walls of the bladder lose their tone. It may be due to affections of the spinal cord, trauma, various forms of cystitis, or to emaciation and general weakness, which allows the bladder to distend enormousty. The urine in some cases will flow out in a small continuous stream after the distention has reached a ccrtain degree.

Compression of some portion of the urethra or neck of the bladder from without, such as neoplasms, acute and chronic prostatitis which is common in old dogs. Stricture of the urethra from injury or surgical operations; compression by distention of the uterus in pregnancy and diseases of this organ; diseases of the penis in the male animal are further causes.

Spasmodic contraction of the sphincter resicx, which may be duc to surden change in temperature especially cold (chills); to some medicinal agent as strychnin; to some diseases, crstitis, tetanus (rare).

Symptoms. - The early indication of retention of urine is ischuria (suppression of urine), or painful micturition with only a small amount of urine passed. In the dog micturition is accompanied by serere straining.

In sensitive small animals there is usually considerable abdominal pain, resembling acute indigestion or colic. 'The back is arched and the gait is stiff and strardling.

In a short time, in case the condition is not reliever, the s.mptoms will increase in intensity until the bladder ruptures, 
in which case the simptoms of pain will disappear for a time until peritonitis and uremia develop. If the bladder ruptures symptoms of uremia will develop in a few hours. (See ('remia.)

In the $\log$ the distention of the bladder will produce a noticeable increase in size of the abdominal cavity. Careful palpation will reveal the distended movable bladder. When rupture oceurs the fluid will be detected free in the abdominal cavity. In such case puncturing the abdominal wall with an exploring trocar will reveal the presence of urine. Care should be observed, however, to determine whether the fluid is in the cavity or still in the bladder, as the distended bladkler can extend well forward in the abdominal cavity.

Diagnosis. - 'The srmptoms should be noted carefully. If the patient shows colic, frequent micturition, with small quantities of urine passed, a careful and thorough examination of the urinary organs should be made. As retention of urine in most cases is secondary to some disease of the urinary. organs, a careful examination should be made to determine the primary condition. The examination may include puncturing the abdominal walls, laparotomy, rectal or vaginal exploration.

Prognosis.- The prognosis depends principally upon the possibility of relieving the primary condition, and whether or not the bladder is still intact. In case of rupture it is unfarorable, especially when simptoms of uremia are present.

Treatment. - The treatmont must be directed toward removing the causes. In cases of paralysis of the walls of the bladder, it is advisable to remove the urine as early as possible. 'This can be done in most cases by catheterization. Simall doses of struchnin (0.0(0), dog) are recommended to give tone to the walls of the bladicr.

In spasms of the splineter vesica, catheterization may be tried; if unsucesstul, puncture the bladder and remove a portion of the urine. A small dose of morphin will overeome the spasmodic contraction, allowing the urine to flow out.

In case an olstruction to the outflow of nine exists, treatment must be applied to remove it.

Iouse-broken dogs should be allowed to rum out of doors at regular intervals to aroid extreme distention. 


\section{INCONTINENCE OF URINE.}

Definition.-A constant discharge of the urine from the bladder; inability to retain urine.

Etiology. - Incontinence may result from several different causes:

(a) Affections of the spinal cord, as degeneration, edema, compression from hemorrhage, etc.

(b) Paralysis of the sphincter vesicæ.

(c) Long standing cases of retention.

(d) Lack of tone of the muscles due to senility.

(e) Some cases of cystitis.

(f) Injury to the sphincter muscles from surgical operations, tumors, calculi, etc.

Symptoms. - Constant dribbling of urine. Examination reveals the bladder empty, and the sphincter vesicæ relaxed.

Prognosis.-Depends upon the primary cause. Usually not considered very favorable as recovery is rarely complete.

Treatment.-Symptomatic. Determine the cause and apply treatment to relieve it.

\section{CATARRH OF THE BLADDER.}

Cystitis. Urocystitis.

Definition. - A catarrhal inflammation of the bladder which may be either acute or chronic.

Etiology.-The principal causes are: 1. Bacteria (infection), or irritants in the form of toxins, drugs, chemicals, etc. Bacteria gain entrance to the bladder in various ways:

(a) Through the genito-urinary tract. Catheterization of animals is a common source of infection, the organisms being introduced directly by an infected catheter. In the female germs are easily introduced into the bladder through the urethra, which is short, and its opening near the vulva. A spread of inflammation from other infected parts of the urinary tract, e.g., pyelitis, nephritis, urethritis may also induce.cystitis. Retention of urine from any cause leads to decomposition of the urine, and lowering of the normal 
resistance of the mucous membrane of the bladder, allowing infection to take place.

(b) Infection may gain entrance to the bladder from the blood. In some of the infectious diseases, as distemper in the dog and cat, the bacteria are carried to the mucosa of the bladder by the blood stream. In digestive disturbances (constipation, etc.) the organisms which accumulate in the bowels enter the blood stream and are eliminated through the urinary passages, often leading to an acute cystitis. The colon bacteria and others more or less closely related are the chief offenders.

(c) The infection may spread from the peritoneum, either from acute or chronic peritonitis, producing in some cases simply a pericystitis, and in others an involvement of the entire bladder wall.

2. Irritating agents in the form of chemicals, or drugs, when eliminated in large quantities, frequently will produce cystitis. Cantharides, turpentine, balsams, and coal tar compounds given internally will produce it; external applications of easily diffusible substances will, by absorption have the same effect, especially when applied over extensive areas.

3. Sudden change in temperature, cold (chills), disturbs the general circulation, and may lead to congestion of the bladder. Extreme cold affecting the body temperature might induce extensive inflammation of the organ.

4. Calculi and other foreign material often produce chronic cystitis by the constant irritation they keep up.

Pathology. - In the early stages of acute cystitis, the mucous membrane will be found reddened, congested, much swollen, and here and there small hemorrhages will be noted. There is usually considerable thick, viscid mucus covering the nembrane, or there may be an admixture of pus. The purulent exudate of ten covers the entire membrane. In the later stages of the disease the mucosa is covered by a croupous or diphtheritic, yellowish membrane. Abscesses of various size may exist between the mucosa and the muscular walls. lirosions and ulcerations on the membrane are often the result of irritating materials.

In the chronic form the pathological changes are principally 
a thickening of the mucosa, which is corrugated, often presenting projecting growths. The muscular walls become contracted, thickened and incapable of distention. 'The apices of the corrugations are darkened, eroded and ulcerated. The bladder is usually empty and contracted. Inflammation of other portions of the urinary tract will be more or less apparent.

Symptoms.-In acute cystitis, the animal will show marked symptoms of difficult micturition, severe straining as if to urinate, with only small quantities of urine passed. Sometimes small quantities of blood follow the attempts to urinate. The animal stands with the back arched, shows pain when forced to move, and stiffness in walking. When moved the patient will cry out; it often assumes the attitude of urinating. An erection of the penis is a frequent symptom. Pressure over the region of the bladder induces pain. A dog being examined in the standing position will often cry out with pain, and try to bite and get away. Digital pressure either through the rectum or vagina produces the same symptoms. In most cases the bladder is found empty. Unless the condition is mild, general symptoms are usually observed. The temperature is elevated in the early stages, depending upon the cause and kind of infection. Later it may be normal or subnormal. Suppression of appetite, thirst, and general depression arc often observed. Uremic symptoms will be found in some cases due to reabsorption of urinc, or lack of elimination in cases where the other urinary organs are involved. 'The urine, passed in small quantities, will be dark in color, contains varying amounts of albumin, sometimes pus, and stringy mucus. Shreds of fibrin and necrotic membrane are passed in the croupous and diphtheritic forms of cystitis. The urine is usually alkaline in reaction but may be acid. It will contain fibrin, pus cells (both the large cells and the long slender variety), crystals of ammonium urate, and numerous bacteria. The urine content assists in confirming the diagnosis. Chronic cystitis produces much the same symptoms but less severe than in the acute form. The most noticeable indications of chronic cystitis are painful micturition, the urine passed containing pus, red corpuscles, 
etc. 'The catheter should be inserted to differentiate from calculi.

Diagnosis. - In acute cystitis a diagnosis can be made by observing the symptoms, making a careful local examination, and by analysis of the urine. Discases of other portions of the urinary tract should be considered. In chronic cystitis the diagnosis is made by the examination of the urine, and the local examination of the patient. Calculi in the bladder and urethra should be excluded.

Prognosis. - In mild cases of acute cystitis the prognosis is usually favorable. In severe cases, owing to the changes which are produced in the walls of the bladder, the prognosis is unfavorable. Chronic eystitis may run a long course without producing any marked symptoms. Complete recovery is rare. By careful treatment considerable improvement can be attained.

Treatment.-Dietetic.-Non-stimulating food slould be given. Milk is perhaps the best as it contains a large percentage of water, which is desirable. Avoid giving meats until the acute symptoms entirely disappear.

Medical.-Much can be done in acute cystitis by the internal administration of antiseptics and disinfectants. Urotropin $(0.25-0.5)$ two or three times daily for dogs; cats should receive about one-fourth the quantity. This preparation produces a disinfectant action owing to the liberation of formaldehyd gas.

Ilelmitol (dog, 1.0-2.0; cat, 0.1-0.5) can also be used for the same purpose, administered either in the form of a powder, or may be given as a subcutaneous injection in 10 per cent. solution.

Salol, salicylic acid, and resorcin may be given for a similar action. In chronic catarrh much the same treatment is recommenled as in the acute form of the disease.

Balsam copaiba, and turpentine in small doses are recommended.

Irrigation of the Bladder. - Irrigation of the bladder, which is easy in the dog, in order to remove infections and irritaling matter, is of great importance in cystitis. In the male animal a catheter is passed into the blarkler, and the urine is allowed 
to flow out. A rubber tube about 2 or 3 feet in length, provided with a funnel, is attached to the catheter. The tube is elevated and a warm, normal salt solution (40.0-70.0) allowed to flow into the bladder. The tube is then depressed to siphon out the fluid. Following the injection, it is advisable to use some of the mild disinfectants as boric acid (2 per cent.), or ichthyol solution (1-2 per cent.). These should be allowed to remain in the bladder five to ten minutes and then removed. The injection should be repeated in eight to twelve hours. In females the injections are made much in the same way. Sometimes in the male, when injections are to be made often, the urethra becomes irritated from the frequent passing of the catheter. In such cases a temporary urethrotomy may be performed at the ischial arch, and the injections made from this point. Thorough application of these solutions will often produce most excellent results.

\section{TORSION OF THE BLADDER.}

This condition has been found to occur occasionally in the dog. It leads to retention of urine and eventually to rupture of the bladder. Death occurs from peritonitis and uremia. Surgical relief should be attempter.

\section{CALCULI IN THE BLADDER.}

Calculi are more common in the dog than in any other small animal. They appear in various sizes and forms. In some cases they are very small and multiple; in others a single, rounded concretion, conforming to the shape of the bladder, is found.

Etiology. - The factors producing concretions in the bladder may be classed under three headings:

(a) Local disturbance in the urinary organs. In this case there will be found degenerative changes from the products of the urine (uric acid), leading to necrosis of the cells, such prorlucts forming the nucleus around which the salts deposit. 
(b) Disturbances of metabolism in which there will be a larger quantity of material eliminated in the form of phosphates, carbonates, oxalates (calcium and ammonium oxalate), uric acid, urates (ammonium urate), etc. The excess of these salts becomes deposited around the organic nucleus, leading to bladder calculus.

(c) 'The administration of foods rich in salts of various kinds will increase the quantity in the borly, and consequently more salts will be eliminated.

Forms and Varieties.-1. Acid Urine Calculi. (a) Uric Acid Calculi (Ammonium Urate).-These are small, hard, smooth calculi, of a reddish or yellowish-brown color. They are perhaps the most common kind found in the dog.

(b) Oxalate Calculi (Calcium and Ammonium Oxalate).In form these calculi are rough on the surface, irregular in shape, and usually when removel are of a dirty white or yellowish color.

(c) Cystin C'alculi.-Soft waxy' bodies, which no doubt result from disturbed metabolism of nitrogenous substances. 'Their color is brown or brownish-yellow. They are soft enough in most cases to be crushed between the fingers.

2. Alkaline Urine Calculi.--There will be found various forms of these concretions, such as phosphates and carbonates predominating, and in combination with other salts, etc. 'These calculi occur either multiple, as particles of sand or grit, or in single, large concretions. 'They are hard, irregular, rough or smooth stones, of a white, yellowish or dirty color, which are usually flattened, oval or oblong.

The recognition of the different varieties of calculi is important from the standpoint of recurrence and treatment following their removal.

Symptoms. - The acid concretions do not, as a rule, produce any marked symptoms, except as they impede the flow of urine.

The large, alkaline stones are most productive of clinical symptoms, and the ones which require the most radical treatment. There will be symptoms of a catarrhal inflammation of the bladder, and pus is discharged with the urine. Micturition is painful, and only small quantities of urine are 
passed. Attention is usually called to the case by the constant dribbling of urine.

Examination of the bladder is necessary to determine the presence of the ealculus. This may be made either by digital examination through the reetum, by abdominal palpation, or by laparotomy. (See Examination of Bladder.)

Prognosis. - Cases when taken early before systemie disturbances make their appearanee from absorption of urine, etc., are favorable. However, the loeal disturbanees in the bladder produced by the calculi should be taken into eonsideration, for sometimes serious alterations diffieult to heal will be found in the mucosa.

Treatment.-Surgical treatment is the only satisfaetory method of removing ealeuli from the bladder. In the male animal the following procedure has been found to be the most efficient.

The animal is prepared for operation by being given an anesthetic, placed on the table in the dorsal position, and the field of operation just anterior to the pubis and lateral to the penis shaved and disinfeeted. The incision should be 2 to 3 inches in length, so that the bladder can be exposed. After exposing the bladder it should be well proteeted with gauze to prevent urine from flowing into the cavity when ineised. 'The incision is made through the walls of the bladder where bloodvessels show the fewest anastomoses. It should be of sufficient size to remove the calculus. After the removal of the calculus the mucosa of the bladder should be examined for smaller stones or deposits, and if any are found they should also be removed with a blunt curette. The mucosa is then swabberl with gauze saturated in an antiseptic solution. 'The wound in the bladder is sutured with a double row of sutures bringing the serous coats in direct approximation. 'The sutures should be placed close together to prevent the urine escaping until adhesion takes place. The laparotomy wound is closed and protected in the usual manner. In the female two methods are employed:

(a) 'The animal, well hoppled, is placed in the ventral position on the table. A vaginal speeulum is used to dilate the vagina. A grooved director is inserted into the urethra 
and with a probe-pointed knife the urethra is incised back to the neck of the bladder. 'The stone, if not too large, is grasped with a suitable forceps and removed. After removal the bladder should be flushed out with a warm boric acid solution (2 per cent.). The vagina should be flushed out daily for a few days.

(b) In case the stone is too large to be removed through the neck of the bladder without injuring the sphincter vesicæ, the operation for cystotomy should be performed as in the male.

'The after-trcatment consists in irrigation of the bladder (sec Cystitis), and feeding plenty of milk and no meat for a week or ten days. In some cases when hemorrhage takes place following the operation, the catheter should be passed daily to remove the urine and any clots which might form.

\section{TUMORS OF THE BLADDER.}

Therc are a few varieties of tumors found involving this organ. 'The most common ones are: Sarcomata, carcinomata, and fibromata. 'They will be recognized by the symptoms of chronic eystitis they produce, by the examination of the urine and of the bladder. If necessary laparotomy may be performed and the bladder examined direct. (Sec Examination of the Bladder.)

Prognosis.-This is not very farorable, especially if the tumor is malignant.

Treatment.-Resection of a portion of the bladiler wall is to be recommended when the tumor formation is localized, otherwise no treatment can be given. Irrigate the bladder in the same manner as in cystitis. (Sec Cystitis.)

\section{PARASITES IN THE BLADDER.}

'The Dioctophyme renale parasite is found occasionally in the bladder. A few cases have been recorded in which species of parasites found in the blood became located in 
the wall of the bladder. Symptoms of catarrhal inflammation of the bladder, with the presence of eggs in the urine, will assist in making the diagnosis. Should eggs be found in the urine, it then becomes necessary to definitely locate the parasite.

Treatment.- Treatment consists in removal of the parasites by cystotomy, and the subsequent irrigation of the bladder with antiseptics. 


\section{CHAP'TER III.}

\section{1)ISEASES OF' 'THE IRE'THRA.}

Examination.-The urethra may be examiner in two ways:

(a) In the male it is possible to palpate from without along its course until it passes over the isehial arch; the part within the pelvis may be palpated through the reetum. Palpation will reveal sensitiveness in cases of urethritis and caleuli lodged at some point along its course. There are three parts of the urethra in which ealeuli are most apt to lodge: At the neck of the bladder; where the prostate glands practically surround it, and at the posterior end of the bone of the penis. At these points, owing to the strueture of the urethra and adjacent parts, any foreign material passed from the bladkler is most apt to become lodged. In the female the urethra ean be palpated through the vagina. In the female, the urethra, as a rule, is free from foreign material, because any substance of this kind small enough to pass from the bladder into the urethra, will be forced out with the urine.

(b) The passage of the catheter or sound is a valuable means of determining the sensitiveness of the mueous membrane, the presenee of caleuli or other foreign material, or strictures at different points along the course of the urethra. Care should be taken in inserting the eatheter not to injure the urethral mucosa; also one should not mistake the normal narrowing of the lumen of the urethra for strictures, etc.

\section{CONGENITAL MALFORMATIONS.}

\section{Occlusion of the Urethra.}

Occlusion of the urethra is oceasionally found in both the male and female. Sometimes an opening exists in some other portion of the urethra through which the urine is discharged. 
Various kinds of abnormalities have bcen observed, such as epispadia and hypospadia.

Symptoms.-Occlusion of the urethra at its outlet is characterized in young animals by a retention of urine, enlargement of the abdomen, and no signs of inicturition. The distended bladder will be found on examination.

Treatment. - Surgical treatment should be given at once. If the occlusion is at the extreme end of the urethra it should be incised at this point and the flaps stitched back to the skin to prevent adhesions taking place. The urine will usually keep the wound open. Should the occlusion be at a point higher up, in a male dog, an artificial opening should be made at the ischial arch through which the urine is allowed to pass. It may be necessary for this opening to be used permanently, in which case the edges of the membrane on either side should be stitched back to the skin, and kept clean for several days until union takes place. In the female the urethra should be opened with a pair of scissors, and kept dilated with a metallic catheter used daily.

\section{WOUNDS OF THE URETHRA.}

It happens occasionally when animals are injured that the urethra will be opened at some point along its exposed portion. It will be made manifest by the presence of a wound through which urine escapes. Internal wounds of the mucosa occur from the careless use of a cathetcr or sound, or by foreign bodies passing from the bladder, or by wceds or straws entering the urethral outlet.

Treatment. - In extensive and severe lacerations of the urethra they should be sutured, and the wound well protected. In a short time union will take place and the urine passed normally. Injuries to the mucosa are treated by injecting mild antiseptic solutions (boric acid 2 per cent).

\section{STRICTURE OF THE URETHRA.}

Definition.-A constriction of the wall of the urethra which narrows the lumen and interferes with the passage of urine. 
Etiology. -This is brought about by a number of conditions which lead to injury of the mucous membrane, and in the healing process to the formation of cicatricial tissue with narrowing of the lumen, and loss of elasticity in the urethral wall. The most common causes of stricture are: ('alculi, tumors, urethritis and torsion of the urethra occurring during copulation.

Symptoms.-Impeded or complete suppression of micturition, with straining and pain. Bladder distended. In some ('ases small quantities of urine will be passed after much straining.

Diagnosis.- The diagnosis is made by noting the symptoms, and the passage of a sound or catheter. Stricture of the urethra should not be confused with calculi and prostatic enlargement.

Prognosis.- Not very favorable, as complete recovery is rare.

Treatment. - The passage of a sound or catheter daily for a time will tend to dilate the urethra. The eatheter or sound should be well disinfected each time to avoid infection in the urethra or bladder. No other treatment has proved of any value.

\section{CALCULI IN THE URETHRA.}

Frequently in the male dog calculi are found at some point along the course of the urethra. These stones are passed from the bladder and are of sufficient size to become lortged in the urethra at the prostate gland and at the os penis. While they usually consist of one or more concretions, in some cases an impacted mass of small stones with blood or fibrin elot forms the stoppage. Occasionally there will be found injury to the mueous membrane, the stones passing out in part into the adjacent tissues.

Symptoms.- When the calculi become lodged in the uretlira marked symptoms develop in a short time. If there is complete stoppage of urine, the animal will soon show distress, frequent attempts at micturition, straddling, stiff gait, cvidences of urinary pain or colic. Examination of 
the bladder will reveal its distended condition. Should the condition continue for several hours the urine will be dammed back to produce distention of all the urinary passages. Passage of the sound or catheter will reveal the obstruction in the urethra. In the female, by inserting the fingers in the vagina, the enlargement can be felt.

Prognosis.-Farorable in case treatment can be given promptly. 'The complications, paralysis or rupture of the bladder, should be taken into consideration, as they are apt to occur if treatment is delayed too long.

Treatment.-Surgical treatment is resorted to promptly to prevent rupture of the bladder. In case rupture threatens, use a long, disinfected, exploring trocar, disinfect the skin in the prepubic region, place the animal in a dorsal position and insert the trocar through the abdominal wall into the bladder. Allow the urine to flow out, remove the trocar and cover the wound with flexible collodion. There is usually but little danger of injuring the bowels, as the distended bladder pushes them to one side. Urethrotomy should then be performed. In the male an anesthetic (morphin or chloroform) should be administered, and the patient placed on the table in a dorsal position with the hind legs brought forward. The sound or catheter is inserted as a guide to locate the calculus, and also to assist in making the incision. The seat of operation will depend upon the location of the calculus. When located just posterior to the os penis, the incision is made at the distal portion of the enlargement. Should the calculus be at the prostatic portion of the urethra the operation should be performed at the ischial arch. The seat of operation should be thoroughly cleaned and disinfected. The incision is marle immediately over the sound which can be distinctly felt. 'This should be made of sufficient size to allow the calculus to be removed without injuring the adjacent tissues. In some cases, when the tissues are lacerated, they become infiltrated with urine and cause considerable trouble following the operation. After the removal of the calculus, if any urine is present, it will flow out; should this fail to occur examine further for other calculi by passing the sound 
beyond the point of operation. When the calculus is in the prostatic portion of the urethra, the sound should be passed as before and the incision made at the ischial arch, rutting down to the catheter or sound. The urethra then is dilated by either passing a larger sound, or by inserting a grooved director and enlarging it with a probe-pointed knife. The calculus is then extracted by using a strong dressing forceps. In some cases it may be crushed with lithotomy forceps and taken out in pieces.

In the female the operation is much more simple. In some cases the calculus can be removed by manipulating it with the finger inserted in the vagina. Should this fail the urethra must be dilated or enlarged sufficiently to admit forceps for its extraction. It is best to use a grooved director and with a probe-pointed knife the urethra is divided up to the stone, where it can be extracted with forceps.

After-treatment consists in flushing out the bladder with some mild antiseptic solution, such as sodium bicarbonate (2 per cent.) or boric acid ( 2 per cent.). 'The wound should be left open and kept clean with antiseptics. The urine will at first pass out through the incision, but as the wound fills in by granulation, eventually it will be voided normally. In the female the ragina should be cleansed daily with antiseptics.

\section{INFLAMMATION OF THE URETHRA.}

\section{Urethritis.}

Lrethritis is not a common primary condition in small animals, but it sometimes accompanies other diseases of the urinary organs. A primary urethritis results from infection due to the introduction of a sound or catheter or from injuries during copulation, etc.

Symptoms.-Painful micturition. Pus and blood can be pressed out of the urethra.

Treatment.-Antiseptic solutions, such as boric acid (2 per (ent.) or sulphate of zine ( 1 per cent.) are to be used as injections into the urethra and prepuce. 



\title{
PART TX. \\ DISEASES OF THE NERYOLS SYSTEN.
}

\author{
CHAPTER I.
}

\section{DISEASES OF' 'THE BRAIN.}

General Considerations.-Diseases of the central nervous systcm are usually, for the purpose of convenience and pedagogy, classified into those affecting the encephalon or brain, those affecting the spinal cord, and those affecting the peripheral nerves.

This seems to be a logical classification, and this method will be followed in presenting the diseases of the nervous systcm. In order to correctly diagnose diseases of this system, a knowlerge of its functions as well as the seat of each function is required. These will, therefore, be considered briefly:

Preliminary remarks on the functions and seat of each:

Cortex.-The cortex of the cerebral hemispheres is the seat of all psychic function, such as thought, the will and sensation, and all efferent nerve fibers originate herc. The voluntary motor fibers also originate in the cortex, pass through the pons to the merlulla oblongata where they cross to the opposite side and communicate with the motor nerves of the extremities. All sensory nerve fibers and fibers of special sense which conduct perceptible impulses to the brain terminate in the cortex. The cortcx of the cercbrum, then, being the seat of the mind and of voluntary movement, it follows that any destructive process affecting this portion of the brain will produce psychic or mental 
disturbances as well as impaired mobility and sensation on the opposite side of the body, the degree of impairment depending upon the extent of the lesion.

The Midbrain (Crura Cerebri, Corpora Quadrigemini and Optic Thalami).-This portion of the brain is the seat of harmony of motion and equilibrium.' As some of the cranial nerves arise here, the fifth pair being the most important of these, any disease, destructive process or undue pressure operating on this part of the brain will produce sensory and motor disturbances in the region of the facc, lips, eyes, ears and part of the tongue, and, if extensive, the entirc organism may be affected. Involuntary movements of the limbs, head, neck and eyes are the most common symptoms resulting.

The Cerebellum.- The functions of the cerebellum or hindbrain are not fully known but it is regarded as being closely connected with locomotion and equilibrium. It is also thought to be the seat of the muscle sense and assists in the coördination of the muscle movements. Each hemisphere of the cerebellum presides over the muscles of the same side of the body and if either half be injured or diseased the animal will exhibit muscular and motor disturbances of the same side, varying in degree from slight muscular incoördination to spasmodic movements, or it may walk in a circle or crowd or roll toward the injured hemisphere.

Examination.-The brain, because of its position, protected by the bones of the cranium, cannot be examined directly. Diseases of this organ can be recognized only by observing the disturbed functions produced after pathological changes have occurred. It is necessary, therefore, to exannine carefully the functions of the brain before one can arrive at definite conclusions.

As the brain is the seat of the mind (thought), feeling, consciousness, sensibility and voluntary movement, any disturbances or impairment of these functions must be attributed to some pathological change in that organ.

Taking up the examination of the brain by cxannining its functions in the order named above, the psvchic function or mental condition will be first considered. 
1. Psychic Disturbances.-Any variation from the normal mental condition is manifested by abnormal exeitability, or abnormal depression.

(a) Mental Excitement.-Abnormal mental exeitement is eaused by eerebral irritation involving particularly the cortex. This may be due to hyperemia, inflammatory changes, exeessive heat or toxie influence. The degree of excitement may vary from restlessness to mania. In these attaeks docile animals may beeome vicious, bite animate or inanimate objects, or even their own flesh; may stand up on their hind legs, froth at the mouth, and the eyes show a vaeant, staring expression, eonjunetiva injeeted. These symptoms may gradually subside or they may terminate in spasms and convulsions.

(b) Mental Depression.- This may be defined as a dulling of the psychic functions and may vary in degree from dulness to coma. Mental depression is shown by the animal taking less interest in its surroundings than usual, drooping of the head and tail, refusing to obey commands or obeys slowly or clumsily; it assumes somnolent or lethargie attitudes or may wander aimlessly about running into objeets, etc. These are the inilder manifestations of depression and are seen in subacute and chronie diseases affeeting the brain chiefly the cortex. 'They may occur in acute infectious diseases, as the early stages of distemper, rabies, in severe febrile diseases, and in icterus and uremia. Other degrees of mental depression are shown by sommoleney, a eondition in which the animal appears to be asleep, but from which it may be roused; or sopor, deep sleep from which it is difficult to rouse the animal, and coma, or complete unconseiousness. These ronditions are produeed by more severe or extensive lesions. They are seen in eompression of the hrain, cerebral hemorrhage and tunors of the brain. 'They' may be accompanied by motor disturbanees in addition to the mental symptoms, since these conditions may involve the eleeper strurtures of the brain as well as the cortex.

‥ SENinilltT:- Nisturbances of sensation may be considered as of two types, viz.: (a) Pathological excitation or hyperesthesia and (b) pathologieal depression or anesthesia. 
Hyperesthesia when observed in small animals is usually due to some of the infectious diseases, and is seen in the early stages of rabies, in tetanus, and in some of the milder diseases of the cerebrum as hyperemia and acute cerebritis. Hyperesthesia is manifested by abnormal movements of the animal which are entirely out of proportion to the stimulus applied. For example, slamming the door or clapping the hands may so excite the animal that it will fall to the floor or ground in spasms. Local or peripheral hyperesthesia is of little or no importance in small animal practice.

Anesthesia.-This is a condition in which there is a complete loss of sensation. Hyperesthesia indicates a condition in which tactile sensibility is merely decreased. Diminished sensibility may be general or complete, affecting the entire animal, as in subacute or chronic inflammatory conditions of the cortex of the brain and its coverings. It may be partial or incomplete, affecting one entire side, having its origin in one hemisphere, that of the opposite side. Or it may be local, circumscribed, when more or less extensive areas of the cerebrum are involved. Depression of sensibility is determined by applying some stimulus, which when applied to the normal animal will cause pain. To test sensibility the skin is pricked with a needle or pin, pinched or burned with a heated instrument. If the animal fails to react, that is, does not show pain by crying out, whining, or trying to get away from the irritant, the area or part tested is anesthetic.

Motility.-Disturbances of motility arising from the brain vary in degree from slight incoördination to complete paralysis. They will vary in extent and character, depending upon the location and size of the lesion. Disturbed motility may be classified into (a) hyperkinesis or exaggerated action as seen in spasms and involuntary movements, and $(b)$ akinesis or decreased action such as occurs in loss of the muscular sense and in paralysis. Disordered motility may arise from pathological changes in the brain or in the spinal cord. In examining disturbances of motility, it!is sometimes impossible to locate definitcly the seat of the lesion. However, if the impaired function is accompanicd 
by mental disturbances, the brain may be regarded as being the seat of the lesion. If, on the other hand, no mental disturbances are noted, it is assumed that the lesion is in the cord. Further, the portion of the animal affected will often point to the origin of the disturbance. Hemiplegia, or paralysis of one-half of the body, and monoplegia, paralysis of a single organ or part, indicate that the disturbance is of cerebral origin, while paraplegia, a paralysis of a portion of both sides, indicates spinal paralysis.

\section{HYPEREMIA OF THE BRAIN.}

Congestion of the Brain.

Definition.-Hyperemia is a condition in which there is all engorgement of the vessels of the brain. The engorgeinent may be active or passive.

Etiology.-Active hyperemia, or congestion of the brain, inay be caused by anything which affects the cerebral arterial circulation. Violent exercise, excitement, especially in young animals, blows and concussion on the head, are causes. It may accompany eruption of the permanent teeth, or abnormal heart action as in hypertrophy of the left ventricle. Excessive heat, as direct sunlight upon the head in hot weather, will also produce active hyperemia.

Infective hyperemia of the brain appears secondarily to some of the infectious diseases, the most common being rabies and distemper.

P'assive IIyperemia.-The causes of passive hyperemia of the brain are chiefly mechanical, and may be anything which inpedes the outflow of the blood from the brain. Tight collars will compress the jugular veins and produce it. 'Tumors and enlarged thrroid glands (goiter), valvular insufficiency of the left heart, chronic diseases of the lungs as interstitial pneumonia, may produce a passive hyperemia.

Pathology. - In severe hyperemia of the brain, the dura mater or outer covering will be found injected and in cases of long standing may be adherent to the bones of the cranial cavity. The pia mater or inner membrane is hyperemic and the bloodvessels engorged. 'The gray matter varies 
from a gray to a pinkish color, and the white matter a yellowish-red. Between the brain and its covering, membrane, and between the membranes themselves there is an abnormal amount of fluid; the brain substance itself is abnormally infiltrated with serum. In very severe hyperemia there may be ecchymoses or petechiæ present cither in the substance of the brain or its membranes.

Symptoms.-The symptoms of active hyperemia of the brain vary, depending upon the severity of the engorgcment and the degree of intracranial tension. In mild cases there may be only symptoms of restlessness shown, the animal frequently changing its position or wandering about in an aimless manner. Excitement and irritability may be seen with a tendency to bite, although the animal is not aggressive. In more severe hyperemia, there may be spasms and convilsions. The conjunctiva is congested, the pupil contracted and the expression vacant or staring. On palpation the head feels warmer than normal. The pulse and respirations are accelerated, appetite lost or variable and the animal may vomit. These symptoms appear quite suddenly but do not persist for long periods. They may disappear in a few hours or may last as long as three or four days.

The symptoms of passive hypercmia are chicfly those of depression, although these may altcrnate with periods of excitement.

Diagnosis.-Except for its shorter coursc and less severc symptoms, hypcremia of the brain cannot be differentiated from cnccphalitis, the symptoms being identical.

Prognosis.-The prognosis should be guarded, as cren apparently mild cases terminate fatally through inflammation of the brain, a common sequel.

Treatment.- Dietetic.-As the animal will not usually take fool dluring the acute stage and forcible fecding aggravates the simptoms, only fresh milk and plenty of fresh water should be offered at frequent intervals. Keep the patient in a cool, quiet, dark place, away from noisc and exciting influences. 
Medical.-In the early stages mild revulsives may be alministered, the object being to divert the blood from the head to the intestinal tract. Magnesium sulphate (8.0-10.0) may be given for this purpose or pilocarpin (0.00324-0.0081), the latter subcutaneously. If the excitemcnt is intense and there are convulsions, morphin sulphate (0.0324-0.1944) may be adninistered subcutaneously. If the animal is depressed, nareotics should not be given, but cerebral stimulants administered: Caffein citrate (0.0324-0.1944) dissolved.in normal salt solution or distilled water; camphor in the form of the spirit $(0.5-1.0)$ given subcutaneously, or, diluted via the mouth. Atropin sulphate $(0.0005-0.001)$ is also useful as a cerebral and heart stimulant, subcutaneously. If the hyperemia is dlue to pressure from enlarged glands or tumors, these must be removed according to the rules of surgery.

Surgical.- If the animal is strong and plethoric, venerection may he performed on the saphena veins withdrawing from 3 to $6 \mathrm{oz}$ of bloorl. Cold applications to the head in the form of cold packs or ice-bags are useful but only in the earliest stages, and should not be employed if the animal is depressed. After convalescence is established, feed lightly griving laxative foods, as mush and liver or oatmeal and milk.

\section{ANEMIA OF THE BRAIN. CEREBRAL ANEMIA.}

Definition.-Anemia of the brain is a condition in which there is a marked decrease from the normal in the amount of blood in the brain and its membranes. It may be acnte or chronic.

Etiology.-Acute anemia of the brain may follow severe hemorrhage, or the too rapirl withrlawal of fluid from the ablominal or thoracic cavities as in paracentesis. It may occur in thrombosis of the carotid arteries or in cardiac cliseases, as stemosis or valvular insufficiency.

('hronic anemia of the brain is seen in chronic constitutional diseases, and in diseases affecting the blood, as general ancmia and leukemia. Helmintliasis is a common cause. 
Pathology.--The brain and its coverings are pale and the vessels quite bloodless. The cortex which is normally pinkish-gray in color is almost white and on section of the brain mass appears to be fused with the underlying white mass, the line of demarcation being indistinct.

Symptoms. - In acute anemia of the brain, the symptoms appear quite suddenly and vary from a slight dizziness to complete insensibility. The pulse is small and weak, the respirations may be slow and labored or accelerated. The mucous membranes of the head are very pale. 'The pupil of the eve is dilated. There may be convulsions from which the animal gradually recovers or these may be followed by death. The symptoms of chronic anemia are milder and in cases which progress slowly, no symptoms of either motor or psychic disturbances occur.

Prognosis.-Depends on the direct cause and the possibility of its removal.

Treatment.-Medical.-In acute anemia of the brain, the treatment is stimulative. Any of the cerebral stimulants, as caffein citrate (0.0324-0.1944) subcutaneously, alcohol (2.0-4.0) diluted, if the animal can swallow, or aromatic spirit of ammonia (2.0-4.0) well diluted.

Surgical.-Artificial respiration and massage should be practiced if the patient requires it. Clysters of normal salt solution are also beneficial, or the solution may be given intraperitoneally.

Chronic anemia of the brain must be treated by removing the primary cause and treating the general anemia by the administration of tonies, particularly the hematinics (iron and arsenic preparations). Also prescribe a full, rich diet.

\section{MENINGO-ENCEPHALITIS.}

Definition.-This is an inflammatory process affecting the brain and its covering membranes. It may be suppurative or non-suppurative. The writer's reason for combining meningitis and encephalitis is, that in practice these diseases rannot be differentiated during the life of the animal, and 
when one exists the other is present at least to some extent. Furthermore, the treatment is essentially the same.

Etiology.-Meningo-eneephalitis is eaused by praetieally the same factors that produce hyperemia of the brain. These are: Violent exereise, exeitement, blows or eoneussions on the head. Excessive heat, direet sunlight, ete., are thermic causes.

Infectious diseases, as rabies and distemper, are at times aecompanied by meningo-encephalitis as are suppurative diseases of the auditory eanal (otitis) frequently seen in the rabbit and oeeasionally in the dog. Other causes are metastatic emboli from infeeted internal organs as the uterus, lungs, heart (endocarditis) and mammary glands (tumors).

Pathology.-In meningo-encephalitis lesions of various size and eharaeter may be seen, depending upon the eause, and may be formed anywhere in the brain or on the membranes. There may be numerous inflammatory areas or perhaps only one. Usually hemorrhagie, eireumseribed or diffuse areas are noted either on or within the hemisphere, or on the eerebral membranes. The color of the areas varies from a clark brown to a greenish-yellow, depending on the age of the lesion. In those eases caused by metastatic emboli, suppurative areas may be found in any part of the brain and are usually multiple.

Symptoms. - As in hyperemia of the brain, there are symptoms of psychic or motor disturbance, or both. Early there is restlessness and timidity, and the dog may howl or bark continuously. If unrestrained the animal will run or wander about in an aimless mamner and frequently run against objects. The head is hot, visible mueous membranes congested, and the eyes have a vaeant, staring expression. 'The pupils may show unilateral contraction or dilatation. While most text-books state that the pupils are eontracted, the writer has observed that in most cases they are either dilated or unequal.

In severe eases of meningo-eneephalitis, spasms and convulsions, followed by unconsciousness, are observed. 'The animal may gradually recover consciousness, or may' die in one of these atticks. When the inflammatory areas 
are caused by metastatic emboli, the temperaturc is elevated about two degrees and there are symptoms of paralysis shown, the parts involved depending upon what portion of the brain is affected. Deafness and blindness are not uncommon, showing involvement of the cranial nerves.

Diagnosis.-Meningo-encephalitis, except for its longer course, cannot be differentiated from acute hyperemia or congestion of the brain. None but the layman would confuse it with rabies (see Rabies).

Prognosis. - The prognosis is unfavorable as less than 20 per cent. of cases fully recover. The others usually die within a few days or become chronic "dummies."

Treatment.-The treatment of meningo-encephalitis varies in no way from that of hyperemia of the brain.

\section{CEREBRAL HEMORRHAGE. APOPLEXY.}

Definition.-This is a hemorrhage involving usually the cortex of the cerebrum, though it may occur in any portion of the brain.

Etiology.-Cerebral hemorrhage is most frequently secn in old dogs in which there is some degenerative process in the walls of the afferent bloodvessels of the brain. It is also seen in distemper and in the arteriosclerosis which may follow rheumatism. 'These are predisposing causes. 'The direct causes are anything which raises the blood pressugre, as excitement, violent muscular exercises, etc.

Pathology.-The hemorrhage occurs usually on the cerebrum from rupture of a capillary. If the hemorrhage is near the surface the membrane covering the brain at that point will be distended and the convolutions will be depressed or flattened.

'The site of the lesion may contain blood, hemoglobin or a serous fluid, depending upon the age of the lesion.

Symptoms. - These appear suddenly, usually after play or cxcitement and are those of parcsis or paralysis. The animal drops to the floor or ground and is convulsed with muscular spasms. 'These may pass off and the animal will rise and walk about in an unsteady manner, or may lose conscious- 
ness. 'Tlye conjunctiva is reddened, and the lieart beat rapid. 'The respirations are slow and regular or they may' be stuporous and irregular, of the Clicyne-Stokes' variety. The temperature is about normal.

If the animal does not die at once, it is usually left witl a partial or complete paralysis, monoplegic or hemiplegic in eliaracter, depending upon the size and location of the hemorrhage. If the hemorrhage is small and away from the cortex, there will be only slight convulsions shom followed by muscular incoördination, the animal stumbling or staggering from side to side and falling.

Diagnosis. - The sudden occurrence, the history, the character of the respiration and the paralysis make the diagnosis not difficult.

Prognosis. - The prognosis should be unfavorable, only the milder cases terminating favorably.

Treatment.-Place the animal in moderately cool, wellventilated quarters away from exciting influences. Cold applications should be applied early to the head, and if there be convulsions, antispasmodics (morphin, 0.0162-0.1944) may be administercd. Give potassium iorlid (0.1-0.8) to resorb the hemorrhagic exudate, and keep the bowels open witl mild purgatives, such as castor oil (15.0-40.0). Later the paralysis may be treated by the administration of stryclinin to almost the toxic point (0.00054-0.00216). The faradic battery is also useful in treating the paralysis.

As this condition is brouglit about by a high blood pressure and is most commonly seen in plethoric animals, it is well to reduce the bloor pressure by depletion methords, such as a restricted diet and occasional bleeding from the sapliena vein, to prevent anotler attack.

\section{TUMORS OF THE BRAIN.}

Tumors of the brain are rare in small animals but are occasionally observed. 'They may involve any part of the brain and its tovering nembrane, and histologically may be of any type. 
The symptoms produced depend upon the location of the tumor and the degree of intracranial tension. They may be those of paralysis, muscular incoördination, rolling or turning movements, deafness or blindness. Unless the symptoms indicate that the tumor is located near the cortex or involves the membranes covering the brain, treatment, which is purely surgical, should not be attempted. 


\section{CHAPTER II. \\ 1) SEASE OF THE SPINAL CORI).}

General Considerations. -Functions of the ('ord.-Briefly stated, the functions of the spinal cord are: $(a)$ A conductor of nerve impulses from the intracranial nerve centers to the periphery (skin and muscles), and from the periphery to the center; $(b)$ it is the great reflex center for muscular roördination, and also contains in the anterior part special reflex centers, which control respiration, the circulation and deghutition, and in the lumbar portion are the centers for defecation, micturition, etc.

Examination.-The cord, like the brain, cannot be examined directly on account of its sheltered position within the vertebral canal, but diseases of the cord can, in a general way, be recognized by examining its functions. 'This is done by essentially the same methods as are employed in making an examination of the brain.

It is difficult in some cases to differentiate between diseases affeceting the cord and those affecting the brain, but since the corl is the seat of the reflex action, diseases affecting it will, in many cases, destroy one or more of the reflex ares repending upon what particular part of the cord the lesion occurs in. 'Therefore, all reflex action will be absent or morlified posterior to the lesion. 'This, together with the fact that in diseases or lesions involving the cord alone no psychic disturbances, as a rule, are present, will serve to differentiate between them.

\section{MENINGOMYELITIS.}

Definition. - This is an inflammation of the spinal cord and its covering membranes. It is quite common in the dog and rabbit but rare in the other small animals. 
Etiology. - Mechanical.-Common causes of meningomyclitis are traumatic injuries, such as blows in the region of the back or loins, being run over by vehicles, ete.

Infectious. - It is also seen during or following the infectious diseases as distemper, rabies, pycmia, etc., and abscesses in the region of the spine, the pus burrowing betwcen the vertebre and attacking the meninges and cord occasion it.

Pathology. - The membranes covering the cord are somewhat thickened and show either diffuse or circumscribed areas of inflammation, and may be adherent to the cord itself. In other cases, depending on the cause, abscesses may be found involving both the membranes and the cord. If the condition is due to traumatic causes, the vertebræ may be broken or splintered with some portion pressing on the cord. 'The spinal fluid is increased in quantity and may be purulent in character.

Symptoms. - The symptoms of meningomyelitis, unless of traumatic origin, appear gradually and become more sevcre as the discase progresses. They may vary from slight motor and sensory disturbances to complete paralysis. 'There is slight twitching of the extremities which is usually the first symptom noted.

1)isturbances of sensation are frequently observed as lryperesthesia, the animal showing pain when handlcd or even when stroked with the hand. Symptoms of paralysis are seen later, except when due to severe traumatic causes, when they may be the first and only symptoms shown. 'The patient has a staggering gait, sways from sidc to side when walking and finally drags its hind limbs. When placed on its feet, it will drop sideways on its hind quarters.

If the lesion in the cord is far forward, the anterior limbs may also be involved. If in the cervical region, howevcr, death usually follows suddenly from respiratory arrest.

The sphincters of the anus and urinary bladder are usually involved causing the feces and urine to pass involuntarily, though there is usually constipation. Progressive paralysis indicates tumors pressing on the cord. If only the inembranes covering the cord are involved, the spinal reflex is present and may be exaggerated. If a portion of 
the cord itself is destroyerl, reflex movement is absent posterior to the lesion. Conseiousness is not disturber.

Diagnosis.-It is usually not difficult to differentiate between diseases of the spinal cord and those of the brain, but to state definitely the character of the lesion and its exact location should not be attempted. In diseases of the nervous system it is generally sufficient to state whether the brain or cord is affected.

Prognosis. - In meningomyelitis, as in other diseases of the brain and cord, the prognosis is generally unfavorable, only a small percentage recovering.

Treatment. - In the early stages give laxatives, as magnesium sulphate $(8.0-12.0)$ or castor oil $(15.0-40.0)$ and apply counterirritants to the spine.

The faradic battery is useful in treating the paralysis, or strychnin almost to the point of intoxication. Iodid of potassium may be given to resorb the exudate.

The animal should be placed under good hygienic surroundings and kept clean and dry.

\section{CONCUSSION OF THE SPINAL CORD.}

\section{Injuries of the Spinal C'ord.}

Etiology.- This condition oceurs quite frequently in the dog and cat from a variety of causes. These animals are subjected to extreme violence often by being run over by fast moving vehicles, by penctration of the spinal eanal by sharp or blunt objects, or from the animal falling. Nany cases such as described result in fracture of the vertebra with clirect injury to the cord. Extreme muscular exertion combined with diseases of the bones (fragilitas ossium) will result in fracture and injury to the corrl. The injuries to the cord with fracture of the vertebre often lead to hemorrhage between the membrancs or in the spinal cord proper. Punture into the spinal canal as has been practiced in crrtain cases, frequently terminates in edema of the cord and membranes from the irritation, or hemorrhage into the spinal canal. 
Pathogenesis.-As soon as the injury occurs, and the cord either crushed or compressed by extravasated blood or serum, it loses its conductivity in proportion to the degree of the injury and compression. The conductivity of the cord may thus be either partially or entirely lost and the function of the nerves will be partially or completely destroyed in the area involved. In minor injuries with simply edema of the membranes or cord the development is gradual and the degree of involvement very slight.

Symptoms. - The symptoms of compression or injury to the spinal cord will depend upon: (a) The location of the injury or portion of the cord affected; $(b)$ the degree of compression or destruction of the cord. When the spinal corr is compressed or crushed in the cervical region the animal, as a rule, does not live over a few minutes or hours. Complete paralysis is observer posterior to the point of injury. The patient may be able to bring the muscles of the head into action for a short period preceding, death. In cases of lesser injury or compression the symptoms are not so pronounced and the patient may be able to move certain groups of muscles. If the compression is due to edema of the inembranes or hemorrhage into the cord or canal the symptoms are milder and gradually disappear in the course of a few days or weeks.

Complete destruction of the cord posterior to the cervical enlargement will produce paralysis and complete loss in sensation in the limbs, tail and body. This is noticeable in the respiration as the ribs remain fixed and the respiratory movements are confined to the diaphragm.

There may be retention of urine and feres, or they may be voided involuntarily.

When the dorsal portion of the spinal cord is affected there will be paralysis of the posterior part of the body. In slight injury or compression there may be only incoordination of movement from the point of injury.

Compression or destruction of the anterior part of the lumbar segment results in paralysis and anesthesia of the hind limbs, tail and muscles of the croup. When the injury is in the midllle or posterior portion of the lumbar 
seginent the symptoms will be modified somewhat owing to the injury of the sacral segment which results in paraly sis of the area supplied by the sciatic nerve. The sphincters of the bladder and anus respectively will be paralyzed and urine and feces discharged involuntarily.

When the injury occurs in small animals spasms of adjacent muscles will be observed. This is due no doubt to the injury producing stimulation to the nerve roots. On examination of the patient the temperature is often ele vated, or it may be subnormal if the sphincters are relaxed and the thermometer inserted in the rectum. Palpation over the region of injury will cause the animal intense pain and frequently convulsions or spasms. Swelling is often present and crepitation may be detecter. Abnormal movement of the vertebræ involved can be determined in the cervical and lumbar segments.

Diagnosis. - This is accomplished only after careful examination and consideration of the parts paralyzed. The determination of the degree of injury is often very difficult. There might be a complete paralysis resulting from edema and hemorrhage greatly resembling cases of destruction of the cord. However, the history of the case will assist in the differential diagnosis.

Course.-In complete destruction of the cord in the cervical segment death may occur in a few moments or may be delayed for several hours. Should there be hemorrhage only and partial paralysis the patient may live for several days and some will make a complete recovery. In involvement of the dorsal and lumbar segments the course will depend upon the degree of injury. In small animals they may live for several weeks or months.

Prognosis. - A definite prognosis is often difficult to arrive at on account of the impossibility to determine the degree of injury in all cases. When there is evidence of complete destruction of the cord the case is hopeless. In cases of hemorrhage or edema most patients will make a complete recovery. At hest the prognosis should be held in reserve until the exact condition can be determincd. 
Treatment. - No treatment will be of any value where the spinal cord is destroyed. If crepitation is present and distinct separation and movement between the involved vertebræ are detected it is advisable to destroy the animal. If in doubt in regard to the actual condition the patient should be given a soft bed and quiet place. Good nourishing food (meat, milk) and gentle massage over the region injured will assist in the resorption. In the secondary stages small doses of strychnin sulphate (0.001) daily and electricity have proved to be beneficial.

\section{COMPRESSION OF THE SPINAL CORD.}

Definition.-A condition in which there is more or less disturbance in the function of the spinal cord from pressure by exostosis, tumors, abscesses, parasites, etc.

Etiology. - Various diseased conditions will produce compression of the cord. 'The following are most common and important: (a) In the log degeneration of the intervertcbral disks in which there is distortion and enlargement projecting into the spinal canal, narrowing its lumen and causing compression of the cord. 'This condition has been observed in certain breeds of dogs and in Belgian hares. 'The cause is no doubt injury to the disks by extreme mobility or concussion. (b) Ossification of the intervertebral disks. This is found in older animals, mainly in old dogs. It may exist in an individual disk or what is more common a number of them will be affected producing rigidity of the rertebral column. The enlargements resulting from the ossification project into the spinal canal directly compressing the cord. The condition usually begins in the most mobile portion of the rertebral column. Fracture of the vertebra without destruction of the cord may bring about a similar condition upon union of the fractured portions. The new bone formation projects into the spinal canal. (c) 'lumors. In the dog sarcomas may cause compression of the rord by the tumor developing in close proximity to the rertebral column and the growth extending through the intervertehal foramina. Other growths rarely produce this 
condition. (d) A few cases have been observed in dogs and in rabbits in which Echinococcus granulosus cysts produced local pressure on the spinal cord. (e) Abscesses developing in the spinal canal are rare, but wlien found near the vertehral column, the pus may burrow in between the intervertebral disks producing infection resulting in edema and inflammation.

Pathogenesis.-Any of the conditions cnumerated may lead to a reduction in the lumen of the spinal canal. The degree of injury or compression of the cord will depend upon the character of the course and the point of involvement of the vertebral column.

Symptoms. - The rigidity of the spine and the careful way in which the animal lies down and gets up are somewhat characteristic. Dogs exhibit considerable pain on moving the spinal column by whining, erying, etc. In movement the animal is very cautious and often if recumbent refuses to arise when called. Examination of the patient reveals the rigid eondition of the spine and the fixation of the muscles of the back. Forced movement of the vertebra induces severe pain. Paralysis gradually develops posterior to the point of compression; sensation is partially or eompletely destroyed, and involuntary passage of urine and feces follows from paralysis of the sphineter muscles conecrued.

Diagnosis. - An early diagnosis is often difficult owing to the gradual development of the primary condition. A careful examination of the vertebral column, its rigidity, evidence of pain on movement, will assist in the diagnosis. It may be confused with muscular rheumatism.

Prognosis. - This must be consiclered unfavorable in all asces. Recovery is very rare.

Treatment.- Treatment is practically impossible. Operable tumors, when its cause, may be removed surgically. Abseesses may be opened and drained, but owing to the complicating infection little can be expeeted in the way of recovery or even improvement. No internal treatment has proverl of any valuc. Small doses of potassium iodid may be tricd in the milder cases. 


\section{CHAPTER III.}

\section{DISEASES OF THE PERIPHERAL NERVOUS SYSTEM.}

\section{INJURIES OF THE PERIPHERAL NERVES.}

Small animals are subjected to a variety of injuries which may involve the individual nerves or nerve endings, such as bruises of the muscles, in which the nerve is crushed against the bones, or between muscles, or stretched or torn or the nerve is cut by sharp objects which is more frequent.

\section{PRESSURE UPON THE PERIPHERAL NERVES. COMPRESSION.}

Most frequently compression results from neoplastic formations (sarcomas, neuromas), from hemorrhagic extravasations, serous effusions into the tissues, enlargement of lymph glands, fractures of bones or abscess formation.

Neuritis undoubtedly occurs in small animals, particularly in dogs, and may result from a variety of causes. Chilling or subjection to extremes of temperature is perhaps most productive of the condition. The inflammation resulting is subscquently followed by paralysis in a number of cases.

\section{PARALYSIS OF THE PERIPHERAL NERVES.}

The following paralyses of peripheral norves have becn noted:

Facial Nerve.-Dogs and rabbits are most of ten affected.

Etiology. - (a) Traumatic influences play an important role in the unilateral form of facial paralysis (monoplegia facialis) by injuring the nerve at the point where it goes around the maxillary bonc. (b) Neoplasms in the parotid region involve 
the nerve and destroy its function either by compression or direct growth into it. (c) Inflammation of the middle ear, caries of the petrous portion of the temporal bone, and tumors in the base of the brain often produce it. (d) Distemper (nervous form) producing an encephalitis will often result in paralysis of the facial nerve. (e) Exposure to cold, such as hunting dogs, or retrievers in which the surface of the body is suiddenly subjected to the extreme temperature.

A bilateral facial paralysis (diplegia facialis) is usually of central origin.

Symptoms. - In case the entire nerve with all its branches is paralyzed there will be paralysis of the ear, eyelids and lips on the side affected. 'The ear will droop, and the animal is unable to elerate it when called or excitcd. The eyelids hang downward and are immovable. The lip will be found soft, flabby and will not react to normal stimuli. When these symptoms are present the lesion is central. On the other hand, should there be a paralysis of the lips only, it would indicate a peripheral form of facial paralysis. Paralysis of the facial nerve arising from the central nervous system may have associated with it paralysis of other cranial nerves. This would complicate the symptoms.

Diagnosis. - The symptoms are characteristic. A differential diagnosis should be made between central facial paralysis and the peripheral form. 'This can be easily determined in most cases by noting the extent of the paralysis.

Prognosis. - When there is complete paralysis of central origin it is considered unfavorable. However, if the condition has only temporarily affected the nerve the animal will recover. 'The exact condition of the nerve is impossible to determine. In the peripheral form the condition of the nerve at the point of injury is important. When not destroyed the prognosis is favorable.

Treatment. - In the peripheral form due to injury or chilling, the symptoms usually disappear quite promptly. The parts should be massaged thoroughly and the electric current applied daily over the region. Stimulating liniments massaged into the tissues are recommended (soap liniment, (amphor liniment, white liniment). If tumors 
or abscesses are present they should be operated at once, care being taken to avoid injuring the nerve. In the central form nerve stimulants (strychnin sulphate 0.001 daily), or electricity should be employed. I'sually in the course of ten days to two weeks improvement will be noticed. - If after one month to six weeks no improvement is noted the chances are that the nerve trunk has been completely destroyed and further treatment is useless. Spasm of the muscles supplied by the facial nerve occurs occasionally, especially in dogs, no doubt due to the infection from distemper producing irritation to the nerve. It may also occur in meningitis and encephalitis. When present the condition is characterized by clonic convulsions of the muscles supplied by the nerve. Sedatives would be indicated to reduce the irritation.

Trigeminal Nerve.-Paralysis of this nerve is observed most often in dogs.

Etiology.-(a) Rabies produces the greatest number of cases. Therefore, all cases of trigeminal paralysis should be handled with caution until the exact cause is known.

Occurs in some cases from distemper. (c) Inflammation of the brain and concussion of the brain also cause it. (d) Injuries in which the motor branch is pressed or erushed. This happens not uncommonly in dogs from extreme opening of mouth, or carrying large heavy objects in the mouth. (e) 'Tumor formations, such as sarcomata in close proximity to the nerve, or abscesses. 'These conditions may either' injure the nerve directly or by external pressure. (f) Rheumatic conditions involving the muscles supplied by the nerve. (g) Neoplasms at the base of the cranium (angioma).

Symptoms. - The most pronounced symptom is dropping of the lower jaw, the mouth remaining open constantly. In such cases rabies should be suspeeted. In unilateral paralysis the animal may be able to close the mouth and masticate on one sille. If all three branches of the nerve are paralyzed mastication and sensibility are lost. In case any individual branch of the nerve is paralyzed that part supplied by that branch only will be affected. When the mouth remains open the tongue will protrude, become dry and discolored. 
Saliva is usuall! profuse and flows from the open mouth. Attempts at eating and drinking fail. Food is swallowed when placed back in the moutl.

Diagnosis. - The only difficulty in diagnosis is to determine the cause of the paralysis. 'The symptoms are so characteristic that the actual condition is easily recognized. A differential diagnosis should be made to determine whether or not the animal is affected with rabies. The general condition of the animal, disturbance in swallowing, change of voice and paralysis in the posterior part of the body in rabies are indicative.

Prognosis. - Should the condition result from trauma then the prognosis is considered favorable, otherwise unfavorable.

Treatment. - If the paralysis is the result of rabies 110 treatment should be attempted. In cases due to other causes, give nourishing food (milk, chopped meat). This is best done either by placing the food well back into the moutl, or by the use of a stomach-tube. Massage the muscles thoroughly, using at the same time a stimulating liniment (soap liniment). Electricity may also be trierl. (ive internally tincture nux vomica $(0.3-0.7)$ or strychnin sulphate (0.(01) daily. Spasms of the muscles supplied by the trigeminal nerve are observed in tetanus and in some cases of the nervous form of distemper. The muscles are either rigicl as in tetanus, or contrating and relaxing rapidly as in some cases of distemper. Nerve sedatives shouli be used to eontrol the spasms. Tetanus antitoxin and distemper sermm respectively may be employed, depending on the condition present.

Auditory Nerve. - Etiology. - The true nerve of hearing (cochlear nerve) is not frequently paralyzed. However, it may be paralyzed from a congenital defect or inflammatory (hanges within the internal ear, or from diseases affecting the merlulla oblongata. P'aralysis of the vestibular nerve is very commonly observed in dogs, rabbits, fowls and pigeons. In practically all cases, however, it is the result of inflammatory changes within the midlle and inner ear. 'These changes may result from chicken pest, cholera, epitheliosis, contagious rhinitis (rabbit), or distemper. Causes of 
minor importance are: Concussion of the brain, hemorrhages in the middle ear, or caries of the petrous portion of the temporal bone.

Symptoms.-Deafness is the prollounced symptom of paralysis of the cochlear nerve. If bilateral and complete the animal will be totally deaf. In vestibular paralysis, when milateral, the patient will assume a peculiar attitude holding the head downward and toward the normal side. In chickens the head is rotated to the degree that the comb will rest on the ground. In dogs and rabbits rolling movements are very prominent symptoms. 'This is so marked in some cases that it is impossible to hold the animal. The least irritation or disturbance will cause them to show it. Rolling movements always take place toward the normal side. Attempts at walking are difficult but if they "succeed will travel in circles, often falling down and rolling over and over. The eyelids are often closed and the eyeball assumes an abnormal position. In case of bilateral vestibular paralysis, the head drops down and the muscles of the neck are limp.

Diagnosis. - This should not be difficult, as the symptoms of deafness are easily manifest and the peculiar movements of the animal in vestibular paralysis are characteristic.

Prognosis. - Should be considered unfavorable except when due to injuries.

Treatment.-If due to injuries the animal should be kept quiet and if nccessary fed artificially in order to inaintain its general condition. The ears should always be examined to determine their condition (disease or parasites). Pigeons, when affected, are isolated and the premises disinfected to guard against contagious meningitis. Internal administration of magnesium sulphate or castor oil as a laxative is advised. No treatment can be applied dircet to the seat of the condition.

Radial Nerve. - Paralysis of this nerve occurs occasionally in the dog and cat.

Etiology.-(a) On account of the position of the radial nerve it is easily injured by traumatism. Animals struck by objects, falling, jumping, etc., very commonly injure 
the nerve with resulting partial or complete paralysis which may be temporary or permanent. (b) May follow muscular rheumatism or subjection to cold. (c) Follows infectious diseases, such as distemper in dogs and cats. (d) Has been observed from injury to the spinal cord. (e) Tumors and abscesses in the muscles may bring about at least a temporary radial paralysis.

Symptoms. - The radial nerve controls the muscles that extend the forelimb; therefore the most prominent symptom is inability to carry the limb forward. The joints are extended below the elbow and flexed above that point. The animal in moving forward drags the toe on the ground and weight cannot be supported owing to the difficulty of properly placing the limb in the normal position. Some weight will be supported on the limb when it is placed in position. The degree of disturbance will depend upon whether the paralysis is complete or partial. Local examination reveals absence of inflammatory changes. There is usually a normal degree of sensitiveness in the skin.

Diagnosis. - A careful examination should be made in the dog for they are inclined to favor the limb in the least disturbance. However, in many cases of injuries, the limb will be carried from the ground while in this case it will be just the opposite, dragged on the ground. Examine for thrombosis of the axillary arteries.

Prognosis. - The larger number of cases recover. 'This is explained in that most cases result from injuries which do not seriously disturb the structure of the nerve. Few cases will be permanent.

Treatment. - Massage the muscles and stimulate them by the use of the elcctric current. Nerve tonics may also be given. If no improvement is apparent in ten days to two weeks the case should be considered unfavorable.

Brachial Plexus. - Paralysis of the brachial plexus occurs most commonly in the dog and cat.

Etiology.-The majority of cases result from injury, from falling or jumping from great heights. Fracture of bones adjacent to the plexus resulting in injury will produce it. 'Tumor formations and abscesses in the axillary region are also causes. 
Symptoms. - The most proninent symptom is a limp, lifeless condition of the limb unable to support any weight. Sensation, as a rule, is lost in the entire limb. If the paralysis is partial only, the symptoms will be less prominent.

Prognosis.-Most cases, inasmuch as they are due to injuries, recover completely in the course of a few weeks.

Treatment.-Massage and nerve stimulants are useful. Keep the animal well nourished.

Sciatic Nerve.-Etiology.-(a) Falling from heights and jumping. (b) Wounds and direct injuries to the nerve. Infectious diseases (distemper). (d) Tumors and abscesses in contact with the nerve.

Symptoms. - There will be paralysis of the biceps femoris, the semitendinosus and the muscles below the stifle joint. In the dog the limb will hang relaxed and during forward movement the toe is dragged on the ground. Cases will be seen where the hair and skin are abraded from the anterior surface of the foot. There may be loss of sensation below the stifle joint. In bilateral sciatic paralysis it resembles lumbar paralysis to a certain degree. A differentiation should be made. Atrophy of the affected muscles will soon be noticeable.

Prognosis.-If due to injuries it is favorable, provided the nerve is not completely destroyed.

Treatment.-Massage and employ nerve stimulants. Protect the feet from injury, give nourishing food and use the electric current.

Paralysis of other nerves is occasionally seen but is of minor importance. 


\section{CHAPTER IV.}

\section{FINCTIONAL NERTOES DISEASES.}

\section{VERTIGO. MEGRIM.}

Definition. - A condition characterized by dizziness and general disturbance of equilibrium (swooning). In small animals it is not very commonly observed, except in dogs, rabbits and pigeons.

Etiology. - In these animals the condition is very seldom found as a primary disease. It is usually secondary to other diseases which it may follow.

(a) Diseases of the brain, such as hyperemia, acute and chronic, or encephalitis, of ten produce the sumptoms of vertigo. (b) 'Tumors, hemorrhage into the brain or membranes, concussion of the brain, or emboli of some of the cerebral bloodvessels may also produce it. (r) Defects of vision, or irregular lighting in which too sudden ehange takes place in the acrommodation of the ere, have been eited as causes. (d) Diseases of the middle or inner ear. (p) sudden change in the circulation of the blood in which there is cerebral anemia. Tight collars may produce the condition, or the dog pulling steadily on the leash may bring it about. ( $f$ ) Reflex conditions from the intestinal tract (parasites or intestinal (atarrh). (g) From poisoning, such as ptomains, rertain poisonous plants, or overdoses of alcohol and other narcotics. (h) l'igeons are sometimes affected by a contagious or infectious disease having as its most prominent symptom vertigo. I arge numbers may be affected at the same time.

Symptoms. - The early indication of vertigo is (haracterized by a sudden staggering gait. The animal falls down, hecones unconscious. It remains in this position quietly for a few moments, arises and soon assumes its normal condition. 'The individual attack is usually of 
short duration, from two to ten minutes. 'The time elapsing between the attacks is variable. The prodromal symptoms are anxiety, staring expression, increascd respiratory movements, and sometimes slight twitching of the muscles. When occurring in pigeons the number affected should be noted to determine whether or not an infectious disease is causing it.

Diagnosis.-A differential diagnosis should be made between vertigo and epilepsy. The main differential feature is the absence of convulsions in vertigo. The symptom vertigo is not so difficult to determine, but its causes may remain quite obscure.

Prognosis. - Should not be considered very favorable as the cause is hard to determine. Individual attacks of the disease usually do not cause any serious disturbance barring accidents and injuries.

Treatment.-During an attack of vertigo the animal should be placed in a comfortable, quiet place and protected from injury. Following the attack the examination should be directed to find out the underlying cause and treatment applied accordingly. When the condition occurs in pigeons as a contagious disease, the entire premises should be disinfected after the removal of all affected birds. Individual treatment in these cases is unsatisfactory.

\section{EPILEPSY.}

Definition.-Epilepsy is a disease of the central nervous system which is characterized by convulsions occurring at irregular intervals, the subject usually being unconscious luring the attack.

Etiology. - The cause of primary true epilepsy is unknown although it is regarded as being hereditary; at least the off'spring of epileptic parents are markedly predisposed to the disease. 'This has been observed in man as well as in the domesticated animals.

Pathology. - No postmortem lesions of any kind have been observed either in animals or man which would account for the disease.

Symptoms. - In epilepsy the attacks come on suddenly, thc animal performing uncontrollable movements. This is 
followed by the subject falling to the ground or floor and in convulsions of a clonic type. Generally every muscle is involved, including the facial muscles. There is champing of the jaws with salivation, the saliva being churned into foam and often blood-stained due to injuries of the tongue by the teeth. The visible mucous membranes are cyanotic; the heart beat is full and strong, and the respirations suspended. The convulsions last but a few seconds and gradually become weaker and finally cease. The animal lies quietly for a few minutes, then rises to its feet, staggers and finally recovers. The attacks do not occur at regular periods. The animal may have two or more in a day, or there may be weeks or months between attacks.

Diagnosis. - It is difficult to differentiate between true epilepsy and secondary or reflex epilepsy which is merely a symptom of some other disease. A history of chronicity and the rather long periods between the attacks point to true epilepsy. Further, true epilepsy may be seen in both old and young animals while secondlary or reflex is usually confined to the young.

Prognosis. - The prognosis is unfarorable, as true epilepsy' is considered incurable.

Treatment. - If treatment is undertaken, the bowels should be kept open by feeding laxative food and, if necessary, the administration of laxative drugs as cascara sagrada, fluidextract $(2.0-8.0)$, or sulphur $(2.0-6.0)$ in the food as required.

T'he periods between the attacks may be lengthened by the administration of bromides in full doses, the bromid of sodium being preferable $(0.5-4.0)$.

Castration is said to have a beneficial action in some cases.

Reflex or Secondary Epilepsy. - This is seen as a symptom of several diseases occurring in small animals and somewhat resembles true epilepsy.

Reflex or secondary epilepsy is sometimes seen in rickets, inflammatory diseases of the digestive tract, some infestation with internal parasites, during the eruption of the permanent teeth, in constipation, and frequently distemper, especially the nervous form.

It is seen chiefly in young animals, being quite rare in 
older, while true epilepsy affects the old as well as the young. 'This will assist in the differentiation between reflex and true epilepsy.

Treatment.- Treatment must be directed toward the primary disease or condition producing the symptoms.

\section{CATALEPSY.}

Definition.-This is a peculiar functional disease of the central nervous system, probably of the cortex of the brain. It is characterized by a suspension of voluntary motion on the part of the subject, but when the position of the animal is passively changed, it will be maintained by the patient for a long time.

Etiology. - The cause of catalepsy is not known, though it is probably of reflex origin.

Pathology. - No pathological lesions of the central nervous system have been demonstrated. Degenerative changes in the muscles have been observed, also small hemorrhages in the stomach and intestines, but these are not constant.

Symptoms. - The attacks come on rather suddenly, the animal becoming rigid, muscles hard and tense. 'The cyes are fixed and dull in appearance, the pupils may be dilated or contracted to the utmost. Sensation seems to be inhibited during the attack. The circulatory and respiratory functions are undisturbed; temperature normal. If the position of the animal's limbs be passively changed it will remain in that position for a long time.

Diagnosis. - This is made chiefly by passively changing the position of the body of the animal or its limbs. If it remains in this position without change for a considerable lengtl of time, the attack is undoubtedly catalepsy.

Course. - The course of the attacks is from four to twentyfour hours from which the animal usually recovers.

Prognosis. - The prognosis is not unfavorable, though the attacks may recur.

Treatment. - The administration of antispasmodics is indicated. Give morphin (0.032-0.2) subcutaneously, or chloral hydrate (2.0-4.0) in emulsion per rectum. This will relieve the attack, but there is a tendency to recurrence. 


\section{CHOREA.}

Definition.-This is a persistent clonic spasm, or twitching of certain muscles, or group of muscles. It is oftenest scen in the dog; rare in other animals.

Etiology.-Chorea results most frequently from acute infectious diseases as distemper to which it is a common sequel. It also occurs in myelitis, and in the early stages of rachitis.

Pathology.-There is no demonstrable lesion observed even in the most careful examination which might account for the symptoms shown. Anemia is the most constant.

Symptoms. - The twitching of the muscles is quite constant and usually involves those of the head and anterior limbs, though often one or both of the pelvic limbs may bc involved. There is a pcculiar dipping morement of the head and shoulders. Often the masseter muscles arc the only ones involved producing a spasmodic movement of the jaws. Consciousness is not disturbed.

These rhythmic spasms are less marked when the animal is alonc and during slcep. 'The pulse and tcmperature arc normal; the appetite unaffected. 'The disease is chronic and may persist for months or years. Young animals frequently recover without treatment.

Diagnosis.-Diagnosis is not difficult. 'The history, the peculiar rhythmic spasms of certain muscles, and the absence of general symptoms point clearly to chorea.

Prognosis. - Prognosis is good so far as the life of the animal is concerncel, but bad from the standpoint of recovery or cure.

Treatment. - Many kinds of treatment have bcen tried but none have given decided results. Arsenic in the form of Fowler's solution (0.1-0.75) once daily has proved helpful.

Recently leukocytic extract has been administered cxperimentally with excellent results. The animal should be given nourishine food, and if anemic, iron preparations as iron and quinin citrate $(0.2-() . \overline{6})$ are useful.

\section{ECLAMPSIA.}

Definition.- Velampsia is a tonoclonic spasm observed in hitches. It is associated, though in some cases quite remotely, 
with parturition. As a rule the animal is conscious during the attack.

Etiology.- The cause of eclampsia is not definitely known. It occurs in pregnant bitches and as late as fifty days after whelping. It has been observed to follow exposure to cold in pregnant bitches, and following grief or anxiety owing to the loss of one or more of the puppies in suckling bitches.

Symptoms. - The disease appears suddenly, usually about the second week after parturition, though it may occur before. Generally small delicate house dogs are affected.

Early in the attack the animal becomes restless and has an anxious facial expression. Later motor disturbences are seen, the animal falls to the ground or floor in spasms of a tonoclonic, or mixed type, the legs sticking out stiffly as in tetanus. The muscles of the body and limbs are hard and tense, occasionally the limbs will relax and immediately become stiff again. The respiration is rapid, and the pulse accelerated, small and hard. Visible mucous membranes are congested. There is some salivation, the saliva being swallowed or dripping from the mouth. The eyes are open, pupil normal, and the corneal reftex present. The animal is conscious but unable to obey commands. The temperature is normal or subnormal.

Diagnosis.-The diagnosis of eclampsia is sometimes difficult. It might be confused with strychnin poisoning, a condition it closely resembles, but in eclampsia there is less hyperesthesia. It might also be confused with tetanus but tetanus comes on gradually, this being about the only differential feature.

Prognosis. -If the animal is presented for treatment early, the prognosis is favorable. Otherwise it is unfavorable, especially when the temperature is more than $1 \frac{1}{2}^{\circ}$ below normal.

Treatment. - Give narcotics as morphin subcutaneously in full doses and keep the animal in a warm, quiet place. Chloroform syrup (1 c.c. of chlorororm to 90 c.c. of simple syrup) in doses of 4.0-8.0 every fifteen minutes until the muscles relax, then at longer intervals as required, may be given.

Puppies must not be allowed to nurse during the acute stage and should be kept away from the dam. 


\section{PART $X$. \\ DISEASES OF THE SKII.}

\section{CHAP.TER I. \\ NON-PARASITIC SKIN DISEASES.}

Examination.-Careful methods are required for an accurate diagnosis of skin diseases. Small diseased areas and the larger parasites, if not very numerous, may casily be overlooked in long-haired individuals, or in birds.

In the majority of cases a microscopic examination is necessary to determine if a skin disease is parasitic or nonparasitic. If the former, it is necessary to identify the parasite in order to give the proper prognosis and treatment.

Some few cases present certain rather characteristic features which indicate the real nature of the disease but the only accurate diagnostic method is by the use of the microscope. The larger parasites may be identified with a reading glass; the mange mites can be seen with a microscope, using low power, while high power is necessary to identify some of the vegetable forms.

Microscopic Examinations. - With a small, sharp curette scrape deeply into the skin at a point where the disease process is active until a mass of moist scrapings the size of a grain of wheat is obtained. 'Transfer this mass directly' to a slide and moisten it with a drop of water. Put on a cover-glass and press down with a rotary motion to evenly distribute the material to the proper density. By this method animal parasites remain active, and their movements readily indicate their presence. For vegetable parasites 
in addition to the scrapings pluck a tuft of hair at the edge of the diseased area and mount as above. Examine with high power for the fungus along the hair near the roots. If the scrapings are to be examined later, seeure a larger amount and put in a clean vial or ointment box. Smear slides cannot be made with this very well after it has dried. I sually it is best to boil it slightly in a 10 pcr eent. solution of KOH. Centrifuge it and withdraw some material from near the bottom with a pipette and mount with eover-glass.

\section{DANDRUFF。}

Definition. - The presence of fine, grayish-white scales on the skin or in the hair, which may affeet the entire skin surface or small circumscribed areas.

Etiology.-A mild superficial inflammation of the skin resulting in excessive exfoliation of the epidermis. Dandruff may be due to several causes, viz: Bath soaps that are too irritating or used too often; irritating medicinal preparations used on the skin, especially parasiticide preparations; dircet sunshinc on short-haired animals, especially when unaccustomed to it or those recently sheared. Parasites are a frequent cause; a mild attack of the demodectie parasites will occasionally produce no other symptoms. Internal disorders, especially digestive diseases favor the condition. Very often no assignable cause can be discovered.

Symptoms. -The disease occurs chiefly on the upper surface of the body, cspecially on the neek, under the collar and along the back. The hair coat is dull and dry and the skin is covered with small grayish-whitc scales or dust. Itching is sometimes present.

Diagnosis. - The prescnce of the scales indicates the condition, and only the absence of parasites distinguishes it from parasitic diseases.

Prognosis.-'The disease runs a rather prolonged course but continued treatment usually effects a cure.

Treatment.-Good nourishment is necessary if digestive disturbances are suspected as the indirect cause. Internal treatment with digestive tonics, especially preparations 
containing arsenic for its action on the skin. 'The external treatment consists in cleaning applications with alkaline solution (sodium carbonate, 2 per cent.) and applications of salicylic ointment (10 per cent.). A solution of resorcin (5 per cent.) is very satisfactory as it does not soil the hair coat, nor cause dirt to adhere to it. It is best to clip the hair and brush the skin well before medicinal treatment is begun, and thereafter at frequent intervals.

\section{ALOPECIA.}

Definition.-A loss of hair, feathers or fur from large or small areas due to causes other than organic diseases or parasites.

Etiology.-Mechanical.-Fowls, especially the males when penned, may pluck the feathers from the breast, denuding a large area. Female rabbits pull out their fur for use in preparing a nest for their young.

Chemical.-Acids or strong caustics by deep action on the skin will destroy the hair follicles and when healing occurs the area is free of hair.

Thermic.-IHot water, often intentionally applied, may act deeply enough to destroy the follicles and denude an area. Burns appcar similar but more diffuse.

The most common cause is deranged nutrition to such an extent that the hair falls out usually in patches over the body. A single arca, so affected, and when the usual etiological factors are wanting, must be ascribed to a disturbance of the trophie skin nerves of that part. In one case the hair was lost each succeeding summer from pigmented parts of the skin. No eause could be given.

Pathology.--The hair appears to loosen in patehes and fall (nut. The skin appears almost normal in some cases but usually somewhat dry and hard. In those cases following serere wounds, sealds, or burns, the skin shows scar formation. 'The microscopic exanination for parasites is negative.

Symptoms. - There appear on the skin small areas demulerl of hair, which gradually become larger. The hair 
at other parts can be readily pulled out. The skin of fowls, when feathers have been plucked, appears apparently healthy, as does that of rabbits which have pulled out the fur. When due to wounds, scalds and burns the skin is thickened, often scaly and the hair around the affected area often distorted from its usual direction of growth, appearing longer at the margins.

Diagnosis. - This is made by negative microscopic examination of skin scrapings, and inspection of skin for scars. Observing or inquiring into the habits of the animal affected, and a careful examination into its general condition are helpful.

Differential Diagnosis.-Alopecia must not be confused with demodectic mange, which frequently causes loss of hair in small but gradually enlarging areas, nor with the depluming mite which acts similarly on fowls.

Prognosis. - Good if due to general nutrition disturbance. If from scar formation the loss is permanent, as is also true of trophic nerve disturbance.

Treatment.-Treatment consists in giving good food in proper amounts, and stimulating metabolism by tonics, especially those containing arsenic for its alterative action on the skin. Scarified areas, if small, may be overcome by complete removal of the areas and the healthy skin approximated by suturing. Feather pulling may be prevented by allowing free range, or by using a device attached around the upper half of the beak which prevents complete closing but does not inconvenience the bird in eating or drinking. Fur pulling of rabbits is not objectionable when their habits are known. It may be overcome in part by supplying proper material for bedding just before parturition.

\section{DERMATITIS.}

Definition.-An acute or subacute, septic or aseptic inflammation of the skin. It may be local or general.

Etiology.-Parasites.-Probably more inflammatory conditions of the skin in small animals are due to small parasites 
than to all other causes combined, therefore, such are considered under parasitic skin diseases.

Mechanical,-Traumatic causes may be pressure, as lying on hard floor, whipping, bites (flies), running through brush as hunting dogs do, excessive wagging of the tail, friction when bathing by use of stiff brush, and shaking the ears.

C'hemical.-Chemical agents as strong caustic soaps or the too frequent use of ordinary soap; irritating medicinal preparations, as parasiticides improperly applied, or vesicatory agents as mustard. Acids intentionally applied, caustics as use of lime in dusting pens for poultry, and bee or wasp stings are also causes.

Thermic.--Thermic causes are: Scalding, burning, freezing or exposure to direct sun rays of animals unaccustomed to it after having been sheared.

Infectious.-Infections of Bacillus necrophorus, especially in suckling young, produce serious necrotic sloughs of the skin. Secondary dermatitis is frequently seen during the course of distemper in dogs.

Pathology. - The mild, acute form reddens the skin, which becomes sensitive, but is otherwise little changed. 'The subacute form is shown by a thickening and hardening of the skin. The skin feels rough and fissured. Any scrous or hemorrhagic discharge soils the hair and forms crusts. The microscopic examination for parasites is negative and the skin itself shows an increase of connective tissue and general infiltration often to the extent of separation of the layers.

Symptoms. - Pruritus is usually the first noticeable symptom which eauses repeated scratching, rubbing on the yround or floor and licking the part. In hunting dogs, especially at the beginning of the season, the skin over the rhest and anterior part of limbs, between the tocs, and on the cnd of the tail, will be reddened and moist, often bleeding, while the dog is being used in the field. In dogs and cats following the use of strong or the too frequent use of bath soaps, and in poultry from dusting them in limc, a mild, acute, diffuse rlermatitis ensues. Its persistence depends on the rontinuance of the causes. Sleeping on hard surfaces without bedding causes a chronic local der- 
matitis, especially in large dogs, which appears in the form of a thickened, roughened, hairless area over the elbow (scleroderma). A persistent form of dermatitis occurs as a result of injuries to the edge of pendulous ears and the irritation induces frequent shaking of the head which serves to aggravate the condition. Fly bites on the ears to the extent of producing severe inflammation are common.

Diagnosis. - Negative microscopic examination with consideration of the several causes assists in making a diagnosis.

Prognosis.-Good, especially in acute conditions when the causes can be removed.

Treatment.-Mild acute forms may be treated successfully with the application of lead water or drying powders. When crusts have formed, soiling the hair, a cleansing wash of a sodium carbonate solution (5 per cent.) repeated daily is very good and will relieve the itching usually present in this form. Chronic forms necessarily require longer treatment with preparations to soften the skin as lanolin or ichthyol ointment. The chronic form which occurs on the cdge of pendulous ears can be successfully treated only by bandaging which prevents the animal shaking the ears. The bandage should be so applied that the affected tips arc exposed for treatment with creolin ointment. This aids healing and prevents further injury from fly bites, the most usual source of this trouble. If the ears are very much thickened it may be advisable to remove the edges evenly and immobilize until complete healing occurs. Gangrenous dermatitis requires prompt treatment by removing the affected parts of skin and applying strong antiseptics.

\section{ACNE.}

Definition.-An inflammation of the glands of the skin with enlargement, appearing as small nodules in the skin. Quite frequently they pass on into pustules.

Etiology.- Irritation of the glands by rubbing, or by the collar, or when there is an obstruction to the glandular openings by accumulations of dirt or medicinal substances which have been applied. Preparations, as creolin, con- 
tinued for a time may produce inflammation of the glands. The bacteria always present in the skin find favorable conditions in an obstructed gland and soon convert it into a pustule. Acne is secondary to an invasion of the hair follicles and glands with parasites. (See Demodex Mange.)

Pathology. - Small elevations appear on the skin. 'They may be scattered or appear in groups. As the condition advances a few show a change to pustular form.

Symptoms. - Small, round elevations varying in size up to that of a pea appear on above-mentioned parts of the skin. Inflammatory symptoms are present and the skin is quitc sensitive. Small, clear vesicles appear and soon become turbid, rupture and their contents dry to form a scab. These scabs fall off and leave a small area denuded of hair for some time. Some nodules may gradually disappear in one to two weeks without disturbance of the skin or hair over them. All stages of the disease may be present at the same time.

Prognosis. - The prognosis is good. Recovery occurs in one to two weeks, either by pustular formation, rupture and escape of the contents, or by gradual reduction of the inflammatory process.

Treatment.-'Thoroughly clcanse the affected parts of the skin with a warm alkaline solution; in the nodular stage salicylic ointment (5 per cent.) may be used. As the nodules become softcned the contents should be squeezed, after opening if necessary, and washed out with antiseptic solutions. Internally the administration of Fowler's solution is usually beneficial. 'The use of tar, phenol, sulphur or salicylic acid preparations is contraindicated in cases of acne due to chemical irritations of the skin as they usually aggravate the condition.

\section{ECZEMA.}

Definition.-A chronic inflammation of the skin in the course of which papules, vesicles and pustules develop. Rupture of these complicate the condition by causing the skin to be moist and eovered with crusts matted in the 
hair. Under these crusts infection flourishes and decomposition occurs, further irritating the skin.

Etiology. - Various irritants which produce dermatitis may be the remote causes of eczema. The most frequent external cause is the accumulation of dirt on the skin, especially when the hair is long. Consequently the regions most commonly affected are the root of the tail, along the back, shoulders, neck and back of the ears. It is more common in long-haired dogs. Digestive disturbance has an indirect relation to eczema. Decomposing food is a common cause, as is overfeeding. Probably the most persisting forms of eczema occur in overfed, fat animals.

Pathology. - The skin may show all the stages of inflammation from acute to chronic. As it is a chronic condition, the acute symptoms are usually induced by scratching or biting the parts.

Symptoms. - The early stages appear as an ordinary dermatitis progressing through the various stages of inflammation until the pustular eruptions occur. Recovery may occur spontaneously at this time, or with proper treatment, but if not it passes on to the eczematous stage. Pustules continue to form and discharge their contents often unnoticed, under the long hair and crusts. If sufficient to keep the skin moist it is commonly classed as weeping eczema. The itching is intense, and the frequent scratching, biting or rubbing removes the matted hair and crusts leaving a raw bleeding surface. The skin lesions may occur in one or more small areas or over a gradually increasing large surface. In consequence of continued efforts to relieve the itching by licking and scratching, the inflammation extends into the deeper layers of the skin. Healing occurs in three or four weeks with recurrence of the condition. The skin becomes thickened and fissured, and bleeds easily. Scales continue to form on these partly healed areas. Some of the hair bulbs atrophy or are destroyed and only a partial growth of hair reappears on the surface. A recurrence of the condition each succeeding summer is quite common in well fed house pets especially among well bred dogs with fine skins, as the poodle. 
Diagnosis. - A diagnosis can only be made by the exclusion of parasitic conditions and the more acute forms of dermatitis and acne.

Prognosis. - Favorable in the earlier stages, but when the skin becomes thickened, hard and fissured, healing is as a rule only temporary, as acute relapses occur. When occurring as result of a chronic internal disease the prognosis is especially unfavorable.

Treatment.-Carefully cleanse the affected parts, remove all the crusts and scabs possible, and clip any hair that may be over the part and for some distance around the margin. Nild soap may be used for washing, also alkaline solution (sodium carbonate 5 per cent.). Lpon the condition of the skin further treatment depend's. If the surface is moist, drying powders can be used, such as talcum, zinc oxid, or boric acid. Liquid preparations of lead and zinc (Burrow's solution) are also very good. Proper internal treatment is important in all cases of eczema as has been shown by marked improvement from this form of treatment alone. Mild purgatives, especially calomel $(0.06-0.12)$ or magnesium sulphate (8.0-12.0), for their antiseptic and laxative action repeated at two- or three-day intervals. Fowler's solution (0.19-0.58) daily for dogs gives the best general results and should be continued for a long time. Calcium chlorate (15.0-30.0) in solution daily acts to relieve the itching. A good diet of easily digested, non-irritating foods is essential. 


\section{CHAPTER II. \\ PARASITIC SKIN DISEASES.}

\section{FLEAS.}

Description.-Fleas are large enough to be seen with the naked eye, but magnification is necessary to distinguish the species. They are a jumping insect without wings, brown in color and about 2 to $4 \mathrm{~mm}$. long. The female is the larger. They obtain nourishment by sucking blood. They readily pass from one animal to another and may reside in dust, filth, etc. The eggs are dropped or deposited on the ground or floor and hatch in six to twelve days. The final transformation into perfect insects requires three to five wecks depending upon the temperature.

Occurrence.-The dog is the most commonly affected of the small animals, with the Ctenocephalus canis, the common dog flea. The Pulex irritans of man will also live on dogs. The cat (Ctenoccphalus felis) is rarely affected except when closely associated with infested dogs. They suffer considerable annoyance but the fleas soon leave of their own accord. Hares and rabbits harbor the rabbit flea (Pulex gonircephahus), but may also be the hosts of the dog and human flea. On fowls, pigeons and other birds the bird flca (Pulex avium) is parasitic.

Symptoms. - Fleas are most common on dogs and pigeons. Scdentery, feeble or young animals, or those nursing young, or birds incubating eggs, are particularly susceptible; also animals kept in close quarters. Flcas are tormenting and harm the animal chiefly by disturbing rest and quict. Large numbers may produce chronic skin eruptions, but the most damage to the skin results from frequent and persistent scratching which produces irritated areas on the skin of the neck, back of the ears, and back of the forcarm. The skin 
is soiled witl the excrenent of the parasite which appears as brown specks seattered through the hair. Birds pick and scratcl the skin to relieve the irritation and pluck at their feathers. Severe infestations in young or feeble animals may finally cause death from exhaustion.

Diagnosis.- The diagnosis is easy by identifying the flea. It is well to be careful not to attribute a severe condition to fleas alone as smaller parasites may also be present.

Treatment. - The destruetion of fleas on dogs and cats is best effected by the use of creolin (1 to 2 per cent.) solution. It is cheap, only slightly toxic and may be applied to the entire skin surfaee at one time, and will destroy all fleas with which it comes in eontact. The odor which persists for two or three days will prevent further infestation only for that period, consequently a thorough cleansing of the premises and the repeated use of the creolin solution in the kemmed are nccessary for permanent relief. Creolin is somewl at irritating to the animal's eyes which may be avoided by careful bathing. Owing to the odor it is not advisable to wse it on house pets. Instead pyrethrum or insecticide powders containing same should be used by dusting it well in the hair or feathers and then brushing it out in fifteen to thirty minutes. Serious results have followed the too liberal and careless use of it. Frequent cleansing of both animals and quarters is discouraging to these pests. The use of powdered tobaceo in the bedding or sprinkling the floor with creolin solution usually serves to prevent further infestation. I) ogs permitted free range will soon become reinfested from assoriation with others. The nests of birds may be kept free from lice by mixing powdered tobaceo with the straw. Gum (amphor may be used in the same way.

\section{LICE.}

Description.- The louse as orlinarily elassed covers all the wingless parasites which do not jump. Lice do not leave their hosts except the fowl louse which drops off after engorgement with blood to return again when the birds return to roost. They are dull white in color except when engorged 
with blood. The eggs, commonly called nits, are firmly glued to the hairs or feathers. The young are similar in shape to the adults but only attain full size after several changes. The females are larger and more numcrous than males. Phthiriasis or lousiness is a term applied to the condition of an animal or bird infested with lice. Two kinds of lice affect small animals: The Linognathus piliferus which obtains nourishment by sucking blood, and the Trichodectes latus which lives by eating the epidermal scales and hair. The former may be recognized by its elongated head; the head of the latter is short and broad. The common louse of the dog is the Linognathus piliferus (Hematopinus piliferus), which may also live on ferrets, and the Trichodectes latus. The cat louse is Trichodectes subrostratus, and a rare species of Linognathus piliferus is sometimes found on rabbits. Several species of blood sucking lice infest fowls and birds, chiefly the Menopum trigonocephalum, while a larger one, the Menopum biseriatum, is most commonly found on the heads of young chicks. By cohabitation of the different species of fowls parasites peculiar to one of the species may be found on others. Other animals or man coming in contact with articles where lousy fowls roost will become infested, but the lice soon leave their illegitimate host and the irritated condition soon ends unless conditions favor further infestation as when straw is used from barns where fowls roost.

Symptoms.-The blood-sucking louse causes the most annoyance. Consequently dogs and fowls are the greatest suffercrs. The dog is not so frequently infested nor does he suffer as much as fowls in which phthiriasis is a serious condition, causing. the birds to lose their rest and become emaciated. It also interferes with the rearing of young. While incubating, when conditions are ideal for a rapid increase in these parasites, fowls suffer to such an extent as to be compelled to leave the nest, or remaining, often die from exhaustion from loss of blood and irritation. Newly hatched chicks are frequent sufferers from large numbers of lice especially the large ones on the head. 'The dog does not seem to resent the presence of lice as much as fleas, hence scratching and rubbing is less pronounced. A mild derma- 
titis may result from both parasitic wounds and scratching especially along under the surface of the neck. Fowls and birds scratch and pick themselves and pluck feathers, and, as the parasites increase gradually, exhibit all the symptoms of cachexia. With increasing numbers of lice emaciation increases, the birds appear droopy and a few may finally die from exhaustion. Canaries and parrots are frequent sufferers with the same symptoms.

Diagnosis. - The diagnosis of lice is easy when the size of the species is known. Numbers sufficient to cause noticeable simptoms are readily seen. Mange in dogs has been occasionally overlooked because the pruritus was ascribed to lice.

Prognosis. - Phthiriasis is serious and obstinate when young or feeble animals are affected, or when the number of animals is large and they have extensive range. Ordinarily it is not serious if treated, as many remedies are efficacious. Extensive obstinate cases reported have often proved to have a coincident infestation with mange mites.

Treatment.-Cleanliness discourages lice. In short-haired animals it is most easily attained. Iong-haired animals should be closely clipped and thoroughly brushed. The use of a creolin solution ( 2 per cent.) is very effective. It may be used as a bath and applied to kennel and runways. For poultry frequent applications of creolin (2 per cent.) or other parasiticides to the roosts and nesting places are sufficient for most of the birds. A small amount of oil of anise applied under the wing has proved of value in the treatment of (anary birds. Sulpluur and lard (.5.0-20.0) has also been used. Those showing severe symptoms should have special treatment. Tobacco or gum camphor placed in the nests is usually necessary to protect incubating fowls and little chichs.

\section{SCABIES. MANGE. RED MANGE. ITCH.}

Definition.-Mange is a contagious disease of the shin due to so-called mites.

Etiology.- 'Two kinds of mites, the sarcoptes and the l)emodex, are the chief ones found on small animals. They 
are quite small and can only be rocognized when highly magnified.

Sarcoptes Mite. - This is the most common mange mite and is distinguished by its tortoisc-like appearance, an elongated horseshoe-shaped head, and four pairs of short, thick legs. In the male, which is the smaller, the first and fourth pairs of legs are provided with a distinct cup-shaped disk, but only the first and second pairs are so provided in the female. The other legs are each supplied with a long bristle. These parasites make burrows in the skin where they live and obtain nourishment and in which the female deposits eggs. The eggs hatch in five to eight days and the parasite is fully dereloped in two to three weeks. They increase rapidly. It is estimated that a million and a half are produced in three months, under favorable conditions, from a single female. The sarcoptes mite varies slightly in size and minor details according to the species of animal upon which it lives. 'This makes several varieties which are sometimes described as so many species. Most of the sarcoptes mites live exclusively on their particular host, but, due to intimate association, may be found occasionally on others.

Pathology.--The skin shows symptoms of chronic inflammation raricd according to the severity of the attack. In mild cases dry crusts and scales cover the surface while the severe ones show a much wrinkled and sometimes moist surface. Fresh, bleeding areas may be associated with the condition as the result of scratching or rubbing the part.

Sarcoptes Mite of the Dog (Sarcoptes scabiei, variety canis). - This is the common mite found on the dog. Sarcoptic scabics may appear on any part of the body but usually around the head. If unchecked it gradually spreads, becoming generalized in four to eight weeks. Scratching and rubbing induced by the intense pruritus are the first noticeable symptoms. Simall red spots appear followed by papules and pustules and these, rupturcd by scratching, lead to formation of moist areas which continue to spread. The areas first invaded soon become dry. Yellowish-gray crusts form and gradually scale off. 'The hair falls out during the process. The skin becomes thickened and shows wrinkles and thick 
folds. The dog gives off an offensive but characteristic odor, and is repulsive in appearance. Emaciation increases, and, if not treated, death occurs from cachexia and exhaustion. In some cases the skin remains dry with profuse desquamation of bran-like scales; it may be and often is mistaken for a non-parasitic condition.

Sarcoptes Wite of the Cat (Notodres cati, var. cati; Sarcoptes minor, var, cati).-Scabies of the cat usually affects the head and neck, only occasionally invading the legs. 'The acute stages are less marked than in the dog, probably due in part to less violent scratching. The process continues with slight pustular eruption and the formation of crusts. Sometimes, especially when irregularly treaterl, it continues for months as a very mild condition with only slight thickening of the skin and scanty crust formation. 'The crusts are most noticeable on the edges of the ears and there is often a slight loss of hair on top of the hearl. In more severe eases the skin becomes thickened, hard and wrinkled. The eyelids become involved resulting in an intense conjunctivitis. The thickening of the skin around the nostril openings may be sufficient to interfere with respiration. The animal gradually becomes emaciated, exhausted and reath occurs usually in four to six months. Young animals die earlicr.

Sarcoptes Mite of the Rabbit (Notoders rati, var. amiculi; Sarcoptes minor, car. cumiculi).-In rabbits scabies attacks chiefly the skin of the head, particularly around the eyes, nose and at the base of the ears. It frequently extends to the hind and sometimes to the forepaws. 'The lesion resembles that seen in cats. Itching is intense inducing continual scratching and rubbing; the fur falls out and thick, gray scabs appear. As the process continues the eyes become surrounderl by masses of dry exudate. ('onjunctivitis is also present. By affecting the lips prehension of food is difficult. Emaciation appears early, and, if untreated, the animals invariably die in three to five months. The disease is highly communicable and serious in young animats.

Sarcoptes Wite of the Ferret (Sarcoptes seadiei, rar. hydrochaeri). This disease in ferrets is usually confined to the head and feet. The affecter skin is ustrally found covered 
with dirty yellow crusts. Crusts also form on the plantar surface of the feet and at the root of the claws which become swollen and distorted. To relieve the intense itching the animal frequently scratches and bites itself, at times gnawing the feet so viciously that they become severely wounded. Walking is difficult, the weight being thrown on posterior parts of the foot. A disagreeable odor is exhaled from the animal.

Sarcoptes Mite of the Fowl (Cnemidocoptes mutans, Sarcoptes mutans, var.gallina). - These parasites burrow under the scales on the featherless parts of the skin of the feet and legs. The resulting exudate elevates the scales and there is a formation of a white granular matter agglutinated by the exuded serum. Irregular masses of crusts separated by fissures continue to form and usually involving all of the skin on both legs. They are firmly adherent and when detached leave the skin raw and bleeding. The crusts become of a soft, spongy consistency due to the numerous furrows excavated by the parasite. Movements of the joints aggravate the condition; walking and even standing are difficult and painful. Occasionally an entire toe may drop off. The course of the disease is slow, the fowls become emaciated and finally dic from exhaustion.

Diagnosis. - An accurate diagnosis is possible only by a careful microscopic examination. Otherwise sarcoptic mange may be mistaken for many other diseases of the skin. It is essential to detect and identify the parasitc before a correct diagnosis can be made.

Prognosis. - Mild cases with local lcsions only may be treated with very good results. This is especially true in cats. Severe cases, where the process affects the entire skin surface, are almost incurable especially if symptoms of emaciation and exhaustion are present.

Treatment. - It is necessary to remove all the hair coat, if long. A possible exception may be made when the diseasc is strictly localized as in cats. ('leanse the skin and remove all scales and crusts by using an alkaline wash (sodium carbonate 2 per cent., or soapy water) applied with a fairly stiff brush. Howcrer, indiscriminate brushing should be 
avoided in localized cases as it may tend to spread the disease to healthy parts of the skin. Care must be observed in washing the cat, rabbit and ferret as they do not endure bathing very well. In some cases softening of the crusts is advisable which may be accomplished by using carbolated oil or glycerin. For dogs, even in the early stages with only small areas affected, vigorous treatment is advisable and careful repeated inspection is necessary to detect new areas. This is especially true in long-haired individuals. Most any of the parasiticides are more or less effective and failures are due as much to improper application as to the preparation used. Alcohol or aqueous preparations are convenient to apply but they are less energetic than those prepared with an oleaginous base. These will adhere to the skin longer and at the same time penetrate to the deeper layers. They should be applied generously and well rubbed in. Care should be taken to avoid irritating the conjunctiva when applying in the region of the eyes. Application may be made immediately after cleaning the skin, and repeated daily for four to six days, followed by another thorough cleansing. This course of treatment must be continued with three or fourday intermissions until itching disappears. Prevent the animal from licking the applications by muzzling or the addition of a bitter substance such as aloes. Many remedies have been used to destroy the mites. The following give good results and are least objectionable.

Sulphur ointment is one of the most common preparations used for the sarcoptes mite. It gives better results when used against the Notœelres cati of the cat and Cnemidocoptes mutans of the chicken. A more active preparation must be used on the dog. The following mixture is recommended:

R-Creolini

Phenol.

15.0

Sulphur fior.

()l. terebinth

60.0

120.0

Aq. ammonii

90.0

Ol. lini .

600.0

Kerosene

1200.0

Misce et fiat emulsio. 
Daily application may be made over small areas but in generalized cases only a part of the body should be treated at a time. The mixture is irritating, hence when the animal shows signs of exhaustion its use should be discontinued for a few days. Other preparations used are balsam of Peru in alcohol (5 to 10 per cent.), creolin ointment, styrax ointment (5 per cent.).

Demodex Mite.-Etiology.-Demodectic scabies is produced by the parasite, Demodex folliculorum. This parasite lives in the hair follicles and sebaceous glands, locations favoring much irritation and making treatment most difficult. The demodex mite is decidedly different in appearance from the sarcoptes. Its body is elongated, and provided with eight short legs emanating from its anterior third. The posterior part tapers to a blunt point. The head is short and thick. The total length is about six times the body width. Some one of the many varieties may be found on all kinds of small animals. The dog, however, is the principal sufferer. Cats are rarely attacked.

Pathology. - The skin in mild cases shows only slight inflammatory symptoms. In severe cases the skin is greatly thickened, and shows intact or ruptured pustules. In microscopic cross-sections the parasite is found within the glands and follicles.

Symptoms. - The early indications of demodex mange are the presence of isolated inflammatory areas from one-half to two inches in diameter. 'The hair covering them appears as though clipped off closely. 'The condition gradually spreads and may cover the entire body. Occasionally demodex mange occurs in the well named squamous form. In this there is only slight irritation and no marked inflammatory symptoms. The hair becomes thin, and gray-white scales cover the skin. This condition may continue without change for months especially when the dog is brushed and bathed frequently as may be done with house pets. The disease makes progress by a gradual peripheral spread over the skin, or less often by the occurrence of new isolated areas over the body. The skin thickens, pustules develop and erupt forming crusts; the hair falls out. Emaciation follows and the 
animal becones cachectic and exlausted. Pruritus is present from the beginning and frequent scratching and rubbing are the nost noticeable symptoms.

Diagnosis. - A positive diagnosis can be made only by idlentifying the parasite under the microscope. As these parasites live deep in the skin it is necessary to make the scraping accordingly.

Prognosis.-Favorable if local and treated in the early stages; unfavorable if generalized. Recovery may be obtained by proper treatment which may require eight to ten wecks. In severe cases with symptoms of cachexia and exhaustion present the treatment usually hastens the fatal termination.

Treatment.-Mild localized cases may be treated without washing or brushing. Generalized cases with crust formations require thorough cleansing with soap or alkaline solutions and all hair removed. As the demodex live deep in the skin glands it is evident that severe and vigorous treatment is required to effect recovery. Every parasiticide known has been used either alone, or in combination but without success. One mixture deserves mention as many cases have recovered after its prolonged use. It is not toxic, and will not be licked off.

\begin{tabular}{|c|c|c|c|c|}
\hline R-Creolini & & & & 30.0 \\
\hline Phenol. & & & & 15.0 \\
\hline Sulphur flor. & . & & & 60.0 \\
\hline Ol. terebinth & & & & 120.0 \\
\hline Aq. ammonii & & & & 90.0 \\
\hline Ol. lini & . & & & 600.0 \\
\hline Kerosene & & . & & 1200.0 \\
\hline
\end{tabular}

Misce et fiat emulsio.

'This may be applied daily over diseased a reas. If the (ntire body is affected, only one-third of it should be treated at a time. A thorough skin washing should be given once a week. If the animal shows ill effects of the treatment after a time, it may be discontinued for a few days and a dressing of oil substituterl. Aroid the eyes in applying it; keep the patient in a warm place. Feed well and stimulate the appetite with tonies. A saturated solution of balsam of Peru in 
alcohol (1.0-5.0) has given good results but the odor is objectionable in house pets. Animals being treated with oily or fatty preparations must be kept in a warm place as the heat radiation from the body is increased by such application.

\section{DERMATOMYCOSIS.}

\section{Vegetable Parasitic Disease of the Skin.}

Definition. - A communicable disease of the skin produced by fungi, somewhat similar to ordinary molds. Several species of fungi have been found to produce skin diseases. From the standpoint of clinical diagnosis they may be divided into two classes. Identification is possible only by their cultural characteristics. These parasites occur on the skin in the form of mycelia or filaments which may or may not be segmented. From these segments spores are formed which in turn, germinate to form new mycelia. The spores are round or oblong and under the microscope strongly refract the light.

Examination.-The mycelia and spores are extremely small and require high power magnification. A smear slide made with scrapings from the crusts or scabs, examined under high power, will show them as small, rounded spores either scattered or in chains. 'The segmented mycelia may also be recognized. It is advisable to pull a few hairs from the diseased area and examine near the roots for masses of fungi.

\section{HERPES TONSURANS.}

\section{Ringworm. Red Itch.}

Etiology.-This disease is produced by the 'Trichophyton tonsurans or other similar forms of fungi which are usually classed as the trichophyta.

Pathology. - The fungi develop around the shaft of the hair and extend down into the follicle but not to the root. 'The hair becomes brittle, splits and breaks off close to the skin. The enormous number of spores that grow in the follicles and beneath the epidermis set up inflammation, especially in the parts of the skin thickly covered with hair. 
Symptoms.-Ringworm occurs in all small animals but is most common in the dog. The lesions may be found on any part of the skin but usually appear on the head and legs, parts frequently in contact with objects harboring the parasite.

Ringworm is characterized by small, well defined, circular areas. $\mathrm{By}$ increasing in size and number they merge to form irregular, large patches. At first slightly reddened, these areas soon are covered with dirty, gray crusts or scabs. Irritation is variable, at times very intense inducing scratching and rubbing which remove the crusts and leave the surface raw and bleeding. Small nodules are sometimes seen, due to swollen hair follicles. Most of the hair is broken off. On those areas where pruritus is absent the (rusts remain, become thicker and agglutinate the hairs. Suppuration proceeds beneath the crusts and an offensive odor is emitted. In general the condition is less severe and more superficial than sarcoptic scabies, but the spread is more rapid. When the condition involves a greater part of the body general disturbance follows. Suppuration beneath the scabs permits of the absorption of toxins which poison, and the constant irritation exhausts the animal.

Diagnosis.-Accurate diagnosis is possible only with the microscope. The well defined, circular areas covered with short hairs in most cases and the rapid progress may be indicative.

Prognosis. - Very good if treated before the animal becomes emaciated and exhausted. Spontaneous recovery is exceedingly rare.

Treatment. - In a generalized case, except in short haired animals, remove all of the hair. Where only isolated spots occur clip the hair well back from the margin. Remove the (rusts, sof tening them if necessary with lard, oil or ointments. ('leanse the skin by washing with a soapy or alkaline (sodium ('arbonate) solution.

'This parasite is not very resistant, hence most of the antiparasitic preparations give good results. Inasmuch as the fungus is somewhat susceptible to fats the medicinal agents should be applied in oils or ointments. These prevent fur- 
ther spread of the fungus and in addition are soothing and protective to the irritated skin.

Creolin or iodin ointment is recommended and gives good results. 'These may' be applied onee daily for a week followed by a thorough cleansing. Continued treatment depends upon the appearanee. Small areas may be treated with pure tineture of iodin, two or three applieations a week. A strong solution of biehlorid of mereury $(1-500)$ is also quite effieient. Careful inspeetion must be made frequently for new disease areas. Powdered aloes will prevent the patient lieking off the ointment.

If other animals are kept near care must be taken to prevent the spread of the disease. All removed hair, erusts, also the bedding used by the patient, should be destroyed. ('leanse the sleeping quarters with a strong solution of hichlorid of mereury. Restriet their range to prevent eontamination of runways and yards. Cleanse and disinfeet all utensils and other artieles used about them.

\section{FAVUS.}

\section{IIoneycomb Ringurom. Comb Disease. White Comb.}

Etiology. - This disease is due to a fungus, the Aehorion sehönleinii. It oeeurs ehiefly in cats, miee and rats, but also in dogs and rabbits. The type affeeting birds is eaused by a speeial fungus similar to the trichophyton and designated as the Lophophyton gallinarum, commonly called "white comb" or "comb disease."

Pathology. - The fungus localizes itself in a hair folliele. As it develops it gradually extends over the surrounding skin forming a yellowish crust. Immediately beneath it the skin atrophies, beeomes moist and around the edge of the lesion appears an inflammatory swelling produeing a eup-shaped depression at the bottom of which the crust is attaeherl. 'This crust is eomposed mostly of the fungus material, mixed with hair or epithelial masses.

Symptoms.-Favus usually appears about the head especially in eats but the feet are also commonly affeeted. 'This 
is due to the infection being acquired from mice and rats affected with the disease. In dogs and cats the lesions appear ats a sulphur-rellow, circular mass depressed in the center and free at the elges, the size of a ten-cent piece. A number of areas in contact do not merge but encroach upon one another producing irregular shaped figures. In birds the crusts are thimner than in mammals and the areas tend to coalesce until a large surface is formed covered by a creviced rrust. When feathered parts of the skin are invaded the feathers fall out. The disease may extend all over the body.

Pruritus is rare and it is seldom that the crusts are rubbed or scratched off.

Diagnosis. - The presence of the sulphur-yellow, favic cup and slow growth make it easily rerognized. In birds the mouldy appearance and progressive growth indicate the (haracter of the disease.

Prognosis. - The prognosis is favorable; the disease may heal spontaneously. If far advanced and generalized in hirds it is usually fatal.

Treatment.- Remove the erusts with a blunt instrument damaging the skin as little as possible. Wash or otherwise cleanse the skin and apply tincture of iodin diluted well with alcohol. Bichlorid of mercury $(1-500)$ is equally good. l)ilute silver nitrate solution (2 per cent.) may also be used. ["sually i) to 6 daily treatments are sufficient. 



\section{PART XI.}

\section{DISEASE OF THE EAR.}

Examination.-The external ear (coneha) in dogs varies greatly in size depending upon the breed and size of the animal. It is easily examined by direct inspeetion. Careful examination should be made for wounds, bruises, hematomas and scars. Long hairs in eertain breeds (cocker spaniels) will interfere to a eertain degree with the examination. In cats and rabbits an inspection of the external ear can be made without difficulty. Note sensitiveness to manipulation.

The external ear eanal can be inspected in ordinary daylight but the examination is facilitated by the use of an ear speculum and mirror to reflect the light to the bottom of the canal. 'The operator should exereise care in the examination by either muzzling or taping the animal. Examine the external canal for inflammation, tumors, foreign bodies, cerumen aceumulations and parasites.

\section{WOUNDS OF THE EAR.}

Various degrees of wounds are observed involving the concha. Bites from other animals are the most common causes. The lesion may be a simple scrateh or cut in the skin or the entire strueture may be split in various degrees. Extensive wounds are often found in all the animals. Constant shaking the head especially in long eared dogs often leads to extensive injury to the outer margin of the eoncha. This mode of injury is often brought about by the parts being irritated by insects (flies) and parasites (Otodeetes eynotis). 
In some animals the thick, heavy, cartilaginous conchal base may be fractured by external violence.

Symptoms. - In dogs careful shaking of the head and holding it to one side, erying and evincing pain when the ear comes in contact with objects are often noted. Careful inspection will reveal the nature and extent of the wound.

Prognosis.-Favorable in most cases. In dogs with long ears, and when the wounds are extensive, it is difficult to prevent their shaking the head and aggravating the wound, which will materially influence the healing process.

Treatment.-In recent wounds the edges should be carefully cleaned, straightened with the scissors if necessary, and approximated with sutures. To facilitate union in some cases it is necessary to bandage the ears over the top of the head using a cap to cover the entire head to hold them in place. Frequent dressing (twice daily) is advisable. Apply boric acid powder as an antiseptic and to prevent adhesions of the parts by the secretions. When extensive tearing of the entire structure is found, it is necessary in some cases to remove a portion of the ear-flap. In this case it is best to remove a similar portion of the normal ear so that the ears will be symmetrical after healing takes place.

\section{ULCERATION OF THE CONCHA.}

This condition oceurs most frequently in dogs and espeeially in the breeds possessing long pendent ears. 'The ulcerous process is found in nearly all cases on the edge of the roncha.

Etiology.-Oecurs in most cases from injuries with more or less constant shaking of the head. 'The irritation thus produced keeps up the injury and interferes with union of the parts. Ilceration is most frequent during the fly season. 'The irritation produced by the flies causes the animal to shake its head violently, injuring still further the wounded parts. 'This is observed especially in older animals. Other diseases such as otitis and eezeina produce it in a similar manner. Ilunting dogs are frequently affecterl from the ears becoming injured while going, through brush, briers, ete. 
Symptoms. - The condition is easily diagnosed as the ulceration is observed on or near the edge of the ear-flap. 'The (onstant shaking and holding the head to one side are the most pronounced simptoms. On examination the part is usially found edematous, hot, painful and more or less hemorrhage present. Dried blood will be found on the margins of the ear-flap. Should the fissuring be deep it may be infeeted with pus.

Prognosis. - Should not be made very favorable as there is usually some loss in the ear-flap, which in some animats would be serious by disfiguring. Further, the condition is often resistant to treatment. The prognosis is less favorable in old animals.

Treatment. - The part should be first washed with an alkaline soap, to remove all dried crusts and other accumulations. Apply silver nitrate solution (2 per cent.) to stimulate granulation. Follow by an antiseptic dusting powder (iodoform; xeroform). One of the essential factors is to protect the ears from further injury and irritation. This is best done by bandaging the ears over the top of the head and using a head ap. Frequent dressing with the above materials is advisable. If the condition is due to insect bites the animal should be protected. In hunting dogs the ears should also be protected from further injury by light head cap or bandage.

\section{HEMATOMA.}

Ilematomas are frequently found in dogs with long pendent ears. Other animals are seddom affected.

Etiology. - This condition is brought about through traumatism. Very often it occurs secondary to otitis or ulceration of the concha. The hematoma results from a rupture of the capillaries and an extravasation of blood or serum hetween the skin and eonchal cartilage.

Symptoms. - As a rule hematoma develops suddonly. 'The animal holds the head sideways, the affected ear down and frequenty shakes its head. The ear-flap is sensitive to the tonch, and a distinet butging of the skin on the upper or lower surface is noted; both surfaces may' be involved. 'The 
enlargement will be found sensitive, hot and fluetuating. When of older standing the acute symptotns will be absent and some organization of tissue will be found around the margins of the enlargement.

Diagnosis. - This is made on the location of the enlargement, the fluctuation and the absence of marked inflammatory symptoms.

Prognosis. - Favorable in most cases.

Treatment.-Several modes of remedial procedure have been used. The principles involved in the treatment in order to be successful consist in the evacuation of the contents, maintaining the separated tissues in contact so that union can take place, and keeping the ear-flap quiet. This is best accomplished perhaps by the following: Shave the hair over the area and thoroughly disinfect the surface by painting with iodin. Make the incision at the most pendent portion of the enlargement and of sufficient length to permit all the serum, blood clots and organized tissue to be removed completely. Press out all the fluid and bring the separated tissues in contact with the cartilage. Retain in contact by suturing with interrupted sutures which pass through the concha. The sutures should be placed so that the skin is held in intimate contact with the cartilage at all points. A dressing of iodoform should be applied and the ears bandaged over the top of the head to ensure a quiet position and sufficient external pressure to prevent any further collection of serum. The dressing should be changed daily. In a week or ten days the sutures may be removed and the bandage discontinued. This method must be rather rigidly adhered to if good results are expected. Other methods such as removing the collection of material and injecting Lugol's solution or tincture of iodin have been used.

\section{OTITIS EXTERNA. OTORRHEA.}

Involvement of the internal portion of the external ear with various degrees of inflammation is very common in dogs with long pendulous ears and in rabbits from parasitic invasion. It is frequently unilateral, but in a number of 
cases both ears are involved. Middle aged or older animals are affected most commonly.

Etiology.--There are a variety of things having to do with the cause of this condition: (a) Accumulations of cerumen and dirt are common causative factors. These substances cause irritation to the skin resulting in inflammation and pyogenic infection. Animals with long pendent ears tend to retain these materials and decomposition and infection are thus formed. (b) Retention of débris from acute attacks leads to further irritation and of ten produces a chronic form of the disease with considerable thickening of the tissues. (c) In young animals the condition is frequently associated with distemper. Secondary infection during the progress of the disease involves the auditory canal. (d) Occasionally in dogs, but more commonly in rabbits, invasion with the parasite Otodectes cynotis occurs. In this case the entire canal is involved, even the intcrnal ear. The parasites produce extensive irritation and an aggravated form of the disease. (e) May result from ulceration of the concha and hematoma by extension of the process from continued irritation.

Symptoms. - In the early stages and when the condition is acoute the animal shows restlessness, shakes and scratches its luead and ears with its paws almost continuously, and rubs them against objects. Along the margins of the ear is of ten noticed a blood-mixed discharge. Direct examination reveals the ear to be hot, sensitive and the surface covered with a thick waxy secretion. Owing to the swelling of the skin and contiguous tissues an ear speculum is often necessary in order to examine thoroughly the lower portion of the (anal. I)uring the later stages ulceration takes place and more or less extensive pus formation is found. Palpating the base of the concha and exerting some pressure a thick, clark colored discharge will be forced out. The head is usually held in a fixed position, and if one side only is affected it will be held to that side. 'The condition may' produce temporary or permanent deafness. In rabbits, when due to parasites, nervous symptoms are very common. This has been observed in dogs. 
In the chronic form there will be extensive proliferation of connective tissuc projecting out into the auditory canal. The mass is irregular in form, often pelunculated, and will in some cases close the entire opening.

Diagnosis. - The characteristic discharge and other symptoms as rescribed make the diagnosis simple. Examination of the discharge should be made carefully to determine whether parasites are present or not.

Prognosis. - In the acute form, favorable. After extensive proliferation of tissue has taken place the prognosis is less farorable and recovery is difficult.

Treatment.-Both ears should be examined carefully to determine the rlegrec of involvement. It is essential in the first place to remove all dirt and cerumen which has accumulated. This is best accomplished by taking pledgets of cotton or gauze on a dressing forceps, saturate them in ether, thoroughly swabbing out the whole canal until all the débris is remored. In this cleansing process all of the small interstices between the swollen masses should be opened and swabbed. The ether solution is nccessary in order to dissolve the cerumen. With the scissors cut away all superfluous hair in the canal. Dry cotton or gauze should then be used in making the surface perfectly dry and also to remore any excess of ether which might be retained. An antiseptic powrler (boric acid; xeriform; tannoform) is worked down (arefully into the canal to produce antiseptic action and to prevent the secretions alhering to the skin surface. This treatment should be applied daily until the inflammation and swelling subside.

In the ehronic form with proliferation of tissue and ulceration the canal should be cleaned as clescriberl and the surface thoromghly cauterized with stick silver nitrate. 'To prevent mulue cauterization follow by salt solution. After the ulcerative process is reduced antiseptic treatment can be substituterl. Several weeks' treatment is often required in this form. Should the animal continue to shake the hearl and aggravate the condition a hearl cap may be emploved for a fex days. In long-eared dogs the cars should be examined "very few weeks as there is danger of recurrence. Should parasites be present treat with antiparasitics. 


\section{OTITIS MEDIA AND INTERNA.}

This condition is not very common in small animals. It oecurs most frequently in rabbits from parasitic invasion. The diagnosis is difficult as the condition is usually compliated with involvement of the auditory nerve, or pachymeningitis. So treatment is of any value. Sedatives would be indicated to control the nerrous stmptoms.

\section{TUMORS. NEOPLASMS OF THE EAR.}

Neoplastic formations are not so common in this loeation. I'apillomata occur commonly on the inner surface of the outer ear, but malignant tumors are rarely seen. Distentions of the sebaceous ducts are occasionally seen.

Papillomata. - These are found on the margins of the skin or in some portion of the vestibule of the ear and appear as single or multiple excrescentes. They are flat, oval or cauliftower-like in their growth. The surface is smooth or roughened and frequently covered by a thick, greasy material.

Symptoms. - The presence of tumors of ten leads to irritation and may produce otitis. (See Otitis.) On examination they are observed either as isolated single tumors or multiple tumors and with smooth or irregular surface. They are often pedunculated and stand out prominently. When in the lower portion of the canal they may completely occlude the opening. 'The diagnosis can be made positive by microseopic examination.

Prognosis. - Favorable.

Treatment. Operation is advisable. When large numbers are present the animal should be anesthetized and the entire surface deaned and disinfected. The tumors are removed as "lose to the base as possible, best by the seissors. 'The base should be cauterized by the actual cautery. The aftertreatment eonsists in keeping the part elean and using an antiseptic astringent powder (tannoform). If necessary a head bandage or eap may be used to keep the ear quiet. 
Sebaceous Tumors or Cysts. - These are recognized by their rounded, well-defined appearance, absence of inflammatory symptoms, and the character of the contents.

Treatment.-The cyst should be opened well, all of the secretions pressed out, and tincture of iodin applied to destroy the gland. It may be necessary in some cases to open into the gland and destroy it by silver nitrate or the actual cautery.

\section{PARASITES IN THE EAR.}

In the rabbit and dog the Otodectes cynotis and the Dermatophagus canis are frequently found producing a more or less intensive form of otitis.

Symptoms. -During the early stage of invasion the symptoms are not very pronounced. Later as the parasites increase in numbers and they extend farther down into the canal, the patient carries its head at an angle, whines, and rubs its ears against objects. Rabbits often show marked nervous symptoms from the irritation of the internal ear and auditory nerve direct. On examination there will be found a similar condition as in otitis. Tiny white or reddish specks will be visible in the discharge and when examined under a low power microscope or reading glass, the parasites are seen and recognized without difficulty.

Diagnosis. - This is made positive by the presence of the parasites in the discharge.

Prognosis.-Favorable. Most cases will recover unless nervous symptoms are present indicating the involvement of the internal ear. Such cases should be considered hopeless, especially in rabbits.

Treatment.-Local applications have proved quite satisfactory in these cases. Remove all crusts, secretions and débris with a solution of alcohol or ether. Dry the surface by using a swab of cotton or gauze. Apply paraffin oil to the entire surface, or a solution of carbolic acid ( 5 per cent.) in paraffin oil. Other oleaginous compounds may be used. Thoroughness in the application is important in order to destroy all parasites present. Daily applications are advisable. 


\section{DEAFNESS.}

Etiology.-This condition is observed from diseases affecting the internal ear, from closure of the external canal or it may be congenital. Paralysis of the auditory nerve may be the result of general infection (distemper) or ptomain poisoning. Deafness is noted very commonly in puppies, sometimes several in a litter will be affected. 'This no doubt is due to improper development of the auditory nerve or other structures of the internal ear. The condition is acquired in some cases from disease processes and infection extending to the internal ear. Complete closure of the external canal will produce at least a temporary deafness.

Symptoms. - The most pronounced symptom is failure to obey commands. Puppies deaf from birth do not bark or make any definite sounds. In congenital deafness the examination will reveal nothing abnormal with the external ear. In acquired deafness in some cases the cause may be found, such as closure of the external canal.

Prognosis. - Lnfavorable in all cases of congenital origin or in cases clue to external infection or parasites entering and destroying the internal ear. It is favorable in some cases of closure of the external canal with cerumen or tumor formation.

Treatment. - In congenital deafness no treatment seems to be of any value. If the deafness is due to paralysis of the auditory nerve small doses of strychnin would be recommended (0.001 once daily). Examination of the external meatus shoukl be made and, if closed, it should be dilated and the cause removed.

\section{EAR FISTULA.}

Etiology.-1)entigerous eysts are occasionally found in the temporal region and have their opening in the auditory canal. Wounds of the cartilage of the ear, either from sharp objects penetrating this region or from other causes may lead to a fistulous opening. 
Symptoms. - The presence of a thick, white, glutinous discharge either into the auditory meatus or to the outside should be regarded with suspicion and the parts thoroughly examined. Often the hair will be removed at the point of discharge and the material collect, become dried and form crusts. Pressure over the region will often reveal the point of discharge. The introduction of a small flexible probe will make the diagnosis positive. There is in nearly all cases some enlargement in the tissues adjacent to the fistula.

Prognosis. - All fistule involving the cartilage of the ear should not be considered in favorable light. As the process is a progressively destructive one, healing is difficult.

Treatment.-The part should be shaved and cleansed thoroughly. Introduce a flexible probe to determine the extent of the fistula and the tissue invaded. A free opening is made and all necrotic tissue removed. Pack the wound with iodin gauze. A head bandage should be employed to keep the pack in position. Dress the wound daily. After a few days it may be treated as an open wound. Some cases will require amputation of the concha. This, however, should be avoided if possible. 


\title{
PAR'T XII. \\ DISEASES OF THE EYE.
}

\author{
CHAP'TER I. \\ DISEASES OF THE EYELIDS.
}

Examination.-This is easily accomplished in ordinary daylight, or artifieial light. 'The following points are worthy of consideration: $(a)$ Note the position of the lids, whether they are normal or the degree of abnormality if present. (b) Note the reaction of the licls to ordinary stimuli. This is important to determine different degrees of sensitiveness. (c) Inspect the margin of the lids to determine whether or not the outline is regular, the presence or absence of the eyelashes. (d) Palpate the external surface for irregularities, tumor's, ete. (e) 'The condition of the hair and surface of the erelid should be inspected for wonnds, denuded areas, ex/ema, mange, ete. $(f)$ Evert the lids noting the resistance of the supporting structure and the condition of the conjunctival surface.

Various positions and reactions of the lids bear an important relation to different forms of paralysis and other diseases.

\section{WOUNDS OF THE EYELIDS.}

Various degrees of wounds are found involving the eyelids. Iacerations of different degrees are quite common from injuries or having the lids caught on sharp objects and torn. These wounds may involve only the margin of the lids, the skin covering the cartilage, or the entire structure. Even 
slight wounds on the lids should be considered important on account of the deformity, entropion or ectropion which might result.

All wounds should be examined thoroughly in order to determine the actual condition present. The wound should be cleaned and washed with boric acid solution ( 2 per cent.). Approximate with sutures and cover the surface with flexible collodion or airol paste. After adhesions have taken place the sutures may be removed and a dusting powder applied. Irritating substances should be avoided to prevent irritation to the eye proper.

When edcma is present as a result of the injury, warm water applications are useful. If abscesses develop they are opened, drainage cffected, and boric acid powder applied. All wounds should be given careful attention to prevent complications in the eye itself.

\section{INFLAMMATION OF THE EYELIDS.}

\section{Blepharitis.}

Definition.-An inflammation of the eyelids often leading to edema and suppuration or abscess formation.

Etiology. - Several factors have to do with producing this condition: (a) Wounds and bruises often develop into abscess formation. (b) Mange, particularly follicular, is a frequent cause in dogs; and sarcoptic mange in cats. Eczema. (d) Exposure to cold winds, water, etc., seen in hunting dogs. (e) Spread of inflammation from the conjunctiva. ( $f$ ) Foreign bodies penetrating the lids, such as brier barbs, etc.

Symptoms. - The condition is sclf-evident. An examination should be made to determine the cause of the inflammation, whether it is due to parasites, trauma, eczema, or other things.

Prognosis. - The prognosis is favorable in most cases unless complications involving the eye are present.

Treatment.-Treatment must be applied to remove the cause of the condition. When due to mange mites nonirritating antiparasitic agents are recommended. Balsam 
of Peru has been very satisfactory for this purpose. In eczema the surface of the lid should be freed of all scales, and other accumulating material by washing, and astringent antiseptics applied either in the form of solution or dusting powder. Abscesses when present should be opened and proper drainage afforded. All foreign bodies present must be removed and the wound treated with boric acid solution (2) per (ent.).

\section{MALPOSITION OF THE EYELIDS.}

Several malpositions of the eyelids are met with, especially in dogs. Certain breeds of dogs are predisposed thereto from their breeding. The abnormalities inost of ten found are: (a) Entropion, (b) ectropion, (c) ptosis, (d) paralysis of the orbicularis, and (e) spasm of the orbicularis.

Entropion.-Definition.-An inversion of the eyelids either in part or the entire lid. Entropion is usually complicated with trichiasis in which the eyelashes cause more or less direct irritation and injury to the conjunctiva and cornea. The condition is prescnt in the upper lid in most cases on account of the difference in form.

Etiology. - It often results from the cieatrization of wounds, chronic inflammation of the lids, and mange.

Symptoms. - The eyelid is turned inwardly against the cornea. The eyelashes coming in contact with the cornca prorluce inflammation, suppuration and in some cases ulccration. There is copious discharge of tcars, and pus. The conjunctiva will be found redkened and congested.

Prognosis. - Favorable. The eondition can be corrected in the majority of cases.

Treatment. - The injury to the cornea or conjunctiva may le treated with boric acid solution (2 per ecnt.). Surgical treatment is necessary in order to afford permancht relief. An elliptical piece of skin is removed from the eyelid, parallel to the margin and about one-eighth to one-quarter inch from the edge of the lisl.

Entropion forceps are used to grasp a sufficient fold of the skin so that when it is removerl and sutured the erlge of the 
lid will be drawn outward or everted. Care should be used to avoid injury to the deeper structures or the margin of the lid. A certain amount of judgment is necessary to determine the amount of skin to remove in each case. After operation the wound is covered with an impervious dressing (flexible collodion, airol paste). In two or three days the sutures may be removed. Recovery is prompt and complete in most cases. Eye complications should be treated according to their condition.

Ectropion.-Definition. - An eversion of the eyelid in part or the whole lid. It is rare in animals. Some breeds are more predisposed than others (bloodhounds, pointers). The lower lid is most frequently involved due to the lesser curvature as compared to the upper.

Etiology.-The common causes of this condition are: Wounds with cicatrix formation, lacerations of the margin of the lid, ulceration and abscess formation of the lid, and inflammation of the conjunctiva. All of these conditions tend to force the margin of the lid outward. Paralysis of the facial nerve allows the lower lid to fall outward.

Symptoms.-The prominence of the conjunctiva of the lower lid and the formation of a more or less deep gutter from the separation of the palpebral edge from the cornea are present in all cases. The conjunctiva is reddened, thickened, and there is a more or less constant flow of tears down over the face.

Prognosis. - The majority of cases can be relieved by surgical means. It might be possible that the operation will need to be repeated to properly approximate the parts.

Treatment.-In mild cases, resulting from congestion of the conjunctiva, relief is afforded by removing the cause and treating with mild astringents and antiseptics ( 0.5 to 1 per cent. zinc sulphate solution).

When the conjunctiva is chronically thickened forcing the palpebral edge outward, it is advisable to remove a portion of the conjunctiva. The resulting contracture of the wound in healing will bring the lid back in position. Codrenin solution should be instilled into the tissues before operating to reduce the pain and control the hemorrhage. 
Several operations have been used successfully in relieving this condition. The removal of a $V$-shaped portion of the lower lid, leaving the margin of the lid free, and bringing the edges of the wound together with sutures has becn successful. This forces the margin of the lid upward and inward. The amount of tissue removed will depend upon the degree of correction to be made.

Another method frequently employed is to remove a triangular piece of skin just outside of the external canthus and on a direct line with the lower lid, the base of the triangle being upward. Sutures are applied and the traction of the sutures and the cicatricial tissue formed keeps the lower lid pulled upwarl. In this case the size of the triangle will depend on the degree of ectropion present.

These operations should be made under strict antiseptic precautions and the parts protected. If necessary a head handlage with eye protector can be userl.

Ptosis.-Blepharoptosis.-Definition.-A dropping or falling of the upper lid so that it continuously covers the eye. It may occur as a symptomatic condition in conjunction with other local affections, such as atrophy of the eyeball, enophthalmos, etc.

Etiology. - The causes are various:

(a) It may be sympathetic, resulting from paralysis of the cervical sympathetic nerves. Wounds and injuries to the nerve, or growths, or cnlarged glands, in its cervical or intrathoracic course will prorluce ptosis.

(b) Ptosis is often paralytic produced by a paralysis of the inuscles of the upper eyelid, either due to a lesion of the muscle or its nerve supply.

(c) It may result from fracture of the orbital rim or process of the frontal bone.

(d) Tumors pressing on the cerebrum or cerebellum have been known to cause it.

(e) Ptosis often follows distemper, and other general diseases.

(f) Very often tumor formation on the upper eyelid will gradually overcome the muscular action and drag the eyelid downwarl. 
Symptoms. - The position of the upper evelid, which hangs downward and partially eovers the eveball, is characteristic. All movements of the animal indicate its inability to move the eyelid to any degree. The most important thing to be considered is the causative factor, and the examination should be made to determine it, if possible.

Treatment. - The treatment should be applied to remove the cause. If sympathetic and the lesion apparent an attempt should be made to correct it. Very often the cause is central and cannot be removed. Potassium iodid in small doses is indicatet. If of paralytic origin nerve stimulants, such as strychnin sulphate (0.001 daily) may be tried. Tumor formations on the eyelid should be removed conserving as much of the lid as possible.

Paralysis of the Orbicularis Nerve.-Definition.-Paralysis of the facial nerve, probably of central origin, may be peripheral in which the eye is held open, the lower lid relaxed falling away from the eyeball.

Etiology. - The cause of paralysis of the orbicularis may be wounds or contusions of the nerve leading to temporary or permanent paralysis. When of central origin it often results from hemorrhages in contact with the nerve at the point where it leaves the brain; or thrombus formation in the left posterior cerebellar artery interfering with the nutrition of the nucleus of the facial nerve. Tumors of the brain, and chronic lesions resulting from distemper have also been found to produce it.

Symptoms. - The lack of symmetry of the two sides of the face is the most apparent symptom of facial paralysis. The side paralyzed has a rclaxed and drooped appearance. 'The evelid will be drooped to a degree and cover a portion of the ryeball but cannot be closed producing a condition of lagophthalmus. There is a very noticeable absence of winking of the lids. Palpation of the lids will at once reveal their condition.

Prognosis. - If of peripheral origin and resulting from simple contusing a farorable termination may be looked for. Howerer, if inflications point to central origin the condition is often permanent. 'The degree of injury to the nerve will 
letermine the prognosis. Unfortunately in many cases this cannot be accurately determined.

Treatment. - As the eye cannot be completely closed, the cornea is exposed and may become dry and injured. An attempt should be made to correct this if possible. Oily collyria should be instilled in the eye two or three times daily. Massage over the course of the nerve trunk with a stimulating liniment (white liniment, soap liniment) often produces good results. Internal administration of strychnin sulphate (0.001-0.0025 once daily) should be continued for a few day's. If no results are obtained from the treatment after a course of two to three weeks further medication should be discontinued.

Spasm of the Orbicularis Nerve.-Blepharospasm.Definition. - A tonic or clonic spasm of the eyelids. It may occur periodically or be continuous. In the tonic form the eye is more or less permanently closed and constitutes usually a reflex symptom of a local eye disease, such as keratitis, panophthalmitis, conjunctivitis, or the presence of foreign bodies in the ere. Naturally the vision is interfered with by the original affection as well as by the spasm of the orbicularis nerve.

Clonic blepharospasm is observed in dogs following distemper when chorea-like movements are a sequel. Rapid contractions and relaxations of the lid (winking) take place. 'Twitching of the inuscles of the face and ear often are associated with the eye symptoms.

Treatment.--An examination of the eye should always be marle to determine the cause of the condition. If the symptoms result from local affections, treatment must be applied to relieve the special condition. If no local involvement can be found, treatment is out of the question. Twitching of the evelid as a sequel to distemper may disappear in a few weeks, or may remain permanently. Treatment has been unsatisfactory.

\section{TRICHIASIS.}

Definition.-An abnormal position or direction of the eyelashes. The condition, as a rule, is not observed in animals 
unless the lashes are directed toward the cornea and more or less injury to this membrane occurs. Trichiasis is often congenital in dogs, although it may be acquired. The smaller breeds of dogs are most often affected (Pekinese and Japanese spaniels). It also occurs in the cat and bird. In the latter fine, small feathers, which take the place of the eyelashes, are misdirected.

Symptoms.--The principal symptoms are lacrimation, keratitis, blepharospasm, and ulceration of the cornea. The eye must be examined by elevating the lids, and after cleansing with tepid water the lashes will be seen taking an abnormal direction. Extensive irritation and injury have been observed in such cases when they have been neglected.

Prognosis. - Favorable, except when the complications lead to corneal ulcer, or panophthalmitis.

Treatment.- Removal of the offending lashes is the first consideration. 'Their removal is effected by pulling them out with small tweezers or forceps. Temporary relief can also be obtained by cutting the lashes close to the margin of the lids. More permanent results will be obtained by performing the entropion operation. Any injury to the structures of the cye should be treated with antiseptics (boric acid 2 per cent. solution in distilled water).

\section{DISTRICHIASIS.}

Definition.-A condition in which there arc two rows of eyelashes present on the margin of the lids. One row is usually normally directed while the other is misclirected against the eyeball. 'The smaller breeds of dogs are most often affectcd. The abnormal lashes grow out from the puncta or outlets of the Meibomian glands.

The symptoms and trcatment are the same as in trichiasis.

\section{ADHESIONS OF THE EYELIDS.}

A normal adhesion of the eyelids is found at birth, and continues for a period of twelve to fiftcen days. At this period the eyelids open and assume their normal function 
unless some abnormality is present. Adhesions form between the margins of the lids which may be partial or complete (ankyloblepharon), or bctween the eyelids and the eyeball, the margins of the lids being free (symblepharon).

Ankyloblepharon. - In complete ankyloblepharon the palpebral fissure has entirely disappeared. An incomplete closure of the palpebral fissure is known as blepharophimosis. This condition, as a rule, is congenital, and is found in the (log and cat. It is diagnosed readily. A sufficient time should be allowed (ten to eighteen days) for the normal adhesions to disappear. Tery often there will be found a small line or groove at the point where the opening should be.

Treatment. - A small opening is made through the lid at the outer canthus, a grooved director inserted and the incision carried clear across to the inner canthus. 'The after-treatment consists in washing the eyes with a boric acid solution ( 2 per cent), and applying vaseline to the lid margins to prevent further adhesions.

In blepharophimosis there is a narrowing of the palpebral fissure which makes the eye appear much smaller than normal. It is often associated with trichiasis or entropion. To correct it the palpebral opening should be enlarged at the external canthus, and the skin sutured back both on the upper and lower lids to prevent adhesions. 'This operation will also correet trichiasis or entropion if either should be present. Keep the wound clean and apply a mild ointment to the recently incised margins.

Symblepharon. - This is an adhesion of the eyelids to the eyeball, and has been observed in dogs and cats. The majority of cases are congenital. Occasionally an acquired symblepharon is found due to adhesions following destruction of the conjunctival membrane during the course of distemper. An examination of the eye will at once reveal the condition. The lids cannot be everted and are found adherent to the eyeball.

Treatment.- Separation of the lid from the eyeball is possible. The animal should be anesthetized. The lids are carefully separated so that the eyeball is left intact. The operation can be done satisfactorily with a fine pair of 
scissors or a very small scalpel. After the operation is completed all hemorrhage must be controlled and an antiseptic ointment applied under the lids. Yellow oxid of mercury ointment has proved satisfactory for this purpose. It should be applied daily.

\section{LAGOPHTHALMUS.}

Definition.-An incomplete closure of the eyelids in which a portion of the cornea is left exposed. When the cornea or conjunctiva is left continuously exposed to the air without the normal secretions covering them, they become irritated, inflamed and serious alterations take place. The membranes are thickened, the cornea opaque and dry, and may even become perforated. The tears follow the angle of the eyelids and flow down over the face. The condition is brought about in most cases by ectropion, exophthalmos, enlargement of the eyeball, and some few cases by paralysis of the orbicularis nerve. Wounds of the lids with extensive loss of substance may also produce it.

Treatment.-The first indication is to remove the cause, if possible. If this cannot be done the only thing left is to protect the conjunctiva and cornea from dessication and eventual destruction. Oily solutions are best for this purpose. Instill them into the eye once or twice daily.

\section{TUMORS OF THE EYELIDS.}

Several varieties of tumors are found on the eyelids of small animals.

Papillomata.-Warts.-These are not uncommon in dogs and birds. The wart-like excrescences found in birds, particularly parrots, are usually tubercular, and are often associated with degenerations and abscess formation. In old logs and cats, horn-like growths are found as outgrowths from the walls of Meibomian cysts. Small warts are also seen developing along the margin of the lid, or on the skin over the evelid. During the course of epitheliosis of birds small horn-like growths appear on the lids.

Treatment.-Small warts appearing on the margin of the lids, or skin, are removed with the scissors. Cauterize the 
base of them with silver nitrate, and follow at once with salt solution to prevent undue caustie action and injury to the eve. 'Tubercular nodules and warts in parrots should be incised and remored as eompletely as possible. Conserve as much of the eyelid as possible. In epitheliosis the warts should be removed with the knife or curette and ioclin applied. Marts projecting out from the Meibomian glands are opened and curetted with a small instrument. 'The base of the warts should be carefully cauterized.

Chalazion.-Meibomian Cyst.-These are small eystic srowths, appearing on the free margin of the lid, or on the skin, standing out prominently. They are sebaeeous cysts having no inflammatory reaction and filled, as a rule, with a thick honey-like material. A true chalazion is a retention of the secretions of the Meibomian glands. 'They are frequently observed in dogs, more eommonly in mature animals. They form a firm, eircumscribed swelling, gradually enlarging until they reach the size of a pea or walnut. A differentiation is difficult to make as they resemble sebaceous eysts. 'Their derelopment often leads to a horny-like excreseence on the surface, and often take on the character of a malignant growth. 'They may involve the free margin of the lid, or extend to the entire structure of the upper eyelid.

Treatment.-Sinall sebaceous cysts are opened and the contents squeezed out. 'The opening is made large enough in order that the gland can be destroyed with iodin or silver nitrate.

In true chalazion it is atvisable to remove a small triangular portion of the lid, including the eyst, with the base of the triangle on the margin of the lid. 'This is best done, under morphin anesthesia, with the scissors. Approximate the wound thus mate with fine silk or linen. Cnion is usually (omplete in ten days to two weeks. Curetting the enlargenent may be tried in cases where the operation is not possible.

Pilosebaceous Cysts. - These are found on the upper eyelicl in dogs, especially bullelogs and pugs with wrinkled skin. 'The contents of the eysts are hair, sebum, and pus, which is usually discharged through a fistulous opening. 
Treatment.- The cyst should be opened, curetted, and the cyst wall destroyed with tincture of iodin.

Lipoma and Sebaceous Tumors in Birds. - These are frequently observed in the different species of birds. The tumors are well defined, of a firm consistency, and vary in size from a pea to a walnut. On opening the tumor fatty tissue may be seen, or which is more common, a vellowish, cheesy, tough material enclosed in a distinct capsule is present. They are situated immediately under the skin. From their nature they are either lipomas or sebaceous cysts. Various conditions are found. Sometimes the cysts will rupture, discharging the material, while in other cases horny growths will partially replace the eyst. Very often a number of birds in a flock will be affected.

Treatment.-Incise the tumor, remove all the contents and apply tincture of iodin to its base. Frec hemorrhage may occur after incision, which can be controlled by cauterizing with silver nitrate.

Enlargement of the Glands of Moll.-These small glands are situated along the free margin of the evelid, their ducts emptying into the glands of the hair follicles. They are enlarged commonly in older animals. The cysts are clear, transparent, and varying in size from a wheat grain and smaller.

Treatment. - The contents of the cysts are discharged. 'This is best done by erushing the small cyst with an artery forceps. Iodin is then applied to destroy the gland and cyst wall.

Granulomas.-Resulting from wounds, granulomatous tissue is often found developing on the free margin of the lids, or on the surface. 'This is easily determined by its general appearance. If there should be rloubt a definite diagnosis may be made with the microscope.

Treatment.-Complete extirpation is indicated. Cauterize the base of the tumefaction with silver nitrate, or actual (autery.

Malignant Neoplasms. - Several varieties of malignant tumors have been found involving the eyelids of small animals. 
Epitheliomata, sarcomata, carcinomata, and tuberculomata are the ones most often observed.

Diagnosis. - A diagnosis is made partly on the character of the growth and finally by microscopic examination.

Treatment.-Complete excision of the tumor should be attempted as early as possible. When the entire eyelid is involved treatment is of very little value. A recurrence of the tumors is liable to occur after operation. In removing the tumor as much as possible of the eyelid should be saved. 


\section{DISEASES OF THE CONJUNCTIVA.}

Examination.-Examination is simple in the dog, eat and bird. 'The animal should be held either by an assistant or' restrained so as to facilitate the examination. Ordinary daylight or artificial light may be used. The lower lid can be pulled downward by placing the thumb on or near the palpebral margin, and the upper lid pushed upward with the index finger of the same hand in a similar position. This manipulation will expose nearly all of the membrane. The examination is made for inflammations, foreign bodies, cysts, tumors, and wounds of various kinds. Vicious animals should be restrained by taping the mouth or by general anesthesia.

\section{CONJUNCTIVITIS.}

Definition.-Inflammation of the conjunctiva. In small animals various types of inflammation are of frequent occurrence. These animals are subjected to injuries which often involve the eonjunctival membrane, and infectious diseases (distemper in dog and cat, epitheliosis in bird) which prorluce various. forms of inflammation. The xerosis bacillus, Staphylococcus albus and S. aureus, streptococcus, diphtheria bacillus and others are more or less present in the eye learling in many cases to local infection and inflammation.

It is noterl further that the conjunctival membrane is very vaseular, rich in lymph cells, and sensitive to outside influences, all of which tend to favor the development of inflammatory conditions.

The following forms of conjunctivitis are recognized:

(a) Catarrhal, (b) purulent, (c) parenchymatous, (d) croupous, $(\rho)$ follicular, and $(f)$ exanthematous. 
Catarrhal Conjunctivitis. - A form of inflammation of very frequent occurrence in all small animals. It is characterized by congestion, swelling, and a discharge which may be serous, mucous, or mucopurulent.

Etiology.-Catarrhal conjunctivitis is often associated with or secondary to other diseases, such as eczema, distemper, epitheliosis, nasal catarrh, bronchitis and some constitutional diseases.

Mcchanical conjunctivitis often occurs in small animals from injuries of various kinds, forcign bodies, dirt or dust getting into contact with the membranes. Cold winds sometimes produce it in hunting dogs.

Irritating collyria instilled too often may produce it. Serious disturbances are frequently observed from too strong solutions.

Infection no doubt plays an important role in producing catarrhal conjunctivitis. 'The ordinary infection is constantly present and develops when the local resistance is reduced.

Symptoms. - There is a distinct change in the color of the membrane and a mucous or mucopurulent discharge. 'The congestion of the membrane produces a pink or reddish roloration which may be accompanied by a slight yellow tinge due to interstitial infiltration.

The secretion, at first serous, soon changes to mucous or mucopurulent and collects around the palpebral margin. The edges of the lids are found edematous.

Some photophobia is present. The animal will show morc or less constant movement of the lids, and holds the lids partially or completely closecl. A careful examination reveals the true nature of the condition.

Prognosis. - As a rule favorable except when secondary to infectious diseases. The inflammation usually subsides in a week or ten days.

Treatment. - A careful examination should be made to determine whether any foreign substances are present which must be carefully removed.

Wash the membranes thoroughly with boric acid (2 per cent.). This can be repeated two or three times daily during 
the first few days. Follow eaeh application of borie aeid with an instillation of one of the following solutions: Zine sulphate (1-1 $\frac{1}{2}$ per cent.); tannie acid (1-2 per cent.); ehinosol (1-500); protargol (2-4 per eent.); or if the condition is persistent solution of silver nitrate $(0.5$ per cent.).

The animal should be isolated and the premises disinfeeted to avoid spreading the disease.

Purulent Conjunctivitis.-Blennorrhea.-Acute Form.Definition.-A purulent secretion present in one or both eyes. In this eondition the discharge is more eopious and the inflammation more intense than in eatarrhal conjunetivitis. Purulent conjunctivitis results very frequently from or aeeompanies distemper of dogs and cats, and epitheliosis in fowls. It often oceurs as an epizoötie or enzoötie in kittens and puppies. Both the acute and chronic forms of the disease are frequently met with in practice.

Etiology. - The majority of cases can be determined quite definitely to be due to infection aeeompanying or following infeetious diseases. It is very common in dog distemper, cat distemper, and epitheliosis in birds. Various kinds of organisms have been isolated from the diseharge but none of them has been proved to be the speeifie cause. The fact that large numbers of puppies, kittens or fowls are affected, when closely assoeiated, indieates the communieable character of the disease. It is possible to have this form of conjunctivitis follow the eatarrhal, owing to the reduced resistance of the membrane which allows secondary infection to develop. It is, however, possible that many cases are produced in fowls and in some of the other animals by a parasite. Several species of parasites have been isolated from the conjunctival sac of birds.

Newly born animals contract the disease from the vaginal infection of the mother at the time of parturition.

Symptoms.-The eonjunctival membrane is of an intense red eolor and more or less uniform over its entire surface. 'The degree of redness depends upon the severity of the infection and the stage of development. In many cases the mucous membrane is prominent from the edematous swelling resulting from the irritation, thus giving it a "pouehing out" 
appearance. The amount of pus present is more or less characteristic. The eye is often entirely filled with a thick, whitish, glutinous pus, which becomes dried around the palpebral borders and forms crusts frequently agglutinating the eyclids. On account of the spread of the infection to the cornea, this organ becomes cloudy or even ulcerated. Owing to the irritant action of the pus the animal will show considerable pain, scratch and rub the eyes either with the paws or against objects. The membrana nictitans is red and protrudes over a portion of the eyeball. Most cases show considerable photophobia.

General.-General symptoms are noted in many cases in the form of duhness, loss of appetite and general weakness. There may be sufficient infection to produce general intoxication and death in birds. In these cases, however, it is quite likely that the infection is found in other parts of the body.

Prognosis. - The prognosis is not always favorable. Due to complications, which may involve the eyeball, partial or total blindness can result. While many cases terminate in complete resolution, others produce ulcerations and perforations of the cornea. When the latter occurs panophthalmitis is the result. In some animals, particularly birds, this would seriously interfere with the obtaining of food. When parasites are the cause of the condition other complications follow, such as infestation of the nasal passages and sinuses, and in some cases invasion of the air sacs.

Treatment. - Thorough disinfection of the premises, houses, ete., should be practised in order to prevent the spread of the infection. 'This can be accomplished either by the use of liquor cresolis compositus (3 per cent.) with which all places and articles in contact with the animals are thoroughly saturated, or the animals are remover from the house or kennel which is then disinfected with formaldehyrl fumes.

The general condition of the patient must be considered and its strength maintained by good, nourishing food and surh tonics as tincture of gentian, or tincture of nux vomica (0.)-0.t twice raily). A solution of iron sulphate (2 per (ent.) in the drinking water has proved of value as a tonic for birds. 
Local treatment is applied at least twice daily in order to remove the irritating material from the conjunctival sac, and also to destroy infection. Many preparations have been recommended and used for this purpose: Boric acid ( 2 per cent.); creolin (2 per cent.); chinosol (1-500); protargol (2-3 per cent.); or in severe cases silver nitrate ( 0.5 per cent.). This treatment must be continued for one to two weeks to obtain the best results.

C'hronic Form.-This form of the disease has been observed very frequently in dogs and cats following infectious and contagious diseases. To recognize it as being separate from the acute form is necessary on account of its persistent and long course. It is very common in old animals and those kept under bad hygienic conditions. Many cases of eczema and mange are associated with chronic purulent conjunctivitis. It may follow the acute form.

Symptoms.- The condition of the conjunctiva and the presence of pus with agglutination of the palpebral margins present the appearance in general of the acute form. On examination the conjunctiva is found to be dull red in color, slightly edematous and the cornea frequently has numerous small bloodvessels over its surface. The general condition of the patient is usually bad.

Prognosis. - Owing to the chronic nature of the disease and its persistency the prognosis is not favorable. The disease may last for months or even the entire life of the animal.

Treatment.-One of the first essentials in the treatment of this disease is to secure good sanitary conditions, giving plenty of nourishing food and do everything possible to increase the strength of the patient. The various antiseptic solutions recommended for the acute form may also be tried.

Good results have followed in some chronic cases by preparing an autogenic vaccine and administering 1 to 2 c.c. subcutaneously every three to five days. 'This in conjunction with the above treatment has proved to be of considerable value.

Parenchymatous Conjunctivitis.--Erysipelatous C'onjunctiritis.-Definition.-An inflammation of the deeper layers of 
the eorjunctiva and its submucous tissue. It oecurs in all small animals, most frequently in birds.

Etiology. - There are many factors producing parenchymatous conjunctivitis. Many of them are the same as those ausing the catarrhal form. Injuries to the mucous membranes and underlying structures can produce it. Chronic agents in the form of irritants, such as irritating collyria, when used too often or in too large quantities; thermic agents in the form of hot water or lot antiseptic solutions. 'The lisease is observet in hunting dogs from exposure, or to foreign borlies getting into the eves while going through briers and brush. Infection plays an important role in the production of this form of conjunctivitis. The infection is often introduced directly into the conjunctival sac where it enters small wouncls and produces intense inflammation of the parenchyma. Numerous organisms have been isolated in these cases. It is evident that no one specific organism produces it. 'The disease may atso develop secondarily (hematogenously) to some of the infectious diseases. It is a common sequel to don distemper or epitheliosis of forts.

Symptoms. 'The early indications of the disease are swelling and puffiness of the mucous membrane. This is so markerl in many cases that the swollen conjunctiva pouches ontwarl over the palpebral borders or the cornea. I) uring this stage the conjunctiva is intensely congested and redened. 'There is considerable laerimation and photophobia during the entire period of the disease. In the second stage there is a purulent discharge from the eye, which may he rery eopions. The general condition of the patient is ms mally not disturbed unless complications develop, or from the at tendine infertious diseases.

Prognosis. - The prognosis in most primary ases is favorable. In the arute form it subsiles in a week or ten rlays. When secondary to infectious diseases, the prognosis is not so favorable. In such instances it may develop into a chronic purukent conjunctivitis.

Treatment. - Prompt treatment is important in preventing the spread of the inflammation. Intiseptic and astringent onlutions should be employed: Borie acid (2 per cent.); 
lead water (2 per cent.); potassium permanganate ( $\frac{1}{4}$ per cent.); tincture opium ( 1 per cent.). If the patient shows considerable pain and irritation with marked congestion of the inembrane, codrenin solution ( 2 per cent.), or a few drops of stovain solution ( 2 per cent.) are recommended. When intense pain is present and the conjunctiva is a dull, red color indicating a subacute inflammation, silver nitrate $(0.5$ per cent.) is of value.

Croupous Conjunctivitis.-Definition.-An inflammation of the conjunctiva characterized by the formation of a pseudomembrane which covers its surface. Croupous conjunctivitis is observed more commonly in the dog and cat. In birds it accompanies epitheliosis, when the eyes are involved.

Etiology.-The majority of cases occurring in the dog and cat arc produced either by chemical irritation or as a result of specific infection following or accompanying infectious discases. In birds, owing to the prevalency of epitheliosis, large numbers in an individual flock may be affected at the same time.

Symptoms.--The conjunctiva is covered with a fibrinous exudate which frequently extends over the cornea. The corneal surface may be entirely covered. In birds a very heavy, thick, fibrinous membrane completely covers the entire anterior surface of the eye. Due to exudate and secrctions collecting under the pseudomembrane, it is forced outwardly protruding betwcen the lids. If the membrane is removed, the eye will be found normal in position and color. There are no general symptoms except as produced by some infectious diseases which the conjunctivitis attends.

Prognosis. - Favorablc in primary cases. When secondary the prognosis depends upon the primary disease it accompanies.

Treatment.-'The animal's general condition should bc obscrved and any necessary treatinent applied. The cye may be flushed out with a warm boric acid solution and the croupous membrane removed with a pledget of cotton or a pincettc. After the membranc is rcmoved, apply antiseptic or astringent collyria. It will probably be necessary to remove fragments of membrame as they later develop. 
Follicular Conjunctivitis. - Definition. - An inflammation of the conjunctiva characterized by the formation of small, gramular elevations over its surface. These granulations are due to swelling of the lymph follicles and to connective tissue proliferation, the result of inflanmation. 'The granulations often attain the size of a grain of millet or larger. Follicular conjunctivitis occurs usually on the inner surfase. of the membrana nictitans. In a large number of dog: examined, nearly one-half were found to be affected with . this disease. House dogs seem more susceptible than others.

Etiology.-The cause is unknown. Many etiological factors have been accused, such as dirt, dust and other irritants which enter the eye. Indoubtedly many cases result from distemper.

Symptoms. - As such a large number of dogs are affected with enlarged lymph follicles on the membrana nictitans one is led to conclude that their presence may not be abnormal. However, in many cases the granules appear also over the entire conjunctiva leading often to ectropion, an outward rolling of the eyelids. On cxamination the mucous membrane will be found to be of a dull red color and irregular on the surface. Small, white granules at their apices stand out quite prominently over the membrane. The membrana nictitans, when elevated with the forceps, will disclose small elevations on its under surface. In case of long standing the palpebral margins become red and the small glands prominent. 'There is some lacrimation and frequently a discharge of pus from the inner canthus. During the later stage small ulcers appear on the apices of the granulations, which, by confluence, form large, ulceraterl surfaces. The eves, kept partly closed and rubbed witl the paws, slow evidence of irritation and pain.

Prognosis.-The prognosis is not very favorable because the disease is usually well developed before the animal shows any marked symptoms. Owing to the chronic character of the disease the course is protracted requiring long continued treatment.

Treatment. - A thorough examination slould be made of the entire conjunctiva in order to determine the extent of 
the disease. 'The ordinary collyria, such as zine sulphate (1 per cent.); silver nitrate (0.5 per cent.), or chinosol (1-500), should be used for a few days to remove all of the surface infection and astringe the membrane. Following this treatment the affected parts of the conjunctiva are cauterized with silver nitrate. Apply immediately a solution of sodium clsorid to prevent injury to the cornea. 'This operation 's, iy be repeated in a few days, if necessary: In the meanve the astringent and antiseptic solutions are to be conued.

It is advisable to remove the membrana nictitans when it hows the pronouneed follieular granulations. This is best .one in the following manner: The animal may be given a general anesthetic, or anesthetize the mombrane locally by instilling 0.8-1.5 of eodrenin solution in the affected eye. If the latter is used the operation should be delayed for ten or fifteen minutes for eomplete anesthesia. The animal is placed on the operating table, held by an assistant, the membrana nietitans grasped with a small forceps and pulled outwardly toward the inner canthus of the eye. It is then cut off carcfully with a curved scissors. Care must be taken to prevent removal of any of the other conjunctival membrane, as this would lead to scar formation and result in entropion. Serious hemorrhage may occur in individual cases. If the hemorrhage is copious a gauze paek should be placed over the eye and held in place by a head bandage. This may be removed in twenty-four lours and the ordinary antiseptic solutions used. Recovery from the operation requires ten days to two weeks.

Exanthematous Conjunctivitis.-Definition.-An exanthematous eruption occurring during the course of certain discases. In small animals this form of conjunctivitis is not very common, but has been observed aceompanying or following distemper in the $\operatorname{dog}$ and cat. Parasitie skin diseases and eezemas often cxtend to the conjunetiva from the skin around the palpebral margins. This frequently results in suppurative inflammation of the glands along the margin of the lids, and in the membrana nictitans. Many cases of favus in fowls extend to the conjunctiva. 
Treatment. - The treatment of exanthematous conjunctivitis is very similar to that of the catarrhal form and its varieties. The original disease must be treated, and antiseptic and astringent solutions applied to the affected conjunctiva. When there is severe lacrimation, calomel has proved to be of considerable value.

\section{PTERYGIUM.}

Definition. - A triangular fold of the eonjunctiva and subconjunctival tissue extending over a part of the cornea. It is derived from the bulbar eonjunctiva and narrows to a point as it extends over the corneal membrane.

Symptoms. - Pterygium develops gradually and does not produce any marked irritation except a slight catarrhal inflammation. When it extends over on the cornea it may affecet rision.

Diagnosis.- The diagnosis is not difficult. The triangular growth, attacherl more or less firmly to the cornea, rieh in bloodvessels, whieh gives it a pink or reddish cast, eharacterizes it.

Prognosis. - I'terygium is not very serious and can usually be operated successfully.

Treatment. - It is advisable to treat the mucous membrane for a few days previous to the operation with antiseptic and astringent solutions. A loeal anesthetic should be administered, or if the animal is nervous, general anesthesia would be preferable. I)isseet off the triangular fold of membrane from the cornea and remove it as near the basc as possible with the seissors. It is further recommended that the base of the growth be dissected out eompletely and the wound sutured. The after-treatment consists in the use of antiseptie and astringent solutions. Recovery is usually prompt, and, if the operation has been thorough, the growth does not return.

\section{TRAUMATIC LESIONS OF THE CONJUNCTIVA.}

Foreign Bodies in the Conjunctiva. - Various kinds of substances are found in the conjunetival sac of sinall animals. 
Dirt, straws, briars, wheat seeds, splinters of wood, etc., have been observed quite commonly. This is particularly true in hunting dogs, and animals that are kept in dusty and unsanitary kennels. In birds, parasites have been found in a number of cases (spiroptera). These substances are frequently found undermeath the membrana nictitans.

Symptoms. - All foreign bodies cause more or less intense reactive symptoms, such as lacrimation, photophobia, serous or mucopurulent discharge, swelling of the eyelids, opaqueness of the cornea, etc. The severity of the symptoms will depend somewhat upon the character of the foreign body and the degree of irritation. The patient usually shows considerable pain, rubs the eyes against objects or with the paws, and thus intensifies the symptoms.

A sudden development of the symptoms cited should lead one to suspect a foreign body in the eye.

Treatment.-Flush out the conjunctival sac with warm water or boric acid solution (2 per cent.). Raise the membrana nictitans and evert the lids so that a close inspection of the entire conjunctival sac can be made. In the majority of cases the foreign body is visible. A reading glass will facilitatc finding small foreign bodies.

After the foreign body is located it is advisable to remove it either by flushing the surface with a boric acid solution (2 per cent.) or by grasping it with a small forceps, or wiping it out with a pledget of cotton. Considerable care should be exercised to see that all of the foreign material is removed. After removal flush the inucous membrane two or three times daily with boric acid solution (2 per cent.).

Wounds on the Conjunctiva. - Very often the conjunctiva is injured by sticks, pieces of straw, briers, etc. In the majority of cases the injury is slight and of little significance. In a few cases, where the lesion is extcnsive, there will be symptoms similar to those caused by foreign bodies in the conjunctival sac. A differentiation is made only by a careful examination.

Treatment.-'The lids slould be everted and the part thoroughly examined to determine the extent of the injury. 
If any shreds of membrame are present, they should be removed with the seissors. Antiseptic and astringent collyria are used until the symptoms subside.

\section{CORROSIONS AND BURNS OF THE CONJUNCTIVA.}

'llise oceur rather commonly in dogs and cats the result of arcident or intent. They may also be due to the use of strong collyria. A common practice, when dogs are fighting. is to separate them by throwing ammonia, pepper, or other irritant substances into their eyes. This often results in a serious inflammation of the conjunctiva and cornea. Thermic agents, such as hot water, also produce extcnsive injury.

Treatment. - A carcful history of the case should be obtained if possible in order to determine the cause of the injury. It would be best to first flush out the eyes with a warm boric acid solution (2 per cent.), and if the injury is produced by strong alkalies, follow with some soothing, lubricating agent, such as warm milk, or a dilute glycerin solution. Oleaginous preparations are also to be recommended. Ophthalmic ointments are useful. If the cauterization has produced an opacity of the cornea, of grcat value is a solution of sucens cineraria maritima compositus (50) jer cent.) used daily.

\section{ULCERATION OF THE CONJUNCTIVA.}

A more or less extensive ulceration of the conjunctival membrane occurs following discases or injuries. It has been observed following burns and corrosions; in birds, tuberculosis produces it.

Treatment.- When ulceration of the conjunctiva occurs, the part should be thoroughly cleaned, curetted, and the hase of the ulcer rauterized with silver nitrate followed by salt solution. This treatment may be repeated in a few days, if necessary. 'The eonjunctival sare should be washed daily' with boric acid (2) per cent.) to present infection and injury to other parts of the eve. 


\section{TUMORS AND GROWTHS ON THE CONJUNCTIVA.}

In small animals, tumors involving the conjunctiva and the membrana nictitans are of frequent occurrence. Several rarieties of tumors have been observed. The principal ones are: Epitheliomas, sarcomas, tubercular nodules, dermoids, adenomas of the glands of Harder, papillomas, fibromas and lipomas. It is very essential that a differential diagnosis be made in order to distinguish the malignant from the nonmalignant varieties. Malignant growths will not only invade the conjunctiva but spread rapidly to the adjacent tissue, and eventually cause the destruction of the entire eyeball.

Treatment. - All enlargements along the margin of the lids, on the conjunctiva or on the membrana nictitans should be removed completely as early as possible. This operation is best accomplished under general anesthesia. The aftertreatment would consist in cauterizing the wound and the application of the ordinary antiseptics.

\section{INF'LAMMATION OF THE MEMBRANA NICTITANS.}

Examination.-An examination of the membrana nictitans is very easily accomplished in small animals by ordinary daylight. Proper restraint of the animal is necessary for a careful and thorough inspection. If the animal is of a nervous temperament, or inclined to be vicious, a general anesthetic should be administered, or the patient hoppled and a tape applied around the mouth. The lids are then everted or held open with the thumb and finger while with the other hand the membrana nictitans can be grasped with a forceps and elevated so that all parts of it can be examined. Examination should be made for inflammation, granular enlargements, tumors, and injuries.

As the inucous membrane of the membrana nictitans is exposed to the same causes of disease as the rest of the ('onjunctiva, it frequently shows lesions.

Symptoms. - Inflammation of the membrana nictitans is characterized by a red, more or less flat enlargement appearing at the inner palpebral fissure. In severe cases the 
membrane stands out prominently and cxtends over a portion of the cornea. A protrusion of the membrana nictitans is observed in tetanus, which should not be mistaken for an inflammatory condition. 'This can easily be distinguished by other symptoms of tetanus appearing in other parts of the body.

Prognosis. - Favorable in most cases. It may terminate in a chronic inflammation in which case the membrana nictitans will remain permanently thickened.

Treatment.-The entire membrane should be inspected closely for foreign bodies. Flush out the membrane daily with boric acid solution ( 2 per cent.).

If in the course of two or three weeks the swelling has not subsided, it would indicate a chronic inflammation. In this case the removal of the entire membrane is recommended.

\section{WOUNDS OF THE MEMBRANA NICTITANS.}

In small animals wounds of the membrana nictitans are produced by the same causes as those producing traumatic conjunctivitis elsewhere. As a rule they are of little importance unless very extensive or followed by marked granulation. Antiseptic and astringent solutions usually suffice to heal them. If extcnsive granulation occur, it may be necessary to extirpate the entire membrane.

\section{TUMORS ON THE MEMBRANA NICTITANS.}

Various tumors have been found on the membrana nictitans. It is adrisable in all ases to remove the membrane surgically. 


\section{DISEASES OF THE LACRIMAL APPARATLS.}

Examination.-This apparatus consists of the lacrimal gland and the lacrimal passages. In small animals it is somewhat difficult to make an examination of the entire apparatus. The lacrimal gland in the dog is flat, light pink in color, of a mixed type and is situated in the periorbit directly under the orbital ligament. In the cat the position is very similar. In birds the gland is a small, round, reddish body against the eveball near the outer canthus and opens upon the wall of the eyelid through a small slit.

The lacrimal openings (puncta lacrimalia) are elliptical fissures and are two in number in the dog and one in the bird. The lacrimal canal extends from the lacrimal gland to an opening in the lower border of the nasal canal. This is a whitish, membranous tube to conduct the excessive secretion into the nasal passages. The entire lacrimal apparatus is of lesser importance in small animals than in horses.

\section{LACRIMATION.}

Epiphore.

Definition. - A term applied to designate a flow of tears over the lid margins. It is not of common occurrence in small animals, except in certain breeds of dogs.

Etiology.- (a) Vcry often due to ectropion or entropion, which may produce a deriation of the puncta lacrimalia, allowing the secretion to flow out over the margin of the lids. (b) Obstruction of the lacrimal duct by foreign substances, mucus, etc. $(c)$ Constriction and obliteration of the duct due to injuries, ete. $(d)$ Lacrimation is of ten seen as a result of irritation or diseases affecting the conjunctival membranes. In this case the secretion forms so rapidly that the lacrimal duct cannot carry it away, the excess flowing over the lid margins. (e) Ordinary lacrimation may be produced by exposure to irritating gases, smoke, dust or cold air. 
Symptoms. - A copious tear flow over the lid nargin. An examination of the surroundings of the patient should be made, and also a close inspection of the eonjunctiva and lacrimal apparatus, to cletermine if possible the cause of the condition. In certain breeds of dogs (spaniels, poodles, Boston terriers) it should not be looked upon as anything serious.

Prognosis.-As soon as the causes are removerl most cases recover promptly. In case the lacrimal passages are obstructed, the prognosis would be unfarorable.

Treatment.- If possible remove the cause of the condition. If in the lacrimal passages, they should be opened by injecting a warm solution of boric acid ( 2 per cent.) or sodium bicarbonate (1 per cent.) with a fine nozzled syringe. As the duct is often very small the operation is not always sueeessful.

\section{DACRYOCYSTITIS.}

Definition.-An inflammation of the lacrimal sac. It is quite common in logs and cats.

Symptoms.- The first symptom is a swelling or bulging in the neighborlood of the inner canthus of the eye. When pressed with the finger the contents are foreed out through the puncta lacrimalia. 'The diseharge may be serous, mueoid, or purulent, depending upon the age of the eondition. The ease often develops suddenly, the skin over the swelling becoming shiny and red. It often perforates and through the opening blood-tinged pus is diseharged. Later the discharge becomes mucoid or serous. A lacrimal fistula frequently results from the continuous tear flow through the abscess opening. I)acryocystitis usually becomes elıronie.

Prognosis.- On aecount of its chronieity and the development of fistula the prognosis is not very favorable. As a rule several weeks are required to bring about complete recovery.

Treatment. In dacryorestitis, lacrimal catarrh and fistula thorough irrigation with warm antiseptie solutions is inclicated. Apply with a syringe with a fine long nozzle. If the fistula persists, open the canaliculus and lacrimal sac by slitting and treat as an open wound. 


\section{CHAP'TER IV.}

\section{DISEASES OF THE CORNEA.}

Examination. - The cornea is normally transparent forming the anterior eovering of the eveball. It is shaped somewhat like a wateh-erystal and in the dog, cat and birel spherieal in form. The surface is smooth and shiny. In examining the cornea employ either daylight or artificial light. The animal may be restrained with an anesthetic or hoppled and tape applied around the mouth. By everting the eyclids with the thumb and index finger the entire eornea is exposed. Its form should be noted, its curvature and its transparency. The curvature of the cornea of onc eye should be compared with that of the other. Of assistance in this regard is a lighted candle. By noting the size of the flame as reflected by the cornea of each eye, comparisons may be made. If the curvature is weak, the flame appears larger; if strong, smaller. In $\operatorname{logs}$ and cats phrsiological variations will be noted in the eurvature of each eornea due to the difference in the shape and size of the eyes. The surface of the cornea should be smooth and even. 'To determine these observe it from the sidc, best using reflected light. 'The eornca should be tested for sensitiveness by gently touching it. It is normally very sensitive. In ulceration and when inflamed sensitiveness is increased; it is reducel in glaucoma. Further examination is made for cuts, scratches, foreign bodies, ete:

\section{KERATITIS.}

Definition.-An inflammation of the cornea. Two types are reeognized: 1. Non-suppurative, and 2, suppurative keratitis. I'nder the former are included the following forms: (a) Superficial, (b) vascular (pamnus), (c) keratitis pigmentosa, $(d)$ keratitis punetata superficialis, $(e)$ parenchy- 
matous, $(f)$ keratitis punctata profunda. Inder the latter are included: (a) Clceration of the cornea, $(b)$ abscess of the cormea, (c) keratitis neuroparalytica, and (d) keratitis from lagophthalmus.

\section{Non-suppurative Keratitis.}

Superficial Keratitis. - Definition.-An inflammation of the cornea which involves the epithelial laver and the superficial layers of the parenchyma. It is very commonly due to accidents, injuries, and infections.

Etiology.-Superficial keratitis may result from $(a)$ a spread of infection from the palpebral or scleral conjunctiva. Wounds on the cornea. (c) Foreign bodies. (d) Chemical irritants, such as too strong silver preparations, antiseptics, etc. The common practice among the laity of using alum, ammonia, powdered glass in turbidity of the cornea obviously often injures it. (e) Spreading of inflammation from adjacent structures, such as the iris and ciliary bodies. (f) Superficial keratitis is often observed to accompany dog distempter and epitheliosis of fowls.

Symptoms. - Lacrimation and photophobia are early and prominent symptoms. Clouding of the cornea in the form of a bluish-white film is seen which may be localized or general. An epithelial desquamation is often produced on the surface of the corneal infiltration. This is so slight in many cases that it is not recognized. The symptoms usually disappear completely in a week or ten rays.

Course. - The course is usually about two weeks.

Prognosis. - Favorable unless complications develop.

Treatment. - A careful examination should be made of all parts of the evelids and cornea to determine the conditions aceurately. Flush the cornea and conjunctiva with boric acid solution (2 per cent.), or chinosol (1-2000 in distilled water). If considerable pain and photophobia are present a cocain and atropin solution $(1-150)$ is recommended. A solution of succus cineraria maritima compositus $(50$ per (ent.) has given most excellent results. Should the condition indicate chronicity, and no ulceration be present, a mild 
solution of silver nitrate will be found useful to change the type of inflammation and to act as an antiseptic and astringent. Daily examination and treatment must be carried out until the opacity disappears.

Vascular Keratitis. - Pannus.-Definition.-The formation of new vascular tissue extending over the cornea just underneath the epithelial covering. Pannus is not frequent in small animals; it is seen occasionally in dogs.

Etiology. - Pannus occurs in most instances from irritation of a severe form extending over a long period of time. In dogs it commonly results from chenical irritation, when strong chemical agents, such as alum, mercury, etc., are used in treating opacities and chronic conjunctivitis. It may be due to trichiasis or districhiasis, and is also a sequel to chronic conjunctivitis, or may occur during the course of dog distemper.

Symptoms. - Pannus usually begins with photophobia and lacrimation. This is soon followed by a gray or white opacity, traversed by numerous bloodvessels which can be easily seen extending over the cornea in the form of reddish, radiating lines. Later, when the cause is removed, the new tissue becomes organized and appears as a white opacity of varying density.

Prognosis.-Complete recovery is difficult to attain in all cases. The prognosis should be guarded as complications may follow.

Treatment. $-A$ careful history of the case should be obtained in order to determine the cause, which should be removed, if possible. If opacity is present a solution of succus cineraria maritima compositus (50 per cent.) is recommended, using a few drops once or twice daily. Some of the various oph thalmic ointments might also be tried. Operative procerlure is recommended in case the ordinary treatment fails. The pamnus may be removed by first giving the patient a general or local anesthetic, dilating the lids with an ere speculum, and carefully euretting the surface with a rather dull curette. ('are must be taken to prevent curetting too deep. After operating apply antiseptics, and in severe cases cover the eye with sterile gauze held in place by a head handage. I)aily dressing is recommended. 
Keratitis Pigmentosa.-Pigmentury Keratitis.-Definition. I chronic disease of the cornea, either inflammatory or legenerative, with which is associated pigment deposit. It has been observerl quite often in certain breeds of dogs and cats. The poodle, small house spaniel, and other house dogs are the most common sufferers.

Etiology.--In most case's pigmentary keratitis is secondary to ulceration of the cornea, other forms of keratitis and corneal opacities. It may occur as a primary condition in dogs having marked pigment deposits along the corneal limbus. In these cases it affects usually only a portion of the cornea, but may extend gradually to other portions. Occasionally the pigmentation may be deposited around the scleral comeal zone, extending toward the center of the cornca. The causes of the primary form are unknown.

Symptoms. l'igmentary keratitis is characterized by deposits of melanin or pigment in the cornea. It usually begins as a fine, triangular network of small, dark lines rarliating from the limbus, the base of the formation being at the limbus and the apex directerl toward the center of the cornea. The triangular pigment formation is often so dense that the part of the cornea traversed by it is almost black. sometimes the pigmentation disappears to reappear later. ('ontinuerl recurrences eventually leave the cornea dull and uneven on its surface. Lacrimation and photophobia are little marked.

Prognosis. - The prognosis is unfavorable. As a rule following frequent recurrences permanent opacity of the (ornea results.

Treatment.-Borie acid (2 per cent.), or ichthyol (2 per ("ent.) is recommended. Pain may be arrested by stovain (2) per cent.), or alypin (2 per cent.), combined with the antiseptics. Internally potassium iodid $(0.012-0.12)$ once daily, or atoxyl in small doses has been used with apparent success. The patient should be fed liberally and given good ("are.

Keratitis Punctata Superficialis.-Facetted Keratitis.Definition. - A chronic inflammation of the superficial layer of the cornea causing opacity and the appearance of small 34 
facets occurring singly or multiple. 'This disease has been observed quite often in certain breeds of dogs but is very rare in cats and birds.

Etiology. - Nothing definite is known in regard to its etiology. Dogs with prominent eyes are often affected. It has been observed to accompany or follow rheumatic affections. It may be due to injuries, infections, specific or general, or exposure.

Symptoms.-Cloudiness of the cornea is the earliest symptom. This is followed soon by small, opaque, white, gray or yellow spots which penetrate into the parenchyma of the cornea. L'lceration of one or more turbid areas may take place causing small facets to appear. Injection of the conjunctiva and scleral membranes is a prominent symptom which may gradually disappear as the case becomes chronic. Lacrimation and photophobia are frequently seen. Owing to the disturbance of the cornea it will have a shagreened appearance.

Prognosis and Course.- The course of the disease is chronic. The symptoms may almost completely disappear only to recur. Some cases show a tendency to become periodic. The prognosis, thereforc, should not be made too favorable.

Treatment. - Flush the cornea with boric acid (2 per cent.), chinosol (1-500), or silver nitrate solution ( $\frac{1}{4}$ per cent.). This should be followed immediately with a few drops of a solution of succus cineraria maritima compositus (50 per cent.). This treatment should be continued for several days or weeks to obtain the best results. Some of the ophthalmic ointments may be tried in obstinate cases.

Parenchymatous Keratitis.-Definition. -An inflammation of the parenchyma of the cornea, characterized by a diffusc infiltration of the interstitial tissue with a vascularization of the sclera. It is accompanied in most cases by iridocyclitis. Parenchymatous keratitis is a very common affection in dogs but rare in cats and birds.

Etiology.-Infection is an important factor. In dogs it follows or accompanies distemper. It may be due to a spread of inflammation from the iris (iritis), choroid (choroiditis), etc. Chronic eczema, mange, etc., when they affect the 
eyelirls, ear, or adjacent parts, may be attended by it. Iirect injury to the cornea may be a cause.

Symptoms. - The cornea becomes turbid, gray or yellow. The normal transparency is lost. The turbidity may not extend over the entire cornea. Very' often a dulness of the cornea is all that is noticed during the eariy stages of the disease.

When the cornea is closely examined there will be found small elevations over the epithelial layer, and a retwork of fine bloodvessels radiating more or less evenly from the periphery to the center. The bloodvessels are often so small that a reading glass is necessary in order to see them. They rum parallel to each other and usually do not anastomose but terminate in a small loop forming a zone entirely around the corneal limbus. These new formed vessels become quite extensive and involve the entire cornea. Lacrimation, photophobia, pain, and congestion of the sclera are present. When examined with an ophthalmoscope there will be evilence of iritis and choroiditis.

Course.--The coursc is usually several days to a few weeks. Abscess or ulceration of the cornea is a rare sequel.

Prognosis. - Owing to the changes that have taken place in the cornea, it requires quite a long time to bring about healing. The tendency to recur makes the prognosis rather unfarorable. Permanent turbidity of the cornea may result with partial or complete loss of sight.

Treatment.-1)uring the early stages of the disease the patient sliould be protected from strong light and everything possible done to prevent irritation of the cornea.

('onstriction of the ressels and reduction of the irritation are best brought about by applying to the cornea three or four times daily corlrenin solution ( 4 per cent.). This is continued for two or three days. When there is evidence of iritis or choroiditis atropin solution ( $\frac{1}{2}$ per cent.) is of value to prevent adhesions (posterior synechia).

If pns is present a mild, non-irritating antiseptic should be userl. Recommenderl are boric acid (2 per cent.), biniodid of mercury $(1-10,000)$. Codrenin solution may be added to the above to constrict the bloodvessels and control the pain. 
When the condition becomes chronic silver preparations are useful. An autogenic vaccine has been used with good results and may be given every four or five days.

Give the patient plenty of good, nourishing food, occasional laxatives, and small daily doses of potassium iodid (0.0160.032 ).

Keratitis Punctata Profunda.-Definition. - An inflammation of the deeper layer of the cornea appearing in the form of multiple, small spots. It is more common in the horse and dog than in other animals.

Etiology. - The cause has not been determined. It may be produced by specific infection and inflammation spreading from adjacent parts.

Symptoms. - Numerous small opacities or spots are noted in the depths of the cornea. They are visible to the naked eye, or are seen better with a reading glass, with oblique or direct illumination. The spots are mostly circular, well defined, evenly distributed, or more or less grouped. In color they are usually white, occasionally darker. The rest of the eye appears normal.

Prognosis. - Lnfavorable.

Treatment. $-N_{0}$ treatment is of avail.

\section{Suppurative Keratitis.}

Ulceration of the Cornea. - Definition. - A destruction of the substance of the cornea, the result of infection with pus. The ulcers vary in size, some are not larger than a pin point; others involye a large part of the cornea.

Etiology.-The causes of ulceration are varied. Wounds of the cornea, even though slight, form ports of entry for infection. In small animals the cornea is frequently injured by foreign bodies which enter the lid sae, by being bitten or scrateher by other animals, or from an inversion of the eyelashes.

The undue exposure of the cornea which accompanes lagophthalmus, exophthalmus, and paralysis of the fifth nerve is a predisposing eause. ['lceration of the eornea accompanying or following infectious diseases, especially 
distemper of the dog and cat, is not infrequent. Purulent infection of the conjunctiva (palpebral, scleral, corneal) may learl to ulceration. The nicroörganisms most frequently found are streptococci, staphylococci, pncumococci, and other pyogenic germs.

The infection producing ulceration is no doubt in many rases carried by the blood to the cornca, and by the lymph into the cornea. Many diseases which reduce corneal resistance predispose to ulceration, such as chronic constitutional diseases, and some infectious diseases. In birds suppuration of the lacrimal passages and conjunctiva leads to ukccration as is frequently noted in epitheliosis. This form commonly leads to perforation of the cornea.

Symptoms. - Corneal ulcers vary considerably in size, form and depth. Any portion of the cornea may be the seat of one or more of them. In most cases, however, ulcers are found at or ncar the center of the cornea. In form they are mostly round with well defined, sharp borders as if cut out witl a sharp instrument. In other eases their form is irregular. 'Tle base of the ulcer may be flat, concave, or convex; rough, vascular, or infiltrated. The cornea surrounding the ulcer is usually turbid, and secondary ulcers not uncommonly develop. I'lceration of the cornea accompanying dog distemper often occurs suddenly, tends to spread rapidly and not infrequently leads to perforation in a few days. Photophobia, lacrimation. and congestion of the adjacent structures are nearly always present. Frequently a (opious discharge of pus occurs from the affected cye. P'ain may or may not be a prominent symptom, depending upon the cause of the ulcer and to what cxtent other structures of the eve are involved.

Course.- The usual course is chronic. Some cases require several weeks for complete disappearance. The ulcer may disappear without trace, or it may lead to perforation, or ricatrization and permanent opacity.

Prognosis. - Clceration of the cornea is always serious. Perforation, panophthalmitis, or opacity often follow it. Its (hronicity and tendency to recurrence make the prognosis always guarded. 
Treatment.-Flush the corneal surface with a mild antiseptic solution (boric acid, 2 per cent.; ichthyol, 2 per cent.). If pain is present, drop into the eye a small amount of cocain, stovain or alypin solution, to reduce the irritation and prevent further injury by the patient rubbing or scratching the affected eye. This treatment will control the general infection preventing further spread of the ulcer. Where pus formation is copious (blennorrhea) an autogenic vaccine may be prepared and used in conjunction with the other treatment.

Direct treatment of the ulcer requires judgment and care. Careful curetting followed by applying protargol solution (5 per cent.) is recommended. Good results have followed the use of succus cineraria maritima compositus (50 per cent.).

When the ulcer shows a tendency to perforate an antiseptic pack should be aprlied to the eye (eyelids closed), and a head bandage to hold it in place. This will exert outside pressure and may prevent perforation, permitting in the meantime new formed granulation tissue to strengthen the defect. Lead and silver solutions should not be used too strong on account of the danger of permanent deposits resulting. Treatment should be applied daily.

Abscess of the Cornea.-Definition.-A collection of pus in the cornea. Corneal abscesses are very common in dogs.

Etiology.-Infection with pus organisms usually through wounds or abrasions. Foreign bodies, bites and scratches pave the way for an infection which results in abscess. Corneal abscesses commonly result from distemper of the (log and cat, and epitheliosis in birds.

Symptoms. - The first symptoms noted are discharge of pus from the affected eye, photophobia, and a tendency to rub the eye with the paws. On examination a yellow, sharply defined abscess from the size of a pin-point to a wheat grain or larger is noted near the center of the cornea. The cornea surrounding the abscess may be transparent but is often turbid. As the abscess ages new formed bloodvessels are projected from the corneal bloodvessels across the cornea to the abscess. Surrounding the abscess a bloodvessel appears. If the abscess is large, or a number of small 
abscesses are present, the curvature of the cornea becomes stronger, sometimes cone-shaped. Conjunctivitis, congestion of the episcleral bloodvessels, and profuse lacrimation accompany the abscess.

Course.-Most cases require several weeks for complete recovery. As a rule the abscess ruptures outwardly; occasionally inwardly, discharging the pus into the anterior chamber causing hypopyon and in some cases suppurative panophthalmitis. Corneal ulcer may result from the abscess.

Prognosis. - On account of the danger of serious complications, the prognosis should be guarded. It is possible for the corneal abscess to heal without trace.

Treatment. - To remove surface infection warm, antiseptic solutions (boric acid, 2 per cent.; ichthyol, 3 per cent.) may be used. Pain is controlled by cocain, stovain, alypin or codrenin. The abscess may be opened under strict antiseptic precautions and the cavity treated with protargol solution (5 per cent.). Treatment should be made daily.

Keratitis Neuroparalytica. -Definition. - A rare affection in animals due to a paralysis of nerves (trigeminal, ciliary) leading to undue exposure of the cornea. (See larger works.)

Keratitis from Lagophthalmus.-Definition.-An inflammation of the cornea resulting from incomplete closing of the eyelids. The portions of the cornea not protected by the lid become dry, opaque, and ulcerated. Hypopyon, iritis and panophthahmitis frequently result.

Treatment.-Besides keeping the parts lubricated with glycerized antiseptics nothing can be done.

\section{FOREIGN BODIES AND WOUNDS OF THE CORNEA.}

Foreign bodies frequently enter the eyes of animals. Occasionally they become imbedded producing usually infected wounds of the cornea. Wounds also result from bites or scratches of other animals.

Symptoms.-The symptoms of lacrimation, photophobia and pain develop suddenly following the injury. The examination of the eye should be made thorough, everting the lids and nictating membrane, in order that foreign bodies will not be overlooked. 
Prognosis.-Providing too mucl injury has not been wrought by the offending foreign body, the prognosis is favorable. In making the prognosis one should bear in mind complications resulting from probable infection.

Treatment.-Flush the eye with mild antiseptic solutions (boric acid, 2 per eent.; ichthyol, 2 per cent.; chinosol, 1-2000). Foreign bodies must be removed. Further treatment will depend upon the progress of the case. 'The affected eve should be watched carefully and threatened complications promptly treated. Some wounds require careful handling in order to prevent perforation.

\section{OPACITIES OF THE CORNEA.}

Most opacities of the cornea result from inflammation. 'Those resulting from other causes are very rare in animals. The majority follow wounds, ulcers, irritant chemicals instilled into the eye, or burns.

Depending upon their density, various terms have been applied to opacities: (a) Nebula, a slight turbidity. The cornea appears blue or slightly foggy. (b) Macula, a denser opacity easily seen in ordinary light. It may be milk white. (c) Albugo, a translucent opacity. (d) Leukoma, the entire cornea becoming turbid, milk white. It may be either congenital or acquired.

Prognosis.-The prognosis depends upon the age of the patient, duration, location, extent and character of the opacity. 'The more recent the opacity, the more favorable the prognosis. Superficial oparities are more readily remover than deeper ones. However, the prognosis slould be guarded.

Treatment. - As most opacities of the cornea are composed of cicatricial tissue complete removal of them is very difficult. However, some of them will entirely disapjear and the cornea reassume its former transparency. 'The following agents are recommended: Dionin solution (i) to 10 per cent.), or in the form of powder, las given exeellent results. Begin with a few drops of a j per cent. solution instilled two or three times daily. 'The strength of the preparation may be 
increased later if necessary. A severe reaction usually follows the first treatments. 'This disappears later when the strength of the solution can be increased. Red or yellow oxid of mercury ointment has also been used with good results. A small quantity is applied once daily. Silver nitrate solution (4 per cent.), or protargol (10 per cent.) is used to stimulate greater cellular activity in the region of the opacity. succus cineraria maritima compositus is also of value. To obtain results treatment must be continued for a long time. As a last resort, when medical treatment does not suffice, iridectony may be tried. The operation consists in making an artificial pupil so situated that light may reach the retina.

\section{ECTASIA OF THE CORNEA.}

Two types of ectasia are recognized: (a) Inflammatory ectasia, and (b) non-inflammatory ectasia.

Inflammatory Ectasia.-Two forms occur in animals, viz.: Staphyloma, and keratectasia.

Staphyloma.-Definition.-A protrusion of the cornea, the result of inflammation (ulceration, perforation, prolapsus of iris). Staphyloma may be partial or complete; in form spherical or conical. In animals it is usually complete the entire cornea protruding from the scleral margin. In color it is blue or black due to cellular infiltration and pigment deposit. 'The protrusion may interfere with the closing of the licls. Iacrimation, photophobia and pain are prominent symptoms. In the incomplete form (conical) only a portion of the cornea protrudes.

Treatment.- Treatment is usually unsatisfactory. 'The rause shouk be removed if possible. Apply gentle pressure to the eye through antiseptic packs retainer with a hearl bandage. When the intra-ocular pressure is too great, some of the fluid from the anterior clamber may be aspirated before applying the pack. Permanent satisfactory results (an harelly be hoped for. When other treatment fails the affected eye should be enucleated.

Keratectasia.-Definition.- Keratertasia involves only the rornea which has become weakened at some point either from 
infiltration or from an ulcer which lias not perforated its entire thickness. In keratectasia the iris is not involved as in staphyloma.

Treatment.-Relieve the intra-ocular tension and apply a compress. Iridectomy may be useful in some cases.

Non-inflammatory Ectasia. - 'Two forms are recognizcd: Keratoconus, and keratoglobus.

Keratoconus.-A cone-shaped protrusion of the cornea which does not become opaque. It results from a weakening of the cornca at its center and an increase in intra-ocular tension. The condition is incurable.

Keratoglobus. - The entire cornea is enlarged as the result of a general increase in size of the entire eyeball. It is seen in hydrophthalmus. 'The cornca retains its transparency. The condition is usually congenital and most often observed in young dogs and young cats.

T'reatment.- No treatment is of any value.

\section{TUMORS OF THE CORNEA.}

Tumors of the cornea are rare in animals. Dermoids are occasionally met with in dogs and cats. Sarcomas and carcinomas have been noted. They usually originate either in some other part of the eye or in adjacent tissues.

Treatment.-Surgical trcatment should be attempted as early as possible. No treatment should be attempted in malignant tumors. 


\section{CHAPTER V.}

\section{I)ISEASES OF THE IRIS AND CILIARY BODY.}

General. - In practice it is very difficult to separate the diseases of the iris and ciliary body of which the iris is practically an extension. The structure of the iris is much the same in all animals. The arrangement of its muscular fibers in different animals accounts for the variation in the shape of the pupil. In the cat the pupil is an elongated slit; in dogs it is spherical or oval.

The color of the iris is due to the quantity of pigment present in the posterior layers and in the membrane proper. Frequently the pigment is entirely absent producing the albino or pink eye. This is common in rabbits and is occasionally observed in other animals. The other colors, such as blue, black or gray eyes, are determined by the amount and distribution of the pigment. A more complete cxamination of the iris can be made if a few drops of eserin are instilled into the eye to contract the pupil. The examination should be made for congenital defects, inflammations, tumors, etc.

\section{CONGENITAL DEFECTS OF THE IRIS.}

A number of defects in the formation and development of the iris has been observed: (a) Occlusion of the pupil, occurring in the dog, cat and rabbit, causing congenital blindness. (b) Ectopia pupillæ, a displacement of the pupil often found accompanying luxation of the lens. ('oloboma, a portion of the iris failing causing a large, irregular-shaped opening. (d) Aniridia, absence of the iris, very unusual in animals. 'I'reatment for these conditions is unsatisfactory. The size and form of the pupil are influenced by light, disease and medication. 


\section{MYDRIASIS.}

Definition.- A rlilatation of the pupil. It may be due to: (a) Paralysis of the third nerve, $(b)$ disease of the central nerrous system, $(c)$ constitutional diseases, $(d)$ poisons, $(e)$ mydriatics.

Mydriasis is produced artificially in order to examinc the interior of the eye.

\section{MYOSIS.}

Definition.-A contraction of the pupil. It is caused by paralysis of the cervical sympathetic nerves, tabes dorsalis, inflammation of the iris, forcign bodies in the cornea and by myotic drugs (morphin, codrenin, eserin). It is frequently noted in rabitanimals.

\section{IRITIS AND CYCLITIS. IRIDOCYCLITIS.}

Definition. - An inflammation of the iris and of the ciliary borly. They usually co-exist and will be therefore considered together (iridocyclitis).

Etiology.-Traumatism. A primary iridocyclitis is not common. Most cases are sccondary to other diseases, such as rlistemper of the dog and eat.

Symptoms. - Iridocyclitis is eharacterized by congestion, discoloration, loss of the normal striations and inability of the iris to react to light or other stimula. The pupil is ustally found contracted. While it is possible for the irilian exurlate to be very limited, the inflammation stopping in the congestive stage, as a rule, it is profuse, falls off the iris and accumulates in the anterior ehamber (hypopyon if purulent). 'The exulate, which is usually fibrinous, may be seren through the cornea as a movable, yellow, sometimes boor-streaked arecumulation floating in the anterior chamber. If an arlhesion between the iris and the eornea results, anterior sprnechia is spoken of; if between the iris and lens, posterior sinechia. Simptoms of lacrimation, photophobia and congestion of the conjunctiva are usually present. The rornea is nearly alwars involver, appearing as if lightly greased over its surface. When the ciliary bodies are 
prominently involved a turbidity of the corneal margins is noterl. Exudate, which has passed through the pupil, reaches the anterior chamber producing the same symptoms as iridian exudate. The iris may be only slightly involved as is determined by its eolor, striations and reaction to light.

Course. - The course in iridocyelitis is usually short. The inflammation rapidly subsides and the exudate is quickly resorbed. A few cases become chronic and lead to posterior sinechia.

Prognosis. - In uncomplicated cases the prognosis is favorable. When the choroid or retina is involved the prognosis is guarded.

Treatment. - When secondary to constitutional diseases, distemper, etc., these should receive attention. Iocal treatment consists in instilling atropin solution $(0 . \overline{5}$ to 1 per cent.) once or twice daily, which paralyzes the aceommodation, relieves pain and congestion and prevents adhesions. Dionin solution ( 4 per cent.) is also useful to stimulate the lymph circulation. Hot applieations in the form of a hot-water eompress are of great serviee. They should be applied for an hour at a time during the first twenty-four to thirty-six lours. Antiseptic and astringent solutions, such as succus cineraria maritima eompositus (50 per cent.), or ichthyol (4 per cent.) may also be used. Complieations should be treated as ther arise.

\section{CYSTS AND TUMORS OF THE IRIS.}

Cysts involving the iris are quite rare. They most of ten result from injury and appear as enlargements on the iris. It is rlifficult to distinguish between cysts and tumors. 'They are treated by puncturing, under antiseptic precautions, with a knife necrlle inserted through the corneal margin.

Both benign and malignant tumors may involve the iris. Melanoma is the most frequent primary tumor. Sarcomas and carcinomas also oecur. They usually extend from aljacent structures which are their primary seats. 'Treatment consists in enucleating the eyeball. 


\section{DISEASES OF THE LENS.}

Examination.-'The lens is best examined after dilating the pupil with atropin. 'The patient should be placed in a good light or light may be reflected with an ophthalmoscope against the lens. Normally the lens is transparent. It should be examined for turbidity, position and form. In old dogs the lens is usually less transparent than in younger animals.

\section{CATARACT.}

Definition.-An opacity of the lens, its capsule or both. Cataract is common in the dog and cat, especially in old animals. The following kinds occur: (a) Symptomatic, $(b)$ traumatic, $(c)$ senile, $(d)$ diabetic, $(e)$ congenital.

Symptomatic Cataract. - This form results from an inflammation of some of the adjacent structures which interferes with the nutrition of the lens. In the dog and cat it often develops from distemper.

Traumatic Cataract.-Traumatic cataract is caused by injury to the lens by sharp objects which penetrate the cornea. Or it may be due to indirect injury, the animal receiving a blow which jars the lens from its fastenings. It may, therefore, result from fracture of the orbit or some of the other bones of the head.

Senile Cataract.-Common in old dogs. It is due to an atrophy of the lens and is usually bilateral. In the early stages senile cataract usually appears as radiating, gray lines which extend from the periphery to the center of the lens. In other cases it may occur as an opaque spot or spots in the lens. The opacity spreads until eventually the entire lens is involved. 
Diabetic C'ataract.-Very rare in animals. A few cases have been noted in dogs.

Congenital Cataract.-Quite common in young dogs. As a rule, the lens only is involved. It is often bilateral. - It may be partial or complete.

Symptoms. - General.-In animals cataract is usually overlooked until it becomes so well marked as to be visible to the ordinary observer. Partial cases are sometimes discovered during an examination of the eye for some other disease. In using the ophthalmoscope in cataract the light should not be too strong. Under subdued light the opacity appears as a dark spot, its color differentiating it from the tapetum lucidum or the red papilla. Cataract should be distinguished from foreign bodics on the cornca, corneal opacities and turbidity of the vitreous humor. In most cases a careful examination with an ophthalmoscope will suffice to differentiate between lens opacities and those in other parts of the eye. When the diagnosis cataract has been madc, the form and cause should be determined whenever possible. The history of the case, the age and condition of the patient, and the appearance of the opacity are indicativc.

Course.-The course in cataract is chronic, usually leading to complete loss of vision. Occasionally a traunatic cataract develops rapidly and undergocs a spontaneous recovery within a short time. Such cases are rare.

Prognosis.-The prognosis is unfavorable.

Treatment.- The only treatment of value is an operation to remove the lens. In veterinary practice the difficulty in keeping the parts ascptic, the dressings in place and the patient quiet, is so great that cataract operations arc seldom employed. There are two operations for cataract: (a) A discission of the lens, and (b) cxtraction of the lens.

Discission.-Discission is practised only in congenital cataract, or when cataract appears early in life, is soft and capable of absorption. The operation is performed under complete anesthesia and strict asepsis. The eye to be operated is flushed out with an antiseptic solution (boric acid, 2 per cent.; bichlorid of mercury, 1-5000), followed 
by a solution of atropin ( 1 per cent.) to dilate the pupil. The lids are held open with an eye speculum. With a special instrument, a small knife-needle, which is passed through the cornea at its margin and pushed diagonally through the lens capsule into the lens proper, the lens is cut and separated. The instrument is then withdrawn carefully so as to avoid injury to the cornea. An absorption of the lens should follow. If not, the operation may be repeated in a few weeks.

Complications, sueh as swelling of the lens and increased intra-ocular tension with severe pain, may follow the operation. Cold packs are recommended to rerluce the swelling. If they afford no relief within a short time the cornea may be punctured at its märgin which relieves the tension. Through the same opening the lens substance may be removed. If iritis result from particles of the lens coming in contact with the iris, instil atropin solution and apply hot packs.

Extraction.- The lens is extracted in all cases when the discission operation is contra-indicated. The technic of the operation is rather difficult and requires mueh practice. It consists briefly in incising the cornea in the sclerocorneal limbus, fixing the eyeball with a special fixation forceps, incising the capsule of the lens, and expressing the lens. The eye should be cleansed and a dressing applied. Many complications may follow the operation in animals: P'anophthalmitis, prolapse of the iris; iritis and cyclitis in a small percentage of cases. Proper and careful dressing of the wound after the operation is espeeially important.

\section{LUXATION OF THE LENS.}

Iuxation is not very frequent in small animals. In most instances it results from traumatism. It may be due to extreme intra-ocular tension in either ehamber of the eye, or from rupture of the suspensory ligaments of the lens. 'The luxation may be partial or complete; into the anterior (ol" posterior chamber.

Treatment. - Treatment is of no value. A removal of the lens: is occasionally employed. 


\section{CHAPTER VII.}

\section{DISEASES OF THE RETINA AND CHOROID.}

THFse membranes can be seen only with the ophthalmoscopc. It is best to dilate the pupil in order to increase the size of the field of the eye's background.

The following pathological changes may be noted on the retina: (a) Hyperemia, (b) cdema, (c) inflammation (retinitis), (d) detachment of the retina, $(e)$ anemia, $(f)$ atrophy.

IIyperemia.-A congestion of the retina. The bloodressels will be found dilated and engorged with blood.

Edema.-Rare in animals. It may result from direct injury, or inflammatory exudate which collects in the retina. The retina appears cloudy.

Inflammation (Retinitis). - An inflammation of the retina. It usually develops during or as a sequel to infectious or chronic constitutional diseases. It is characterized by hyperemia, edema, partial or complete obliteration of the papilla and hemorrhage.

Detachment of the Retina.-Rare in animals. It may result from an accumulation of exudate or transudate between the retina and choroid, which causes the retina to become detached and float loose in the vitreous humor. It causes partial or complete blinelness.

Inemia.-Retinal anemia usually results from general hemorrhage; obriously it accompanies general anemia. It may have a local origin, the bloodvessels supplying the retina becoming blocked and pressed upon by tumors or inflammatory growths. The retina appears pale and the bloodvessels reduced in size.

Itrophy. - C sually follows extensive and severe retinitis; or when the nutrition of the retina has become reduced. The bloodvessels appear unusually small; in some cases harrlly visible.

The discases of the choroirl are so intimatcly interwoven with those of the other membrancs that a separate description is unnecessary. 


\section{CHAPTER VIII.}

\section{DISEASES OF THE OPTIC NERVE.}

Usually when the optie nerve is affected diseases of other struetures, partieularly of the retina, eo-exist.

The prineipal pathological ehanges affecting the optic nerve are: (a) Wounds, $(b)$ hemorrhages, $(c)$ inflammation, (d) tumors.

The optie nerve is also affeeted by inflammation of adjaeent struetures, and diseases of the central nervous system.

The diseases of the optie nerve usually noted are: (a) Papillitis, (b) retrobulbar neuritis, (c) atrophy. Clinically amblyopia and amaurosis are recognized.

\section{PAPILLITIS.}

Definition.-An inflammation of the papilla. It may be unilateral or bilateral. It is usually eaused by poisons, traumatism and diseases of the central nervous system. Viewed with the ophthalmoscope the papilla appears either enlarged and engorged with blood or, on the other hand, too pale-even white in color. Its outline is usually indistinct and striations are seen radiating from its center. When due to tumors and marked eongestion is present, the papilla appears intensely red ("ehoked disk").

Prognosis. - The prognosis is unfarorable. Partial or complete blindness will usually result.

\section{RETROBULBAR NEURITIS.}

Definition.-An inflammation of the optie nerve just posterior to its entrance into the eyeball. It may result from injuries, infection through wounds involving the orbit; or attend nasal catarrh, or dog distemper which has 
attacked the sinuses of the head. 'The patient is partially or totally blind. The papilla will appear congested. Many eases will recover when the cause is removed.

\section{ATROPHY OF THE OPTIC NERVE.}

Definition. - It may result from inflammation or be a simple atrophy. Blindness is a prominent symptom.

Prognosis. - The prognosis is unfavorable.

\section{AMBLYOPIA.}

Definition.- Partial blinduess. No lesion can be determined. In animals it usually results from poisoning. As a rule when the cause is removed sight is restored.

\section{AMAUROSIS.}

Definition.-Blindness without visible lesion of the eye. Anaurosis is a symptom and not a disease. The term is falling into disuse as ophthalınoseopy becomes better developed. It may be congenital or acquired. I)iseases of the optic nerve, retina, brain and certain poisons are its principal causes. The symptoms are blindness, abnormal dilatation of the pupil which does not react to light. In the early stages the eye appears normal but in time the globe becomes atrophice. Ophthalmeseopic examination may be negative, although usually changes cau be noted on the retina or papilla. 'There is no treatment of value. Cases due to poisons may recover. 


\section{CHAPTER IX.}

\section{DISEASES OF THE GLOBE AND ORBIT.}

InJuries to the eyeball are very common. They occur as wounds, lacerations and contusions. Sometimes the cyeball is ruptured. Diseases involving the globe and orbit are also frequent. The following are the most important: (a) Panophthalmitis, (b) glaucoma, (c) hydrophthalinus, (d) exophthalmus, $(e)$ luxation of the eyeball, $(f)$ enophthalmus, $(g)$ strabismus, $(h)$ nystagmus, $(i)$ parasites, (j) fracture of the orbit, $(k)$ inflammation of the orbit, $(l)$ tumors of tlie orbit.

\section{PANOPHTHALMITIS.}

Definition. - An inflanmation of the cntire eyeball. It is usually due to injury with infection, or may develop during the course of infectious diseases, the infection being carried to the eye by the blood or lymph.

Symptoms. - The initial symptoms will vary, depending upon whether the infection enters through wounds (exogenctic) or is carried by the blood or lymph (endogenic). When panoplithalmitis begins in the postcrior part of the cye it may escape attention until the anterior portion is involved. In cases of exogenetic origin usually a wound through the cornea or sclera is found out of which pus dischrarges. In endogenic infection a general congestion of the cycball is an early symptom. As the disease progresses perforation usually through the cornea with prolapsus of the iris and sometimes the lens follows. As a rule, the eyeball is destroyed.

Diagnosis. P'anophthahmitis should be differentiated from phlegmonous conjunctivitis and inflammation of other 
parts of the eye. These sometimes present symptoms confusingly like it. The eye should be earefully examined to avoid error in this regard. Should a perforating wound be found the diagnosis is simplified.

Prognosis. - Lnfavorable. Almost every case leads to destruction of the eyeball.

Treatment. - An effort should be made to arrest the spread of the infection. Obviously as the deeper structures are involved this is difficult to accomplish. The eye should be flushed with warm antiseptic solutions (boric acid, 2 per ceut.; ichthyol, 3 per cent). Subconjunctival injections of 1-2 c.c. of a solution of cyanide of inercury ( 0.5 per cent.) are recommended. Suppurating wounds should be drained and flushed out. I sually enucleation of the eyeball becomes necessary.

\section{GLAUCOMA.}

Definition. - A term applied to a number of diseases of the eve marked by intense intra-ocular tension, atrophy of the papilla and blindness. It is rare in animals. It is supposed to be due to some disturbance in the lymph or blood circulation of the eyeball which may be congenital or acquired.

Symptoms.-Claucoma develops gradually without signs of inflammation and with little evidence of pain. The early stages are often overlooker. As the disease progresses the pupil dilates and a marked hardness of the eveball develops due to increased intra-ocular pressure. The cornea may be clear or cloudy. With the ophthalmoscope the optic nerve appears cupped. The vision is gradually destrojed. In some cases (inflammatory glaucoma) acute inflammatory symptoms suddenly develop).

Prognosis.- Bad.

Treatment.-Eserin or pilocarpin should be tried; if unsuccessful relieve the intra-ocular pressure by paracentesis of the anterior chamber of the ere. 'The results are usually unsatisfactory. 


\section{HYDROPHTHALMUS.}

Definition.-An cnlargement of the cyeball in all its dimensions. It is common in pups and kittens. Hydrophthalmus develops slowly, the eyeball enlarging, the eurvature of the eornea becoming wcaker, the pupil dilated, intra-ocular pressure increased, and the papilla cupped. The condition is usually congenital.

Treatment.-Escrin and piloearpin are recommended, but they do little good. Paracentesis of the anterior chamber will relieve intra-oeular pressure as in glaucoma. Iridectomy may be tried.

\section{EXOPHTHALMUS.}

Definition.- In abnormal protrusion of the eyeball. It should not be confused with normally prominent eyeballs of some breeds of dogs.

Etiology. - Fracture of the orbital areh, the displaced bones forcing the eveball outward; edema, abscess or hematoma in the postbulbar tissue; retrobulbary cellulitis; intra-orbital tumors; tubereulous growths in the orbit. Exophthalmus is a prominent simptom of exophthalmic goiter in logs.

Symptoms.-One or both eyes appear unduly prominent. As the eyelids do not entirely cover the eye the surface of the ("ornea becomes dry and ulcerated.

Prognosis.- The prognosis should be guarded.

Treatment.-Treatment is only successful when the cause can be removerl. 'Tumors should be opcrated, enlarged thyroids remover and the affected eyeball proteeted.

\section{LUXATION OF THE EYEBALL.}

Common in the dog and cat. Dogs with prominent ares are predisposed. It results from injury, fighting, bereming caught in doors, ete.

Prognosis.-The prognosis will depend upon how long the eye has been prolapsed, the degree of injury to the optic nerve and to the eveball.

Treatment.--The patient should be anesthetized and the prolapserl eve flusherl with a warm antiseptic solution 
(boric acid, 2 per cent.). 'Try replacement by picking up the eyelids, retracting them as much as possible, at the same time gently but firmly pressing the eyeball back into its socket. It may be necessary to enlarge the palpebral slit by cutting the outer canthus. After replacement two or three stitches will retain the eyeball in position. The after-treatment consists in fomenting the eye with warm water and keeping it lubricated with dilute glycerin or liquid vaselin. Should panophțhalmitis or hydrophthalmitis develop, or the luxation reappear, enucleation of the eycball should be practised. 'The operation is as follows: The patient should be given a general anesthetic and the eyeball thoroughly washed with an antiseptic. The lids arc held apart by a retractor, or with dressing forceps held by an assistant. Nake an incision through the conjunctiva at the corneal margin, dissect back to the muscles, cutting them off close to their attachment to the eyeball. Keep as close to the eyeball as possiblc. When all of the muscles have becn cut away pull the eyeball downward and excisc the optic nerve. Control hemorrhage, pack the sockct with sterilc gauze, or iodoform gauze, put retaining sutures in the lids, and cover the whole with an antiseptic pack held in place with a head bandage. In twenty-four hours remove the pack and treat with antiseptic dusting powder. Healing is usually prompt.

\section{ENOPHTHALMUS.}

Definition.-An abnormal sinking of the eyeball into the orbit. It is rare in animals.

Etiology. - It may be congenital. Most cases, however, are acquired and due to an atrophy of the retrobulbar fat cushion, general emaciation, spasms of the muscles of the eye.

Symptoms. - The eyeball appears retracted into its socket. It should be distinguished from normal eyes which are unusually small. 'The general condition of the patient suffices for differentiation. 


\section{Prognosis.-1)epends upon the cause.}

Treatment.-Determine the cause and eliminate it by proper treatment.

\section{STRABISMUS.}

Definition. -A deviation of one of the eyes from its normal direction so that the risual axes cannot be focussed simultaneously on the same objective point.

Etiology.-It may be due to a mechanical interference with the movement and position of the eyeball, paralysis of the muscles of the eye, intracranial paralysis, spasms of the eye muscles, cerebral hemorrhage, and poisoning.

Diagnosis. - The diagnosis is not difficult. One eye will be turned inward or outward while the fcllow one is directed straight ahead.

Treatment.-A palliative treatment consists in applying a counterirritant to the region of the orbit and administering internally small doses of iodid of potash. A radical treatment is to perform a tenotomy, severing one of the tendons of the cye muscle at its insertion into the sclera. The particular tendon to be divided is determined by the individual case. If the strabismus is convergent the internal rectus is cut; if divcrgent, the external. The operation is briefty as follows: Give a general anesthetic. Flush the eye with antiseptics and follow by a solution of codrenin to control the hemorrhage. Cut through the conjunctiva and carefully dissect down to the tendon which grasp with a blunt hook, pull forward and cut off with a scissors. Inless the tendon is entirely severed the results will not be satisfactory. 'The wound in the conjunctiva may be left open. Flush out daily with antiseptic solution.

\section{NYSTAGMUS.}

Definition.-A continuous rolling movement of the eyeball. It occurs occasionally in logs. It very often accompanies cpilcpsy, convulsions, parasitic invasion of the ear, catarrh of the ear and sometimes accompanies chloroform narcosis. 
Treatment. - No treatment beyond rectifying the primary condition of which it is a symptom is recommended.

\section{PARASITES OF THE EYE.}

Lice (pediculi) are often found on the margins of the lids and on the skin over the orbital region. Mange mites also invarle the lids. The demodex mite may enter the Meibomian glands, conjunetiva and lacrimal apparatus. The F̈llaria oeuli canini is occasionally found, and spiroptera have been noted in the eyes of birds.

\section{FRACTURE OF THE ORBIT.}

Common in animals. Careful palpation will reveal (repitation.

Treatment. - 'Treatment should follow the general principles of surgery. Possible injury to the eyeball demands first rminsideration.

\section{INFLAMMATION OF THE ORBIT.}

I"sually results from traumatism. Due to the abundance of fat, inflammation of the orbit spreads rapidly and always endangers the optie nerve and eyeball. It is usually diffi(cult to obtain proper drainage or to apply antiseptics to stop the progress of the inflammation. An attempt should be made, however, to secure drainage and keep the parts rlean.

\section{TUMORS OF THE ORBIT.}

Infrequent. Sarcomas, earcinomas, epitheliomas and osteomas have been noted in this region. It is usually necessary to enucleate the eyeball in order to remove them. Malignant tumors are apt to recur. 



\section{PART XIIT.}

\section{HERNIA.}

Definition. - The term hernia is applied to a protrusion of a portion of the abdominal contents through a normal or an abnormal opening in the abdominal wall. The larger number of hernias is found under the skin, the smaller through the diaphragm.

Occurrence.-Hernia is very frequent in the dog but rather rare in other small animals.

General Remarks. - Hernia may consist of a portion of the bowel (enterocele), a section of the omentum (epiplocele), or a combination of both (entero-epiplocele); further, a portion of the uterus may be protruded (metrocele), or the uterus and its ligamentous attachments (metro-mesometrocele). Some of the other organs are occasionally found in the hernial sac, such as the liver (hepatocele), etc. In the dog it is possible to find almost any one of the organs of the abdominal cavity present in the hernial sac. The number and forms of hernia are quite variel.

'The following parts are distinguished in a hernia: IIernial sace, (b) hernial ring, $(c)$ hernial contents.

(a) The hernial sac consists of the skin, subcutaneous rellular tissues, and in most cases the peritoneum. Sometimes the peritoneum is ruptured and when this occurs the skin and subcutancous tissues constitute the hernial sac.

(b) The hernial ring is the opening through which the contents pass from the abdominal cavity. In recent hernias the ring is marle up of the margins of the muscular tissue, and its size is determined by the rent in the abdominal wall. In 
old cases connective tissue elements form around the margins which results in a distinct, firm ring. Palpation of a hernia will of ten reveal a well defined, firm enlargement which will serve to differentiate recent from long standing cases.

(c) The contents of a hernia are quite varied and will depend somewhat on its location. In most cases they consist of a portion of bowel, or omentum, or both. In a smaller number a portion of one of the other abdominal organs is present, such as the liver, stomach, spleen, uterus or bladder. When the contents fluctuate on palpation it may be due to the fluid content in the loop of bowel, or to serum which accumulates from a venous stagnation of the imprisoned contents.

From a practical standpoint it is important to classify hernias into: (a) Reducible, and $(b)$ irreducible.

(a) Reducible hernias are those in which the contents can be readily replaced in the abdominal cavity. This may be done by manipulation, or is often accomplished by changing the position of the patient. Such hernias present certain characteristic symptoms: They are enlargements, usually appearing on some portion of the abdominal wall, non-inflammatory (usually), casily replaced in the (avity, and the ring readily distinguished. It is possible in many cases to determine the character of the hernial contents by palpation. Allhesions will take place in some cases bctween the hernial sac and its contents which will interfere with complete reduction of the enlargement. In this case, as soon as pressure is removed from the outside, the hernial contents will again reappear in the sac. Practically all reducible hernias return unless outside pressure is maintained.

(b) Irreducihle hernias are those which cannot be returned by manipulation to the abdominal cavity. This condition may be brought about by adhesions between the different parts of the hernia, by swelling around the hernial ring, or by strangulation with subsequent swelling of the hernial contents. When a loop of bowel is present in the hernial sac strangulation frequently occurs from fecal matter accumulating and distending the prolapsecl bowel. It is very important to distinguish between strangulated and non-strangulated hernias. The differentiation is made very definitely 
by the symptoms shown by the patient and the local examination of the hernia. Marked symptoms of pain, vomiting, and local inflammation indicate strangulation. During the secondary stages of its development the hernial sac becomes rold, doughy, and non-sensitive to the tonch. Fecal fistulæ (intestinal fistulæ) occur in some cases from a sloughing of a portion of the intestine.

Forms. - The following are the most common hernias found in small animals: (a) Limbilieal, (b) ventral, (c) inguinal, (d) femoral, (e) diaphragmatic, and $(f)$ perineal.

Umbilical Hernia.-Etiology.-This form occurs either rongenitally (usual) or a short time after birth. The hernial ring is formed by an improper closure of the umbilicus, or the fibrous organization being of insufficient strength allowing the abdominal contents to pass through. Occasionally umbilical hernia occurs accirlentally in which case the peritoneum forms the inner portion of the hernial sac.

The hernial contents may consist of omentum, small or large intestines, or both. In most cases in puppies the sac rontains only omentum.

Symptoms. - The presence of an enlargement at the umbilicus which may be soft or firm, depending on the contents and local conditions. In most cașes the contents can be readily returner to the abdominal cavity. Occasionally adhesions are present which prevent this. Strangulation is very rare in this hernia.

Treatment.-In puppies many cases disappear spontaneously. Several methods of procedure in treatment have been recommenderl:

(a) Pressure bandage, or adliesive tape, applied over the part for a few days has proved satisfactory in many cases. This method keeps the contents in the eavity, allowing time for fibrous tissue organization to close the ring.

(b) Operation. - When adhesions are present or the ring is of considerable size, it is arlvisable to perform herniotomy. 'The animal is anesthetizerl, placed in a dorsal position on the table, the hair removed from the area and painted with tincture of iodin. An incision is mate through the skin of sufficient length, dissecting the hernial contents from the 
adjacent tissues, if necessary, carefully so as to avoid injuring the bowel. Return the contents to the abdominal cavity. Remove a small portion of the hernial ring on either side making a fresh wound surface to facilitate union of the parts. Suture the wound and apply after-treatment as in laparotomy (see Laparotomy).

Ventral Hernia.-Definition.- Tentral hernia is a term applied to a subcutaneous rupture of the abdominal muscles which permits the abdominal contents to pass through. This may occur at any point in the abdominal walls. The hernial sac consists of the peritoneum, subcutaneous tissue and the skin in the majority of cases. Sometimes the peritoneum is also ruptured allowing the contents to lie immediately under the skin.

Etiology. - The cause of ventral hernia is usually traumatic, or intra-abdominal pressure. In some instances when incomplete union of the abdominal muscles takes place following surgical operations, a hernia will develop.

Symptoms. - The sudden development of an enlargement appearing at some point in the abdominal wall. The size of the hernia will depend upon the extent of the rent in the abdominal muscles. Palpation of the enlargement will reveal a soft, fluctuating or elastic mass which can be reduced in most cases except when strangulated. When reduction is brought about the opening through the abdominal inuscles can be easily determined and the margins of the hernial ring felt. Changing the position of the patient will bring about reduction except when adhesions are present or the parts strangulated. Unless the hernia is very recent or strangulated, there will be no inflammation nor pain present on manipulation. It is necessary to differentiate recent hernia from abscess. This can be clone by careful palpation or by explorative operation. Some difficulty will be experienced in distinguishing between incarcerated hernia and tumors. However, the consistency, location and an explorative operation if necessary, will serve to make the distinction. A strangulated ventral hernia will be characterized by synptoms of inflammation, doughy consistency, pain on palpation and the general reaction of the patient. 
Treatment. - After the examination has been made carefully to determine the exact conditions, then it is possible to decide on the method of procedure. Treatment in ventral hernia should be operative. There is very little danger providing the usual precautions are observed in opening the abdominal cavity.

In ventral hernia without strangulation or incarceration the operation is performed as follows: The animal is given a laxative and fasted for twenty-four hours. The field of operation is shaved and an antiseptic pack applied for the same period. The animal is then given an anesthetic, preferably morphin, placed on the operating table in an advantageous position, the pack removed and the surface painted with tincture of iodin. A longitudinal incision is marle immediately over the hernia through the skin and parallel to the rent in the abdominal wall. The hernial contents are returned to the abdominal cavity. It is arlvisable to open the hernial sac in order to determine the condition of the hernial contents. When they are found normal and no evidence of injury to the structures the hernial sac can be trimmed off with the scissors and the rent in the abdominal wall sutured. If the hernial ring indicates fibrous tissue formation, it is advisable to trim off the margins with a scissors or knife to produce a fresh wound surface for approximation. The abdominal wound is then closed as in laparotomy (see Laparotomy).

In strangulated ventral hernia treatment should be attempted as early as possible. The same precautions should be observed as above and the contents examined to determine the cause of the strangulation and the condition of the rontents of the hernial sac. If the strangulation is produced by a constricted ring it should be enlarged with a probepointed knife sufficiently to allow the contents to be returned to the abdominal cavity. The wound in the wall is closed in the usual manner. If the hernial contents have been strangulated and retained until gangrenous then proper treatment should be employed. A section of bowel or omentum may be removed without difficulty and suceessfully when properly done. Adhesions when present should be 
(arefully broken down to allow the contents to be returned. Some of these cases may present special problems, and therefore a careful examination of the contents should always be madc. The after-treatment would consist of keeping an antiseptic pack in contact with the wound for a few days until union takes place. There is a possibility of a reeurrence of the condition if union of the abdominal wall is incomplete. leoperation is recommended when this occurs.

Inguinal Hernia.- It is necessary to divide this form of hernia into two classes on account of the anatomical differences in the female and male animal: 1. Inguinal hernia in the female, and 2. inguinal and scrotal hernia in the male.

1. Inguinal Hernia in the Female.-Etiology.-A very common form of hernia. The inguinal eanal in the female is very short and the diameter usually greater than in the male animal. During pregnancy considerable strain is thrown upon these structures resulting in a hernia. It may result also from inereased intra-abdominal pressure, from aseites, distention of the organs, or hypertrophy. Congenital inguimal hernia in the female has been observerl.

The hernial contents consist of the round ligament with peritoneum, or one or both cornua of the uterus. In a smaller number of eases, other abdominal organs may be present.

Symptoms. - An enlargement appearing just posterior to the inguinal mammæ. It varies in size from a small, rounded mass to one of sufficient proportions to reach the ground. The consistency of the hernia will depend on its contents, sometimes fluctuating, at other times firm. When the animal is pregnant the fetuses may be palpated in the hernial sac. The contents, if no adhesions are present, may be rasily pushed back into the abdominal cavity and the hernial ring distinctly felt. Placing the dog in a dorsal position with the hind limbs elevated often will effect replacement. liurther, there is no symptom of inflammation and the parts are non-sensitive on palpation. All enlargements appearing in this loeation should be examined from the standpoint of leernia as it is not ahways possible to reduce them and palpate ther ring. 
Trentment.-Operation is advised in all cases. Herniotomy is performed in the following manner: 'The animal should be properly prepared by fasting and administration of a laxative twenty-four hours in advance. The operative field should be shaved and disinfected. Make an incision longitudinally over the enlargement through the skin and hernial sac. If no adhesions are present and the contents capable of being replaced this should be done at once. If adhesions are present preventing reduction they must be carefully broken down when the contents will return easily. Sometimes the blarkler is encountered considerably distended with urine. If this is the case, introduce a trocar and draw off the urine which facilitates replacement. When the gravid uterus is found in the sac it will be necessary to remove the fetuses in the usual manner and return the cornua to the abdominal cavity. After reduction has been brought about the hernial sac should be ligated as close to the cavity as possible and removed, pushing the stump into the cavity. Suture the ring by inserting the sutures close together, keeping away from the pudic veins. The extra skin which will be present should be properly trimmed off with the scissors and sutured. An antiseptic pack is applied, renewed daily, and kept in position until union is complete. The external sutures should be removed when the wound is healed.

2. Inguinal and Scrotal Hernia in the Male.-Etiology.Inguinal hernia is not as common in the male animal although it is met with occasionally. Dilatation of the inguinal canal from any cause will allow the intestines or omentum to pass through carrying a portion of the peritoneum with it forming an inguinal hemia.

Sirotal hernia is far more common in the male animal. The hernial sac is formed by the processus vaginalis, and the rontents rensist of omentum or a loop of bowel protruding out into the scrotum in contact with the testicle. The hernia mạy be unilateral or bilateral.

Sympterims.- Inguinal hernia is characterized by an enlargement appearing to one side of the penis. It is usually soft, fluctuating, and reducible. The ring can be palpated in most cases. Occasionally some difficulty in diagnosis 36 
might be met with when adhesions are present. Explorative opcration would be recommended. Scrotal hernia is recognizerl as an elongated enlargement in the scrotum. Palpation will usually revcal the dilated canal and the contents can be easily returned to the abdominal cavity. Holding the animal up by the hind limbs often effects replacement. Differentiation should be made from other scrotal enlargements. As a rule no particular difficulty will be encountered.

Treatment.-In inguinal hernia the operation would be practically the same as in the female. The same care should bc exercised to avoid complications.

Scrotal hernia may be operated in two ways: (a) Inguinal operation, and $(b)$ scrotal opcration.

(a) Inguinal Operation.-Prepare the log the same as in the female. Make an incision through the skin over the inguinal canal down upon the internal ring. Open the processus vaginalis and pull the bowel or omentum back into the abdominal cavity. Suture the internal ring but allow sufficient room for the spermatic cord and vessels. A certain amount of swelling will take place after the operation which should be considered when applying the sutures. This opcration is especially advised when the male is to be kept in the sturl.

(b) Srrotal Operation.-This operation is performed by opening the scrotum, removing the testicle, returning the omentum or bowel to the abdominal cavity, and suturing the extcrnal ring. Retaining sutures should be placed in the serotum for a few days to a void prolapsus of the bowel in case the other sutures tear out.

Femoral Hernia. - This form of hernia is not common in small animals. It has been observer in a few instances. It ronsists in a portion of the viscera, in most cases intestine with peritoncum, which passes through the femoral canal in (llosc proximity to the femoral vessels.

Symptoms. - A soft enlargement is found on the inncr part of the thigh which interferes with bringing the limb forward prorlucing lameness. Inless strangulated there are no inflammatory symptoms. Any enlargement in this location should be suspecterl of bcing a femoral hernia. 
Treatment. - It is possible to reduce femoral hernia by the usual hernia operation but care should be taken to avoid the large vessels in the immediate vicinity.

Diaphragmatic Hernia.-Definition. - A hernia taking place through the diaphragm. It may be either congenital or acquired. This hernia is characterized by the passage of a portion of the abdominal viscera into the thoracic cavity either with or without the peritoneum.

Etiology.-Most cases of acquired diaphragmatic hernia oc'ur as a result of violence in which the cliaphragm ruptures allowing abdominal viscera to pass through the rent. Strangulation is rare.

Symptoms. - No symptoms are observed in most cases of congenital diaphragmatic hernia. In the acquired form the simptoms come on suddenly and consist of severe dyspnea, restlessness, pain, etc. 'The patient as a rule does not live but a short time. A few cases have been observed where the patients lived for several months showing dyspnea and marked circulatory disturbance. A diagnosis is in most cases difficult.

Treatment. - No treatment cau be given.

Perineal Hernia. - Definition. - A hernia appearing in both males and females and characterized by an enlargement on one or both sides of the anus.

Etiology.-The perincal tissue is easily ruptured or torn allowing some of the abdominal organs or omentum to protrucle at the sides of the rectum. Perineal hernia is often observerl in trick (logs which are required to walk a great dral on their hind legs. 'The mnatural position and the presiure of the abdominal organs no doubt lead to rupture of the perineal tissuc. It may also result from strain from (op)rostasis, prostatic enlargement, ete. Strangulation is rare in this form of hernia. In some aases torsion of the blakkler accompanies it.

Symptoms.--This hernia is recognized as an enlargement appearing at one or both sides of the rectum. The majority' of eases are unilateral. The anus is pushed to one side and often there is difficulty in defecation. In the female the colargenent is usually slightly lower than in the male. 
The consisteney of the hernia will depend upon the contents. Perineal hernias are usually soft, easily reduced and the hernial ring readily palpated. Holding the patient up by the hind limbs often effects reduction. Should the bladder be in the hernial sac the patient will show dysuria and the enlargement feel cystic. A positive diagnosis can be made by puncturing the swelling with a trocar and obtaining some of the contents. A differential diagnosis is necessary between the hernia and enlargement of the anal pouches. A careful examination will reveal the difference between them.

Treatment. $-\mathrm{A}$ careful consideration of the symptoms should be taken into account in order to determine definitely the conditions so that proper treatment can be applied. If the bladder is in the hernial sac replacement should be brought about as soon as possible. This may be done by carefully manipulating the parts, or if this fails open the hernial sac, empty the bladder with a fine trocar and push it back into the abdominal cavity. Insert the sutures rather deep in the tissues in order to effect deep adhesions.

In the female, if the uterus is in the hernial sac, ventrofixation is recommended. Many eases, when of ordinary size and no particular disturbance present, should not be treated. Castration is recommended when enlarged prostates are present. In some cases it may be necessary to remove the glands.

Other hernias have been observed but they are so rare that no attempt will be made to describe them. 


\title{
PART XIV. \\ INFE(TIOL'S DISEASES.
}

\author{
CHAPTER I. \\ AC'I'TE GENERAL INFECTIOLS DISEASES.
}

\section{DISTEMPER OF DOGS.}

Definition.-An acute, infectious, communicable disease which in most cases affects young dogs.

Occurrence.-This is one of the most common diseases affecting dogs, and is known in every country where dogs are found. It is particularly a disease of young animals, the majority of dogs contracting it at some time during the first year of their lives. Whole litters of puppies, or all the animals in a kennel may become affected at one time. It occurs in districts as an enzoötic. In cities it is more prevalent than in the open country. The season of the year has some influence on its prevalency and distribution, the fall and winter months being most productive of the disease. Highly bred animals are more commonly affected than those bred by natural selection.

Etiology. - There seems to be considerable difference of opinion in regard to the exact etiology of dog distemper. fome claim it to be produced by a specific microörganism (Bronchosepticus canis, Torrey, Ferry, McGowan), while others think it is due to a filtrable virus. In each case evidence has been produced which seems to substantiate the claim made. 'T'wo distinctive factors are recognized from a clinical standpoint: (a) A primary infectious agent which 
produces the nuaked initial symptoms of the discase. (b) secondary organisms which produce many varied and serious complicating conditions. A large number of organisms have been isolated from animals affected with distemper, but so far they have proved to be simply secondary invaders.

Natural Infection. - Natural infection takes place in several ways. It may be either direct or indirect, the animals coming into immediate contact with each other, ol through intermediary agents, the virus being taken into the digestive tract with the food or drink. 'There are a number of factors which tend to favor the development of the rirus, such as influence of any kind which reduces the general resistance of the animal, in the way of poor food, insufficient food, colds, ete., or various diseased conditions interfering with the assimilation of food. Puppies with wak constitutions are especially susceptible. The development of the clisease is ordinarily in animals from three months to one vear of age. Older animals are very seldom affected and if so take the disease in a mild form. This may be explained by the immunity the dog possesses or by its having had the disease in a mild form. House dogs which have been pampered and petted, or those of the finer breeds are morc susceptible, and usually take distemper in a more severe form.

It has been determined quite conclusively that the specific virus produces an acute or peracute condition with a high temperature followed in a few days by secondary changes due to other bacteria resulting in various complications, such as oecur in the skin (pustules), respiratory passages, digestive tract, nervous system, ete.

Necropsy. - Owing to the variety of forms of distemper in dogs the lesions fomnd on examination are of many kinds, varying with the complieations due to secondary infection. In peracute and acute eases there will be effusions of fluid from the serous membranes particularly in the pericardial sac, the thoracic and abdominal eavities. Small hemorrhages are observed in some of the organs (hcart, liver and kirlneys). In most cases of distemper the lungs will be afferted, either as a capillary bronchitis, congestion or 
bronchopneumonia. The pleura is usually eongested and sometimes covered with fibrinous deposits.

The intestinal tract shows marked ehanges, in most cases a catarrhal inflammation, which may be primarily in the stomach, or involving the entire tract. The glands in the mucosa are swollen. Erosions and ulcerations are frequently noted in the subacute or chronic forms. The lymph glands of the mesentery are enlarged and edematous. The liver is usually congested, or inflamed, frequently showing degenerative changes. The kidney's are enlarged, the capsule easily removed and the eortex markedly changed.

The central nervous system is often involved and there will be found eongestion of the membranes and the cortex of the brain. Small hemorrhages will be observed in some cases. Other pathological changes of a minor eharacter are noted, such as conjunctivitis, keratitis and more rarely panophthalmitis. Pustules in the skin are eommon.

Symptoms. - There is quite a variation in the symptomatology depending upon the form whieh the disease takes. 'The most prominent manifestations are those of an infectious catarrh, involving the membranes of the eye, the respiratory and digestive systems. The eatarrhal symptoms are often complicated with those of severe disturbance of the brain and cord, pustular eruptions on the skin, and very frequently bronchopneumonia. For elearness in the description of the stmptoms it is best to consider them under the following headings, depending upon the part affected:

1. Initial Symptoms. - The period of incubation is usually from three to five days. 'This period will vary considerably, depending upon various faetors. Some few cases have been known to develop the disease in two to three days, while in others it required two to three weeks. The peracute type of distemper is ushered in by marked constitutional disturbances, such as great depression, fatigue, total loss of appetite, a very high temperature $\left(106^{\circ}-107^{\circ} \mathrm{F}\right.$.) whieh in the course of several hours drops to normal, and later to subnormal. 'This form of the disease takes a very rapid course, the animal soon passing into a comatose state. 
The acutc form usually begins with a rather high temperature $\left(103^{\circ}-106^{\circ} \mathrm{F}\right.$.) and remains elevated for several days or weeks. Some cases show a marked variation in temperature, beginning with a high temperature which in a few days drops to normal or in some cases subnormal where it remains until either reeovery or death takes placc. In some cases due to complications the fever is remittent. The owner will notice in the inception of the disease a change in the disposition of the animal. Instead of being lively it will be stupid, does not answer the call or obey commands given it. 'The hair coat becomes rough, quickly loses its gloss and the animal in general shows a dejected appearance. On examination of the nosc, it will be found hot and dry; the mucosa becoming irritated induces rather violent sneezing. After one or two days more pronounced symptoms make their appearance.

2. Symptoms Shoun by the Eyes. - In a large number of cases conjunetivitis is a prominent symptom. In the early stages it begins as a serous conjunctivitis, which soon becomes purulent from secondary infection. 'The diseharge from the eycs consists of a thick mucus, or whitish or yellowisl pus. 'This diseharge is usually found collecting chiefly at the inner canthus of the eye, soiling the edges of the eyelids, or from the exudate drying, crusts form causing the eyelids to adhere. Usually in a few days lesions and ulcers appear on thc cornea as a result of the irritant exudate, the patient rubbing its eves or pawing them with the forefeet, or by the swelling which interferes with local nutrition. These ulcers are usually small, pin-point, funnel-shaped and extend downward in a straight direction; their base usually covered with a pus-like material. 'They heal by a proliferation of ressels from the edges of the cornea. In some cases the ulceration leads to partial perforation of the cornea, with a protrusion of the Descemet's membranc, prolapsus of the iris (staphyloma). The resulting cicatrization causes permanent white spots on the cornea (leukoma). In other cases the entire cornea becomes opaque (parenehymatous keratitis) which gives the eyes the appearance of ground glass. Rarely does the entire eyeball become inflamed 
(panophthalmitis). Opacity of the cornea in a number of cases remains for wceks and cren months.

3. Symptoms Shoun by the Digestive Tract.-Vomiting is an early symptom in a large number of cases. The vomitus often consists of particles of food, frothy mucus stained with bile in cases where the romiting is persistent. The mucous membrane of the mouth is hot,.dry and congested. The animal drinks freely indicating the involvement of the mucosa of the stomach and bowels. Constipation is invariably the rule during the early stages of the disease, followed by diarrhea in the course of a day or so. The feces in the latter case are very fetid, often shiny, frothy and even bloody indicating hemorrhagic intestinal catarrh. The intensity of the gastro-intestinal symptoms varies from mild catarrh to a severe hemorrhagic gastro-enteritis. Symptoms of icterus are prescnt in some cases due to the catarrhal duodenitis (catarrhal icterus).

4. Symptoms in the Respiratory Tract.-During the early stages of the disease the nose becomes dry and hot. There is nasal discharge which is at first serous but later thicker and mucopurulcnt in character. During the early stages there is much sncezing and sniffling due to the attending rhinitis. The dog shows much distress and makes frequent attcmpts to clear the nasal passages by sudden expiratory cfforts, rubbing the nose against objects and clawing at the llasal openings with the forepaws. The discharge accumulates around the nasal openings in the form of crusts; when these are removed the skin and mucosa often show excoriations. In severe cascs the discharge is often so copious that the nostrils will be found partially or completely occluded.

Catarrh of the larynx is nearly always associated with this condition and is manifested by a cough, which usually occurs in paroxysms; it is at first dry and harsh, and later becomes moist and considerable mucus is coughed up, which is immediately swallowed by the animal. 'These paroxysms of coughing frequently end in gagging and vomiting. The (atarrhal inflamination spreads quite rapidly to the trachea and bronchi. 'Tlie resulting bronchitis causes a cough, which 
is deep and explosive. The respirations are accelerated. Auscultation reveals coarse, sharp vesicular murmurs, and rhonchi. As the inflammation proceeds it involves the bronchioli (capillary bronchitis) which produces increased, labored respirations. At this stage there is usually a feeble harassing cough, most noticeable when the dog is made to move, or if the thorax is manipulated. 'The auscultatory sounds become increased, and there will be noted dry or moist, crackling, or fine rales. In very weak, or young dogs, in which the exudation accumulates in the bronchioli, it is very sure to produce a bronchopneumonia on account of the secretions being drawn into the alveoli. The resulting pneumonia will be recognized by: (a) The great increase in the temperature; $(b)$ the excessive dyspnea (inflation of cheeks at each expiration); (c) the sitting posture of animals with elbows spread apart to facilitate respiration; $(d)$ irregular vesicular murmur; $(e)$ irregular dulness and tympany on percussion; $(f)$ the bronchial breathing which will be audible. The cough at this stage becomes very dull and weak. 'The nasal discharge becomes very fetid, and often has a greenish cast. I) uring the last stages as the heart becomes weak, symptoms of edema of the lungs appear in the form of serere dyspnea, and bubbling rhonchi.

5. Syuptoms Shown by the Nervous System.-This disease in practically all eases is accompanied by some nervous phenomena. It often begins, especially in weak individuals, witl marked dulness and depression. These symptoms una be all that the animal will show. However, in a great many cases, the nervous simptoms develop early in the course of the disease, and are manifested by excitement, restlessness, relping, cries, even simulating some of the s.rmptoms of furous rabies. In many instances tremors of muscles, tonic and clonic spasms, which may involve certain groups of muscles or the entire muscular system are observerl. I,ocal spasms and twitchings of the muscles are frequently observed involving the muscles of the face, ()ver the region of the liearl and neck. The masseter muscles are frequently afferterl, resulting in chattering teeth and foaming at the mouth. Sipasms of the muscles of the neck 
and limbs cause regular or irregular movement of the head or limbs. 'These movements may be present constantly or periolically. Convulsive contractions of certain groups of muscles are frequently observed, in which the animal at first becomes restless, excited, runs aimlessly about, is suddenly seized by tonic and clonic spasms, the head and ne'ck usually drawn backward, falls down as if from epilepsy, barks, cries and becomes unconscious with relaxation of the sphincter muscles. In some cases the animal will die during these convulsions; in others there is a gradual return to consciousness and in one or two minutes the animal is able to rise. Some cases pass into a long-continued state of coma. These convulsive seizures may become less frequent, and entirely disappear; or they may terminate in partial or complete paralysis. Paralysis is a very common sequel to this disease. It very rarely begins at the onset of the disease, but appears in most cases during the latter period. The paralysis may be confined to certain groups of muscles, in fact the posterior limbs are most of ten affected. The paralisis of the sphincter muscles is a very frequent serguel. Some cases begin with a paralysis of the posterior limbs, and result subsequently in complete recovery, while others show a progressive ascending paralysis, resulting in death from general paralysis. Various paralytic compli(ations are often observed following the nervous form of distemper, such as deafness, amaurosis, hemiplegia laryngis, aphasia, loss of sense of smell, hrodrocephalus, etc.

i. Symptoms shown by the Shin. - In a large number of (ase's there will be noted a characteristic pustular exanthema. sinall, red spots appear along on the abdomen, on the inner surface of the thighs, occasionally on the skin of the face, aromind the eyes and on the internal surface of the external (ar. In twenty-four to thirty-six hours these red spots are transformed into miliary nodules, each surrounded by a rert ring. 'These nodules change very rapidly into vesicles which become infected to form pustules. The pustules vary but are usually the size of a pea. They stry up either into a yellow, brown crust, or rupture and leave reddened, moist places's to become tovered later by a scab. When the 
pustular eruption becomes extensive the body gives off a peculiar, offensive, sweetish odor. Healing of the pustules takes place usually in about six to eight days, leaving bright pigmented, reddish spots, which remain for several weeks. In some eases the pustular form of distemper may spread, the pustules become eonfluent, forming a scabby eczema, which may be localized around the eyes, over the abdomen, or thighs, or it may be general over the entire body. Complications, such as septicemia and septicopyemia develop occasionally from this form of the disease. When the pustular eruption forms around the margin of the lips, it often spreads rapidly to the mucosa producing a severe gangrenous stomatitis. Involvement of the external ear in the form of an otitis is observed in a large number of cases. 'This is characterized by a thin flud secretion, which accumulates on the surface of the ear, dries and forms a vellowish-brown crust or scales.

7. General. Symptoms.-The initial high temperature is usually followed by a remittent or subnormal temperature. Some cases do not show much abnormality in temperature. Normal temperature in this disease, when other marked simptoms are present, does not always inclicate a favorable termination. As the disease progresses the animal becomes emaciated, weak, and the action of the heart is more or less disturberl, depending upon the severity of the ease. 'The mucous membrames are pale, the patient becomes very weak, staggers or retains a recumbent position.

Diagnosis. - During the early stages of distemper it presents some difficulty. It becomes necessary to decide whether we have distemper to deal with, or the beginning of some other condition, such as catarrhal inflammation of the eres, nose, lungs, stomach or bowels. An accurate diagnosis cannot always be made during the early stages of the discase. Just as soon, however, as there appear symptoms of general depression, high temperature, the implication of sereral organs, especially in a young dog, distemper shoull be susperoted. Should skin pustules be present they will assist materially in making the diagnosis.

In catarrhal inflammation of the bowels there is usually 
very little rise in temperature, and catarrhal symptoms of the eyes, nose, ctc., fail. The symptoms of cerebral irritation sometimes found in distemper make it necessary to differentiate it from rabies. The characteristic aggressive tendency of animals with rabies, the change in the voice, the facial expression, and the absence of eatarrhal symptoms usually make the differentiation easy. The eruption on the skin might be confused with mange, but negative results on microscopic examination would at once show the difference. There should be but little difficulty experienced in differcutiating it from eczema as the lesions and their location differ and the other symptoms of distemper usually present. 'The convulsions oceurring in the nervous form of distemper are distinguished from those of epilepsy by the acute character, oecurring at frequent intervals, and the local muscular twitchings, which occur even between the convulsive attacks.

Prognosis. - The prognosis is not favorable, even in the milder forms of the disease. Owing to the variety of forms the disease assumes, and the frequent complications, the mortality is high, amounting to approximately 50 per cent. 'The prognosis depends to a certain extent upon the type of the disease, and whether or not the animal is strong and vigorous. 'The purely exanthematous form is most favorable. If the disease confines itself to this form the majority of the cases will completely recover in two to three weeks.

The catarrhal forms, involving the respiratory or digestive systems, are less favorable owing to serious complications which attend them.

'The highest mortality occurs when the nervous system is involved. When severe nervous phenomena are present the prognosis is bad, only is to 10 per eent. recovering. Where there is a continuous, high temperature the prog- nosis is not favorable as there is danger of lieart complications. A rapid fall in temperature to below normal is also a grave sign; in most cases it indicates the approach of death.

Treatment.-Dietetic- It is very essential in distemper to supply the patient with easily digesterl, nourishing food. Perhaps the best food is raw meat chopped fine, or scraped 
into a pulp. Patients will be tempted by this food when they will not eat anything else. In cases where the appetite is lost, and in order to maintain the animal's strength, beef brotll is most excellent. This may be given with warm. milk, or milk with an egg beaten up in it. Other foodstuff's may be given, depending upon the progress of the case, and the needs of the patient. In the digestive form of the disease only small amounts of liquid foods should be given via the mouth. Rectal fecding with warm milk and meat broth is recommenderl in these cases.

II ygienic. - The animal must be well protected from exposure to extremes in temperature, or cold draughts of air. They should be placed in a clean, morlerately warm wellventilated room. The berlding should be kept clean at all times. Supply plenty of fresh water.

Medical. - Owing to the various complications the medical treatment must be essentially sumptomatic. During the carly stages of the disease calomal $\left(0.075^{-0.10)}\right.$ is recommended as a purgative and bowel disinfeetant. This dose may be repeated in twenty-four hours if necessary. 'To overeome the general depression, which is an early symptom, nerve stimulants, alcohol, aromatic spirits ammonia, nux vomica tincture $(0.4-0.7)$, anyone of which should be given well diluted, and repeated as the case demands. As an abortive treatment subcutaneous injections of, trichlorid of iodin (.3 or 4 times daily, 2-4 c.c. of a solution of 1-1000) have been used with very good results. This treatment when given early in the course of the disease has a very beneficial effect in regulating the temperature. The patients seem brighter, and the catarrhal symptoms diminished. The injections should be made at different points owing to the danger of skin necrosis. Further medical treatment will vary depending upon the part affected.

(a) When the disease involves the eyes, they should be washed with an antiseptic once or twice daily, boric acid (2 per cent.), creolin (1.5 per cent.), to prevent undue injury to the cornea and other structures from the infection. In obstinate cases with excessive pus formation and dis(charge, silver nitrate solution ( 0.5 to 1 per cent.) has been found very efficacious. When there is extensive parenchy- 
matoms keratitis solution of stovain (1 to 2 per cent.) is usceful to prevent irritation and subsequent injury to the cyes from rubbing them against objects, or with the paws. ()pacities of the cornea when of some standing may be treated with a silver nitrate solution (2 per cent.), followed hy a normal salt solution as a wash, or suceus cineraria naratima compositus $(0.4-0.7)$ dropped into the eye laily. This latter preparation has provel to be of great value in the eye complications of clistemper.

(b) The respiratory sistem should be treated by removing the secretions from the nasal openings, spraying the nasal passages with (reolin (2 per cent.), or silver nitrate solution (0.25 per cent.). Warm vapors, such as steam, or solutions containing alkalies (bicarbonate of sorlium) given as inhalations are highly recommended. When there is a painful, dry, harsh cough the following formula is very heneficial:

R-Morphinii sulphatis

0.15

Aqua amygdala amare

12.00

Acqua dest.

150.00

Misere et fiat solutio.

Sig. - Give teasponnful once or twice daily.

In the presence of profuse secretions, sedatives are eontraindicated. Instearl ammonium chlorid $(0.1-0.5)$ is given as an expectorant twice daily to dissolve the inucus and other secretions. If symptoms of pneumonia are present, counterirritation to the thoracic walls with oil of mustard and glicerin $(1-20)$. Heart stimulants are also arbiserl. (See 'Treatment of Bronchopneumonia.)

(c) The digestive complieations are treated according to the conditions found. If the animal is constipated it should be relieverl by the arlministration of a purgative; if severe diarrhea is present the irritating bowel contents are first remover by a laxative followed by astringents and sedatives. The following formula will be found beneficial in the latter ('onclition:

F-Tincturæ opii

Aquæ foeniculi

Misere et fiat solutio.

Sig.-Give teaspoonful two or three times daily. 
Is a bowel disinfeetant use small doses of salol $(0.2-0.5)$ twice daily. If vomiting is persistent it must be controlled with eold water containing soda ( 2 per cent.), salicylate of bismuth (0.3), or in extreme eases by small doses of opium $(0.05-0.15)$.

(d) For the stimulation of the digestion small doses of hydroehloric aeid $(0.1-0.5)$ diluted in water and pepsin $(0.075-0.5)$ is the best treatment. It is advisable to stimulate the mucosa further with tineture of gentian $(0.05-0.9)$, or tineture of nux vomica $(0.4-0.7)$ given once daily. Fluid cxtract of echinacex $(0.5-1.0)$ is also used.

(e) When nervous symptoms are present, it is important that they be controlled by anodynes and sedatives (morphin $0.03-0.09$, or sodium or potassium bromid 1-150 in water) given in teaspoonful doses four to six times daily. Some cases, when spasms and eonvulsions are severe, will require maximum doses to control them. When paralysis is present, electrieity is highly recommended, also strychnin (0.001) once or twice daily. Subcutaneous injeetions of veratrin $(0.01-0.05)$ are sometimes used.

(f) The temperature in this disease is rarely treated, and only in exceptional cases where a very high temperature remains for several days and threatens to become dangerous to the heart, would it bc advisable to use antipyreties. Phenacetin, acetanilid $(0.25-0.50)$ may be used for this purposc.

(g) In the skin form, when pustules are present, they should be opened, and washed with creolin (2 per cent.). For the exanthema the skin should be washed with an antiseptic soap (germicidal, or tar soap), dried, and a llying powder or zinc oxid ointment applied. 'The latter is preferable.

Sera and I'accines. - This disease has been treatcd quite cxtensively during the past two or thrce years with vaccines and sera. Good results have been obtained by their use by some, others have reported less favorably. 'The great advantage no doubt in the use of thesc preparations is to control the secondary infection which produces the complicating conditions. Varying degrees of immunity have been claimed by the use of vaccines. 
Prevention.-Animals with distemper should be isolated and kept from healthy young dogs. Thorough disinfection of all utensils, bedding, rooms, etc., should be attended to promptly.

Preventive inoculation has proved of value in a large number of cases and is highly recommended.

\section{DISTEMPER OF CATS.}

Definition.-An acute contagio-infectious disease found more commonly in young cats. Older animals are less often affected, probably due to either an acquired immunity (having harl the disease in a mild form) or to a greater natural resistance against the infection. The disease in cats is not nearly so common as in dogs.

Etiology.-According to the best information obtainable it is produced by the same causes as canine distemper.

Pathology.-'The pathological lesions are principally those found in canine distemper, except that the disease seems to be confined mainly to the respiratory and digestive tracts. In the respiratory form there is an inflammation involving the nasal passages (rhinitis), larynx (laryngitis), the bronchi and bronchioli (capillary bronchitis), and the lungs (bronchopneumonia). 'The digestive tract shows ratarhal inflammation. 'The mucosa is covered with a heavy mucous exulate which in some cases is mixed with blood (hemorrhagic catarrhal gastro-enteritis). Some of the other organs (kidneys, liver and heart) show degenerative clianges.

Symptoms.--The initial symptoms arc those of inappetence, rlcpression, vomiting in some cases and moderately higl, temperature $\left(103^{\circ}-104^{\circ} \mathrm{F}\right.$.). When the respiratory passages are primarily affected the animal will sneeze frequently, there is a clischarge from the nose which is at first thin, but later becomes thicker and glutinous. This will collect around the nasal openings and in some cases occlucle them completely.

Conjunctivitis is a very prominent symptom. At first the discharge is serous, but later it becomes mucopurulent. 
The cornea often presents the characteristic changes noted in dog distemper. The bowel form is observed frequently, and is characterized by vomiting, abdominal pain and later severe diarrhea. The animal becomes emaciated, there is marked weakness and a fall in temperature, which often becomes subnormal. Convulsions and other nervous phenomena are not commonly observed.

Diagnosis. - The diagnosis depends upon the existence of catarrhal symptoms involving the eyes and nose, the temperature and the rapid emaciation and weakness. A differential diagnosis should be made from hemorrhagic septicemia. In this disease no catarrhal symptoms are present, as a rule, but there is marked gastro-enteritis, and the course is peracute. The mortality in hemorrhagic septicemia is much higher.

Prognosis. - The prognosis is bad. The mortality, however, is lower in cats than in dogs. The catarrhal form involving the eyes, nasal passages and lungs is more favorable than the bowel form.

Treatment.-IIygienic.-The animal should be kept in a moderately warm place, free from draughts of air. The room should be well ventilated, and every precaution taken to prevent unnecessary exposure.

Dietetic.-The appetite can be tempted by offering small amounts of fresh lean meat, or liver at frequent intervals. Milk should be allowed in small quantities: In case the animal refuses food, it is advisable to give small quantities of beef broth, or warm milk to maintain strength.

Medical. - A laxative is given at the onset of the disease (castor oil 4.0-12.0, or sulphur in milk 1.0-2.0). The eyes sliould be cleansed daily with boric acid solution (2 per (cent.) to fontrol the blennorrhea. The nasal passages can be cleansed with a similar antiseptic solution. Small doses of opium (tincture of opium 0.10-0.15) are often beneficial in preventing the spread of the inflammation in the stomach and bowels and to control the diarrhea. Sulphocarbolate tablets are to be recommended as a bowel disinfectant $(0.1-0.2)$. When pneumonia threatens inhalations of warm medicated solutions are valuable. Atropin sulphate (0.003) 
is useful to sustain the heart action. Good care and nourishing food plays a very important role in the treatinent of cat distemper.

\section{TYPHUS OF DOGS.}

\section{C'anine Typhus. Ilemorrhagic G'astro-entertis.}

Dog Plague. Black Tongne.

Definition.-An acute, infectious disease occurring in an (pizoötic form, characterized by a severe gastro-enteritis, stomatitis, and in some cases severe nervous simptoms.

Occurrence.-I) uring the past few years this risease has appeared as an cpizoötic in various sections of the Inited States, producing extensive losses, particularly in old dogs. In sone districts it has made the breeding and handling of dogs prohibitive. The disease has been disseminated by dog shows, and follows in the wake of such exhibitions.

Etiology.- The nature of the disease indicates that it is produced by some specific infection. Ip to the present time the infections agent has not been isolated. Experimental inoculations with various organisms, which have been isolated, have not proved that any of them are constant in reproducing the disease in healthy animals. In a number of outbreaks in this country, old dogs were as commonly affeeted as young. Further, dogs which have had distemper severely come down with this disease in a severe form. 'The breed, or sex of the animal seems to have nothing to do with its susceptibility. The infectious agent is probably taken in with the food or drink. The disease is spread by colabitation, or by the infection being carried he intermediate agents. The period of incubation is from three to five days.

Pathology.- 'The autopsy in this discase presents a rather (onstant picture. 'The digestive tract is mainly involved. Tlec mucosa of the mouth is often inflaned; ulcers are fomel in a number of cases, particularly moticeable along the malrgin of genms. In some rases extensive necrosis of the huccal mucosa is fomul. 'The tongue is frequently thick- 
ened, swollen and dark red or bluish in color. In the stomach the lesions are very prominent, and consist of an intense inflammatory condition. The mucosa is dark red, or almost black, corrugated and at the apex of the corrugations the membranc is eroded.

Hemorrhages take place in the membrane and give it a more or less mottled appcarance. Some cases present extensive hemorrhages over the entire membrane. The surface of the mucosa is covered by a mucus of a dark brown, or chocolate color, which can be readily scraped off. 'The contents of the stomach have a peculiar offensivc orlor. The intestines show similar changes, but in most cases not. so extensive. The cecum and small intestines show the most prominent lesions. The peritoneum covering the bowels is congested and the mesenteric lymph glands are enlarged. 'The spleen is often much enlarged and shows evidence of acute inflammation. The liver and some of the other organs are hyperemic. The lungs in most cases do not show much on examination. They are usually slightly congested, and edematous. The heart is pale in color and very friable. The brain and its membranes mav he inarkedly hyperemic, or show but slight change. 'The lesions found on autopsy are indicative of a more or less intense gastro-enteritis.

Symptoms. - The initial symptom, which is quite constant, is a very persistent vomiting. The vomitus at first consists of food particles; later mostly mucus mixed with blood and bile. The attacks of vomiting often begin without any previous indication of illness, and suddenly become very severc, producing in a short time marked weakness and prostration. The appetite is lost; there is rapid cxhaustion and staggering gait. The progress of the disease is quite rapid. Frequently in one to three days it has reached its characteristic form. Examination of the mouth reveals morc or less extensive lesions of the mucous membrane. Along the margin of the gums will be noticed degencrative or necrotic changes, ulccrs and at the margins of the lips there will be present the charactcristic chocolate-colored discharge. The tongue will be affected; it is sometimes partially or 
completely paralyzed, swollen and of darkbluish color. A very unpleasant, peculiar odor is emitted from the mouth. As the disease progresses, the degenerative and necrotic processes eontinue until in many cases the ulcers become confluent, forming cxtensive ulcerative surfaces. The tonguc usually in the later stages is dry, chapped, loses its scnsitiveness, and becomes necrotic or gangrenous. Necrosis of the lip is observed in some cases, usually most prominent at the commissures of the mouth. Palpation over the region of the stomach, which often induces vomiting, produces marked pain. Manipulation of the bowels is also painful in most cases. Constipation is present during the early period of the disease to be followed later by diarrhea. The discharges from the bowels are mixed or streaked with blood, and emit a very offensive odor. The mucous membranc of the rectum is inflamed and shows degenerative changes. The conjunctiva shows similar discoloration to that of the mouth (brownish-red), strongly injected, but the absence of a purulent discharge. The tempcrature does not rise, as a rulc, at any stage of the disease. With the appearance of depression and coma the temperature falls rapidly to subnormal, the rule in this disease. Therc is no cough and in most cases no indication of lung involvement until the later stages of the diseasc, when in some cases acute edema, or a foreign body pneumonia producing dyspnea. develops. The urinary secretion is nearly always diminished or suppressed. Nervous simptoms of cxcitement and convulsions are occasionally observed, but in most cases somnolenec, lassitude and coma are characteristic. Modification of these symptoms may be found, depending upon the screrity of the eondition.

Diagnosis.--There are some very characteristie symptons which materially assist in establishing a diagnosisthe sudden and persistent vomiting, the inappetence, the presence of ulcerations on the mucous membranes of the moutl, the great depression, the character of the vomitus, the characteristic injection of the conjunctiva, and the normal or subnormal temperaturc. A differential diagnosis must be made from canine distemper. In canine distemper 
there are invariably present the characteristic catarhal symptoms involving the eves and nasal passages. The period of incubation is usually longer in distemper, five to nine days. Further, distemper is more common in young animals, and the course of the disease is acute. This disease must also be differentiated from ptomain and other poisonings. In some cases this is quite difficult, owing to the close similarity of the symptoms, gastro-enteritis appearing in both conditions. The necrosis of the membranes and ulcers are absent in ptomain poisoning, and in the majority of mineral poisonings. The development of the disease is different from ptomain, or other poisonings. Canine typhus usually appears as an epizoötic which would assist in establishing a rliagnosis. Differentiation between this disease and ulcerative stomatitis should present no great difficulties. The absence of the general depression, romiting and gastroenteritis in ulcerative stomatitis is indicative. Scorbutus develops slowly, and is attended by neither romiting nor general simptoms.

Course. - The average duration of the lisease is four to six days; in milder cases often twelve to fourteen rlays. A few cases run a much more rapirl course followed by death in one to three days. At the beginning of an outbreak it seems to run a more rapid course than later.

Prognosis. - The prognosis is unfarorable; the mortality from 50 to 75 per cent. Young animals suffer less severely than older ones. When the disease develops gradually, indicating low virulence of the infection, or high resistance on the part of the animal, the prognosis is more favorable; on the other hand when the development of the disease is rapirl and the symptoms prominent, the outlook is grave. In some cases recovery takes place quite rapidly, and when this does oceur there is usually no complication, and even the ulcerative processes chisappear completely.

Treatment.-Dietetic.-During the early stages of the disease, no food should be given. After the acute simptoms have begun to subside and the animal reaches the convaleseent stage, nutritious foor can be allowed. Meat broth would be most applieable as the sensitive mucous 
membrane of the stomach would not retain solid food. later small amounts of meat chopped fine could be given. should it be impossible for the animal to retain food in the stomach, rectal feeding is advisable, using concentrated ineat broth warmed to the body temperature. This should be injected high up in the rectum, small amounts given every three or four hours.

Medical.-At the onset of the disease, when romiting is severe and persistent, it would be best to wash the stomach with solium bicarbonate solution (2 per cent.). This may ho repeated in ten to twelve hours. To allay vomiting, serlatives (morphin subcutaneously, 0.016-0.12), or small amounts of cold water at frequent intervals are useful. Hot packs applied to the epigastrium are beneficial. Rectal injections of warm water, or even washing the entire alimentary tract with sodium bicarbonate solution (2 per cent.) when liarrhea is present, will remove irritating material and infection. In obstipation, warm rectal infusions may he used in preference to laxatives or purgatives. Ichthargan has been highly recommended for typhus in the following formula:

R--Ichthargan,

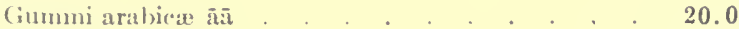

Acquie chloroformi . . . . . . . . . 60.0

Misce et fiat mist.

Sig.- Give teaspoonful every three or four hours.

Simulants (atropin sulphate, 0.075-0.15 subcutaneously) to sustaiu the heart action and to overcome the general depression would be advised. 'The mucous membrane of the mouth should be washed at least twice daily with boric acid solution (2 per cent.); potassium permanganate (0.25 per cent.), or therapogen (2 per cent.). 'The animal should be well protected in a quiet place. In a great many cases the treatment has proved unsatisfactory owing to the peracute course it of ten assumes.

\section{PURPURA HEMORRHAGICA OF DOGS.}

'This disease has been observed in logs, but as it is very rare it will not be rlescribed here. (See other works.) 
FOWL CHOLERA.

Cholera Gallinarum. Pasteurellosis Avium. Fowl
Typhoid.

Definition. - This is an acute contagious disease of fowls, usually occurring in an epizoötic form, and affecting particularly chickens, geese, pigeons, ducks, quail, sparrows and pheasants. It is characterized by a general infection, and the prominent symptom of diarrhea.

Occurrence.-Fowl cholera is found in most sections of the Cnited States, causing extensive losses to poultrymen. The finer breeds of fowls seem to be more susceptible to infection, probably because of exposure to the disease at poultry shows and other public exhibitions. It occurs as an epizoötic, sometimes destroying entire flocks. The losses are serious from an economic standpoint. The monetary loss, which is great when a large number of birds die; the loss in egg production, and the interference with breeding operations make fowl cholera a formidable disease. The disease often spreads over a large area, producing enormous losses.

Etiology. - It is produced by the Bacillus avisepticus (bipolaris). This bacillus is one of the smallest bipolar organisms.

Pathogenesis. - The transmission of the disease from one bird to another is very easy. Directly inoculating the blood from a sick fowl to a healthy one by scarifying the skin or membranes, produces the disease in practically every attempt. Subcutaneous and intramuscular inoculations bring similar results. Inoculations with the infected blood of any of the birds into other species will produce the disease. Instillation of the infection into the conjunctival sac of fowls produces the diseasc. Feeding infected meat to other fowls, or cultures of the organism will in the majority of cases result in the bird coming down with the disease. Other animals-guinea pigs, mice and rats-are susceptible to the disease by inoculation with 
the infected blood or witlı cultures of the organism. Some of the domesticated animals are susceptible to intravenous injections of highly virulent cultures of the organism. The disease is not produccl in dogs, cats or hogs from eating the infected meat of fowls. The virulence of the organism may be increased by repeatedly passing it through chickens, guinea pigs, pigeons or sparrows. The organism may be attenuated by subjecting it to sunlight, drying and exposure to the air. The attenuation may be sufficient to prevent further spread of the disease.

Natural Infection. - Natural infection may take place in numerous ways: (a) The droppings of fowls contaminate the feed and water given other fowls and the infection is thus introduced immediately into the digestive tract.

(b) In most cases the flock is infected through newly acquired cholera-sick birds, or birds returned from shows or exhibitions where they have been in contact with the infection.

(c) Pigeons and sparrows play an important part in the dissemination of cholera, by carrying the infection from flock to flock. 'This accounts for isolated outbreaks of the disease where no exchange of birds has taken place.

(d) The infection is frequently carried on clothing, shoes and other objects.

(e) It may he spread by carelessness in disinfecting cars in which cases have bcen transported. 'This has proved to be the source of infection in a number of instances.

$(f)$ Eggs have been the carriers of the infection in some few cases reported.

The infection in most cases is taken directly in the digestive tract with the food or drink. The bacilli penetrate the mucous membrane and enter the lymph spaces of the walls of the intestines. They are soon taken into the circulation where they multiply very rapidly. In some ases when the infection is introduced into wounds it is taken into the cireulation nore promptly, and consequently the course of the discase is more rapid. Death results in these cases no doubt from the large amount of toxins formed. 
Pathology. - The nost pronounced pathological ehanges "are found in the intestines, heart and lungs. In the acute form the following lesions are found:

(a) The intestines show marked pathological changes. The mueous meinbrane throughout the entire tract is highly congested and covered in numerous places with darker areas. 'The intestines contain a thin, watery, frothy, mucopurulent and yellow exudate often mixed with blood and bile giving them a dark greenish tinge. Dcfects in the inucosa are often present, the apex of the intestinal villi are often eroded, and in some cases croupous exudatc is present, in others ulceration. All of these changes in the mucosa may be observed in the same case. The mucous nembranes of the pharynx, crop, colon and rectum show the inost pronounced lesions. The mesenteric lymph glands are ncarly always enlarged, edematous and show numcrous petechise.

(b) 'The heart shows, as a rule, distinctive changes. It is covered with small, dark red, pin-point dots, and the bloorlvessels congested. The cpicardium is often covered by a fine fibrinous nembrane. The pericardial fluid is nearly always cloudy, containing some fibrinous flakes. In the peracute form of the disease thesc changes arc not so prominent.

(r) The lungs are found congested, thickened, dark red in color, very heary (hepatized); the milder type will show rongestion and edema. In the subacute form fibrinous pleuritis is frequently noted. The larynx, trachea and bronchi show congestion and redrlening (catarrh). Some of the other organs (spleen, liver, kidncys) show parenchymatous degoneration. In the subacute and chronie cases the lesions are caseous foci in the lungs, liver, on the mucosa of the intestine and occasionally on the epi- and endoardimm. Fibrinous pleuritis is frequent in the chronic form. 'The joints of the limbs arc often involved, showing exurlation and more or less marked arthritis. The mus('nlature in the chronic form will be grayish in color and flably. 
Symptoms. - The period of incubation raries somewhat, no doubt due to the morle and virulency of the infection. It is usually one to four dars; oecasionally longer, four to reren days. Fowl cholera is characterized by its sudden mset and the rapidity of its course. When it first appears in a flock, birds will drop dead apoplectically without showing symptoms. They may be found dead beneath the roost, or on the nest. Some of them when attempting to walk or fly will drop suddenly and die after a brief struggle. After the discase has been in a flock for a few day's, the less susreptible will show some marked symptoms. The birds lose their appetite, become weak and greatly depressed. 'The wings are usually pendent, the neek curved and the head pulled downward. The entire plumage beeomes ruffled and the loirds walk with a weak, staggering gait. 'The temperature is elevated 2 to $4^{\circ} \mathrm{F}$. above normal. I)iarrhea, aceompanied by intense thirst, beeomes a prominent symptom. The droppings are at first of a yellowish-gray eolor, later becoming very thin, watery, fetid, of a greenish east or dlark green. The feathers around the eloaca become soiled and matted with the bowet discharge. Marked dyspnea sets in, the lird opening and closing its mouth (luring respirations; wheezing and rhonchi are frequently heard. The comb and wattles are dark bluish (cyanotic). The bird finally becomes very weak, staggers about, falls down, and dies in coma or convulsions. In the chronie form of the disease the course is mueh longer. 'The bird grows anemic, emaciated and cachectic. Frequently the joints are enlarged; the joint capsule may rupture and dis(harge a seropurulent material. I) eath results from exbaustion. The course in this form may extend over a period of rerreal weeks.

Diagnosis. - Tlere are a fow conditions in birds that might be confused with cholera, as the symptoms are similar. An arcurate diagnosis is only possible on finding the typical hacilli. However, the simptoms and pathological findings shoukl assist in making a diagnosis. A differentral diagnosis must be made from ptomain poisoning which is very eommon in birds, and which frequently kills a large number in 
a flock. The postmorten examination and the microscopie findings will be suffieient to make the distinetion between them. Parasites in the bowels sometimes produce similar symptoms, particularly Trichosoma collare, but an examination of one or more of the affected birds would make a elear differentiation. Chronie eases are sometimes mistaken for tubereulosis and arthritis uriea, but the autopsy and mieroscopic examination would at once make clear the difference. Inoculations with the blood into healthy birds may be made to assist in making the diagnosis .

Prognosis.-The mortality is 80 to 95 per cent. Frequently the entire flock will die. In some outbreaks the disease runs a milder course, the virulence of the infcction not being high.

Treatment.-Medical.-Treatment eonsists in the administration of bowcl antisepties and disinfectants. The course of the disease is usually too rapid for any medical treatment to be of value. It is advisable to administer to the entire flock a solution of iron sulphate ( 1 per cent.), which they should be allowed to drink of freely; or a solution of tannie acid ( 0.5 to 2 per eent.), which may be administered with a medieine dropper (1.0-2.0) to each bird twice daily. Some have elaimed cxcellent results with sulphoearbolates $(0.2-1) .6)$ once daily. Various other medicines have been recommended but none has proved to be speeific.

I accines and Sera.-The treatment of sick fowls with vaccines and scra has not been suecessful to date.

An immune serum has been made which produees a passive immunity lasting for a period of onc to two weeks. 'This is reconmended as a prevent:ve when the disease makes its appearance in a flock, or when a flock of birds is exprosed to infeetion.

Varcoines (autogenic and polyvalent) have been used. 'J'he results have been unsatisfactory where the birds were alrealy infected. On the other hand, very good results have becu reported from the use of autogenie and polyvalent vaccines to prevent the disease in the healthy birds of an infected flock. Vaceines seen to establish immunity in hoalthy flocks, preventing the disease or reducing the 
mortality. More expcrimentation, lowever, is nccessary before definite statements can be made regarding the value of vaceines and sera in the treatment of fowl cholera.

Prevention.-Preventive treatment is by far the most important. In applying it the following should be considered:

(a) All newly acquired birds should be isolated for at least ten days before being allowed to come in contact with the regular flock.

(b) When the disease makes its appearance in the neighborhood, precautions should be taken in regard to the carriers of the infection. Arminister antiseptics. (See Medical 'Treatiment.)

(c) When the disease appears in the flock, radical measures must be taken promptly. All hcalthy birds must be removed to new, uninfected quarters. The sick birds should be destroyed at once, and either burned or buried in lime. All litter and droppings should be treated in a similar manner. I)isinfect the houses, coops, ground and vards with liquor cresolis compositus, using onc gallon to thirty gallons of water. L'se a spray pump so that the disinfectant will be forced to the ceiling, walls and all parts of the building. The buildings and coops should then be allowed to stand open for a few days to allow the air to come in contact with all parts of it. It would be advisable to start with a new flock in the now quarters after a few weeks. A second disinfection would be recommended bcfore allowing birds to be placed in the buildings.

\section{FOWL PEST.}

\section{Foul Plague. Pestus Avium.}

Definition.-An acute, contagious, infectious disease of fowls. Very similar in many respects to chicken cholera.

Occurrence.-'This disease las been reported from different sections of the Inited States, occurring in the form of an cpizoötic. It has been very prevalent during the past ten years in some of the European countries (Germany, 
Belgium, Italy and France). There is no doubt but that it has frequently been mistaken for fowl cholera.

Etiology. - This disease is produced by an ultramicroscopie virus. The exaet nature of this virus has never been determined. It is contained in the blood, the droppings, the exurdate found in the serous cavities, the bile and in the nasal discharges. The filtrate, when passed through porcelain filters and injected into healthy chickens will protuce the characteristic symptoms rather constantly.

Pathogenicity. - Practically all fowls are susceptible to the virus which is most commonly found in chickens, turkey's, sparrows and pheasants. Water fowls and pigeons are less susceptible to infection. Mammalia are not susceptible to the virus.

Natural Infection.-Cohabitation of birds so that they rome in contact with the nasal discharges and droppings from the sick is the most likely way in which infeetion is spread through a flock. Ingestion of food contaminated with the virus, or the eating of infected viscera, or blood, introduces the virus immediately into the digestive tract where it develops and produces the disease. Infection takes place no doubt in some cases from the infection entering the tissmes through lesions in the skin or mucous membranes. The transmission of the disease may be brought about by the virus being carried through intermediate objects.

Pathology. - The lesions found on antopsy are similar in some respeets to those found in fowl cholera. In the peracute cases the lesions may be very slight. The principal lesions are those found in ordinary septicemia, especially hemorrhages in the serous membranes (pleura, peritonemin, rpi- and corlocardimm), and in the mucous membranes of the digestive trart. 'The finding of a fibrinous exudate on the peritonem and plenra has been frequently reported. Jyperemia of the spleen, liver and kidner's is usually present. The (omb) and wattles are lark bluish in (olor (cyanotic). ('atarrhal inflammation of the eonjunetiva and bueeal membrane, with collections of frothy mucus, is often noterl. In the digestive tract changes orenr on the mucous membrane strch as hemorrhages, collections of gravish-white, or yel- 
lowish-green fecal material. Catarrhal inflammation of the intestinal mucosa, in varying degrees of intensity, is found throughout its course. 'The lungs are but slightly involved in the majority of cases.

Symptoms. - These develop suddenly after a period of incubation of three to five days. They consist chiefly of depression, marked dulness, plumage becomes rough, comb and wattles bluish-red; drowsiness, coma, paralysis. The fowl will seek dark places, sit with head down in a crouching attitnde, and refuse to move except when forced to do so. 'They will show a staggering gait, and as the disease progresses will not be able to walk. There is nearly always a profuse discharge from the eyes, the conjunctiva inflamed and swollen. Collections of a similar secretion are found in the nasal passages, mouth and throat. On opening the mouth the membrane will show injection; in some cases hemorrhages and a thick glutinous collection over its surface. The bird often shakes its head and sneezes frequently to discharge the exudate which has collected. The bowel discharges may be normal in appearance, or thin, watery and have a greenish tinge. Sometimes hemorrlages occur in the bowels which give the feces a red or brown color.

Is the disease progresses the bird becomes much weaker, shows marked dyspnea and dies from paralysis. Some (ases show spasms and convulsions shortly before death.

The course of the disease is usually three to five days. subacute cases last five to eight days.

Diagnosis. - There is alway's some difficulty in differentiating between fowl pest and fowl eholera. The symptoms and autopsy are so similar that it is necessary to make a microscopic examination to determine whether or not the hacilli of fow cholera are present. In fowl pest no organisms are found on microscopic examination, nor on cultures.

Differential Diagnosis.- The differential diagnosis will lepencl upon: (a) Microseopic findings; (b) the infectio(ontagious character of the disease (to distinguish from ptomain poisoning); (c) the well-known infectionsness of cholera virus to pigeons which are not susceptible to fowl pest. 
Prognosis. - As most cases terminate fatally, the prognosis is bad.

Treatment. - No medical treatment is known that has any material effect in controlling this disease after the fow is once infected. Preventive treatment is by far the most important, and should consist of radical measures of isolation and disinfection. (See Fowl Cholera.) 


\section{ACI'TE INEEC'TIOLS DISEASES IVITH LOCALIZA- TION IN CER'TAIN ORGANS.}

\section{EPITHELIOSIS INFECTIOSA AVIUM.}

\section{('ontagious Epithelioma. Chicken-pox. Diphtheria. Roup. Canker.}

Prevalency and Distribution of the Disease. -The disease affects nearly all birds, especially chickens, turkeys, peafowls, pheasants, guineas, pigeons, sparrows, quail, etc., but is not so frecpuently observer in ducks, geese and swans. 'The malady is widely distributed, appearing in praetically all countries where birds are found in any considerable numbers. In the I'nited States infeetious epitheliosis is (unite prevalent, especially in the northern states during the colder months. In the fall when the cold, damp weather begins outbreaks orcur quite commonly, and poultrymen often refer to the early inception of the clisease as a "coll.." The intensity of the disense usually increases until the warm weather makes its appearance, when it diminishes and lies dormant, to reappear at the first cool, damp weather in the fall when the birls are more dosely confined.

Etiology. Evilene obtained by experiments indicates that a filtrable virus is the primary causative agent, and that the more or less severe complicating conditions are indureel by secondary infection, of which a bacillus, probably the Barillus diphtheria columbarum of Loeffler, seems to be the most constant.

Pathogenesis. - Transmission of this disease is not very diffirult. I"sually about 70 per cent. of healthy birds will slow srmptoms of the clisease after associating with an affected one for a short time. Actual contact is not neces- 
sary, as is shown by the spread of the disease at poultry shows. A bird with mucous membrane lesions may transmit the disease to a susceptible bird in the cutaneous form, and vice rersa.

Emulsions of scrapings from either cutaneous or mucous membrane lesions injected subcutaneously, submuçously or applied to scarified areas on the skin, would in some cases produce the generalized form of the disease. Similar results were produced by using an emulsion of a twenty-four-hour agar growth of cultures taken from, lesions and injected subcutaneously.

It is possible at times by scarifying the mucous membrane of apparently healthy birds to produce at the point of injury local lesions not unlike those of this disease, but they disappear in a few days without manifestation of general symptoms. Incomplete experiments by using the blood of sick birds in an attempt to produce the disease gave negative results, which is contrary to that claimed by a few other investigators.

Natural Infection. - There are a variety of ways by which natural infection takes place. Cohabitation is one of the most common, an infected bird introducing the disease into an entire flock. The exfoliated membranes, or the exudate given off while sneezing or coughing, act as intermediary carriers to the healthy birds. 'The infection in this way will find lodgment on the skin where there are slight abrasions, or on the mucosa of the eyes or mouth, where it develops either to form the pox lesions or the various other forms found on the mucous membranes.

It is quite possible to have the disease develop in the eyes by the infection gaining entrance, and the bird irritating the skin and conjunctiva by rubbing them against objects or by scratching them with their feet. The disease is frequently spread by food or drink which is contaminated with infective material.

A very common source of the disease is the poultry show where infected birds are exhibited, and conditions exist farorable to reduce the bird's resistance. It very frequently happens that on the return of birds from the show 
the clisease develops in the course of a week or ten days. sparrows and pigeons are common carriers, of ten transporting the infeetion great distances. This is a very important method of transmission of ten overlooked. The disease has been observed in sparrows in a number of cases and exhibiting in them the same characteristics as in other hirds. Sparrows and pigeons come in direct contact with poultry at feeding time and thus infeet them. It is sometimes spread by such intermediary agents as infected coops, ctothing, ete. Young birds are more susceptible to the infection than older ones. Pigeons and chickens seem to be far more susceptible than other birds.

It has been observed that onc attack of the discase will produce immunity for a eertain period. Either the skin form or involvement of the mueous membranes confers equal immunity against the opposite form.

Pathology.-'The postmortem findings vary with the inmnerlate enuse of death. In rare cases when death is due to suffocation early in the discase only the acute conditions are noted. 'The body appears fairly well nourished, the mucolls membranes are covered with a thick slimy mucus which may oeclude the upper air passages. In the larrix, or somewhere along the trachea, possibly in the larger bronchi, will be found the mass of dense, tough, rellowish material obstructing the lumen.

ln some aases when both eves are involved death results from starvation early in the disease. 'The body is emaciated. The mucous membranes of the eyes, and of ten of the mouth and respiratory passages show inflammation, and usually there is a matss of this same vellow material in the conjunctival sac distorting or obsemring the eyes. 'The skin aromel the eyelids may also be involved.

'The majority of the fatal cases die in two to five weeks. In these the body shows all the ssmptoms of a toxemia, cmaclation is prononnced, and the serons membranes stndcled with petechiar. In the rutaneons form the skin presents all the symptoms of a severe inflammation, each nodule heing a distinct process. Some of them show a similarity to tunor formation, especially on the comb where 
the skin is thickest, but on the wattles and feathered skin around the head this feature is less pronounced. Masses of tough yellow material often distend the suborbital fossæ, accumulate in the external ear, in the lung substance and along the digestive tract.

Symptoms. - The disease assumes various forms, depending upon whether it involves the mucous membranes of the mouth, the nasal passages, the eyes, the intestinal tract, or the skin. It is necessary from a clinical standpoint to consider these various types of the disease. It is this fact whieh has led to so much confusion among the poultrymen, as they have been inclined to consider these forms scparate diseases.

Nasal Passages. - The early symptoms are similar to those of an acute nasal catarrh, but the bird shows more duhness and prostration and there is present a peculiar offensive odor. The odor is very characteristic. The discharge from the nasal passages is at first thin, serous and later lecomes thicker and glutinous, causing the affected birds to snecze and shake their heads in an effort to clear the nasal passages. 'The nasal secretions collect around the openings, forming dirty, yellowish crusts. The infra-orbital fosse become inflamed and distended with pus, forming an enlargement on one or both sides of the head below the eres. Owing to the oeclusion of the nasal passages the bird breathes with open mouth.

Eyes. - At first a thin serous secretion is observed flowing from one or both eyes; later it becomes turbid, thicker, arlheres to the edges of the evelids and dries to form crusts rompletely closing the eve. 'The retained yellow material becomes thicker and often accumulates to such an extent that it causes a pronounced bulging of the eyelids. Masses of this material as large as a walnut are often removed from beneath the lids. Sometimes infection develops posteriorly to the eyes in the form of abscesses which force the eyes partly out of position. 'The cornea is turbid and often ulcerated. Complete destruction of one or both eyes is often observed in severe cases. 
Mouth.-Involvement of the mucous membrane of the mouth begins as a local disturhance. 'The discase is ushered in by a slight redness or congestion of isolated areas, or may involve the entire surface. In the conter of the cons gested areas there will appear in a day or so small round or oval vellowish-white spots, which rapidly spread and eventually form extensive collections of glutinous pus, laving somewhat the appearance of diphtheritic memhrane. The nature of the collected material on these areas led to the name "diphtheria" for this form. As the disease progresses this process often extends over the cntire mucosa of the mouth, tongue and throat. When the larynx is involved, or even the trachea, as is occasionally noted, there will he marked symptoms of dyspnea. Asphyxia is frequent when the lesions cxtend to the larynx and trachea. () removal of the deposits the mucosa prescnts a red, granulating surface, which bleeds easily, showing in some rases extensive submucous swelling and edema. 'The commissures of the mouth are frequently involved as is the skin and contiguous tissucs, a characteristic pox lesion developing in the skin. Other complications are frequently found spreading from the oral eavity, $i$. $e$, to the pharynx, esophagus and (rop), prorlucing severe diarrhea and other bowel disturbances.

Slin.-Lesions on the skin are found in quite a few cases, more commonly on the (omb), wattles and other portions of the skin not covered with feathers. It is first noticed as a very fine gray vesicle, which soon develops into small rlevations of a reddish-gray color, which later become more grinyish. Microscopically the nohnles are composed of degeneraterl epithelial cells. These nodules in some eases become quite large, warty in appearance, dry anct hard. In severe cases large numbers of these nodules are found listributed over the skin on exposect surfares. On renoving the surface of the nodules a raw granulating area is noterl. fone eases show distinct degeneration of eontiguous tissues.

A mixed rariets of the disease is of frequent occurrence, the murosa showing the characteristic deposits and the stin pox lesions. It is quite evirlent in these case's that 
the disease spreads from one location to the other, and the difference in the lesions is due to the difference in the strueture of the tissue involved.

General Symptoms.-In the early stages before much secondary infection takes place, there are no marked general symptoms. Iater marked general symptoms appear. 'The birds show dulness, assume a sitting posture, wings are held pendent, plumage becomes rough and the patients show much depression. 'The comb and wattles grow bluish-red in color, later pale and cold. In the colder climates the disease often assumes a subacute or chronic form, while in warmer climates the acute form is more often observed. Frequently, however, the disease assumes the character of a chronic catarrh.

Diagnosis. - The disease usually makes its first appearance in the fall and often occurs as the cutaneous form. It may be overlooked, especially if the birds are on the range. 'The nucous membrane form usually makes its appearance soon after housing for the winter. 'The sneening, mouthbreathing, occluded nostrils and an occasionally inflamed eye are significant, especially when rapidly spreading through the flock. Soon after a few will refuse food and appear depressed.

It must also be suspected when similar symptoms appear after ardling new birds to a healthy flock or birds returned from shows.

A peculiar characteristic and offensive odor is associated with this disease and poultrymen familiar with it often recognize the discase from the odor alone. The same odor is given off by cultures.

Differential Diagnosis. - Wounds on the skin around the head, usually pick-inflicted, appear suspicious, but these heal rapidly without extensive thickening. An injury to the eve, even though serious, will not cause the formation of the characteristic vellow deposit. 1)ifficult respiration, rarcly seen in more than one bird in a flock, may be due to sereral causes, and those observed persisted for some time without afferting the general health of the bird. 
Prognosis.-'The cutaneous form usually runs a more favorable course, apparently recovering in one to three weeks, when the nodules become dry and scale off. The mucous membrane form is less favorable, the mortality varying from 10 to 50 per cent., depending on the care and sanitary surroundings.

Aside from the actual number that die we find there is ronsiderable loss resulting from the chronic effects. After the disappearance of all visible lesions the birds do not scem to regain their former good condition for months. E,gg production is far below normal, and young birds have their growth checkel.

Treatment. - There have been a great many drugs recommended in the treatment of this disease, and so far none of them have proved very satisfactory. Daily treatment of individual birds is a tedious and laborious task, especially where large flocks are affected.

The lesions, whether on the skin or in the mouth, should loe washed with antiscptics, boric acid solution ( 2 per cent.), potassium permanganate (0.25-1 per cent.), or any other rqually efficient antiseptic. The deposits should be removed with a eurette or dull knife before applying the antiseptic.

laccine. - Vaccination has proved very satisfactory in this disease. One c.c. is administered subeutaneously, and in severe cases it should be repeated in four to six days.

Prevention. - All newly acquired birds should be examined and isolated for at least ten days before allowing them to rome in contact with the regular flock.

As soon as the disease is recognized, it is recommended that all birds be vaccinated immediately to eheck it. Ilealthy flocks and those intended for exhibition purposes may be vaccinated to establish immunity. 'This has proved very satisfactory. The inmunity established will last for at least six months to one year.

\section{ANTHRAX.}

This disease is essentially found in large animals. (See other works.) 


\section{FOOT - AND - MOUTH DISEASE.}

Aphtha Eipizoötica.

Foot-and-mouth disease rarely occurs in carnivora, but has been observed in a few cases in dogs. Dogs kept on infected premises in contact with infected cattle, especially those that are used for herding animals are the ones most liable to be affected. Dogs are not very susceptible to the infection, and take it in a mild form. 'The principal symptoms are vesicles on the mucous membranes of the lips, sometimes forming ulcers, which penetrate more or less deeply into the tissues. Sometimes the feet become involved, when there will appear a vesicular exanthema on the balls of the feet and between the toes. 'The feet will be swollen, lot and sensitive, and the patient very lame. General srmptoms, such as elevation of temperature, diarrhea, loss of appetite and vomiting have been observed. The disease has made its appearance in fowls, but only in a few instances has it been recorded.

\section{HEMORRHAGIC SEPTICEMIA OF CATS.}

Infections Gastro-enteritis.

History. - This disease has been reported as occurring in various sections of the Cnited States and Europe. So far but little is known about it as the disease is a comparatively new one.

Occurrence.- ('at septicemia occurs enzoötically, especially among cats congregated as in catteries and shows.

Etiology.- The disease seems to be produced by a specific organism (Bacterium felisepticus bipolaris of Huse and (oleman). It is described as a short, rod-shaped organism, taking the bipolar stain readily with the common stains, but is (iram-negative.

Pathogenicity. - The work up to the present time indicates the pathogenicity of this organism is confined to cats, rabbits and guinea pigs. l logs and birds show no tendency toward natural infection. 
Pathogenesis. - The organisms are evidently taken into the digestive traet with the food and drink. 'The organisms multiply very rapidly and induce in a very short period of time a severe hemorrhagic gastro-enteritis. The severity of the disease depends upon the natural resistanee of the animal and the virulency of the infection.

Symptoms. - The period of incubation is from two to five days. 'Tle development of the symptoms is very rapid. 'The disease is ushered in by a sudden suppression of the appetite, vomiting, retehing, marked depression and general wcakness. The animal shows a tendency to seck dark eool places and lies stretched out on its abdomen. The temperature rises rapidly after the onset reaching $103-106^{\circ} \mathrm{F}$. in twelve to twenty-four hours. 'The temperature remains high during the early part of the disease, but later becomes nornal or subnormal. Emaciation eomes on rapidly, a profuse diarrhea is present, the feees having a characteristic. fetid odor. As the disease progresses the animal beeomes weak, eyes retracted and staring. In three to four days coma develops, and death follows in the eourse of a few lours. The eourse is very rapid in acute cases. Some few cases assume a subacute form, and the symptoms are nilder but progressive.

Pathology. -1 more or less serere gastro-enteritis hemorrhagica is typical of this discase. The entire mucosa of the digestive tract is highly congested and dotted over the surface with peteehia and numerous patches of eechymoses. The membrane is covered with a catarrhal exudate whieh in the majority of cases contains some blood. 'The serous rovering of the intestines shows marked congestion, but no petechiat. 'The presence of gas in the stomach and bowels is quite constant. 'The stomach partieularly is nearly always found distended. 'The heart musele, liver and kidneys show signs of rapid degeneration. The gall-bladder is greatly distended, and the bile is dark colored, thick and tenaceous. 'The lungs may show congestion, and in subacute attacks pneunonic areas. Other eases may not show changes in the lungs. 
Diagnosis. - The disease is recognized by its contagious character, its rapid, severe course and the profuse diarrhea. The absence of catarrhal symptoms of the air passages and eyes would assist in differentiating it from distemper. Ptomains or mineral poisons will produce similar symptoms, but the micrescopic examination, the contagious character and the investigation of the food supply, history of poisoning, etc., will serve to make clear the differentiation. It is very important to be able to make an accurate diagnosis for the protection of other animals.

Prognosis.-The prognosis is bad. Even in the early period of the disease, and in the apparently mild cases, it should be looked upon as a very serious condition. 'The mortality is very high, from $\$ 0$ to 90 per cent. of the cases terminating fatally.

Treatment.-'There is no treatment known that has much, if any, effect on the course of the disease. Symptomatic treatment is the only thing that can be done. A vaccine is being used as a prophylactic treatment, but at present it is difficult to say what the results will prove to be. An antisera is also being used at present and gives considerable promise of sucress as a curative and preventive agent. 'The progress of the disease, after it once breaks out in a hospital or cattery can be curtailed only by removing all sick and exposed animals, and thoroughly disinfecting the rooms with formaldehyd gas. All utensils, litter, etc., should be rendered innoxious. Prevention is far more important than treatment. 


\section{CHAP'TER I I I.}

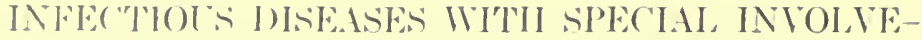 MENT OF TIE NERTOTS STSTEM.}

\section{RABIES.}

IIydrophobia. I.yssa.

Definition.-An alcute infectious disease, fatal in the majority of cases, and (dharaterized dinieally by disturbance of consciousness, marked irritability and later by symptoms of paralysis.

Occurrence. - Rabies is one of the oldest known diseases and has been observerl in all countries. It is most commonly noted in dogs, eats, wolves and foxes. Other animals are less commonly affected. It is estimated that about 90 per cent. of the cases of rabies oceur in dogs. 'The disease often oecurs enzoötically, which may be at any scason of the vear. 'The larger number of outbreaks in the linited states has been in the colder months of the year. Outbreaks are naturally more frequent where dogs congregate, and in communities or cities where there are no proteretive regulations. In some eountries and distriets rabies is decreasing, due to proper police regulations which restrict the munber of dogs rumning at large.

Etiology. - Rabies is produced by an ultramicroseopie virus. This virus is found to be the most virulent when taken from the central nervous sistem (brain and cord), less so from the peripheral nerves, salivary and other glands and their secretions. The infection does not seem to be present in the blood of affeeted animals. The infectious agent is fixed, non-volatile and is an obligatory or fixed parasite.

Pathogenicity.- The disease is readily transmitted to dogss, rabbits, guinea pigs and other animals by inoculating 
them witl the virulent saliva, or an emulsion of the comu anmonis, medulla oblongata, and some other portions of central nerve tissue. The most satisfactory results are obtained by injecting some of the virus under the dura mater of the brain or cord. 'The same inoculation may be marle into the anterior chamber of the eye, or into the muscles, but the results are not so certain. Injection of the virus into or around the peripheral nerves has produced the disease quite constantly. Scarification of the skin and rubbing the virus on the area has produced positive results only in about 40 per cent. of the cases. Other methods of inceulation lave been less suecessful. Birds may be infected hỵ inoeulation.

Natural Infection.-Rabies is produced in practically all (atses by the victim being bitten by a rabid animal, introducing the virulent saliva into the wound, to come in contact with the nerves, muscles, or subcutaneous tissues. liarely are eases produced by the infectious agent being licherl from wounds, or from contact with lesions on the skin.

Pathogenesis.- When the virus is introduced into some part of the body through a bite by another animal, or by incoulation artificially, it is supposed that it follows the ronrse of peripheral nerve fibers along which it is carried to the central nervous system. The explanation of the devalonment of the two types of the disease is explained hy the path of travel to the central system, whether it reaches the brain, medulla oblongata, or the spinal cord. Ifiter the virus reaches the central nervous system to produre irritation on rertain groups of cells, it is then centrifugally distributed to different parts of the body. It reaches the alivary glands, which favors the development of the virus, increasing the sorertion of the glands, and then is finther disioninatert through the saliva. The period of incubation of rabios is quite variable. The virus after hoing introlnerel into a wound may remain in a dormant sate for soms time before reaching the central nervous rytem. 'l'he vintiation in susceptibility of animals in the virulary of the infection, and the mothorl of the dissemination of the virus in the borly naturally make the period of 
incubation rather long. 'Three to seven weeks is the usual time for development in dogs. However, some cases develop only after several months. The period of incubation in eats and fowls is usually shorter than in dogs. The period of incubation is usually shorter in young animals. The toxicity of rabies virus and the pathogenic action on the tissues are no doubt due to some chemical product which up to the present has not been determined.

Pathology.-The disease is really characterized by the absenee of any marked gross pathological findings. The lesions vary somewhat in individual cases. The digestive tract shows congestion and hyperemia. The stomach may be contracted and empty, or is frequently partially filled with foreign material sueh as stones, straw, sticks, splinters of wood, hair, feathers, ete. 'The folds of the mucous membrane are markerly eongested on their summits. Hemorrhage sometimes oecurs from the foreign material cutting or tearing the mucosa. The mueosa of the pharynx and larynx shows congestion and is covered with mucus. An acute eatarrhal inflammation of the respiratory passages is frequently found. The brain and its coverings are often injeeted with small hemorrhages. No other lesions of any importanee are observed.

Symptoms. - From a clinical standpoint it is necessary to divide rabies into two types, viz: (1) Furious rabies, and (2) dumb rabies. 'The different train of symptoms depends entirely upon whether the brain or spinal cord is primarily involved. However, cases are observed occasionally where an animal affected with dumb rabies suddenly develops the furious type. 'The humb or mute rabies seems to be the most common form in the Lniter States.

1. Furlous RaBies. - In this form three stages of tevelopment are rather sharply defined: (a) Melancholic or prodromic stage; $(b)$ maniacal or irritative stage; $(c)$ paralytic stage.

(a) Stage of Melancholy.-In the stage of melancholy or in the beginning of the disease, the first thing usually noted by the owner is the altered behavior of the animal. It becomes sullen, afraid, seeks dark places, is easily irritated, restless and 
obstinate (often loes not obey its master). 'The patient is also inclined to hirle itself, alternately gets up and lies ،оки frequently, makes sudden, unusual movements, sueh as snapping or biting at imaginary objeets, or barks suddenly without any eause. During the early stage of the disease the appetite is not mueh changed, but later the manner of eating and the kind of food chosen are greatly modified. Dogs are inclined to eat indigestible objects, such as pieces of woor; cat their own feces and lap up their own urine, ete. A very noticeable symptom is the tendeney to grasp objects as if to eat them, then suddenly let them fall from the mouth. Constipation is usually present in this stage. I luring the latter part of this stage the patient is excitable. 'The patient shows a marked ehange in disposition, sllaps at objects, its master, other persons or other animals. 'This exeitability is more noticeable toward strange persons or animals. Sometimes the patient manifests no cluange in disposition toward its master, familiar persons or animals. 'The excitability gradually, or very rapilly, increases. 'The nervous reflexes beeome sensitive and the patient is easily startled by sudden noises, touehing objeets or by strong light. 'The pupil of the eye dilates and the facial expression is changed. Dyspnea is prominent at times. 'The patient will often scrateli or bite the place of infection, sometimes doing extensive injury to the area. 'The appotite is entirely suppressed but the animal seeks water and attempts to drink, but, on aceount of the beginming paralysis of the larynx and pharynx, does not suceed. 'The salivary secretion is increased, and saliva hangs from the month in long strands. This stage usually lasts from one to three days.

(b) Stugr of Irritution. - In this stage the symptoms rearh at higher degree of development. The excitement increases; hallueinations, which bring about violent fury or rage, are som apparent. The voice of the animal changes deeidedly from the nommal. 'This is a very characteristic symptom of rabies, and no loubt results from the congestion and paralysis of the vocal corcls. 'The owner's attention is always attraceter hẹ the unusual anount of barking and 
the elhange in the voice. At this stage they become vicious, and if confined show a tendency to chew objeets and tear them to pieces. Rugs, carpets, or other objects with which they come in contact are torn and destroyed. At this time if they are not confined they will usually wander away from home, biting other animals or persons that happen to come in their path. Often during a short period of time they will travel long distances before returning, which they invariably do. Upon their return they show emaciation, exhaustion, often wounds from being bitten by other animals, and in general have a dejected appearance. The desire to bite aimlessly objects and animals is quite characteristie of this stage. A rabid dog in this stage may inoculate a large number of animals unless confined. When confined in a cage or room they show at times intense fury and viciousness. If irritated, by placing a stick between the bars of the cage, the dog will attack it viciously, but shows the peculiar symptom of simply biting instead of holding on to the stick as non-rabid dogs ordinarily do. As this stage progresses the periods of fury become shorter, and soon marked depression is noted. Paralysis of some of the special nerves derelops, shown by the inerease in the change of the roice, which becomes a peculiar wail or howl, and the difficulty in swallowing. The eyes are congested, and also the mucous membranes of the mouth. This stage of fury lasts usually two to four days.

(c) Paralytic Stage.-The symptoms of paralysis become more pronounced, and the patient is less vicious. Complete loss of voice and the inability to swallow, with paralysis of the tongne and masseter muscles, are early svmptoms of this stage. The month is held open, the dry tongue is protruded and completcly paralyzed. 'The eves are retracterl, lose their expression and luster; the pupils beeome dilated, in some cases unequally. The animal shows marked general emaciation, the hair coat rough and there is much exhaustion. 'The general paralysis asserts itself, beginning in most cases at the tail and posterior limbs. 'The animal has difficulty in walking or standing; later there is complete paralysis of the posterior part of the body. 'The paralysis rapidly ascends to the fore parts and central nerrous sys- 
tem. causing death in most cases on the fifth to eighth day of the attack. 'The tempcrature in rabies has not been recorled in many cases. During the early stages it is elevated to $104^{\circ}-106^{\circ} \mathrm{F}$; later becomes subnormal. Variations in the symptoms of furious rabies are observed, but most cases present a rather constant clinical picture.

The symptoms of furious rabies in cats are very similar to those of dogs. 'They often become very vicious, and bite and seratch everything that they come in contact with. 'The change of voice is also quite noticeable. The course of the disease is usually shorter, lasting from three to six rays.

2. Demis RaBies. - This form is characterized by a paralysis of the lower jaw, tongue, larynx and pharynx. 'This simptom develops early in the discase and is the most prominent one until general paralysis manifests itself. 'The owner is often led to suspeet that the animal has a bone or some other object lodged between its teeth, or in its throat. All such cases should be looked upon with suspicion, and all precautions taken in making the examination. In the majority of eases no nerrous or excitable symptoms are shown; the paralytic symptoms gradually becoming more pronounced until the animal succumbs to general paralysis. Cases are observed occasionally where symptoms of excitement levelop during the course of the disease. One such ase was observed in which the early symptoms indicated dumb rabies but in twenty-four hours all the symptoms of the furious form had reveloped. Animals with this form of the disease usually seek rlark, cool places and lie almost constantly.

'There is the same pecoliar expression from the eyes as in the other form. 'The patient often attempts drinking or ating which the paralysis prevents. There is usually no tculenry to bite nor viciousness manifested.

Rabies in rabbits nearly always assumes the paralytic form. Fowls show the characteristic symptoms of furious rilhies in some rases, such as excitement, ehange of voice, rufflal phumage, attacking other fowls, people, etc. In other intances the paralytie form is observerl.

Diagnosis. - In furious rabies the diagnosis does not 
present any great difficulty where the symptoms are pronounced and the animal observed through the different stilges. 'The positive proof of a bite inflicted previously by another animal is of considerable importance in making the diagnosis. An accurate diagnosis is of great importance for two reasons, viz.: (a) In case human subjects are bitter, or in any way inoculated, it will give them an opportunity to receive the antirabic treatment early; and $(b)$ to protect other animals and persons from becoming inoculated by quarantining and confining the animal. Under no circumstances should an animal showing symptoms of rabies, or suspected of having the disease, be killed. It should be put in quarantine and observed. It has been demonstrated that animals destroyed early in the disease often do not show positive findings on microscopic examination. The characteristic symptoms of irritation, tendency for biting, change of voice, paralysis, etc., generally show evidence enough to the experienced clinician to establish the diagnosis. The detection of Negri bodies on microscopic examination will make the diagnosis positive. In dumb rabies the diagnosis is made on the characteristic symptoms of paralysis of the lower jaw, the somnolence in most cases, the expression and the paralysis of otlier parts of the body in the course of two to four days. All cases showing the symptoms of the lower jaw hanging down should be diagnosed dumb rabies until ample proof is shown that it is some other eondition. Microscopic examination of the brain should be made to establish the diagnosis as in furious rabies. Intracranial inoculations of rabbits have been used extensively in making a positive diagnosis. This method requires from fourteen to seventeen days, and is falling into disuse. Inoculations of the virus in guinea pigs have also been used extensively as a diagnostic measure.

Prognosis and Course.-The disease is fatal in practically all cases. The possibility of recovery in very mild cases cannot be denied. However, no authentic case of recovery has been recorded. It has been estimated that only about 17 to 20 per cent. of the animals bitten by known rabid aninals develop rabies. 'This percentage is higher in dogs than in any other snall animal. 'The course of the disease 
is quite constant in the different animals, although variations occur occasionally. Five to seven days is the usual course; it may be in exceptional cases as short as two days or as long as ten or eleven days. 'There is but little difference in the course of the two forms; usually dumb rabies runs a longer course than the furious type.

Differential Diagnosis.-'There are quite a number of diseases in small animals which might be confused with rabies: (a) Diseases of the brain, particularly inflammations involving the meninges and the cerebral substance itself. In these cases irritability and nervousness are present, but the tendency to bite, the aggressiveness, change in voice and development of the paralysis in the same way are absent. If there should be any doubt the animal must be confined and observed for a few days when the differentiation will not be difficult. (b) Canine distemper (nervous form) sometimes simulates rabies, but the history of the case, the other symptoms of distemper and the length of the course of the disease will reveal the difference. Parasites in the intestinal tract often produce certain nervous phenomena, but of different development and type than rabies. Many of the characteristic symptoms of rabies are absent. $(d)$ Other parasites (Linguatula rhinaria, I)ioctoplyme renale, Spiroptera sanguinolenta, Otorlectes (eynotis) may produce certain nervous symptoms, but careful examination and observation of the animal will readiky distinguish the lifference. (e) Foreign bodies lodged between the teeth, around the tongue or in the pharynx all induce salivation, hoarseness from the accompanying colema and cause the mouth to remain open. 'These cases should be examined very carefully to differentiate them from rabies. In most of these cases it is impossible to close the mouth, which is not true in rabies, and there are no general symptoms. ( $f$ ) 'Trigeminal or facial paralysis is observed occasionally in the course of distemper, or as an independent condition. In these eases there are no other symptoms of paralysis, nor any general rmintoms. The course is longer and the animal remains otherwise normal. (g) Epilepsy might be confused with 
mabies. But this condition comes on suddenly and lasts only a few minutes to disappear completely until the next attack. (h) Eclampsia is differentiated by its occurrence in bitches at or near the time of whelping, clonic spasms which affect the entire body and none of the other chararcteristic symptoms of rabies. (i) Infectious bulbar paralrsis in which the symptoms are quite similar to rabies. It is distinguished from rabies by the absence of the furious attacks, viciousness and paralysis of the lower jaw. The blood and brain tissue are infectious and the saliva not. This disease develops suddenly and runs a more rapid course than rabies. Finally, there are a few conditions in which the animal will show more or less nervousness, but none of the other symptoms of rabies. Animal inoculation and the microscopic examination of the brain should bc made in all cases of doubt.

Treatment.-After rabies has once developed in an animal no treatment will have any effect on the disease. Bitten wounds that have been caused by a rabid animal should be treated promptly. They should be opened freely, and if hemorrhage takes place it is all the better, as it will tend to remove the virus from the wound. The wound should be washed freely and thoroughly with potassium permanganate solution ( 1 per cent.), or bichlorid solution ( 1 to 1000). Cauterization of the wound with silver nitrate is also recommended. The success of this treatment in preventing the development of the disease will depend upon the length of time which has elapsed after the bite, and the thoroughness in which the antiseptic or caustic is applied. I)isinfection of the wound alone should not be depended upon for the prevention of rabies.

Preventive Inoculation.--Preventive inoculation is bcing used with great success in animals, and is highly rccommended in all cases where there is any doubt about the animal being infected.

\section{INFECTIOUS BULBAR PARALYSIS.}

\section{Pseudorabies.}

Definition. - An infectious disease affecting dogs, cats, rats, horses and cattle resembling rabies in many respects. 
Etiology.-This discase is produced by a specific virus, the nature of which has not been determined. All animals except fowls are susceptible to inoculations with the virus which is found in the blood, central nervous system, and in somc of the other tissues. The saliva, bile and urine are frec from the virus.

Pathogenicity.-Most animals are very susceptible to infection by inoculating them with only minute quantities of the blood, or central nerve tissues, from those affected with the disease. At the point of inoculation, the tissues become red and inflamed; later, necrotic. The inoculated wound show's intense irritation, and the animal bites and scratches the part almost continuously. Dogs and cats contract the disease readily after being fed on organs or tissules containing the virus.

Natural Infection. - This has not been definitely established, but probably in most cases is due to eating infected meats, or bcing inoculated by infected mice or rats.

Pathology.- No marked lesions are found in this diseasc, except at the point of inoculation. The meninges of the brain, and the brain substance, usually show hyperemia, and blood extravasations. When infection takes place in cats and rogs from cating infected meat, the stomach and small intestines are congesterl, and in some cases markedly inflanerl. Icmorrhages under the mucosa, and frequently petedia on the serous membranes of the stomach and intestines are noted. No other lesions are found.

Symptoms. - The period of incubation varies from two to nine lays. 'The onset of the disease is characterized in the dog and cat by a sudden change in disposition, tendency to serek dark, cool places, where the patient lies quietly or mantains a crouding attitude. They often cry or mew as if in severe pain. lomiting is more or less persistent, there is romplete loss of appetite, and marked salivation. cevere itching is manifested in most cases by the animal sratching and hiting itself, sometimes so severely that the skin is entirely removed over the involved area, usually around the lips or face. In some few cases this symptom may 
be absent. As the disease progresses the animal shows more pain by howling, crying, or groaning. The muscles around the head and face often show marked twitching, which may he periodical or constant. The nervous system is affected as is shown by increased reflex excitability, paralysis of the pharynx and larynx, and increased muscular sensitiveness. There is marked dyspnea. The temperature is usually normal or subnormal; toward the end the subnormal temperature is very marked. The animal usually dies in about thirty-six to forty hours.

Diagnosis. - There might be some difficulty in establishing an accurate diagnosis. However, the symptoms, which are quite constant, and the rapid, fatal course, would assist.

Differential Diagnosis. - In the dog and cat it is necessary to distinguish this disease from rabies. The chief points of difference are the following: (a) Rabies (furious) is characterized by symptoms of rage or fury, aggressiveness; in dumb rabies by paralysis of the lower jaw. Both are absent in bulbar paralysis. (b) The saliva in rabies is highly infectious while in this disease it is non-infectious. (c) The period of incubation is usually from two to nine days, while in rabies, although variable, it is much longer. On account of its rapid course, it might casily be mistaken for some acute poisoning. should the symptom of itching and irritation be absent a differentiation would be quite difficult. In such cases experimental inoculation with the blood of the affected animal into healthy ones will assist. Frequently, a number of animals in a neighborhood will become infected at the same time, the disease appearing in the form of an enzoötic.

Prognosis. - Very unfavorable. The majority of cases die.

Treatment. - Treatment is unsatisfactory. Antiseptics for the digestive tract may be tried. Thorough disinfection is necessary in order to check the disease. All dead animals sliould be burned or buried in lime to destroy the virus.

\section{TETANUS. LOCKJAW.}

Definition. - An acute, infectious disease, characterized by involvement of the nervous system, resulting in spasmodic 
contractions of certain groups of muscles, or the entire musculature of the animal's boly, without that consciousness is disturbed.

Occurrence. - Tetanus is of rare occurrence in small animals. It is observed occasionally in dogs and birds. It is far more (o)mmon in the large rlomesticated animals.

Etiology.-It is produced by a specific bacillus (Bacillus tetani of Nicolaier).

Natural Infection. - Infection takes place by the baeilli or spores gaining entrance to wounds in the skin or mucous membranes. The most common intermediate carrier of the infection is the soil or earth which comes in contact with the wounds. Rarely is the disease spread from one animal to another.

Pathology. - The postmortem is usually negative. The affecterl muscles are usually pale, or may show oeeasional hemorrhages. 'The condition of the blood indicates in most cases that death is due to suffocation. Other lesions are inconstant and of minor importance.

Symptoms. - General tetanus is of rare occurrence, although observerl occasionally. It is characterized by a stiff, stilty gait, extcnded head, ear's stiffy erected, protrusion of the memblana nictitans, skin wrinkled over the forehear, trismus. The muscles of the back and posterior limbs are stiff and harl; tail erected and stiff. 'The reflexes are greatly accelerated as shown when the animal is touched or hears sudden noises. There is frequently opisthotonus, or orthotonus. Very characteristic is the facial expression of dogs suffering from tetamus. Perhaps in the greater number of cases in dogs and hirds tetanus is local and involves only certain groups of muscles, usually the masseters, the muscles of the ars, skin and muscles oxer the foreheal, membrana nictitans, and the muscles of the neck, all of which are contrarterl and the hearl held fixed. In birds the symptoms of stiffiness of the neck, wings held in a fixed position, ruffled fratlers, stilty gait are the most prominent.

Diagnosis. - This is made nainly on the characteristic symptom of tonic spasms of groups of muscles, with normal ronsciousiless and temperature, and the subacute course.

Differential Diagnosis. - (a) Differentiation must be made 
between tetarus and strychnin poisoning which show very similar symptoms. In strychnin poisoning the spasms develop more rapidly, and are of much greater severity and the membrana nictitans is not usually affected. The extremely increased reflex excitability in strychnin poisoning as compared to tetanus will also serve to differentiate them.

(b) 'Tetanus may also be mistaken for cerebrospinal meningitis, but in the latter disease other symptoms, such as dulness and paralysis of certain cerebral nerves, are present which will assist in the diagnosis.

(c) Muscular rheumatism is differentiated by the absence of reflex excitability; the muscles are painful and swollen rather than contracted as in tetanus.

(d) There are quite a number of conditions (rabies, eclampsia, epilepsy, articular rheumatism, etc.) which show some svmptoms of tetanus, but there are always other symptoms present which are sufficient for differentiation.

Prognosis.-The prognosis in dogs, providing the symptoms are localized, is quite favorable. Should the disease, however, become general it is unfavorable. The course in the $\log$ is usually subacute or chronic.

Treatment.-Dietetic.-When trismus is present, preventing the animal from taking food, rectal feeding is recommended. Concentrated beef broth at body temperature is perhaps best. Two to four ounces should be given 3 to 4 times daily. During the later stages milk can be allowed as they will lap it in small quantities.

Medical. - Small and repeated doses of morphin sulphate $(0.05-0.2)$ once or twice daily to control spasms of the muscles; or chloral hydrate administered per rectum ( 1 to 4 with acacia and glycerin), using $6.0-12.0$ of the mixture once or twice daily. Subcutaneous injections of a diluted carbolic acid solution have been recommenderl, but are of doubtful value.

Serum.--Tetanus antitoxin has proved to be of greater value as a prophylactic than a curative treatment. Large doses might be userl, 1500 units daily for three to four days.

Surgical. - In case a wound is found indicating the focus of infection, it should be thoroughly curetted and strong antiseptic solutions applied (mercuric chlorid 1 to 1000; carbolic acid solution 5 per cent.). 


\section{('HRONIC' INFEC'TIOUS DISEASES.}

\section{TUBERCULOSIS OF BIRDS.}

\section{Tuberculosis Avium. Avian Tuberculosis.}

Definition. - A chronic infectious disease of chickens, ducks, pigeons, parrots and ceanaries. It is characterized by a slow, progressive enaciation, which ends in death from exhaustion.

Occurrence. - Avian tuberculosis is a very common disease of chickens and parrots. Oecurring enzoötically among the former, enormous losses to the poultrimen result. During the past few years it has made its appearance in practically all seetions of the Lnited States. In a number of instances entire flocks of birds have succumbed to the disease, or become so inferterl and emaciated that their destruction was necessary. The economic inportance of the disease is not entirely confined to those that die from the disease lirectly, but it has an important bearing on the egg production.

Etiology.- 'This disense is produced by the Bacillus tuberculosis arium. Its biological characteristics are similar in many respects to the Bacillus tuberculosis of other animals (mammalian tuberculosis).

Pathogenesis. - The harilli, picked up with the food, are passerl directly to the intestinal tract, where they pass through the limph follicles into the intestinal wall, either to berome localized at this point, or to pass into the portal vein and liver. Or they may gain acess to the general circulation and through it become distributed to various parts of the horly. It is possible that the intestines may not berome affecterl by the bacilli passing through the walls, but in a great many (ases tubercular nodules are found in this 
location. When the bacilli are taken into the general circulation specifie lesions will be found in the splecn, lungs, joints, etc. 'The large number of norlules found in the intestinal wall derclop in most eases from the infection following along the course of the lymph nodes, or from the baeilli beeoming lodged between the serous and museular coats of the bowel, producing a reactive inflammation and the formation of tubereles. The toxie aetion of the ehemieal products of the bacilli produees a gradual emaeiation, eachexia, and ultimately death.

Natural Infection. - Most cases of tubereulosis in fowls no doubt result from eating foods eontaminated with bowel discharges from affeeted birds. Baeilli are found in the feces of affeeted birds in large numbers, partieularly in those (ases where ulcerations are present on the intestinal mucosa. Another very eommon source of infeetion is when healthy birds eat the organs of those that have sueeumbed to the disease. Carelessness in disposing of dead birds makes this a very common source of infeetion. There is a possibility of birds eontraeting the disease from the feeal diseharges of cattle affected with tubereulosis. This is a disputed point at the present time. 'There is eonsiderable doubt also about their contraeting the disease from the sputum of the human subject. Parrots seem to eontraet the disease from this source. Numerous eases have been reeorded where fowls contracted tuberculosis, and at the same time the attendants liarl the disease. Whether transmission of the disease is brought about in this way is in dispute. Flocks are usually infected from newly aequired tubercular birds. Pigeons may (arry the disease from flock to flock.

Pathology.--The chief anatomieal ehanges are found in the liver, spleen, intestines, joints, and in parrots in the joints, and skin about the head and neck. The surfaee of the liver is covered with numerous small nodules and on cross-section they are found to extend into or through the entire structure. sometimes the tubercles become confluent and form large masses, white, gray, or grayish-white in color, the ecnters of which are caseous. The liver is frequently mueh enlarged, very friable inclicating fatty degeneration. The spleen is 
similarly affected. "The intestinal lesions are very eomnon, and consist of a large number of small nodules found on the mueosa. By confluence large areas appear as do ulcers. On the serous coat of the intestines are found nodules varying in size from a millet seed to a hazel nut. The mesentery may show similar lesions. The abdominal lymph glands are usually enlarged and caseated. In some cases on opening the abdominal cavity a striking picture is presented as the entire visceral peritoneum is eovered by hundreds of very small white or gravish nodules. The lesions in the lungs are similar, although not usually so marked as in the liver and intestines. The affeceted joints are enlarged, contain a eheesy, yellowish mass and the articular eartilages are eroded. 'The other organs and tissues are not often involved, although nodules are found oceasionally in the kidneys, ovaries, testides, pericardium, heart and gizzard. In parrots the disease is usually localized around the head and in the mouth. Jodules varying in size and eondition are found eontaining the same dheesy, rellowish material.

Symptoms. - In the early stages of avian tubereulosis the symptoms are very indefinite. The disease is ehronie and (haracterized by a gradual emaciation without any speeifie rlinical simnptoms. 'The emaciation continues in spite of good appetite. 'l'he appetite persists as a rule until the beginning of a general toxemia, when it diminishes to disappear entirely during the latter stages of the disease. Symptoms of anemia develop as evidenced by paleness of the mucous membranes, (omb and wattles, dulness, and rough phumage. During the latter stage the fowls are listless, much emaeiated, and show all the simptoms of asthenia. Diarrhea sets in, and eomplete rxhanstion is followed in a few days by death. There is eonsiderable variation in the period of time required for the development of the general symptoms. When the bones and joints are affected, there is usually an involvement of the joints of the wings, and the tarsal joints. At first the joint shows swelling which develops gradually. 'The swelling is firm at first, but in nearly all cases beeomes soft and fluetuating, and when opened clischarges a yellow, caseous material. 'The birl is lame and the wings are held pendent. 
In some cases small nodules are found beneath the skin on various parts of the body. Parrots are affected in the majority of cases locally around the eyes, face, or on the neck, or the articulations may be involved. The lesions are small, round, or oval nodules developing on some part of the skin or mucous membrane. Later they become caseous. 'Their characteristics vary somewhat, depending upon whether they arc located in the skin, or in the mucous membrane of the mouth. The nodules when irritated often become quite large, rupture, and leave an extensive ulcerous surface. When the joints are involved the same symptoms are observed as in fowls. General symptoms oceur occasionally in parrots.

Diagnosis. - The diagnosis from the symptoms presents considerable difficulty. However, when we consider that a number of fowls in a flock are affecter with a disease causing cmaciation, exhaustion and death, we should suspect tuberculosis. Several methods of diagnosis have been recommended of which the following have given the best results: (a) An affected bird is killed and an autopsy made. The tubercular lesions are quitc characteristic and the tubercle bacilli may be found under the microscope. (b) By use of avian tubereulin. This method has bcen employed quite cxtensively of late. It has been found quite reliable in most "ases. 'Tle tubereulin is injected, using about 0.003 per hird, into the deeper layers of the skin of the comb or wattles, using a small dental syringe. Carc should be observed that the needlc is not inserted too deeply, or the tuberculin will enter the subcutaneous tissues. On the other hand, it must not be too superficial or the epithelial layer will rupture and allow the tubereulin to escape. The reaction consists in a rounderk swelling which develops in twenty-four to seventytwo lrours at the point of injection. The degree of reaction varies with individual cases.

Differential Diagnosis. - There are scveral conditions whieh might be confused with avian tuberculosis, viz: (a) Asthenia ("going light") which is characterized by gradual emaciation, and cxhaustion, but negative findings on autopsy. Microscopie examination will aid in revealing the difference. External parasites when affecting a number of birds in a 
flock will produce emaciation and a general unthrifty condition. Finding the parasites in large numbers, and negative autopsy will suffice for differentiation. (c) Rheumatic urthritis, or arthritis urica may resemble joint tuberculosis. In rloubtful cases a microscopic examination (tubercle bacilli) may be resorted to.

Prognosis. - I'nfavorable.

Treatment. - No treatment is of any value after the disease has once berome established.

Surgical.-Localized abscesses as they occur in parrots should be incised freely, and the contents thoroughly removed with a curette. 'Tincture of iodin is applied to abscess eavities.

Prevention.-On account of the disease being difficult to liagnose until well established, separation of the sick from the healthy birds is not feasible. In valuable birds the tuberculin test with segregation should be tried. Removing the reactor's will assist in controlling the disease. If this cannot be done, it is recommended that the entire flock be killed, all diseased fowls burned, and the healthy ones sold for food. 'The premises should be thoroughly disinfected, buildings, coops, etc., washed with bichlorid solution (1 to 20(0)(0). 'The soil in the runs should be saturated with liquor cresolis compositus' (3 per cent.).

I new flock of birds should not be obtained before at least six months have elapsed. 'Thoroughness in the cleaning and disinfection is very important if good results are to be experted.

\section{TUBERCULOSIS OF DOGS AND CATS.}

Occurrence and Form.-'Tuberculosis is not very often ohorervel in rlogs and cats. As the pulmonary form is most (")mmon, the infection is probably transmitted to the lungs (n) inhaled particles of dust. In some cases, primary involvemont of tlre digestive tract is found, indicating that the germs were probably taken in with the food. Rarely do we find inferetion has taken place througl any other channel. 
Pathogenesis.-Nost of the cases no doubt result from association with tubercular human beings, or contact with infected rooms, etc. It may also result from ingesting meat containing the bacilli.

Pathology.-Two distinct types are found on necropsy: (i) Pulmonary form, and (b) digestive form.

(i) In the pulmonary form the lungs show caseous foci, or small miliary nodules. A chronic, indurative bronchopneumonia or a chronic interstitial pneumonia is frequently found. Adhesions are common between the lungs and parietal pleura. Aside from these lesions there are very often present edema and emphysema of the lungs, bronchitis and bronchiectasis. The pleura often shows evidence of a serous or serofibrinous pleuritis with considerable fluid present in the thoracic cavity; or in other cases a dry, granular adhesive pleuritis in which the parietal and visceral pleura are adherent. Numerous small nodules are often found on the pleura. The bronchial lymph glands are more or less enlarged.

(b) 'The intestinal lesions are mainly in the mesenterie lr'mph glands, which are enlarged; the walls of the intestines show miliary tuberculosis, particularly on the serous eovering; the liver usually presents similar lesions. The spleen and kidneys often show miliary tuberculosis. The cadaver in general shows evidence of cachexia and emaciation.

Symptoms. - The disease usually runs a chronic course the symptoms developing gradually. General emaciation is apparent in spite of a fairly good appetite, the animals become easily exhausted, are dyspneic (lung form), and weak. After enaciation begins to be a prominent symptom, the form of the disease, whether pulmonary or intestinal, will assert itself. 'The pulmonary form is characterized by a short, dry cough, which later becomes moister and is accompanied by a discharge of a mucopurulent secretion which in most cases is swallowed. The respirations grow labored; dry or moist rales are heard on ausculation. Percussion reveals areas of flatness, and usually hydrothorax. An atypical fever is present during the course of the disease. In the intestinal form the symptoms of chronic intestinal catarrh are most 
promincut with diarrhea during the latter stages. Rapid emaciation is generally followed by death in a short time.

Diagnosis. - Is tubereulosis is not very conimon in dogs and cats, and the symptoms not particularly eharacteristie, a diagnosis is not often made during life. 'The history of the (ase and its ehronicity might lead one to suspect it. 'Tuberculin (0.15 to 0.20$)$ might be used subeutaneously. The reaction is more prompt but the test is not so reliable as in large animals.

Prognosis. - Infavorable.

Treatment. - In advanced cases no treatment should be attempted. 1)uring the early stages good nutritious food, and tonic's are hest. Inlaalations of creolin vapor are recommendable.

\section{GLANDERS.}

In dogrs and eats glanders is not very common. It is occasionally observed among earnivora fed meat or organs from horses affected with glanders. In zoölogical gardens glanders may be enzoötic among meat eating animals, especially when horse meat is fed. Glanders usually assumes an acute form in these animals. 'The early symptoms are those of an interse inflammation of the mucous membrane of the nasal pastages, larynx, trachea, and the conjunetiva. In a short time (two to five days) appears a greenish-gray or bloodstained secretion from the eyes and nose. 'The respirations are labored on accomnt of the intense congestion and swelling of the respiratory mueous membrane. 'The symptoms rapidly become aggravated, the tissues about the head become swollen, nodules appear at different points in the skin, which open and form irregular shaped ulcers. Diarrhea is a promincont symptom. "The animal beeomes emaciated, and sucerumbs in the course of eight to fourteen days. When such symptoms occur, the animal should be isolated so that further spread of the discase is controlled. Thorough disinfection of the premises, and proper disposition of the carlavers are very important. 


\section{PSEUDO-ACTINOMYCOSIS OF DOGS.}

\section{Streptotrichosis Camum. Actinomyces Canis.}

Definition.-A specific risease, caused by the Actinomyces ('allis, (haracterized by an inflammation of serous membranes, and abscess formation of the subcutaneous tissues.

Occurrence.-This disease is not of frequent occurrence in dogs. A few cases have also been noted in cats.

Etiology.-The specific virus, Actinomyces canis, produces lorg rlivided filaments, which are easily stained by the GramWeigert method. Sometimes they form club-shaped bodies. On artificial media the fungus grows at the body temperature.

Pathogenicity. - When pure cultures are injected intraperitoneally into mice, they produce at the point of inoculation nodules of varying size (pea to bean) containing pus. Subcutaneous injections into rabbits cause at the point of injection nodules which form abscesses. The same condition may be produced in dogs by subcutaneous injections.

Pathology. - The lesions usually found are in the pleura or peritoneum. They consist of an exudate of reddish color collected in the body cavity, containing numerous small, white, pin-point nodes. On the serous membrane will be a number of small nodules, and on the pleura fibrinous exudate. 'The lungs often contain a number of pea-sized nodes with caseous centers.

Abscess formation occurs in different parts of the borly, particularly in the subparotid region, the vagina, or in the subperitoneal connective tissue of the pelvis. The abscesses contain a grayish turbid mass in which are many of the characteristic granules. The abscesses usually heal after discharging their contents. 'The spleen, kidneys and heart muscles often show nodular lesions. Arthritis with pus formation is seen in some cases.

Symptoms. - The disease during the early stages does not present any characteristic symptoms. Later, however, there will be evidence of chronic inflammation of the serous membranes with collections of fluid in the thoracic and abdominal cavities. As the disease progresses, tle animal shows general emaciation, weakness, and the presence of 
abscesses in various parts of the body. The inflammation of the lumgs develops graduatly and is not attended by any marked change in temperature. I)spnea is a prominent symptom. The animal gradually grows weaker and dies from exhaustion.

Diagnosis.--The diagnosis is established only after finding the characteristic granules or filaments in the discharge from the abseresses. The symptoms alone would not be sufficient for an accurate diagnosis.

Prognosis. - When the disease shows evidence of general involvement of the body, the prognosis is unfavorable. Nore favorable are those cases of localized infection.

Treatment. - Abseesses should be opened, drained, and the ravity painted with tincture of iorlin. Potassium iorlid (dog (1.0.5) 0.15; (cat 0.00.5-(0.0.5) given once daily is recommended. So further treatment would be of any value. 


\section{CHAPTER V.}

\section{INFEC'TIOUS DISEASES PRODUCED BY PROTOZOA.}

\section{SPIROCHETOSIS OF FOWLS.}

Spirochatosis Avium. Spirillosis of Chickens.

Definition.-A fatal septicemia of chickens, ducks, geese, and pigeons.

Occurrence.-This disease has made its appearance in several countries in enzoötic form. A few outbreaks have been observed in the southern part of the United States. Up to the present time the disease has not attained economic importance in this country. It occurs most commonly in chickens where it seriously interferes with breeding.

Etiology. - The Spirochete gallinarum (Spirillum anserum) is now recognized as the causative agent. This is a blood parasite, probably a flagellated protozoön. It appears as fine threads, 10 to 20 microns long, spirally formed, and provided with one or more cilia which show active movements. These parasites leave the blood rapidly after the fowl's death.

Pathogenesis.-The disease is easily produced by injections of virulent blood from one fowl to another of the same species. Older fowls are less susceptible than younger ones. The disease develops rapidly following inoculation. After the second day larger numbers of the spirillæ are found in the blood.

Natural Infection. - The disease is transmitted by ticks; mainly by the Argas miniatus, in some cases by the Argas persicus and Argas reflexus, and in others by the Ornithodorus moubata. 'The ticks exist in waste places or in wooded districts. Fowls become infested by roosting where the ticks oceur. 'The ticks attach themsclves to the body, and suck 
its blood, thus directly inoculating the fowl. The spirillæ are soon found in the blood.

Pathology. - The most characteristic lesions on neeropsy are enlarged spleen and liver, both organs showing numerous areas of degeneration and neerosis. The heart musele shows evidenee of inflammation. No other lesions of importance are found.

Symptoms. - The period of incubation is from eight to ten day's. Some cases develop in four to six days after the ticks have been placed on the fowl. The early symptoms are those of suppression of appetite, high temperature $\left(108^{\circ}-111^{\circ} \mathrm{F}\right.$.), somnolence, weakness. Later diarrhea sets in; the comb and wattles beeome bluish-red; marked weakness and paralysis. During the later stages of the disease the temperature beeomes normal or subnormal. Death follows in a short time in most cases under symptoms of convulsions and paralysis. The majority of cases are acute; a few have been observed to assume a ehronie type. The eourse of the disease is usually from four to six days in the aeute form and ten to eighteen days in the ehronie.

Diagnosis. - This is made by noting the enzoötie eharacter of the disease; the charaeteristic lesions on autopsy, and the finding of the Spirochetes in the blood of siek birds.

Prognosis.- The prognosis is unfavorable when the disease is well establisherl. Some cases recover.

Trea tment. - I toxyl ( 0.1 per orem for two consecutive doses) has been recommended, and has proved to be highly effieient in produeing immunity from infection in normal fowls. Atoxyl $(0.05)$ as an intramuscular injeetion is recommended for infeeted birds. In two to three days the parasites disappear from the blood. Atoxylate of mercury has also been used in a similar manner. 'This treatment when properly anlministered will produce excellent results.

Immunization of fowls with the blood of siek ehickens, which has been allowed to stand for forty-eight hours, has proved of great value. After this period the blood is no longer infertious and may be injected subcutaneously into h(althy fowls. 'This establishes an immunity' against infections. 
$A$ liorse serum has been produced (by hyperimmunization of the horse with repcated injections of live spirillæ). In doses of 3 to 5 c.e. per bird it has given good results. Immunization of the flock by this method would be recommended as carly as possible.

\section{ENTEROHEPATITIS.}

\section{Blackhead.}

Definition.-A disease peculiar to turkeys. It is occasionally observed in other birds, particularly chickens.

Occurrence.-Blackhead is very prevalent in various sections of the United States, occurring of ten in an enzoötic form destroying whole flocks, and seriously interfering with the raising of turkeys in many districts. It is especially fatal in young birds.

Etiology. - It is produced by a protozoön, Amœba meleagridis. 'This parasite is microscopic in size and is found in large numbers in the liver, ceca and other portions of the intestinal tract. Some authorities claim that a coccidium is the cause of the disease. When coccidia are present, however, the above parasite also coexists which makes it probable that the amoba rather than the coccidia cause the disease.

Pathogenesis.-Blackhead is spread by the bowel discharges of the affected birds which contain the parasites in large numbers. Food and water polluted with infested fecal mattcr are taken into the digestive tract of well birds. When the amoba reach the intestinal tract they develop rapidly, producing irritation to the mucosa and ultimately lead to a marked inflammation. 'The liver as a rule is invaded during the progress of the disease showing intense hepatitis. 'The parasites may enter the liver via the lymphatic system or directly through the bile duct. Intermediate agents (other hirds, sparrows, pigeons, etc.) are often responsible for the transmission of the disease to other flocks.

Necropsy.-The autopsy findings are confined mostly to the liver and intestines. The liver is found much enlarged (often thrce to four times normal), the surface studded with whitish or yellowish spots, which when opened show degener- 
ated areas fillerl with a soft cascated material. On crosssection the liver will show darkened spots often surrounded by degenerated areas, giving it a peculiar spotted or marbled appearance. The organ is soft, friable, easily crushed between the fingers. The infection produces a more or less rapid necrosis of the entire liver substance. The ceca are much enlarged and the mucosa swollen; later cases show ulceration and degeneration of the membrane. Chronic cases exhibit cxtensive ulcerations on various portions of the mucosa. The fecal matter in the lower bowels is mixed with blood, giving it a dark tarry appearance. The scrous membranes (perieardium, pleura, peritoneum, ete.) show evidence of inflanmation and cffusion of fluid. The cavities (thoracic and abdominal) may be partially filled with exudate. In chronic cases dropsical swellings are often found in the cavities and extremities.

Symptoms.-'The disease is most often observed in young turkeys, although in some outbreaks older birds are affected. The early indications of the disease are purplish discoloration of the comb and wattles, dulness, rough plumage, drooping of the tail and wings, eomplete loss of appetite in most cases, and a tendency for the bird to remain in a sitting posture for long periods. During this period there is nearly always a severe diarrhea. The discharges, being greenish-yellow in color, liave a very offensive orlor. The birds gradually grow weaker and become exhausterl in a few days. Death occurs in most cases in three to five days. In less severe, or chronic cases, the birds lose their appetite or it becomes irregular, and they gradually grow emaciated and weak. Some cases will partially recover, and the bird will droop around for several weeks. Other cases recover completely from the disease.

Diagnosis. - This is marle by considering the enzoötic character of the lisease, its symptoms, and autopsy findings. 'There is 110 other clisease common to turkeys producing such serious losses. Whenever the disease is suspected a microserpic examination should be made of the discharges and cecal contents. 
Prognosis.-Owing to the high mortality of the disease the prognosis is unfavorable. Its rapid spread from one bird to another, and to other flocks, makes the disease difficult to control. 'The mortality may reach from 50 to 90 per cent.

Treatment.-Intestinal antiseptics are indicated. Sodium sulphocarbolate $(0.2-0.4)$ two or three times daily has given good results. Also salol in the same doses. Administer sulphatc of iron in the drinking water, using one ounce of the sulphate of iron to one gallon of water. This has proved to be of value as a bowel disinfectant. As soon as an outbreak appears, radical means should be adopted at once to prevent the spread of the disease. Disinfect the coops and houses thoroughly with lysol solution (3 per cent.), or lime solution. Clean up, all droppings two or three times daily and either burn them or bury in lime. Disinfect all (lrinking fountains and feed pans daily. Extreme care must be used by attendants to prevent carrying the infection to other birds or flocks. Good results often follow when these details are carried out conscientiously.

\section{INFECTIOUS DIARRHEA OF CHICKS.}

\section{White Diarrhea.}

Definition.-An infectious disease affecting young chicks, characterized by a severe diarrhea, prostration and high mortality. Owing to the fact that it affects chicks shortly after being hatched, and such large numbers die, it is one of the most important diseases with which the poultryman has to contend. The losses to the poultry industry through its ravages are cnormous. In some districts it is becoming very difficult to rear young chicks.

Etiology. - It is necessary to recognize two distinct etiological factors, viz: (a) A form produced by the Bacterium pullorum, and $(b)$ a protozoal form caused by the Coccidium tenellum. Both of these forms are frequently met with in practice. The reason the conditions should be classified together is because they appear in the same aged birds, produce much the same symptoms, and it has been found 
that the two diseases often coexist in the same ehick. It is necessary to differentiate the two eonditions by examination of the eceal contents for the coecidia, or the liver, spleen and kidneys for the bacterimm.

Necropsy. - In the form produced by the Baeterium pullorum the principal lesions are found in the liver, kidneys, spleen and intestinal tract. 'The liver shows areas of congestion and fatty degeneration. The kidneys are usually normal in size but show some evidenee of congestion and eloudy swelling. 'The involvement of the intestinal traet is as a rule of minor importance. Therefore, but slight ehanges are found in the majority of cases. The other tissues in the body are pale, anemic and show evidenee of emaeiation. In the form produced by the Coccidium tenellum, the most prominent lesions will be present in the intestinal traet. 'The mucosa is congested and distinet uleerations are usually present in the ceca. The liver, kidneys and musculature show similar changes as found in the other form. The intestinal eontents indicate partial or eomplete loss in funetion.

Pathogenesis. - In the baeillary form it is quite evident that the eggs are frequently eontaminated in their formative stage. When latched the infeetion is already present in the rhick. 'This no doubt aeeounts for the large number of ehicks that succumb to the disease so soon after they are hatched. 'This mode of infection is explained by the fact that the laying hens carry the infection in some of the organs, probably the ovaries, which permit the organisms to pass to the eggs during the early stage of their development. 'The bacilli have been fonnd in the yolks of eggs. It is quite possible to lave other modes of transmission of the infection, sueh as the infection being carricd on the outside of the egg shell and when the bird is liberated it beeomes infeeted. Many casies are infected from the water, feed, or diseharges from the diseased rhicks. The infeetion, when introdueed into the delicate digestive tract of the chiek, develops rapidly and interferes with the early digestion of the food. Naturally assimilation is practically stopped and the bird rapidly heromes woak and cmaciated. It has been proved that during the first few days of the chick's life its prineipal 
source of food supply is from the yolk of the egg from which it is hatched. The infection is carried to the other organs by the blood or lymph systems.

The coccidial form develops in a similar manner. Numerous examinations have proved that laying hens are often chronic carriers of the coccidia which are passed out either with the eggs, or with the discharges. The food, water, litter, etc., are contaminated and therefore easily picked up by the chick during the first week or ten days of its life. The coccidia, when introduced into the digestive tract, develop in a few hours and penetrate into the mucosa which accounts for the digestive disturbances. Both forms of the disease result in intestinal irritation, congestion, and disturbed function.

Symptoms. - The early indication of the disease is the presence of a whitish, or whitish-brown, frothy discharge from the bowels. Examination of the chick reveals the soft, pasty mass adhering around the cloaca and on the feathers. 'They are dull, wings held pendent, head held down and more or less constantly emitting a peculiar "peeping" sound. The appetite is suppressed or entirely lost. The chicks rapidly become weak, often fall down when urged to move, and show every cvidence of general cachexia. Death follows often in a few days following hatching. Other cases when infected later often are normal during the first few days or week only to takc the disease in two to four weeks, showing practically the same symptoms. The mortality is 50 to 90 per cent. of the hatch. In order to determine the presence of the bacilli or coccidia, a microscopic examination should be made of the discharges, cecal contents, or cultures taken from the organs.

Diagnosis. - This is not considered difficult as the disease makes its appearance soon after the chicks are hatched, and the fact that a number of them are affected at the same time. 'The symptoms arc also quite charactcristic. There is no similar discase affecting young chicks at this age, except possibly ptomain poisoning. A microscopic examination will assist in making the diagnosis. 
Prognosis.-Due to the character of the disease and the high mortality the prognosis is unfavorable. It will depend to some extent on the age of the chicks and the virulency of the infection.

Treatment.-Dietetic. - Withhold all food from the chicks during the first two or three days. 'They have sufficient food from the rolk of the egg to sustain them for this periorl. Afterward feed small amounts of egg yolk, or bread and milk. Buttermilk or sour milk is highly recommended on account of the acid it contains producing antiseptic action on the bowel contents. A small amount of lactic acid added to sweet milk will have a similar action. Buttermilk tablets are recommended so that a uniform quantity of sour milk may be kept for their use daily. 'These when added to sweet milk bring about sufficient fermentation in twelve to twenty-four hours. It is quite important that they do not receive too much food during the first week to bring on digestive disturbances, thereby lowering the natural resistance of the chick.

Medical.-Zinc sulphocarbolate or sodium sulphocarbolate has given excellent results. It is best given by dissolving the preparation in water and allowing the chicks to drink of the solution freely. Fach chick should receive from three to five grains of the compound one or twice daily. When the diarrhea is severe iron sulphate in the drinking water (30.0 to 4000.0$)$ is beneficial as an antiseptic and astringent.

In the coccidial form the following formula is highly rrecommenterl:

13-Potassi dichromate

Water

250.0

Misce et fiat solutio.

Sig.- C'se one teasponful to 1 quart of water for chicks one week old. Younger chioks lialf the quantity. Allow them to drink of this solution fre.ely.

Prevention.- Much can be done to prevent the disease developing in young rhicks: (a) All incubators should be thoroughly disinfected by washing all parts of them with a livpuor cresolis compositus solution (3 per cent.). Allow them to dry ont thoromglily before using. Another very effective 
method is to close them up tight and introduce formaldehyd gas. Incubators have proved to be common carriers of the infection, therefore attention to this matter is especially important. (b) All eggs selected for hatching should be (leanerl and disinfected in the following manner: Saturate a cloth in 50 per cent. alcohol and wipe each egg carefully before placing it in the incubator. Do not use an excess of alcohol as it will penetrate the egg shell. This treatment will effectively remove all surface infection. (c) All coops, houses, nests, etc., should be disinfected at regular intervals. (d) When possible it would be advisable to select hens as lavers that are free from the disease. This can be done quite efficiently by examining samples of eggs, discharges, agglutination test, etc. (e) All litter from infected houses, coops, etc., should either be burned or treated with lime to prev'nt the perpetuation of the disease.

\section{PIROPLASMOSIS OF THE DOG.}

Infectious Jaundice. Biliary Fever. Malignant Jaundice.

Definition. - A malignant or infectious disease of the dog produced by the I'iroplasma canis or Piroplasma commune.

Etiology. - Piroplasmosis of the dog has been reported in several different countries. It is produced by two species of piroplasma or Babesia: Piroplasma canis and Piroplasma commune. Morphologically these parasites are identical with P'iroplasma bigeminum. 'The Piroplasma canis is 2-4 microns in diameter, the free organisms spherical, and those contained within the corpuscles are pear-shaped or contain many angles. Multiplication is by direct division. 'The P'iroplasma commune is similar, round or pear-shaped. The round type is 1.0-1.5 microns, and the pear-shaped 1.5-2.5 microns in diameter. The former is not transmissible to any other animal while the latter has been transmitted to the guinea pig and cat.

I ogs become infecterl from ticks and fleas (Ixorles ricinus, IRhipicephalus sanguineus, Dermacentor variabilis, Haemaphysalis Leachi, Ctenocephalus canis), which have been found to be common carriers of the disease. Young animals 
(puppies) are most susceptible and of ten an entire litter will develop the disease. Older animals are partially or completely immune. The disease may be readily transmitted hx injecting young animals with virulent blood. 'The initial simptoms develop in two to three rlays. Natural infection takes place from animals coming in contact with fleas or ticks infested with the piroplasm. 'The period of incubation from natural infection is from seven to ten days.

Pathology. - In the acute form the disease process is often so rapid that but little will be found on postmortem examination. The liver is found congested, the bile of dark color and thick. The spleen is enlarged often two or three times its normal size; the color is dark, the borders rounded. 'The kidneys are congested, and small hemorrhages are noted on the surface. 'The heart muscle is pale, and small petechiæ and ecchymoses are observed on the endo- and epicardium. The lungs are usually edematous, and ecchymoses occur on the membranes. 'The bladder contains a reddish-brown colored urine, especially in the very acute form of the disease. The skin and mucous membranes are greenish in color, which in some cases may be absent, the membranes very pale and anemic. In the chronic form the postmortem lesions are those of an anemia, with a paleness of all the tissues and organs. 'The liver is found intensely congested, the bile of a syrupy consistency and very dark in color. 'The blood from the spleen will contain large numbers of the parasites. The kidneys, heart and lungs are congested and show numerous small hemorihages on their surfaces. 'The marrow of the bones is intensely congested, and of a dark reddish rolor. In the rhronic form icteric symptoms are also observed. 'The blood has a thin, watery appearance, the serum practically colorless.

Symptoms. - The initial simptoms of the acute form are those of a severe infectious disease: Depression, loss of appetite, often severe vomiting. Paralysis of the posterior ('xtremities often takes place early (in cighteen to twentyfonr hours) and ascends rapidly producing a general paralysis in three to four days. In the more subacute form the simptoms are less pronounced, presenting all the evi- 
dences of an acute anemia, the mucous membranes becoming pale, colorless, or bluish. 'The most common change.in the mucous membranes, however, is icteric discoloration. A (haracteristic symptom is the blood coloring matter in the urine, which turns it pale red or reddish-brown. The hemoglobin found in the urine is rather high (5 per cent.). 'The temperature in the early stages is elevated $\left(103^{\circ}-105^{\circ} \mathrm{F}\right.$.). It remains elevated usually for twenty-four to forty-eight hours, when it drops suddenly and becomes subnormal. In young (logs the temperature drops very rapidly, as low as $95^{\circ}-96^{\circ} \mathrm{F}$. The pulse is increased, weak and wiry; the respirations 36 to 60 and often labored.

The blood is thin and paler than normal. The number of the red corpuscles is greatly reduced (from 5-7 million to 2 million per e.), the white corpuscles greatly increased (from $6-7000$ to 40,000 per c.).

In the chronic form the symptoms are less pronounced. 'There are evidences of anemia, the animals become weak and indifferent to their surroundings. The mucous membranes are usually pale or yellowish, and sometimes of a bluish color. The urine contains blood-coloring matter, which seens to be a constant symptom. The blood when examined will be found to have a great reduction in the number of red corpuseles, but less than in the acute form, and the white corpuscles are about in the same ratio as in the acute form. Most of the white corpuscles are found to be polynuclear, and are often almost completely filled with the parasites. The course in this form may be long, lasting for dlays or weeks, the animals gradually recovering or dying of marasinus.

Diagnosis. - The diagnosis is made by carefully observing the symptoms, the fact that the disease occurs in several animals at the same time, and the microscopic examination of the bloorl to determine the ratio between the red and white corpuscles, and the presence of the specific parasite. Sometimes it is difficult to find the parasite from a clinical case under the microscope. In such cases it is of advantage to confinn the diagnosis by inoculating a young puppy with some of the blood of the affected animal. 'The virulent 
bhod should be injected into the circulation to obtain the lnost accurate and rapid results.

Prognosis. - In the acute form, the prognosis is very unfavorable, the animals grow weak rapidly, and die from exhaustion in from three to six days. In the chronic form reevery often takes plate after several weeks.

Treatment. - In the aeute form, treatment has but little effect on the course of the clisease. Symptomatic treatment must be used. When there is extreme weakness and subnomal temperature stimulants, such as strychnin sulphate (0.0)(1 subcutaneously), or diffusible stimulants should be given as often as necessary to keep up the eirculation and general condition of the animal. Quinin $(0.3-1.0)$ two or three times daily has been highly recommended.

'The treatment in the chronie form is very similar. Plenty of grood nutritious food should be given to conserve the strength of the patient. 


\section{EQUIVALENTS OF APOTHECARIES IN METRIC MEASURES.}

Cubic

Minims.

1
2
3
4
5
6
7
8
9
10
11
12
13
14
15
16
17
18
20
30
40
50

('entimeters. Fluidrams.

0.061

0.123

0.185

0.246

0.308

0.370

0.431

0.493

0.554

0.616

0.678

0.739

0.801

0.863

0.924

1. 00

1.06

1. 12

1.23

1. 84

2. 46

3. 08
1 .

2

3

4

5

6

7

Fluidounces.

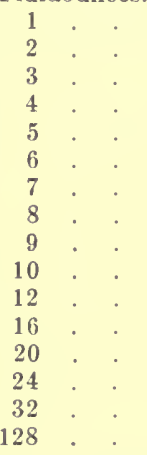

Cubic

Centimeters.

3.7

7. 39

11. 09

15. 00

18.50

22.50

26.00

Cubic

Centimeters.

29.57

59.14

89.00

118.29

148. 00

177.42

207.00

236.59

266.16

295. 73

355.00

473. 17

591.50

710.00

946.35

3785.43

EQUIVALENTS OF APOTHECARIES IN METRIC WEIGHTS.

Grain.
$1-1000$
$1-500$
$1-250$
$1-200$
$1-150$
$1-120$
$1-100$
$1-75$
$1-60$
$1-50$
$1-40$
$1-30$
$1-25$
$1-20$
$1-12$
$1-10$
$1-8$
$1-6$
$1-5$
$1-4$
$1-3$

\begin{tabular}{llll} 
Grams. & \multicolumn{2}{c}{ Grain. } \\
.000065 & $1-2$ & &. \\
.000129 & 1 &. &. \\
.000258 & 2 &. &. \\
.000324 & 3 &. &. \\
.00043 & 4 &. &. \\
.00054 & 5 &. &. \\
.00064 & 6 &. &. \\
.00086 & 7 &. &. \\
00108 & 8 &. &. \\
.00129 & 9 &. &. \\
00162 & 10 &. &. \\
.00216 & 11 &. &. \\
.00259 & 12 &. &. \\
.00324 & 13 &. &. \\
00540 & 14 &. &. \\
00649 & 15 &. &. \\
.0081 & 20 &. &. \\
0108 & 25 &. &. \\
.0129 & 30 &. &. \\
0162 & 35 &. &. \\
.0216 & 40 &. &. \\
& & &
\end{tabular}

Grams.

.0324

.0648

.1296

.1944

.2592

.3240

.3888

.4536

.5184

.5832

.6480

.7128

.7776

.8424

.9072

.9720

1. 2960

1. 6200

1. 9440

2. 2680

2. 2920 


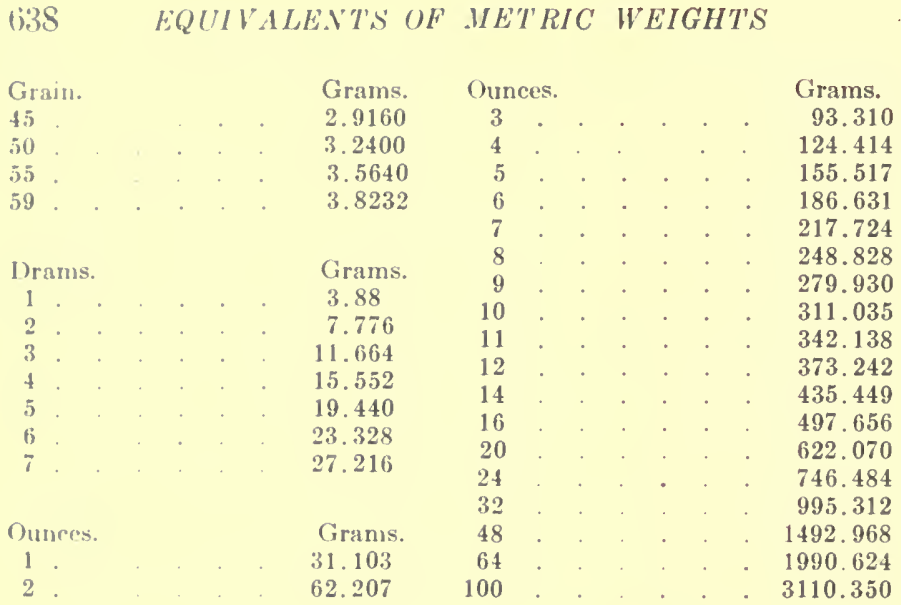




\section{N D E X.}

A

Abscess of cornea, 534

of kidney, 396

of liver, 236

renal, 397

Acne, 468

definition, 468

etiology, 468,469

pathology, 469

prognosis, 469

symptoms, 469

treatment, 469

Actinomyces canis, 623

Adhesions of eyelids, 504,505

ankyloblepharon, 505 treatment, 505

symblepharon, 505 treatment, 505,506

Alopecia, 465

definition, 465

diagnosis, 466 differential, 466

etiology, 465

pathology, 465

prognosis, 466

symptoms, 465,466

treatment, 466

Alveolar periostitis, 108

Amaurosis, 547

Amblyopia, 547

Amyloid kidney, 407

liver, 242

definition, 242

etiology, 242

pathology, 242, 243

symptoms, 243

Anal glands, suppuration of, 223, 224

Anemia, 319

of brain, 437
Anemia of brain, definition, 437

etiology, 437

pathology, 438

prognosis, 438

symptoms, 438

treatment, 438

cerebral, 437

course, 321

definition, 319

diagnosis, 321

etiology, 319,320

occurrence, 319

pathology, 320

prognosis, 321

symptoms, 320,321

treatment, 321, 322

Animal parasites in blood, 330 in kidney, 407

in trachea and bronchial tubes, 42

Ankyloblepharon, 505

Ankylostoma canina, 207

Ankylostomum stenocephalum, 207 trigonacephalum, 207

Anthrax, 599

Aphtha, 100

Aphthæ epizoöticæ, 600

Apoplexia hepatis, 246

Apoplexy, 440

definition, 440

diagnosis, 441

etiology, 440

pathology, 440

prognosis, 441

symptoms, 440,441

treatment, 441

Arthritis, 383

urica, 354

Articular rheumatism, 363

Articulations, dislocation of, 378 caudal vertebræe, 383 
Articulations, dislocations of, cauldal coxofemoral, 381 etiology, 381 prognosis, 381 symptoins, 381 treatment, 382

humero-radio-ulnar, 379, 380 etiology, 380 prognosis, 380 symptoms, 380 treatment, 380 patellar, 382 etiology, 382 prognosis, 382 symptoms, 382 treatment, 382, 383 phalangeal, 381 radio-ulnar-carpal, 380 diagnosis, 380 symptoms, 380 treatment, 380, 381 scapulohumeral, 379 etiology, 379 prognosis, 379 symptoms, 379 treatment, 379 temporomaxillary, 37s etiology, 378 prognosis, 378 symptoms, 378 treatment, 378

(ibiotarsal, 383 vertebral, 379 inflammation of, 38.3 sprains and injuries to, 377 course, 377 prognosis, 377 symptoms, $37 \mathrm{~T}$ treatment, 377 wounds of, 376

diagnosis, 376

rtiology, 376

prognosis, 376

symptoms, 376

treatment, 376,377

Inciarilar, 201

Iscites, 255

definition, 257

diagnosis, 259, 260)

refislogy, 257, 258

pathology, 2.5s

prognosis, 260

-ymptoms, 2.58, 259
Ascites, trealment, 260, 261

Asperyillosis, 40

Asthenia, infectious, of birds, 191 definition, 191 diagnosis, 191, 192 etiology, 191 pathology, 191 prevention, 192 prognosis, 192 symptoms, 191 treatment, 192

Atroplyy of liver, 240 definition, 240 diagnosis, 241 etiology, 240,241 pathology, 241 prognosis, 241 symptoms, 241 treatment, 241 of optic nerve, 547 definition, 547 prognosis, 547

Auditory nerve, paralysis of, 453 Avian tuberculosis, 616

\section{B}

BaLANitis, 265

Basedow's disease, 343

Belascaris marginata, 202 myxtax, 202

Benign neoplasms of mouth, 102 tumors of mammary glands, 317 fibromata, $31 \overline{7}, 318$

Biliary fever, 633 lipoma, 318

Black tongue, 579

Blackhead, 627

Bladder, calculi in, 420

catarrh of, 416

cliseases of, 410 examination, 410, 411, 412 by laparotomy, 411

by palpation, 410,411 of urine, 411,412

incontinence of urine in, 416 parasites in, 42:3, 424

retention of urine in, 414

rupture of, 412

torsion of, 420

tumors in, 423 
Bladder, wounds of, 412

Blennorrhea, 512

13lepharitis, 498

definition, 498

etiology, 498

prognosis, 498

syinptoms, 498

trcatment, 498, 499

Blcpharoptosis, 501

Blcpharospasm, 503

Bothriocephalus felis, 197

Brachial plcxus, paralysis of, 455

Brain, anemia of, 437

congestion of, 435

diseases of, 431

examination, $432,433,434,435$

psychic disturlances, 433

sensibility, 4333, 434, 435

general eonsiderations, 431,432 cercbellum, 432

cortex, 431, 432

midbrain, 432

hyperemia of, 435

tumors of, 441,442

Brittleness of bones, 362

Bronchial catarrh of birds, 38

tubes and trachea, animal parasites in, 42

Bronchitis, 38

and trachcitis, 34

aeute, 34

chronie, 44

13ronchocele, 336

Bronchopneumonia, 53

definition, 5:3

diagnosis, 56

etiology, 53, 54

pathology, 54, 55

prognosis, 56

symptoms, 55, 56

treatment, 56

l3ulbar paralysis, infectious, 611

\section{C}

Calcull ill bladder, 420

ctiology, 420, 421

forms and varieties, 421

acid urine calculi, 421

alkaline urine calculi, 421

prognosis, 422

symptolns, 421,422

41
Caleuli in bladder, treatment, 422 , 423

in kidney, 402

definition, 402

diagnosis, 403

etiology, 402

pathology, 402

prognosis, 403

symptoms, 402, 403

treatment, 403, 404

in urethra, 427

prognosis, 428

symptoms, 427,428

treatment, 428,429

Canine typhus, 579

Canker, 593

of mouth, 97

Caponizing, 276, 277, 278

Carcinomata of bladder, 423

of eyelids, 509

of mammary glands, 318

of penis and prepuce, 269

of serotum and testes, 273

Caries of teeth, 109

definition, 109

etiology, 109

pathology, 109, 110

symptolns, 110

treatment, 110

Castration, 273

of birds, $276,277,278$

of cat, 275,276

of dog, 274,275

eryptorehid, 275

monorehid, 275

Catalepsy, 460

eourse, 460

definition, 460

diagnosis, 460

etiology, 460

pathology, 460

prognosis, 460

symptoms, 460

treatment, 460

Cataract, 542

eourse, 543

definition, 542

forms, 542

eongenital, 543

diabetie, 543

senile, 542

symptomatic, 542

traumatic, $\mathbf{5 4 2}$ 
Cataract, prognosis, 543 symptoms, 543 treatment, 543,544 discission, 543, 544 extraction, 544

Catarrh of bladder, 416 definition, 416 diagnosis, 419 etiology, 416,417 pathology, 417, 418 prognosis, 419 symptoms, 418, 419 treatment, 419, 420 bronchial, of birds, 38 definition, 38 diagnosis, 39 etiology, 39 prognosis, 39 symptoms, 39 treatment, 39, 40 chronic, of stomach, 146 of crop in birds, 137 definition, 137 diagnosis, 138 etiology, 137, 138 prognosis, 138 symptoms, 138 treatment, 138, 139 nasal, acute, 19 definition, 19 diagnosis, 19 etiology, 19 prognosis, 19 symptoins, 19 treatment, 19,20 chronic, 23. definition, 23 diagnosis, 24 etiology, 23 pathology, 23 prognosis, 24 symptons, 23,24 treatment, 24 of rabbits, 20 infectious, 20 definition, 20 diagnosis, 21 etiology; 20, 21 necropsy, 21 prognosis, 21 prophylaxis, 22 symptoms, 21 treatment, 21
Catarrh, nasal, of rabbits, parasitic, 22 definition, 22 diagnosis, 23 etiology, 22 prevention, 23 prognosis, 23 symptoms, 22 treatment, 23

preputial, 265 definition, 265 etiology, 265, 266 prognosis, 266 symptoms, 266 treatment, 266 simple, of stomach, 142

Catarrhal conjunctivitis, 511 pneumonia, 53 stomatitis, 94

Caudal vertebræ, dislocation of, 383

Cerebral anemia, 437 hemorrhage, 440

Cestoda, 193, 194, 195 species, 195 tæniæ, 195 in intestine of birds, 198 of cats, 197 of dogs, 195, 196, 197 of rabbits, 197,198

Chalazion of eyelids, 507

Chicken-pox, 593

Choanotænia infundibuliformis, 198

Cholelithiasis, 245

symptoms, 245 trcatment, 245

Cholera, fowl, 584 gallinarum, 584

Chorea, 461

definition, 461 diagnosis, 461 etiology, 461 pathology, 461 prognosis, 461 symptoms, 461 treatment, 461

Chronic catarrh of stomach, 146 coryza, 23 dilatation of stomach, 154 dyspepsia, 146 enteritis, 173 gastritis, 146 interstitial hepatitis, 243 pneumonia, 57 
('hronie metritis, 296

nasal catarrh, 23

peritonitis, 255

pharyngitis, 125

rhinitis, 23

traeheit is and bronchitis, 44

('irrhosis of liver, 243

definition, 243

diagnosis, 244

etiology, 243

pathology, 243

prognosis, 244

symptoms, 243,244

treatment, 244

of lungs, 57

definition, 57

diagnosis, 58

etiology, 57, is 8

pathology, 58

prognosis, 58

symptoms, $5 \mathrm{~s}$

treatment, 58

(ittotænia dentieulata, 197

Coeeidia in kidney, 409

Coceidium tenellum in kidney, 409

Comb disease, 484

white, 484

Compression of peripheral nerves, 450

of spinal cord, 448

definition, 448

diagnosis, 449

etiology, 448,449

pathogencsis, 449

prognosis, 449

symptoins, 449

treatment, 449

('oneha, uleeration of, 488

Concussion of spinal cord, 445

course, 447

diagnosis, 447

etiology, 445

pathogenesis, 446

prognosis, 447

symptoms, 446,447

treatment, 448

('ongenital defeets of iris, 539

inalformations of penis and prepuce, 265

of urethra, 425

of vagina and vulva, 309

('ongestion of brain, 435

of kidney, 387
Congestion of liver, 231

active, 231

eourse, 232

definition, 231

diagnosis, 232

etiology, 231

pathology, 231

prognosis, 232

symptoms, 232

treatment, 232, 233

passive, 233

definition, 233

diagnosis, 234

etiology, 233

pathology, 233, 234

prognosis; 234

symptoins, 234

treatment, 235

of lungs, 49

active, 49

diagnosis, 50

etiology, 49

pathology, 49

prognosis, 50

symptoms, 49,50

treatment, 50

passive, 50

diagnosis, 51

etiology, 50, 51

pathology, 51

prognosis, 51

symptoms, 51

treatment, 51,52

of mammary glands, 316

of thyroid glands, 334,335

Conjunctiva, corrosions and burns of, 521

diseases of, 510

examination, 510

foreign bodies in, 519,520

growths on, 522

traumatic lesions of, 519

tumors on, 522

ulceration of, 521

wounds on, 520

Conjunctivitis, 510

definition, 510

forms, 510

eatarrhal, 511

etiology, 511

prognosis, 511

symptoms, 511

treatment, 511,512 
Conjunctivitis, forms, croupeus, Corrosions and burns of conjunc516

(lefinition, 516

etiology, 516

progmosis, 516

symptoms, 516

treatuent, 516

ervipelatous, 514

exunthematous, $51 \mathrm{~s}$

definition, 518

trentment, 519

follicular, 517

definition, 517

etiology, 517

prognosis, 517

symptoms, 517

treat ment, 517,518

parenchymatous, 514

definition, 514, 515

(ctiology, 515

prognosis, 515

symptoms, 515

treatment, 515, 516

purulent, 512

aeute, 512

definition, 512

etiology, 512

prognosis, 51:3

symptoms, 512,513

trontment, 513, 514

cluronic, 514

proguosis, 514

symptons, 514

treatment, 514

Constipation, 18:

definition, 182

diagnosis, 18:3

rtiology, 182

patlrolngy, 18:2

prognosis, 18:3

symptoms, 182, 18:3

treatment, 18:3, 184, 18;

('ontagious cpithelioma, 593

(oruea, abscess of, 5;34

clikeases of, 526

('xamination, 52()

rectasia of, 5337

foreign bodies of, .335

opacities of, .5.36

tumors of, $53 \mathrm{~s}$

uleeration of, 5i32

wounds of, 5i35

('ornua uteri, torsion of, 302

tiva, 521

treatment, 521

Coryza, 19

elironic, 19

C'oxofemoral disloeation, 381

('ranial bones, fracture of, 368

Crop, eatarrh of, in birds, 137

hard, 139

obstruction of, in birds, 139 soft, 137

Croupous conjunctivitis, 516 enteritis of cats, 190

pharyngitis of birds, 127

Ctcuocephalus canis of dog, 472, (i.33

felis of cat, 472

Cuterebra emaseulator, 273

Cyclitis, 540

Cystic goiter, 339

kidney, 405

Cysticcrcus eellulosa in kidney, 409

Cystitis, 416

Cysts of ear, 494

of eyelids, 507

Meibomian, 507

piloscbaceous, 507

of iris, 541

of ovaries, 284

retention, in mouth, 103

of uterine tubes, 291

D

DACRYOCYSTITIS, 525

definition, 525

prognosis, 525

symptoms, 525

trcatinent, 525

Dandruff, 464

definition, 464

diagnosis, 464

etiology, 464

prognosis, 464

symptoms, 464

treatment, 464, 465

Davainea cesticillus, 198

erassula, 198

echinobothrida, 198

proglottina, 198

tetragona, 198

Deafness, 495 
De:afness, etiology, 495

prognosis, 495

symptoms, $49 \overline{5}$

treatment, 495

Defects, congenital, of iris, 539 aniridia, 539

coloboma, 539

cetopie pupillae, 539 ocelusion of pupil, 539

Demodex follieulorum, 480 mite, 480

Dermacentor variabilis, 633

Dermatitis, 466 definition, 466 diagnosis, 468 etiology, 466, 467 pathology, 467 prognosis, 468 symptoms, 467,468 treatment, 468

Dermatomycosis, 482 definition, 482 examination, 482

Diabetes, 347 definition, 347 insipidus, 347 eourse, 349 definition, 347 diagnosis, 349 etiology, 347, 348 occurrence, 347 pathology, 348 prognosis, 349 symptoms, 348, 349 treatment, 349,350 mellitus, 350 course, 351 definition, 350 diagnosis, 351 etiology, 350 oceurrenee, 350 pathology, 350 prognosis, 351 symptoms, 350, 351 treatment, 351,352

Diaphragmatic hernia, 563 Diarrhea, $17 \mathrm{~s}$ definition, 178 diagnosis, 181 etiology, 178, 179, 180) infeetious, of chicks, 629 definition, 629 diagnosis, 631
Diarrhea, infectious, of chicks, etiology, 629, 630 necropsy, 630 pathogenesis, 630,631 prevention, 632,633 prognosis, 632 symptoms, 631 treatment, 632 prognosis, 181 symptoms, 180 treatment, 181 white, 629

Dieranotænia sphenoides, 198

Dilatation and divertieula of esophagus, 136

definition, 136

diagnosis, 137

etiology, 136, 137

prognosis, 137

symptoms, 137

treatment, 137

of stomach, 152

acute, 152

diagnosis, 154

etiology, 152, 153

pathology, 153

prognosis, 154

symptoms, 153, 154

treatment, 154

chronie, 154

diagnosis, 157

etiology, 154, 155

pathology, 155, 156

prognosis, 157

symptoins, 156

treatment, 157

Dioctophyme renale, in bladder, 423 in kidney, 407

Diphtheria, 593

Dipylidium caninum, 195

Dirofilaria immitis in blood, 330

Disloeation of articulations, 378

Dispharagus nasutus in stomach of birds, 163

spiralis in stomaeh of birds, 163

Distemper of cats, 577

definition, 577

diagnosis, 578

etiology, 577

pathology, 577

prognosis, 578

symptoms, 577, 578

treatment, 578, 579 
Distemper of dogs, 565 definition, 565

diagnosis, 572,573

ctiology, 565,566

natural infection, 566

neeropsy, 566,567

occurrence, 565

prevertion, 577

prognosis, 573

symptoms, 567, 568, 569, 570, 571,572

treatment, $573,574,575,576$

rabbit, 20

Districhiasis, 504

Diverticula of esophagus, 136

Dochmius trigonacephalus, 207

Dog plague, 579

Dropsy of kidney, 405

definition, 405

diagnosis, 406

ctiology, 405, 406

pathology; 406

prognosis, 406

symptoins, 406

treatment, 406

of pericardium, 76

Dyspepsia, acute, 142

clironic', 146;

Dystocia, 305

definition, 305

diagnosis, 307

etiology, 305, 306

prognosis, 307

symptoms, 306, 307

treatment, $307,30 \mathrm{~s}$

\section{E}

Eate, diseases of, 487 exumination, 487

fistula, 495

etiology, 49.5

prognosis, 496;

symptons, 496

treatment, 496

neoplasnis of, $49: 3$

parasites in, 494

wounds of, 487,488

Fohinococecus granulosus, 197

Johimorlıynchus canis, 202

Filampin, $4(i) 1$

definition, $46 ; 1$ liclampsia, diagnosis, 462

etiology, 462

prognosis, 462

symptoms, 462

treatment, 462

Eetasia of eornea, 537

inflammatory, 537

forms, 537

kerateetasia, 537 definition, 537, $53 \mathrm{~s}$

treatment, 538

staphyloma, 537 definition, 537

treatment, 537

non-inflaminatory, 538

forms, 538

keratoeonus, 538

keratoglobus, 538 treatment, 538

Eetropion, 500

Eezema, 469

definition, 469

diagnosis, 471

etiology, 470

pathology, 470

prognosis, 471

symptoms, 470

treatment, 471

Edema of lungs, 52

pulmonary, 52

Eimeria avium in kidney, 409

Empyema of infraorbital fossæ of birds, 20 definition, 20 etiology, 20 prognosis, 20 symptoms, 20 treatment, 20

Endoearditis, acute, 85 definition, 85

diagnosis, $\$ 8$

etiology, 85, 86

necropsy, 86,87

prognosis, 88

symptoms, 87,88

treatment, 88,89

Enophthalmus, 551

definition, 551

etiology, 551

prognosis, 552

symptoms, 551

treatment, 552

Enteritis, 167 
Enteritis, acute, 167 definition, 167,168 diagnosis, 171 etiology, 168, 169 pathology, 169 prognosis, 171 symptoms, 169, 170, 171 treatment, $171,172,173$ chronic, 173

definition, 173

diagnosis, 174,175

etiology, 173

pathology, 173, 174

prognosis, 175

synnptoms, 174

treatment, 175

eroupous, of eats, 190

definition, 190

diagnosis, 190

etiology, 190

pathology, 190

prognosis, 190

symptoms, 190

treatment, 191

membranous, 190

Enterohepatitis, 627

definition, 627

diagnosis, 628

etiology, 627

neeropsy, 627,628

occurrence, 627

patlrogenesis, 627

prognosis, 629

syniptoms, 628

treatment, 629

Enterorrhagia, 175

Entropion, 499

Epilepsy, 458
definition, 458
diagnosis, 459
etiology, 458
pathology, 458
prognosis, 459
reflex, 459,460
treatment, 460
seeondary, 459,460
symptoms, 459
treatment, 459
Epiphora, 524
Epistaxis, 24
definition, 24
etiology, 24,25
prognosis, 25

Epistaxis, symptoms, 25 treatinent, 25

Epithelioma, contagious, 593

Epitheliomata of eyelids, 509 of mouth, 104

of penis and prepuee, 269

of pharynx, 129

Epitheliosis infectiosa avium, 593 diagnosis, 598

etiology, 593 natural infeetion, 594,595 pathogenesis, 593,594 pathology, 595, 596 prevaleney and distribution, 593

prevention, 599

prognosis, 599

symptoms, $593,597,593$

treatment, 599

Esophagismus, 135

definition, 135

etiology, 135

prognosis, 135

symptoms, 135

treatment, 135

Esophagitis, 130

course, 131

definition, 130

etiology, 130

pathology, 130, 131

prognosis, 131

symptoms, 131

treatment, 131

Esophagus, dilatations of, 136

diseases of, 130

examination, 130

diverticula of, 136

foreign bodies in, 131

neoplasms of, 141

obstruetion in, 131

strieture of, 135

Eustrongylus gigas, 407

Eversion of uterus, 300

diagnosis, 301

prognosis, 301

symptoms, 301

treatment, 301,302

Exanthematous conjunetivitis, 518

Exophthalmic goiter, 343

Exophthalmus, 550

definition, 550

etiology, 550

prognosis, 550 
Exophthlahnus, svmptoms, 500 treatiremt, 5io)

Fye, parasites of, 553

Eyeball, luxation of, 550)

Eyclidls, allhesions of, 504,506 diseases of, 497

examination, 497

inflarnmation of, 498

malposition of, 499

tumors of, 506

wounds of, 497,498

\section{F}

FacetTed keratitis, 529

Farial nerve, paralysis of, 450

Fatty liver, 241

definition, 241

etiology, 241, 242

pathology, 242

symptoms, 242

Favis, 484

diagnosis, 48.5

etiology, 484

pathology, 484

prognosis, 485

svmptons, 484, 485

treatment, 48.5

Featler eating in bircls, 357

ct iology, 357

symptoms, 357 treatment, $357,35 \mathrm{~s}$

pulling in lirds, 357

Fenoral hernia, 562

lemur, fracture of, 374

Fetid stomatitis, 96

Fibromata of bladder, 42:3

of mammary glands, 317, 318

of mouth, 102

of scrotum and testes, 272,273

of uterus, 303, 304

of vulva and vagina, 313

Fïbrous goiter, 346)

Filaria immitis in bloorl, 330

Filariclat, 3:30

Fïstula, ear, 495

salivary, 118

refinition, His

diagnosis, 119

(tiology, 11s

jatloology, ins

progunsis, 119
Fistula, salivary, symptoms, 118, 119

treatment, 119

Fleas, 472

description, 472

diagnosis, 473

occurrenee, 472

Ctenoeephalus eanis of $d \mathrm{log}$, 472

felis of eat, 472

Pulex avium of bird, 472

gonivcephalus of rabbit, 472

irritans of man, 472

symptoms, 472,473

treatment 473

Follieular eonjunctivitis, 517

Foot-and-mouth disease, 600

Foreign-body pneumonia, 58

Foreign bodies and wounds of eornea, 535

prognosis, 536

symptoms, 535

treatment, 536

in eonjunetiva, 519,520

in esophagus, 131

diagnosis, 133

etiology, 131, 132

prognosis, 133

symptoms, 132, 133

treatment, 1333, 134, 135

in mouth, 106

symptoms, 106

treatment, 106

in pharynx, 126

diagnosis, 126

prognosis, 126

symptoms, 126

treatment, 126, 127

in stomach, 148

diagnosis, 149, 150

etiology, 148, 149

prognosis, 150

symptoins, 149

treatment, 150, 151, 152

Fowl cholera, 584

definition, 584

(liagnosis, 587, 588

etiology, 584

natural infeetion, 585

occurrence, 584

pathogenesis, 584, 585

pathology, 586 
Iowl cholera, prevention, 5s9 prognosis, 588 symptoms, 587 treatment, 588, 589 pest, 589

definition, 589

diagnosis, 591

etiology, 590

natural infection, 590

occurrence, 589,590

pathogenicity, 590

pathology, 590,591

prognosis, 592

symptoms, 591

treatment, 592

plague, 589

typhoid, 584

Iracture of bones, 368

cranial, 368

etiology, 368

prognosis, 369

symptoms, 368,369

treatment, 369

femur, 374

diagnosis, 375

etiology, 374

prognosis, 375

symptoins, 375

treatment, 375

humerus, 372

diagnosis, 372

etiology, 372

prognosis, 372

symptoms, 372

treatment, 372

inferior maxilla, 369

etiology, 370

prognosis, 370

symptoms, 370

treatment, 370

metacarpal and phalangeal, 373

diagnosis, 373

treatment, $37: 3$

patella, 375

pelvis, 373,374

etiology, $: 374$

progimosis, 374

symptoms, 374

treatment, 374

radius and ulna, 372,373

etiology, $37: 3$

syinptoms, 373
Fracture of bones, radius and ulna, treatment, 373

ribs, 371

etiology, 371

prognosis, 371

symptoms, 371

treatment, 371

scapula, 371

etiology, 372

symptoms, 372

treatment, 372

tibia and fibula, 375

vertebræ, 370

etiology, 370

prognosis, 371

symptoms, 370,371

treatment, 371

of orbit, 553

treatinent, 553

of teeth, 108

treatment, 108

\section{G}

Gali-stones, 245

Gangrene of lungs, $5 \mathrm{~S}$

of tongue, 112

definition, 112

diagnosis, 113

etiology, 113

pathology, 113

prognosis, 113

symptoms, 113

treatment, 113, 114

Gangrenous glossitis, 112 stomatitis, 97

Gapes in chickens, 42

Gastritis, 142

acute, 142

definition, 142

diagnosis, 144

etiology, 142, 143

pathology. 143

prognosis, 144

symptoms, 143

treatment, 144, 145, 146

chronic, 146

definition, 146

diagnosis, 147

etiology, 146

pathology, 146

prognosis, 147 
Gisstritis, ehronic, symptoms, 146 treatment, 147,148

(instro-enteritis, hemorrlagic, 579 infectious, 600

Gustrophilus intestinalis in stomach of $\log , 163$

(ilanders, 622

(ilands of Moll, enlargement of, 508 sub)nuxillary and sublingual, dise:ises of, 118 symptoms, 118 treatinent, 118

(ilaucoma, 549 defuition, 549 prognosis, 549 symptoms, 549 treatment, 549

Globe and orbit, diseases of, 548

(ilossitis, 111

definition, 111

etiology, 111, 112

gangrenous, 112

pathology, 112

prognosis, 112

symuptoms, 112

treatment, 112

Goiter, 3:36

eystir, 339

definition, 339

diagnosis, 339

prognosis, 339

symptoms, 339

treatment, 339, 340

definition, 33:36

exophthahnic, 343

definition, 343

(liagnosis, 345

etiology, 34:3

pathogenesis, 343

pathology, 34:3

prognosis, 345

symptoms, 34

treaturent, 3.4.5

fibrotis, 340

Arfinition, 340

dianguosis, 340

prognosis, 340, 341

symptous, 3:40)

treatment, $3 \$ 1$

maliguant, 342

defunition, 342

diagnosis, 3.42, 3.43

prognosis, 343
Goiter, malignant, symptoms, 342 treatment, 343

occurrence, 336

parenchymatous, 336

definition, 336

diagnosis, 337,338

occurrence, 336

prognosis, 338

symptoms, 336, 337

treatment, 338

vascular, 341

definition, 341

diagnosis, 341,342

prognosis, 342

symptoms, 341

treatment, 342

Gout, 354

course, 356

definition, 354

diagnosis, 356

etiology, 354,355

occurrenee, 354

pathogenesis, 355

pathology, 355

symptoms, 355,356

treatment, 356,357

Granulomas of eyelids, 510

Graves's disease, 343

\section{H}

HemapHYSALIS LEACHI, 633

Hæmostrongylus vasorum in blood, 332

Hard crop, 139

Heart, diseases of, 78

hypertrophy and dilatation of, 89 rupture of, 92

Helminthiasis, 193

Hematemesis, 160

definition, 160

diagnosis, 161, 162

etiology, 160

pathology, 160, 161

prognosis, 162

symptoms, 161

treatment, 162

Hematoma, 489

diagnosis, 490

etiology, 489

prognosis, 490

symptoms, 489, 490 
IIcrnatoma, treatment, 490

Henratozoön Lewisi in blood, 332

Hemopericardium, 77

definition, 77

etiology, 77

symptoms, 77

treatment, 77

Henophilia, 328

Hemorrhage, eerebral, 440

intestinal, 175

definition, 175, 176

diagnosis, 177

etiology, 176

prognosis, 177

symptoms, 176,177

treatınent, 177

Hemorrhagic gastro-enteritis, 579 septieemia of eats, 600

diagnosis, 602

etiology, 600

occurrence, 600

patlogenesis, 601

pathogenicity, 600

pathology, 601

prognosis, 602

symptoms, 601

treatment, 602

IIemorrhoids, 216

definition, 216

diagnosis, 217

etiology, 216

pathology, 216

prognosis, 217

syinptoms, 216

treatinent, 217

IIemothorax, 67

definition, 67

diagnosis, 68

etiology, 67

pathology, 67

prognosis, 68

symptoms, 67

treatment, 68

Ifepatitis, 235

clronic interstitial, 243

definition, 235

diagnosis, 236

etiology, 235

pathology, 235

prognosis, 236

suppurative, 236

definition, 236,237

diagnosis, 239
Hepatitis, suppurative, etiology, 237

pathology, 237, 238

prognosis, 239

symptoms, 238, 239

treatment, 239,240

symptoms, 235, 236

treatment, 236

Hernia, 555

definition, 555

diaphragmatic, 563

definition, 563

etiology, 563

symptoms, 563

treatment, 563

femoral, 562

symptoms, 562

treatment, 563

general remarks, 555, 556, 557

hernial contents, 556

ring, 555,556

sac, 555

irreducible hernias, 556, 557

reducible hernias, 556

inguinal, 560

in female, 560

etiology, 560

symptoms, 560

treatment, 561

in male, 561

etiology, 561

symptoms, 561,562

treatment, 562

oeeurrence, 555

perineal, 563

definition, 563

etiology, 563

symptoms, 563, 564

treatment, 564

scrotal, 561

treatment, 562

umbilical, 557

etiology, 557

symptoms, 557

treatment, 557,558

ventral, 558

definition, 558

etiology, 558

symptoms, 558

treatment, 559,560

Herpes tonsurans, 482

diagnosis, 483

etiology, 482 
IIerpes tonsurans, pathology, progumosis, 4\$:3

symptoms, $48: 3$

treatment, 453,484

Heterakiclac, 202

Heterakis eompressa, 203 crassa, 20:3

- differens, 20:3

dispar, 20:3

inflexa, 20:3

lineata, 203

muculosa, 203

papillosa, 202, 203

perspicillum, 203

vesicularis, 202, 203

Hodgkin's disease, 327

Honeycomb, ringworm, 4S4

Hookworm, 206

IIumero-radio-ulnar dislocation, 379,350

Humerus, fracture of, 372

Hydrometra of uterus, 304

Iydronephrosis, 405

IIydropericardium, 76 definition. 76

etiology, 76

symptonis, 76

treatinent, 76

Hydrophobia, 603

Hydrophthatmus, 550

Ilefinition, 5050

treatument, 550)

Hydrops aludominis, 2.57

ascites, 257

peritonei, 257

IIydrothorax, 6j

(lefinition, 6.5

diagnosis, 66

ctiology, 65)

pathology, 65

jorognosis, 66 ;

symptoms, 6 f)

troutment, 16

Hyprermia. 387

ancuter, $3 \times 7$

(+tiology, :357

pit hology, 387. 335

proguosis, $38 \mathrm{~s}$

symptoms, 385

troatment. 38s

of lorinin, 4.35

lefinition, 135

diagnosis, $4: 36$
482 Hyperemia of brain, etiology, 435 pathology, 435. 436

prognosis, 436

symptoins, 436

treatment, 436.437

of lungs, 49

passive, 388

etiology, 388

pathology, 388

prognosis, 388

symptoms, 388

treatment, 388

Hypertrophy and dilatation of lieart, 89

definition, 89

diagnosis, 91

etiology, 89.90

necropsy, 90

j)rognosis, 91

symptoms, 90,91

treatment, 91

of prostate gland, 280

\section{I}

ICTERUS, 225

definition, 225

diagnosis, 229

etiology, 225, 226

patliology, 226, 227

prognosis, 229

symptoms, 227, 228, 229

treatment, 229,230

Incontinence of urine in bladder, 416

definition, 416

etiology, 416

prognosis, 416

symptoins, 416

treatment, 416

Inerustations of tartar, 108

Infectious asthenia of hirds, 191

bulbar paralysis, 611

diarrhea of chicks, 629

gastro-enteritis, 600

j:unnclice, 6:3:3

Jeukemia of ehickens, 325

nasal catarrl of rabbits, 20

Inferior maxilla, fracture of, 369

Inflamnation of eyelids, 498

of kidneys, 388

of membrana nictitans, 522 
Inflammation of membrana nictitans, examination, 522 prognosis, 523

symptoms, 522,523 treatment, 523

of orbit, 553

of ovaries, 283

definition, 283

diagnosis, 284

etiology, 284

prognosis, 284

symptoms, 284

treatment, 284

of renal pelvis, 398

of synovial membrane and articulations, $38: 3$

definition, 383

etiology, $38: 3$

prognosis, 38:3

symptoms, 38.3

of urethra, 429

treatment, 383,384

Inflummatory eetasia of cornea, 537 forms, 537 kerateetasia, 537 staphyloma, 537

Influenza, rabbit, 20

Infraorbital foss:e of lirds, empyema of, 20

Inguinal hernia, 560

in female, 560

in male, 561

Injuries of peripheral nerves, 450 of spinal eord, 445

Insuffieiency, valvular, 78

Interstitial hepatitis, 243 ehronic, 243

pneumonia, 57

chronic, 57

Intestinal hemorrhage, 175 obstruetion, 182

Intestines, diseases of, 167 examination, 167

parasites in, 193

round worms in, 202

of birds, 202, 203

of cat, 202

of dog, 202

of rabbit, 202

strongylidx in, 207

of cat, 207

of dog, 207

of rabbit, 207
Intestines, tænia in, 195

of birds, 198

of cats, 197

of dogs, 195, 196, 197

of rabbits, 197, 198

trichinellidx in, 209, 210, 211

of birds, 210,211

of $\operatorname{dog}, 210$

of rabbit, 210

wounds of, 188

Intussuseeption, 186

definition, 186

diagnosis, 187

etiology, 186

pathology, 186, 187

prognosis, 187

symptoms, 187

treatment, 187,188

Inversion of uterus, 300

Iridoeyclitis, 540

course, 541

definition, 540

etiology, 540

prognosis, 541

symptoms, 540,541

treatment, 541

Iris and ciliary body, diseases of, 539

eongenital defeets of, 539

cysts of, 541

tumors of, 541

Iritis, 540

Itch, 475

red, 482

Ixodes rieinus, 633

\section{J}

JAUNDICE, 225

infectious, 633

inalignant, 633

\section{$\mathbf{K}$}

\author{
Keratectasia, 537 \\ lieratitis, 526 \\ definition, 526,527 \\ non-suppurative, 527 \\ keratitis pigmentosa, 529 \\ definition, 529 \\ etiology, 529
}


Keratitis, non-suppurative, keratitis pigmentosa, prognosis, 529

svimptoms, 529

treatment, 529

punetata profunda, 532 definition, 532

etiology, 532

prognosis, 532

symptoms, 532

treatment, 532

superficialis, 529

course, 530

definition, 529,530

etiology, 530

prognosis, 530

symptoms, 530

treatment, 530

parenehymatous, 530

eourse, 531

definition, 530

etiology, 530, 531

prognosis, 531

symptoms, 531

treatment, 531, 532

superficial, 527

eourse, 527

definition, 527

etiology, 527

prognosis, 527

synnptoms, 527

treatment, 527, 528

viscular, 528

definition, 528

etiology, 528

prognosis, 528

symptoms, 528

treatment, 528

suppurative, 527

abscess of eornea, 534

course, 535

definition, 534

etiology, 534

prognosis, 5.35

symptoms, 534, 535

treatment, 535

keratitis from lagophthalınos, 535

definition, 535

treatment, 535

neuroparalytica, 535

definition, 535

ulceration of eornea, 532
Keratitis, suppurative, ulceration of cornea, course, 533 definition, 532 etiology, 532, 533 prognosis, 533 symptoms, 533 treatment, 534

Keratoeonus, 538

Keratoglobus, 538

Kidney abscess, 396

amyloid, 407

animal parasites in, 407

eoceidia, 409

Coeciclium tenellum, 409

Cysticercus cellulosæ, 409

Dioctophyme renale, 407

Eimeria a vium, 409

Eustrongylus gigas, 407

ealeuli in, 402

congestion of, 387

eystic, 405

diseases of, 385

examination, $385,386,387$

dropsy of, 405

inflammation of, 388

tumors in, 407

$\mathbf{L}$

LaCrimal apparatus, diseases of, 524

examination, 524

Laerimation, 524

definition, 524

etiology, 524

prognosis, 525

symptoms, 525

treatment, 525

Lagoph thalmos, 506

definition, 506

treatment, 506

Laryngitis, 30

acute, 30

definition, 30

diagnosis, 31

etiology, 30

pathology, 30, 31

prognosis, 31

symptoms, 31

treatment, 31

ehronie, 31 
Laryngitis, chronic, definition, 31 diagnosis, 32 etiology, 31 pathology, 32 prognosis, 32 symptoms, 32 treatment, 32,33

Larynx, diseases of, 30 examination, 30

Iens, diseases of, 542 examination, 542 luxation of, 544

Leukemia, 322 course, 325 definition, 322, 323 diagnosis, 325 etiology, 323

infectious, of chickens, 325 course, 326 definition, 325 diagnosis, 326,327 etiology, 325,326 occurrence, 325 pathogenesis, 326 pathology, 326 prognosis, 327 symptoms, 326 treatment, 327

lymphatic, 322

myelogenous, 322

occurrence, 323

pathology, 323

prognosis, 325

symptoms, $323,324,325$ treatment, 325

Lice, 473

description, 473,474

diagnosis, 475

kinds, 474

Linognathus piliferus, 474

Menopum biseriatum, 474 trigonocephalum, 474

Trichodectes latus, 474 subrostratus, 474

prognosis, 475

symptoms, 474,475 treatment, 475

Linognathus piliferus, 474

Lipoma of eyelids, in birds, 508 of mammary glands, 318

Liver, abscess of, 236 amyloid, 242 atrophy of, 240
Liver, cirrhosis of, 243 congestion of, 231 diseases of, 225

- examination, 225 fatty, 241 neoplasms of, 244 rupture of, 246

Lockjaw, 613

Lungs, cirrhosis of, 57 congestion of, 49 active, 49 passive, 50 diseases of, 47 examination, $47,48,49$ auscultation, 47,48 percussion, 49 respiration, 47 thorax, 47

edema of, 52 gangrene of, 58 hyperemia of, 49

Luxation, 378

of eyeball, 550

prognosis, 550

treatment, 550, 551

of lens, 544

treatment, 544

Lymphadenitis, 120

Lyssa, 603

\section{M}

Malfollmations, congenital, of penis and prepuce, 265 of urethra, 425

symptoms, 426

treatment, 426

of vagina and vulva, 309 of teeth, 107

treatment, 107

Malignant goiter, 342 jaundice, 633 neoplasms of eyelids, 508, 509 of mouth, 104

tumors of mammary glands, 318 carcinomata, 318 sarcomata, 318 in nasal passages, 29

Malposition of eyelids, 499 blepharoptosis, 501 blepharospasm, 503 ectropion, 500 
Malposition of eyelids, cetropion, Mange, mites, sarcoptes of cat, definition, 500

etiology, 500

prognosis, 500

simptoms, 500

treatment, 500,501

entropion, 499

definition, 499

etiology, 499

prognosis, 499

symptoms, 499

treatment, 499,500

paralysis of orbicularis nerve, $50)^{*}$

definition, 502

etiology, 502

prognosis, 502, 5033

symutoms, 5 (t)'

treatment, 503

ptosis, 501

(lefinition, 501

ctiology, 501

symptoms, 502

treatment, 502

spism of orlieularis nerve, 503 definition, 50:3 treatment, 50:3

Mammary glandls, congestion of, 316

disenses of, 315

examination, 315

tumors of, 317

wounds and injuries of, 315

. Inmnitis, 3I6

definition, 316

ctiology, 316

prognosis, 317

symptoms, 316,317

treatment, 317

Mange, 47j

definition, 47.)

elemolectic, 4s()

diagmesis, tsi

(tiology, 4si)

pathology, 4so)

prognosis, 18 ]

srmptoms, 48(), 4si

treatument, 4\$1, 482

etiolegy, 47.5, 476

mites, 476

demolex, $1 \mathrm{ing}$

folliculormm, 4\$0

satreoptes, 476

red, 475

$$
477
$$

Sarcoptes minor, var. cati, 477

Notoedres cati, var. cat, 477

of dog, 476,477

Sarcoptes scabici, var. canis, 476,477

of ferret, 477,478

Sarcoptes scabiei, var. hydrochæri, 477,478

of fowl, 478

Cnemidocoptes mutans, var. gallinæ, 478

Sareoptes mutals, 47S of ralbbit, 477

Noterleses cati, var. cuniculi, 477

Sircoptes Ininor, var. cuniculi, 477

sarcoptic, 475

diagnosis, $47 \mathrm{~S}$

pathology, 476

prognosis, 478

treatment, $478,479,480$

Mastitis, 316

Megrim, 457

Meibomian cyst of eyelids, 507

Membrana nietitans, inflammation of, 522

tumors on, 523

wounds of, $52: 3$

Membranous enteritis, 190

Meningo-encephalitis, 438

definition, $43\{, 439$

diagnosis, 440

etiology, 439

pathology, 439

prognosis, 440

symptoms, 439,440

treatment, 440)

Meningonyelit is, 44:3

definition, 44:3

diagnosis, 445

etiology, 444

pathology, 444

prognosis, 445

symptoms, 444,445

treatment, 445

Menopum biseriatum, 476

trigonocephaluin, 476 
Metacarpal and phalangeal bones, fracture of, 373

Metastrongulinæ, 330

Metritis, 293

acute, 293

definition, 293

diagnosis, 295

etiology, 293, 294

pathology, 294, 295

prognosis, 295

symptoms, 295

treatment, 295, 296

chronic, 296

definition, 296

diagnosis, 298

etiology, $296 ; 297$

pathology, 297

prognosis, 298

symptoms, 297, 298

treatment, 298

definition, 293

Moniezia denticulata, 197

Mouth, benign neoplasms of, 102 canker of, 97

diseases of, 93

examination, 93, 94

abnormal conditions noted, 93,94

foreign bodies, 94

mucous membranes, 94 neoplasms, 94 odor, 93 secretions, 93, 94

foreign bodies in, 106 malignant neoplasms of, 104 sore, 96

Multiceps multiceris, 196 serialis, 196, 197

Mumps, 115

Muscular rheumatism, 365

Mycotic pneumonia, 40

Mydriasis, 539, 540 definition, 539

Myocarditis, 82 acute, 82

definition, 82

diagnosis, 84

etiology, 82,83

necropsy, 8.3

prognosis, 84

symptoms, 83,84

treatment, 84

chronic, 84
Myocarditis, chronic, definition, 84 etiology, 85 necropsy, 85

Myomata of uterus, 304

Myosis, 540

definition, 540

\section{$\mathbf{N}$}

NASAL catarrh, acute, 19 chronic, 23

of rabbits, 20, 21

infectious, 20, 21

parasitic; 22

passages, diseases of, 17

examination, 17,18

abnormal conditions noted, 18,19

discharge, 19

foreign bodies, 18

hemorrhage, 18

infectious, 18

malformations, 18,19

parasites, 18

tumors, 18

neoplasms of, 28

benign tumors, 28

papillomata, 28 treatment, 28

polypoid fibromata, 28

treatment, 28,29

malignant tumors, 29

diagnosis, 29

prognosis, 29

symptoms, 29

treatment, 29

parasites of, 25

definition, 25

diagnosis, 27

etiology, 25

life cycle, 26

necropsy, 27

prognosis, 27

symptoms, 27

treatment, 27

Nematoda, 201

species, 201

ascaridæ, 201

hookworm, 206

round worms, 201

in intestine of birds, 202, 
Nematoda, species, round worms, in intestine of cat, 202

of dog, 202

of rablit, 202

strongylidx, 206

in intestine of cat, 207

of $\operatorname{dog}, 207$

of rabbit, 207

trichinellid:e, 209, 210

in intestine of birds, 210 of $\operatorname{dog}, 210$ of rabbit, 210

whipworm, 209, 210

Neoplasms of ear, 493

cysts, 494

papillomata, 493

prognosis, 493

symptoms, 493

treatment, 493

sebaceous tumors, 494 treatment, 491

of esophagus, 141

diagnosis, 141

prognosis, 141

symptoins, 141

treatment, 141

of evelids, 508, 509

of liver, 244

syinptoms, 244

treatment, 244

of mouth, 102

benign, 102

fibromata, 102

symptoms, 103

treatment, 103

osteoma, 103

symptoms, 103

treatment, 103

papillomata, 102

symptoms, 102

treatment, 102

retention eysts, 103 treatment, 104

malignant, 104

epitheliomata, 104

symptons, 104, 105

treatment, 105

sarcoinata, 105

symptoins, 105

treatment, 105

of nasal passages, 28

of pharvin, 125
Neoplasms of pharynx, epitheliomata, 129

symptoms, 129

treatment, 129

polypoid growths, 128

symptoms, 128

treatinent, 128

in rectum, 222

diagnosis, 223

prognosis, 223

symptoms, 222, 223

treatment, 223

in stomach, 165

prognosis, 165

symptoms, 165

treatment, 165,166

Nephritis, 388

acute, 388

course, 392

definition, 388, 389

diagnosis, 391, 392

etiology, 389, 390

pathology, 390

prognosis, 392

symptoms, 391

treatment, 392

chronic, 392

definition, 392, 393

diagnosis, 395

etiology, 393

pathology, 393, 394

prognosis, 395

symptoms, 394, 395

treatment, 396

purulent, 396

definition, 396

diagnosis, 398

etiology, 396, 397

pathology, 397

prognosis, 398

symptoms, 397,398

treatment, 398

Nephrolithiasis, 402

Neuritis, retrobulbar, 546,547

Non-inflammatory ectasia of cornea, 538

forms, 538

keratoconus, 538

keratoglobus, 538

Non-suppurative keratitis, 527

forms, 526,527

keratitis pigmentosa, 529

punctata profunda, 532 
Non-suppurative keratitis, forms, Optic nerve, wounds of, 546 keratitis pigmentos a, Orbicularis nerve, paralysis of, 502 punctata superficialis, 529 spasm of, 503

parenchymatous, 530

superficial, 527

Nystagmus, 552 vascular, 528

definition, 552

treatment, 553

\section{o}

OBEsITy, 352

course, 353

definition, 352

etiology, 352, 353

pathology, 353

prognosis, 353

symptoms, 353

treatment, 353,354

Obstipation, 182

Obstruction of crop in birds, 139

definition, 139

etiology, 139

prognosis, 140

symptoms, 139, 140

treatment, 140, 141

in esophagus, 131

intestinal, 182

Occlusion of rectum and anus, 212, 213

artificial, 213

congenital, 212, 213

diagnosis, 213

prognosis, 213

symptoms, 213

treatment, 213,214

of urethra, 425

Ollulanus tricuspis in stomach of cat, 163

Oöphorectomy, 285, 286

in cat, 289

in dog, 286, 287, 288, 289

Oöphoritis, 283

Opacities of cornea, 536 prognosis, 536 treatment, 536, 537

Optic nerve, atrophy of, 547 diseases of, 546 hemorrhages of, 546 inflammation of, 546 tumors of, 546

Orbit, fracture of, 553 inflammation of, 553 tumors of, 553

Orchectomy, 273

Orchitis, 271

definition, 271

etiology, 271, 272

prognosis, 272

symptoms, 272

treatment, 272

Osteoma of mouth, 103

Osteomalacia, 362

Otitis externa, 490, 491

diagnosis, 492

etiology, 491

prognosis, 492

symptoms, 491, 492

treatment, 492

interna, 493

media, 493

Otorrhea, 490, 491

Ovariectomy, 285, 286

Ovaries, diseases of, 283

examination, 283

inflammation of, 283

tumors of, 284 t

Oxyuridæ, 221

Oxyuris ambigua, 202, 222

compar, 221

vermicularis, 221

\section{$\mathbf{P}$}

Pannes, 528

Panophthalmitis, 548

definition, 548

diagnosis, $\mathbf{5 4 8}, 540$

prognosis, 549

symptoms, 548

treatment, 549

Papillitis, 546

definition, 546

prognosis, 546

P'apillomata of ear, 493

of eyelids, 506

of mouth, 102

of nasal passages, 28

of penis and prepuce, 269

of vulva and vagina, 314 
Paralysis, infectious bullar, 611 definition, 611 diagnosis, 612 etiology, 612 nat ural infection, 612 pathogenicity, 612 pathology, 612 prognosis, 613 symptoms, 612, 613 treatment, 613 of orbicularis nerve, 502 of peripheral nerves, 450 auditory, 453 diagnosis, 454 etiology, 453, 454 prognosis, 454 symptoms, 454 treatment, 454 brachial plexus, 455 etiology, 455 prognosis, 456 symptoms, 456 facial, 450 treatment, 456

diagnosis, 451 etiology, 450, 451 prognosis, 451 symptoms, 451 treatment, 451, 452 radial, 454

diagnosis, 455 etiology, 454, 455 prognosis, 455 symptoms, 455 treatment, 455 sciatic, 456 etiology, 456 prognosis, 456 symptoms, 456 treatment, 456 trigeminal, 452 diagnosis, 453 etiology, 452 prognosis, 453 syunptoms, 452,453 treatment, 453

of pharynx, 127

Paraphimosis, 267 definition, 267 etiology, 267 prognosis, 26\% syinptoms, 268 treatment, 268
Parasites, animal, in blood, 330

Dirofilaria immitis, 330 diagnosis, 332 etiology, 330, 331 occurrence, 330 pathogenesis, 331 pathology, 331 symptoms, 331,332 treatment, 332

Filaria inmitis, 330 Hæmostrongylus vasorum, 332 diagnosis, 333 symptoms, 332 treatment, 333

Hematozoön Lewisi, 332 Spiroptera sanguinolenta, 333

in kidney, 407

coccidia, 409

Coccidium tenellum, 409

Cysticercus cellulosæ, 409

Dioctophyme renale, 407 diagnosis, 409 life cycle, 408 prognosis, 409 symptoms, 408,409 treatment, 409

Eimeria a vium, 409

Eustrongylus gigas, 407 in bladder, 423, 424

Dioctophyme renale, 423 treatment, 424

in ear, 494

diagnosis, 494

prognosis, 494

symptoms, 494

treatment, 494

of eye, 553

in intestines, 193

of nasal passages, 25

in rectum, 221

Oxyuridx, 221

Oxyuris ambigua, 222

compar, 221

vermicularis, 221

symptoms, 222

treatment, 222

in scrotum and testes, 273

in stornach, 163

of birds, 163

Dispharagus 163 
Parasites in stomach of birds, Dis- Pericardium, diseases of, examipharagus spiralis, 163

Trichosomum contortum, 163 of cat, 163

Ollulanus tricuspis, 163

Tænia tæniæformis, 163 diagnosis, 164

of dog, 163

Gastrophilus intestinalis, 163

Spiroptera sanguinolenta, 163

prognosis, 164

of rabbits, 163

Strongylus strigosus, 163

syrnptoms, 164

treatment, 165

in trachea and bronchial tubes, 42

Parasitic nasal catarrh of birds, 22 of rabbits, 22 stomatitis, 100

Parenchymatous conjunctivitis, 514 goiter, 336

keratitis, 530

Parotitis, 115

definition, 115

diagnosis, 117

etiology, 115, 116

pathology, 116

prognosis, 117

symptoms, 116,117

treatment, 117

Passolurus ambigus, 202

Pasteurellosis avium, 584

Patella, fracture of, 375

Patellar dislocation, 382

Pelvis, fracture of, 373,374

Penis and prepuce, congenital malformations of, 265

diseases of, 263

examination, 263

tumors of, 268

wounds of, 263,264

Pericarditis, 73

definition, 73

diagnosis, 75

etiology, 73,74

necropsy, 74

prognosis, 75

symptoms, 75

treatment, 75,76

I'ericardium, diseases of, 69

examination, $69,70,71,72,73$

dropsy of, 76 nation, heart, 71

auscultation, $71,72,73$

endocardial bruits, 72 , 73

pericardial bruits, 73

palpation, 71

percussion, 71

pulse, 69,70

frequency, 69,70

quality, 70

rhythm, 70

Pericementitis, 108

Perineal hernia, 563

Periodontitis, 108

Periostitis, alveolar, 108

definition, 108

etiology, 108, 109

pathology, 109

symptoms, 109

treatment, 109

Peripheral nerves, compression of, 450

injuries of, 450

paralysis of, 450

pressure on, 450

Peritoneum, diseases of, 248 general remarks, 248,249

Peritonitis, 250

acute, 250

course, 253,254

definition, 250

diagnosis, 254

etiology, 250, 251

pathogenesis, 251

pathology, 251, 252

prognosis, 254

symptoms, 252, 253

treatment, 254, 255

chronic, 255

definition, 255

diagnosis, 257

etiology, 255, 256

pathology, 256

prognosis, 257

symptoms, 256

treatment, 257

Pestus avium, 489

Phalangeal dislocation, 381

Pharyngitis, 123

acute, 123

definition, 123 
Pharyngitis, acute, diagnosis, 124 etiology, 123

pathology, 124

prognosis, 124

symptoms, 121

treatment, 125

chronie, 125

definition, 125

etiology, 125

pathology, 125

prognosis, 125

symptoms, 125

treatment, 125,126

croupous, of birds, 127

definition, 127

etiology, 127

pathology, 127

prognosis, 128

symptoms, 128

treatinent, 128

Pharynx, diseases of, 123 examination, 123

foreign bodies in, 126

neoplasms of, 128

paralysis of, 127 .

Phimosis, 266

definition, 266

etiology, 266

symptoms, 266, 267

treatment, 267

Phlegmonous stonatitis, 99

Phthiriasis, 474

Pigmentary keratitis, 529

Piles, 216

Pilosebraceous cysts, 507

P'iroplasma bigeminun, 633

eanis, 633

eommune, 633

Piroplasmosis of clog, 633

definition, 633

diagnosis, 635, 636

etiology, 633, 634

pathology, 634

prognosis, 636

symptoms, 634, 635

treatinent, 6.36

I'lague, dog, 579

fowl, 589

rabbit, 20

I'leura, diseases of, 60

P'leurisy, 60

J'leuritis, 60

definition, 6io
Plcuritis, diagnosis, 64

etiology, 60, 61

pathology, 61, 62

prognosis, 64

syinptoms, 62,63

treatincnt, 64,65

Pneumonia, catarrhal, 53

chronic interstitial, 57

foreign body, 58 definition, 58

diagnosis, 59

etiology, 58, 59

pathology, 59

prognosis, 59

symptoms, 59

treatment, 59

mycotic, 40

definition, 40

diagnosis, 41

ctiology, 40

pathology, 40, 41

prevention, 41

prognosis, 41

symptoms, 41

treatment, 41

Pneumothorax, 66

definition, 66

diagnosis, 67

etiology, 66

pathology, 66

prognosis, 67

symptoms, 67

treatment, 67

Podagra, 354

Polyarthritis rhcumatica, 363

Polypoid fibromata of nasal passages, 28

growths of pharynx, 128

Poulardizing the female chicken, 289,290

I'reputial catarrh, 265

Prcssure on peripheral nerves, 450

Proctitis, 214

definition, 214

diagnosis, 215

ctiology, 214

pathology, 214, 215

prognosis, 215

symptoms, 215

treatment, 215

Prolapse of rectum, 217

definition, 217

diagnosis, 218 
Prolapse of rectum, etiology, 217, Puerperal septicemia, pathology,

\section{8}

prognosis, 218,219

symptoms, 218

treatment, $219,220,221$

of uterus, 300

of vagina, 311

diagnosis, 312

etiology, 311

prognosis, 312

symptoms, 312

treatment, 312,313

Prostate gland, diseases of, 279 examination, 279

hypertrophy of, 280

tumors of, 280

Prostatitis, 279

definition, 279

etiology, 279

prognosis, 280

symptoms, 279, 280

treatinent, 280

Pseudo-actinomyeosis of dogs, 623 definition, 623

diagnosis, 624

etiology, 623

occurrence, 623

pathogenieity, 623

pathology, 623

prognosis, 624

symptoms, 623, 624

treatment, 624

Pseudoleukemia, 327

definition, 327

diagnosis, 327

etiology, 327

oeeurrence, 327

pathology, 327

prognosis, 327

symptoms, 327

treatment, 327

Pseudorabies, 611

Pterygium, 519

definition, 519

diagnosis, 519

prognosis, 519

symptoms, 519

treatment, 519

Ptosis, 501

Puerperal septicemia, 298 definition, 298, 299

diagnosis, 299, 300

etiology, 299
299

prognosis, 300

symptoms, 299

treatment, 300

Pulex avium of birds, 472

gonivcephalus of rabbits, 472

irritans of man, 472

Pulmonary edema, 52

definition, 52

diagnosis, 53

etiology, 52

pathology, 52

prognosis, 53

symptoms, 52

treatinent, 53

Purpura hemorrhagiea of dogs, 583

Purulent eonjunetivitis, 512

Pyelitis, 398

definition, 398

diagnosis, 399

etiology, 398, 399

pathology, 399

prognosis, 399

symptoms, 399

treatment, 400

Pyometra, 296

Pyosalpinx, 291

\section{$\mathbf{R}$}

RABBIT distemper, 20

influenza, 20

plague, 20

Rabies, 603

eourse, 609, 610

definition, 603

diagnosis, 608 differential, 610,611

confused with eanine distemper (nervous form), 610

diseases of brain, 610

celampsia, 611

epilepsy, 611

foreign bodies, 610

infectious bulbar paralysis, 611

parasites in intestinal tract, 610

trigeminal or facial paralysis, 610,611 
Rabies, etiolugy, 603

natural infection, 604

occurrence, 603

pathogenesis, 604,605

pathogenicity, 603, 604

pasthology, 605

preventive inoculation, 611

prognosis, 609,610

syilptoms, 605, 606, 607

treatment, 611

Rachitis, 359

course, 361

definition, 359

etiology, 359,360

occurrence, 359

pathology, 360

prognosis, 361

symptoms, 360, 361

treatment, 361,362

Radial nerve, paralysis of, 454

Radio-ulnar-carpal dislocation, 380

Raclius and ulna, fracture of, 372, 373

Ranula, 103

Rectum and anus, diseascs of, 212 cxamination, 212

neoplasins in, 222

occlusion of, 212,213

parasitcs in, 221

prolapse of, 217

Red itch, $4 \triangleright 2$

mange, 475

Reflcx epilcpsy, 459

Renal abscess, 397

pelvis, inflammation of, 398

Reproductive organs, discases of, 263

Retcntio urinæ vesicalis, 414

Retcntion cysts in mouth, 103 of scrotum and testes, 273

of urine in bladder, 414

definition, 414

diagnosis, 415

ctiology, 414

prognosis, 415

symptoms, 414, 415

treatinent, 415

Retina and choroid, diseases of, 545

pathologieal clanges, 545

ancmia, 5t:5

istrophy, 545

detachinent of retina, 545

(rdema, 545
Retina and choroid, pathological changes, hyperemia, 545 inflammation (retinitis), 545

Retrobulbar neuritis, 546, 547

Rheumatism, 363

articular, 363

course, 364

definition, 363

diagnosis, 364

etiology, 363

pathology, 363

prognosis, 364,365

symptoms, 364

treatment, 365

muscular, 365

course, 367

definition, 365

etiology, 365,366

pathology, 366

prognosis, 367

symptoms, 366,367

treatment, 367

Rhinitis, 19

chronic, 23

coccidiosa, 22

Rhipicephalus sanguineus, 633

Ribs, fracture of, 371

Rickets, 359

Ringworm, 482 honeycomb, 484

Roundworms, 201 diagnosis, 204

in intestine of birds, 202, 203

Hcterakis compressa, 203 crassa, 203

differens, 203

r dispar, 203

inflexa, 203

lineata, 203

muculosa, 203

papillosa, 202, 203

perspicillum, 203

vesicularis, 202, 203

of cat, 202

Belascaris mystax, 202

of $\mathrm{dog}, 202$.

Belascaris marginata, 202

Echinorhynchus canis, 202

Toxascaris limbata, 202 marginata, 202

of rabbit, 202 
Roundworms in intestine of rabbit, Oxyuris ambigua, 202

Passolurus ambigus, 202 pathology, 293, 204 prevention, 205, 206 prognosis, 205 symptoms, 204 treatment, 205

Roup, 593

Ruptura hepatis, 246

Rupture of bladder, 412 prognosis, 413

symptonis, 412,413 treatment, 413

of heart, 92 etiology, 92 symptoms, 92 treatment, 92 of liver, 246 diagnosis, 246 ctiology, 246 pathology, 246 prognosis, 246, 247 symptoms, 246 treatment, 247 of uterus, 303 prognosis, 303 symptoms, 303 treatment, 303 of vagina, 313

\section{S}

Salivary fistula, 118 glands, discases of, 115 cxamination, 115

Salpingitis, 291 definition, 291 ctiology, 291 symptoms, 291 treatment, 291

Sarcomata of bladder, 423 of cyelids, 509 of mammary glands, 318 of mouth, 105 of penis and prepuce, 269 of scrotum and testes, 273 of vulva and vagina, 314

Sarcoptes mite, 476 of cat, 477

Notodres cati, var. cati, 477 Sarcoptes minor, var. cati, 477
Sarcoptes mite of dog, 476,477 Sarcoptes scabiei, var. canis, 478,479

of ferret, 479,480

Sarcoptes scabiei, var. hydrochaeri, 477,478

of fowl, 478

Cncmidocoptes mutans, var. gallinæ, 478

Sarcoptes mutans, 478

of rabbit, 477

- Notoedres cati, var. cuniculi, 477

Sarcoptes minor, var. cuniculi, 477

Scabies, 475

Scapula, fracture of, 371

Scapulohumeral dislocation, 379

sciatic nerve, paralysis of, 456

scorbutus, 328

Scrotal hernia, 561

Scrotum, diseases of, 271

parasites in, 273

tumors of, 272

wounds and injuries of, 271

Seurvy, 328

definition, 328

diagnosis, 329

etiology, 328

occurrence, 328

pathology, 328,329

prognosis, 329,330

symptoms, 329

treatment, 330

Sebaceous tumors in birds, 508 of ear, 494

Secondary epilepsy, 457

septiccmia, hemorrhagic, of cats, 600

puerperal, 298

Simple catarrh of stomach, 142

Skin diseases, non-parasitic, 463

examination, 463,464 microscopic, 463, 464

parasitic, 472 vegetable 482

Soft crop, 137

soor, 100

Sore mouth, 96

Spasm of orbicularis nerve, 503

Spinal cord, compression of, 448 concussion of, 445

diseases of, 443 
Spinal eord, diseases of, examina- Stomatitis, gangrenous, symptoms, tion, 443

general eonsiderations, 443 functions of eord, 443

injuries of, 445

Spirillosis of ehiekens, 625

Spiroehætosis avium, 625

of fowls, 625

definition, 625

diagnosis, 626

etiology, 625

natural infeetion, 625,626

oceurrenee, 625

pathogenesis, 625

pathology, 626

prognosis, 626

symptoms, 626

treatment, 626,627

spiroptera sanguinolenta, 333

in stomach of $\log , 16.3$

Sprains and injuries to artieulations, 377

Staplyyloma, 537

Stenosis, 78

Stomaeace, 96

Stomach, ehronic eatarrh of, 146

dilatation of, 152

aeute, 152

ehronie, 154

diseases of, 142 examination, 142

foreign bodies in, 148

neoplasnis in, 16.5

parasites in, 16.3

simple eatarrh of, 142

ulceration of, 157

Stomatitis, 9.1

eatarrhal, 94

definition, 94

diagnosis, 95

etiology, 94, 95

pathology, 95

prognosis, 96

symptoms, 9j

treatinent, 96

fetid, 96

gangrenous, 97

definition, 97

diagnosis, 98

etiology, 97,98

pathology, is

prevention, 99

prognosis, 98, 99

98

treatment, 99

parasitie, 100

definition, 100

diagnosis, 101

etiology, 100, 101

pathology, 101

prognosis, 101

symptoms, 101

treatinent, 102

phlegmonous, 99

definition, 99

diagnosis, 100

etiology, 99

pathology, 99

prognosis, 100

syniptoms, 100

treatment, 100

uleerative, 96

definition, 96

diagnosis, 97

etiology, 96

pathology, 96

prognosis, 97

syinptoms, 96,97

treatment, 97

Strabismus, 552

definition, 552

diagnosis, 552

etiology, 552

treatnent, 552

Streptotriehosis eanum, 623

Strieture of esophagus, 135

definition, 135

etiology, 136

prognosis, 136

symptoms, 136

treatment, 136

of urethra, 426

definition, 426

diagnosis, 427

etiology, 427

prognosis, 427

symptoms, 427

treatment, 427

Strongylidax, 206

diagnosis, 208

in intestine of eat, 207

Ankylostomum trigonoeephalum, 207

of $\operatorname{dog}, 207$

Ankylostomum eanina, 207 
Strongylidæ in intestine of dog, Trenia serrata, 195, 196

Ankylostomun stenocephalum, 207

Dochmius trigonocephalus, 207

Uncinaria stenocephala, 207 trigonocephala, 207

of rabbit, 207

Strongyloides longus, 207

Strongylus strigosus, 207

pathology, 208

prognosis, 209

symptoms, 208

treatment, 209

Strongyloides longus, 207

Strongylus strigosus, 207

in stomach of rabbits, 163

Struma, 336

Submaxillary and sublingualglands, $11 \mathrm{~s}$

Superficial keratitis, 529

Suppuration of anal glands, 223,224 diagnosis, 224

treatment, 224

Suppurative hepatitis, 236

keratitis, 532

forms, 532

abscess of cornea, 534

keratitis from lagoplithalmos, 535

neuroparalytica, 535

ulceration of cornea, 532

Symblepharon, 505

Syngamosis, 42

Synovial membranc, inflammation of, 38.3

Synovitis, 383

\section{$\mathrm{T}$}

TABLE of equivalents in weights and measures, 6.37

'Tænia, conurus, 196

crassicollis, 197

cucumerina, 19;

erhinococcus, 197

elliptica, 197

hydatigena, 196

marginata, 196

pisifornis, 195

Iscudo-elliptica, 197

serialis, 196 tanixformis in stomach of cat, 193

Tæenix, 193

in intestine of birds, 198

Choanotania infundibuliformis, 198

Davainea cesticillus, 198 crassula, 198 echinobothrida, 198 proglottina, 198 tetragona, 198

Dicranotænia sphenoides, 198

Tænia cantaniani, 198 of cats, 197

Bothriocephalus felis, 197

Tæenia crassicollis, 197 elliptica, 197 pseudo-elliptica, 197 tæniæformis, 197

of dogs, 195

Dipylidium caninum, 195

Echinococcus granulosus, 197

Multiceps multiceps, 196 serialis, 196,197

Tænia cœnurus, 196

cucumerina, 195

echinococcus, 197

hydatigena, 196

marginata, 196

pisiformis, 195, 196

serialis, 196,197 serrata, 195, 196

of rabbits, 197, 198

Cittotænia denticulata, 197

Moniezia denticulata, 197

life history, 194, 195

Treniasis, 193, 194, 195

in birds, 198

in cats, 197

diagnosis, 200

in dogs, 195

pathology, 198, 199

prognosis, 200

in rabbits, 197,198

symptoms, 199,200

treatment, 200, 201

Tartar, incrustations of, 108

Teeth, caries of, 109

diseases of, 107

cxamination, 107 
Teeth, fractures of, 108 malformations of, 107

'Temporomaxillary dislocation, 378

'Testes, diseases of, 271 parasites in, 273

tumors of, 272

wounds and injuries of, 271

Tetanus, 613

$$
\begin{aligned}
& \text { definition, } 613,614 \\
& \text { diagnosis, } 614 \\
& \text { etiology, } 614 \\
& \text { natural infection, } 614 \\
& \text { occurrence, } 614 \\
& \text { pathology, } 614 \\
& \text { prognosis, } 615 \\
& \text { symptoms, } 614,615 \\
& \text { treatment, } 615 \text {, }
\end{aligned}
$$

'l'lurush, 100

'Thyroid glands, congestion of, 334, 335

diseases of, 334 examination, 334 general considerations, 334

Thyroiditis, acute, 335

(lefinition, $33 \dot{j}$

etiology, 335

prognosis, 335

symntoms, 335

treatment, 335,336

Tibia and fibula, fracture of, 375

'Tibiotarsal dislocation, 383

'T'ongue, diseases of, 111 exanination, 111

gangrene of, 112

Tonsillitis and lymphadenitis, 120 definition, 120

etiology, 120, 121

pathology, 121

prognosis, 121

symptoms, 121

treatment, 121, 122

Tonsils, diseases of, 120

examination, 120

Torsion of bladder, 420

of cornua uteri, 302

symptoms, 302

treatment, 302

Toxascaris limbata, 202

marginata, 202

Trachea and bronchial tubes, aninial parasites in, 42 definition, 42 diaguosis, 43
I Trachea and bronchial tubes, animal parasites in, etiology, 42

prevention, 43

prognosis, 43

symptoms, 42,43

diseases of, 34

treatment, 43

examination, 34

Tracheitis and bronchitis, 34

acute, 34

definition, 34

diagnosis, 37

etiology, 34, 35, 36

pathology, 36

prognosis, 37

symptoms, 36,37

treatment, 37,38

chronic, 44

definition, 44

diagnosis, 45

etiology, 44

pathology, 44

prognosis, 45

symptoms, 44,45

treatment, 45,46

Traumatic lesions of conjunctiva, 519

foreign bodies, 519,520

symptoms, 520

treatment, 520

wounds, 520

treatment, 520,521

Trichiasis, 503

definition, 503, 504

prognosis, 504

symptoms, 504

treatment, 504

Trichinellid:e, 209, 210

diagnosis, 211

in intestine of birds, 210, 211

Trichosomum annulatum, 210

brevicolle, 211

collare, 210

retusum, 210

tennissimum, 211

of $\log , 210$

Trichuris depressiusculus, 210

of rabbit, 210

Trichurus unguiculatus, 210 pathology, 211 
Trichinellidæ, prognosis, 211

symptoms, 211

treatment, 211

Trichodectes latus, 474

subrostratus, 474

Trichosomum annulatum, 210

brevicolle, 211

collare, 210

contortum, 163

in stomach of birds, 163

retusum, 210

tennissimum, 211

Trichurus depressiusculus, 210 unguiculatus, 210

Trigeminal nerve, paralysis of, 451

Tuberculomata of eyelids, 511

Tuberculosis, avian, 616

avium, 616

of birds, 616

definition, 616

diagnosis, 619

etiology, 616

natural infection, 617

occurrence, 616

pathogenesis, 616,617

pathology, 617, 618

prevention, 620

prognosis, 620

symptoms, 618,619

treatment, 620

of dogs and cats, 620

diagnosis, 622

form, 620

occurrence, 620

pathogenesis, 621

pathology, 621

prognosis, 622

symptoms, 621,622

treatment, 622

Tumors of bladder, 423

prognosis, 423

treatment, 423

varieties, 423

carcinomata, 423

fibroinata, 423

sarcomata, 423

of brain, 441,442

symptoms, 442

on conjunctiva, 522

treatment, 522

of cornea, 538

treatment, 538

of ear, 493
Tumors of eyelids, 506

varieties, 506

chalazion, 507

treatment, 507

cysts, meiboınian, 507

pilosebaceous, 507

enlargement of glands of Moll, 508 treatınent, 508

granuloinas, 508 treatment, 508

lipoma in birds, 508 treatment, 508

malignant neoplasms, 508, 509

diagnosis, 509

treatment, 509

varieties, 509

carcinomata, 509

epitheliomata, 509

sarcomata, 509

tuberculomata, 509

Meibomian cysts, 507

papillomata, $\tilde{506}$

treatment, 506, 507

pilosebaceous cysts, 507

treatment, 508

sebaceous, in birds, 508

warts, 506

of iris, 541

in kidney, 407

treatment, 407

in nasal passages, 29

benign, 28

malignant, 29

of mammary glands, 317 benign, 317

fibromata, 317 prognosis, 318 symptoms, 318 treatinent, 318

lipoma, 318

prognosis, 318

symptoms, 318

treatment, 318

malignant, 318

carcinomata, 318

prognosis, 318

symptoms, 318

treatment, 318

sarcomata, 318

diagnosis, 318

symptoms, 318 
Tumors of mammary glands, malignant, sarcomata, treatment, 318 on membrana nictitans, 523 of orbit, 55.3 of ovaries, 284

varieties, 284

cysts, 281

diagnosis, 285 prognosis, 285 treatment, 285

other tumor formations, 285 of penis and prepuee, 268

varieties, 269,270

carcinomata, 269

epitheliomata, 269

papillomata, 269

symptons, 269

treatment, 269

sarcomata, 269

diagnosis, 269

symptoms, 269

treatment, 269

venereal granulomata, 269

diagnosis, 270

prognosis, 270

symptoms, 269,270

treatment, 270

of prostate gland, 280

diagnosis, 281

prognosis, 281

symptoms, 281

treatment, 281, 282

of scrotum and testes, 272

carcinomata, 273

fibromata, 272,273

retention cysts, 273

sareomata, 273

treatment, 273

of uterine tubes, 291

of uterus, 303

fibromata, 303, 304

diagnosis, 304

prognosis, 304

symptoms, 304

treatment, 304

hydrometra, 304

definition, 304

diagnosis, 305

etiology, 304, 305

progmosis, 305

symptoms, 305

treatment, 305
Tumors of uterus, myomata, 304 of vulva and vagina, 313

fibromata, 313 diagnosis, 313 prognosis, 313 treatment, 314 papillomata, 314 treatment, 314 sareomata, 314 treatment, 314 venereal granulomata, 314 treatment, 314

Typhoid, fowl, 584

Typhus, eanine, 579

of dogs, 579

course, 582

definition, 579

diagnosis, 581, 582

etiology, 579

oecurrenee, 579

pathology, 579, 580

prognosis, 582

symptoms, 580,581

treatment, 582,583

\section{U}

Cliceration of eoneha, 488 etiology, 488

prognosis, 489

symptoms, 489

treatment, 489

of eonjunetiva, 521

treatment, 521

of eornea, 532

of stomach, 157

definition, 157, 158

diagnosis, 159

etiology, 158

pathology, 158

prognosis, 159

symptoms, 158, 159

treatment, 159

Uleerative stomatitis, 96

Ulcus ventriculi, 157

Umbilical hernia, 557

Uncinaria stenoeephala, 207 trigonoeephala, 207

Uremia, 400

definition, 400

diagnosis, 401

etiology, 400 
Uremia, prognosis, 401

symptoms, 400

treatment, 401,402

Uretlira, calculi in, 427

congenital malformations of, 425 diseases of, 425 examination, 425

inflammation of, 429

occlusion of, 425

stricture of, 426

wounds of, 426

Irethritis, 429

symptoms, 429

treatment, 429

Urine, incontinence of, in bladder, 416

retention of, in bladder, 414

Urocystitis, 416

Uterine tubes, cysts of, 291 cliseases of, 291 examination, 291

tumors of, 291

Uterus, diseases of, 292 examination, 292,293

eversion of, 300

inversion of, 300

prolapse of, 300

rupture of, 303

tumors of, 303

\section{V}

$V_{\text {AGINA, diseases of, } 309}$ examination, 309

malformations, congenital, 309 prolapse of, 311 rupture of, 313 tumors of, 313 and vulva, 309

congenital malformations of, 309

Vaginitis and vulvitis, 309

definition, 309

diagnosis, 311

etiology, 310

prognosis, 311

symutons, 310

treatment, 311

Valvular insufficiency and stenosis, 78

definition, 78,79

insufficiency, 78,79
Valvular insufflciency and stenosis, definition, insuffleiency, imperfect closing of valves, 78,79

stenosis or contraction of openings, 79

diagnosis, 81

etiology, 79

necropsy, 80

prognosis, 81

symptoms, 80,81

treatment, 81,82

Vascular goiter, 341

keratitis, 528

Vegetable parasitic clisease of skin, 482

Venereal granulomata of penis and prepuce, 269

of vulva and vagina, 314

Ventral hernia, 558

Vertebræ, fracture of, 370

Vertebral dislocation, 379

Vertigo, 455

definition, 455

diagnosis, 456

etiology, 455

prognosis, 456

symptoms, 455,456

treatinent, 456

Volvulus, $185^{\circ}$.

definition, 185

diagnosis, 185, 186

etiology, 185

pathology, 185

prognosis, 186

symptoms, 185

treatment, 186

Vulva, diseases of, 309

examination, 309

tumors of, 313

Vulvitis, 309

\section{W}

WARTS on eyelids, 506

Whipworm, 209, 210

White comb, 484 diarrhea, 629

Wounds of articulations, 376

of bladder, 412

on conjunctiva, 520

of cornea, 535 
Wounds of ear, $48 \pi, 488$ prognosis, $4 \$ \mathrm{~S}$ symptoms, $4 \mathrm{SS}$ treatment, 488

of evelids, 497,498

and injuries of mammary gland 315

treatment, 315

of testes and scrotum, 271

prognosis, 271

symptoms, 271

treatment, 271
Wounds of intestines, 188

definition, 188

etiology, 188

symptoms, 188, 189

treatment, $\mathbf{1 8 9}$

of membrana nictitans, 523

of penis and prepuce, 263, 264 prognosis, 264

symptoms, 264

treatment, 264, 265

of urethra, 426

treatmert, 426 



\section{UNIVERSITY OF CALIFORNIA LIBRARY}

\section{Los Angeles}

This book is DUE on the last date stamped below.

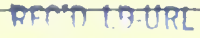 \\ JAN 131984}

PSD $2343 \quad 9 / 77$ 

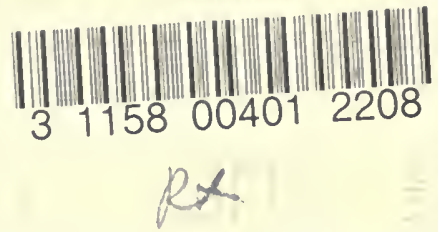

A 0011077450 
